\title{
Sabine Könninger
}

Genealogie
der Ethikpoiftik

Nationale Ethikkomitees

als neue Regierungstechnologie

Das Beispiel Frankreichs

[transcript] s c i encestudies 
Sabine Könninger

Genealogie der Ethikpolitik 
Sabine Könninger (Dr. phil.) lebt und arbeitet als Politikwissenschaftlerin in Berlin. Ihre Forschungsschwerpunkte sind Ethikpolitiken sowie Biomedizin- und Nanotechnologiepolitiken, Wissenschafts- und Medizingeschichte. 
SABINE KÖNNINGER

\section{Genealogie der Ethikpolitik}

Nationale Ethikkomitees als neue Regierungstechnologie.

Das Beispiel Frankreichs

[transcript $]$ 


\section{(c) (1) ()}

Dieses Werk ist lizenziert unter der Creative Commons Attribution-Share Alike 4.0 Lizenz (BY-SA). Diese Lizenz erlaubt unter Voraussetzung der Namensnennung des Urhebers die Bearbeitung, Vervielfältigung und Verbreitung des Materials in jedem Format oder Medium für beliebige Zwecke, auch kommerziell, sofern der neu entstandene Text unter derselben Lizenz wie das Original verbreitet werden. (Lizenz-Text:

https://creativecommons.org/licenses/by-sa/4.o/deed.de)

Erschienen im transcript Verlag 2016

\section{(c) Sabine Könninger}

\section{Bibliografische Information der Deutschen Nationalbibliothek}

Die Deutsche Nationalbibliothek verzeichnet diese Publikation in der Deutschen Nationalbibliografie; detaillierte bibliografische Daten sind im Internet über http://dnb.d-nb.de abrufbar.

Umschlagkonzept: Kordula Röckenhaus, Bielefeld

Druck: Majuskel Medienproduktion $\mathrm{GmbH}$, Wetzlar

Print-ISBN 978-3-8376-3286-6

PDF-ISBN 978-3-8394-3286-0

Gedruckt auf alterungsbeständigem Papier mit chlorfrei gebleichtem Zellstoff. Besuchen Sie uns im Internet: http://www.transcript-verlag.de Bitte fordern Sie unser Gesamtverzeichnis und andere Broschüren an unter: info@transcript-verlag.de 


\section{Inhalt}

Vorwort | 11

\section{EINLEITUNG | 13}

1 Zur Entstehung der Untersuchung | 15

2 Von gouvernementalen Ethikregimen und vom magistère bioéthique | 16

3 Forschungsfragen $\mid 19$

$4 \quad$ Fallstudie und Untersuchungsmaterial $\mid 22$

5 Aufbau der Studie 123

I Stand der Forschung | 29

1 Untersuchungen zu Ethik, Biomedizin und Biotechnologie I 30

2 Untersuchungen zu Ethik und Nanotechnologie I 35

3 Zur kritischen Diskussion normativer Tendenzen in den Science and Technology Studies 139

\section{ANALYTISCH-METHODISCHER RAHMEN:}

DAS KONZEPT DER GOUVERNEMENTALITÄT UND DIE GENEALOGIE । 43

1 Die Gouvernementalitätsstudien $\mid 43$

1.1 Das Konzept der Gouvernementalität |45

1.2 Die Perspektive der Gouvernementalität 148

2 Der Abstieg: die genealogische Herangehensweise 150

2.1 Problematisierungen als »struggle over meaning « $\mid 51$

2.2 Ethik als Frame I 53

3 Der Aufstieg: Ethik als neoliberale Gouvernementalität? I 57

3.1 Vom governing at a distance und dem reflexive government I 59

3.2 Die delegierte Biopolitik I 60 


\section{Die Sprache der EthIK ALS ANTWort AUf Problematisierungen MEdizinISCHER AUTONOMIE I 63}

1 Das Ethikkomitee des Institut National de la Santé et de la Recherche Médicale 164

1.1 Zur Entstehung des Institut National de la Santé et de la Recherche Médicale: Vichy-Regime und nationales Hygieneinstitut 165

1.2 Von Hygiene zu biomedizinischer Forschung I 81

1.3 Die Verwissenschaftlichung der Medizin 182

1.4 Forschungspolitik in der Nachkriegszeit - moderne Technokratie I 84

1.5 Der Institut National de la Santé et de la Recherche Médicale I 87

2 Wofür war das Ethikkomitee des Institut National de la Santé et de la Recherche Médicale die Lösung? I 87

2.1 Ethik als vertrauensbildende Maßnahme? Von »Experimentieranstalten « und Medikamentenskandalen 190

2.2 Medizinische Ethik gegen Humanexperimente 195

2.3 Die »moralische Bildung des Arztes « zum Schutz wissenschaftlicher Objektivität 198

2.4 Medizinische Moral und Nation I 103

2.5 Medizinische Ethik und individuelles Gewissen । 105

2.6 Ethik als »shifted responsibilities « und als »management of living «? | 108

2.7 Mai 1968 und Medizinkritik । 111

2.8 Der Abtreibungskonflikt - Politische Selbstbestimmung versus Moral | 114

2.9 Ein Weniger an medizinischer Macht? I 118

3 Zusammenfassung $\mid 120$

IV Die Sprache der Ethik als ANTWORT AUF Problematisierungen (Gen)WISSEnschaftLicher AUTONOMIE | 123

1 Die Ethikkommission der Délégation Générale à la Recherche Scientifique et Technique | 123

2 Der internationale genwissenschaftliche Diskurs der 1970er Jahre Austausch in einem »exklusiven Club « | 127

2.1 Der Hintergrund der US-amerikanischen Kontroverse um Gentechnologie: Öffentliche Kontextualisierung wissenschaftlichtechnologischer Entwicklungen | 127 
2.2 Die new critical science movement: Wissenschaftliche Verantwortung als politische Perspektive? I 130

2.3 Die genwissenschaftliche Community und ihre Zwickmühle | 135

2.4 Der Asilomar-Prozess (1973-1975) । 136

2.5 Ethik im Asilomar-Prozess I 142

3 Ethikkommission und Risikoabschätzung: Die Auswirkungen des AsilomarProzesses in Frankreich I 150

3.1 Technokratiekritik und Wissenschaftsskepsis in der französischen Öffentlichkeit I 151

3.2 Die Konflikte um Atomenergie | 152

3.3 Die Kontroversen um Gentechnologie im Kontext des Asilomar-Prozesses । 159

4 Zusammenfassung | 167

\section{Die Sprache der EthiK als Ursache für} Problematisierungen BIOLOGISCHER UND LEBENSWISSENSCHAFTLICHER AUTONOMIE । 169

1 Der Mouvement Universel de la Responsabilité Scientifique | 170

1.1 Die internationale Tagung »Biologie und die zukünftige Entwicklung des Menschen « I 173

1.2 Ein »Ort des Zusammenflusses«? । 175

1.3 »Moral and ethical aspects « - »partly emotional « I 176

1.4 Wissenschaftliche Verantwortung $=$ Wohltaten und Risiken I 180

1.5 Mehr »shifted responsibilities« und weniger Moral | 181

$1.6 »$ Responsibility to and for science «? | 183

2 Der Mouvement Universel de la Responsabilité Scientifique:

Lösung wofür? I 186

2.1 Technokratiekritik: »Die Wissenschaft de-moralisieren« | 188

2.2 Vertrauensverlust der Öffentlichkeit - eine »natural evolution«? | 190

3 Reflexion und Dialog als Antwort auf ethische Problematisierungen und zum Schutz der Forschungsfreiheit I 195

3.1 Der Bericht »Lebenswissenschaften und Gesellschaft« | 195

3.2 Die Lebenswissenschaften und die Befreiung der Frau । 197

3.3 Die Lebenswissenschaften und ihre »humanitäre Rolle« | 198

3.4 Die Lebenswissenschaften und die »Gunst der Öffentlichkeit« | 199

4 Zusammenfassung | 201 


\section{Die Sprache der Ethik als Antwort auf PROBLEMATISIERUNGEN LEBENSWISSENSCHAFTLICHER AUTONOMIE I 203}

1 Die Forschungspolitik zu Beginn der 1980er Jahre »ein absolut anderer Esprit« | 204

1.1 »Der Weg aus der Krise « | 205

1.2 »Bewegung von unten«: das nationale Kolloquium zu Forschung und Technologie I 206

1.3 Ethik - vom gesellschaftlichen Problem zur Aufgabe der Bürgerin und des Bürgers 1209

1.4 Das Verschwinden der wissenschaftskritischen Debatte I 212

1.5 Dialog und Innovation als Krisenlösung - eine Form des advanced liberal government? I 214

2 Der Comité Consultatif National d'Éthique 1216

2.1 Aufgaben 1216

2.2 Zusammensetzung 1217

2.3 Arbeitsweise 1218

2.4 Öffentlichkeitsarbeit 1219

3 Wofür war der Comité Consultatif National d'Éthique die Lösung? I 220

3.1 Das erste Reagenzglasbaby - Eine Bedrohung der sozialen Ordnung? | 221

3.2 Ethik als Lösung für die »schwindelerregenden Fortschritte« | 227

4 Der Comité Consultatif National d'Éthique und sein Verständnis von ethischen und nicht ethischen Themen 1229

4.1 Zur Aufteilung von facts und values 1229

4.2 Von medizinischer Praxis und medizinischer Forschung eine thematische Aufteilung 1235

5 Der Comité Consultatif National d'Éthique und die Bedeutung von Ethik I 238

5.1 Die Moral der Ethik I 238

5.2 Definitionslose Ethik 1240

5.3 Verantwortung für alle | 241

5.4 Das Referenzwissen für die »wirklichen Probleme« $\mid 242$

5.5 Reversible Ethik I 243

5.6 Die Unabhängigkeit der Ethik । 244

5.7 Moralisierung oder Ethisierung? 1247

6 »Fragen ethischer Ordnung auf gesellschaftlichem Niveau « I 248

6.1 Die Journées annuelles d'éthique 1250 
7 Ethik im Wandel? $\mid 255$

8 Zusammenfassung $\mathbf{2 5 7}$

\section{Die Sprache der Ethik als ANTWORT aUf Problematisierungen NANOTECHNOLOGISCHER AUTONOMIE I 259}

1 Das Problem mit der Definition 1260

2 Forschungspolitischer Hintergrund:

Die Schlüsseltechnologie des 21. Jahrhunderts | 261

3 Der Nanotechnologiediskurs: Ethik und $\gg$ The Coming Era of Nanotechnology $\mid 263$

3.1 Nano-boundary work: von unrealistischen Fiktionen und realistischen Entwicklungen $\mid 265$

3.2 Die »standard story« über die Nanotechnologie | 267

4 Der Nanotechnologiediskurs in Frankreich: von Risiko-Problemen und ethischen Implikationen 1268

4.1 »Nekrotechnologie« | 271

4.2 Von $»[$ E $]$ thischen $<$ Zierereien $\ll \mid 273$

$4.3 »$ Ethics lags behind «? । 274

5 Die Nanoethik des nationalen Ethikkomitees | 277

5.1 » Training in ethics « 1277

5.2 Mehr Forschung und mehr Ethik! I 279

5.3 »Echte« ethische Ziele | 280

5.4 Die Bedeutung von Nanoethik - Alles gesund? | 281

6 Ethische Ausblicke 1283

7 Zusammenfassung | 285

VIII RESÜMEE | 287

\section{ABKÜRZUNGEN | 295}

LITERATUR । 297 



\section{VORWORT}

Die vorliegende Studie ist eine überarbeitete Fassung meiner Dissertationsschrift, die im Januar 2015 von der Philosophischen Fakultät der Leibniz Universität Hannover (LUH) angenommen wurde.

Ohne vielfältige Formen der Unterstützung kann das Erstellen und Publizieren einer wissenschaftlichen Arbeit kaum gelingen. Ich danke meiner Doktormutter Prof. Dr. Kathrin Braun für ihre Betreuung. Sie hat mir mit ihren fachlichen Anregungen, ihrer konstruktiven Kritik und ihren wertvollen Ratschlägen neue Denkräume eröffnet und es gleichzeitig geschafft, dass ich ein Licht am Ende des Tunnels sehe. Mein Promotionsvorhaben entstand aus einem Forschungsprojekt an der LUH unter ihrer Leitung. Es bot mir zu Beginn einen inspirierenden und fördernden Rahmen. Auch die fruchtbaren Diskussionen, das Auseinandernehmen von Gedankensträngen und das Querdenken im ehemaligen Doktorandinnen- und Doktorandenkolloquium »Transformation von Biopolitik und Körperverständnis« an der LUH haben mein Projekt auf seinen Weg gebracht. Angeleitet wurde es von Prof. Dr. Kathrin Braun und Prof. Dr. Barbara Duden. Bei ihnen wie auch bei Dr. Svea Luise Herrmann, Dr. Isabella Jordan, Dr. Helen Kohlen, Dr. Marion Schumann, Torben Klußmann und dem Berliner >Ableger $<$ Dr. Susanne Schultz bedanke ich mich. Zudem gilt mein Dank Janine Doerry, Ralf Steckert, Stefan Warnken und Jana Otto aus dem historischen und sozialwissenschaftlichen, selbstorganisierten Kolloquium in Hannover für die bereichernden, kritischen Diskussionen und für die Solidarität. Elisabetta Romeo-Vareille und Dr. Bernard Schmid danke ich für die praktische Hilfe in Paris, die zum Gelingen meiner Forschungs- und Interviewreisen beigetragen hat. Ich danke Daniel Scherf, dessen Unterstützungen im Alltag und dessen linguistische Expertise für meine Arbeit sehr wichtig waren. Bedanken möchte ich mich herzlich bei Dr. Anke Neuber für die inhaltlichen Korrekturen und die Tipps sowie bei Jörg Meyer für den stilistischen Feinschliff. Monika Spitzer danke ich nicht allein dafür, dass sie die Dissertation in ihre layoutete Form brachte, sondern auch dafür, dass sie während der Höhen und Tiefen der Promotionsphase für mich da war. Auch meinen Eltern möchte ich herzlich danken. Ohne sie wäre diese Studie nie entstanden. Sie haben immer zu mir gehalten. Leider konnte ich mich nicht mehr gemeinsam mit meinem Vater über das Ende dieser Arbeit und die Veröffentlichung freuen. Allen, die mich in dieser Zeit begleitet haben, danke ich dafür, dass sie mir Mut zugesprochen haben, offene Ohren hatten, den Rücken frei gehalten und den selbigen immer wieder gestärkt haben.

Nicht zuletzt gilt mein Dank dem Ethik-Institut an der Philosophisch-Theologischen Hochschule Vallendar, das diese Publikation finanziell durch einen Druckkostenzuschuss gefördert hat. 



\section{Einleitung}

Seit den 1980er Jahren wird insbesondere in westlichen Gesellschaften eine Fülle nationaler Ethikinstitutionen staatlich initiiert, sei es in Form von Ethikkomitees, Ethikkommissionen oder Ethikräten. Sie werden von der Politik eingerichtet, um über einen ethisch vertretbaren Umgang mit Entwicklungen in den Lebenswissenschaften zu reflektieren. Die Ergebnisse werden in Form von Empfehlungen, öffentlichen Tagungen oder Dialogveranstaltungen Politik und Öffentlichkeit zur Verfügung gestellt. Aber nicht nur Ethikinstitutionen werden eingerichtet, sondern ebenso Meinungsumfragen durchgeführt, Bürgerinnen- und Bürgerkonferenzen oder öffentliche Debatten zu ethischen Aspekten und Implikationen im Bereich der Lebenswissenschaften abgehalten. Es ist eine Ausweitung von Ethikpolitiken, den ethischen Steuerungsmodi auf Ebene der Politik zu beobachten und damit einhergehend eine gesteigerte Einbeziehung der Öffentlichkeit. Über diese Entwicklung im Kontext der Lebenswissenschaften hinaus werden zunehmend weitere Themen als ethische angesehen. So wird in Deutschland im Jahr 2011 nach der Reaktorkatastrophe von Fukushima die Ethik-Kommission Sichere Energieversorgung ins Leben gerufen (vgl. Ethik-Kommission Sichere Energieversorgung 2011). In Frankreich beschäftigt sich das nationale Ethikkomitee im Jahr 2007 mit Nanotechnologie, und in Österreich publiziert die Bioethikkommission beim Bundeskanzleramt im selben Jahr einen »Katalog ethischer Probleme und Empfehlungen« zu Nanotechnologie (vgl. CCNE 2007; Bioethikkommission beim Bundeskanzleramt 2007). Nationale Ethikinstitutionen scheinen zunehmend selbstverständlich die Lösung für ethische Probleme im Bereich wissenschaftlicher und technologischer Entwicklungen insgesamt zu werden.

Allein ein Blick zurück in die 1970er und 80er Jahre zeigt jedoch, dass Themen wie Atomtechnologie nicht immer als explizit ethische Themen verstanden wurden. In den Konflikten um Atomtechnologie in Europa wurde maßgeblich von Risiko-Problemen gesprochen. Zwar lagen diesen Konflikten - wie jedem anderen Konflikt auch - Werte zugrunde, jedoch wurden die Auseinandersetzungen nicht in 
ethischen, sondern in Risikobegriffen geführt. Auch in den Konflikten um die neuen Reproduktionstechnologien in den 1980er Jahren wurden, wie bspw. von westdeutschen Feministinnen, nicht ethische Fragen, sondern bevölkerungspolitische Probleme thematisiert. Ein moralisches Problem stellte für Lebensschützerinnen und Lebensschützer in Frankreich Mitte der 1970er Jahre die Möglichkeit der Invitro-Fertilisation dar. All diese Themen, die mittlerweile in Ethikinstitutionen besprochen werden, waren nicht immer ethisch gerahmt, es gab unterschiedliche Rahmungen, Problemdefinitionen und Lösungsvorschläge.

Im Bereich sozial- und geisteswissenschaftlicher Forschung zu Wissenschaft und Technologie ist die Ausweitung von Ethikpolitiken nicht unbemerkt geblieben. Die Wissenschaftsforscherin Sheila Jasanoff stellt einen »ethical turn « auf Ebene der Politik fest (Jasanoff 2011: 633). Von einem »boom in ethics commitees« spricht die Wissenschaftsforscherin Helga Nowotny (2003: 154). Ethisierungen sprich, dass viele technik- und wissenschaftspolitische Fragen als ethische Herausforderungen verstanden werden - behandelt der Sozialwissenschaftler Alexander Bogner in seiner Untersuchung zu nationalen Ethikinstitutionen (2013: 51). Die Politikwissenschaftlerin Kathrin Braun und ihre Kolleginnen und Kollegen arbeiten vor dem Hintergrund, dass nationale Ethikinstitutionen lebenswissenschaftliche Themen behandeln, heraus, für welche Problematisierung nationale Ethikinstitutionen eine Lösung darstellen (Braun et al. 2010a; Braun et al. 2010b).

Wie kommt es jedoch, dass nationale Ethikinstitutionen als adäquate Lösung für Probleme im Bereich wissenschaftlicher und technologischer Entwicklungen insgesamt erscheinen? Welche unterschiedlichen Problematisierungen und Lösungsvorschläge gab es? Diese Fragen sind bisher unbeantwortet geblieben. Wenn Ethik in Form von nationalen Ethikinstitutionen zu einem selbstverständlichen Rahmen geworden ist, um über biomedizinische, biotechnologische, nanotechnologische und auch atomtechnologische Themen zu sprechen, sind daran anschlieBende Fragen: Wie hat sich dieser Rahmen durchgesetzt? Und wie wird diese Form der politischen Steuerung themen-flexibel?

Diesen Fragen gehe ich in der vorliegenden Studie anhand eines Beispiels nach, und zwar anhand des französischen nationalen konsultativen Ethikkomitees für Lebenswissenschaften und Gesundheit, dem Comité Consultatif National d'Éthique pour les sciences de la vie et de la santé (CCNE). ${ }^{1}$ Anhand seiner Ent-

1 Das französische Genus der Eigennamen wird in der vorliegenden Publikation für die Wahl des deutschen Artikels übernommen. Da sich dies durchzusetzen scheint und es hierfür kein Regelwerk gibt, habe ich mich für diese Variante entschieden. Deutlich wird dies aktuell (leider) am Beispiel Front National. Es wird nicht mehr von die Front National, sondern von der Front National gesprochen. 
stehungsgeschichte und Institutionalisierung untersuche ich die Entwicklung, in deren Verlauf Ethik in der Politik zu einem selbstverständlichen Rahmen wird, welche Problematisierungen und Lösungsvorschläge sichtbar und welche unsichtbar werden und inwiefern sich diese Form der Steuerung von biomedizinischen und -technologischen auf weitere, insbesondere nanotechnologische Themen ausdehnen kann. Berücksichtigt werden in der Untersuchung insbesondere Konflikte um wissenschaftliche, medizinische oder technologische Entwicklungen mit einem Augenmerk auf die Problematisierungen sozialer Proteste. Die Studie hat somit zum Ziel, zur Forschung ethischer Institutionen und Diskurse im Bereich sozialund geisteswissenschaftlicher Forschung zu Wissenschaft und Technologie, den Science and Technology Studies beizutragen.

\section{Zur ENTSTEHUNG DER UNTERSUCHUNG}

Die Untersuchung knüpft an ein Forschungsprojekt zu nationalen Ethikinstitutionen in Deutschland, Großbritannien und Frankreich an, an dem ich als wissenschaftliche Mitarbeiterin für die Fallstudie zu Frankreich beteiligt war. Es handelt sich um das Projekt »Ethical Governance? Wissen, Werte und politische Entscheidungsfindung « (EGo), das an der Leibniz Universität Hannover unter der Leitung der Politologin Prof. Dr. Kathrin Braun durchgeführt wurde. ${ }^{2}$ Das EGo-Projekt fand vor dem Hintergrund statt, dass diese Institutionen ausschließlich biomedizinische und biotechnologische Themen behandeln. Erst mit dem Ende der Projektlaufzeit zeigte sich, dass eine dieser Institutionen, der CCNE, darüber hinaus zu Nanotechnologie arbeitet. Die Publikation des CCNE erschien im Jahr 2007. Aus dieser Beobachtung kam die Frage auf, wie sich Ethik-Rahmungen am konkreten Beispiel des Diskurses um Nanotechnologie ausdehnen. Da sich im Kontext der Nanotechnologie in Frankreich soziale Konflikte abzeichnen, entwickelte sich aus dieser weiteren Beobachtung die Idee, soziale Konflikte insgesamt im Entstehungsprozess des CCNE herauszuarbeiten. Der empirischen Untersuchung des Nanotechnologiediskurses in Frankreich konnte ich während meiner Mitarbeit in einem weiteren Forschungsprojekt nachgehen. Es handelt sich um das interdisziplinäre, ländervergleichende Projekt »>Converging Institutions?< How do regional

2 Das Projekt wurde in den Jahren 2004-2006 durchgeführt und stand im Rahmen des Science-Policy-Programms der Berlin Brandenburgischen Akademie der Wissenschaften. Die Forschung wurde durch das Bundesministerium für Bildung und Forschung gefördert (Fördernr. 07SP31). Aus der Studie ist eine Reihe an Publikationen hervorgegangen, vgl. Braun et al. (2010a; 2010b; 2009; 2008; Herrmann/Könninger 2008). 
institutions stimulate the innovation process of nanotechnologies in economy and society? A German-French comparison«. Es wurde unter der Leitung des Soziologen Prof. Dr. Christian Papilloud und der Wirtschaftswissenschaftlerin Prof. Dr. Ingrid Ott am Karlsruher Institut für Technologie und der Leuphana Universität Lüneburg durchgeführt. ${ }^{3}$ Die Forschungsfragen, auf die ich im Folgenden zu sprechen komme, entwickelten sich aus den Untersuchungsergebnissen der EGoStudie. Damit schließt die vorliegende Untersuchung an dieses Projekt inhaltlich an; die Ergebnisse des Projektes erweitere und ergänze ich im Hinblick auf die französische Fallstudie. Neben den Ergebnissen der EGo-Studie wird zudem die Untersuchung der französischen Politikwissenschaftlerin Dominique Memmi zum magistère bioéthique und der delegierten Biopolitik des CCNE einbezogen.

\section{Von GOUVERNEMENTALEN ETHIKREGIMEN UND VOM MAGISTĖRE BIOÉTHIQUE}

Das EGo-Projekt untersucht unter der Bezeichnung »gouvernementale Ethikregime« staatlich initiierte oder geförderte Institutionen, Praktiken und Diskurse, deren Aufgabe es ist, die politische Willensbildung und/oder Entscheidungsfindung an Beratungen über Fragen anzubinden, die als ethische Fragen gelten (Braun et al. 2010b: 511; Braun et al. 2008: 221). Nationale Ethikkomitees und Ethikkommissionen ebenso wie öffentliche Anhörungen oder Bürgerinnen- und Bürgerkonferenzen, die seit den 1970er und 80er Jahren entstehen, werden in dieser Studie in den Blick genommen. Die regierungsanalytische Perspektive ist die der Gouvernementalität, wie sie in Anlehnung an Michel Foucault von den Gouvernementalitätsstudien fruchtbar gemacht wird. Die Untersuchung fokussiert darauf, inwiefern sich gouvernementale Ethikregime als Form des governing at a distance im Rahmen des advanced liberal government nach Nikolas Rose und Peter Miller (Rose 1996; Rose/Miller 1992) sowie als Form des reflexive government verstehen lassen. Der verwendete Begriff reflexive government und mit ihm ein bestimmtes Konzept der Problematisierung geht auf den australischen Soziologen Mitchell Dean zurück (Dean 1999a). Dean verwendet den Begriff, um eine weniger direkte Form der Regierung zu erfassen, die er in den 1980er und 90er Jahren hauptsächlich im ökonomischen Bereich aufkommen sieht. Seine Hypothese ist, dass die

3 Das Projekt wurde von der Initiative »Innovationsprozesse in Wirtschaft und Gesellschaft « der Volkswagenstiftung in den Jahren 2007-2011 gefördert (Fördernr. II/83 568). $\mathrm{Zu}$ den Publikationen, die im Kontext des Projektes entstanden sind, vgl. Ott et al. (2008), Papilloud (2010), Könninger et al. (2010, 2008), Könninger (2011). 
Gouvernementalisierung des Staates, durch welche der Staat die Aufgabe für die Bevölkerung und Individuen übernimmt, gegenwärtig (teilweise) dahingehend transformiert wird, dass Regierungsmechanismen selbst zum Gegenstand der Problematisierung werden (Dean 1999b: 38ff). In der EGo-Studie wird aufgezeigt, dass die gouvernementalen Ethikregime insofern als reflexive government verstanden werden können, als sie in einem Moment entstehen, in dem neue Entwicklungen in Wissenschaft und Technologie auftreten, die Beunruhigung auslösen, während gleichzeitig die Selbststeuerung der Wissenschaft problematisch wird. Vor diesem Hintergrund, so ein Ergebnis der Studie, steht die Politik vor einem klassisch liberalen Dilemma, nämlich die wissenschaftliche Entwicklung zu fördern und sie gleichzeitig zu begrenzen:

»[S]ie soll einerseits die Freiheit, nämlich die Freiheit des Individuums und die Freiheit der Wissenschaft fördern und andererseits die Bedenken der Öffentlichkeit ernst nehmen und die Gesellschaft und ihre Mitglieder vor möglichen Risiken und Gefahren schützen, die aus der Ausübung dieser Freiheit erwachsen könnten«(Braun et al. 2008: 238).

Das Ethikregime begegnet diesem Dilemma des Regierens, indem es einen Rahmen für das Management von Konflikten bietet. Dabei handelt es sich nicht bzw. nicht vorrangig um politische Technologien, mittels derer der Staat direkt Prozesse der Wissenschafts- und Technologieentwicklung steuert, sondern um ein governing at a distance, also um Technologien, mit deren Einsatz nicht Entscheidungen herbeigeführt werden sollen, sondern die darauf gerichtet sind, Wissenschafts- und Technikkonflikte zu managen. Sie sind somit nicht Instrumente des klassischmodernen Interventionsstaates, die dazu dienen würden, biomedizinische und -technologische Entwicklungen zu regulieren. Sie sind als governing at a distance Teil der Werkzeugkiste des advanced liberalism: Sie steuern den Diskurs über diese Entwicklungen, indem das Sprechen über die Probleme dieser Prozesse angeleitet und in einer bestimmten Art und Weise gerahmt wird (Braun et al. 2008: 238; Braun 2013: 95).

Zur Frage der Rahmung bzw. zur Analyse der diskursiven Ebene greift das EGo-Projekt auf die Frame-Analyse und das Frame-Konzept nach den USamerikanischen Sozialwissenschaftlern Martin Rein und Donald Schön zurück (Rein/Schön 1993; Schön/Rein 1994). Ethik wird in der EGo-Studie als Frame analysiert. Mit der Frame-Analyse zeichnet die Studie nach, was in einem bestimmten Kontext unter Ethik verstanden wird, wann und wo die Sprache der Ethik aufkommt, d.h. wann und wie Probleme als ethische Probleme, ethische Implikationen oder ethische Fragen gerahmt werden. Die Begriffe Ethik oder ethisch werden in einem strikt nominalistischen Sinn verwendet, um nicht von der Frage 
abzulenken, welche Bedeutungen die Akteure und Akteurinnen dem Terminus im jeweiligen Kontext geben, wie sich die Bedeutungen verschieben und ob dem Terminus Ethik überhaupt eine präzise Bedeutung zugeschrieben wird (Braun et al. 2008: 222).

In der EGo-Studie zeigt sich anhand von Interviews, dass die Mitglieder der nationalen Ethikinstitutionen Ethik nicht definieren, es gibt keine exakte Vorstellung darüber, was Ethik ist. Das Verständnis von Ethik zeichnet sich vielmehr dadurch aus, wie nicht gesprochen werden soll: Es geht nicht darum, andere zu überzeugen oder um das einzig Richtige oder Falsche. Es geht auch nicht um dauerhafte Grenzen oder darum, wissenschaftlich-technologische Entwicklungen in Frage zu stellen oder zu blockieren. Das Sprechen im Ethik-Rahmen ist nicht durch den Kampf verschiedener Interessen oder durch gegnerische politische Positionen charakterisiert. Ethik zeigt sich eher als Rahmen für eine gemäßigte, individuelle, selbstreflektierende Art und Weise des Sprechens und Denkens über wissenschaftliche und technologische Entwicklungen, dessen Output nicht auf einzig richtigen Handlungsempfehlungen basiert. Das Ethikregime demonstriert vielmehr ein Modell des »richtigen Sprechens«, also eines Sprechens, das durch Offenheit für neue Themen, Ansichten und neue Grenzen gekennzeichnet ist. Es bietet, so zeigt diese Studie, eher den Rahmen für die Produktion von Diskursen als einen substantiell normativen Handlungsrahmen (ebd.: 238f; 2009: 46; Braun et al. 2010b: 515).

In ähnlicher Weise bezeichnet die französische Politikwissenschaftlerin Dominique Memmi die Art des Regierens des CCNE, nämlich als delegierte Biopolitik, die mit einem governing through speech einhergeht (Memmi 2003c, b). Gegenstand der Untersuchungen von Memmi ist nicht allein der CCNE, sondern insbesondere der Beginn und das Ende des Lebens (Reproduktion, Schwangerschaftsabbruch, Sterbehilfe) (Memmi 2004, 2003a). Memmi rekurriert in ihrer Analyse hauptsächlich auf Foucaults Konzept der Biopolitik (vgl. Foucault 2005a). Mit der delegierten Biopolitik bezeichnet Memmi einen biopolitischen Mechanismus, der die Transformation von der Disziplinierung der Bevölkerung zu einer begleiteten Selbstregierung des Individuums bezeichnet. Sie untersucht diesen Mechanismus im Rahmen der Transformation politischer Kontroll- und Steuerungsmodi, die in den 1960er und 70er Jahren beginnt, und analysiert die sich in diesem Kontext verändernden Formen des Umgangs des Individuums mit seinem Körper. Sie entwickeln sich von Verbot und Strafe hin zu einer zunehmenden Entkriminalisierung und Bioindividuation. Diese neue Art der Regierung richtet sich insbesondere auf die Regierung der körperlichen Führung, auf das »Bio« oder »Soma« (Memmi 2003a: 292). Diese von Memmi so bezeichnete Bioindividuation beinhaltet, körperliche Bedingungen auszubalancieren, Risiken zu kalkulieren und die Kosten der eigenen Gesundheit und ihrer Verbesserung zu evaluieren (ebd.: 292f). 
Die Führung dieser Art der Selbstregierung findet durch eine von Expertinnen und Experten angeleitete Art und Weise des Sprechens statt. Memmi nennt diese angeleitet Art und Weise des Sprechens, das eine individuelle und »rationale Kontrolle« stimuliert, das governing through speech (Memmi 2003c: 656/Übers. S.K.; 2005). Das governing through speech findet sich sowohl am Anfang und am Ende des Lebens, etwa im Kontext der Abtreibung oder der Sterbehilfe, als auch beim CCNE. In seinem Kontext spricht Memmi auch vom magistère bioéthique, einer Art bioethischem Lehramt (Memmi 2003a: 3). Die Bioethik bestätige die Transformation von der Disziplinierung der Bevölkerung zu einer begleiteten Selbstregierung des Individuums. »>[B]ioethical< regulation confirms the tendency to decriminalize the relationship to the body«, so Memmi (2003c: 656/Herv. i.O.). Dies zeigt sich darin, dass der CCNE nur Meinungen abgibt und selbst darauf besteht, über keine politische oder rechtliche Autorität zu verfügen. Die ethische Reflexion bedeutet dementsprechend »a growing interiorization of rational control or better still, of >reasonable conduct $<$ - in social practices« (ebd./Herv. i.O.). Während ethische Meinung eine Verpflichtung zur Selbstkontrolle befördert, kann, so Memmi, eine rechtliche oder politische Autorität Widerstand hervorrufen.

\section{FORSCHUNGSFRAGEN}

Wesentliche Überschneidung der EGo-Studie und Memmis Arbeit ist der Foucault'sche Regierungsbegriff. Beide Untersuchungen arbeiten die spezifische Form des Regierens des CCNE als indirekte, nicht-direktive Form heraus. Merkmal dieser Regierungsform ist das durch Expertinnen und Experten angeleitete Sprechen oder das »richtige Sprechen«, ein offenes, gemäßigtes, selbstreflektierendes Sprechen. Eine weitere Überschneidung besteht darin, dass diese Untersuchungen vor dem Hintergrund stattfinden, dass diese Ethikkommissionen lebenswissenschaftliche Themen behandeln. Beide Untersuchungen richten den Fokus nicht explizit auf soziale Konflikte.

Ein Ergebnis der EGo-Studie ist, dass dem Terminus Ethik ein diffuses Verständnis zugrunde liegt. Es wird nicht definiert, was Ethik ist, sondern eher, was sie nicht ist. Liegt gerade in diesem diffusen Verständnis eine Möglichkeit für die Ausdehnung dieser Form des governing at a distance auf weitere Themen? Denn, wenn nicht gesagt wird, was Ethik ist, können beliebige Themen als ethische Themen verstanden werden. Dass dieses diffuse Verständnis die Ausdehnung von ethischer Steuerung auf andere Themen ermöglichen kann, vermutet auch Kathrin Braun in einer Arbeit zur Ethik-Kommission Sichere Energieversorgung (Braun 
2013). Voraussetzung für diese Möglichkeit ist allerdings, dass dieses diffuse Verständnis auch im Kontext der Nanotechnologie zum Tragen kommt.

Eine weitere Frage ergibt sich aus der Arbeit von Memmi. Hier zeigt sich, dass die delegierte Biopolitik in Form des CCNE mit einer Bioindividuation verbunden ist, d.h. es geht um ein angeleitetes Sprechen über den Körper und die individuelle Gesundheit. Das Verständnis von Ethik ist in diesem Sinn insofern nicht diffus, als es explizit mit dem Sprechen über den Körper und über die individuelle Gesundheit verknüpft ist. Unter dem Begriff Nanotechnologie und ihren Anwendungsbereichen werden zwar auch biomedizinische und biotechnologische Bereiche verstanden, jedoch ebenso Energie- und Umwelttechnologie, die Bereiche Lebensmittel, Kosmetik oder der Textilbereich (vgl. Wullweber 2008b: 30). Auch wenn Nanotechnologie nicht (nur) eine Technologie ist, die in direktem Bezug zum Körper steht, besteht eine Möglichkeit, sie in die Bioindividuation zu integrieren, indem sie als individuelles gesundheitsrelevantes Thema verstanden wird. Wenn es sich im Kontext der Nanotechnologie weiterhin beim CCNE um eine Form der delegierten Biopolitik handelt, wird sie dann als individuelles gesundheitsrelevantes Problem thematisiert? Anders formuliert, überprüfe ich, ob es sich gegenwärtig noch um ein magistère bioéthique oder sozusagen um ein magistère nanoéthique handelt.

Ein weiteres Ergebnis der EGo-Studie ist, dass gouvernementale Ethikregime in einem Kontext entstanden sind, in dem wissenschaftliche und technologische Entwicklungen Beunruhigungen hervorgerufen haben und in dem die Selbststeuerung dieser Entwicklungen problematisch geworden ist. Allerdings muss diese Problematisierung nicht für den Kontext nanotechnologischer Entwicklungen zutreffen. Nur wenn diese spezifische Problematisierung vorliegt, handelt es sich um ein reflexive government. Liegt diese spezifische Problematisierung im Kontext der Nanotechnologie vor? In der vorliegenden Studie geht es also um die Frage, ob es sich im Kontext der Ausdehnung ethischer Rahmungen und Problematisierungen auf Nanotechnologie um eine Form des reflexive government handelt. Für die Untersuchung der Entstehung des CCNE ist diese Problematisierung wiederum anleitend. Wenn es diese Problematisierung ist, für die die Institutionalisierung des CCNE als Lösung erscheint, stellt sich die Frage, welche anderen Problematisierungen und Lösungsvorschläge es in seinem Entstehungsprozess gibt.

Zur Bearbeitung der Forschungsfragen habe ich mich für die regierungsanalytische Perspektive der Gouvernementalität entschieden. Sie ist insofern fruchtbar, als sie nicht allein die ausschließenden Wirkungen, sondern gerade auch die produktiven Seiten von Regierungspraktiken beleuchtet. Regieren bedeutet in dieser Perspektive nicht, die Freiheit und Handlungsfähigkeit der Menschen zu negieren, sondern vielmehr sie zu strukturieren (Rose 1999: 4). Diese Perspektive ist für die 
Untersuchung insofern geeignet, als die Antwort auf die Frage der Ausdehnung nicht unbedingt allein in ausschließenden Wirkungen von Regierungspraktiken zu finden ist, sondern auch in dem, was sie ermöglichen. Zudem erlaubt diese Perspektive, die Ergebnisse der EGo-Fallstudie zu Frankreich anhand der Frage der Ausdehnung ethischer Rahmungen zu überprüfen und sie um die Frage der verschiedenen Problematisierungen zu erweitern. Darüber hinaus kann Memmis Konzept in die Untersuchung integriert und das möglicherweise Spezifische dieser ethischen Regierungsform, die Bioindividuation, herausgearbeitet werden.

Die Erweiterung der EGo-Studie besteht insbesondere darin, die Problematisierungen aus sozialen Konflikten und Protesten zu berücksichtigen, und zwar sowohl im Kontext der Entstehung des CCNE als auch im Kontext der Ausdehnung ethischer Rahmungen. Um die unterschiedlichen Problematisierungen, die im Kontext der Entstehung und Institutionalisierung des CCNE aufkommen, fassen zu können, stütze ich mich auf eine genealogische Herangehensweise im Sinne Foucaults. Diese Herangehensweise beginnt mit der Problematisierung zu einem bestimmten Zeitpunkt in einem bestimmten Kontext und verfolgt historisch die Problematisierungen und Lösungsvorschläge zurück. Mit der Frage, wofür der CCNE eine Lösung ist bzw. worauf seine Publikation zu Nanotechnologie antwortet, verfolge ich die Rahmungen und Problematisierungen zurück. Mit der genealogischen Herangehensweise wird im Hinblick auf Konflikte um wissenschaftliche, medizinische oder technologische Entwicklungen herauspräpariert, welche Problematisierungen und Lösungsvorschläge verloren gehen und welche sich durchsetzen. Um nicht allein auf die spezifische Problematisierung zu fokussieren, die in der EGo-Studie zentral ist, verwende ich ein offenes Konzept der Problematisierung. Problematisierung verstehe ich als »a conflict-ridden, discursive process in which various actors struggle over the meaning and definition of >the problem < and in which >the problem < is constantly reframed « (Herrmann 2011: 163/Herv. i.O.). Zur Analyse der diskursiven Ebene dient mir die bereits in der EGo-Studie erprobte FrameAnalyse. Im Unterschied dazu berücksichtige ich allerdings nicht nur ethische Rahmungen, sondern gerade auch nicht ethische Rahmungen. Kurz gesagt, ist der theoretisch-methodische Rahmen in der Untersuchung die Perspektive der Gouvernementalität gekoppelt mit einer genealogischen Herangehensweise, die mit diskursanalytischen Konzepten der post-positivistischen interpretativen PolicyAnalyse kombiniert werden. 


\section{Fallstudie Und Untersuchungsmaterial}

Das französische nationale Ethikkomitee dient in der vorliegenden Studie aus mehreren Gründen als Beispiel. Auslöser für die Wahl ist die Beobachtung, dass sich das Komitee augenscheinlich mit nicht-lebenswissenschaftlichen Themen beschäftigt und sich ethische Rahmungen auf nanotechnologische Themen ausdehnen. Ausschlaggebend ist zudem, dass es in Frankreich seit Beginn der 2000er Jahre gesellschaftliche Konflikte um die Nanotechnologie gibt. An ihnen lässt sich untersuchen, inwiefern sich die ethische Rahmung ausdehnt. Zwar hat ebenfalls in Österreich die Bioethikkommission beim Bundeskanzleramt im Jahr 2007 Empfehlungen zu Nanotechnologie publiziert, jedoch gibt es in Österreich keine wesentlichen Konflikte um diese Technologie. Die Kombination aus >Konflikten $<$, >nicht-lebenswissenschaftliches Thema < und >nationale Ethikinstitution< gibt es zu Beginn dieser Untersuchung in keinem anderen europäischen Land. ${ }^{4}$ Hinzu kommt, dass mit diesem Beispiel weiterführende Fragen, die sich aus dem EGo-Projekt ergaben, nachgegangen werden kann und ich sowohl auf das Untersuchungsmaterial dieser Studie wie auch auf das von mir gewonnene Material aus dem Projekt »Converging Institutions « zurückgreifen kann.

Bei dem Material handelt es sich um Dokumente, das Protokoll einer teilnehmenden Beobachtung sowie Aufnahmen und Protokolle 13 leitfadengestützter Interviews mit Expertinnen und Experten. ${ }^{5}$ Ergänzt wird es durch weitere Dokumente zu sozialen Konflikten. Zu den Dokumenten zählen Gesetze, Dekrete, Presseartikel, Publikationen des CCNE und seiner Mitglieder (Empfehlungen, Berichte, Artikel), Internetauftritte der Institutionen, forschungspolitische Berichte und Tagungsberichte, Publikationen sozialer Bewegungen bzw. kritischer Akteure und Akteurinnen sowie Nichtregierungsorganisationen (NGOs) wie Appelle, Flugblätter, Zeitschriftenartikel, auch graue Literatur. Die Interviews wurden mit (ehemaligen) Mitgliedern des CCNE und/oder mit forschungspolitischen Akteurinnen und Akteuren durchgeführt. Die Expertinnen und Experten für die Interviews habe ich aufgrund ihres Praxis- und Erfahrungswissens ausgesucht (vgl. Bogner et al.

4 Die Ethikkommission zur sicheren Energieversorgung in Deutschland wird nicht berïcksichtigt, weil sie erst während meiner Untersuchung eingerichtet wurde.

5 Alle Interviews wurden aufgezeichnet, protokolliert und von mir vom Französischen ins Deutsche übersetzt. Die Interviews wurden für die vorliegende Publikation anonymisiert, da nicht alle Gesprächspartnerinnen und -partner namentlich erwähnt sein wollten. Die Interviews sind in römischen Ziffern durchnummeriert. Es sind aus dem Kontext der Zitate nur Rückschlüsse auf diejenigen Interviewpartnerinnen und -partner möglich, die einer namentlichen Benennung zugestimmt haben. 
2005). Die teilnehmende Beobachtung wurde bei der jährlich stattfindenden öffentlichen Tagung des CCNE, den Journées annuelles d'éthique (16./17. November 2004) an der Université Paris V René Descartes von mir durchgeführt.

Die Dokumente analysiere ich im Folgenden danach, wann, wie und von wem Themen als ethische Probleme, ethische Implikationen oder ethische Fragen gerahmt werden, wann also die Sprache der Ethik auftaucht. Für die Untersuchung ordne ich die Dokumente nach Diskursen, wobei diese nicht nur als Sprache, sondern auch als gesellschaftliche Praktiken und Institutionen verstanden werden nach Diskursen also, in denen die Sprache der Ethik auftaucht, bzw. in denen die Ethikinstitutionen, die forschungspolitischen Berichte, Tagungen oder Konferenzen in Erscheinung treten, die als Vorgänger des CCNE gelten, bzw. denen ein Einfluss auf seine Einrichtung zugesprochen wird. Mit der Frage, wofür die jeweiligen Ethikinstitutionen eine Lösung sind, bzw. worauf die jeweilige Tagung oder der jeweilige Bericht eine Antwort ist, werden die Rahmungen und Problematisierungen in den Diskursen zurückverfolgt. Anleitend ist hier, wann die Sprache der Ethik in Erscheinung tritt, was problematisiert wird und daran anknüpfend, welche weiteren Problematisierungen auftreten. Berücksichtigt wird also nicht allein, wann, warum und von wem etwas als ethisches Problem oder Thema gesehen wird, sondern auch, welche anderen Problematisierungen es gibt. Im Wesentlichen habe ich folgende Diskurse identifiziert und untersucht: den medizinischen Diskurs, den Gentechnologiediskurs, den Diskurs um Biologie und Lebenswissenschaften sowie den Nanotechnologiediskurs.

\section{Aufbau der Studie}

Die Darstellung der Untersuchung erfolgt in Form der Diskurse und nicht als kontinuierliche, chronologische Erzählung. Der Untersuchungszeitraum der jeweiligen Diskurse richtet sich danach, wann die jeweilige Ethikinstitution eingerichtet wird, die jeweilige Tagung stattfindet bzw. der jeweilige Bericht publiziert wird und wann die jeweiligen Rahmungen und Problematisierungen auftauchen. Im medizinischen Diskurs werden die (ethischen) Rahmungen von 1974 bis in die Nachkriegszeit zurückverfolgt. Die Entstehung einer Institution verfolge ich bis in das Vichy-Regime. Der Gentechnologiediskurs ebenso wie der Diskurs um Biologie verdichtet sich in den 1970er Jahren bis zur Einrichtung des CCNE im Jahr 1983. Die Untersuchung des Nanotechnologiediskurses geht punktuell in die 1980er Jahre zurück, konzentriert sich im Wesentlichen vom Beginn der 2000er Jahre bis zur Publikation über Nanotechnologie des CCNE im Jahr 2007. 
Die Studie unterteilt sich in acht Kapitel. Kapitel I und II beschäftigen sich mit dem Stand der Forschung sowie dem analytisch-methodischen Rahmen. Im Kapitel zum Forschungsstand geht es um die Beantwortung der Frage, wie ethische Themen im Kontext nanotechnologischer, biotechnologischer und biomedizinischer Entwicklungen bisher in sozial- und geisteswissenschaftlicher Literatur zu Wissenschaft und Technologie untersucht werden. Darüber hinaus geht es um die Beantwortung der Frage, welche Untersuchungen mit welchen Ergebnissen zu nationalen Ethikinstitutionen vorliegen. Aufgezeigt werden Anknüpfungspunkte ebenso wie Forschungslücken. Daran anschließend werden weitere Fragen für die Untersuchung entwickelt. Darüber hinaus wird verdeutlicht, welchen Beitrag diese Studie zur Untersuchung von Ethikdiskursen in den Science and Technology Studies leisten kann. Im daran anschließenden Kapitel zum analytisch-methodischen Rahmen werden Foucaults Konzept der Gouvernementalität und die Gouvernementalitätsstudien vorgestellt, ebenso die genealogische Herangehensweise. In diesem Zusammenhang nehme ich eine Präzisierung der Untersuchungsperspektive vor und konkretisiere zentrale Konzepte und Begriffe.

Der empirische Teil der Untersuchung umfasst fünf Kapitel (III-VII). Die Kapitel III bis VI beschäftigen sich mit der Entwicklung und Entstehung des CCNE, das Kapitel VII mit der Ausdehnung ethischer Rahmungen am Beispiel der Nanotechnologie. In Kapitel III gehe ich auf die Entstehung des ersten staatlich initiierten Ethikkomitees im Jahr 1974 am nationalen Institut für Gesundheit und medizinische Forschung, dem Institut National de la Santé et de la Recherche Médicale (INSERM), ein. Der INSERM entsteht aus einem Hygieneinistitut, das im VichyRegime eingerichtet wird. Sein Ethikkomitee entsteht im Kontext eines Diskurses um medizinische Forschung und insbesondere um Humanexperimente. In diesem Diskurs zeigen sich verschiedene Problematisierungen dieser Praktiken. Sie rekurrieren auf die medizinische Forschung im Zweiten Weltkrieg und insbesondere im Nationalsozialismus. Um diesen Vergangenheitsdiskurs zu verstehen, wird zuerst die Entstehung und Entwicklung des INSERM, beginnend mit dem Hygieneinstitut im Vichy-Regime bis zur Einrichtung des Ethikkomitees, berücksichtigt. AnschlieBend verfolge ich, ausgehend von der Institutionalisierung des Komitees, die Sprache der Ethik in die Diskurse um Humanexperimente bis in die Nachkriegszeit zurück und arbeite heraus, in welchem Vergangenheitsdiskurs sich das Ethikkomitee verortet. Dabei gehe ich mit der Frage, ob und inwiefern die Sprache der Ethik in weiteren Diskursen relevant ist, die in den 1960er und 70er Jahren den öffentlichen Raum prägen, auf weitere Diskurse ein: auf solche um Medikamentenskandale, um die Legalisierung des Schwangerschaftsabbruchs sowie auf den medizinkritischen Diskurs, der aus den 1968er-Bewegungen hervorgegangen ist. Ethik in Form des Ethikkomitees vom INSERM erscheint als Antwort auf das Infragestellen und die 
Verteidigung medizinischer Autonomie. Diese Diskussionen werden nicht allein durch soziale Bewegungen initiiert, sondern finden auch innerwissenschaftlich statt. Es zeigt sich die Abkehr von einer als überholt empfundenen, medizinischkonservativen Moralvorstellung, mit der bestimmte Forschungen blockiert werden könnten, hin zu einer ethischen Rahmung, die Forschungsfreiheit sichert.

Eine weitere, im Jahr 1974 eingerichtete Ethikinstitution wird in Kapitel IV behandelt: Die Ethikkommission der Délégation Générale à la Recherche Scientifique et Technique, einer Vorgängerin des französischen Forschungsministeriums. Eingerichtet wird diese Ethikkommission im Kontext der Kontroversen um Gentechnologie. Diese Kontroversen werden in den 1970er Jahren nicht alleine in Frankreich, sondern international geführt und sind stark von der US-amerikanischen wissenschaftlichen Community geprägt. Um die Auswirkungen der US-amerikanisch geprägten, internationalen Debatte auf die ethischen Rahmungen in Frankreich in die Untersuhung einbeziehen zu können, wird die Sprache der Ethik auch im US-amerikanischen Raum nachgegangen. In diesen Kontroversen um Gentechnologie ist eine Kritik entscheidend, die von den Konflikten um Atomtechnologie beeinflusst ist. Überprüft wird, inwiefern diese Verknüpfung zum AntiAtomdiskurs im französischen Diskurs um Gentechnologie besteht. Die Ethik in Form der Ethikkommission kann als Bemühung der Wissenschaftler und Wissenschaftlerinnen verstanden werden, einen ethischen Umgang mit ihrer Forschung zu zeigen. Dieser Umgang erscheint als Antwort auf eine Kritik, die allerdings nicht ethisch gerahmt ist und insbesondere die politischen Entscheidungen über wissenschaftliche und technologische Entwicklungen angreift. In diesem Sinn zeigt sich Ethik in Form der Ethikkommission eher als Sicherung wissenschaftlicher Autonomie.

Kapitel V umfasst eine forschungspolitische Tagung, die in Paris ebenfalls im Jahr 1974 stattfindet, sowie einen forschungspolitischen Bericht aus dem Jahr 1979. Die Tagung trägt den Titel »Biologie et devenir de l'homme« - »Biologie und die zukünftige Entwicklung des Menschen«, der Bericht den Titel »Sciences de la vie et société« - »Lebenswissenschaften und Gesellschaft«. Kennzeichen sowohl der Tagung als auch des Berichtes ist, dass die Sprache der Ethik nicht mehr jeweils im Kontext gentechnologischer oder medizinischer Fragen in Erscheinung tritt, sondern insofern interdisziplinär ist, als diese Fragen als biologische und lebenswissenschaftliche Themen verhandelt werden. Im Kontrast zu den EthikRahmungen im medizinischen und gentechnologischen Diskurs zeigen sich diese nicht mehr als Antwort auf Problematisierungen wissenschaftlicher und medizinischer Selbststeuerung, sondern als deren antizipiertes Problem. Dieses Problem wird von wissenschaftlichen Akteuren und Akteurinnen auf gesellschaftlicher Seite verortet. Die vorgeschlagene Lösung gesellschaftlicher ethischer Probleme, die ei- 
ne Sicherung der Forschungsfreiheit ermöglicht, liegt im Dialog mit der Gesellschaft und in Reflexionsgruppen - Konzepte, die sich in der Planung und Umsetzung des CCNE wiederfinden.

In Kapitel VI wird die Einrichtung des CCNE im Kontext der sich wandelnden Forschungspolitik zu Beginn der 1980er Jahre betrachtet. In diesem Zusammenhang entstehen bereits unabhängig von der Institutionalisierung des CCNE Formen des governing at a distance. Die Sprache der Ethik erscheint nun als Lösung im Umgang mit wissenschaftskritischen Bewegungen. Darüber hinaus entsteht in diesem forschungspolitischen Kontext im selben Jahr wie der CCNE eine parlamentarische Technikfolgenabschätzungsbehörde. Um herauszuarbeiten welche Themen als ethische Themen verstanden werden, wird der Aufgabenbereich dieser Behörde mit dem des CCNE kontrastiert. Sowohl die Technikfolgenabschätzungsbehörde als auch der CCNE beschäftigen sich mit Gentechnologie. Dementsprechend wird untersucht, inwiefern die damit einhergehenden unterschiedlichen Rahmungen Auswirkungen auf die Kritik an Gentechnologie haben. Ein Fokus des Kapitels liegt auf der Frage, inwiefern neue Problematisierungen bei der Planung und Einrichtung des CCNE angesprochen werden. Unter Berücksichtigung der herausgearbeiteten Problematisierungen aus den vorangegangenen Kapiteln wird zudem untersucht, welche Themen noch angesprochen und welche nicht mehr berücksichtigt, unsichtbar werden. Zudem werden das »richtige Sprechen« und das governing through speech ausgeführt und damit die Frage beantwortet, wie im institutionalisierten Ethik-Rahmen gesprochen werden kann. Ethik in Form des nationalen Ethikkomitees erscheint im Kontext zweier gegenläufiger Tendenzen - dem Infragestellen und der Stabilisierung wissenschaftlicher Selbststeuerung, die sie löst: Sie stellt eine Modifikation wissenschaftlicher Steuerung dar, ohne die wissenschaftliche Entwicklung zu verhindern.

Am Beispiel des Nanotechnologiediskurses untersuche ich im letzten Kapitel der empirischen Untersuchung (VII) die Ausdehnung ethischer Rahmungen und Problematisierungen anhand der Publikation des CCNE zu Nanotechnologie. Der Diskurs um Nanotechnologie ist in Frankreich sowohl geprägt von Konflikten, die sich zuerst im US-amerikanischen Diskurs um Nanotechnologie entwickeln, als auch von Konflikten, die in Frankreich entstehen. Unter Berücksichtigung der Frage, inwiefern die Sprache der Ethik im US-amerikanischen Diskurs um Nanotechnologie auftaucht, wird überprüft, ob und inwiefern sich diese Rahmungen im französischen Diskurs wiederfinden. Anschließend werden die Kontroversen in Frankreich dargestellt und die Rahmungen herausgearbeitet. Anhand der Publikation des CCNE zu Nanotechnologie zeige ich auf, auf welche Problematisierungen dieser antwortet und inwiefern sich ethische Rahmungen und Problematisierungen ausdehnen. Die Ausdehnung ist möglich, da dieses governing at a distance wie im 
biotechnologischen und -medizinischen Diskurs auf einem diffusen Verständnis von Ethik basiert, das eine Themen-Flexibilität ermöglicht. Themen-flexibel ist dieses allerdings unter der Voraussetzung, dass Nanotechnologie als individuelles gesundheitliches Thema aufgefasst wird. Insofern kann im Kontext des CCNE weiterhin von einer delegierten Biopolitik gesprochen werden. Wesentlicher Unterschied zum biomedizinischen und -technologischen Diskurs ist allerdings ein Konflikt darüber, ob Nanotechnologie als ethisches Problem zu verstehen ist. Entsprechend wird das ethische governing at a distance selbst in Frage gestellt. Die Lösung für diese Problematisierung liegt nicht allein im CCNE. Indem er empfiehlt, dass weitere Akteure und Akteurinnen ethisch ausgebildet werden und eine gesteigerte Einbeziehung der Öffentlichkeit in Debatten stattfinden soll, stellt er vielmehr die Lösung zur Lösung von Konflikten dar.

Im abschließenden achten Kapitel resümiere ich die Untersuchung und reflektiere ihren methodisch-analytischen Rahmen im Hinblick auf die empirischen Forschungsergebnisse. 



\section{Stand der Forschung}

In der sozial- und geisteswissenschaftlichen Forschung zu Wissenschaft und Technik, den Science and Technology Studies, entwickelt sich sichtbar seit den 1980er Jahren eine Fülle an Literatur zu ethischen Themen, Problemen, Implikationen oder Ethikinstitutionen. Unter der Bezeichnung Science and Technology Studies (STS) versammeln sich seitdem Untersuchungen zu Wissenschaft und Technik verschiedener Disziplinen, die meist sozialkonstruktivistisch geprägt sind ${ }^{1}$ (Ilyes 2006: 2). Auf diese Untersuchungen im Kontext der Biomedizin und Biotechnologie sowie im Kontext der Nanotechnologie, ihre Anknüpfungspunkte und Forschungslücken im Hinblick auf die Forschungsfragen dieser Studie gehe ich im Folgenden ein.

1 Unter STS wird z.T. auch Science, Technology and Society verstanden. Anstatt des Akronyms STS wird manchmal STSS verwendet. Es steht für Science, Technology and Society Studies. Etwas allgemeiner gehalten wird auch von Science Studies gesprochen (Ilyes 2006: 2f). Länderspezifische Unterschiede zeigen sich in den jeweiligen Ausprägungen der beteiligten Disziplinen: Während bspw. in Frankreich hauptsächlich die Soziologie vertreten ist, in Deutschland zudem Geschichte, Anthropologie und Philosophie, sind vor allem in den USA auch Politikwissenschaften, Cultural Studies, Feminismus- und Postkolonialismusforschung vertreten (ebd.: 4). Zu den Ursprüngen und Entwicklungen der STS vgl. David Edge (1995) sowie Sheila Jasanoff (2010). Für einen jüngeren Überblick über gegenwärtige Forschungsrichtungen und Ansätze vgl. Sergio Sismondo (2008). 


\section{Untersuchungen ZU ETHIK, BIOMEDIZIN UND BIOTECHNOLOGIE}

Untersuchungen zu nationalen Ethikinstitutionen, ihrer Entstehung und ihrer Bedeutung im Kontext biomedizinischer und -technologischer Entwicklungen liegen in großer Fülle vor, auch zum nationalen Komitee Frankreichs. Zu den bestehenden Typen nationaler Ethikinstitutionen und Verfahren, inklusive dem CCNE, existieren einige Überblicke (Fuchs 2005, 2001a; Braun et al. 2002; Gill/Dreyer 2001; Galloux et al. 2002; Le Bris 1993; Moulin 1990). Gerade die Überblicksarbeiten sind allerdings aufgrund der zum Teil temporär begrenzten Verfahren und Praktiken, der raschen Ausdehnung der Institutionenlandschaft und der Weiterentwicklungen der bestehenden Institutionen schnell überholt. ${ }^{2}$

Darüber hinaus gibt es eine Fülle an Beiträgen, die Aufgabe und Sinn von nationalen Ethikgremien diskutieren, allerdings eher auf einer normativen und/oder anwendungsorientierten Ebene (Lenoir 1997; Düwell 2000; Düwell/Steigleder 2003; Kettner 2000; Ach/Runtenberg 2002). Hierzu können auch solche Beiträge über den CCNE gezählt werden, die meist von dessen (ehemaligen) Mitgliedern verfasst wurden (vgl. u.a. Ambroselli 1987, 1990; Questiaux 2003; Braibant et al. 1993; Quéré 2003; Kenis/Heuskin 1990). Darüber hinaus zählen dazu die Beiträge zur Entstehung des CCNE, seiner Arbeitsweise, seinem legalen Status und der Entstehung der Bioethik in Frankreich (vgl. u.a. Debru 2003; Martinez 2004; Michaud 1990, 2002, 2003; Bernard 1986/1987, 1988; Lenoir 1991; Durand 1999).

$\mathrm{Zu}$ den Arbeiten, die explizit den CCNE im Untersuchungsfokus haben, gehören zudem eine Reihe französischer sozialwissenschaftlicher Diplomarbeiten und Dissertationen. Die Politikwissenschaftlerin Myriam Bachir-Benlahsen untersucht das Aufkommen der comités de sages - Komitees der Weisen in den 1980er Jahren, zu denen sie den CCNE zählt, und fragt, wie sie die Politik legitimieren. Sie zeigt auf, dass die Legitimität dieser Komitees prozeduraler Natur ist und auf Deliberation basiert. Es handelt sich um eine Form der Beratung, die insofern nicht mehr technokratisch ist, als sie nicht hinter verschlossenen Türen stattfindet. Vielmehr geht sie mit Transparenz einher und bezieht die Zivilgesellschaft ein (BachirBenlahsen 1995). Die Frage der politischen Effizienz des CCNE und seiner demokratischen Perspektiven stellt der Soziologe François Alias. Diese Frage untersucht er unter Berücksichtigung der konzeptuellen und historischen Einschreibung des CCNE sowohl in eine medizinische und wissenschaftliche Logik als auch im Kon-

2 Eine Auflistung über die weltweit bestehenden Ethikkommissionen, -komitees und -räte bietet das Deutsche Referenzzentrum für Ethik in den Biowissenschaften: http://www. drze.de/bioethik-links, 13.03.2014. 
text der Menschenrechte. Er zeigt auf, dass der CCNE weniger dazu dient, eine konstruktive öffentliche Debatte zu initiieren, als vielmehr dazu, den Gesetzgebungsprozess zu unterstiutzen (Alias 1991). Aus politikwissenschaftlicher Perspektive untersucht Alix Didrich-Tabaste am Beispiel des CCNE die soziale Konstruktion einer Autorité Administrative Indépendante ${ }^{3}$ (Didrich-Tabaste 1994). Die Politikwissenschaftlerin Myriam Duteil analysiert das institutionelle Aufkommen von Ethik in Form des CCNE und fragt nach seiner Bedeutung im politisch liberalen System Frankreichs (Duteil 1990). Abgesehen von diesen Qualifizierungsarbeiten untersuchte ebenso im französischsprachigen Raum die Sozialwissenschaftlerin Virginie Tournay den CCNE. Sie betrachtet die Bioethik, die sie als Teilhaberin am Dreh- und Angelpunkt der Medikalisierung des Sozialen sieht, als die institutionelle Aufnahme eines bestimmten Typs der sozialen Führung des biologischen Körpers. Der CCNE trägt aus ihrer Perspektive als konsultative Instanz mit seiner deliberativen Aktivität zur Regulation der wissenschaftlichen Normativität bei und zur Neuverhandlung medizinischer und wissenschaftlicher Praktiken (Tournay 2002, 2003). Die Quelle der sozialen Autorität der Mitglieder des CCNE untersuchte die französische Politikwissenschaftlerin Dominique Memmi, wobei sie eine »soziale Topografie« ihrer professionellen und sozialen Identität erstellt hat. Charakteristisch für die Ethik-Experten und Ethik-Expertinnen ist nach Memmi, dass sie in der Öffentlichkeit als moderat, unparteiisch und nicht als Interessenvertreter oder -vertreterinnen bekannt sind und die Fähigkeit zur individuellen Reflexion besitzen (Memmi 1996).

Diese Arbeiten geben einen Einblick, wie und warum nationale Ethikkomitees und insbesondere der CCNE entstanden sind, welche Aufgaben und welche Bedeutung das Komitee in den verschiedenen Perspektiven hat. Die Arbeit von Memmi gibt Anhaltspunkte für meine Frage, wie über die jeweiligen Themen gesprochen und reflektiert werden kann. Jedoch ist ihre Untersuchung genauso wie die anderen Analysen vor dem Hintergrund entstanden, dass nationale Ethikinstitutionen biomedizinische und biotechnologische Themen behandeln. Die Frage nach der Ausdehnung ethischer Steuerung, insbesondere in den Bereich der Nanotechnologie, wurde nicht berücksichtigt. Zudem liegt in den bisher genannten Arbeiten der Fokus nicht auf Diskursen sozialer Proteste oder Kontroversen. Solch eine Untersuchungsperspektive findet sich nach meiner Recherche vor allem außerhalb des französischen Sprachraums. Diese Untersuchungen können weitere Anhaltspunkte geben, wie im Ethik-Rahmen gesprochen werden kann.

3 Es handelt sich hierbei um eine Art Mittlerorganisation, im Englischen als quango - quasi Nichtregierungsorganisation bekannt. Quasi insofern, da diese Organisationen einen Regierungsauftrag haben oder/und von der Regierung finanziell unterstützt werden. 
Diejenigen sozialwissenschaftlichen Arbeiten, die in der Analyse der Entstehungsgeschichte nationaler Ethikinstitutionen in Europa oder einzelnen europäischen Ländern Kontroversen berücksichtigen, benennen als Schlüsselereignis insbesondere die Debatte um Gentechnologie sowie um das erste Reagenzglasbaby ab Mitte/Ende der 1970er Jahre (Galloux et al. 2002; Jelsøe et al. 2006; Lindsey et al. 2001; Braun et al. 2008, 2009; Braun et al. 2010a; Braun et al. 2010b; Herrmann/Könninger 2008; Könninger 2010, 2011, 2013). Die Historikerin Tina Stevens sieht die Ursprünge der Bioethik in den USA bereits in den 1960er Jahren als Folge der responsible science movement, die aus der Kritik an Atomtechnologie entsteht (Stevens 2000). In ihrer Untersuchung kommt Stevens zu dem Ergebnis, dass die Bioethik eher als Hebamme für neue medizinische und technologische Forschungen dient als dazu, diese Entwicklungen zu kritisieren (ebd.: 45ff). Zwar wird auch in Untersuchungen zur Entstehung des CCNE die In-vitro-Fertilisation als Schlüsselereignis benannt, der Diskurs, der sich aus der Kritik an Atomtechnologie entwickelte, blieb bisher allerdings unberücksichtigt. Hatte er keinen Einfluss und wenn doch, inwiefern?

Nicht nur Stevens, die die Bioethik als Hebamme für neue medizinische und technologische Forschungen und Entwicklungen sieht, sondern auch weitere Untersuchungen betrachten die Bioethik als Helferin biomedizinischer Entwicklungen: als Form des Akzeptanzmanagements oder als Legitimationsinstanzen (Feuerstein/Kollek 1999; Barben 1997). ${ }^{4}$ Der US-amerikanische Sozialwissenschaftler John Evans untersucht die öffentliche bioethische Debatte im Kontext der Humangenetik von den 1970er Jahren bis Mitte der 1990er Jahre in den USA (Evans 2002). Er zeigt auf, wie die professionelle Bioethik aufkommt und wie sich mit ihr die bioethische Debatte rationalisiert, ausdünnt, technischer, mehr formal als substantiell wird. Es entsteht eine Debatte, in der es nicht mehr möglich scheint, über die Ziele humangenetischer Forschung und Entwicklung zu sprechen. Nicht nur die Möglichkeit, über Ziele humangenetischer Forschung und Entwicklung zu sprechen, scheint im Ethik-Rahmen nicht gegeben, sondern über den wissenschaftlichen Fortschritt an sich, wie die deutsche Politikwissenschaftlerin Svea Herrmann anhand der ethischen Debatte um Stammzellforschung in Großbritannien und Deutschland zeigt. Sie analysiert, inwiefern diese Debatte Basis für gesellschaftliche Kontrolle und politische Steuerung bietet. Sie zeigt auf, dass in beiden Ländern Diskurse, die in ethischen Begriffen geführt werden, ein Medium sind, in

4 In diese Richtung gehen weitere Untersuchungen wie die des britischen Politologen Brian Salter und der britischen Sozialgerontologin Charlotte Salter zur Bioethik als »global moral economy« im Kontext embryonaler Stammzellforschung (Salter/Salter 2007; vgl. Salter/Jones 2005). 
dem der Imperativ des wissenschaftlichen Fortschritts nicht in Frage gestellt wird (Herrmann 2009; Herrmann 2011). Mit der Marginalisierung moralischer Reflexion und der Marginalisierung moralischer Probleme durch Ethik befassen sich Untersuchungen zum Verhältnis von Biotechnologie, Politik und Öffentlichkeit der britischen Wissenschaftsforscherinnen und -forscher Alan Irwin (2006), Brian Wynne (2001), Les Levidow und Susan Carr (1997). Sie untersuchen u.a. die Erweiterung von Risiko- durch Ethikdiskussionen im biotechnologischen Agrarbereich. Sie kommen zu dem Schluss, dass die Einbeziehung von Ethik eine öffentliche oder auch eine wissenschaftsinterne moralische Reflexion technisch-wissenschaftlicher Entwicklungen eher verhindert als anregt. Denn in dem von ihnen untersuchten Bereich wird Ethik als von der Wissenschaft getrenntes issue gerahmt. Ethik als Komponente des policy-making am Beispiel der Policy einer Biobank in Schweden untersucht der Anthropologe und Mediziner Klaus Hoeyer. In seinem untersuchten Fall wurde die ethicspolicy aus Gründen des Marketing entwickelt, um mit befürchteter gesellschaftlicher Kritik umzugehen. Darüber hinaus kommt er zu dem Ergebnis, dass die Einbeziehung moralischer Probleme durch die ethicspolicy limitiert wurde (Hoeyer 2005). Um Marginalisierung geht es in einer soziologischen Untersuchung von Alexander Bogner zu nationalen Ethikkommissionen in Österreich, der Schweiz und Deutschland (Bogner 2009). In seiner Untersuchung zur Mikropolitik des Wissens in Ethikräten zeigt er auf, dass gerade Fachethik marginalisiert wird. Um Ausgrenzung bestimmter Akteurinnen und Akteure geht es in der Untersuchung des britischen Soziologen Alfred Moore. Er untersucht public bioethics als Form der deliberativen Demokratie und spezifiziert so die Dynamiken der Demokratisierung von Expertise (Moore 2010). Sein Untersuchungsgegenstand sind public bioethic bodies in Großbritannien. Darunter werden sowohl Institutionen als auch Prozeduren wie nationale Ethikräte, parlamentarische Ethikkommissionen oder öffentliche Anhörungen zu ethischen Themen verstanden, die dazu dienen, über politische Entscheidungsfindung zu informieren. Moore betrachtet das Ausmaß, in dem public bioethics bodies deliberative Ideale realisieren und umgekehrt, was public bioethics über deliberative Demokratie sagen können. Einige Probleme der Expertinnen- und Expertenherrschaft werden in den $p u b$ lic bioethic bodies reproduziert, in dem bspw. verschiedene Öffentlichkeiten kategorisiert werden. Ausgeschlossen sind vor allem »partisan publics « wie Interessengruppen, Lobbyisten und Lobbyistinnen oder Mitglieder von NGOs. Probleme der Expertinnen- und Expertenherrschaft tauchen u.a. auf, da es eine erzieherische Dimension in den Kommissionen gibt, sowohl in Bezug auf die Mitglieder als auch auf die Öffentlichkeit, da von beiden angenommen wird, dass sie wenig Wissen von technischen Aspekten haben. 
Weitere Untersuchungen analysieren Ethikinstitutionen im Hinblick darauf, wie sie die Grenze zwischen Politik und Expertise stabilisieren oder manifestieren. Es handelt sich insbesondere um die Arbeiten der Soziologen Alexander Bogner und Wolfgang Menz ${ }^{5}$ (Bogner et al. 2008; Bogner/Menz 2010). Sie beziehen sich in ihren Untersuchungen auf den von dem US-amerikanischen Soziologen Thomas Gieryn geprägten Ansatz des boundary work (vgl. Gieryn 1983). Allerdings geht es ihnen nicht wie in Gieryns Ansatz darum, wie sich eine Wissenschaft mittels Abgrenzungsaktivitäten durchsetzt, sondern wie sich die Grenze zwischen Expertise und Politik manifestiert. Sie zeigen auf, dass die Empfehlungen, die aus nationalen Ethikräten in Österreich und Deutschland hervorgehen, meist dissensual sind. Der Dissens ist, wie Bogner an andere Stelle ausführt, ein Kennzeichen der Ethisierung. Bogner versteht unter Ethisierung, dass viele technik- und wissenschaftspolitische Fragen als ethische Herausforderungen verstanden werden (Bogner 2013: 51). Im Kontrast zur Moralisierung, in der Wertkonflikte als Wahrheitsproblem verhandelt werden, geht die Ethisierung mit der Anerkennung des Pluralismus und der »Dissensgewissheit« einher (ebd.: 54). Dass Entscheidungsträgerinnen und -träger mit divergierenden Empfehlungen von Expertinnen und Experten umgehen müssen, scheint aber kein Problem im Politikprozess zu sein. Es wird nicht (mehr) erwartet, dass Expertise Wahrheit produziert. Die Funktion des Dissens für die Politik besteht, so Bogner und Menz, darin, Handlungs- und Legitimationsmöglichkeiten zu sichern und zu eröffnen. Für die Politik ist es möglich, Entscheidungen in Bezugnahme auf die Expertise zu legitimieren, sie muss sich aber nicht festlegen. Politik wird so als Politik sichtbar. Es lässt sich eine Grenzziehung zwischen den Bereichen des Beratens und des Entscheidens erkennen (Bogner et al. 2008: 263). Die »Dissensgewissheit«, die Bogner und Menz für die Ethikinstitutionen in Österreich und Deutschland feststellen, trifft in Frankreich nicht zu. Hier sind die Empfehlungen meist konsensual. Handelt es sich deshalb um eine Moralisierung oder ist der Dissens nicht unbedingt Kennzeichen einer Ethisierung im Sinne Bogners?

Diese Ergebnisse, also die Marginalisierung moralischer Reflexion technologischer und wissenschaftlicher Entwicklungen, die Limitierung der Einbeziehung moralischer Probleme durch Ethikpolitiken, die Antizipation von Kritik, die Aus-

5 Vgl. darüber hinaus die Untersuchungen der US-amerikanischen Sozialwissenschaftlerinnen Mary Leinhos (2005) und Susan Kelly (2003). Sie beforschten Ethikinstitutionen auf Staatsebene in den USA im Hinblick darauf, wie sie die Grenze zwischen Wissenschaft und Politik festigen (»border guards«) (Kelly 2003: 358). Sie arbeiten mit dem Ansatz der boundary organizations, der aus dem Ansatz der boundary work nach Gieryn hervorging. Zum Ansatz der boundary organizations vgl. Guston (1999: 90f; 2000: 2). 
klammerung solcher Positionen, die den wissenschaftlichen Fortschritt in Frage stellen, habe ich für den französischen Untersuchungskontext berücksichtigt. Meine Perspektive richtet sich nicht allein auf Marginalisierungen und Ausgrenzungen durch ethische Rahmungen oder Ethikinstitutionen. Im Hinblick auf die Frage, wie sich die ethische Rahmung auf das Thema Nanotechnologie ausdehnen kann, geht es mir auch darum, wie gesprochen werden kann. Darin knüpfe ich an die EGoStudie und die Untersuchung von Dominique Memmi an, die beide aufzeigen, dass es sich um ein offenes, gemäßigtes Sprechen handelt, das, wie insbesondere in der EGo-Studie deutlich wird, auf einem diffusen Verständnis von Ethik basiert. An dieses Ergebnis der EGo-Studie anknüpfend habe ich die Untersuchungsfrage formuliert, ob und inwiefern dieses diffuse Verständnis und die Offenheit ermöglichen, dass der CCNE verstanden als Form des governing at a distance themenflexibel wird.

\section{UNTERSUCHUNGEN ZU ETHIK UND NANOTECHNOLOGIE}

Im Unterschied $\mathrm{zu}$ früheren neuen Technologien wie Nuklearenergie, Informationstechnologie und selbst Biotechnologie zeichnet sich in sozial- und geisteswissenschaftlichen Untersuchungen zur Nanotechnologie ein hohes Maß an »ethicsrelated activities « ab, wie der deutsche Wissenschaftshistoriker und -philosoph Alfred Nordmann und sein niederländischer Kollege Arie Rip feststellen (Nordmann/Rip 2009). Ein ausschlaggebendes Moment für diese Aktivitäten ist der Artikel von Anisa Mnyusiwalla, Abdallah Daar und Peter A. Singer der Universität von Toronto, USA, aus dem Jahr 2003 (Mnyusiwalla et al. 2003). »As the science leaps ahead, the ethics lags behind « - »Mind the gap«, lautet der Appell des Aufsatzes. Um ein Moratorium zu verhindern, das von der nanotechnologiekritischen NGO Action Group on Erosion, Technology and Concentration (ETC Group) gefordert wurde (ETC Group 2003), seien Untersuchungen zu ethischen und sozialen Implikationen nanotechnologischer Entwicklungen notwendig, so die Autorinnen und Autoren. Nicht allein das Moratorium sollte verhindert werden, sondern auch, dass sich wissenschaftliche Entwicklungen verlangsamen (Mnyusiwalla et al. 2003: R9). Mittlerweile existiert eine Fülle an Untersuchungen zur Frage ethischer Aspekte der Nanotechnologie, eine Zeitschrift mit dem Titel »NanoEthics. Ethics for Technologies that Converge at the Nanoscale «, ${ }^{6}$ diverse Forschungsnetzwerke und -projekte. Der Ethik-Boom ist in diesem Bereich so auffällig, dass in Anbetracht

6 Vgl. http://www.springer.com/social+sciences/applied+ethics/journal/11569, 31.03.2013. 
dieser Entwicklung vor einigen Jahren gefolgert wurde: »as ethics leaps ahead [...] current science is left behind « (Nordmann/Rip 2009; vgl. Ebbesen et al. 2006).

Diskutiert wird in den Untersuchungen zur Ethik im Kontext der Nanotechnologie, ob und inwiefern es eine spezifische Nanoethik gibt und/oder wie sie gestaltet werden kann (Grunwald 2004, 2005; Baumgartner 2008), und auch ob eine spezifische Nanoethik oder Nanoethiker notwendig wären (Holm 2014). Diskutiert wird zudem, welche Lehren aus der Biotechnologie und ihrer Kontroversen gezogen werden können. Und da sich bisher wenige nationale Ethikkomitees mit dem Thema Nanotechnologie beschäftigt haben, gibt es Untersuchungen dazu, welche Aufgaben sie in diesem Bereich übernehmen sollten: Untersuchungen, die danach fragen, welche Lehren für die Nanotechnologie aus den Erfahrungen mit der Biotechnologie (meist im Agrarbereich) gezogen werden können, richten ihren Fokus vor allem darauf, wie gesellschaftliche und ethische Aspekte so früh wie möglich auf die wissenschaftliche und technische Agenda gesetzt werden können (Kearnes et al. 2006; Macnaghten et al. 2005; Kenneth/Thompson 2008; Kuzma/Besley 2008; Sandler 2014). Die kanadischen Kommunikationswissenschaftlerinnen Edna Einsiedel und Linda Goldenberg meinen bspw.: »If there is anything that the evolutionary path of biotechnology has taught us, it is the greater danger of keeping the social at bay when developing or discussing technology« (Einsiedel/Goldenberg 2004: 32; Mehta 2004). Auch vom ELSI-Programm soll gelernt werden. Das Programm ELSI wird im Jahr 1990 im Kontext der Humangenomforschung zur Untersuchung der Ethical, Legal and Social Implications vom US-amerikanischen National Human Genome Research Institute entwickelt. ${ }^{7}$ Wie bereits im Kontext der Humangenomforschung sollen ethische und soziale Implikationen ebenfalls im Kontext der Nanotechnologie beforscht werden, um die Ergebnisse in Forschung und Entwicklungen zu integrieren (Fisher 2005). Der Ansatz, ethische und soziale Themen in nanotechnologische Forschung und Entwicklung zu integrieren, findet sich darüber hinaus in von der Europäischen Kommission geförderten Projekten wie dem Projekt Deepening Ethical Engagement and Participation in Emerging Nanotechnologies (DEEPEN) oder dem Projekt The Ethical GovernAnce of emergIng technologieS (EGAIS). Ein Ziel von DEEPEN, das in den Jahren 2006-2009 durchgeführt wird, ist es, Empfehlungen zu entwickeln, »for articulating and deliberating ethical reflection in nanoscience practice and governance processes « (DEEPEN o.J.). Auch bei EGAIS, durchgeführt in den Jahren 2009-2012, liegt ein Ziel darin, Empfehlungen über »good practices« zu entwickeln. Ein Schwerpunkt der von EGAIS untersuchten emerging technologies sind Nanotechnologien. In

7 Insbesondere die ELSI-Forschung in den USA war von Bioethikerinnen und Bioethikern sowie von Juristinnen und Juristen geprägt (vgl. Hedgecoe/Martin 2008: 829f). 
dem Projekt wird untersucht, wie ethische Fragen in bestehenden institutionellen und organisatorischen Bedingungen von Technikfolgenabschätzungsprozeduren aufgenommen und angesprochen werden können (Rainey/Goujon 2011). Ein Fokus liegt zudem darauf, wie ethische und soziale Themen bereits in die Umsetzung von Forschungsprojekten integriert werden können (Rainey et al. 2012). Zu den Empfehlungen zählt, dass ethische Themen durch eine breite Partizipation in Foren berücksichtigt werden sollen (Forscherinnen und Forscher, soziale Akteurinnen und Akteure, politische Entscheidungsträgerinnen und -träger, Industrielle etc.). Eine »dialogical, open, legitimate and effective force « ist das Merkmal dieser ethical governance (ebd.: 144).

Von EGAIS wurden zudem verschiedene Ethikinstitutionen und Verfahren evaluiert, darunter nationale Ethikkomitees. Sie werden als ein Element ethischer Steuerungsinstrumente verstanden. Wichtig sei, die Öffentlichkeit breiter einzubeziehen und gerade die Rolle ethischer Expertise öffentlich zu kommunizieren. Diese Expertise solle nicht eine Quelle der Normativität sein oder als Instanz verstanden werden, die zwangsläufig das letzte Wort hat. Ethik-Expertise in Form nationaler Komitees solle vielmehr eine Möglichkeit darstellen, einen ethischen Diskurs zu provozieren (ebd.: 134f). Die Frage der aktiven Beteiligung zeigt sich in einer weiteren Untersuchung zu nationalen Ethikkomitees im Kontext neu entstehender Wissenschaften und Technologien wie Bio-, Nano-, Informations- und Kommunikations- oder Neurotechnologien. Der slowenische Wissenschaftsforscher Franc Mali, der deutsche Politologe Christopher Coenen und ihre Kolleginnen und Kollegen untersuchten die Rolle und Funktion nationaler Ethikkomitees in Bezug auf die Steuerung ethischer Kontroversen. Sie analysierten 32 nationale Ethikkomitees (EABs - National Ethics Advisory Bodies) in Europa. Zentral in ihrer Studie ist die Frage, ob es sich bei den EABs um klassische technokratische Expertinnen- und Expertengremien handelt oder ob sie eine frühe gesellschaftliche Mitgestaltung von Wissenschaft und Technologie ermöglichen, die durch solche partizipatorische Mechanismen ermöglicht wird, die verantwortliche Akteurinnen und Akteure hervorbringen »with respect to the ethical acceptability, sustainability and societal desirability of the progress of science and technology« (Mali et al. 2012: 168). Im Kontext der Ethikkomitees stellen sie Defizite fest: »There are many deficiencies with regard to public participation in the work of EABs. [...] Many new and emerging issues in science and technology are highly complex, requiring active scientific citizenship to achieve meaningful participation« (ebd.: 181), so das Ergebnis.

Eine Randerscheinung im Kontext der Forschung zu Ethikinstitutionen, die sich mit Nanotechnologie beschäftigen, ist die Untersuchung von Alfred Nordmann und seiner Kollegin Astrid Schwarz. Sie beschäftigen sich mit der kanadi- 
schen Ethikkommission und ihrer Empfehlung zu Nanotechnologie (Nordmann/Schwarz 2010: 364). Eine Randerscheinung ist die Untersuchung insofern, als Nordmann und Schwarz nicht anwendungsorientiert untersuchen, was die Aufgabe dieser Institutionen sein sollte. Die Empfehlung der Ethikkommission sehen sie als eine von mehreren charakteristischen Beispielen anhand derer sie ein Rahmenwerk entwerfen, um die Frage der Macht in gegenwärtigen Regimen der Wissensproduktion im Kontext der Nanotechnologie zu untersuchen. Rekurrierend auf den französischen Philosophen und Soziologen Jean Baudrillard, sehen sie in diesen gegenwärtigen Regimen der Wissensproduktion insbesondere eine »Power of Seduction « (ebd.: 364). Anhand der Empfehlung der Ethikkommission zeigen sie auf, dass dieser ein eher schwacher Begriff von Ethik zugrunde liegt: Grenzen nanotechnologischer Entwicklungen werden nicht spezifiziert, Verpflichtungen oder Verbote nicht benannt. Zwar wird in der Publikation der kanadischen Kommission thematisiert, dass wenig über die Nanotechnologie bekannt sei, indem sie allerdings im Rahmen einer »responsible development« dargestellt wird, erscheint ein Nein zu nanotechnologischen Entwicklungen unmöglich. Insofern entstehe ein »lure of the Yes«, eine Art »zwangloser Zwang « (ebd.: 259/Herv. i.O.). Einerseits zeigt diese Untersuchung, dass Ethik ähnlich dem diffusen Verständnis im Kontext der Biotechnologie und -medizin auch im Kontext der Nanotechnologie ein schwaches Verständnis zugrunde liegt, und dass es kaum möglich scheint, sich gegen diese Entwicklungen zu stellen. Andererseits hat diese Kommission jedoch das Mandat, sich mit ethischen Themen wissenschaftlicher und technologischer Entwicklungen zu beschäftigen und nicht explizit, wie im Kontext des CCNE, mit den Lebenswissenschaften. Hier stellt sich in Anknüpfung an Memmis Untersuchung weiterhin die Frage, ob und inwiefern eine Ausdehnung der ethischen Rahmung in den Bereich der Nanotechnologie möglich ist, weil sie als gesundheitsrelevantes Thema verstanden wird.

In den $»^{\mathrm{NANO}_{-} \mathrm{STS}}$ « (Baird et al. 2004:1) scheint sich eine Tendenz abzuzeichnen, Ethik unhinterfragt als selbstverständlichen Bestandteil wissenschaftlicher und technologischer Entwicklungen aufzufassen. Ethische Themen sollen so früh wie möglich in die Steuerung wissenschaftlicher und technologischer Entwicklungen integriert werden; es wird untersucht, wie nationale Ethikinstitutionen verbessert werden können. Kritisiert wird solch eine (implizit) normative Tendenz in den STS von dem französischen Wissenschaftshistoriker Dominique Pestre. Er fordert dazu auf, diese Tendenz zu hinterfragen. Seine Kritik und seine Aufforderung formuliert er allerdings nicht in Bezug auf Ethikinstitutionen oder auf Diskurse um Ethik, sondern in Bezug auf die Diskurse um Partizipation. Jedoch, so möchte ich behaupten, lässt sich diese Aufforderung auf meine Untersuchung übertragen. Inwiefern meine Untersuchung an diese Aufforderung anknüpft und als Beitrag in 
den STS verstanden werden kann, Ethik-Rahmungen (selbst-)kritisch zu hinterfragen, wird im Folgenden ausgeführt. Dafür werde ich zunächst Pestres Kritik im Kontext der Partizipation erläutern.

\section{ZUR KRITISCHEN DISKUSSION NORMATIVER TENDENZEN IN DEN SCIENCE AND TECHNOLOGY STUDIES}

Das wachsende Interesse an Fragen der Partizipation von nicht-wissenschaftlichen Akteurinnen und Akteuren ist in den STS nicht erst in den letzten Jahren aufgekommen. Es entwickelt sich aus einer bestimmten historischen Konstellation vor dem Hintergrund der Wissenschaftspolitik der Nachkriegszeit, so der schweizerische Wissenschaftshistoriker Martin Lengwiler (2008: 193). ${ }^{8}$ Als ein Faktor für diese Entwicklung kann die Technologiekritik neuer sozialer Bewegungen der 1960er und 70er Jahre, wie sie etwa von Seiten der Frauen-, der Anti-Atom- oder der Umweltbewegung formuliert wird, gesehen werden. Diese Kritiken führen bereits in den 1970er Jahren zu ersten Formen der Partizipation in der Wissenschaftsund Technologiepolitik. Parallel gerät die Wissenschaftspolitik der Nachkriegszeit zunehmend unter Druck: Die »honeymoon period of public funding « geht dem Ende entgegen (ebd.: 193f). Mit ihr, so die US-amerikanische Wissenschaftsforscherin Sheila Jasanoff, endet auch die Vorstellung des Pipeline-Modells: die Vorstellung, dass technologische und wissenschaftliche Entwicklungen über kurz oder lang zu nationalem Wohlstand und Fortschritt führen, sowie die Annahme, dass die Umsetzung von Grundlagenwissen in Anwendungen linear verlaufe und als unproblematischer Prozess anzusehen sei (Jasanoff 2003: 227f). Das »speaking truth to power model«, das Modell, in dem wissenschaftliches Wissen als wahr und objektiv angesehen wird, wird als überholt angesehen. Als Grund werden Fehler in den selbstregulierenden Mechanismen von Forschung und Wissenschaft gesehen, die sich in einer Reihe an Forschungsskandalen zeigten, sowie ein wachsender Vertrauensrückgang der Öffentlichkeit in wissenschaftliche Expertise und in eine expertinnen- und experten-basierte Wissenschaftspolitik. Der Vertrauensrückgang ist durch diverse Krisen, wie die Reaktorkatastrophe in Tschernobyl Mitte der 1980er Jahre oder die BSE-Krise in den 1990ern beeinflusst (Lengwiler 2008: 194; Jasanoff 2003: 223; Callon 1999: 38f). Jasanoff schreibt: »The wider public responsibilities of science, as well as changes in modes of knowledge-making, de-

8 Zur historischen Entstehung partizipatorischer Fragen in der Wissenschaftspolitik ab dem späten 19. Jahrhundert vgl. Lengwiler (2008). 
mand new forms of public justification « (Jasanoff 2003: 226). Mittlerweile wird von einem participatory turn gesprochen (ebd.). Es ist gegenwärtig anerkannt, sei es von Bürgerinnen und Bürgern, NGOs, Regierungen oder der Wissenschaft, dass »increased participation and interactive knowledge-making may improve accountability and lead to more credible assessments of science and technology « (ebd.: 243). Zwar haben sich eine Reihe kritischer Studien zu partizipatorischen Praxen entwickelt, zur Frage, wie die Öffentlichkeit oder auch Problemdefinitionen konstruiert und Themen gerahmt sind und welche Wirkung dies auf den Output, die Empfehlungen und Lösungen hat, kurz zu Defiziten und Begrenzungen partizipatorischer Praxen (vgl. u.a. Irwin/Wynne 1996; Irwin 2001; Wynne 2001; Levidow/Carr 1997; Fischer 2000; Braun/Schultz 2010; Felt/Fochler 2010; Stirling 2008; Wehling 2012; Stilgoe et al. 2014). Jedoch bleibt Partizipation ein mehrheitlich unhinterfragtes, auch als hegemonial bezeichnetes Konzept im Bereich der STS (Braun/Schultz 2010; Pestre 2008).

Einer der prominentesten Kritiker dieses unhinterfragten Konzeptes in den STS ist der französische Wissenschaftshistoriker Dominique Pestre. Seine Kritik im Kontext der Partizipation richtet sich insbesondere auf das Werk zur »technical democracy« der französischen Soziologen Michel Callon, Pierre Lascoumes und Yannick Barthes (2009). Die Autoren entwickelten das Konzept der »hybriden Foren « - hybrid bezeichnet hierbei die Heterogenität der Teilnehmenden eines Forums (Expertinnen und Experten, Politikerinnen und Politiker, Laien etc.). Sie zielen mit ihrem Konzept auf eine dialogische Demokratie, die die delegierte Demokratie prozedural bereichern könnte. Pestre interpretiert die Haltung der Autoren und insgesamt die Tendenz in den STS insofern als (implizit) normativ, als sie mittels Partizipation den besten oder einen besseren Weg suchen, mit technologischen und wissenschaftlichen Fragen umzugehen. Dieser Fokus auf die besten Prozeduren der Partizipation verstelle den Blick darauf, dass in marktorientierten Demokratien partizipatorische Prozesse bzw. eine dialogische Demokratie für technowissenschaftliche Regulierungen nicht zentral sind, sondern immer noch ökonomische Akteure und Akteurinnen wie die Weltbank, die außerhalb einer dialogischen Ordnung stehen (Pestre 2008: 103ff). Unberücksichtigt bleibe zudem, dass es sich bei partizipatorischen Mechanismen um Regierungsinstrumente handelt (Pestre 2004b: 363f; 2004a: 3). Auf diesen blinden Fleck weist Pestre in Anlehnung an das Werk »Der neue Geist des Kapitalismus« der französischen Sozialwissenschaftlerin Ève Chiapello und des Sozialwissenschaftlers Luc Boltanski und deren Konzept von Kritik hin (Boltanski/Chiapello 2003). Boltanski und Chiapello sehen Kritik als einen Motor für Transformationen des Kapitalismus. Kritik fordert den Geist des Kapitalismus heraus, bringt ihn aber auch voran, indem sie vereinnahmt wird. Pestre meint, es werde in den STS übersehen, dass die einstige Kritik an 
Technokratie und die Forderung nach Partizipation und Dialog in Regierungsinstrumente transformiert wurde: »[S]uch demands for dialogic democracy and the questioning of expert knowledge (notably promoted by STS) have today become cynically instrumentalized on an unprecedented scale by people who use them as an instrument of government « (Pestre 2004a: 3). Dieses Regierungsinstrument, das er in Anlehnung an die Gouvernementalitätsstudien als zentrales Element einer neoliberalen Gouvernementalität sieht, »stresses the duty of self-governance but also develops unprecedented (mainly economic and managerial) procedures which bear on many kinds of subjects - consumers, patients, workers, professionals, etc. « (Pestre 2008: 103/Herv. i.O.; vgl. Blondiaux/Sintomer 2002: 33). Pestre ruft dazu auf, gerade diese Gouvernementalität zu untersuchen, die implizit normative Tendenz in den STS (selbst-)kritisch zu betrachten und danach zu fragen, inwiefern die STS-Diskurse um Partizipation, auch um Dialog und governance, Teil dieser neoliberalen Gouvernementalität sind (Pestre 2004a; vgl. Braun 2009). ${ }^{9}$ Diese (Selbst-)Kritik soll nicht dazu führen, sich von Konzepten der Partizipation oder des Dialogs zu verabschieden: »keeping the space of dialogue alive - but at the same time being able to analyze, suggest, and support competing forms of politics that are based on the premise that this space of dialogue is a decoy and often constitutes just another form of government of people and things « (Pestre 2004a: 4). Zweck - »What should be the future shape of our society? « - und Mittel - »What should be the democratic tools? « - sollten immer mitgedacht werden (Pestre 2009a: 62). Für solch eine kritische Analyse schlägt Pestre eine genealogische Herangehensweise vor, denn »[i]n short, genealogical analysis aims to question the >givens $<$ of a present that has forgotten the underlying basis of its origins, in other words the historical circumstances that made these values socially obvious « (Pestre 2009b: 253f/Herv. i.O.).

An Pestres Aufforderung zur Untersuchung dieses blinden Flecks knüpft meine Studie an. Allerdings liegt mein Hauptaugenmerk nicht auf Diskursen um Partizipation: Nicht allein Partizipation und Dialog haben sich in den STS zu dominanten Diskursen entwickelt, welchen eine bessere, legitimere Art zugeschrieben wird, technologische und wissenschaftliche Entwicklungen zu steuern. Ethik, insbesondere im Kontext der Nanotechnologie, fügt sich m.E. in diese Reihe ein - sie wird gefordert, erwartet, untersucht. Ihr wird eine »dialogical, open, legitimate and effective force« zugeschrieben (Rainey et al. 2012: 144). Mit ihr ist eine aktive

9 Dazu, inwiefern Praktiken der Partizipation im Diskurs der Wissensgesellschaft innerhalb einer neoliberalen Gouvernementalität verstanden werden können, vgl. insbesondere die Publikation des Soziologen Torsten Junge »Gouvernementalität der Wissensgesellschaft (Junge 2008). 
Bürgerinnen- und Bürgerschaft verbunden (Mali et al. 2012: 181). Eine frühe gesellschaftliche Mitgestaltung von Wissenschaft und Technologie, die durch nationale Ethikkomitees realisiert werden kann, wird als Kennzeichen und Gegengewicht zu klassischen technokratischen Expertinnen- und Expertengremien gesehen (ebd.: 168). Die Forderungen oder Empfehlungen der Nano-STS nach mehr Ethik und Partizipation zeigen sich auf politisch-institutioneller Ebene: Von der Europäischen Kommission oder auch von nationalen Ethikkomitees wird eine frühe Einbeziehung der Gesellschaft, der Dialog mit der Öffentlichkeit unter Berücksichtigung ethischer Themen gefordert (vgl. bspw. Kommission der europäischen Gemeinschaften 2004; CCNE 2007).

Der participatory turn beginnt mit einer Technologiekritik von Seiten neuer sozialer Bewegungen. Wie Pestre ausführt, wird diese einstige Kritik an Technologie und Technokratie und die Forderung nach Partizipation, gefördert durch die STS, in Regierungsinstrumente transformiert. Die Diskurse um Ethik und Partizipation sind zwar verknüpft, aber welche Kritik und welche Forderungen werden hier transformiert? Indem in dieser Untersuchung herausgearbeitet wird, wie sich der CCNE, verstanden als Regierungsinstrument, herausbildet und insbesondere welche Problematisierungen und welche Lösungsvorschläge sozialer Proteste in diesem Prozess verloren gehen oder unsichtbar werden, kann sie, in einem übergeordneten Rahmen betrachtet, zu einer (selbst-)kritischen Diskussion innerhalb der STS beitragen. Die vorliegende Studie zeigt also am Beispiel des CCNE auf, wie das, was in den Nano-STS selbstverständlich gefordert wird, nämlich ein Mehr an Ethik, das als legitim gilt, überhaupt selbstverständlich werden konnte und welche Wirkungen diese Entwicklung hat.

Zwar verfolge ich, wie auch Pestre vorschlägt, eine genealogische Herangehensweise, jedoch gehe ich nicht davon aus, dass es sich beim CCNE als Regierungsinstrument per se um eine Form der neoliberalen Gouvernementalität handeln muss. Auf das Konzept der Gouvernementalität und die genealogische Herangehensweise wird im folgenden Kapitel zum analytisch-methodischen Rahmen eingegangen. 


\section{Analytisch-methodischer Rahmen: Das Konzept der Gouvernementalität und die Genealogie}

Für die Studie wird die regierungsanalytische Perspektive der Gouvernementalität übernommen, wie sie in Anlehnung an Michel Foucault von den Gouvernementalitätsstudien (GS) fruchtbar gemacht wurde. Verknüpft habe ich diese, ebenfalls in Anlehnung an Foucault, mit einer genealogischen Herangehensweise. Letztere wird mit diskursanalytischen Konzepten der post-positivistischen interpretativen Policy-Analyse kombiniert. Im Folgenden stelle ich zuerst die GS, das Konzept der Gouvernementalität wie auch ein wesentliches Konzept der GS, die Problematisierung vor, das die Vorgehensweise der Untersuchung verdeutlicht. Daran anschließend werden die genealogische Herangehensweise präzisiert und weitere Konzepte der Studie ausgeführt wie die Frame-Analyse und die Ethisierung. Im Anschluss daran stelle ich die Konzepte des governing at a distance von Miller und Rose und des reflexive government von Dean vor sowie das Konzept der delegierten Biopolitik von Memmi und führe aus, wie sie in der Untersuchung verwendet werden.

\section{Die Gouvernementalitätsstudien}

Der französische Philosoph, Historiker und Sozialwissenschaftler Michel Foucault (*1926-†1984) stellt das Konzept der gouvernementalité - Gouvernementalität ${ }^{1}$ gegen Ende seines Schaffens in seinen Vorlesungen vor, die er in den Jahren 1978-

1 Verwendet wird der Begriff bereits in den 1950er Jahren von dem französischen Poststrukturalisten Roland Barthes (*1915-†1980). Foucault übernahm den Begriff, löste ihn aber aus seinem semiotischen Kontext (Bröckling et al. 2011: 1). 
1979 am Collège de France in Paris abhält (Sennelart 2004a, b). ${ }^{2}$ In diesen Vorlesungen zur Genealogie des modernen Staates geht Foucault dessen Spuren von der griechischen Antike bis zum modernen Neoliberalismus nach.

In den 1970er und 80er Jahren erscheinen einige Forschungsarbeiten von Foucault-Schülerinnen und -Schülern, die sich direkt auf Foucaults Vorlesungen beziehen und die begleitend zu den Seminaren am Collège de France durchgeführt wurden. Die foucaldiens und foucaldiennes, wie Foucault-Schülerinnen und -Schüler im französischen Sprachraum gerne genannt werden, beschäftigten sich vor allem mit der sich verändernden Gouvernementalität im 19. Jahrhundert (Lemke et al. 2000: 17; Bröckling et al. 2011: 8). Ab den 1990er Jahren breiten sich Studien zur Frage der Gouvernementalität über die Grenzen Frankreichs hinaus aus. Diese Expansion findet vor allem im englischen Sprachraum statt, initiiert durch die Publikation »The Foucault Effect. Studies in Governmentality« im Jahr 1991 (Burchell et al. 1991). In Anlehnung an Foucaults Konzept der Gouvernementalität bilden sich im Anschluss die Governmentality Studies - Gouvernementalitätsstudien heraus und verhelfen so dem Konzept auch international zu einiger Prominenz. ${ }^{3}$ Ab Ende der 1990er Jahre zieht das Konzept der Gouvernementalität auch in Deutschland zunehmendes Interesse auf sich. Es entsteht eine Vielzahl an Studien in einem Spektrum von Disziplinen, das von der Soziologie über die Erziehungs- und Geschichtswissenschaften bis hin zur Politologie reicht (Bröckling et al. 2011: 10). Auch in Frankreich, wo Untersuchungen in Anknüpfung an Foucault lange Zeit marginal waren, entwickelt sich in den letzten Jahren eine wachsende Aufmerksamkeit für sein Werk (ebd.), wenn auch nicht mit demselben Schwerpunkt auf dem Konzept der Gouvernementalität wie im deutschen und englischen Sprachraum, sondern auf Formen der Biopolitik. ${ }^{4}$ Genannt seien hier insbesondere die Arbeiten zu Gesundheit, Bioethik, Körper, Geburt und Tod des Anthropologen und Soziologen Didier Fassin, der Politikwissenschaftlerin Dominique Memmi und von ihren Kollegen und Kolleginnen (vgl. u.a. Fassin 2011; Fassin/Memmi 2004; Memmi 2003a, 1999, 2000, 2004).

2 Zum ersten Mal formuliert er den Begriff der Gouvernementalität in der vierten Vorlesung im Jahr 1978 (Foucault 2004 (1978)-a).

3 Vgl. u.a. die Arbeiten von Mitchell Dean (1999a), Wendy Larner (2000), Nikolas Rose (1993; 1996; 1999), Rose et al. (Rose/Miller 1990; Barry et al. 1993, 2001) sowie Graham Burchell (1993). Für einen Überblick vgl. Thomas Osborne (2004) und Thomas Lemke (2000).

$4 \mathrm{Zu}$ der Frage, wie es kommt, dass sich die Governmentality Studies vorrangig im englischen Sprachraum herausgebildet haben, vgl. das Interview von Jacques Donzelot mit Colin Gordon (2008). 
Die GS sind weniger historisch-genealogisch ausgerichtet und richten ihren Blick vor allem auf die gegenwärtigen gesellschaftlichen Transformationsprozesse, insbesondere auf die sich seit den 1970er Jahren herausbildende neoliberale Gouvernementalität (Bröckling et al. 2011: 9; Lessenich 2003: 80; Lemke et al. 2000: 7). Gemeinsam ist den GS, dass sie auf Foucaults Begriff der Regierung zurückgreifen, wie er ihn in seinen Vorlesungen zur Gouvernementalität weiterentwickelt hat.

\subsection{Das Konzept der Gouvernementalität}

Mit dem Konzept der Gouvernementalität reflektiert Foucault selbstkritisch seine eigene, wie er 1977 meint, bis dahin traditionelle Auffassung von Macht (Foucault 2005b: 126). Er distanziert sich damit sowohl vom juridischen Machtkonzept als auch von der Hypothese Nietzsches. Dies bedeutet, er analysiert Macht bis dahin vor allem in Begriffen von »Kampf, Krieg und Eroberung « oder Konfrontation (Lemke et al. 2000: 8; Lemke 1997: 104; Bröckling et al. 2011: 1; Sennelart 2004a: 528f). Foucault zufolge war das juridische Machtkonzept insofern defizitär, da es »weder der Komplexität der Mechanismen noch ihrer Spezifizität« Rechnung trägt (Foucault 2005b: 131; Lemke 1997: 100). Nach Foucaults Auffassung geht es gerade darum, der historischen Veränderung der Machtmechanismen theoretisch nachzukommen. Die politische Analyse sollte sich von der Vorstellung der Souveränität, des rechtlichen Codes und der im Mittelpunkt stehenden Figur des Königs lösen (s. Lemke 1997: 107; Sennelart 2004a: 553). Foucault formuliert es folgendermaßen: »Im politischen Denken und in der politischen Analyse ist der Kopf des Königs noch immer nicht gerollt« (Foucault 1999: 110). Ein Schwerpunkt der Weiterentwicklung der Machtanalyse ist der Begriff der Regierung (Sennelart 2004a: 554, 565f; Lemke et al. 2000: 8). ${ }^{5}$ Die deutschen Sozialwissenschaftlerinnen und

5 Foucaults Machtanalytik und ihre Entwicklung wird in der Diskussion um sein Werk teils als Weiterentwicklung verstanden, teils auch als Korrektur, als Paradigmenwechsel, als Wende und als »theoretische Neuorientierung « (Lemke 2000: 7f; Muhle 2008: 264ff). Im deutschen und englischen Sprachraum ist im Kontext von Foucaults Untersuchung des Liberalismus auch von einem Bruch die Rede (Muhle 2008: 266). Diese Diskussion soll an dieser Stelle nicht aufgegriffen werden. Ich schließe mich der von Lemke et al. beschriebenen Weiterentwicklung sowie der Ausführung des französischen Philosophen Michel Sennelart an. Sennelart spricht von einer »Verschiebung von der Macht zur Regierung« und somit der »Ausdehnung auf ein neues Objekt«, den Staat (Sennelart 
-wissenschaftler Thomas Lemke, Susanne Krasmann und Ulrich Bröckling schreiben, es handle sich insofern um eine »neue Dimension« der Machtanalyse, da Machtbeziehungen in der Perspektive der »Führung « untersucht werden (Lemke et al. 2000: 8).

»F Führung $<$ heißt einerseits, andere (durch mehr oder weniger strengen Zwang) zu lenken, und andererseits sich (gut oder schlecht) aufzuführen, also sich in einem mehr oder weniger offenen Handlungsfeld zu verhalten. Machtausübung besteht darin, >Führung zu lenken<, also Einfluss auf die Wahrscheinlichkeiten von Verhalten zu nehmen« (Foucault 2005c: 256/Herv. i.O.).

Führung bedeutet somit sowohl, andere zu lenken als auch, sich in einem Handlungsfeld zu verhalten (Selbstführung).

Die Bedeutung, in der Foucault Regierung versteht, kommt in dieser Form im 16. Jahrhundert auf (Foucault 2005c: 256). Foucault geht von der These aus, dass sich der moderne westliche Staat aus einer komplexen Verbindung von »politischen « und »pastoralen« Machttechniken entwickelt hat (Lemke et al. 2000: 10f). Unter Pastoralmacht begreift er die christliche Konzeption von der Beziehung zwischen Hirt und Herde (ebd.: 11), die sich ab dem zweiten, dritten Jahrhundert nach Christus zu entwickeln beginnt (Foucault 2004 (1978)-b: 218; 2004 (1978)-c: 240; vgl. Sennelart 2004a). Sie ist auf das Seelenheil ausgerichtet, die Sorge um das Heil im Jenseits (Foucault 2005c: 248). Die Eigenheit des christlichen Pastorats besteht in der »Entwicklung von Analysemethoden, Reflexions- und Führungstechniken « (Lemke et al. 2000: 11). Zentral ist die Technik der Beichte. Die Führungsinstanz, der Pastor, kümmert sich um die Gemeinschaft ebenso wie um jeden Einzelnen in der »Herde « (Foucault 2005c: 247f). Er ist qua kirchlichem Amt Vermittler zwischen Gott und Individuum. Die pastorale Machtausübung »setzt voraus, dass man das Bewusstsein des Einzelnen kennt und zu lenken vermag « (ebd.: 248). ${ }^{6}$ Diese Art der Führung setzt zudem ein bestimmtes Wissen über den oder die zu Führende voraus. Dieser oder diese unterliegt einer Verpflichtung zur Wahrheit und zur Selbsterkenntnis, um das Heil im Jenseits, das ewige Leben zu erreichen:

2004a: 554). Mit Sennelart verstanden, erweiterte sich Foucaults Perspektive, und es entfaltet sich ein neues Forschungsfeld: die Genealogie des modernen Staates (ebd.: 551ff).

6 Foucault spricht im Kontext des Pastorats nicht allein von der Kunst des Führens bzw. von führen (frz. conduire) oder von lenken (frz. diriger), sondern auch von leiten (frz. mener) oder anleiten (frz. guider) (vgl. Foucault 2004 (1978)-c: 241). 
»Jeder hat die Pflicht zu erkennen, wer er ist, das heißt, er soll ergründen, was in ihm vorgeht, er muss versuchen, Fehler, Versuchungen und Begierden in sich selbst ausfindig zu machen, und jedermann ist gehalten, diese Dinge entweder vor Gott oder vor den anderen Mitgliedern der Gemeinschaft zu enthüllen, also öffentlich oder privat gegen sich selbst auszusagen« (Foucault 1993b: 52).

Die Führungstechniken, die sich innerhalb des Christentums entwickeln, so Foucault, dehnen sich ab dem 16. Jahrhundert aus und säkularisieren sich; sie treten in die Politik ein (Foucault 2004 (1978)-d: 334ff). Foucault nimmt in seiner Regierungsanalyse an, »dass die pastoralen Führungstechniken Subjektivierungsformen hervorbrachten, auf denen der moderne Staat und die kapitalistischen Gesellschaften aufbauten« (Lemke et al. 2000: 11). Ab dem 18. Jahrhundert sind Pastoralmacht und politische Macht nicht mehr strikt getrennt, wie Foucault in »Subjekt und Macht« sagt: Der Machttyp des Pastorats findet sich modifiziert in verschiedenen Bereichen wie Familie, Medizin, Bildungswesen, Psychiatrie oder in Arbeitsverhältnissen (Foucault 2005c: 249). Die Überlagerung der politischen Macht durch die Pastoralmacht geht mit einer gewissen Transformation von Regierung einher. Dies führen Lemke et al. anhand der Wesenszüge der säkularisierten Pastoralmacht aus: »Das Eigentümliche dieser spezifisch modernen Formen von Regierung - der Regierung von Menschen im Unterschied zur Regierung der >Seelen < besteht zum einen in der Notwendigkeit einer Reflexion auf die Voraussetzungen, den Gegenstand und die Ziele von Regierung (Lemke et al. 2000: 11/Herv. i.O.). Und das Eigentümliche der modernen Formen von Regierung bestehe zum anderen darin, dass vormals religiöse Zielsetzungen, wie Glück, Heil oder Wohlstand innerhalb einer politischen Problematik des Staates neu formuliert werden (ebd.). Es geht nun nicht mehr um das Heil im Jenseits, sondern um das irdische Heil, das nun verschiedene Bedeutungen annimmt, wie »Gesundheit, Wohlergehen (im Sinne eines angemessenen Lebensstandards und ausreichender Ressourcen), Sicherheit und Schutz vor Unfällen aller Art (Foucault 2005c: 248f). In diesem Kontext spricht Foucault von einer »komplexe[n] Verbindung zwischen Techniken der Individualisierung und totalisierenden Verfahren« (ebd.: 247). Der moderne Staat ist sowohl eine rechtlich-politische Struktur als auch eine neue Form der Pastoralmacht (Lemke et al. 2000: 12).

Foucault versteht den Staat somit als »Resultante gesellschaftlicher Kräfteverhältnisse« (vgl. ebd.: 8). Er untersucht moderne Staatlichkeit nicht innerhalb einer Logik der einheitlichen historischen Entwicklung mit einem Verständnis, das den Staat als institutionelle-administrative Struktur begreift, sondern analysiert die heterogenen und diskontinuierlichen » Regierungskünste«« (ebd.: 10/Herv. i.O.). 


\title{
1.2 Die Perspektive der Gouvernementalität
}

Foucaults Arbeit zur Gouvernementalität blieb aufgrund seines frühen Todes fragmentarisch. Den im Anschluss an Foucaults Werk entstandenen GS ist insbesondere gemeinsam, dass sie nach den Regierungskünsten fragen, mit einem weit gefassten, über den Bereich staatlicher Intervention hinausgehenden Regierungsbegriff im Sinne Foucaults. In diesem Sinn eint sie vor allem die Forschungsperspektive auf Regierung. Sie verfügen nicht über ein integriertes Forschungsprogramm oder ein einheitliches Methodeninventar, sondern greifen auf Foucaults »Werkzeugkiste« zurück (Foucault 1976: 53; Bröckling/Krasmann 2010: 32). Ein Instrument aus dieser Kiste ist das Konzept der Problematisierung, das ich in meine Untersuchung aufnehme.

\subsubsection{Das thinking problematically}

\begin{abstract}
»Gouvernementalitätsanalysen präparieren heraus, wie sich bestimmte Annahmen darüber, was als wahr bzw. vernünftig anzuerkennen ist, mit spezifischen Problemdiagnosen verbinden, [sic!, mit, S.K.] denen wiederum spezifische Strategien zu ihrer Bewältigung korrespondieren, an die dann bestimmte Techniken und Verfahren anschließen, die ihrerseits neue Objekte und Subjekte des Regierens hervorbringen können« (Bröckling/Krasmann 2010: 24).
\end{abstract}

In einem Artikel aus dem Jahr 1970 fordert Foucault dazu auf: »think problematically« (Foucault 1998 (1970): 359). Foucault verwendet das Konzept der Problematisierung in zweierlei Hinsicht. Er beschreibt damit zum einen seine »Analysebewegung « (Foucault 2005 (1984): 733). Zum anderenr verwendet er den Begriff, um auf den historischen Prozess Bezug zu nehmen, der Objekte des Denkens produziert (Bacchi 2012: 1; vgl. Lemke 1997: 339ff). Foucault sagt, bei der Problematisierung handelt es sich um »eine kritische Analysebewegung, über die versucht wird herauszufinden, wie die verschiedenen Lösungen für ein Problem erstellt werden konnten, aber auch, wie diese verschiedenen Lösungen zu einer spezifischen Problematisierungsform gehören« (Foucault 2005 (1984): 733). Es geht ihm nicht darum, die eine richtige Antwort auf ein Problem zu finden, sondern zu untersuchen, wie ein bestimmtes Thema zu einem bestimmten Zeitpunkt und unter bestimmten Bedingungen hinterfragt, analysiert, klassifiziert und reguliert wird (Bacchi 2012: 1). Um bspw. den Status französischer Gefängnisse in Frage zu stellen, betrachtet er, wie Systeme der Bestrafung in der Vergangenheit problematisiert 
wurden. So kann er verfolgen, wie gegenwärtige Gefängnispraktiken zu diesen früheren Problematisierungen in Beziehung stehen. Foucault geht davon aus, »daß es eine Beziehung zwischen der problematisierten Sache und dem Prozeß der Problematisierung gibt. Die Problematisierung ist eine >Antwort< auf eine konkrete Situation« (Foucault 1996: 179/Herv. i.O.). In seiner Untersuchung der »Geschichte des Wahnsinns « fragte Foucault bspw. danach, wie und warum etwas in der Welt als Geisteskrankheit gekennzeichnet und behandelt wird (Bacchi 2012: 2; Foucault 1977). Es ermöglichte ihm, die Elemente sichtbar zu machen, die für eine Problematisierung relevant sind: »It was a matter of determining the role of politics and ethics in the establishment of madness as a particular domain of scientific knowledge [connaissance], and also of analyzing the effects of the latter on political and ethical practices « (Foucault 1997b: 116). Foucault betrachtete Wahnsinn, Sexualität, Macht oder Staat nicht als wahr oder real (Bacchi 2012: 1). Er bezeichnete sich selbst als historischen Nominalisten (Foucault 1991: 86). Entsprechend schreibt er in »Sexualität und Wahrheit« zu dem Begriff Macht: »Zweifellos muß man Nominalist sein: die Macht ist nicht eine Institution, ist nicht eine Struktur, ist nicht eine Mächtigkeit einiger Mächtiger. Die Macht ist der Name, den man einer komplexen strategischen Situation in einer Gesellschaft gibt « (Foucault 1999: 114). Indem Macht oder Wahnsinn bzw. Geisteskrankheit nicht als wahr oder real angesehen werden, ist es möglich, sowohl die komplexen Beziehungen zu untersuchen, die sie hervorgebracht haben, als auch die Frage zu beantworten, welche Wirkungen daraus hervorgehen (Bacchi 2012: 1).

Das Konzept der Problematisierung im Sinne Foucaults umfasst einen zweistufigen Prozess, so die australische Politikwissenschaftlerin Carol Bacchi: die Frage danach, wie und warum etwas (Phänomene, Prozesse etc.) zum Problem geworden ist und wie es als Objekt des Denkens geformt wird (Bacchi 2012: 1). Diesem zweistufigen Modell, Foucaults »Analysebewegung «, folge ich in meiner Untersuchung. Dies bedeutet, meine Untersuchung folgt sowohl einer absteigenden als auch einer aufsteigenden Analyse: ich verfolge die Problematisierungen zurück und arbeite anhand der Problematisierungen die jeweiligen Regierungstechniken heraus. Ebenso nehme ich Foucaults »historisch nominalistische Methode « in die Untersuchung auf (Lemke 1998). Bevor diese wie auch das in der Untersuchung verwendete Konzept der Problematisierung spezifiziert werden und die Frage beantwortet wird, wie das Frame-Konzept im Hinblick auf die Untersuchung von Ethik verwendet wird, komme ich auf die genealogische Herangehensweise zu sprechen. 


\section{Der Abstieg: die genalogische HERANGEHENSWEISE}

Die Genealogie ist ein historisches Narrativ, das der Produktion von Diskursen nachgeht, so Kathrin Braun in ihrem Artikel zur Diskurstheorie und -analyse (Braun 2014: 94). Die Genealogie spürt dieser Produktion als historisches Geschehen nach. »Dieses historische Geschehen ist immer auch ein Geschehen zwischen Diskursen, ein Prozess der Verdrängung, Überlagerung, Überwindung oder Verbindung von Diskursen im Verlauf der Zeit« (ebd./Herv. i.O.). Im Kontrast zu teleologischen Annahmen liegt der Genealogie ein Verständnis von Geschichte zugrunde, dass diese als konflikthaftes, diskontinuierliches und kontingentes Geschehen begreift (ebd.; Bevir 2008: 264, 267). So äußert sich Foucault im Jahr 1976: »Ich betrachte die Geschichte als eine Abfolge von Fragmenten, eine Abfolge von Zufällen, Gewalttaten und Brüchen« (Foucault 2003 (1976)-a: 109). »Für die kritische Analyse geht es darum, diese Denksysteme und Praktiken als mehrdeutige Produkte eines kontingenten und konflikthaften Prozesses verstehbar zu machen«, so Braun (2014: 94).

Die genealogische Herangehensweise konzentriert sich auf bestimmte Praktiken, Ideen, Denksysteme und Wissensformen, die gegenwärtig bestimmend sind, die sich als notwendig und alternativlos oder auch als »glorreiche zivilisatorische Errungenschaften« darstellen (ebd.). Genealoginnen und Genealogen »continually question, exposing the particularity of perspectices that appear to be universal or timeless truths«, so der britische Politologe Marc Bevir (2008: 270). Als Narrativ verstanden, kann die Genealogie denjenigen Perspektiven, die als wahr oder universal gelten, die Selbstverständlichkeit, das Gegebene nehmen (Braun 2014: 94f). In diesem Sinn ist sie eine »denaturalizing form of critique (Bevir 2008: 272; vgl. Saar 2008: 313). Eine Kritik, die auch die Selbstkritik des Forschenden einbezieht: »genealogists may question their own narratives, and accept that the genealogical stance is a particular one that arose historically, without thereby rejecting their narratives or the genealogical stance « (Bevir 2008: 270).

Zur genealogischen Herangehensweise sagt Foucault: »I set out from a problem expressed in the terms current today and I try to work out its genealogy. Genealogy means that I begin my analysis from a question posed in the present « (Foucault 1990: 262). Entsprechend Foucaults genealogischer Herangehensweise beginne ich mit der Analyse des Problems in der Gegenwart. Die institutionalisierte Ethik betrachte ich als Produkt eines konflikthaften, diskontinuierlichen, kontingenten Geschehens; ein Prozess der Verdrängung, Überschneidung oder Verbindung von Diskursen. Um den Status von Ethikinstitutionen in Frage zu stellen, betrachte ich, wie und was in der Vergangenheit problematisiert wurde - worauf war 
Ethik die Antwort, wofür war die Einrichtung eines Ethikkomitees die Lösung und welche anderen Lösungsvorschläge gab es?

\subsection{Problematisierungen als "struggle over meaning « ${ }^{7}$}

Wie die deutsche Politikwissenschaftlerin Svea Herrmann in ihrer Untersuchung zur Problematisierung der Reprogenetik in Deutschland und Großbritannien (Herrmann 2011; Herrmann 2009) verbinde ich das Foucault'sche Konzept der Problematisierung mit post-positivistischen, diskursanalytischen Ansätzen, insbesondere aus dem Bereich der interpretativen Policy-Analyse (IPA).

Die IPA ist ein Entwicklungsstrang der Policy-Analyse. Die Entstehung der Policy-Analyse geht in die US-amerikanischen Nachkriegsjahre des Zweiten Weltkriegs zurück. In der Bundesrepublik wird sie ab den 1980er Jahren rezipiert (vgl. deLeon/Vogenbeck 2007; Schubert/Bandelow 2009, 3, 12; Saretzki 2007). Was gegenwärtig unter dem Begriff policy verstanden wird und entsprechend unter der Policy-Analyse, auch Policy-Forschung oder Politikfeldanalyse genannt, ist nicht unumstritten (Fischer/Forrester 1993; Herrmann 2009: 21ff). Eine Aufschlüsselung der verschiedenen Ansätze führt an dieser Stelle allerdings zu weit. ${ }^{8}$ Allgemein gesagt, beschäftigt sich die Policy-Analyse mit Politikinhalten: ihrem Zustandekommen, ihrer Ausgestaltung sowie mit ihren Effekten in unterschiedlichen Politikfeldern, wie Sozialpolitik oder Arbeitsmarktpolitik. Policies können bspw. Gesetze, Verordnungen oder Maßnahmen sein (Schubert/Bandelow 2009: 3f).

Ab den 1990er Jahren ist in Europa von einer argumentativen oder interpretativen Wende der Policy-Analyse die Rede, initiiert durch die Publikation von Frank Fischer und John Forrester »The Argumentative Turn in Policy Analysis and Planning« im Jahr 1993 (Fischer/Forrester 1993; vgl. Schubert/Bandelow 2009: 14; Saretzki 2009). Mit der argumentativen/interpretativen Wende wird die Rolle der Sprache und der Argumentation im Politikprozess zu einem Fokus der PolicyAnalyse, wobei die Konzepte von Argumentation, Diskurs und des kommunikativen Handelns meist in Anlehnung an Jürgen Habermas verwendet werden (Braun 2014: 82; Pülzl/Wydra 2011: 396). Zentral ist hier das Verständnis, Politiken weniger als rationale Antworten auf objektive Probleme zu sehen, sondern vielmehr als sprachliche Konstrukte. Sprache wird nicht als neutral oder beschreibend betrach-

7 Braun 2014: 79.

8 Zur unterschiedlichen Verwendung und Entstehung der Policy-Analyse vgl. Herrmann (2009: 21ff) und Fischer et al. (2007), weiterführend zur kritischen Policy-Analyse vgl. Fischer et al. (2015). 
tet, ihr kommt eine »>politische « Rolle« zu, sie ist formierend im Politikprozess (Pülzl/Wydra 2011: 396/Herv. i.O.). Die Analyse kann auch soziale Praktiken beinhalten, wobei Argumentation als Kommunikationsprozess in einem spezifischen gesellschaftlichen und historischen Kontext gesehen wird (Hajer 1993: 45; Herrmann 2009: 28f; Braun 2014). Das Augenmerk der IPA liegt darauf, wie etwas überhaupt als ein Problem definiert wird, auf der diskursiven Aushandlung sowie darauf, verschiedene Lösungsmöglichkeiten sichtbar zu machen. Die Zahl der Beiträge zur IPA ist in den letzten Jahren gestiegen; Konzepte, Theorien und Methoden sind differenzierter geworden (vgl. Fischer/Gottweis 2012). Als Merkmal der IPA ist nicht ein gemeinsamer Gegenstandsbereich oder ein gemeinsames Methodeninventar ersichtlich, wie Braun ausführt, sondern vielmehr eine gemeinsame Philosophie.

»Deren Kern besteht in der Zurückweisung eines szientistischen Verständnisses von Politikund Sozialwissenschaften, das den Anspruch erhebt, sich von einem neutralen Standpunkt aus mittels wertneutraler Methoden auf objektive Fakten zu beziehen und so zu allgemeingültigen Aussagen in Form von Korrelationen oder Kausalbeziehungen zu gelangen « (Braun 2014: 83; vgl. Gottweis 2007: 238).

Entsprechend gibt es im Sinne der IPA keine uninterpretierte soziale Realität. An diesem Punkt unterscheidet sie sich nicht unbedingt von diskursanalytischen Ansätzen in den Geistes- und Sozialwissenschaften (Braun 2014: 84). Merkmal der IPA ist allerdings, und hier zeigt sich ihre politikwissenschaftliche Spanne, dass mit ihr verdeckte politische Dimensionen herausgearbeitet werden können, in dem Sinn, dass Politik als Kampf oder Auseinandersetzung um Bedeutung verstanden wird (ebd.). Entscheidend ist dabei, wie Probleme als solche bestimmt werden, wie sie diskursiv ausgehandelt werden und welche Lösungsmöglichkeiten daraus resultieren. »Politik ist ein Kampf um Bedeutung - a struggle over meaning« (ebd.: 79/Herv. i.O.). In diesem Sinn ist Problematisierung, wie es Herrmann formuliert »a conflict-ridden, discursive process in which various actors struggle over the meaning and definition of >the problem < and in which >the problem< is constantly reframed « (Herrmann 2011: 163/Herv. i.O.). Dieses Konzept der Problematisierung korrespondiert m.E. mit der genealogischen Herangehensweise insofern, als es die Problematisierung als konflikthaften, kontingenten Prozess begreift. Im Unterschied zu den GS, die nicht so sehr danach fragen, welche Akteure und Akteurinnen, sondern welche jeweiligen »Strömungen « ziehen, mitreißen oder bremsen (Bröckling/Krasmann 2010: 28), richtet sich mein Blick auch auf Akteurinnen und Akteure. Den Raum, in dem Problematisierungen auftauchen, verstehe ich im Sinne der IPA als umkämpft und wandelbar, als einen Raum, in dem verschiedene Ak- 
teure und Akteurinnen ein Problem unterschiedlich interpretieren können, vor dem Hintergrund unterschiedlicher Rationalitäten und Wissensbestände. Die Frage ist, welche Problematisierung sich durchsetzt, welche nicht und wie sie sich im Prozess verändern (vgl. Herrmann 2011: 163). In Bezug auf meine Untersuchung ist die Frage nicht zuletzt, für welche Problematisierungen eine institutionalisierte Ethik als Lösung hervorgeht.

Die in der vorliegenden Studie behandelten Fragen, welche Problematisierung sich durchsetzt und wofür Ethik-Rahmungen eine Lösung sind, widerspricht genau genommen der Aufforderung von Dominique Pestre. Wie in Kapitel I zum Forschungsstand aufgeführt, besteht seine Aufforderung darin, zu untersuchen, wie Kritik in Regierungsinstrumente transformiert wurde. Pestre formulierte seine Aufforderung in Anlehnung an Boltanski und Chiapello. Letztgenannte sehen in ihrem Werk »Der neue Geist des Kapitalismus « die Kritik sozialer Bewegungen als einen der »wirkungsmächtigsten Motoren des Kapitalismus« (Boltanski/Chiapello 2003: 86). In der Perspektive der GS gibt es allerdings nicht nur eine Hauptströmung, die mitreißt oder bremst, es geht darum, »die konfligierenden Kräfte, die Brüche und Widerstände sichtbar zu machen, die Regierungsanstrengungen provozieren« (Bröckling/Krasmann 2010: 28). Mit Boltanski und Chiapello gesprochen, aber in Perspektive der GS gedacht, verstehe ich soziale Proteste und ihre Kritik als einen Motor von vielen. Dessen Leistung - letztlich den Geist des Kapitalismus zu verändern - setze ich allerdings ebenso wenig voraus wie, dass es sich bspw. im Kontext der Nanotechnologie beim CCNE per se um eine Regierungsform des advanced liberal government (Rose 1996; Rose/Miller 1992) handeln muss. Es geht also darum, Problematisierungen, die aus sozialen Protesten oder Bewegungen entstehen, zu berücksichtigen und zu überprüfen, inwiefern Ethik-Rahmungen als Antwort darauf zu verstehen sind.

\subsection{Ethik als Frame}

Wesentliche Überschneidungen der IPA, Foucaults und entsprechend der GS liegen darin, dass in diesen Perspektiven diskursive und nicht-diskursive Praktiken miteinander verknüpft sind und es nicht die Vorstellung einer uninterpretierten sozialen Realität gibt. Foucault meint im Jahr 1976: »Es gibt kein absolut Erstes, dass zu interpretieren wäre, denn im Grunde ist immer schon alles Interpretation « (Foucault 2001 (1967): 734). Entsprechend geht es ihm damals und später auch den GS nicht darum, eine bestimmte historische Wahrheit oder das einzig und eigentlich Richtige herauszustellen, sondern um »die Problematisierung der Art und Weise, wie wir heute über bestimmte Gegenstände urteilen und denken, um sie ihrer 
>Selbstverständlichkeit< oder >Natürlichkeit< zu entreißen und neue Erfahrungen zu ermöglichen« (Lemke 1998/Herv. i.O.). Foucault verdeutlicht seine »historisch nominalistische Methode« (ebd.) wie folgt

»wenn ich sage, daß ich die >Problematisierung< von Wahnsinn, Verbrechen oder Sexualität studiere, so ist das keine Art und Weise, die Realität solcher Erscheinungen zu leugnen. Im Gegenteil, ich habe versucht zu zeigen, daß gerade etwas wirklich in der Welt Vorhandenes in einem gegebenen Augenblick das Ziel sozialer Regulierung war. Ich stelle folgende Frage: Wie und warum wurden unterschiedliche Dinge in der Welt zum Beispiel unter dem Begriff >Geisteskrankheit< zusammengefasst?«(Foucault 1996: 179/Herv. i.O.).

Ähnlich wie Foucault Wahnsinn oder Verbrechen nominalistisch versteht, verstehe ich Ethik in meiner Untersuchung nominalistisch. Dies bedeutet nicht, dass ich davon ausgehe, dass gesellschaftspolitische Themen keine ethischen oder normativen Fragen implizieren: Die Atomkonflikte der 1970er und 80er Jahre in Europa sind zum Beispiel durch Werte wie Verantwortung gegenüber zukünftigen Generationen gekennzeichnet. Jedoch wird keine explizite staatliche Ethikinstitution eingerichtet, und Kontroversen werden nicht in ethischen Begriffen geführt, sondern in Risikobegriffen. Ich betrachte Ethik vielmehr nominalistisch, um das Selbstverständliche zu entschlüsseln. Das bedeutete, sie als etwas zu begreifen oder auch, wie sich im Lauf der Untersuchung herausstellte, als etwas Seltsames. Denn man kann es durchaus seltsam finden, dass innerhalb eines Ethikkomitees nicht darüber gesprochen wird, was Ethik ist. Ebenso seltsam kann es anmuten, dass im selben diskursiven Rahmen über nanotechnologisch bearbeiteten Autolack oder selbstreinigende Fenster gesprochen wird wie über Präimplantationsdiagnostik oder Organspende. Diese Perspektive ermöglicht m.E. eine Offenheit dafür, was Ethik bedeutet und wie in diesem Rahmen gedacht werden kann. Ethik nominalistisch zu verstehen, bedeutet also, dass ich nicht definiere, was Ethik ist oder nicht ist. Stattdessen versuche ich zu verstehen, was Ethik in einem bestimmten (historischen) Kontext bedeutet und was die verschiedenen Akteure und Akteurinnen darunter verstehen (vgl. Braun et al. 2008; Könninger 2009). Zudem wird so, wie in der EGoStudie, nicht von der Frage abgelenkt, welche Bedeutungen die Akteurinnen und Akteure dem Begriff geben, ob und wie es zu Bedeutungsverschiebungen kommt, oder ob ihm überhaupt eine präzise Bedeutung zugeschrieben wird (Braun et al. 2008: 222). Anstatt Ethik als etwas zu verstehen, das bio- und nanotechnologischen Themen inhärent ist, betrachte ich, wie bereits das EGo-Projekt, Ethik als Rahmen (frame). Wenn ich im Folgenden von einem Ethik-Rahmen spreche, so meine ich damit das Phänomen, dass Probleme und Streitfragen explizit in einer Sprache der Ethik formuliert werden, also als ethische Fragen oder Probleme, die 
ein Nachdenken über ethische Implikationen, ethische Dilemmata etc. erfordern. Ich beziehe mich dabei auf die Frame-Analyse von Rein und Schön (Rein/Schön 1993; Schön/Rein 1994), verwende deren Frame-Konzept aber in etwas modifizierter Form.

\subsubsection{Re-framing der Frame-Analyse}

Die Frame-Analyse nach Schön und Rein ist einer von mehreren Ansätzen der IPA. Sie kann zu den dialogisch orientierten Ansätzen gezählt werden, die an die Habermas'sche Diskurstheorie anknüpfen (Braun 2014: 89). Die Anknüpfungen bestehen allerdings nicht darin, mittels der Habermas'schen Konzeption von Diskurs Politiken zu verstehen, sondern im Anspruch der »Entwicklung einer dialogischeren, reflektierteren und demokratischeren Form von Politik« (ebd.). In diesem Sinn ist sie praxisorientiert. Die Protagonistinnen und Protagonisten dieses Ansatzes verstehen ihre Rolle darin, Verständigungsprozesse zwischen Bürgerinnen und Bürgern und Politik bzw. zwischen Problemdeutungen, die unvereinbar scheinen zu ermöglichen, so Braun (2014, 2015b). Die Frame-Analyse im Sinne von Rein und Schön hat das ideale Ziel, dass alle an einem Policy-Diskurs Beteiligten die konfligierenden Rahmen erkennen und reflektieren (frame-reflection), es zu einem re-framing und letztlich zur Lösung von Kontroversen kommt. Der Forscher oder die Forscherin ist in diesen Reflexionsprozess eingeschlossen und eher unterstützend tätig (Schön/Rein 1994: 39ff).

In der Gouvernementalitätsperspektive geht es allerdings nicht darum, eine bessere oder ideale Antwort auf divergierende Problemdeutungen oder Rahmen zu finden oder eine dialogischere Form der Politik zu entwickeln (vgl. Bröckling/Krasmann 2010: 28). Die GS fragen eher danach, wie gerade diese Form der Politik des Dialogs zu einem bestimmten Zeitpunkt und unter bestimmten Bedingungen aufkommt. Entsprechend verwende ich das Frame-Konzept von Rein und Schön in diesem Sinn nicht praxisorientiert, sondern zur Analyse von Ethik. Praxisorientiert verwende ich die Frame-Analyse nur insofern, als sie in meiner genealogischen Untersuchungspraxis dazu dient, aufzuzeigen, wie etwas selbstverständlich wird und sich daraus die Praxis einer anderen Denkweise ergeben kann. Denn gerade hier zeigen die GS laut Bröckling und Krassmann ihr Analysepotential: wenn sie irritieren, ein Korrektiv, aber keine neue Schule sind. In diesem Sinn können GS verstanden werden als »eine Art sanfte[r], wenn nicht provokative[r], wirksame[r] konzeptuelle[r] Hebel, um neue Denkweisen zu entwickeln, vertraute Wege zu verlassen, manche zu demontieren und andere zu erneuern«, so der britische Sozialwissenschaftler Thomas Osborne (2004: 35).

In der Gouvernementalitätsperspektive gedacht, ermöglicht die FrameAnalyse, den Raum sichtbar zu machen, innerhalb dessen Probleme überhaupt erst 
auftauchen, als ethische Probleme benannt und in einer bestimmten Art und Weise behandelbar werden. Mit der Frame-Analyse kann der Raum sichtbar gemacht werden, in dem bestimmte Arten und Weisen des Denkens und Sprechens ermöglicht werden. Es zeigt sich aber auch, was nicht thematisiert wird bzw. nicht mehr thematisiert werden kann. Frames bringen bestimmte Elemente der Wirklichkeit zum Vorschein und erlauben besondere Arten des Sprechens, während andere ausgeschlossen werden (vgl. Fischer 2003: 144). Mit der Frame-Analye kann herausgearbeitet werden, wie und welche Sprecher- und Sprecherinnenpositionen entworfen oder formiert werden und auch welche Akteure und Akteurinnen sowie Adressaten und Adressatinnen (Subjekte) entworfen und angesprochen werden sollen und welche nicht.

Ein weiterer Aspekt dialogischer Ansätze ist, dass ihr Untersuchungszeitraum begrenzt ist. »[H]istorische Analysen fallen aus dem Theorierahmen insofern hinaus, als mit den Protagonistinnen früherer Diskurse kein praktischer Dialog mehr möglich ist « (Braun 2014: 89). Die Studien konzentrieren sich eher auf die Jetztzeit. In meiner Untersuchung werde ich das Frame-Konzept in eine historische Analyse oder genauer, in eine genealogische Praxis einbinden. Dies bedeutet, ich verwende das Konzept, um ethische Rahmungen über die Zeit sichtbar zu machen. Dies bedeutet zudem, dass ich davon ausgehe, dass der Ethik-Frame an frühere Diskurse anknüpft, Ethik unterschiedliche Bedeutungen haben kann oder es zu Bedeutungsverschiebungen kommen kann. Darüber hinaus gehe ich nicht davon aus, dass institutionalisierte Ethik die Antwort auf ethische Problematisierungen sein muss, auf etwas, das als explizit ethisches Problem gerahmt ist. Es können ebenso andere Problematisierungen und Rahmungen auftauchen, die aber gerade sichtbar werden, indem sie nicht ethisch gerahmt sind.

\subsubsection{Ethisierung}

Von Ethisierung ${ }^{9}$ spreche ich, um kenntlich zu machen, dass vormals andere, nicht als ethisch gerahmte Themen und Probleme als ethische Probleme aufgefasst werden. Bspw. wenn einige Akteurinnen und Akteure Gentechnologie als RisikoProblem thematisieren, andere dies zu einer ethischen Frage erklären und sich die ethische Rahmung insofern durchsetzt, als sie z.B. in den institutionalisierten Ethik-Rahmen integriert wird. Ob und inwiefern auch im Kontext der Nanotechno-

9 Der Begriff Ethisierung taucht bereits zu Beginn der 1920er Jahre im deutschen Sprachraum auf. Viktor Engelhardt schreibt 1922 in seiner Publikation »Weltanschauung und Technik«, dass eine Ethisierung der Technik notwendig sei (Engelhardt 1922). Ernst Cassirer bezieht sich 1930 in seiner Schrift »Form und Technik « positiv auf die von Engelhardt geforderte Ethisierung (Cassirer 2004 (1930)). 
logie von einer Ethisierung gesprochen werden kann, wird in dieser Studie nachgegangen. Es handelt sich um eine offene Frage, und insofern spreche ich in diesem Kontext vorab von Ausdehnung. Diese Auffassung des Begriffs der Ethisierung zeigt einerseits Übereinstimmungen mit dem Ethisierungsbegriff, wie er von Alexander Bogner verwendet wird, andererseits auch Unterschiede. Bogner versteht unter Ethisierung, dass viele technik- und wissenschaftspolitische Fragen als ethische Herausforderungen verstanden werden (Bogner 2013: 51) - soweit die Übereinstimmung. Allerdings beinhaltet der von mir verwendete Begriff zudem die Frage, wie es genau dazu kommt und welche Problematisierungen auf dem Weg dahin verloren gegangen sind. Bogner spezifiziert den Begriff wie folgt: »Manche politisch regulierungsrelevanten Technologien werden öffentlich aus ethischer Perspektive problematisiert - es wird darüber gestritten, ob eine Technologie oder eine Forschungsoption moralisch vertretbar ist oder nicht«. Und er schreibt weiter: »Ethisierung soll heißen: Über Wissenschaft und Technik wird nach Maßgabe der Unterscheidung von gut und böse debattiert - und nicht (vorrangig) entlang der Unterscheidungen von riskant versus sicher oder ökonomisch nutzlos versus gewinnbringend « (ebd.: 51). In meiner Untersuchung setze ich dies nicht voraus. $\mathrm{Ob}$ im Ethik-Rahmen die moralische Vertretbarkeit von Forschungsoptionen, die Unterscheidung »von gut und böse« in den Debatten eine Rolle spielt oder nicht, hängt davon ab, was die Akteure und Akteurinnen jeweils selbst unter Ethik verstehen. Dies wiederum kann sich über die Zeit verändern.

Die Frage, ob von Ethisierungen gesprochen werden kann, inwiefern sich ethische Rahmungen durchsetzen, lässt sich erst in der aufsteigenden Untersuchung beantworten, auf die ich nun zu sprechen komme.

\section{Der Aufstieg: Ethik ALs NeOLIBERALE GOUVERNEMENTALITÄT?}

In der aufsteigenden Untersuchung greife ich, wie einleitend erwähnt, auf das Konzept der delegierten Biopolitik und darüber hinaus auf die Konzepte des reflexive government und des governing at a distance zurück. Insbesondere letzteres stellt gerade die weichen Machtmechanismen einer neoliberalen Regierung bzw. des fortgeschrittenen Liberalismus heraus sowie das »Regieren über Freiheit«. Bevor ich auf die Konzepte zu sprechen komme, wird die neoliberale Gouvernementalität in ihren wesentlichen Zügen ausgeführt.

Neoliberalismus wird im Sinne der Gouvernementalitätsperspektive nicht als Realität verstanden, sondern als politische Rationalität. Diese Rationalität ermöglicht, das Soziale zu ökonomisieren, (wohlfahrts-)staatliche Handlungen zu redu- 
zieren und Sicherheitssysteme mit einem wachsenden Ruf nach persönlicher Verantwortung und Selbstsorge zu verbinden (Lemke 2001: 203). Mit Foucault verstanden ist Neoliberalismus eine bestimmte Art und Weise der Problematisierung, so Lemke. Sie definiert einen politisch-epistemologischen Raum oder ein $»$ Möglichkeitsfeld « (Lemke et al. 2000: 20; vgl. Foucault 2005c: 255). Der Rückzug des Staates, die Reduktion (wohlfahrts-)staatlicher Interventionen werden entsprechend dem Foucault'schen Regierungsverständnis nicht als ein Weniger an Regierung verstanden. Stattdessen werden sie, wie Lemke in einem Artikel zur neoliberalen Gouvernementalität aus dem Jahr 2001 schreibt, als Restrukturierung staatlicher Regierungstechniken begriffen. Dabei behält der Staat traditionelle Aufgaben, übernimmt aber auch neue Aufgaben und Funktionen (Lemke 2001: 202f). Ein Element der Restrukturierung staatlicher Regierungstechniken liegt darin, die Regulierungskompetenz des Staates auf Individuen zu verschieben, die als verantwortlich und rational entworfen werden (auch Familien, Kollektive, Gruppen etc.) (ebd.: 202f). Die Verantwortung für soziale Risiken wie Krankheit oder Arbeitslosigkeit wird in einen Bereich verschoben, in dem das Individuum verantwortlich ist, und wird so zu einem Problem der Selbstsorge. Die Besonderheit der neoliberalen Rationalität liegt, wie Lemke schreibt, darin, dass sie zwischen einem verantwortlichen Individuum und ökonomisch-rationalen Akteurinnen und Akteuren eine Übereinstimmung zu erreichen versucht. Es handelt sich um Akteurinnen und Akteure, die in einer Kosten-Nutzen-Analyse und in Abgrenzung zu möglichen Alternativen ihre Entscheidungen abwägen (ebd.: 202f; 2000: 38). Da in dieser Rationalität die Wahl zwischen verschiedenen Handlungsmöglichkeiten Ausdruck für den freien Willen, für eine selbstbestimmte Entscheidung ist, ist das Subjekt allein für seine Konsequenzen verantwortlich. Der Neoliberalismus antwortet damit auf eine Nachfrage nach mehr Selbstbestimmung und Autonomie, so Lemke, indem er es ermöglicht, aktiv an der Lösung bestimmter Probleme und Themenbereiche zu partizipieren; dies geschieht allerdings um den Preis, hierfür selbst verantwortlich zu sein (ebd.: 202; vgl. Burchell 1993: 276). Der freie Wille, die freie Entscheidung, kurz die Freiheit ist zentrales Charakteristikum der neoliberalen Gouvernementalität. Regieren ist »an activity that shapes the field of action and thus, in a sense, attempts to shape freedom « (Dean 1999a: 13). Es bedeutet nicht, die Freiheit und Handlungsfähigkeit der Menschen zu negieren, sondern vielmehr sie zu strukturieren (Rose 1999: 4).

Die neoliberalen Regierungsformen weisen nicht nur direkte staatliche, sondern auch indirekte Techniken auf, Techniken des governing at a distance, wie sie von Rose und Miller bezeichnet werden. Auf das governing at a distance wie auch auf das Konzept des reflexive government nach Dean greift, wie bereits erwähnt, die EGo-Studie in ihren Untersuchungen der gouvernementalen Ethikregime zu- 
rück. In der vorliegenden Studie werden diese Konzepte in spezifischer Hinsicht für die Untersuchung übernommen und zudem um das Konzept der delegierten Biopolitik von Memmi erweitert. Bevor ich konkretisiere, wie die Konzepte in meiner Untersuchung eingesetzt werden, werden sie in den wesentlichen Zügen vorgestellt.

\subsection{Vom governing at a distance und dem reflexive government}

Liberale Regierung ist insofern immer problematisch, so Rose und Miller, als es sich um ein Regieren in Distanz handelt (Rose/Miller 1992: 181). Bereits der klassische Liberalismus, verstanden als Rationalität, basiert auf der Annahme, dass der Staat Subjekten gegenübersteht, die mit Rechten ausgestattet sind, in die der Staat nicht eingreifen darf. Und der klassische Liberalismus basiert darauf, dass »rulers are faced with a realm of processes that they cannot govern by the exercise of sovereign will because they lack the requisite knowledge and capacities« (Rose 1993, 289). Einerseits konnte der Staat im Sinne des Liberalismus Bereiche wie die Familie oder den Markt nicht einfach durch direkte Interventionen regieren - er war immer in Gefahr des »governing too much «, wie Foucault meint (Foucault 1997a: 76). Andererseits waren diese Bereiche immer anfällig für Krisen, und Bürgerinnen und Bürger mussten durch den Staat gegen möglichen Schaden geschützt werden, der durch einen exzessiven Gebrauch der Freiheit verursacht war. Um diese beiden Seiten auszubalancieren, war der klassische Liberalismus auf die Produktion von Wissen über die unbändigen sozialen Bereiche angewiesen sowie auf Expertinnen und Experten, die zwischen Individuen und dem Staat vermitteln und so das Problem direkter staatlicher Steuerung kompensieren. Der gegenwärtige fortgeschrittene Liberalismus (advanced liberalism), so Rose, hat im Vergleich zum klassischen Liberalismus neue Strategien entwickelt, um mit dem Problem, zu viel zu regieren, umzugehen. Fortgeschrittene liberale Strategien suchen Regierungstechniken, die eine Distanz zwischen den Entscheidungen formaler politischer Institutionen und anderen sozialen Akteurinnen und Akteuren schaffen. Die sozialen Akteurinnen und Akteure werden hierbei in neuer Art als verantwortliche, autonome Subjekte konzipiert. Die fortgeschrittenen liberalen Strategien suchen danach, auf die Akteurinnen und Akteure einzuwirken, indem sie ihre Freiheit formen (Rose 1996: 53f).

Auch Dean verwendet den Begriff reflexive government, um eine weniger direkte Form der Regierung zu erfassen. Diese sieht er in den 1980er und 90er Jahren hauptsächlich im ökonomischen Bereich aufkommen (Dean 1999b: 39f). Seine Hypothese ist, dass die Gouvernementalisierung des Staates, durch welche der Staat die Aufgabe für die Bevölkerung und Individuen übernommen hat, gegen- 
wärtig (teilweise) dahingehend transformiert wird, dass Regierungsmechanismen selbst zum Gegenstand der Problematisierung werden. Problematisierungen im Sinne Deans sind: »a calling into question of how we shape or direct our own and other's conduct. Problematizations might thus equally concern how we conduct government and how we govern conduct« (Dean 1999b: 27). Dies versteht Dean als Gouvernementalisierung der Regierung und $»$ What results might be called >reflexive government « (ebd.: 38/Herv. i.O.).

\subsection{Die delegierte Biopolitik}

Memmis Ansatz der delegierten Biopolitik ist zwar nicht explizit in den GS zu verorten, jedoch weist er eine wesentliche Überschneidung mit dem Konzept des governing at a distance nach Rose auf. Die Überschneidung liegt vor allem in der indirekten Art der Regierung, bei Memmi governing through speech genannt. Dieses governing through speech, das mit einer Bioindividuation einhergeht, die insbesondere auf die Regierung der körperlichen Führung zielt, sieht sie nicht erst mit dem CCNE aufkommen. Es kommt bereits in den 1960er und 70er Jahren im Kontext der Legalisierung von Verhütungsmitteln und des Schwangerschaftsabbruchs in Frankreich auf. Mit der Legalisierung des Schwangerschaftsbruchs Mitte der 1970er wird ein Thema der (staatlichen) Regierung zunehmend in die Verantwortung des Individuums verlagert oder delegiert, ohne dass der Staat komplett verschwindet. Soziale Bewegungen haben eine wesentliche Bedeutung in diesem Transformationsprozess, so Memmi. Bereits mit der Legalisierung der Kontrazeptiva Mitte der 1960er Jahre sind durch die französische Bewegung für Familienplanung private Verhütungspraktiken (Kräuter, Kondome, Abstinenz etc.) und Entscheidungen, die gegen staatliche Regulierungen und gegen die Kirche stattgefunden haben, überhaupt öffentlich geworden. Das Ende der 1960er Jahre ist dadurch gekennzeichnet, dass der Prozess der Bioindividuation, der erst gegen die Kirche und den Staat gewendet war, sich nun an den Staat richtet. Ebenfalls an den Staat richtet sich einige Jahre später die feministische Bewegung mit ihrer Forderung der Legalisierung des Schwangerschaftsabbruchs. Eine vormals heimliche Praxis wurde so zur öffentlichen Angelegenheit und zur Forderung an den Staat: »l'histoire des résistances n'a pas commencé par des paroles, mais par des actions silencieuses et clandestines « - »die Geschichte des Widerstands begann nicht mit Worten, sondern mit stillen und heimlichen Handlungen «, so Memmi (2005: 11/Übers. S.K.). Im Kontext der Legalisierung des Schwangerschaftsabbruchs im Jahr 1975 werden Worte - das Sprechen - gesetzlich verankert: das Gesetz sieht seitdem für schwangere Frauen, die einen Schwangerschaftsabbruch möchten, eine 
medizinische Beratung vor. In dieser Beratung wird die Art und Weise des Sprechens durch den Mediziner oder die Medizinerin angeleitet bzw. überwacht (ebd.: 2). Die Frau, die durch die gesetzlich festgelegte Abtreibungsfrist unter Zeitdruck steht, wird zum Sprechen angeregt, es wird von ihr erwartet, ihre Entscheidung zu begründen, auch um eine Kostenübernahme des Schwangerschaftsabbruchs zu erhalten. Nicht allein die schwangere Frau, ebenso der Mediziner oder die Medizinerin muss sprechen, informieren, erklären. Er oder sie kann nicht ohne Zustimmung der Frau über ihren Körper verfügen, sondern muss die Impulse gegenüber der Patientin beherrschen (Memmi 2003c: 648; 2005: 3). Die Entscheidung über eine Abtreibung wird vom Staat durch das Gesetz an die individuelle Frau delegiert. Es handelt sich um eine Regierung, die sich wenig autoritär gibt und die auf eine begleitende Selbstkontrolle durch eine gelehrte Expertise (Mediziner oder Medizinerin) setzt (Memmi 2005: 3). Dieses governing through speech, die von Expertinnen und Experten angeleitete Art und Weise des Sprechens, findet sich sowohl am Anfang und am Ende des Lebens, wie im Kontext der Abtreibung oder der Sterbehilfe (Memmi 2005, 2003a), als auch beim CCNE - dem magistère bioéthique (Memmi 2003a: 3; 1996; vgl. Memmi 1999, 2000). Die Bioethik bestätige die Transformation von der Disziplinierung der Bevölkerung zu einer begleiteten Selbstregierung des Individuums und »>bioethical< regulation confirms the tendency to decriminalize the relationship to the body«, so Memmi (2003c: 656/Herv. i.O.). Die bioethische Reflexion und das Verhältnis zwischen Arzt oder Ärztin und Patient oder Patientin haben in diesem Sinn eines gemeinsam: »a growing interiorization of rational control - or better still, of >reasonable conduct $<-$ in social practices «, so Memmi (ebd.: 656/Herv. i.O.).

Die aufsteigende Untersuchung beinhaltet, dass ich die Regierungsform des CCNE nicht per se als Form des reflexive government (Dean 1999a) und des advanced liberal government (Rose 1996; Rose/Miller 1992) verstehe, wie dies in der EGo-Studie herausgearbeitet wurde, sondern dies überprüfe. Zwar wird in dieser Studie im Kontext biomedizinischer und -technologischer Entwicklungen mit Deans Konzept der Problematisierung herausgearbeitet, dass gouvernementale Ethikregime in einem Kontext entstehen, in dem die Selbststeuerung wissenschaftlicher und technologischer Entwicklungen problematisch geworden ist, es muss aber nicht im Kontext nanotechnologischer Entwicklungen zutreffen. Ob es zutrifft, hängt wiederum davon ab, ob und inwiefern Regierungsmechanismen zum Gegenstand von Problematisierungen werden. Mit dem Begriff der Problematisierung knüpfe ich somit nicht von vornherein an Deans Definition an. Dies würde bedeuten, dass ich nach einer einzigen Problematisierung und somit auch eher nach einer einzigen Lösung suche: dem Ethikregime als Form des advanced liberal government. Es käme einer Art self-fulfilling prophecy gleich (vgl. Bröck- 
ling/Krasmann 2010: 33). Es kann aber immer verschiedene, auch widersprüchliche Lösungs- und Bearbeitungsstrategien für Probleme geben (vgl. Lemke 2000: 32). Ebenso von den Problematisierungen abhängig ist es, inwiefern der CCNE im Kontext der Nanotechnologie als magistère bioéthique verstanden werden kann, als eine delegierte Biopolitik mit der damit einhergehenden Bioindividuation im Sinne Memmis. Dies setzt m.E. voraus, dass Gesundheit in irgendeiner Form problematisiert wird. Meine Perspektive liegt also darin, nach Problematisierungen und den jeweiligen Lösungs- und Bearbeitungsstrategien zu suchen. Beide Konzepte, das governing at a distance wie auch die delegierte Biopolitik, sind aber für die Analyse der Entstehungsgeschichte des CCNE hilfreich. Hier werden bspw. auch die Ethikinstitutionen berücksichtigt, die dem CCNE voran gehen. Mit den Konzepten kann das Spezifische der Regierungsformen dieser ethischen Steuerungen herausgearbeitet werden: zielen sie auf eine Führung der körperlichen Führung, wird das Sprechen angeleitet, inwiefern wird Regierung problematisiert?

Zusammenfassend gesagt, folgt die Untersuchung im Sinne des thinking problematically einer absteigenden und einer aufsteigenden Untersuchung. Absteigend ist sie in dem Sinn, dass die Sprache der Ethik genealogisch in den jeweiligen Diskursen zurückverfolgt wird, im medizinischen Diskurs, im Gentechnologiediskurs, in den Diskursen um Biologie und Lebenswissenschaften sowie im Nanotechnologiediskurs. Hier werden die (ethischen) Rahmungen und Problematisierungen sichtbar gemacht. Aufsteigend ist die Untersuchung in dem Sinn, dass nicht auf ein Großnarrativ - die neoliberale Gouvernementalität - fokussiert wird (vgl. Bröckling/Krasmann 2010: 33), sondern in Abhängigkeit der Problematisierungen die Regierungstechniken herausgearbeitet werden. In diese aufsteigende Untersuchung werden die Konzepte des governing at a distance (Miller und Rose), der delegierten Biopolitik (Memmi) und der Problematisierung nach Dean aufgenommen und zudem Ethisierungen berücksichtigt. Die Frage, inwiefern sich die Regierungsform des CCNE auf das Thema der Nanotechnologie ausdehnen kann, wird in Abhängigkeit der Art und Weise der Problematisierungen und der Rahmungen gesehen. 


\section{Die Sprache der Ethik als Antwort auf Problematisierungen medizinischer Autonomie}

In der Publikation aus dem Jahr 1991 »Aux frontières de la vie. Une éthique biomédicale à la française « - »An den Grenzen des Lebens. Eine biomedizinische Ethik französischer Art « (Lenoir 1991: 16/Übers. S.K.) ${ }^{1}$ wird die Entstehung von Ethikinstitutionen wie folgt hergeleitet: »Das erste Bewusstwerden über diese ethischen Probleme geht auf den Zweiten Weltkrieg zurück und noch genauer auf die Enthüllung der medizinischen Nazi->Experimente< beim Prozess von Nürnberg« (ebd.: 16/Herv. i.O., Übers. S.K.). Die Autorin, Noëlle Lenoir, französische Juristin und 1992-1998 Leiterin des Bioethikkomitees der United Nations Educational Scientific and Cultural Organization (UNESCO) begründet so die Entstehung von Ethikinstitutionen, sowohl auf Politikebene wie dem CCNE als auch an Forschungsinstituten oder Kliniken. Auch der französische Mediziner Jean Bernard gibt die Medizin im Nationalsozialismus (NS) als einen von zwei Gründen für den Ursprung der Ethik der Biologie und der Medizin an. In seinem autobiografischen Werk aus dem Jahr 1994 »Médecin dans le siècle« - »Mediziner im Wandel eines Jahrhunderts « benennt er auf der einen Seite die Revolutionen in Medizin und Biologie und

»[a]uf der anderen Seite, Hitler. Innerhalb von ein paar Jahren haben Hitler und die seinigen eine neue Barbarei hervorgebracht, eine Barbarei, die von der Verachtung der Person getragen war und von einer abwegigen Wissenschaft [...]. Es gilt daran zu erinnern, dass die skandalösen Experimente von Hitlers Medizinern, die gefangene, deportierte Frauen, Männer und

1 Es handelt sich um einen Bericht, der von Michel Rocard, damaliger sozialistischer Premierminister (1988-1991), in Auftrag gegeben wurde. 
jüdische Kinder wie Versuchstiere benutzt haben, dass diese Experimente keinen einzigen wissenschaftlich gültigen Fortschritt zugelassen haben«(Bernard 1994: 321/Übers. S.K.).

Der hier zitierte Jean Bernard (*1907-†2006) wird im Jahr 1974 Präsident des ersten Ethikkomitees an einem Forschungsinstitut. Dieses konsultative Komitee wird beim nationalen Institut für Gesundheit und medizinische Forschung, dem Institut National de la Santé et de la Recherche Médicale (INSERM) unter dem Titel Comité d'Éthique d'INSERM ${ }^{2}$ eingerichtet. Bernard ${ }^{3}$ wird zudem Mitglied einer weiteren Ethikinstitution, die ebenfalls Mitte der 1970er Jahre eingerichtet wird, und zwar bei der Délégation Générale à la Recherche Scientifique et Technique, einer Vorgängerin des Forschungsministeriums. Sie wird im nächsten Kapitel (IV) behandelt. Darüber hinaus wird Bernard im Jahr 1983 Präsident des nationalen Ethikkomitees, dem CCNE. Abgesehen von diesen Tätigkeiten ist er in den Jahren 1983-1984 Präsident der Académie des Sciences - der Wissenschaftsakademie und 1983-1992 der Académie Nationale de Médecine - der nationalen Medizinakademie.

\section{DAS ETHIKKOMITEE dES INSTITUT NATIONAL DE LA SANTÉ ET DE LA RECHERCHE MÉdICALE}

Mit dem Comité d'Éthique d'INSERM, der als Vorgänger des CCNE gilt, erscheint der Begriff Ethik in Frankreich zum ersten Mal im Titel eines staatlich initiierten Komitees. Die Entstehungsgeschichte des INSERM, an den das Ethikkomitee angegliedert ist, reicht in die Zeit des Zweiten Weltkriegs zurück. Hier liegt, der Äußerung Lenoirs und Bernards folgend, die Frage nahe, ob und inwiefern der Entstehungskontext des INSERM im Zweiten Weltkrieg für die Einrichtung des Comité

2 Das Ethikkomitee wird in der Literatur unterschiedlich bezeichnet. Es wird vom Comité consultatif d'éthique médicale - konsultativen medizinethischen Komitee gesprochen (Brief von J.-P. Chèvenement an Ph. Lazar, 26.02.1982, zit. nach Memmi 1996/Übers. S.K.), vom Comité consultatif d'éthique de l'INSERM - konsultativen Ethikkomitee vom INSERM (Lazar 1993, Editorial de la revue INSERM Actualités, zit. nach DidrichTabaste 1994: 95/Übers. S.K.) oder auch, wie auf der Homepage des INSERM, vom Comité d'éthique - Ethikkomitee des INSERM (http://www.inserm.fr/, 14.07.2015). Die letztgenannte Bezeichnung wird im Folgenden verwendet.

3 Zu Bernards Werdegang als Mediziner und Forscher vgl. Christelle Rigals Aufsatz zur Reorganisierung medizinischer Forschung nach dem Zweiten Weltkrieg in Frankreich (Rigal 2008: 513ff). 
d'Éthique d'INSERM relevant war. In diesem Kapitel wird berücksichtigt, wie und wann die Sprache der Ethik in Erscheinung tritt und inwiefern die Erläuterung Bernards und Lenoirs, der Zusammenhang zwischen dem »Bewusstwerden über ethische Probleme « durch den Zweiten Weltkrieg und insbesondere durch die Humanexperimente im NS zutrifft. Ausgehend von der Entstehungsgeschichte des INSERM und der Institutionalisierung des Ethikkomitees wird die Sprache der Ethik im Folgenden in den Diskursen um Humanexperimente zurückverfolgt. Jedoch liegt das Augenmerk nicht ausschließlich auf diesen Diskursen, sondern auch auf der Frage, ob und inwiefern die Sprache der Ethik in weiteren Diskursen auftaucht, die in den 1970er Jahren den öffentlichen Raum prägen, wie die Diskurse um Medikamentenskandale, um die Legalisierung der Abtreibung oder wie der medizinkritische Diskurs, der aus den 1968er-Bewegungen hervorgeht. Für welche Problematisierungen ist die Einrichtung dieses Komitees die Lösung?

\subsection{Zur Entstehung des Institut National de la Santé et de la Recherche Médicale: Vichy-Regime und nationales Hygieneinstitut}

Im Februar des Jahres 1974 entsteht der Comité d'Éthique d'INSERM. Der INSERM, das nationale Forschungsinstitut, geht im Jahr 1964 aus dem Institut National d'Hygiène (INH) - dem nationalen Hygieneinstitut hervor, der während des Zweiten Weltkriegs, im Kontext des Vichy-Regimes (1940-1944) entstanden ist. Wenn es ausgelöst durch den Zweiten Weltkrieg und die NS-Medizin eine Verknüpfung zwischen der Einrichtung von Ethikinstitutionen und dem »Bewusstwerden über ethische Probleme « gibt, stellt sich die Frage, inwiefern dies auf die Einrichtung des Ethikkomitees vom INSERM zutrifft. Spielt dann die Vorgeschichte des INSERM, also der Institution, an der schließlich eines der ersten Ethikkomitees eingerichtet wird, und seine Entstehung während des Vichy-Regimes, für die Einrichtung des Ethikkomitees eine Rolle und wenn ja, inwiefern? Um diese Frage zu beantworten, werde ich zuerst darauf eingehen, wie der INH entstanden ist und welche Aufgaben er hatte.

Die Antwort auf die Frage nach der Entstehung des INH bringt ein Problem mit sich. In den bestehenden Arbeiten über den INH wird die politische Dimension des Vichy-Regimes kaum berücksichtigt. Insbesondere der französische Historiker Jean-François Picard hat mit seiner Untersuchung zur Entstehung des INH (Picard 1999, 2001, 2003) »Pionierarbeit« geleistet, so die französische Wissenschaftshistorikerin Ilana Löwy - der INH wurde zuvor nicht explizit untersucht (Löwy 2003: 29/Übers. S.K.). Von dem US-amerikanischen Historiker William H. Schneider 
liegt ebenfalls eine jüngere Untersuchung über den INH vor (Schneider 2003). Picards Anliegen ist es, zu zeigen, dass die Vichy-Regierung, die die Last der beschämenden Niederlage in der französischen Geschichte trägt, »receives credit for some of its achievements « (Picard 2001: o.S.). Er formuliert u.a. in Anlehnung an Schneider und dessen Untersuchung zur Eugenik in Frankreich (Schneider 1990b) die Hypothese, dass ein autoritäres System eher fähig sei, eine Gesundheitspolitik umzusetzen als eine parlamentarische Demokratie (Picard 2001: o.S.). Picard nimmt m.E. eine Perspektive ein, in der Gesundheitspolitik oder Gesundheit positiv gewertet ist und in der es wichtig scheint »achievements « - -Leistungen « oder »Erfolge « eines autoritären Systems herauszustellen. Nicht thematisiert wird, was während des Vichy-Regimes überhaupt unter Gesundheit verstanden wird und kaum thematisiert wird, auf welcher Politik bzw. Politiken sie basiert: Picard und Schneider zeigen auf, dass der INH maßgeblich aus den Initiativen der Rockefeller Stiftung in Frankreich hervorgeht, die bereits vor dem Zweiten Weltkrieg beginnen, und nicht aus dem Kontext der Kollaborationspolitik zwischen dem VichyRegime und dem nationalsozialistischen Deutschland (Schneider 2003; Picard 2003). Die Rockefeller Stiftung ist eine US-amerikanische, international ausgerichtete, philantropische Stiftung, die im öffentlichen Gesundheitswesen, in der medizinischen Ausbildung und der wissenschaftlichen Forschung fördernd tätig ist. Auch historische Arbeiten zum Bereich der öffentlichen Gesundheit nach 1945 in Frankreich, wie die des französischen Historikers Luc Berlivet, knüpfen an dieses Ergebnis an - die Entstehung des INH aus den Initiativen der Rockefeller Stifung (Berlivet 2008: 473). Dass die Initiativen dieser Stiftung für die Entstehung des INH wichtig sind, auch dass nicht die Kollaborationspolitik ausschlaggebend ist, zeigen die Arbeiten auf, jedoch bleibt beinahe unberücksichtigt, inwiefern die Politik des Vichy-Regimes die Einrichtung des INH ermöglichte. Dessen Politik bestand ja nicht ausschließlich in der Kollaboration mit dem nationalsozialistischen Deutschland. Formal betrachtet unterstand ganz Frankreich, die besetzte und die unbesetzte Zone, der Vichy-Regierung von Marschall Philippe Pétain. In der von Nationalsozialisten und -sozialistinnen besetzten Zone waren für Dekrete, Verordnungen und Gesetze der Vichy-Regierung Bestätigungen der Militärverwaltung notwendig. Die französische Verwaltung wiederum war verpflichtet, den Anordnungen der deutschen Besatzung zu folgen (Fekl 2006a: 681). Beinahe unberücksichtigt bleibt diese politische Dimension in den Untersuchungen des INH. Sie wird nur punktuell eingeblendet. So heißt es bspw. bei Schneider: »Given the Vichy regime's slogan of national renewal and the family, the way was clear for a greater emphasis on health. This explains why the Rockefeller efforts were so well received « (Schneider 2003: 22). Inwiefern sich jedoch die Philantropie der Rockefeller Stiftung und die Gesundheitspolitik des Vichy-Regimes überschneiden - der 
INH wird beim Gesundheitsministerium in Paris eingerichtet - bleibt unberücksichtigt. Zwar entsteht der INH nicht im Rahmen der Kollaborationspolitik mit dem nationalsozialistischen Deutschland, aber letztlich wird er in diesem Rahmen auch nicht aufgelöst, wobei formal gesehen, eine Bestätigung der deutschen Militärverwaltung für dessen Einrichtung per Gesetz notwendig war. ${ }^{4}$ Das heißt, der INH wird im Rahmen der Kollaborationspolitik in irgendeiner Form akzeptiert, auch wenn oder gerade weil er nicht thematisiert wird. Darum stellt sich zudem die Frage, ob und wenn ja, inwiefern es zwischen den Vorstellungen und Tätigkeiten des INH oder auch der Rockefeller Stiftung, der Politik des Vichy-Regimes und letztlich der Kollaborationspolitik mit dem nationalsozialistischen Deutschland Überschneidungen gibt.

Dass die politische Dimension in der Arbeit von Picard nicht berücksichtigt wird, kritisiert Ilana Löwy (2003) anhand von Picards Publikation über die Entstehung des INH »Aux origines de l'Inserm« - »Zu den Anfängen des Inserm« (Picard 2003/Übers. S.K.). Löwy meint in ihrem Kommentar, es soll nicht vergessen werden, dass es während des Vichy-Regimes »Kategorien von Personen« gibt, denen die Eigenschaft als Bürger oder Bürgerin abgesprochen wurde und die somit von einer »Verbesserung der Gesundheit der Bevölkerung « ausgeschlossen waren (Löwy 2003: 29/Übers. S.K.). Ausschluss ist in Anbetracht dessen, wie viele Menschen in französischen Lagern inhaftiert und/oder in deutsche Konzentrations- und Vernichtungslager deportiert und ermordet wurden, m.E. ein seltsam anmutendes Wort. Allerdings wird die Begriffswahl Löwys insofern schlüssig, als in der (französischen) Fachliteratur über das Vichy-Regime allgemein von »exclusion« »Ausschluss « die Rede ist, und zwar im Unterschied zur »extermination « - »Vernichtung « oder »Ausrottung «, die der Politik der deutschen Besatzung entsprochen habe. Diese Unterscheidung wird in jüngeren Studien, wie der des französischen Historikers Laurent Joly zur antisemitischen Politik des Vichy-Regimes in Frage gestellt (Joly 2006). Denn, so Joly, diese Aufteilung in zwei Kategorien, in die der deutschen und der französischen Politik, verhindere eine Betrachtung der Zwischenpositionen, die führende Persönlichkeiten des Vichy-Regimes eingenommen

4 Ob eine Bestätigung bedeutete, dass der Gesetzestext von Besatzerinnen und Besatzern unterzeichnet wird, ist unklar. Der Gesetzestext, der im Journal officiel de l'État français publiziert ist, ist nicht von ihnen unterzeichnet (Loi n 4968 1941). Picard benennt zwar das Gesetz vom 30.11.1941, das zur Einrichtung des INH führte, führt allerdings nicht auf, ob es von den Besatzerinnen und Besatzern bestätigt wurde (Picard 2001: o.S.). Zwar wird auf der Homepage des INSERM die Geschichte des Instituts wie auch des INH dargestellt und der Gesetzestext von 1941 zusammengefasst wiedergegeben, die Information ist jedoch auch hier nicht zu finden (vgl. INSERM o. J.). 
haben (ebd.: 15). Um diese Zwischenpositionen zu verstehen, sei es im Sinne Jolys von führenden Persönlichkeiten oder von Institutionen wie eben dem INH, ist es m.E. notwendig, jenseits dieser Kategorien zu denken und insbesondere zu fragen, in welcher Gesundheitspolitik der INH eingebettet ist und was unter Gesundheit verstanden wird. Mit Löwy gedacht, sind Fragen des Ausschlusses bestimmter Menschen, sei es aus der Gesundheitspolitik eines Landes, einer Nation oder eben auch einer Institution sicherlich wichtig. Aber um zu verstehen, was die Gesundheitspolitik beinhaltet, in deren Rahmen der INH entsteht, soll auch die Frage der Inklusion in diese Politik berücksichtigt werden, genauso wie die Frage danach, welche Subjekte oder Adressaten und Adressatinnen entworfen und angesprochen werden.

Die Ergebnisse von Picard und Schneider - die Rolle der Rockefeller Stiftung bei der Entstehung des INH - werden im Folgenden tiefer in den politischen Kontext der Zwischenkriegszeit und des Vichy-Regimes gestellt. Zur Kontextualisierung liegen einige Arbeiten vor, wie die Untersuchung des Medizinhistorikers Paul Weindling zu den Initiativen der Rockefeller Stiftung im Bereich Medizin und Gesundheit zwischen den beiden Weltkriegen in Europa (Weindling 1993, 1997) sowie die Untersuchungen der französischen Historiker Jean-Paul Gaudillière und Patrick Zylbermann sowie der Historikerin Ilana Löwy zur Medizin- und Gesundheitspolitik der Rockefeller Stiftung bis 1945 (Löwy/Zylberman 2000; Gaudillière 2000). Zum Vichy-Regime liegt die einflussreiche Arbeit des US-amerikanischen Historikers Robert Paxton vor (Paxton 1982), die zu Beginn der 1970er Jahre erscheint. Sie bedeutet insofern eine Wende in den historischen Untersuchungen des Vichy-Regimes, als sie dem bis dahin dominierenden Widerstandsmythos einer französischen Mehrheit begegnet und weitere Untersuchungen jenseits einer heroischen Interpretation ermöglicht (Lackerstein 2012: 3). ${ }^{5}$ Hierzu gehört die jüngst erschienene Arbeit der australischen Historikerin Debbie Lackerstein über die »policies and ideas« des Regimes (Lackerstein 2012). Dazu zählt auch die historische Arbeit von Miranda Pollard über die Rollen von Frauen im Vichy-Regime (Pollard 1985, 1993, 2000). Zudem wird auf die Arbeit der französischen Historikerin Anne Carol zur Geschichte der Eugenik in Frankreich zurückgegriffen (Carol 1995), auf »The Oxford Handbook of The History of Eugenics« (Bashford/Levine 2010) und die Untersuchungen des französischen Politikwissenschaftlers PaulAndré Rosenthal und des Historikers und Demografen Hervé Le Bras zur Bevölkerungspolitik in Frankreich (Rosenthal 2003; Le Bras 1999). Berücksichtigt werden zudem weiterführende Fallstudien wie die Arbeit der Demografin Virginie

$5 \mathrm{Zu}$ den Untersuchungen in Frankreich zum Vichy-Regime vor und nach Paxtons Veröffentlichung vgl. Stanley Hoffmann (2000). 
De Luca zur pro-natalistischen Politik in Frankreich am Beispiel des Kampfes gegen Geschlechtskrankheiten (De Luca 2003), die Arbeiten des argentinischen Historikers Andrés Horacio Reggiani und des französischen Historikers und Soziologen Alain Drouard zur Eugenik und Bevölkerungsforschung am Beispiel der Alexis Carrel Stiftung (Reggiani 2002; Drouard 1990).

\subsubsection{Die gesundheitspolitischen Initiativen der Rockefeller Stiftung}

Die Rockefeller Stiftung ist eine seit 1913 bestehende NGO mit dem Ziel »to promote the well-being of mankind throughout the world « (The Rockefeller Foundation 1915: 7). Das »Wohlbefinden« soll durch Förderungen im öffentlichen Gesundheitswesen, der medizinischen Ausbildung und der wissenschaftlichen Forschung erreicht werden. Zur Umsetzung ihrer Förderung suchen die Repräsentanten und Repräsentatinnen der Stiftung in jedem Land Kooperationspartner und -partnerinnen. Hierzu zählen sowohl Institutionen als auch Individuen (Weindling 1993: 254). Wie der Medizinhistoriker Paul Weindling ausführt, hat die Rockefeller Stiftung im Zwischenkriegseuropa das Ziel, wissenschaftliche Forschung und medizinische Programme zu unterstützen, um insgesamt das Gesundheitsniveau in Europa zu verbessern (ebd.: 253). Die Lösung für Krankheit und Armut wird von der Stiftung in der Wissenschaft gesehen. Hierzu gehören nicht nur Medizin, sondern auch die Sozialwissenschaften oder die Molekularbiologie ${ }^{6}$ (Weindling 1997: 271). Medizinischer Fortschritt bedeutet für die Führungskräfte der Rockefeller Stiftung das Ende von Epidemien und Infektionskrankheiten und »was the key to the end of poverty and that it could be achieved by social and organizational means, among which public-health campaigns were of critical value « (Gaudillière 2000: 491). Hygieneinstitute sind für die Stiftung, wie Weindling meint: »bulwarks against the spread of epidemic deseases« (Weindling 1993: 254). Voraussetzung für eine »gute Gesundheit« (ebd.: 253/Übers. S.K.) ist in der Vorstellung der Rockefeller Stiftung, so Weindling, eine demokratische Ordnung: »Convinced that a democratic political order was a prerequisite for good health, the foundation began a more ambitious scheme of social reforms in order to improve general levels of health« (ebd.: 253). Die Vorstellung einer stabilen »demokratischen Ordnung « besteht vor allem in einem Europa »based upon a recognition of even justice with international team play substituted for German domination«, so Weindling. Zudem besteht sie darin, eine Ausdehnung des Kommunismus, ausgehend von der Sow-

6 Zur Rockefeller Stiftung und ihrer Politik der Sozialwissenschaften in Frankreich vgl. Ludovic Tournès (2008), zu ihrer Förderung im Kontext der Molekularbiologie vgl. Lily Kay (1997), in den USA und Europa, hauptsächlich Frankreich, Deutschland und Großbritannien, vgl. Herbert Gottweis (1998). 
jetunion, abzuwenden (ebd.: 253). Hygiene und Gesundheit sollen nicht nur zum allgemeinen »Wohlbefinden « beitragen, sondern darüber hinaus zu einer Amerikanisierung der Gesellschaft in Frankreich: »RF [Rockefeller Foundation, S.K.] officials sincerely believed that advance in hygiene and public health, improvement in the understanding of natural phenomena, and furtherance of the control of human behaviour would all lead to the development of a happier human society, and also one which would be closer to the American model« (Löwy/ Zylberman 2000: 267). Ausgehend von der Annahme einer zunehmenden »social dysfunction « seit den 1920er Jahren - darunter wird die Zunahme an Ehescheidungen, Kriminalität, Schwachsinn, Prostitution und Alkoholismus verstanden -, liegt in den 1930er Jahren der Fokus der Rockefeller Stiftung auf der »control of human behaviour « mit dem Konzept der »new science of man « (Kay 1997: 289). Der damalige Direktor der Rockefeller Stiftung, Warren Weaver, meint 1933:

»the welfare of mankind depends in a vital way upon man's understanding of himself and his physical environment [...]. Important questions are: Can we obtain enough knowledge of the physiology and the psychobiology of sex so that man can bring this aspect of life under rational control? [...]. Can we [...] hope to breed a superior race of men? Can we solve the mysteries of various vitamins so that we can nurture a race sufficiently healthy and resistant? In short, can we rationalise human behaviour and create a new science of man? (The Rockefeller Foundation 1933: 198f).

Mit der »new science of man« kommt ein Diskurs auf, so die Wissenschaftshistorikerin Lily Kay, der auf »sex and reproduction« fokussiert, »where the politics of the body and the management of its behaviour promised to reinforce the foundations for a science based, rationalised social control (Kay 1997: 239). Inwiefern zeigt sich dieser Diskurs in der Umsetzung der Initiativen der Stiftung in Frankreich?

\subsubsection{Die Initiativen der Rockefeller Stiftung in Frankreich}

Die ersten Initiativen der Rockefeller Stiftung in Frankreich, aus denen der INH im Jahr 1942 entsteht, beginnen bereits kurz vor Ende des Ersten Weltkriegs (19141918) (Murard/Zylberman 2000; Schneider 2003: 4; Berlivet 2008: 474). Ab 1917 unterstützt die Stiftung sowohl Kampagnen gegen Tuberkulose (Murard/ Zylberman 2000: 467f), zur Verhinderung von Geschlechtskrankheiten oder zur Senkung der Kindersterblichkeit (De Luca 2003: 486f) als auch Hygieneinstitute. Das erste von der Rockefeller Stiftung initiierte und subventionierte Hygieneinstitut in Frankreich geht im Jahr 1924 aus einer Anti-Tuberkulose-Kampagne hervor: der Office National d'Hygiène Sociale - das nationale Amt für soziale Hy- 
giene. Der Office, der beim Hygieneministerium angesiedelt ist, existiert bis in die 1930er Jahre und stellt seine Tätigkeit aufgrund fehlender staatlicher Mittel ein (Schneider 1982: 282f; Tournès 2008: 1379). Pläne und Initiativen der Rockefeller Stiftung, ein nationales Hygieneinstitut in Paris einzurichten, konkretisieren sich im Jahr 1940, dem Beginn des Vichy-Regimes. Das Institut, das mit der Unterstützung US-amerikanischer Rockefeller-Experten und -Expertinnen aus den Bereichen Epidemiologie, Bakteriologie, Gesundheits-/Sanitärtechnik [engl. sanitary engineering, S.K.] und Ernährung komplettiert werden sollte, wird jedoch nicht ins Leben gerufen. Schneider führt folgende Gründe aus: Durch die mehrfache Umstrukturierung des französischen Gesundheitsministeriums in dieser Zeit fehlen die nötigen Ansprechpartnerinnen und -partner für die Rockefeller Stiftung. Zudem sind Probleme wie die medizinische Versorgung in Frankreich drängender als ein nationales Hygieneinstitut. Und nicht zuletzt ist, so Schneider, die deutsche Besatzung insofern eine Hürde für die Durchführungen der Studien, als seitens der Deutschen Bewilligungen nötig sind, um durch die Besatzungszone zu reisen, was für Studien unerlässlich ist (Schneider 2003: 11f). Die Alternative, die kurz darauf realisiert wird, ist, in der unbesetzten Zone, im südfranzösischen Marseille ein Institut einzurichten. Es handelt sich nicht um ein nationales Institut, sondern um ein Institut, das an der Universität in Marseille bei dem Mediziner André Chevallier und dem Endokrinologen Daniel Kuhlmann angesiedelt ist, der Institut des Recherches d'Hygiène - das Institut für Hygieneforschung. Sowohl Kuhlmann als auch Chevallier werden in ihrer beruflichen Tätigkeit bereits vor der Einrichtung des Instituts von der Rockefeller Stiftung unterstützt (ebd.: 13f). Aufgrund der Erfolge des südfranzösischen Institut des Recherches d'Hygiène, so Berlivet, insbesondere im Bereich der Ernährungsforschung gleich zu Beginn seines Bestehens, wird es auch durch das Vichy-Regime unterstützt. Letzteres plant schließlich ein ähnliches, aber nationales Hygieneinstitut in Paris. Dieser Plan wird bereits Ende des Jahres, im November 1941, in Form des INH umgesetzt (Berlivet 2008: 474). Zwar sind die Repräsentantinnen und Repräsentanten der Rockefeller Stiftung zu diesem Zeitpunkt nicht mehr in Frankreich - sie werden zur Rückkehr in die USA angewiesen, da der Kriegseintritt der USA absehbar scheint -, jedoch erhalten beide Institute weiterhin Unterstuitzungen durch die Stiftung (Schneider 2003: 19; Picard 2003, 12f). Direktor des INH, das beim Gesundheitsministerium in Paris angesiedelt ist, wird André Chevallier. Er ist nicht nur einer der Leiter des Hygieneinstituts in Marseille, sondern zudem bereits als Gesundheitsexperte für Ernährungsfragen für das Vichy-Regime tätig (Schneider 2003: 14; Berlivet 2008: 474f; Picard 2003: 12f). Die Tätigkeitsfelder des INH sind in Sektionen unterteilt: Ernährung, gesellschaftliche Krankheiten [frz. maladies sociales, S.K.], Hygiene und Epidemiologie. In der Ernährungssektion werden vor allem Untersuchungen zu Ernährung wäh- 
rend der Schwangerschaft und Kinderernährung durchgeführt, in der Sektion gesellschaftliche Krankheiten Studien zu Alkoholismus, Geschlechtskrankheiten und Tuberkulose. Die Sektion zu Hygiene und Epidemiologie arbeitet zur Wasserversorgung (Berlivet 2008: 475).

\subsubsection{Der »spirit « ${ }^{7}$ der Rockefeller Stiftung in Frankreich}

$\mathrm{Ob}$ und inwiefern die jeweiligen Politiken der besetzten und unbesetzten Zone in Frankreich bei der Entscheidung zur Einrichtung des Institutes für die Rockefeller Stiftung eine Rolle spielen, führen Picard und Schneider nicht aus. Welches Ziel überhaupt in der Stiftungsarbeit im Kriegskontext gesehen wird, wird anhand der damaligen Jahresberichte der Rockefeller Stiftung deutlich. Die von der Stiftung geförderten Wissenschaftler und Wissenschaftlerinnen: »represent the trained intelligence without which a war cannot be won, or a lasting peace achieved « (The Rockefeller Foundation 1941: 7). Kennzeichen dieser »trained intelligence« ist ihre »Objektivitä««, die als Kontrast zu einer totalitaristischen Denkweise gesehen wird: »the scientist's objective search for truth will outlive all the regimented thinking of totalitarianism« (ebd.: 6). Die »trained intelligence« erscheint als Mittel zum Zweck des Sieges der Demokratie. Auf die Rolle des Hygieneinstituts in Paris wird nicht konkret Bezug genommen, allerdings gehe ich davon aus, dass mit der »trained intelligence « alle von der Rockefeller Stiftung geförderten Wissenschaftler und Wissenschaftlerinnen gemeint sind. Somit sollen auch die Wissenschaftler und Wissenschaftlerinnen des INH mit ihrer »objektiven « und an »Wahrheit« orientierten Wissenschaft den Nutzen haben, zu Frieden und zum Sieg der Demokratie beizutragen. Dass nicht mehr alle geförderten Wissenschaftler und Wissenschaftlerinnen dazu beitragen können, wird im Jahresbericht der Stiftung von 1940 thematisiert: »Jewish professors in France were discharged as a result of the September decrees issued from Vichy « (The Rockefeller Foundation 1940: 10). In dieser von Nutzen geprägten Denkweise erscheint es logisch, lediglich den Ausschluss jüdischer Wissenschaftlerinnen und Wissenschaftler zu thematisieren, da sie nicht mehr für den Sieg der Demokratie arbeiten können. Thematisiert werden nur wissenschaftliche, nicht aber nicht-wissenschaftliche Berufsgruppen oder Menschen, die ausgeschlossen werden oder insgesamt die Politik der so genannten inneren Säuberung des Volkskörpers in Frankreich von »Mikroben« (Baruch 1999: 56/Übers. S.K.; vgl. Paxton 1982: 170).

Diese innere Säuberung beinhaltet u.a. die Internierung in Frankreich lebender Ausländerinnen und Ausländer (bestehende Einbürgerungen werden ab 1940 aufgehoben) wie spanische Republikanerinnen und Republikaner, deutsche Antifa-

7 The Rockefeller Foundation 1942: 8. 
schistinnen und Antifaschisten sowie Jüdinnen und Juden. Zu den so genannten inneren Feinden zählen, auch wenn sie keine Ausländerinnen und Ausländer sind, Kommunistinnen und Kommunisten, Protestantinnen und Protestanten, Freimaurerinnen und Freimauer sowie Jüdinnen und Juden (Paxton 1982: 169ff; vgl. Fekl 2006b: 1003). Noch bevor das nationalsozialistische Deutschland die so gennante Judenpolitik Frankreichs beeinflusst, setzt das Land eigene Maßnahmen um, die die spätere Vernichtungspolitik ausgehend von Deutschland erleichtern (Paxton 1982: 184): Am 3. Oktober 1940 erlässt die Vichy-Regierung das erste so genannte Statut des Juifs - Judenstatut. Diese Statute finden nicht allein in der besetzten und unbesetzten Zone Frankreichs Anwendung, sondern zudem in Algerien, Marokko und Tunesien - Ländern des nordafrikanischen Maghreb, die unter der Kolonialherrschaft Frankreichs stehen. »Ebenso wurden die Verwaltungen der französischen Kolonien, des Protektorats und der Länder ohne Mandat in Madagaskar, Vietnam, Französisch-Guinea, im Senegal, der Elfenbeinküste etc. von der Vichy-Regierung aufgefordert, das >Statut des Juifs $<$ auf die dortigen Juden anzuwenden« (Reichelt 2011: 398f/Herv. i.O.). Damit werden jüdische Menschen aus öffentlichen Ämtern, von Positionen in der Armee, in der Justiz und im kulturellen Leben wie Presse, Unterricht oder Film ausgeschlossen. Ab dem 4. Oktober 1940 sind Präfekte authorisiert, ausländische Jüdinnen und Juden zu internieren (Paxton 1982: 174). In der von Deutschen besetzten Zone gibt es im September 1940 eine Anordnung, Jüdinnen und Juden auszusondern. Eine weitere Anordnung im April 1941 ermöglicht es der provisorischen Verwaltung, jüdisches Eigentum an Arierinnen und Arier zu verkaufen, wovon entsprechend die Französinnen und Franzosen in der besetzten Zone profitieren. Im Jahr 1941 stimmt die französische Polizei zu, bei den ersten Masseninternierungen in der besetzten Zone zu kooperieren. Erste systematische Deportationen von Jüdinnen und Juden in Konzentrations- und Vernichtungslager aus Lagern in der besetzten Zone beginnen 1942 und dehnen sich in der unbesetzten Zone aus (ebd.: 181f). Die größte Massenverhaftung findet im Juli 1942 statt, die so genannte Rafle du Vél d'Hiv - Razzia des Vél d'Hiv (Vélodrome d'Hiver), der Pariser Winter-Radrennbahn, der auch so genannte schwarze Donnerstag (frz. jeudi noir). Geplant und durchgeführt wird sie von der Schutzstaffel der Nationalsozialistischen Deutschen Arbeiterpartei (NSDAP) und der französischen Polizei. Rund 13.000 Menschen werden verhaftet und nach Auschwitz deportiert, 8000 werden vor der Deportation im Vélodrome d'Hiver zusammengepfercht. Fast alle werden nach der Deportation ermordet (Weigel 2011: 413ff). ${ }^{8}$

8 Von staatspolitischer Seite und öffentlich wird die französische Kollaboration erst 1995 thematisiert, und zwar von Staatspräsident Jacques Chirac im Rahmen der Einweihung des Mahmals am Vél d'Hiv (Weigel 2011: 413ff). 
Konzentrationslager werden von der Rockefeller Stiftung als vorübergehendes Phänomen angesehen. Im Jahresbericht der Stiftung von 1944 heißt es: »A freedom thirsty world cannot be kept permanently in chains. Universities in exile, concentration camps, and bonfires for books are temporary phenomena. Men who have tasted liberty never forget the taste « (The Rockefeller Foundation 1944: 6). Ihre »responsibility in this stern days « sieht die Stiftung wie folgt: »We must of necessity serve the war effort, for there is no future for what we most desire in a world dominated by fascism. But we have a responsibility equally compelling to preserve the treasures of the spirit which we hold in trust from the past for the benefit of the generations to come« (The Rockefeller Foundation 1942: 8). Konzentrationslager scheinen für die Stiftung kein tiefgreifendes Problem darzustellen, sie sind ein vorübergehendes Phänomen. Letztlich werde der »spirit«, die objektive Wissenschaft der »trained intelligence« wie die der Wissenschaftlerinnen und Wissenschaftler des INH überleben und zu Sieg und Frieden beitragen.

Der Historiker Schneider geht davon aus, dass durch den Slogan des VichyRegimes, den der nationalen Erneuerung und der Familie, Gesundheit stärker betont wird und so die Bemühungen der Rockefeller Stiftung gut aufgenommen werden (Schneider 2003: 22). Welche Familien- und Gesundheitspolitik setzt sich im Vichy-Regime durch und inwiefern fügt sich hier die »trained intelligence « des INH, der »spirit« und die Vorstellung einer »new science of man« der Stiftung ein?

\subsubsection{Vichy-Politik und Gesundheit}

Die Gesundheitspolitik des Vichy-Regimes ist an der Gesundheit der Nation ausgerichtet. Als Keimzelle der Nation wird die Familie angesehen. $\mathrm{Zu}$ den drei Grundpfeilern des Regimes zählen »Travaille, Famillie, Patrie « - ^Arbeit, Familie, Vaterland«. Gesundheits- und Familienpolitik sind eng verknüpft. Die Familienpolitik ist stark pro-natalistisch und an demografischen Entwicklungen ausgerichtet - die Geburtenrate war zu Beginn des 20. Jahrhunderts gesunken (Lackerstein 2012: 156; Paxton 1982: 166). Die ersten Familienprogramme wie Kampagnen zur Förderung kinderreicher Familien beginnen zwar schon in den 1920er Jahren, expandieren jedoch im Vichy-Regime (Paxton 1982: 166). Die Familienpolitik wie die Politiken des Regimes - der so genannten nationalen Revolution - im Allgemeinen werden im Kontrast zur »Dekadenz« der Zwischenkriegszeit (Dritte Republik) begründet: »National Revolution literature attributes the fall in the birth rate to matirialism, frivolity and a denial of natural and devine law. Often Catholic, it condemned the Republic for its failure « (Lackerstein 2012: 157; vgl. Pollard 1985: 37). Die Familienpolitik, die sich durchsetzt, basiert nicht auf einem Familienkonzept einer einzelnen politischen Partei: »Vichy's portrayal of the family as the basic cell of an organic society, supported by the traditional feminine role, reflected 
attitudes and programmes that could be found in all the mainstream parties, left and right, many conservative organisations and even the extremist leagues « (Lackerstein 2012: 157). Frauen wird in der Keimzelle, der Familie, eine zentrale Rolle zugeschrieben, insbesondere durch die Fähigkeit, Kinder zu gebären. Die Mutterschaft zu Hause wird glorifiziert und aufgewertet (ebd.: 222): »Kraft ihrer Mutterschaft selbst ist die Mutter die privilegierte Arbeiterin der nationalen Sanierung « und »nur die Fruchtbarkeit kann das Wohl der Rasse sichern«, so heißt es in einer Propagandabroschüre gegen Geburtenrückgang aus dem Jahr 1942 (Brochure »Un Fléau National: la Dénatalité« (1942): o.S., zit. nach Pollard 1985: 39/Übers. S.K.). Dem Muttertag kommt große Bedeutung zu, denn die Mutter zu ehren, heißt das Vaterland zu ehren (ebd.: 43). Medizinerinnen und Mediziner, die mehrheitlich die Thesen der nationalen Revolution vertraten, erhalten während des VichyRegimes besonders Beachtung. Die » hussards blancs « - »)weißen Husaren« « dienen der Förderung der Propaganda; Familienärztinnen und -ärzte bekommen besondere soziale Anerkennung (Simonin 1997: 160/Herv. i.O., Übers. S.K.). Ihre Rolle ist insofern bedeutend, so Pollard, als sie Schwangerschaften als Gesundheitsattribut herausstellen und dies wissenschaftlich begründen: »Professor Pinard, einer der großen Lehrherren auf dem Gebiet der Geburtshilfe, liebte es zu sagen, dass eine Frau im Allgemeinen vier Schwangerschaften im Laufe ihres Lebens haben muss, um eine normale Gesundheit zu haben « (Alliance Nationale contre la Dépopulation (1944): o.S., zit. nach Pollard 1985: 44/Übers. S.K.). Väter kinderreicher Familien werden u.a. durch einen besseren Arbeitsplatz oder Steuererleichterungen gefördert (Paxton 1982: 167). Der Schwangerschaftsabbruch wird als »crime against the French people« (Lackerstein 2012: 222) angesehen; ihm wird mit einem hohen Strafmaß bis hin zur Todesstrafe begegnet (Pollard 1993: 174ff; vgl. Pollard 2000). Ehescheidungen werden erschwert und erst nach drei Jahren einer Ehe erlaubt (Lackerstein 2012: 222, 160; Paxton 1982: 166ff). Kritisiert wird die Familienpolitik Vichys von der kollaborationalistischen Presse in Paris als realitätsfern und am eigentlichen Problem vorbei. »Whereas the traditionalists placed greatest emphasis on the family as a social cell and a vital aspect of the health of the nation, the collaborationists saw the revitalisation of the race as remaining the most pressing question« (Lackerstein 2012: 161). Abgesehen von den Kollaborateuren und Kollaborateurinnen und ihrer »Revitalisierung der Rasse « gibt es biologisierende Strömungen, Individuen und Gruppen, insbesondere Mediziner und Medizinerinnen, Eugeniker und Eugenikerinnen, Ethnografen und Ethnografinnen sowie Pseudo-Wissenschaftler und -Wissenschaftlerinnen, so Lackerstein, die eine Politik der moralischen, sozialen und rassischen Hygiene befürworten (ebd.: 225). 


\subsubsection{Biologisierende Strömungen}

Ein zentrales Beispiel dieser biologisierenden Strömung ist die Fondation Carrel die Carrel Stiftung. Hinter der nach ihrem Direktor Alexis Carrel genannten Stiftung steht die im Jahr 1941 eingerichtete Fondation Française pour l'étude des problèmes humains - Stiftung für das Studium der Probleme des Menschen. Aus dieser Stiftung geht nach 1945 das Institut National d'Etudes Démographiques das nationale Institut für demografische Studien hervor, das bis heute existiert (Reggiani 2002: 335, 354). Die als unabhängig bezeichnete Carrel Stiftung, die allerdings ihr Budget vom Gesundheitsministerium erhält, hat u.a. die Aufgabe »Maßnahmen zur Verbesserung und Entwicklung der französischen Bevölkerung « zu untersuchen (Loi du 14 janvier 1942: art. 1, zit. nach Drouard 1990: 1/Übers. S.K.). Alexis Carrel, politisch konservativ, Katholik und Eugeniker, Chirurg und Nobelpreisträger für Medizin (1912), der am Rockefeller Institute for Medical Research in New York (USA) Karriere gemacht hat, wird von Marschall Pétain zum Direktor der Stiftung benannt. Für Carrel bietet die Stiftung, so Reggiani, die Möglichkeit zur »Regenerierung « des Individuums und der »Rasse « beizutragen (Reggiani 2002: 344). Sie bedeutet für ihn, so Rosenthal, eine Art Erfüllung, um weiter an seiner eugenischen Vision zu arbeiten (Rosenthal 2003: 70f; vgl. Lackerstein 2012: 227; Fogarty/Osborne 2010: 338f). Bekannt wird Carrel durch sein 1935 erschienenes Buch »Der Mensch, das unbekannte Wesen « - international ein Bestseller. Der »Best-Selling Eugenicist « plädiert in seiner Publikation u.a. für die humane und ökonomische Euthanasie von Kriminellen und Schwachsinnigen durch Gas (Reggiani 2002: 338, 340f; Baier 2001). Mit der Fondation Carrel entwickelt sich in Frankreich, so der französische Historiker und Demograf Le Bras, »das Programm der negativen Eugenik [...] in bisher noch nicht dagewesener Breite « (Le Bras 1999: 143). Der Begriff Programm bezieht sich dabei auf die Idee der negativen Eugenik und bedeutet nicht, dass sie in Frankreich zum staatlich initiierten Programm wurde. Es gab allerdings staatlich initiierte eugenische Maßnahmen wie das Gesetz zur vorehelichen Untersuchung.

\subsubsection{Eine frühe Form der delegierten Biopolitik - die voreheliche Untersuchung}

Auf staatspolitischer Ebene wird im Jahr 1942 von der Vichy-Regierung ein Gesetz zur verpflichtenden vorehelichen ärztlichen Untersuchung verabschiedet. Es ist Teil eines Gesetzes zum Schutz der Mutterschaft und des ersten Kindes, das u.a. eine gewisse Anzahl pre- und postnataler medizinischer Untersuchungen vorsieht (Carol 1995: 330f). Initiativen für solch eine voreheliche Untersuchung gibt es bereits ab 1900 von Medizinern und Medizinerinnen (ebd.: 61ff). Explizite Gesetzesinitiativen gibt es in Form einer Kampagne ab 1926 seitens der französischen Eu- 
genik-Gesellschaft. Letztgenannte gründet sich kurz nach dem internationalen Eugenik-Kongress, der im Jahr 1912 in London abgehalten wird (vgl. ebd.: 77ff; Schneider 1982: 275ff). ${ }^{9}$ Wie Schneider schreibt, kennzeichnet die Kampagne von 1926 »a shift away from the positive, social hygiene program toward negative measures designed to discourage procreation of the unfit« (Schneider 1990a: 79). Das Gesetz von 1942 schließlich ist eine eugenische Maßnahme, durch die Eheschließungen allerdings nicht verboten oder Sterilisationen erlaubt werden können (Reggiani 2002: 349; Lackerstein 2012: 228). Sie ist damit eher eine Maßnahme von pädagogischem Charakter, wie auch Carol meint. Denn, so heißt es in dem Gesetzestext, das Gesetz habe »nur zum Ziel, die zukünftigen Ehegatten mit ihrem Gewissen und ihrer Verantwortung zu konfrontieren« (Loi $\mathrm{n}^{\circ}$ 941, Bulletin du sécretariat de l'État à la Famille et à la Santé (1942): 301, zit. nach Carol 1995: 332/Übers. S.K.). Wie der Historiker Marius Turda in seiner Untersuchung der Eugenik in Europa schreibt, zielten solche Gesetze auf »a general biotypological investigation of the nation«. Mit solchen Gesetzen zur vorehelichen Untersuchung wird eine biologische Definition zur Norm (Turda 2010: 115). Es handelt sich um eine Norm, die sich m.E. sowohl auf den individuellen Körper bezieht als auch auf die Bevölkerung bzw. Nation (Foucault 1993a: 40). Mit Memmi gedacht, kann die voreheliche Untersuchung auch als frühe Form der delegierten Biopolitik diskutiert werden (Memmi 2003a: 271ff; 2005), denn ein Anliegen des Staates - die Gesundheit der Nation - wird in individuelle Verantwortung übergeben. Die delegierte Biopolitik, die Memmi vor allem in den 1960er und 70er Jahren in Frankreich im Kontext des Schwangerschaftsabbruchs und der Kontrazeptiva aufkommen sieht, geht allerdings mit einer Transformation der staatlichen Kontroll- und Steuerungsmodi einher und entwickelt sich von Verbot und Strafe hin zu einer zunehmenden Entkriminalisierung und Bioindividuation. Im Jahr 1942 ändern sich aber die Steuerungsmodi im Kontext der vorehelichen Untersuchung nicht im Sinne einer Entkriminalisierung. Es kann eher gesagt werden, dass sich medizinische Steuerungsmodi zu staatlichen transformieren. Denn die vorehelichen Untersuchungen werden bereits vor der Einführung des Gesetzes als Teil der Initiativen für ein Gesetz von Medizinern und Medizinerinnen praktiziert und sind in den 1930er Jahren von der öffentlichen Fürsorge autorisiert (Carol 1995: 325ff). Es handelt sich um einen medizinischen Steuerungsmodus oder eine Form der Regierung der körperlichen Führung, die ab 1942 damit einher geht, seine körperlichen Bedingungen und die des potentiellen Ehepartners bzw. der potentiellen Ehepartnerin im Hinblick auf die Gesundheit der Nation auszurichten. In Frankreich besteht das

9 Ausführlich zum Eugenik-Kongress von 1912 vgl. Stefan Kühl in seiner Publikation zur internationalen Bewegung für Eugenik und Rassenhygiene (Kühl 1997: 26ff). 
Gesetz zur vorehelichen Untersuchung mit einigen Modifikationen bis Ende des Jahres 2007 (vgl. Loi n ${ }^{\circ}$ 2007-1787 2007; Carol 1995: 347ff). Allerdings ist bei dieser Form der delegierten Biopolitik keine Reflexionszeit, kein durch Mediziner und Medizinerinnen angeleitetes Sprechen vorgesehen, wie etwa im Kontext des Schwangerschaftsabbruchs ab 1975. Die Entscheidung zu heiraten oder nicht wird an die Individuen delegiert, wobei dieser Entscheidung vermutlich ein medizinischer Rat voran geht.

Lackerstein und Reggiani verdeutlichen, dass das Gesetz von 1942 ein Kompromiss zwischen konservativen, pro-natalistischen Forderungen und der Forderung des Eugenikers Alexis Carrel ist, der in solch einem Gesetz die Möglichkeit zur Umsetzung seiner rassistischen Ideen sieht (Lackerstein 2012: 228; Reggiani 2002: 349). Der Kompromiss besteht darin, dass das Gesetz nicht wie von Carrel gefordert, die Heirat von ») unfit< couples « verhindert (Lackerstein 2012: 228/ Herv. i.O.; vgl. Reggiani 2002: 349). Der Historiker Schneider wiederum zählt das Gesetz zu den "gesetzgeberischen Hinterlassenschaften « des INH, so in einem Aufsatz aus dem Jahr 2003 (Schneider 2003: 20/Übers. S.K.). In seinem Buch aus dem Jahr $1990 »$ Quality and Quantity« zur Eugenik in Frankreich (Schneider 1990b) beschreibt er zwar die Entstehung des Gesetzes ausführlich, erwähnt jedoch ebenso wie Lackerstein und Reggiani den INH im Kontext des Gesetzes nicht. Ob der INH de facto bei der Entstehung des Gesetzes relevant war, ist schwer nachzuvollziehen, eine entsprechende Quellenangabe fehlt bei Schneider. Auch die französische Historikerin Carol beantwortet diese Frage nicht abschlieBend und verweist auf eine schlechte Quellenlage (Carol 1995: 329f). Wie kann der INH vor diesem Hintergrund verstanden werden?

\subsubsection{Das Hygieneinstitut im gesundheitspolitischen Kontext des Vichy-Regimes und des New-science-of-man-Diskurses}

Das Konzept von Gesundheit des Vichy-Regimes ist an der Gesundheit der Nation ausgerichtet, die auf der Keimzelle Familie beruht, in der der Frau eine zentrale Rolle zukommt. »Vichy itself attempted to institutionalise a paternalist and reactionary definition of women's role and status, within the family and within French society« (Pollard 1985: 36). Frauen sollen sich im Sinne der Vichy-Politik auf ihre reproduktiven Fähigkeiten, Haushalt und Familie konzentrieren. Im Mittelpunkt steht die Gesundheit der Mutter bzw. der schwangeren Frau insbesondere im Hinblick auf die pro-natalistische Politik. Viele Schwangerschaften sind in dieser Politik gleichbedeutend mit einer als normal bezeichneten Gesundheit. Diese Definition wird insbesondere durch Mediziner und Medizinerinnen manifestiert. Im Rahmen dieser wissenschaftlich gestützten, paternalistischen und reaktionären Politik ist m.E. der INH insofern verortet, als einer seiner Schwerpunkte auf Studi- 
en zur Ernährung von Schwangeren liegt und somit auf ihrer Gesundheit und in der Gesamtsicht auf der Verbesserung der Gesundheit der Nation. Die Historikerin Ilana Löwy kritisiert, wie eingangs ausgeführt, anhand der Untersuchung Picards, dass er nicht berücksichtigt, dass es während des Regimes »Kategorien von Personen« gibt, denen die Eigenschaft als Bürger bzw. Bürgerin abgesprochen wird und die von einer »Verbesserung der Gesundheit« ausgeschlossen sind (Löwy 2003: 29/Übers. S.K.). Mit Löwy gedacht, ist die so genannte Anti-France - Ausländerinnen und Ausländer, Jüdinnen und Juden, Kommunistinnen und Kommunisten, Freimaurerinnen und Freimaurer von diesem Konzept der Nation ausgeschlossen sie werden interniert; Jüdinnen und Juden insbesondere ab 1942 deportiert und ermordet (Paxton 1982: 169ff, 179ff); damit waren sie nicht nur von der Nation, sondern m.E. auch aus der Gesundheitspolitik des Vichy-Regimes ausgeschlossen. ${ }^{10}$ Einer Nation, die darüber hinaus zunehmend von »Mikroben « (Baruch 1999: 56/Übers. S.K.) »gereinigt« war: »Vichy was totally consistent in its drive to establish an exclusively French, pure national community and rid it of all foreign, degenerate elements« (Pollard 1985: 39). Für diese »pure national community«war die Kategorie Rasse weniger zentral, sie war insgesamt in der Politik des VichyRegimes, auch in der französischen Eugenik nicht derart verankert wie in der nationalsozialistischen Politik Deutschlands (Paxton 1982: 174ff; vgl. Taguieff 1991; Taguieff 1994). Das Ziel einer Verbesserung der Rasse wird von Kollaborateuren und Kollaborateurinnen und insbesondere auch von Medizinern und Medizinerinnen sowie von Eugenikern und Eugenikerinnen gefordert wie Alexis Carrel. Dieses Ziel überschneidet sich punktuell mit dem New-science-of-man-Diskurs der Rockefeller Stiftung. Zur Verbesserung der Rasse wird von der Stiftung u.a. eine wissenschaftsbasierte rationale soziale Kontrolle vorgeschlagen, die auf Reproduktion konzentriert ist. Dies ist ähnlich der Forderung Carrels in Bezug auf das Gesetz zur vorehelichen Untersuchung. Nur eine punktuelle Überschneidung mit den Forderungen Carrels gibt es insofern, als das New-science-of-man-Konzept keine negativ-eugenischen Maßnahmen beinhaltet. Denn Carrel vertritt zudem die humane und ökonomische Euthanasie von Kriminellen und Schwachsinnigen durch Gas (Reggiani 2002: 338, 340f). Abgesehen von dem Ziel der Verbesserung

$10 \mathrm{Ob}$ und inwiefern Frauen der Anti-France von dem Verbot der Abtreibung betroffen waren und im Sinne Vichys ebenso ein Verbrechen gegen das Vaterland verübten wie Französinnen, ist unklar. In dem Gesetzestext wird dies nicht spezifiziert, es wird von schwangeren Frauen gesprochen (vgl. Loi n 300 1942). Pollards Untersuchungsergebnisse zur Anwendung des Abtreibungsgesetzes zeigen, dass das Gesetz vor allem in der besetzten Zone angewendet wird und diese Repression vor allem Arbeiterinnen und Kleinbürgerinnen betrifft (Pollard 1993: 192). 
der Rasse, zeigt sich diese wissenschaftsbasierte rationale soziale Kontrolle zum einen durch das Gesetz zur vorehelichen Untersuchung. Die Kontrolle wird hier insofern auf das Individuum übertragen, als die potentiellen Eheleute auf Basis ihres Gewissens selbst entscheiden sollen, ob sie aufgrund der Untersuchungsergebnisse heiraten oder nicht. Zum anderen zeigt sich die wissenschaftsbasierte rationale Kontrolle auch anhand der Untersuchungen zur Ernährung des INH während der Schwangerschaft. Allerdings zielt ein besseres Ernährungsverhalten im Kontext des INH nicht auf die Verbesserung der Rasse, sondern auf die Verbesserung der nationalen Gesundheit. Die »Leistungen« oder »Erfolge« der Gesundheitspolitik des Vichy-Regimes, die der Historiker Picard anhand des INH sieht, basieren also auf einer paternalistischen und reaktionären Frauen-Politik und gelten nur für die Menschen, die in dem Konzept von Nation inbegriffen waren.

Bevor ich ausführe, ob und inwiefern die Vichy-Vergangenheit für die Einrichtung des Ethikkomitees relevant ist, gehe ich näher auf die Entstehung des INSERM und des Ethikkomitees ein.

\subsubsection{Das Hygieneinstitut und die Libération}

In der Nachkriegszeit der Libération, der Befreiung Frankreichs von der deutschen Besatzung durch die Alliierten und durch die Résistance, findet erneut eine so genannte Säuberung statt. Dieses Mal findet nicht eine Säuberung Frankreichs von der Anti-France statt, sondern von Kollaborateuren und Kollaborateurinnen mit dem NS. Im Kontext dieser Säuberung bleibt der INH nicht unkritisiert, schreibt Berlivet, steht allerdings weniger im Rampenlicht als die Fondation Carrel (Berlivet 2008: 475). Inwiefern der INH oder die Carrel Stiftung der Kollaboration beschuldigt wird, wird von Picard, Schneider und Berlivet nicht konkretisiert. Berlivet schreibt, dass der Direktor des INH, André Chevallier, die Vorwürfe, die ihm entgegen gebracht werden, abwenden konnte, aber seine »Meinungsverschiedenheiten « mit François Billoux, dem kommunistischen Gesundheitsminister (1944-1945), ihn wahrscheinlich dazu bewegte, seinen Posten als Direktor zu verlassen (ebd.: 475/Übers. S.K.). Schneider und Picard schreiben, dass der INH durch sein Renommee international Fürsprache für seine exzellente Arbeit erhält (Schneider 2003: 21). Fürsprecher und Fürsprecherinnen hat nicht nur der INH, sondern auch die Fondation Carrel, wie Reggiani und Drouard schreiben: Über ihre positive Bedeutung insbesondere im Bereich der Demografie äußert sich u.a. der Résistance-Kämpfer und Mediziner Robert Debré, der in seiner Laufbahn von der 
Rockefeller Stiftung unterstützt wurde (Reggiani 2002: 354; Drouard 1990: 13f). ${ }^{11}$ Im Jahr 1946 wird für den INH ein Direktor gefunden, dessen Ansehen, so Schneider, höher ist als das von Chevallier, da er in der Résistance aktiv war. Es handelt sich um Louis Bugnard, der in den 1930er Jahren ebenfalls durch die Rockefeller Stiftung gefördert wurde (Schneider 2003: 21). Präsident des INH wird der o.g. Robert Debré. Der von der Stiftung geförderte »spirit« überlebte den Zweiten Weltkrieg m.E. in dieser Weise, da in der Nachkriegszeit leitende Posten mit ehemaligen Stipendiaten und Stipendiatinnen besetzt werden.

\subsection{Von Hygiene zu biomedizinischer Forschung}

Im Nachkriegsfrankreich wird die Ausrichtung des INH auf die Gesundheit der Bevölkerung schrittweise zugunsten der medizinischen Forschung marginalisiert (Gaudillière 2002a: 449; Berlivet 2008: 478). Dem INH kommt eine zentrale Rolle in der Entwicklung medizinischer und biologischer Forschung zu (Gaudillière 2002b: 391, 397). »The INH vision of biomedecine consisted in a form of laboratory work that targeted the causes and biological features characteristic of human deseases. The basic practice was to mobilize biological techniques to build up models or to experiment on human samples collected in the clinic « (ebd.: 410; vgl. Berlivet 2008: 476). Zu der Laborarbeit gehört in den 1950er Jahren nicht nur der Schwerpunkt Biologie, sondern auch Biochemie, Physiologie, Genetik und Physik (Gaudillière 2002b: 397). Diese Laborarbeit zielt allerdings nicht auf die Produktion von anwendungsorientiertem medizinischem Wissen, bspw. für Ärzte und Ärztinnen (Quirke/Gaudillière 2008: 446; Gaudillière 2002b: 414). Diese Distanzierung zur Produktion von anwendungsorientiertem Wissen resultiert aus der so genannten Demedikalisierung und der Biologisierung der Medizin, Forderungen von Biologinnen und Biologen sowie Klinikerinnen und Klinikern in der Nachkriegszeit, die sich in Frankreich durchsetzen. Die Demedikalisierung wird hauptsächlich von Biologen und Biologinnen aus dem Bereich der Grundlagenforschung gefordert, so am Institut Pasteur bspw. von den Molekularbiologen André Lwoff $(* 1902-\dagger 1994)$, François Jacob $(* 1920-\dagger 2013)$ und Jacques Monod (*1910$\dagger$ 1976) (Gaudillière 2002b, 404f). Die Kollegen Monod und Jacob, beide Biochemiker und/oder Genetiker, erhalten für ihre gemeinsame Genforschung im Jahr 1964 den Medizin-Nobelpreis. In der Nachkriegszeit sehen sie ihre Disziplin als

11 Robert Debré, als jüdischer Mediziner von den Judenstatuten des Vichy-Regimes betroffen, war im medizinischen Widerstandskomitee aktiv. Ausführlich zu diesem Komitee vgl. Anne Simonin (1997). 
unterentwickelt an. Die Unterentwicklung begründen sie in der Dominanz medizinischer Interessen (ebd.: 393, 404f). Die Biologisierung der Medizin wird von einer neuen Generation an Klinikern und Klinikerinnen befürwortet, den »néo-cliniciens« (Rigal 2008: 511; Gaudillière 2002b: 410).

\subsection{Die Verwissenschaftlichung der Medizin}

Die Neo-Kliniker und -Klinikerinnen betonen die Notwendigkeit eines institutionellen sowie eines intellektuellen Wandels der medizinischen Forschung. Vor dem Zweiten Weltkrieg gibt es kaum Forschungslabore an Krankenhäusern oder medizinischen Universitäten, zudem ist die finanzielle Förderung gering. In der direkten Nachkriegszeit existieren nur einige wenige und zudem schlecht ausgerüstete Laboratorien an Krankenhäusern mit geringem Budget (Rigal 2008: 529). Unter dem intellektuellen Wandel der medizinischen Forschung wird von den Neo-Klinikern und -Klinikerinnen sowohl die Stärkung der Rolle der Statistik als auch die der Biologie verstanden (ebd.: 512). »[C]ontemporary medicine [...] is animated by the spirit and rigour of biology [...] Nothing is more outdated than the scholastic dispute about the nature of medicine that divided those who took it for an art, and those for whom it was a science. Medicine is assuredly a science « (Jean Hamburger (1977), in: Nouvelle Revue Française d'Hématalogie, 18, 2, zit. nach ebd.: 527/ Übers. Rigal). So verdeutlicht der Mediziner Jean Hamburger die Position von einem der bedeutendsten Neo-Kliniker, Jean Bernard, später Präsident des Ethikkomitees vom INSERM und erster Präsident des CCNE. Die Neo-Klinikerinnen und -Kliniker treffen sich in den 1950ern zuerst als »Club de Treize « - „Club der Dreizehn« (ebd.: 527/Übers. S.K.). Da ihre Anzahl wächst, organisieren sie sich mit 40 Mitgliedern im Cercle d'Etudes Cliniques et Biologiques - Arbeitskreis biologischer und klinischer Studien. Unter ihnen ist der Mediziner Robert Debré, ehemaliger Fürsprecher der Fondation Carrel während der Säuberung im Kontext der Libération und Präsident des INH. Ihre Vorstellung einer wissenschaftlichen Medizin verdeutlichen sie im Jahr 1956 in der von ihnen ins Leben gerufenen Zeitschrift »Revue française d'études cliniques et biologiques « - »Französische Zeitschrift für klinische und biologische Studien« (Übers. S.K.):

»[M]edical research is different from pure clinical medicine as well as from the so-called basic sciences. It differs from the former, not so much by the methods employed (the examination of a blood test, of an electrical recording, or of an electrophoretic curve, is as much >clinical as the four classical operations of inspection, palpation, percussion, auscultation), as by a constant attempt at induction. From the latter - the physical, biochemical, and bacte- 
riological sciences - it differs by a permanent human concern, medical research being immediately and directly governed by questions arising from disease (Revue française d'études cliniques et biologiques (1956), 1, 15, zit. nach ebd.: 528/Herv. i.O.).

Basierend auf diesem Konzept der wissenschaftlichen Medizin, entwickeln die Neo-Kliniker und -Klinikerinnen ein Konzept der klinischen Forschung. Bis zum Zweiten Weltkrieg beziehen sich die Begriffe Klinik oder klinisch - Begriffe, die bereits 300 Jahre zuvor in den französischen Wortschatz kommen - auf die Untersuchung am Krankenbett. Diese Bedeutung existiert, so Rigal, bis zum Ende des 20. Jahrhunderts parallel zu der Bedeutung, die sich ab den 1960er Jahren durchsetzt. Letztere bezieht sich auf die Quelle, sei es Biologie, Mathematik, Chemie, Psychologie oder Soziologie, die für Diagnosen, Prognosen, Behandlungen oder für die Prävention verwendet werden können (ebd.: 528f). ${ }^{12}$ Den Neo-Klinikern und -Klinikerinnen sowie den Biologen und Biologinnen ist gemeinsam, dass sie es für notwendig halten, dass Medizin mehr von Virologie, Bakteriologie, Immunologie, Embryologie und Biochemie beeinflusst werde sowie dass sie Orte fordern, an denen klinische Fragen in Versuchssysteme überführt und untersucht werden können (ebd.: 511). Auch Louis Bugnard, Direktor des INH, vertritt diese Vorstellung. Im Jahr 1953 wendet er sich mit einem Entwicklungsplan für sein Hygieneinstitut an den Conseil Économique et Social - wirtschaftlicher und sozialer Beirat. Der Beirat, der nach dem Krieg von Charles de Gaulle wiederbelebt wird, versammelt Interessenvertreter und -vertreterinnen, wie Arbeitgebervertreter und -vertreterinnen, Vertreter und Vertreterinnen von Gewerkschaften oder bestimmter Berufsgruppen. Bugnard betont vor dem Conseil die schlechte Entwicklung der medizinischen Forschung in Frankreich im Vergleich zu den USA und Großbritannien sowie die Notwendigkeit von Fördergeldern aus öffentlichen Mitteln (Picard 1999: 176). Dieser Appell wird mit der Association Claude Bernard im Jahr 1953 umgesetzt, einer Pariser Organisation für biologische und medizinische Forschung. Sie ist ein Zusammenschluss verschiedener Institutionen wie dem INH und dem Centre National de la Recherche Scientifique (CNRS) - dem Nationalen Zentrum für wissenschaftliche Forschung sowie der Pariser Stadtverwaltung und der Sécurité social - der Sozialversicherung (Rigal 2008: 529f). Laboratorien in Kran-

12 Zur Entstehung der modernen Klinik im 18. Jahrhundert, zu ihrer Abkoppelung von ärztlichen Praktiken und dem Aufkommen eines neuen, rationalen Umgangs mit Krankheit und Tod, vgl. Foucaults »Geburt der Klinik« (Foucault 1988). An diese Untersuchung anknüpfend, zur Entwicklung und Transformation der Klinik, insbesondere der Transformation von Krankheit und Wissen vgl. Jean-Paul Gaudillière »La médecine et les sciences « - »Die Medizin und die Wissenschaften« (Gaudillière 2006b/Übers. S.K.). 
kenhäusern aufzubauen und die Zusammenarbeit mit dem INH sind die Ziele, die umgesetzt werden (Picard 1999: 176). Diese Entwicklung hätte allerdings nicht ohne den Aufschwung der Forschungspolitik in Frankreich stattgefunden, der sich in den 1950er Jahren vollzieht.

\subsection{Forschungspolitik in der Nachkriegszeit - moderne Technokratie}

Die Forschungspolitik der Nachkriegszeit entsteht im Kontext einer Staatspolitik, die meist als colbertistisch bezeichnet wird, so der französische Wissenschaftshistoriker Christophe Bonneuil in einem Aufsatz zu den Transformationen in der Beziehung von Wissenschaft und Gesellschaft in Frankreich. Gemeint ist eine staatlich gelenkte Forschung und Wissenschaft, die eng mit der Wirtschaft zusammenarbeitet (Bonneuil 2004: 22). Charakteristisch ist, dass sich Entscheidungsprozesse im Inneren der Ministerien und der Führungsspitzen der großen nationalen Unternehmen vollziehen. Entscheidungen werden zwischen Forscherinnen und Forschern sowie ökonomischen und politischen Entscheidungsträgerinnen und -trägern getroffen, die ähnliche Werte teilen und über eine ähnliche Ausbildung verfügen (ebd.: 18) - also meist in den Grandes Écoles ausgebildet wurden. Grandes Écoles sind Hochschulen, die für eine Eliteausbildung stehen wie die École polytechnique oder die École nationale supérieure des mines de Paris. Technischen Kompetenzen wird dort eine hohe Bedeutung zugeschrieben, die V. Republik »émancipe les technocrates modernisateurs « - »emanzipiert die modernisierenden Technokraten ${ }^{13}{ }^{3}$ (ebd.: 18). Es handelt sich um eine wissenschaftliche und politische Elite, die nach dem Zweiten Weltkrieg entstand und größtenteils aus der Résistance hervorging. Sie geht von der Notwendigkeit der Modernisierung des Landes aus. Diese sei nur durch einen von der Wissenschaft aufgeklärten Staat umsetzbar, der stark in Wirtschaft und Gesellschaft eingreift (ebd.: 16f).

Schlüsselereignis für den Aufschwung der Forschungspoltik ist der »colloque sur la recherche et l'enseignement scientifiques « - das »nationale Kolloquium über wissenschaftliche Forschung und Bildung (Équipe des Cahiers de la République 1956/Übers. S.K.). Es findet im Jahr 1956 an der nordfranzösischen Universität

13 Zur Technokratie und dem Aufkommen eine technokratischen Rationalität vgl. Frank Fischers Publikation »Technocracy and the Politics of Expertise« (Fischer 1990: 59ff). Insbesondere zur französischen Technokratie vgl. F. Ridley (1966). Zur Genealogie der französischen Technokraten und Techokratinnen als soziale Gruppe in den 1920er Jahren vgl. Olivier Dard (2000). 
von Caen statt. An dem meist nach seinem Veranstaltungsort genannten »Colloque de Caen « nehmen rund 250 Politikerinnen und Politiker, Wissenschaftlerinnen und Wissenschaftler, Industrielle sowie Gewerkschafterinnen und Gewerkschafter teil (Duclert 2004: 549; 2006: 82, 89). Auf dem Kolloquium wird die Bedeutung wissenschaftlicher Forschung für das nationale Ansehen und den Wohlstand in Frankreich anerkannt und zum staatspolitischen Programm (Strasser 2002: 538; vgl. Gottweis 1997: 324). Im Abschlussbericht des »Colloque de Caen« heißt es: »Considering that the immediate and massive expansion of scientific research is an indispensable condition for the maintenance of the intellectual influence and intellectual rank of France, the development of its agriculture and its industry, the rise of its standard of living and of the well being of its people « (Résolutions adopteés à la séance de clôture du novembre (1956), zit. nach Strasser 2002: 538). ${ }^{14}$ Forschung wird allerdings nicht nur mit Wohlstand verknüpft, sondern darüber hinaus auch mit Humanität. In einem Grußwort René Billières, des damaligen sozialistischen Bildungsministers (1956-1958), das während des Kolloquiums verlesen wird, heißt es, dass sich die Laboratorien vermehren sollen, da es sich um »temples de l'avenir, de la richesse et du bien-être « - »Tempel der Zukunft, des Reichtums und des Wohlstands « handelt. »C'est là que l'humanité grandit, se fortifie et devient meilleure « - »Hier wird die Humanität wachsen, stärker und besser werden « (Équipe des Cahiers de la République 1956: o.S./Übers. S.K.). Wissenschaftlicher und technologischer Fortschritt wird zu der Zeit in Frankreich wie auch in anderen europäischen Ländern als Kernelement für die nationale Entwicklungsfähigkeit gesehen, die sich in ökonomischem Wachstum oder medizinischen Entwicklungen darstellt (Gottweis 1998: 52). Fortschritt beinhaltet in Frankreich allerdings nicht allein ökonomisches Wachstum, sondern beinhaltet darüber hinaus ein als moralisch bezeichnetes Wachstum in Form von Humanität - wie anhand des Zitats des Bildungsministers deutlich wird. Nach dem Zweiten Weltkrieg wird der liberale Kapitalismus für die ökonomischen Probleme der Zwischenkriegszeit und für den »nationalen Verfall« für verantwortlich gehalten. Diese Rahmung steuert zur Reorganisierung des Staatsprojektes bei - mit den Zielen der Modernisierung, der Effizienz, der Produktivität und der Wettbewerbsfähigkeit. Mit Charles de Gaulle, Staatspräsident von 1959 bis $1969^{15}$, und seiner Modernisierungspolitik wird die Forschungsförderung zum Staatsprogramm (ebd.: 54f). »Everybody believed [...] that investing in pure science was economically, militarily and medically sound « (Gaudillière 2002b: 409). Der Modernisierungsdiskurs in Frankreich folgt dem

14 Ich vermute, dass es sich bei dem Zitat um eine Übersetzung Strassers ins Englische handelt. Die Quellenangabe bezeichnet das französische Originaldokument.

15 1944-1946 war er bereits Präsident der provisorischen Regierung Frankreichs. 
science push-Konzept - der Hoffnung, dass die staatliche Unterstützung der Forschung zu Anwendungen und Innovationen im industriellen Bereich führt (Gottweis 1998: 60). Seine Umsetzung findet das Konzept in der planification scientifique. Hierzu zählen staatliche Initiativen zur Forschungsförderung, u.a. die Einrichtung eines interministeriellen Verwaltungsorgans für die Steuerung der wissenschaftlichen und technischen Forschung - die Délégation Générale à la Recherche Scientifique et Technique (DGRST) (Gaudillière 2002a: 293). Die DGRST wird 1961 von Michel Debré, Premierminister (1959-1962) während der Präsidentschaft Charles de Gaulle per Dekret eingerichtet. Ihr Ziel ist es, die nationale Forschung neu zu strukturieren (Duclert 2004: 648, 653). Beraten wird die DGRST von Robert Debré, Vater von Michel Debré, Neo-Kliniker und Präsident des INH (ebd.: 654). Sie ist für die Vorbereitung der Entscheidungen über die staatlichen Forschungsausgaben zuständig und Förderinstanz verschiedener Schwerpunktprogramme, den actions concertées (Gaudillière 2002a: 293). Zu den ersten actions concertées gehören die Förderung der Molekularbiologie, Medizin und Genetik (Duclert 2004: 655; Bidault 1993: 21f). Im Jahr 1981 geht die DGRST in der Struktur des Forschungsministeriums auf, das unter Staatspräsident François Mitterrand eingerichtet wird (Duclert 2004: 648). Zu den weiteren staatlichen Aktivitäten zählt die so genannte Réforme Debré aus dem Jahr 1958, die Medizin- und Krankenhausreform. Sie wird nur gelegentlich als Medizin- und Krankenhausreform bezeichnet, und meist nach ihrem Initiator Robert Debré benannt. Sie kann in doppelter Hinsicht als Debré-Reform verstanden werden, da der Sohn Robert Debrés, Michel, im Jahr 1959 Premierminister unter Charles de Gaulle wird und die Reform 1963 umsetzt (Rigal 2008: 531). Mit der Reform erlangen die Laboratorien in Krankenhäusern, die mit medizinischen Fakultäten zusammenarbeiten und die in den 1950er Jahren von der Association Claude Bernard initiiert werden, einen offiziellen Status (Gaudillière 2002a: 292). Die Centres Hospitaliers Universitaires (CHU) - Krankenhaus- und Universitätszentren - sind laut Verordnung von 1958: »Zentren der Pflege, wo in Achtung der Kranken öffentliche medizinische Bildungseinrichtungen und post-universitäre Einrichtungen ebenso wie [...] weitere Forschungs- und Bildungseinrichtungen [...] organisiert sind « (Ordonnance $\mathrm{n}^{\circ}$ 58-1373 1958/Übers. S.K.). Krankenhäuser und medizinische Fakultäten sind so gemeinsam für medizinische Pflege, Forschung und Lehre verantwortlich (Rigal 2008: 531; Gaudillière 2002a: 292). 


\subsection{Der Institut National de la Santé et de la Recherche Médicale}

Die staatliche Förderung nationaler Forschungsinstitute ist ein wesentliches Merkmal der planification scientifique (Gaudillière 2002a: 292). Der CNRS wird nach dem Krieg das wichtigste Zentrum für Grundlagenforschung. Er verfügt über eigene Laboratorien, und Hunderte an Vollzeitwissenschaftlerinnen und -wissenschaftler sind dort beschäftigt (Gaudillière 2002b: 397). ${ }^{16}$ Auch der INH ist in der planification scientifique einbegriffen, sein Budget wächst immens (Gaudillière 2002a: 292). Im Jahr 1964 entsteht schließlich aus dem nationalen Hygieneinstitut, dem INH, der INSERM, das nationale Institut für Gesundheit und medizinische Forschung. Es untersteht dem Gesundheitsministerium. Zu seinen Aufgaben zählt, die Regierung über den Gesundheitszustand des Landes zu informieren, Studien zur menschlichen Gesundheit und zur medizinische Forschung durchzuführen und zu fördern, einen Beitrag zur Ausbildung in der medizinischen Forschung zu leisten sowie die Publikation seiner Tätigkeiten und Studien (Chambefort o.J.). Zwischen 1964 und 1968 wachsen die Forschungslabore nach eigenen Angaben von 55 auf ungefähr 90 an. Die meisten Labore sind an den CHU angesiedelt (INSERM o.J.; Chambefort o.J.). Die Forschungstätigkeiten sind in die actions concertées eingebunden, die von der DGRST lanciert werden (Chambefort o.J.). Der INH bzw. INSERM entwickelt sich zu einem biomedizinischen Institut, dessen Ausrichtung auf die Gesundheit der Bevölkerung schrittweise marginalisiert wird und der medizinischen Forschung im Sinne der Neo-Kliniker und -Klinikerinnen Raum bietet (Gaudillière 2002a: 449; Berlivet 2008: 478). Hier wird nun im Jahr 1974 ein Ethikkomitee eingerichtet.

\section{WOFÜR WAR DAS ETHIKKOMITEE DES INSTITUT NATIONAL DE LA SANTÉ ET DE LA RECHERCHE MÉDICALE DIE LÖSUNG?}

Das Ethikkomitee vom INSERM wird durch die Entscheidung des damaligen Generalsekretärs des Instituts, Constant Burg, im Jahr 1974 eingerichtet (Alias 1991: 129). Die erste Sitzung wird 1976 abgehalten. Präsident des Komitees wird der Neo-Kliniker Jean Bernard, der, wie bereits erwähnt, im Jahr 1983 der erste Präsident des nationalen Ethikkomitees wird. Beim Comité d'Éthique d'INSERM han-

16 Zum CNRS und seiner Rolle im französischen Forschungssystem vgl. Gerhard Krauss »Forschung im unitaristischen Staat« (Krauss 1996). 
delt es sich damals um ein Expertinnen- und Expertenkomitee, das sich aus vier Klinikerinnen und Klinikern, zwei Biologinnen und Biologen, einem Amtsarzt oder einer Amtsärztin, einem Facharzt oder einer Fachärztin für Medizinrecht und einem Vertreter oder einer Vertreterin des Institutpersonals zusammensetzt. Zu seinen Aufgaben zählt nicht die Politikberatung. Diese Aufgabe hatte nur der INSERM (und nicht sein Ethikkomitee), damals in Form der Information des Gesundheitsministeriums, seit dem Jahr 1981 zudem des Forschungsministeriums. Zu den Aufgaben des Komitees gehört es, Wissenschaftler und Wissenschaftlerinnen im Hinblick auf die Ethik der Biomedizin zu sensibilisieren und Stellungnahmen zu ethischen Fragen von Forschungsplänen im biomedizinischen Bereich abzugeben (ebd.: 131). Zu dem Bereich zählen die Perinatologie, die Pädiatrie, die Neuropsychiatrie und die Onkologie. Die Forschungspläne werden von Wissenschaftlern und Wissenschaftlerinnen auf freiwilliger Basis vorgelegt: Bei negativer Bewertung der Pläne wird die Finanzierung der Projekte in Frage gestellt (ebd.: 133; vgl. Ambroselli 1984: 8; Maio 1995: 291; 2000: 77). Vor der Diskussion im Ethikkomitee wird der Forschungsplan im Hinblick auf seine Methode geprüft. Das Ergebnis der Diskussion innerhalb des Komitees war in den meisten Fällen konsensual (Alias 1991: 132).

In der Folgezeit wenden sich auch Forscher und Forscherinnen, die nicht am INSERM angebunden sind, an das Komitee, denn immer mehr wissenschaftliche Zeitschriften setzen solche Stellungnahmen als Bedingung für eine Veröffentlichung voraus, so der Freiburger Philosoph und Mediziner Giovanni Maio in seiner Studie zur Diskussion von Humanexperimenten in der französischen Nachkriegszeit: »Immer lauter wurde der Ruf nach weiteren Institutionen dieser Art (Maio 2000: 77f; vgl. Bateman Novaes 1998: 14). Für eine Anrufungspflicht bei Forschungsvorhaben tritt die nationale Medizinakademie 1977 ein und 1979 die Standesvertretung für Ärzte und Ärztinnen, der Ordre des médecins. An mehreren privaten und öffentlichen Forschungseinrichtungen entstehen solche Ethikkommissionen oder -komitees zur Beurteilung von Forschungsprojekten (Maio 1995: 291; 2000: 78). Inwiefern scheint die Einrichtung eines solchen Ethikkomitees beim INSERM notwendig oder anders gesagt: Wofür ist es die Lösung?

Die Einrichtung des Comité d'Éthique d'INSERM im Februar 1974 geht auf die Initiative von Simone Veil zurück, Gesundheitsministerin von 1974-1979 während der Präsidentschaft von Valéry Giscard d'Estaing (1974-1981) (Alias 1991: 129). Die konkreten Beweggründe Veils, ein Ethikkomitee zu initiieren, sind schwer rekonstruierbar. In den entsprechenden Dokumenten wird zwar erwähnt, dass sie das Komitee initiiert hat, jedoch nicht, inwiefern die Einrichtung notwendig schien (vgl. Ambroselli 1984: 8; Alias 1991: 129). Für meine Untersuchung hat 
Simone Veil ein Interview zweifach abgelehnt ${ }^{17}$ und auch in den realisierten Interviews gab es auf diese Frage keine präzise Antwort. Allerdings gibt es für die Beantwortung der Frage vier Anhaltspunkte: Erstens, wie zu Beginn dieses Kapitels erwähnt, erklärt Noëlle Lenoir in ihrer Publikation »An den Grenzen des Lebens« (Lenoir 1991/Übers. S.K.) die Entstehung von Ethikkomitees in Frankreich mit dem »Bewusstwerden von ethischen Problemen« durch den Zweiten Weltkrieg und insbesondere durch die Humanexperimente im NS. Auch Jean Bernard nimmt in seinem Buch »Mediziner im Wandel eines Jahrhunderts« auf »die skandalösen Experimente von Hitlers Medizinern« Bezug (Bernard 1994: 321/Übers. S.K.). Zweitens ändert sich in diesem Zeitraum auf Anraten von Simone Veil der Aufgabenbereich des Instituts. Während bis zum Jahr 1974 der Schwerpunkt im Bereich der biologischen Forschung liegt, soll das Institut seine Aktivitäten in der medizinischklinischen Forschung und somit im Bereich der Versuche am Menschen weiterentwickeln (Alias 1991: 129ff). Drittens wird in einem von mir geführten Interview das Komitee als »Comité de protection«, als »Komitee zum Schutz« gegenüber der Kritik an den Forschungsaktivitäten vom INSERM bezeichnet (Int F X/Übers. S.K.). Viertens äußert sich Simone Veil im Jahr 1974 zur biomedizinischen Forschung des Instituts und der Kritik daran. In ihrem Vortrag anlässlich des 10. Jahrestages vom INSERM, sagt sie:

»Entgegen derer, die die Unvollkommenheit und die Unsicherheiten der Medizin aufbauschen und daraus übereilt ein Scheitern der Medizin folgern, bin ich der Ansicht, dass die biomedizinische Forschung [...] alle großen wissenschaftlichen Fortschritte durchdringen, beeinflussen, lenken - in einem Wort humanisieren - und den Menschen auf seine Zukunft vorbereiten kann«(Veil 1974/Übers. S.K.).

Für die Gesundheitsministerin gehört medizinische Forschung verknüpft mit einer »humanisierenden« Wirkung zum Basisbestand des wissenschaftlichen Fortschritts. Dies erinnert an das Grußwort des Bildungsministers René Billières, das im Jahr 1956 auf dem »Colloque de Caen« verlesen wird. Darin heißt es, dass sich die Laboratorien vermehren sollen, da es sich um »Tempel der Zukunft, des Reichtums und des Wohlstands « handelt. Und weiter heißt es: »Hier wird die Humanität wachsen, stärker und besser werden « (Équipe des Cahiers de la République 1956: o.S./Übers. S.K.). Die Gesundheitsministerin knüpft mit der »humanisierenden« Wirkung, die sie der biomedizinischen Forschung zuschreibt, sowohl implizit als auch explizit an den Diskurs der Nachkriegszeit an. Denn sie sagt weiter, dass »Wir« seit Ende der 1950er Jahre »schrittweise in die Gruppe der Länder eingetre-

17 Die Anfragen für ein Interview wurden brieflich am 6. und 16.12.2005 gestellt. 
ten [sind], die einen wahrhaftigen Beitrag zum medizinischen Fortschritt leisten « (Veil 1974/Übers. S.K.). Die Verknüpfung von Forschung und Wohlstand, wie sie sich beim »Colloque de Caen « zeigt, taucht in Veils Rede knapp 20 Jahre später nicht auf. Jedoch zeigt sich insofern eine Verknüpfung mit nationalem Prestige, da Veil die führende Rolle Frankreichs im Bereich der medizinischen Forschung betont. Medizinische Forschung wird von der Gesundheitsministerin m.E. nicht nur als Teil des wissenschaftlichen Fortschritts gesehen. Der medizinischen Forschung kommt in der Perspektive Veils darüber hinaus eine Schlüsselfunktion in der Lenkung, eine Führungsposition der zukünftigen wissenschaftlichen Entwicklungen zu. Zudem spricht Veil von Kritik an Medizin und bezeichnet sie als aufgebauscht und übereilt. Hierdurch stellt sie ihren eigenen Standpunkt ins Licht des Rationalen und macht ihn zu einem, der ohne Übertreibungen auskommt und nicht voreilig, sondern überlegt ist. Indem sie der biomedizinischen Entwicklung eine »humanisierende « Wirkung zuschreibt, zeigt sie implizit auf, dass sich die Kritiker und Kritikerinnen solch einer moralischen Haltung verschließen. Wer eine »humanisierenden«, sprich menschenwürdiger machende Entwicklung kritisiert, kann demnach nur eines sein: inhuman.

Die Anhaltspunkte zu der Frage, inwiefern die Einrichtung eines Ethikkomitees notwendig scheint - die Verschiebung des Aufgabenschwerpunkts vom INSERM hin zur Forschung am Menschen, die zeitlich parallele Einrichtung des Ethikkomitees, das als Schutzkomitee vor Kritik an Forschungsaktivitäten bezeichnet wird und die Bezugnahme auf Medizinskepsis in der Rede Simone Veils - lassen auf einen Zusammenhang zwischen der Entwicklung medizinischer Forschung und ihrer Kritik schließen. Aber was beinhaltet die Kritik an den medizinischen Entwicklungen und insbesondere der Versuche am Menschen, wer sind die Akteurinnen und Akteure in diesem Diskurs? Wurden ethische Probleme benannt und inwiefern stehen diese in Verknüpfung zum Zweiten Weltkrieg, zur NS-Medizin und zur Entstehung des INH im Kontext des Vichy-Regime?

\subsection{Ethik als vertrauensbildende Maßnahme? Von »Experimentieranstalten ${ }^{18}$ und Medikamentenskandalen}

»[D]ie Forschung am Menschen [wurde] in der breiten Bevölkerung auch in den achtziger Jahren nach wie vor negativ konnotiert«, so Giovanni Maio in seiner Analyse der Diskussion über das Humanexperiment in Frankreich nach 1945 (Maio 2000: 60), auf die ich mich im Folgenden häufig beziehe. Maio arbeitet die 
Argumentationslinien dieser Diskussion innerhalb der medizinischen Community heraus. Er konzentriert sich in seiner Untersuchung zwar insbesondere auf die Diskussionen innerhalb der medizinischen Community. Indem er aber aufzeigt, dass die innermedizinische Diskussion über das Humanexperiment als Reaktion auf das öffentliche Misstrauen gegenüber Humanexperimenten zu sehen ist, bietet er einen konkreten Anhaltspunkt dafür, in welchem Kontext sich ein öffentliches Misstrauen manifestiert. Ein Ereignis, in dessen Folge sich eine kritische und skeptische Haltung in der französischen Öffentlichkeit zeigt, ist die Réforme Debré im Jahr 1958, die Krankenhaus- und Medizinreform. Im Rahmen dieser Reform entstehen mehr Forschungsmöglichkeiten an öffentlichen Krankenhäusern (ebd.: 60f). Sie wird nach dem »Colloque de Caen« im Jahr 1956, durch das sich die Bedeutung wissenschaftlicher Forschung für das nationale Ansehen und den Wohlstand manifestiert, ins Leben gerufen und im Jahr 1963 unter Staatspräsident Charles de Gaulle umgesetzt. Mit dieser Reform werden die CHU, Universitätskliniken, eingerichtet. Bis dahin ist die klinische Forschung in Frankreich nicht institutionalisiert (ebd.: 61). Mit der Institutionalisierung der klinischen Forschung geraten die öffentlichen Krankenhäuser in den »Ruf von Experimentieranstalten«, so Maio:

»Denn die finanzielle Förderung der Forschung und ihre quantitative Zunahme in den öffentlichen Kliniken [Maio verwendet die Begriffe Klinik und Krankenhaus synonym, S.K.] bei gleichzeitiger Dominanz privater Anstalten, deren kommerzielle Ausrichtung die Forschung am Menschen geradezu verbot, mußte dazu führen, daß die öffentlichen Krankenhäuser und darunter insbesondere die Centres Hospitaliers Universitaires - zunehmend in den Ruf von Experimentieranstalten kamen« (ebd.).

In Frankreich ist die Anzahl privater Kliniken höher als die öffentlicher. Wenn Maio sagt, dass die kommerzielle Ausrichtung privater Anstalten die Forschung am Menschen verbot, ist von ihm m.E. nicht gemeint, dass die Forschung am Menschen an privaten Kliniken illegal ist, sondern illegitim. Die kommerzielle Ausrichtung in privaten Kliniken, so verstehe ich Maio, verbietet die Forschung am Menschen nicht im juristischen Sinn, sondern im moralischen: Sie ist illegitim, weil in der Logik der Privatkliniken der Patient oder die Patientin eher als Kunde oder Kundin und somit als König oder Königin betrachtet wird. Sie kann zudem nur illegitim sein, da es eine eigene gesetzliche Regelung des Humanexperiments erst im Jahr 1988 gibt. In dem Jahr wird das nach dem Senator Claude Huriet ${ }^{19}$, der das Gesetz maßgeblich ausgearbeitet hat, benannte Loi Huriet (Loi ${ }^{\circ}$ 88-1138 1988)

19 Huriet, Senator (1983-2001) und Mediziner, Präsident des Institut Curie und Mitglied des Bioethikkomitees der UNESCO war 1996-2001 Mitglied des CCNE. 
erlassen. Einen expliziten medizinischen Ethikkodex gibt es in Frankreich seit dem Jahr 1947, den code de déontologie médicale. Diesem ebenso wie dem Nationalrat der ärztlichen Standesvertretung, dem Conseil National de l'Ordre des Médecins (Conseil National de l'Ordre 2012) ${ }^{20}$ unterstehen die Mediziner und Medizinerinnen. Mit diesen »Experimentieranstalten «, den CHU, steht der INSERM in Verbindung: Zwischen den Jahren 1964 und 1969 errichtet das Institut neue Laboratorien. Die meisten dieser Forschungseinheiten werden an den CHU angesiedelt (Chambefort o.J.).

Abgesehen von dem misstrauischen Klima in der französischen Öffentlichkeit gegenüber »Experimentieranstalten«, das im Kontext der Réforme Debré entsteht, ist, so mein Rechercheergebnis, ein weiteres Thema öffentlicher Diskurse Medikamentenskandale. Zu diesen zählen die affaire du Stalinon - Stalinon-Affäre und insbesondere der Contergan-Skandal. Die Stalinon-Affäre wird im Jahr 1954 bekannt. Durch das Präparat Stalinon, ein Furunkulosemittel, werden 117 Menschen vergiftet und rund 100 Menschen sterben in Frankreich und den nordafrikanischen französischen Gebieten (Bonah/Gaudillière 2007: 127; Maio 2001: 1184). Stalinon wird zuvor von der französischen Arzneimittelkommission freigegeben; im Jahr 1953 wird vom Gesundheitsministerium das Zertifikat für die Fabrikantin, die Firma Février et Decoisy, ausgestellt. Der Verkauf von Stalinon wird von den zuständigen Behörden erst Wochen nach den ersten Todesfällen gestoppt. Auch Warnungen der Behörden an Ärztinnen- und Ärzteschaft und Bevölkerung kommen verspätet (Maio 2001: 1184). Contergan kommt 1956/57 durch die westdeutsche Firma Grünenthal auf den Markt, zunächst als Grippemittel, dann als Sedativum. Die Einnahme des Medikaments von Schwangeren führt weltweit bei rund 5000 Neugeborenen zu gravierenden Missbildungen. ${ }^{21}$ Trotz vieler Hinweise von Ärzten und Ärztinnen an die Firma Grünenthal zieht diese ihr Präparat erst 1961, nach einem Artikel in der deutschen Wochenzeitung Die Welt am Sonntag mit dem Titel »Missgeburten durch Tabletten? «urück (Düsseldorf 1961; Maio 2001: 1184). Die Stalinon-Affäre führt in Frankreich, der Contergan-Skandal in der BRD und den USA zu Neuregelungen in der Arzneimittelfreigabe.

20 Zur Entstehung des Ethikkodes vgl. George Weisz Aufsatz zu den Ursprüngen der Medizinethik in Frankreich sowie den Artikel von Robert Nye zu Medizinern und Medizinerinnen, Medizinethik und Staat in Frankreich bis 1947 (Weisz 1990; Nye 2006). Für einen Überblick zur Entstehung des Conseil National d'Ordre des Médecins und der Bestrebungen ab dem 19. Jahrhundert, solch eine Institution einzurichten, vgl. Jean Pouillard (2005).

21 Zur gegenwärtigen Situation um Schadensersatz der Menschen, die mit Conterganschäden leben, vgl. Oliver Tolmein (2013). 
Wie die beiden Medikamentenskandale gerahmt sind, zeigen der französische Historiker Jean-Paul Gaudillière und der französische Medizinhistoriker Christian Bonah anhand ihrer Untersuchung zu diesen Skandalen in Frankreich und den USA (Bonah/Gaudillière 2007). Ihre Untersuchung basiert auf einer Presse- und Dokumentenanalyse (Tages- und Wochenzeitungen, medizinische und juristische Literatur, Dokumente der Assemblée nationale und von Betroffenengruppe etc.). Die Stalinon-Affäre in Frankreich, so ihr Ergebnis, wird als Fehler oder auch als Unfall gerahmt, wie der Contergan-Skandal. So heißt es bspw. im Jahr 1954 in der Tageszeitung Le Monde: »il est donc à peu près impossible d'éviter que ne surviennent de tels accidents « - »schier unmöglich ist es also zu vermeiden, dass sich solche Unfälle unerwartet ereignen« (Le Monde 8.07.1954: o.S., zit. nach ebd.: 127/Übers. S.K.). Während in der Rahmung als Fehler die individuelle Inkompetenz des Pharmazeuten, der Pharmazeutin, des Arztes oder der Ärztin vorgeschriebene Regeln einzuhalten, zentral ist, steht in der Rahmung als Unfall die Gesundheitsverwaltung im Mittelpunkt, so Gaudillière und Bonah. Einem Fehler kann mit besseren Evaluationen im Labor begegnet werden, einem Unfall mit staatlichen Regelungswerken zur Medikamentenfreigabe (ebd.: 125, 148). Der Contergan-Skandal bzw. die Thalidomid-Affäre - in Frankreich wurde Contergan als Thalidomid bekannt - ist, obwohl das Präparat in Frankreich nicht zugelassen war, zum Referenzrahmen für die Geschichte der Medikamente und ihrer Regulation geworden, so Bonah und Gaudillière (ebd.: 137). Zwar ist die Stalinon-Affäre der erste große, eigene Medikamentenskandal im Nachkriegsfrankreich, sie wird aber nicht zum Referenzrahmen. Wie die Stalinon-Affäre im »kollektiven Gedächtnis « Frankreichs in Vergessenheit geraten konnte, bleibt eine offene Frage, so die Autoren (ebd.: 137/Übers. S.K.). Ein Anhaltspunkt für eine Antwort auf diese Frage bietet m.E. ein Blick auf die französische Regulierung der Medikamentenfreigabe. Ab dem Jahr 1959 verfügt Frankreich als Konsequenz der Stalinon-Affäre über ein strengeres Arzneimittelgesetz als die BRD (Maio 2001: 42; Chauveau 2004: 106). Ein strengeres Arzneimittelgesetz bedeutet für die Pharmafabrikantinnen und -fabrikanten, wie die Historikerin Sophie Chauveau in ihrem Artikel zur Genese des Gesundheitschutzes in Frankreich meint, Vertrauensbildung gegenüber der Öffentlichkeit (Chauveau 2004: 106). Contergan führt erst nach der StalinonAffäre und nach der Verabschiedung eines neuen Arzneimittelgesetzes in Frankreich mit der Veröffentlichung in Die Welt am Sonntag im Jahr 1961 zum Skandal. Contergan bzw. Thalidomid ist durch dieses Gesetz in Frankreich verboten. Im Rampenlicht des Skandals stehen insbesondere die BRD und die USA. Entsprechend besser kann sich, so meine These, Frankreich präsentieren, da sich das Arzneimittelgesetz als sicher darstellte. 
Wie Bonah und Gaudillière auf Grundlage ihrer Presse- und Dokumentenanalyse zeigen, sind also die Medikamentenskandale in Frankreich als Fehler oder Unfall gerahmt - nicht als ethisches Problem. Verknüpft ist der Diskurs nicht mit den »Experimentieranstalten « oder der Thematisierung von Versuchen am Menschen. Ein Misstrauen gegenüber den »Experimentieranstalten« entsteht erst nach der Stalinon-Affäre mit der Réforme Debré, die 1963 umgesetzt wird. Es gibt zwar keinen öffentlichen Diskurs, der Medikamentenskandale und Versuche am Menschen verknüpft, allerdings sind m.E. die hohen Verkaufszahlen des Bestsellers mit dem Titel »Guide des médicaments les plus courants« - »Führer der gängigsten Medikamente (ebd.: 108f/Übers. S.K.) ein Indiz dafür, dass nach den Skandalen das Misstrauen gegenüber Medikamenten und Medizin zunimmt. Das Buch wird im Jahr 1974 von dem Arzt und Toxikologen Henri Pradal veröffentlicht. Er beschreibt für jedes Medikament, woraus es besteht, wie es indiziert ist, wie es verschrieben und angewendet wird und welche Nebenwirkungen es hat (ebd.: 109). $\mathrm{Da}$ es in Frankreich ein Bestseller war, scheint es auf ein Problem zu antworten, auf ein Bedürfnis oder Interesse daran, sich selbst informieren zu wollen, sich Wissen anzueignen, um medizinischer Autorität nicht ausgeliefert zu sein. Ein weiteres Zeugnis dafür, sich informieren zu wollen, sind m.E. die Gruppen zum Schutz von Konsumentinnen und Konsumenten, die sich in den 1960er und 70er Jahren vervielfachen, sowohl auf lokaler als auch nationaler Ebene, ebenso wie entsprechende Zeitschriften. Letztere beschäftigen sich u.a. auch mit medizinischen Themen (Chavot/Masseran 2003d: 435f). Die hohe Verkaufszahl des Medikamentenführers zeigt, so Chauveau, das sich ändernde Verhalten der Patientinnen und Patienten in den 1970er Jahren hin zu Konsumentinnen und Konsumenten (Chauveau 2004: 109). Das sich ändernde Verhalten kann, so meine ich, auch anhand der Gruppen zum Schutz von Konsumentinnen und Konsumenten gesehen werden. Allerdings geht es nicht allein um das Verhalten der Patientinnen und Patienten, denn Pradal meint »qu'il est plus facile de traiter un malade informé qu'un malade qui ne l'est pas « - »dass es einfacher ist, einen informierten Kranken zu behandeln, als einen Kranken, der es nicht ist « (Pradal (1974): o.S., zit. nach ebd.: 108/Übers. S.K.). Der Medikamentenführer ist parallel auch ein Zeugnis für ein sich veränderndes ärztliches Verhalten, das m.E. weniger paternalistisch ist.

Die Befürchtung, an öffentlichen Krankenhäusern zum Versuchsobjekt zu werden (Maio 2000: 61), das individuelle Fehlverhalten des Arztes, der Ärztin, des Pharmazeuten oder der Pharmazeutin oder die Unfälle der Gesundheitsverwaltung im Kontext der Medikamentenskandale sind nicht explizit ethisch gerahmt. Ob es in der öffentlichen Debatte um Medikamentenskandale der 1950er und 60er Jahre oder um »Experimentieranstalten« de facto eine Verknüpfung zwischen den CHU und dem INSERM gibt, oder auch, ob eine strategische Entscheidung getroffen 
wird, um mit der Einrichtung des Ethikkomitees einer Medizinkritik zuvor zu kommen, ist unklar. Die öffentlichen Diskurse um Experimentieranstalten und Medikamentenskandale sind jedoch nicht die einzigen Diskurse, die während der Einrichtung des Komitees aktuell sind. Sie sind nicht losgelöst von weiteren, insbesondere medizinischen Diskursen zu sehen, in denen Humanexperimente und medizinische Autonomie thematisiert werden. Inwiefern taucht hier die Sprache der Ethik auf und was bedeutet dies im Hinblick auf die Einrichtung des Ethikkomitees vom INSERM?

\subsection{Medizinische Ethik gegen Humanexperimente}

Die Thematisierung von Humanexperimenten von medizinischer Seite und mit ihr die Thematisierung medizinischer Autonomie ist in den 1970er Jahren als Reaktion auf die öffentliche, medizinskeptische Thematisierung zu verstehen. Wie der Philosoph und Mediziner Giovanni Maio in seiner Literaturanalyse herausgearbeitet hat - Maios Untersuchung basiert auf der Analyse medizinischer Literatur aus den »allgemeinen medizinischen Zeitschriften« und der Analyse von Standesliteratur von Ärztevertetern und -vertreterinnen ${ }^{22}$ (Maio 2000: 37) - sind die Ärzte und Ärztinnen in Frankreich in diesem misstrauischen Klima gegenüber medizinischer Forschung am Menschen gleichsam dazu gezwungen, Humanexperimente zu verurteilen (ebd.: 61f). Die Bestrebungen der Regierung, Humanexperimente gesetzlich zu regeln, sowie die Negativpresse zu Humanexperimenten veranlassen den Ordre des médecins, die französische ärztliche Standesvertretung, im Jahr 1974 dazu, eine Pressekonferenz einzuberufen. Auf dieser Konferenz nimmt der Ordre explizit Position gegen Humanexperimente. Die Stellungnahme hat den Titel: »L'Ordre des médecins contre l'expérimentation humaine - »Der Ordre des médecins gegen Humanexperimente (Le Bureau du Conseil national de l'ordre des médecins 1974/Übers. S.K.). Die Stellungnahme wird in allen großen Medizinzeitschriften wie La Nouvelle Presse Médicale und Le Concours Médical publiziert (Maio 2000: 73). In der Stellungnahme ist zu lesen: Der »Conseil national de l'Ordre des médecins erinnert daran, dass jeder Versuch am Menschen dem Prinzip der Achtung des menschlichen Lebens widerspricht, das im deontologischen Code eingeschrieben ist und an erster Stelle steht « (Le Bureau du Conseil national de l'ordre des médecins 1974/Herv. i.O., Übers. S.K.). Und weiter heißt es: »dieser

22 Um welche Zeitschriften und Standesliteratur es sich konkret handelt, wird nicht umfassend benannt. Dies wird nur an der jeweiligen Textstelle bzw. den Zitaten deutlich, deren Quellen hier entsprechend wiedergegeben werden. 
Versuch wird die Tür für Praktiken öffnen, von denen manch historisches Beispiel in der Erinnerung aller präsent is« (ebd./Übers. S.K.). Zwar benennt der Ordre nicht explizit die Medizin im NS, sondern spricht von einem historischen Beispiel, das in der Erinnerung aller präsent ist. Jedoch gehe ich mit Maio davon aus, dass mit dieser Umschreibung der NS bzw. die NS-Medizin gemeint ist (Maio 2000: 72ff). Die Thematisierung von Humanexperimenten richtet sich allerdings nicht auf Humanexperimente insgesamt, sondern, wie im obigen Zitat benannt, nur auf »diese Versuche«. Gemeint sind die so genannten nicht-therapeutischen Versuche am Menschen. Unter nicht-therapeutischen Versuchen werden in Frankreich solche Versuche verstanden, deren Ziel nicht der Heilungsversuch eines Patienten oder einer Patientin (therapeutische Versuche) ist, sondern denen vorrangig ein wissenschaftliches Interesse zugrunde liegt (ebd.: 48). Durch diese Aufteilung und Fokussierung des Diskurses auf einen Aspekt von Humanexperimenten, den nichttherapeutischen, bleiben die therapeutischen unangetastet. Der Diskurs konzentriert sich somit auf die medizinische Forschung, also auf die nicht-therapeutischen Versuche und ihr wissenschaftliches Interesse. Durch die Verknüpfung nicht-therapeutischer Versuche mit NS-Medizin werden diese Versuche durch den Ordre des médecins tabuisiert. Nach Maio äußert sich der Ordre so vehement gegen diese Humanexperimente, da er selbst während des Vichy-Regimes entsteht (ebd.: 55f; Fette 2012: 162ff): Seine Einrichtung wird per Dekret am 7. Oktober 1940 beschlossen. Der Ordre ist aktiv an der so genannten inneren Säuberung beteiligt. Er hatte die Aufgabe, Zulassungsanfragen jüdischer Ärzte und Ärztinnen zu beurteilen und sie entsprechend abzulehnen - seit einem Gesetz vom 11. August 1941 durften maximal zwei Prozent der praktizierenden Ärzte und Ärztinnen jüdisch sein (Evleth 2006: 217, 222; Maio 2000: 56f). Nach dem Krieg wird der Ordre im Jahr 1945 wiedergegründet und gut ein Drittel der Funktionäre und Funktionärinnen bleiben im Amt (Maio 2000: 57; Fette 2012: 213). Erst im Jahr 1997 äußert sich der Ordre zu seiner Vergangenheit im Vichy-Regime und zwar dahingehend, dass einige Mitglieder an der Umsetzung der antisemitischen Politik des Regimes beteiligt gewesen seien (Evleth 2006: 224; Fette 2012: 212). Die eigene Vergangenheit im Vichy-Regime, die damit einhergehende Verknüpfung mit dem nationalsozialistischen Deutschland und der NS-Medizin führt zu einer, zugespitzt formuliert, anti-nazistischen Argumentation des Ordre gegen Humanexperimente. Indem der Ordre des médecins in seiner Stellungnahme von 1974 Humanexperimente in den Kontext des NS stellt, distanziert er seine Institution von einer Verknüpfung mit Humanexperimenten (Maio 2000: 72ff). Er versucht das Vertrauen der Öffentlichkeit nicht zu verlieren und seine Autorität zu schützen, so Maio (ebd.: 55ff). Diese Autorität wird im Zuge weiterer dominanter Diskurse auf staatlicher Ebene 
und in der Öffentlichkeit in Frage gestellt, wie später in diesem Kapitel noch ausgeführt wird.

Die Abgrenzung der Mediziner und Medizinerinnen von Humanexperimenten im NS entsteht allerdings nicht erst in den 1970er Jahren und allein durch den Ordre. Sie geht in die französische Nachkriegszeit zurück. Carol zeigt in ihrer Studie zur Geschichte der Eugenik in Frankreich auf, dass sich gleich in der unmittelbaren Nachkriegszeit im medizinischen Diskurs der Fokus auf Humanexperimente im NS herausbildet und insbesondere auf Humanexperimente in vivo (Carol 1995: 342). Im Schatten der Auseinandersetzung stehen eugenische Maßnahmen, auch Zwangssterilisationen. In französischen Publikationen zur Eugenik in den 1950er Jahren sind Sterilisationen, die von NS-Medizinern und -Medizinerinnen durchgeführt wurden, bspw. unter den Sterilisationen aufgeführt, die in Skandinavien und Estland realisiert wurden. Sie werden nicht als eigenständiger Bereich oder Problem behandelt. Während des NS werden in den Jahren 1934-1945 auf Basis des Gesetzes zur Verhütung erbkranken Nachwuchses ungefähr 400.000 Menschen zwangssterilisiert (Weingart et al. 1996, 464ff). ${ }^{23}$ Zwischen 1929 und 1940 führen mehrere europäische Länder Sterilisations-Gesetze ein. Sie erlaubten es, Sterilisationen unter bestimmten Bedingungen, auch ohne Zustimmung des betroffenen Menschen, durchzuführen. ${ }^{24}$ Wenn Sterilisationen in französischen Publikationen der Nachkriegszeit thematisiert werden, so stehen ihr Nutzen und ihre Wissenschaftlichkeit im Zentrum: »[L]a stérilisation est beaucoup moins efficace que l'interdiction des mariages consanguins « - »die Sterilisation ist viel weniger effizient als das Eheverbot für Blutsverwandte «, so heißt es in einer Publikation aus den 1950er Jahren (Audit et Tisserand-Perrier (1953) »L'Eugénique et l'euthénique«, 204, zit. nach Carol 1995/Übers. S.K.). Oder: »L'eugénique hitlérienne s'écarta complètement du sens galtonien puisqu'elle se mit uniquement au service d'une race « - »Die Hitler'sche Eugenik hat sich komplett vom galtonschen Sinn entfernt, da sie sich einzig in den Dienst der Rasse stellte« (Sutter (1950) »L'Eugénique, problèmes, méthodes, résultats«, 236, zit. nach ebd.: 346/Übers. S.K.). Zwar werden die riskanten Praktiken oder die grausamen Umstände bemängelt, die Legitimität eugenischer Praktiken wie die der Sterilisation wird allerdings nicht in Frage gestellt, so Carol (ebd.: 343): »Die deutsche Erfahrung öffnet also den Medizinern die Augen für die Gefahren des Rassismus', die Risiken des Autoritarismus', für die Irrtümer einer Medizin, die ausschließlich im Dienst des Staates steht, für den ideologischen Fanatismus, aber sie führt sie ganz selten dazu, die Gültigkeit des

23 Ausführlich dazu vgl. »Rasse, Blut und Gene« von Weingart et al. (1996: 464ff).

$24 \mathrm{Zu}$ den entsprechenden Entschädigungsdebatten insbesondere in der Bundesrepublik, Norwegen und Tschechien vgl. Braun (2015a) sowie Braun et al. (2012). 
eugenischen Konzeptes ausdrücklich in Frage zu stellen « (ebd.: 346f/Übers. S.K.). Die Mediziner und Medizinerinnen in Frankreich, so Carol, »vergaßen im umfassenden Sinn über die deutsche Erfahrung nachzudenken und über die Beteiligung der Mediziner an dieser, selbst die intellektuelle« (ebd.: 343/Übers. S.K.). Wenn in der Nachkriegszeit vergessen wird, über die Eugenik und Sterilisationen umfassend nachzudenken, sie kein grundlegendes Problem darstellen, was wird dann im Kontext der Humanexperimente diskutiert?

\subsection{Die »moralische Bildung des Arztes ${ }^{25}$ zum Schutz wissenschaftlicher Objektivität}

Maio stellt anhand seiner Analyse der Diskussion um das Humanexperiment in Frankreich fest, dass es bereits in der Nachkriegszeit kaum eine medizinische oder medizinisch-standespolitische Veröffentlichung gibt, die nicht darauf verweist, dass ein rein wissenschaftlicher Versuch am Menschen illegitim ist (Maio 1994: 37). In vielen Publikationen werde zudem betont, $\gg$ dass der wissenschaftliche Alltag nichts mit den Versuchen unter der Nazi-Herrschaft zu tun habe« (Maio 2000: 44). Die Rolle des NS für die medizinische Nachkriegsdebatte zeigt sich auch daran, so Maio, dass die Académie Nationale de Médecine aufgrund der »atrocités «»Gräuel « von 1939-1945 (Voncken 1949: 236/Übers. S.K.) im Jahr 1949 die Empfehlung für eine »Kommission zur Förderung der moralischen Bildung des Arztes « ausspricht. Denn, so heißt es im Nachrichtenblatt der Akademie, »in unserer Zeit ist die moralische Bildung des Arztes wichtiger denn je« (Académie Nationale de Médecine 1949: 231/Übers. S.K.; vgl. Maio 2000: 44). Eine verbreitete Schlussfolgerung in den von Maio analysierten Dokumenten ist, dass die Humanexperimente im NS nicht nur moralisch zu verurteilen sind, sondern auch ihre Wissenschaftlichkeit angezweifelt werden müsse (Maio 2000: 45).Was unter Wissenschaftlichkeit verstanden wird, wird in einem Zitat deutlich, das aus einer medizinischen Abschlussarbeit aus dem Jahr 1974 stammt:

»Die Nazi-Experimente im Zweiten Weltkrieg? Da haben wir die Klippe mit der jede Forschung in der Medizin mit Humanexperimenten zusammenprallt. [...] Dieses Andenken hat für die ganze spätere Zeit nach dem Zweiten Weltkrieg gleichwohl den Begriff `Experiment< selbst in Verruf gestürzt und jeden objektiven Ansatz zu dieser Frage heikel werden lassen. Eine zusätzliche Ursache für die Medizin, nicht zu offen auf Humanexperimente in der Medizin Bezug zu nehmen, muss man demnach im >üblen Geruch von Konzentrationslagern<

25 Académie Nationale de Médecine 1949/Übers. S.K.. 
sehen, der dem Begriff >Experiment< anhaftet, aber auch in Folge des systematischen Misstrauens in der Öffentlichkeit« (Lullien, Jean (1974) »L'aspect juridique de l'expérimentation médicale et thérapeutique«. Mémoire de fin d'assistanat. Paris, École Nationale de la Santé Publique: 9, zit. nach ebd.: 45/Herv. i.O., Übers. S.K.).

Wie in der Abschlussarbeit, die Mitte der 1970er Jahre entsteht, deutlich wird, stellt der Autor die Objektivität der NS-Medizin in Frage: Experimente seien aufgrund der NS-Medizin »heikel« geworden, selbst »objektive Ansätze«. Eine objektive Wissenschaft wird im Kontrast zur NS-Medizin gesehen. Die Wissenschaftlichkeit der NS-Medizin wird angezweifelt. Wissenschaftlichkeit und die Sprache der Moral - die »moralische Bildung des Arztes « - sind in der Auseinandersetzung um Humanexperimente zentral. Wie Carol im Kontext der Diskussion eugenischer Maßnahmen in der französischen Nachkriegzeit meint, geht es nicht um die Frage der Beteiligung der französischen Mediziner und Medizinerinnen an diesen, auch nicht um die intellektuelle (Carol 1995: 343). Dies zeichnet sich ebenso im Kontext der Diskussion um das Humanexperiment ab. Moral oder die »moralische Bildung des Arztes« bzw. der Ärztin werden in der Nachkriegszeit als etwas gesehen, das vor medizinischen Verbrechen wie solchen der NS-Medizin schützen kann nicht eine Aufarbeitung, die die Frage der (intellektuellen) Beteiligung an der NSMedizin einschließt. Mit der moralischen Rahmung wird eine Aufarbeitung, etwa Fragen nach der Beteiligung französischer Mediziner und Medizinerinnen, den internationalen Verflechtungen einer eugenisch und/oder rassistisch motivierten Medizin nicht notwendig; diese Fragen rücken in Distanz. Inwiefern zeichnet sich dies im Kontext des Ethikkomitees vom INSERM ab? Wie eingangs erwähnt, wird von Lenoir und Bernard die Einrichtung von Ethikinstitutionen im Zusammenhang mit dem »Bewusstwerden über ethische Probleme«, ausgelöst durch den Zweiten Weltkrieg und die NS-Medizin, gesehen.

Auch hier zeichnet sich m.E. eine Distanzierung vom Vichy-Regime, der NSMedizin und dessen Aufarbeitung ab. Sie zeigt sich vor allem auf symbolischer Ebene, zum einen durch die Persönlichkeit des Präsidenten des Ethikkomitees, Jean Bernard, zum anderen durch die Persönlichkeit der Initiatorin des Ethikkomitees, die Gesundheitsministerin Simone Veil. Bernard, vormals Kämpfer in der Résistance, gehört in der Nachkriegszeit zu den Neo-Klinikern und -Klinikerinnen, die maßgeblich an der Neukonzeption der medizinischen Forschung beteiligt sind. Er gilt als »Papst der französischen Medizin«, wie mir in einem Interview gesagt wurde (Int. F IX, Übers. S.K.; vgl. Rigal 2008, 513ff: 523); Veil, Jüdin, überlebt das Konzentrations- und Vernichtungslager Ausschwitz-Birkenau (Veil 2007: 391f). Sie setzt sich seit der Nachkriegszeit gegen Antisemitismus und für die Erinnerung an die Shoa ein. Wie sich Veil Mitte der 1970er zum Vichy-Regime und 
dessen Aufarbeitung positioniert, wird in ihrer Autobiografie »Une vie« $-»$ Ein Leben $\ll^{26}$ deutlich (ebd./Übers. S.K.). Hier nimmt sie auf die öffentliche Auseinandersetzung um den Film »Le chagrin et la pitié« - »Leid und Mitleid «Bezug (ebd.: 326ff/Übers. S.K.). Im deutschsprachigen Raum erscheint der Film mit dem Titel »Das Haus nebenan«. Der vierstündige Dokumentarfilm von Marcel Orphül, der im Jahr 1969 fertig gestellt wird, behandelt am Beispiel der Stadt ClermontFerrand Besatzung und Widerstand mittels Interviews mit Zeitzeuginnen und -zeugen aller Spektren. Er wird in einer Zeit fertig gestellt, in der in Frankreich begonnen wird, über die Vichy-Vergangenheit, die »schuldhaften Verstrickungen der Kriegsjahre « und den Widerstandsmythos zu sprechen. Dies beginnt vor allem mit den Nachwirkungen der 1968er-Bewegungen und dem Ende der Präsidentschaft von De Gaulle (Wächter 2006: 13). »Le chagrin et la pitié« ist damals ein »coup de boutoir« - »eine Offensive« (Wieviorka 1989: 115/Übers. S.K.). Er ist ein »cineastischer Paukenschlag«, der die bis dahin dominierende Geschichtsbildung hinterfragt, so Christoph Vatter in seinem Buch zu Holocaust und Kollaboration im deutschen und französischen Film (Vatter 2009: 73). Der Film ist ein »Novum«, wie Klaus Peter Walter in einem Aufsatz zur Vergangenheitsbewältigung des Vichy-Regimes im französischen Film schreibt:

»Zumindest für den Bereich Leinwandproduktionen bedeutet es ein Novum, dass hier die Verantwortung für die Gräuel der Judenverfolgung und -vernichtung nicht gleichsam automatisch auf die Zuständigkeit der Nazi-Besatzer und ihres Rassenwahns abgeschoben wird, sondern zum ersten Mal die vernichtende Dynamik eines originär französischen Antisemitismus, wie er sich unter den fatal-günstigen Bedingungen der Zusammenarbeit mit den Okkupanten verwirklichen konnte, auf erschütternde Weise im Original-Ton sowie durch den Kontrast zwischen Entschuldigungsbehauptungen und den Aussagen jüdischer Opfer dokumentiert wird «(Walter 2000: 136).

Der Dokumentarfilm ist ursprünglich für das öffentlich-rechtliche Fernsehen konzipiert, wird aber von Politikerinnen und Politikern sowie von Medienvertreterinnen und -vertretern als derart brisant eingeschätzt, so Walter, dass er nach heftigen Diskussionen 1971 im Kino ausgestrahlt wird. Der Leiter des Office de Radiodiffusion Télévision Française (ORTF), der öffentlich-rechtlichen Rundfunkanstalt, und ehemaliger Résistance-Kämpfer, Jean-Jacques de Bresson, begründet zu der Zeit die Entscheidung damit, dass der Film Mythen zerstöre, die die Französinnen und Franzosen noch bräuchten - gemeint sind die Widerstandsmythen. Während »Le

26 Im deutschsprachigen Raum erscheint Veils Autobiografie mit dem Titel »Und dennoch leben« (Veil 2009). 
chagrin et la pitié« in der BRD, den USA, der Schweiz und den Niederlanden nach seiner Fertigstellung im Fernsehen ausgestrahlt wird, ist er in Frankreich dort erst im Jahr 1981, nachdem die Sozialistinnen und Sozialisten die Regierung übernommen haben, zu sehen (Vatter 2009: 71; Walter 2000: 135f). Simone Veil, zu dieser Zeit im Aufsichtsrat des ORTF, äußert sich in ihrer Biografie mißbilligend über den Film und schreibt, dass sie damals ihren Rücktritt aus dem Aufsichtsrat angekündigt habe, sofern der Film im Fernsehen gezeigt würde (Veil 2007: 327). Ihres Erachtens habe sich in den 1970er Jahren das dominante Bild eines heldenhaften und widerständigen Frankreichs während des Vichy-Regimes zwar verändert, das neue Bild aber sei ebenso vereinfachend gewesen. Das neue Bild suggerierte, dass in Frankreich Denunzierungen allgegenwärtig gewesen seien und allein die Kommunistinnen und Kommunisten Widerstand geleistet hätten. Den Dokumentarfilm verortet Veil in diesem neuen, vereinfachenden Bild. Er sei für dieses »concert d'autoflagellation« - »Konzert der Selbstquälereien« wie gerufen gekommen, »et c'est à ce titre que je trouvais ce film injuste et partisan « - »und aufgrund dessen fand ich diesen Film ungerecht und parteiisch« (ebd.: 326f/Übers. S.K.). Der Film zeige eine »pseudo-vérité « - »Pseudo-Wahrheit«, so Veil, denn er sage insbesondere kein Wort zu den Französinnen und Franzosen, die Jüdinnen und Juden versteckt hielten (ebd.: 328/Übers. S.K.). Sie wollte nicht, dass dieser Film auf dem Rücken solcher Familien ausgestrahlt wird, die Jüdinnen und Juden versteckt hielten, so heißt es weiter in Veils Autobiografie (ebd.: 328f). Welche Bedeutung das Ausstrahlen des Dokumentarfilms im Fernsehen bspw. für die Menschen gehabt hätte, die denunziert wurden, interniert oder Konzentrationslager überlebten, die Jahrzehnte mit einem dominanten Widerstandsmythos konfrontiert waren, thematisiert sie nicht. Die Persönlichkeit Simone Veil steht in den 1970ern Jahren in der französischen Öffentlichkeit m.E. als Symbol gegen den Antisemitismus, und sie steht für eine nicht »vereinfachende«, sondern »wahre « Sichtweise auf die Vichy-Vergangenheit. Ihre Vergangenheitspolitik besteht darin, die positiven Handlungen von Französinnen und Franzosen hervorzuheben. Indem Simone Veil als symbolische Persönlichkeit ein Ethikkomitee an einem Institut initiiert, das seine Wurzeln im Zweiten Weltkrieg hat, wird m.E. eine Verknüpfung zwischen der Entstehung des Instituts und der antisemitischen Politik des Vichy-Regimes erschwert, wie auch eine Aufarbeitung der vielfältigen Schattenseiten des Regimes und seiner eugenischen Politik. In ihrem Vergangenheitsdiskurs der 1970er Jahre sollen die positiven Handlungen im Vordergrund stehen, nicht eine »Selbstquälerei«. Für Bernard haben »Hitler und die seinigen« eine »neue Barbarei« erschaffen: Sie haben Experimente durchgeführt, die auf einer »abwegigen Wissenschaft « basieren, die keinen wissenschaftlichen Fortschritt hervorgebracht haben (Bernard 1994: 321/Übers. S.K.). Ebenso wie im Kontext der moralischen Rahmung wird 
auch hier die Wissenschaftlichkeit der NS-Medizin angezweifelt. Indem Bernard von »Hitler und den seinigen« oder auch »Hitlers Medizinern« spricht, bleibt m.E. die Frage nach der Entstehung, der Beteiligung, den internationalen Verflechtungen von Medizinern und Medizinerinnen und der Förderung einer eugenisch motivierten Medizin außen vor - das Problem wird einzig im nationalsozialistischen Deutschland lokalisiert und an der Figur Hitler manifestiert. Eine Verknüpfung von NS, Vichy-Regime und medizinischer Forschung beim INSERM war somit nahezu ausgeschlossen. Bernards Betonung der »abwegigen Wissenschaft« ohne Fortschritt impliziert m.E., dass in Bernards Vorstellung Wissenschaft auf dem richtigen, ethischen und damit fortschrittlichen Weg ist. Sie entspricht damit auch Veils Auffassung von einer wissenschaftlich konzipierten und »humanisierenden « medizinischen Entwicklung. Betrachtet man darüber hinaus die Homepage des INSERM und dort die Rubrik Geschichte, so findet man Daten zur Entstehung des INH, einen Auszug aus dem entsprechenden Gesetzestext, die Aufgabenbereiche oder das damals verfügbare Budget des Instituts, aber keine Ausführung zum politischen Kontext (INSERM o.J.-a). Allerdings finden sich Literaturhinweise auf die Untersuchungen des französischen Historikers Jean-François Picard. Picard leistete, wie eingangs erwähnt, Pionierarbeit mit seiner Untersuchung über die Entstehung des INH. Anliegen seiner Untersuchung ist es aufzuzeigen, dass die VichyRegierung, die die Last der beschämenden Niederlage in der französischen Geschichte trage, »credit for some of its achievements « (Picard 2001: o.S.) erhalte. Der Historiker vermutet, dass ein autoritäres System eher fähig sei, eine Gesundheitspolitik umzusetzen, als eine parlamentarische Demokratie (ebd.). Gesundheitspolitik oder Gesundheit wird von dem Historiker positiv gewertet. Nicht thematisiert wird, was überhaupt unter Gesundheit verstanden wurde und kaum thematisiert wird, auf welcher Politik bzw. Politiken sie basierte. Mit dem Verweis auf Picards Untersuchung beteiligt sich der INSERM bis heute an einem Vergangenheitsdiskurs, der die politische Dimension des Vichy-Regimes und die internationalen Verflechtungen einer eugenisch motivierten Medizin ausblendet. Es sind also zwei unterschiedliche Umgangsformen zweier Institutionen mit ihrer Verwicklung in das Vichy-Regimes zu beobachten: Der Ordre des médecins, der während des Vichy-Regimes gegründet wurde, distanziert sich von nichttherapeutischen Experimenten, indem er sie in den Kontext des NS stellt und so seine eigene Institution legitimiert. Beim INSERM wird durch die Einrichtung des Ethikkomitees erreicht, die Reflexion über die Arbeit des Instituts während des Vichy-Regimes zu umgehen. Humanexperimente im NS werden zwar verurteilt, mit der ethischen oder moralischen Rahmung kann aber ohne eine grundlegende historische Aufarbeitung über Humanexperimente gesprochen werden. Ethische und moralische Rahmungen erscheinen im Diskurs um Humanexperimente nicht nur 
im Kontext einer bestimmten Vergangenheitspolitik, sondern auch im Kontext einer bestimmten nationalen Positionierung der französischen Forschung.

\subsection{Medizinische Moral und Nation}

Die Distanzierung von Humanexperimenten bietet auch den Raum zur nationalen Besserstellung der französischen Forschung im Kontrast zu der Forschung in den USA und in der BRD. Dies geschieht vor allem mit der Sprache der Moral, wie anhand der Ergebnisse von Maio deutlich wird. Die Sprache der Moral wird zur Verurteilung des Humanexperiments als »nationalistische Abgrenzungsrhetorik « verwendet (Maio 2000: 46ff). Im Vergleich zu Deutschland erscheint Frankreich so integrer aufgrund der Humanexperimente im Dritten Reich. So heißt es im Jahr 1964 in einem Artikel in der medizinischen Zeitschrift Cahiers Laënnec: »die grauenvollen Experimente, die von den Nazi-Medizinern im Widerspruch zu unseren moralischen Gesetzen gemacht wurden, hatten in der Praxis kein einziges gültiges wissenschaftliche Ergebnis« (Laroche, Claude (1964), in: Cahiers Laënnec, 1, 40, zit. nach ebd.: 45/Herv. i.O., Übers. S.K.). Von der Forschung in den USA wird sich aufgrund der öffentlich gewordenen Menschenversuche, u.a. an Gefangenen, abgegrenzt. So meint ein Chirurg im Jahr 1957: »Der Versuch an Freiwilligen wird im Ausland in großem Maßstab praktiziert, der Prozess von Nürnberg hat es uns gelehrt: in den amerikanischen bundesstaatlichen Gefängnissen wurden ungefähr 800 Gefangene >freiwillig< von Malaria befallen« (ebd.: 46/Herv. i.O., Übers. S.K.). Bei dem genannten Versuch handelt es sich aller Wahrscheinlichkeit nach um die so genannte Stateville-Studie, da diese im Rahmen des Prozesses von Nürnberg genannt wird, und es sich um eine Malaria-Studie handelte. Die Studie wird in den 1940er Jahren vom medizinischen Institut der Universität von Chicago durchgeführt. Unterstützt wird sie von der US-amerikanischen Armee (Hornblum 1997: 1438f; Alving et al. 1948). ${ }^{27}$ Verteidiger und Verteidigerinnen der NSMediziner und -Medizinerinnen beziehen die Studie während des Prozesses von Nürnberg ein, um herauszustellen, dass es keinen Unterschied zwischen diesem Versuch und den Versuchen in den Konzentrationslagern gegeben hätte (Hornblum 1997). Die USA werden im medizinischen Diskurs zudem gleichgesetzt mit einer Orientierung an wissenschaftlichen Interessen, die wiederum mit einem Kollektivinteresse gleichgesetzt werden, während Frankreich für die Individualrechte stehe und für das Wohl der einzelnen Patientin oder des Patienten (Maio 2000: 46). Ab-

27 Alving et al. sowie Hornblum sprechen nicht von 800 sondern von 400-500 Gefangenen. 
gesehen davon, dass die Menschenversuche im NS als unvereinbar mit den französischen moralischen Gesetzen dargestellt werden und die Versuche in den USA im Kontrast zur französischen Integrität, werden die USA mit einer ungebremsten Forschung assoziiert, die auf Kosten der Menschenrechte gehe (ebd.: 46). Diese antikollektivistische Grundhaltung der französischen Ärzte und Ärztinnen lässt sich nach Maio wie folgt erklären: Sie liegt in der Begriffsbestimmung des Humanexperiments selbst, da eine strikte Trennung von therapeutischem und nichttherapeutischem Versuch vorgenommen wird. Dieser Differenzierung liegt eine Polarisierung zugrunde: auf der einen Seite steht das Interesse des Patienten oder der Patientin, auf der anderen Seite das Interesse der Gemeinschaft oder der Wissenschaft. Entsprechend dieser Polarisierung wird in der Nachkriegszeit der Heilungsversuch positiv und das nicht-therapeutische Humanexperiment negativ bewertet (ebd.: 48).

Im Kontext dieser Abgrenzung von wissenschaftlichen Interessen, die den USA zugeschrieben werden, sieht der französische Soziologe François Alias die Entstehung des Ethikkomitees vom INSERM. Er bezieht sich hierbei in seiner Untersuchung auf die Deklaration von Helsinki. Die Deklaration wird im Jahr 1964 mit dem Titel »Ethische Grundsätze für die medizinische Forschung am Menschen« von der Generalversammlung des Weltärztebundes verabschiedet. Sie ist das erste internationale Dokument, welches ethische Richtlinien für die Forschung am Menschen formuliert. Die Deklaration soll zu Beginn der 1970er Jahre überarbeitet werden, auch im Hinblick auf die internationale Zusammenarbeit im Bereich medizinischer Forschung. In der im Jahr 1975 überarbeiteten Version von Tokio wird die Einrichtung unabhängiger Ethikkommissionen zur Bewertung von Forschungsvorhaben vorgeschlagen (WMA 2013: Art. 23). In Frankreich wird die Deklaration von Helsinki, so Alias, mit einer Orientierung an Wissenschaft assoziiert, da sie hauptsächlich von den USA inspiriert gewesen sei. Alias meint, dass mit der Einrichtung des Ethikkomitees der Deklaration von Tokio, der Nachfolgerin der Deklaration von Helsinki, und damit der US-amerikanischen Einflussnahme zuvorgekommen wird. Es erlaubt dem INSERM, so Alias, möglichen Problemen im Bereich der nicht-therapeutischen Versuche am Menschen zuvorzukommen und sich davor zu schützen (Alias 1991: 131). Um welche Probleme es sich handelt und wer konkret eine US-amerikanische wissenschaftsorientierte Einflussnahme sieht, führt Alias allerdings nicht aus. Seine Ausführung ist aber insofern schlüssig, als eine Forschungskritik an den USA auch weitere Mediziner und Medizinerinnen und der Ordre des médecins kundtun (Maio 2000: 46ff), wie auch insbesondere Jean Bernard, Präsident des Ethikkomitees vom INSERM und späterer erster Präsident des CCNE. Er sagt auf dem zweiten internationalen Kongress zur medizinischen Moral im Jahr 1966: »[E]s ist verboten Pseudo-Freiwillige zu benutzen, Ge- 
fangene, die durch vage Versprechen angelockt werden, denen man skandalöser Weise krebskrankes Gewebe spritzt« (Bernard, Jean (1966) »Progrès de la médecine et responsabilité du médecin «, in: Ordre national des médecins (Hg.): Deuxième congrès international de morale médicale, Paris: 272, zit. nach ebd.: 46/Übers. S.K.). Bei dem angesprochenen Experiment an Gefangenen handelt es sich meiner Vermutung nach um die von dem US-amerikanischen Virologen Chester Southam zu Beginn der 1950er Jahre durchgeführte Studie. Es ist die einzige Krebsstudie mit Gefangenen, die in dieser Zeit bekannt wird. Southam injiziert über 100 Gefangenen im Ohio State Prison Krebszellen, um den Krankheitsverlauf zu beobachten. Die zu großen Teilen afroamerikanischen Insassen werden nicht darüber aufgeklärt, um welche Zellen es sich handelt. Einige Jahre später wiederholt Southam das Experiment am Brooklyn Jewish Chronic Disease Hospital an über 20 afroamerikanischen Frauen (Hornblum 1997: 1440; Beecher 1966: 371). Mit Alias verstanden ist die Einrichtung des Ethikkomitees ein Symbol eines reflektierten, französisch-moralischeren Umgangs mit nicht-therapeutischen Versuchen. Es steht in Opposition zu dem skandalösen, ungebremsten US-amerikanischen Forscherdrang. Das Ethikkomitee ist in diesem Sinn nicht allein ein »Comité de protection « vor öffentlicher Kritik, sondern auch vor einer Amerikanisierung der Forschung. Vor diesem Hintergrund betrachtet, ermöglicht die Distanzierung von Versuchen in den USA und im nationalsozialistischen Deutschland eine moralische bzw. ethische Selbstrepräsentation französischer medizinischer Forschung.

\subsection{Medizinische Ethik und individuelles Gewissen}

Eine weitere Argumentationslinie im Diskurs um Humanexperimente der Nachkriegszeit beinhaltet nach Maio das »standespolitische Arztbild« (Maio 2000: 49). Die Betonung liegt in dieser Rhetorik auf der »profession libérale « - dem »freien Beruf $«^{28}$, der »indépendance du médecin « - »Unabhängigkeit des Mediziners« oder dem »individualisme médical « - »medizinischen Individualismus« (ebd.: 50/

28 Unter »profession libérale « wird in Frankreich die Gesamtheit der Berufe verstanden, die durch eine Person individuell, unabhängig und unter persönlicher Verantwortung ausgeübt wird. Im Fall des ärztlichen Berufes handelt es sich um eine »profession libérale réglementée«. Diese zeichnet sich u.a. durch das Erfordernis eines höheren Universitätsabschlusses aus und durch einen Ethikkodex, dessen Einhaltung verpflichtend ist. Wie bereits erwähnt, unterstehen die Ärzte und Ärztinnen dem code de déontologie médicale und dem Nationalrat der ärztlichen Standesvertretung, dem Conseil National de l'Ordre des Médecins (Conseil National de l'Ordre 2012). 
Übers. S.K.), den damit einhergehenden freiheitlichen Idealen des Berufes im Kontext des Verhältnisses zwischen Ärztin oder Arzt und Patientin oder Patient: Dieses wird als vertrauliches Verhältnis verstanden, in dem die unabhängige Persönlichkeit des Arztes oder der Ärztin, seine oder ihre freie Entscheidung zum Heilungsprozess gehört. Mit der Betonung der Unabhängigkeit wehren sich die französischen Ärzte und Ärztinnen und insbesondere ihre Standesvertretung, der Ordre des médecins, gegen eine staatliche Gesundheitspolitik in den 1950er und 60er Jahren (ebd.: 50ff; vgl. Weisz 1990: 149f). Sie wehren die Gesundheitspolitik oder die staatliche Kontrolle ab, indem sie darauf verweisen, dass die Einmischung des deutschen Staates im NS dazu geführt habe, dass Ärzte und Ärztinnen das Wohl der Patientinnen und Patienten verletzten.

» $\mathrm{Zu}$ dieser auf therapeutische Autonomie ausgerichteten Strategie gehörte es, sich der ethischen Terminologie zu bedienen. Um die Bedeutsamkeit der freien Entscheidung des Arztes $\mathrm{zu}$ unterstreichen, wurde kurzerhand ärztliche Autonomie zu einem moralischen Gut an sich erhoben. Nur jener Arzt könnte dem Patienten helfen, der niemand anderem als seinem Gewissen verpflichtet sei (Maio 2000: 52f).

Unabhängigkeit und das ärztliche Gewissen werden »als einzige effektive Instanzen betrachtet, die verhindern konnten, dass sich Experimente wie im Dritten Reich wiederholten« (ebd.: 64). Auffällig ist m.E., dass sich die Ärztinnen und Ärzte vor allem von den Experimenten im Dritten Reich als Zeitraum abgrenzen. Nicht thematisiert wird aber bspw. die Freiheit des ärztlichen Gewissens unter demokratischen Bedingungen: Bereits Anfang der 1920er Jahre, zu Zeiten der Weimarer Republik, sind ein Viertel der deutschen Ärzte und Ärztinnen in der NSDAP und verfolgen rassenhygienische Ideen. Darüber hinaus war zwar die Ideologie der Rassenhygiene ein speziell deutscher Ansatz, nicht aber die der Eugenik insgesamt. ${ }^{29}$ Rassenbiologie entwickelt sich ab der Jahrhundertwende zu einer inter-

29 Zur Entwicklung der Rassenhygiene unter der Weimarer Republik vgl. Anahid Rickmann (2002: 22-46). Über die Weimarer Republik hinaus vgl. das Standardwerk von Peter Weingart et al. »Rasse, Blut und Gene« (1996). Die sozialwissenschaftliche Studie untersucht die Geschichte der Eugenik in Deutschland, hier vor allem als Rassenhygiene bezeichnet, und zeigt die Entwicklungen bis hin zur Humangenetik auf. Vergleiche werden vorrangig zu England und den USA gezogen. Das thematische Hauptaugenmerk der Studie liegt auf der »Geschichte der wissenschaftlichen Strategien der Menschenzüchtung und ihrer politischen Indienstnahme« (ebd.: 15). Zu den internationalen Verflechtungen rassenhygienischer und eugenischer Bewegungen im 20. Jahrhundert vgl. »Die Internationale der Rassisten« des Soziologen und Historikers Stefan Kühl 
national anerkannten Wissenschaft, über die auch während des NS ein internationaler Austausch stattfindet. ${ }^{30}$ Ebenso entsteht Eugenik nicht erst im Kontext des Zweiten Weltkriegs. Dies zeigen allein die eugenischen Ideen eines Alexis Carrel, dessen Buch »Der Mensch, das unbekannte Wesen« im Jahr 1935 erscheint und international ein Bestseller wird. Die französischen Mediziner und Medizinerinnen distanzieren sich also lediglich von einem räumlich und zeitlich begrenzten politischen System, nicht aber von einer bestimmten wissenschaftlichen Rationalität. Auch die Frage nach der Beteiligung, nach den internationalen Verflechtungen und der Förderung einer rassistischen und/oder eugenisch motivierten Medizin wird nicht gestellt. Nicht eine Aufarbeitung der (Selbst-)Einbindung der Medizin in menschenverachtende Praktiken ist eine Lösungsmöglichkeit, sondern Ethik in Form eines institutionalisierten Komitees, das Forschungsvorhaben prüft bzw. das als moralisch bezeichnete Gut des ärztlichen Gewissens.

Im Kontext der Gesundheitspolitik seit den 1960er Jahren und der Abwehr staatlicher Kontrolle erscheint das Ethikkomitee vom INSERM - dem nationalen Gesundheitsinstitut - als Zwischeninstanz, als Institution zwischen Staat und medizinischem Forscher oder Forscherin bzw. zwischen staatlichen Interessen und medizinischem Gewissen. Neu ist, dass dieses Gewissen jedoch nicht mehr allein individuelle Angelegenheit des Mediziners oder der Medizinerin ist, sondern durch das Komitee sowohl überprüft als auch kollektiviert wird. Diese Kollektivierung des ärztlichen Gewissens sowie die Verschiebung der individuellen ärztlichen Verantwortung sind allerdings nicht einzigartig, wie im Folgenden gezeigt wird. Sie finden in weiteren Kontexten und Ländern statt.

(1997). Zur Eugenik als transnationales Phänomen und ihrer Geschichte vom 19. Jahrhundert bis in die Zeit nach dem Zweiten Weltkrieg vgl. »The Oxford Handbook of the History of Eugenics« (Bashford/Levine 2010). Zur Entwicklung der Eugenik in der Wissenschaft und Medizin Frankreichs unter Berücksichtigung internationaler Einflüsse vgl. u.a. die Untersuchung des französischen Sozialwissenschaftlers und Philosophen PierreAndré Taguieff (1991, 1994) und der Historikerin Anne Carol (1995). Zur Geschichte und Entwicklung der Eugenik in Frankreich vgl. William Schneider (1990b). Für einen Überblick vgl. der Artikel zu Frankreich in »The Oxford Handbook of the History of Eugenics $\ll$ (Fogarty/Osborne 2010).

$30 \mathrm{Zu}$ internationalen Ärztinnen- und Ärztekongressen und weiterführend zu internationalen Besuchen in der Führerschule der deutschen Ärztinnen- und Ärzteschaft vgl. Thomas Maibaum (2007) und Anahid Rickmann (2002). 


\subsection{Ethik als "shifted responsibilities « $^{31}$ und als »management of living ${ }^{32}$ ?}

Als »shifted responsibilities « bezeichnet die deutsche Sozial- und Gesundheitswissenschaftlerin Helen Kohlen die Verschiebung hin zu kollektiven Entscheidungsfindungen innerhalb von Ethikkomitees (Kohlen 2011: 127). Ihre Untersuchung bezieht sich nicht auf Ethikkomitees an staatlichen Forschungsinstituten sondern an Krankenhäusern in den USA, die sich mit Patientinnen- und Patientenfällen beschäftigen. ${ }^{33}$ Kohlens leitende Frage ist, wie die »Ethik am Krankenbett« dem rationalistischen Rahmenwerk der Bioethik gewichen ist. In diesem Transformationsprozess, so zeigt sie, spielen institutionalisierte Komitees eine bedeutende Rolle (ebd.: 127/Übers. S.K.): Die Idee der Entscheidungsfindung durch lokale Komitees treiben in den 1970er Jahren insbesondere der so genannte Quinlan-Fall und die Diskussion um Sterbehilfe voran. Karen Ann Quinlan ist eine 22-jährige USamerikanische Komapatientin, deren Eltern vor Gericht einklagen, ihre Tochter sterben lassen zu dürfen. Im Jahr 1976 wird geurteilt, dass es in diesem Fall erlaubt sei, die lebensverlängernden Maßnahmen einzustellen. Die Ärzte und Ärztinnen setzen die künstliche Beatmung, die Quinlan erhält, allerdings nur langsam ab. Ihre Atmung stabilisiert sich eigenständig und sie lebt bis 1985. Ihre Eltern kümmern sich bis zu deren Tod um ihre Tochter. ${ }^{34}$ Der Quinlan-Fall fördert nicht nur die Diskussion um Sterbehilfe und wirft die Idee der Entscheidungsfindung durch lokale Komitees auf. Er ist zudem ein Ereignis, so Kohlen, durch das Bioethik graduell in den medizinischen Bereich eindringt. Bioethik, die Helen Kohlen als eine umstrittene Disziplin versteht, deren historische Wurzeln und Aufgaben nicht eindeutig sind, ist in den USA seit den 1960er Jahren zu einem interdisziplinären Unternehmen angewachsen. Bioethiker und Bioethikerinnen werden als Experten und Expertinnen in medizinischen, rechtlichen, politischen oder auch industriellen Bereichen in Anspruch genommen (ebd.: 127). Ethikkomitees werden zur standardisierten Organisationsform, um Patientinnen- und Patientenfälle in einem kollektiven Entscheidungsfindungsprozess zu besprechen. Dafür werden neue Regeln und Methoden formuliert, und Gesundheitspersonal wird darin ausgebildet, mit ethi-

31 Kohlen 2011: 127.

32 Arney/Bergen 1984.

33 Der Artikel, auf den ich mich hier beziehe, basiert auf Kohlens veröffentlichter Dissertation, in der sie nicht nur US-amerikanische sondern auch deutsche Ethikkomitees untersucht hat (Kohlen 2009).

34 Vgl. ausführlich in Kohlen (2011: 139ff). 
schen Dilemmata umzugehen. Dadurch dringe, so Kohlen, Bioethik in den medizinischen Bereich:

»Medical morality gave way to bioethics by adopting not only its interdisciplinary approach, but also its rational analytical style and a technical procedure of principle-based ethics. Hereby, the responsibility, which was once under the authority of the medical profession, rooted in the Hippocratic oath, began shifting into a collective decision-making process by a team of (bio-)ethics experts, physicians, lawyers, nurses, chaplains and social workers (ebd.: 128).

Vor dem Einzug von Bioethik in den medizinischen Bereich, liegen die Entscheidungen in der Verantwortung der individuellen Ärztin oder des individuellen Arztes, meint Kohlen. Das Verhältnis zwischen Ärztin oder Arzt und Patientin oder Patient basiert auf Vertrauen, die Entscheidungsfindung auf Nähe. Die Kenntnisse des Arztes oder der Ärztin über den Patienten oder die Patientin sowie die Situation bestimmen über ethische Fragen am Krankenbett. Mit der Bioethik ändern sich Stil und Substanz der medizinischen Entscheidungsfindung (ebd.). Die »shifted responsibilities «, die Kohlen beschreibt, die Verschiebung der ärztlichen individuellen Verantwortung sind allerdings nicht nur hin zu einer kollektiven Verantwortung wie im Kontext der Ethikkomitees zu sehen. Wie die US-amerikanischen Soziologen William Arney und Bernard Bergen in ihrer Untersuchung medizinischer Fachliteratur aus den 1950er und 60er Jahren zeigen, hat zudem eine Verschiebung der ärztlichen Verantwortung im Kontext des Verhältnisses zwischen Ärztin oder Arzt und Patientin oder Patient stattgefunden (Arney/Bergen 1984). Indem zunehmend psychologische, ökologische oder gesellschaftliche Aspekte in den Entscheidungsprozess einbezogen werden und Gesundheit als System gedacht wird, hat die Medizin ihren Kompetenzbereich ausgedehnt. Kybernetisch definiertes Leben wird das neue medizinische Objekt und es zu managen, das Ziel. Um dieses umfassende »management of living « (ebd.) zu ermöglichen, wird der Patient oder die Patientin neu konzeptioniert. Er oder sie wird aufgefordert, aktiv am medizinischen Unternehmen teilzuhaben und selbst Entscheidungsträgerin oder -träger zu werden (ebd.; vgl. Samerski 2011: 104).

Die Ergebnisse beider Untersuchungen zusammengedacht, bedeutet dies, dass die medizinische Entscheidungsfindung nicht mehr allein auf der individuellen Verantwortung des Arztes oder der Ärztin beruht, wovon Kohlen im Kontext ethischer Fragen idealtypisch ausgeht. Der Patient oder die Patientin treten als Entscheidungsträger oder -trägerin hinzu. Darüber hinaus werden gesellschaftliche oder ökologische Aspekte in den Entscheidungsfindungsprozess integriert. Hinzu kommt die übergeordnete Entscheidungsinstanz in Form des Ethikkomitees im Krankenhaus, in der wiederum die ärztliche Entscheidungsbildung thematisiert und 
überprüft wird (vgl. Kohlen 2011). Ärztliche Entscheidungsbildung wird nicht nur in ein Mehrebenensystem überführt, sondern weitere Akteurinnen und Akteure wie der Patient oder die Patientin oder die Mitglieder des Ethikkomitees werden in den Entscheidungsfindungsprozess integriert. In diesem Mehrebenensystem wird die Gesundheit der Patientin oder des Patienten selbst als System gedacht, indem ökologische oder gesellschaftliche Aspekte berücksichtigt werden - ein System im System sozusagen.

Was bedeuten diese Untersuchungsergebnisse im Hinblick auf das Ethikkomitee vom INSERM? Ob und, wenn ja, inwiefern Gesundheit in Frankreich in den 1970er Jahren als System gedacht wird und inwiefern dies eine Verschiebung der ärztlichen Verantwortung mit sich bringt, kann hier nicht abschließend beantwortet werden. Wie Rigal meint, setzt sich ab den 1960er Jahren eine Bedeutung von klinischen Untersuchungen durch, die vom Krankenbett weg führt. Klinische Untersuchungen beziehen sich jetzt auf die Quelle, sei es Biologie, Mathematik, Chemie, Psychologie oder Soziologie, die für Diagnosen, Prognosen, Behandlungen oder für die Prävention verwendet werden können (Rigal 2008: 528f). Damit werden weitere Aspekte in den Entscheidungsprozess einbezogen, die auch für Frankreich vermuten lassen, dass Gesundheit als System gedacht wird und sich der medizinische Kompetenzbereich erweitert. Allerdings ist in Frankreich insofern von einem starken medizinischen Paternalismus auszugehen, da das Selbstbestimmungsrecht des Patienten oder der Patientin im Kontrast zu den USA nicht besonders betont wird (Maio 2000: 49). Somit ist vermutlich der Patient oder die Patientin nicht in ähnlichem Umfang als Entscheidungsträger oder -trägerin konzeptioniert. Der Begriff Patientenrecht macht sich bspw. erst mit den neuen Reproduktionstechnologien bemerkbar (Nye 2006: 35). Ein weiterer Aspekt an dem ab den 1970er Jahren eine Verschiebung von Verantwortung sichtbar wird, ist m.E. der Medikamentenführer von Pradal. Er wird im Jahr 1974 veröffentlicht und ist in Frankreich ein Bestseller. Dies zeigt sowohl, dass zu dieser Zeit ein Bedürfnis der (möglichen) Patientinnen und Patienten besteht, selbstbestimmter sein zu wollen, als auch dass Ärztinnen und Ärzte eine informierte Patientin oder Patienten bevorzugen, da sie »einfacher« zu behandeln seien (vgl. Chauveau 2004: 108f/Übers. S.K.). Weiter zeigt sich diese Tendenz in den Gruppen zum Schutz von Konsumentinnen und Kosumenten, die sich in den 1960er und 70er Jahren vervielfachen und sich u.a. auch mit medizinischen Themen beschäftigen (Chavot/Masseran 2003d: 435f). Die Gruppen oder der Medikamentenführer scheinen in Frankreich auf ein Problem zu antworten, auf ein Bedürfnis oder Interesse daran, sich selbst zu informieren, Wissen anzueignen, um medizinischer Autorität nicht ausgeliefert zu sein. Von »shifted responsibilities « kann auch im Kontext des Ethikkomitees vom INSERM gesprochen werden, da nicht mehr allein die Verantwortung des Medizi- 
ners oder der Medizinerin ausschlaggebend für das Durchführen eines Versuchs ist, sondern nun auch eine übergeordnete Instanz in Form eines Expertinnen- und Expertenkomitees. Das individuelle Gewissen des Forschers oder der Forscherin wird in ein übergeordnetes kollektives Gewissen verschoben. Es handelt sich um eine Art antipaternalistische Entzerrung des Verhältnisses zwischen der Forscherin oder dem Forscher und der Patientin oder dem Patient in dem Sinn, dass eine weitere Instanz hinzukommt. Die Forschungspläne werden zwar kontrolliert und transparenter, jedoch nur innermedizinisch. Die Freiwilligkeit, den Forschungsplan vorzulegen, wird finanziell angereizt. Wird er vorgelegt und bleibt er im ethischen Rahmen, wird er finanziert. Die französische Medizinerin Claire Ambroselli unterstreicht vor allem die pädagogische Rolle des Komitees: »das Ethikkomitee hatte bei den Forschern, die für die Ethik der Forschung sensibel waren, eine pädagogische Rolle, besonders im Krankenhausmilieu, indem sie ihre Methodik gemäß den Empfehlungen des Komitees nachgebessert haben« (Ambroselli 1984: 8/ Übers. S.K.). Das Komitee hat also eine pädagogische, eine anleitende, bildende, erzieherische Rolle. Angeleitet oder geführt wird nicht durch Verbot oder Strafe, sondern mit dem Anreiz der Finanzierung. Der Mediziner oder die Medizinerin muss sich gegenüber dieser Instanz, sofern er oder sie eine Finanzierung möchte, als Individuum richtig verhalten, das heißt, gemäß der geforderten Standards. Die Finanzierung und damit die Möglichkeit zu forschen, setzt eine Selbstdisziplinierung voraus, die eine Reflexion fordert. Zwar wird das Verfahren der medizinischen Selbstkontrolle durch das Hinzukommen des Komitees ausgedehnt; sie wird durch diese Instanz jedoch gleichzeitig gesichert.

Die Entzerrung des paternalistischen Verhältnisses zwischen Mediziner oder Medizinerin und Patient oder Patientin, die Abschwächung des individuellen ärztlichen Gewissens als einzige Kontrolle, spielt jedoch auch in weiteren Diskursen eine Rolle, die in den 1970er Jahren aktuell sind: dem medizinkritischen Diskurs, der aus der 1968er-Bewegungen hervorgeht sowie dem Diskurs um die Legalisierung des Schwangerschaftsabbruches.

\subsection{Mai 1968 und Medizinkritik}

Die Ereignisse des Mai '68 erschüttern die französische Medizin, so die französische Soziologin Claudine Herzlich und ihre Kollegin (Herzlich/Pierret 2010: 130). Die Revolten des Mai '68 wenden sich gegen den allmächtigen Staat, für den der damalige konservative Staatspräsident Charles de Gaulle steht. Sie beinhalten insbesondere eine Gesellschaftskritik, die auf die wirtschaftliche und politische Organisation der Gesellschaft zielt sowie ihre hierarchischen und autoritären Strukturen 
(vgl. Doll 2006: 607). Bildungseinrichtungen und Universitäten werden zu einem Ort sozialer und politischer Kämpfe. Fragen nach dem Zusammenhang von Wissen und Macht rücken in den Fokus (vgl. Lemke 1997: 58); auch in den Fokus politischer Medizinerinnen und Mediziner. Zu Beginn der 1970er Jahre erscheinen eine Reihe an Publikationen von jungen Medizinerinnen und Medizinern sowie Psychiaterinnen und Psychiatern. Sie behandeln Themen, wie die »Medizin des Kapitals« oder die »Medikalisierung der Gesellschaft« (Herzlich/Pierret 2010: 130/Übers. S.K.). Krankheit, Gesundheit und Körper werden nicht mehr außerhalb des Sozialen und Politischen gesehen; Medizin wird im Rahmen des kapitalistischen Systems gedacht (ebd.: 129). Und nicht zuletzt erscheint im Jahr 1975 auf Französisch Ivan Illichs Buch zur Gesundheits- und Medizintechnokratie »Némésis médicale. L'expropriation de la santé « - »Die Nemesis der Medizin. Die Enteignung der Gesundheit« (Illich 1976). Das kritische Werk stößt auf ein »großes Echo«, wie Foucault es 1976 in einem Vortrag formuliert (Foucault 2003 (1976)-b: 54). Im Kontrast dazu stehen Publikationen wie die Jean Bernards aus dem Jahr 1973 zur »Grandeur et tentations de la médecine « - »Größe und Verlockung der Medizin«, in denen die komplexen Entwicklungen der modernen Medizin betont werden (Herzlich/Pierret 2010: 130/Übers. S.K.). In Anlehnung an den von Michel Foucault zusammen mit dem Historiker Pierre Vidal-Naquet 1971 ins Leben gerufene Groupe d'Information sur les Prisons - Gefängnisinformationsgruppe - und geprägt von den 1968ern wird der Groupe d'Information Santé (GIS) - Gesundheitsinformationsgruppe - im Jahr 1972 gegründet (ebd.; Garcia 2005: 107; Lemke 1997: 62ff; Reynaud 1978: 105). Der GIS setzt sich vor allem aus jungen linken Ärztinnen und Ärzten sowie Medizinstudierenden, aber auch einigen NichtMedizinerinnen und -Medizinern zusammen, insgesamt etwa hundert an der Zahl (Pavard 2009: 82; Gaudillière 2006a: 244; Reynaud 1978: 125f). Dazu zählen der Gynäkologe René Frydman, der im Jahr 1982 einer der so genannten Väter des ersten französischen Reagenzglasbabys wird, und der Arzt Jean Carpentier. Carpentier wird im Jahr 1971 wegen eines Flugblatts mit dem Titel »Apprenons à faire l'amour « - »Lernen wir uns zu lieben ${ }^{35}$ vom Conseil de l'Ordre Médecins für ein Jahr von der Ausübung des ärztlichen Berufes suspendiert (Giami 2007: 14/Übers. S.K.; Reynaud 1978: 9). Das Flugblatt, das zusammen mit Schülerinnen und Schülern entsteht und das an einem Gymnasium verteilt wird, soll zur sexuellen Befreiung beitragen und zu sexuellen Aktivitäten ermutigen (Giami 2007: 14). Darüber hinaus gehört Carpentier zu einer Gruppe, die von 1973-1977 die medizinkritische Zeitschrift Tankonalasanté publiziert. Die Zeitschrift hat eine Auflage von 4000

35 Das Flugblatt findet sich in einer Veröffentlichung von Jean Carpentier mit demselben Titel (Carpentier 2001). 
Exemplaren und ca. 800-1000 Abonnements (Reynaud 1978: 31). Ziel ist es, dass »les malades [...] reprennent le pouvoir sur leur maladie, sur leur corps et leur esprit« - »die Kranken [...] wieder die Macht über ihre Krankheit, ihren Körper und ihren Verstand übernehmen« (Tankonalasanté (1974), n8 »La ligne du journal«: o.S., zit. nach ebd.: 12/Herv. i.O., Übers. S.K.). Das Kampfterrain liegt für den GIS in der außerparlamentarischen sozialen Bewegung und im Klassenkampf (Gaudillière 2006a: 245; vgl. Foucault 1976). Die Gruppe konzentriert sich darauf, »gegen die verlogene Propaganda zu kämpfen, die die Gesundheit an einen mehr oder weniger mythischen Fortschritt koppelt« (Flugblatt (1972), GIS ohne Quellenangabe, zit. nach Herzlich/Pierret 2010: 130/Übers. S.K.; vgl. Artières 2002: 38). Die Vorstellung des GIS von Medizin oder der Ausübung des ärztlichen Berufes besteht nicht darin, dass Medizinerinnen und Mediziner Entscheidungen über jemanden treffen. Sie besteht darin, dass sie ihr Wissen in den Dienst der Menschen stellen (Gaudillière 2006a: 245): »inspiriert von Foucaults Analysen der Beziehungen zwischen >Wissen und Macht $<$ hat er [der GIS, S.K.] die Ambition, die Beziehungen zwischen Medizinern und Patienten umzustürzen« (Garcia 2005: 107/Herv. i.O., Übers. S.K.). Wie Lemke ausführt, rückt mit dem Mai '68 »die politische Frage nach dem Zusammenhang von Wissen und Macht« und so die Frage nach Organisation und Verteilung von Wissen in den Mittelpunkt Foucaults Auseinandersetzungen (Lemke 1997: 57f). »Wie vollzieht sich die Aneignung und die Verteilung des Wissens? Wie kann ein Wissen in einer Gesellschaft seinen Platz einnehmen, sich in ihr entwickeln, seine Kräfte mobilisieren und sich einem Wirtschaftssystem unterordnen?«, so fragt Foucault zu Beginn der 1970er Jahre (Foucault (1970), in: Le Nouvel Observateur, 9.-15.2.: o.S., zit. nach ebd.: 58). Mit dem Konzept eines medizinischen Wissens im Dienste der Menschen, das vom GIS vertreten wurde, zeigt sich bereits das Konzept des »intellectuel spécifique « - des »spezifischen Intellektuellen«, das Michel Foucault kurze Zeit später, im Jahr 1976 ausarbeitet, wie der Historiker Jean-Paul Gaudillière meint (Gaudillière 2006a: 245). Foucault führt es ein, um seine eigene Praxis und die der Informationsgruppen zu untermauern (ebd.). Der »intellectuel spécifique« beinhaltete die Idee eines oder einer Intellektuellen, der oder die sich politisch einmischt, sich in seinem oder ihrem Spezialgebiet engagiert, sein oder ihr Wissen eines Gebietes verwendet, um dessen Praktiken anzuprangern (Artières 2002: 38). Im Kontrast zum »intellectuel >universel «« und dem damit verbundenen Merkmal des »>juste-et-le-vrai pour tous « - » Richtigen-und-Wahren für alle«« sollte es darum gehen, so Foucault zur politischen Aufgabe des Intellektuellen, sich spezifischen Problemen zuzuwenden, sei es im Krankenhaus, im Labor oder in der Universität (Foucault 2001 (1976): 109/Herv. i.O., Übers. S.K.; Artières 2002: 38). In Folge der 1968er und mit dem GIS kommt ein medizinkritischer Diskurs auf, der den Fortschrittsmythos ebenso 
thematisiert wie die Beziehung von Macht und Wissen, insbesondere im Verhältnis zwischen Ärztin oder Arzt und Patientin oder Patient. Verknüpft ist damit die Frage, wer über medizinische Fragen entscheidet. Dies sind Fragen, die der GIS auch im Konflikt um die Legalisierung der Abtreibung einbringt.

\subsection{Der Abtreibungskonflikt - Politische Selbstbestimmung versus Moral}

Zwar steht die Institutionalisierung des Ethikkomitees vom INSERM durch die Thematisierung der medizinischen Forschung in engem Zusammenhang mit dem medizinischen und öffentlichen Diskurs insbesondere um Humanexperimente. Jedoch taucht Mitte der 1970er Jahre die Frage medizinischer Autorität, die insbesondere den Ordre de médecins betrifft, auch im Diskurs um die Legalisierung der Abtreibung auf. In diesem Diskurs ist nicht die Sprache der Ethik zentral, sondern die einer (Anti-)Moral.

In den Kontroversen um die Legalisierung der Abtreibung ${ }^{36}$ wird vor allem die Moral der Abtreibungsgegner und -gegnerinnen kritisiert. Unter den letztgenannten sind viele konservative Mediziner und Medizinerinnen und auch der Ordre des médecins. Bereits durch die Legalisierung der Kontrazeptiva wird die Autorität konservativer Mediziner und Medizinerinnen und insbesondere die des Ordre ab den 1960er Jahren geschwächt, so Maio (2000: 55). Im Jahr 1967 tritt das so genannte Neuwirth-Gesetz in Kraft. Durch das Gesetz wird die Verwendung der AntiBaby-Pille legalisiert. Bis dahin ist die Empfängnisverhütung in Frankreich verboten. Geschwächt wird die Autorität konservativer Medizinerinnen und Mediziner zudem im Kontext des Diskurses um Abtreibung. Die Schwächung dieser konservativ-medizinischen Autorität steht in Wechselwirkung zu den Auseinandersetzungen um Humanexperimente und damit zu der Einrichtung des Ethikkomitees.

In den 1960er Jahren gewinnt eine französische Reformbewegung gegen das bestehende Abtreibungsrecht an Boden. In der Vorkriegszeit werden in Frankreich, aufbauend auf den restriktiven Gesetzen aus den Jahren 1920 und 1923, der Versuch, die Beihilfe zur und die Durchführung der Abtreibung unter ein hohes Strafmaß gestellt: Es reicht von Geldstrafen bis hin zu einer Gefängnisstrafe zwischen

36 Eine Chronologie der Ereignisse mit einem Überblick der beteiligten Akteure und Akteurinnen bieten Anne-Marie Devreux und Michèle Ferrand-Picard (1982). Eine aktuellere Chronologie des Konfliktes um die Legalisierung des Schwangerschaftsabbruchs ab 1967 findet sich auf der Homepage von La documentation Française (http://www. ladocumentationfrancaise.fr/dossiers/ivg/chronologie.shtml, 21.07.2015). 
sechs Monaten und fünf Jahren (Rucht 1994: 348f; Schulz 2002: 121f). Während des Vichy-Regimes verschärft sich diese Regelung bis hin zur Todesstrafe (Rucht 1994: 349; vgl. Pollard 2000). Die bestimmende Kraft dieser Reformbewegung gegen das Abtreibungsgesetz ist der französische Verband zur Familienplanung, der Mouvement français pour le planning familial - vormals Maternité heureuse glückliche Mutterschaft (Rucht 1994: 350f; Memmi 2005: 7). In dieser Zeit nimmt zudem die Frauenbewegung Gestalt an, die sich schließlich zu Beginn der 1970er Jahre des Abtreibungsthemas annimmt (Laborie 1995: 1). Entscheidender Anstoß für die öffentliche Diskussion über das bestehende Abtreibungsrecht und für die Formierung einer breiten Bewegung ist u.a. ein im April 1971 in der linksintellektuellen Wochenzeitung Nouvel Observateur herausgegebenes Manifest. 343 Frauen erklären darin, abgetrieben zu haben. Darunter sind auch Prominente wie die Schriftstellerin und Philosophin Simone de Beauvoir, die Autorin und Filmregisseurin Marguerite Duras oder der Filmstar Jeanne Moreau (Abba-Sidick et al. 1971; Rucht 1994: 351f). Dem französischen Vorbild folgen solche Selbstbezichtigungskampagnen auch in der BRD. Weiterer, entscheidender Anstoß für eine öffentliche Diskussion ist der so genannte Prozess von Bobigny im Jahr 1972. In diesem Prozess wird der Fall einer Minderjährigen verhandelt, die nach einer Vergewaltigung abgetrieben hat (Garcia 2005: 103ff). Abgesehen von dem Manifest, gehören zu weiteren Aktionen u.a. die öffentliche Aussprache für die Legalisierung der Abtreibung von verschiedenen Fachleuten, Wissenschaftlerinnen und Wissenschaftlern, sowie Kampagnen des Mouvement pour la liberté de l'avortement et de la contraception (MLAC), einer Allianz von radikalen Reformgruppen (Rucht 1994: 351ff; Devreux/Ferrand-Picard 1982: 504ff). Ab 1973 zählt zum MLAC auch der Groupe d'Information Santé (GIS). Sein Ziel ist nicht eine Gesetzesreform, sondern die Aufhebung des Abtreibungsgesetzes (Gaudillière 2006a: 245; Reynaud 1978: 112ff). Auch im Kontext der Abtreibung besteht seine Vorstellung über die Ausübung des ärztlichen Berufes darin, das Wissen in den Dienst der Menschen zu stellen, hier in den Dienst der Frauen (Gaudillière 2006a: 245). Als Abtreibungsmethode befürworten und praktizieren sie die Karman-Methode, eine Absaugmethode, die schnell, mit wenig Aufwand und ohne Krankenhausaufenthalt realisierbar ist (Garcia 2005: 107ff). Wie Gaudillière in einem Aufsatz zum medizinischen Diskurs im Abtreibungskonflikt aufführt, bleibt mit dem Konzept des GIS, medizinisches Wissen in den Dienst der Menschen zu stellen, die wissenschaftlich-medizinische Kompetenz unangetastet. Seine Aktionen werden durch ein Wissen gerechtfertigt, das die Mitglieder sich an der medizinischen Fakultät angeeignet haben. Der Inhalt dieses Wissens wird aber kaum kritisiert, er wird lediglich als Kampfmittel eingesetzt, um es denen zur Verfügung zu stellen, die es brauchen (Gaudillière 2006a: 245f). Den Kampf gegen das Abtreibungsgesetz sieht 
der GIS im Rahmen des Kampfes für die Befreiung der Frau und weiter im Rahmen des Klassenkampfes und wendet sich gegen eine bürgerliche Moral (ebd.: 245). Der GIS veröffentlicht im Jahr 1973 die Broschüre »Oui nous avortons!« »Ja, wir treiben ab!«(Groupe d'information santé 1973/Übers. S.K.; Artières 2002: 38). Die Gruppe erreicht im selben Jahr, dass der Nouvel Observateur in Anlehnung an das »Manifest der 343« einen Text publiziert, in dem 330 Mediziner und Medizinerinnen erklären, Abtreibungen durchzuführen (Gaudillière 2006a: 245). Die Definition der medizinischen Moral, wie sie durch den Ordre des médecins verkörpert wird, beginnt, so Sandrine Garcia, ins Wanken zu geraten (Garcia 2005: 102) und zwar nicht allein durch das Manifest der Frauen, sondern darüber hinaus durch das Manifest der Mediziner und Medizinerinnen.

Der Bewegung zur Liberalisierung der Abtreibung stehen ab dem Jahr 1972 verschiedene Gruppierungen und Verbände entgegen, dazu gehören vor allem konservative Mediziner und Medizinerinnen wie auch der Ordre des médecins. Die abwehrende Haltung des Ordre gegenüber der Abtreibung ist vor allem in der Gefährdung seiner Autonomie begründet. Diese sieht er bereits im Kontext der Kontrazeptiva in Gefahr. Hinter seiner abwehrenden Haltung steht, so Maio, »die Sorge zu einer reinen Verschreibungsinstanz instrumentalisiert zu werden « (Maio 2000: 55). Ebenso ablehnend steht der Ordre des médecins der Legalisierung der Abtreibung gegenüber. In seiner Abwehrrethorik zeigt sich der Versuch seiner Autonomie- und Autoritätssicherung: Im Jahr 1974 richtet sich der Präsident des Ordre des médecins mit einem Schreiben an die Gesundheitsministerin Simone Veil. Darin ist zu lesen: »Die Abtreibung zu liberalisieren, das heißt, die Verhütung aufgeben, das heißt eine verantwortungsvolle Geburtenkontrolle durch Verantwortungslosigkeit zu ersetzen« (Bulletin de l'Ordre des médecins (1962), 1, 44, zit. nach ebd.: 58; vgl. Horellou-Lafarge 1982: 402). Der Begriff »verantwortungsvoll« wird hier eingesetzt, um die Kontrolle, die eigene Autorität zu sichern. Das geschieht hier vor allem indem die Entscheidungsfähigkeit der Frauen als unverantwortlich gekennzeichnet wird, als unvernünftig oder irrational im Hinblick auf die Geburtenkontrolle. Die »verantwortungsvolle Geburtenkontrolle« des Ordre kann sich allerdings nicht durchsetzen: Veil ruft den Ordre des médecins zur Raison, indem sie ihn darauf hinweist, dass er den Gesetzen der Republik unterstehe. Kurz nach dem Gesetz zur Liberalisierung der Abtreibung von 1975 gibt es einen Gesetzesvorschlag zur Auflösung des Ordre des médecins. Später, nach dem Regierungswechsel von 1981, gibt es diesbezüglich weitere Bestrebungen. Die neue sozialistische Regierung unter François Mitterrand begründet ihr Vorgehen mit dem Misstrauen, das in der Öffentlichkeit gegenüber dem Ordre vorhanden sei und kritisiert ihn aufgrund seiner veralteten und undemokratischen Strukturen (Maio 2000: 55, 58f; Didrich-Tabaste 1994: 99f). Zu den weiteren Abtreibungsgegnern und -gegne- 
rinnen zählt die Lebensschutzorganisation Laissez-les vivre - Lasst sie leben, die im Jahr 1970 von dem konservativen Mediziner und Genetiker Jérôme Lejeune $(1926 *-\dagger 1994)$ ins Leben gerufen wird (Schulz 2002: 122). Im Jahr 1971 lanciert dieser radikale Flügel der Abtreibungsgegner und -gegnerinnen eine öffentliche Stellungnahme gegen Abtreibung, die 100 Unterschriften zählt (ebd.). Laissez-les vivre fordert u.a. eine moralische und staatsbürgerliche Erziehung der Abtreibungsbefürworterinnen und -befürworter (Ferrand-Picard 1982: 387). Auch die katholische Kirche äußert sich und sagt u.a., dass kein moralisches Prinzip einen Schwangerschaftsabbruch begründen könne (Ladrière 1982: 424). Es gelingt den konservativen Strömungen allerdings nicht, die konservativen politischen Parteien für sich zu gewinnen (Rucht 1994: 355f). In diesem angespannten Klima stehen die politischen Parteien unter Handlungsdruck. Nach mehreren diskutierten Gesetzesentwürfen kommt es im Jahr 1974 schließlich zu einem Entwurf der damaligen Gesundheitsministerin Veil, der im Januar 1975 trotz parlamentarischer Proteste verabschiedet wird. Er erlaubt, sofern die Frau in einer so genannten Notsituation ist, die Abtreibung in den ersten zehn Schwangerschaftswochen und nach einer medizinischen Beratung. Nach diesem Zeitraum wird ein Schwangerschaftsabbruch dann erlaubt, wenn das Leben der Mutter gefährdet oder eine so genannte schwere Schädigung des Fötus zu ersehen ist. Das so genannte Veil-Gesetz soll dem Parlament nach fünf Jahren erneut zur Abstimmung vorgelegt werden und wird darum auch als Probegesetz bezeichnet (ebd.: 356ff; vgl. Loi ${ }^{\circ}$ 75-17 1975).

\subsection{1 "Kein moralischer Druck! « ${ }^{37}$}

Zentrale Parole im Abtreibungsdiskurs ist »un enfant quand je veux - si je veux «»ein Kind wann und wenn ich will«. Angesprochen werden mit dieser Parole der Abtreibungsbefürworterinnen und -befürworter Entscheidungsfreiheit und Selbstbestimmung vor allem über den eigenen Körper. Es wird »la libre disposition de notre corps « - »die freie Verfügung über unseren Körper« gefordert, so im Manifest der 343 Frauen aus dem Nouvel Observateur. Weiter heißt es darin: »ob sie Katholiken heißen, Fundamentalisten, Demografen, Mediziner, >verantwortliche Männer<, Debré, Peyret, Lejeune, Pompidou, Chauchard, der Papst ${ }^{38}$ - wir sagen, dass wir sie demaskiert haben. [...]. Dass wir ihnen verbieten, den Begriff $>$ Achtung vor dem Leben< zu verwenden, der eine Obszönität in ihrem Mund ist« (Ab-

37 Abba-Sidick et al. 1971/Übers. S.K.

38 Bei den Genannten handelt es sich um Abtreibungsgegner: Paul Chauchard und Jérôme Lejeune bspw. waren aktive Lebensschützer. Michel Debré, ehemaliger Premierminister unter Charles de Gaulle, sah in dem Gesetz eine Katastrophe für die Geburtenzahl Frankreichs (Ferrand-Picard 1982: 392; Thalmann/Dhoquois 1995). 
ba-Sidick et al. 1971/Herv. i.O., Übers. S.K.). Die Frauen wenden sich in ihrem Manifest explizit gegen die Verwendung des normativen Begriffes »Achtung vor dem Leben« seitens der Abtreibungsgegnerinnen und -gegner, und wehren sich gegen einen »moralischen Druck«: »Ich werde ein Kind gebären, wenn ich dazu Lust habe, kein moralischer Druck, keine Institution, kein ökonomisches Gebot kann mich dazu zwingen. Das ist meine politische Macht« (ebd./Übers. S.K.). Die Selbstbestimmung über den eigenen Körper (»la libre disposition de notre corps« (ebd.)) wird zur politischen Macht. Es handelt sich um einen politischen Selbstbestimmungsbegriff, der einer Moralisierung der Abtreibungsthematik gegenübergestellt wird. Anders gesagt, die Frauen wenden sich gegen eine Moralisierung des Konfliktes um Abtreibung vor allem durch Instanzen, denen zu der Zeit eine Hoheit über die Definition von Moral zugesprochen wird, wie Mediziner und Medizinerinnen, den Papst oder Experten und Expertinnen, und stellen dagegen ein politisches Konzept der Selbstbestimmung (vgl. Del Re 2000). Dies stellt einen Angriff auf die ärztliche Autorität dar, den damit einhergehenden Paternalismus und ihren bisherigen Kompetenzbereich - die Schwangerschaft und die Geburtenkontrolle.

\subsection{Ein Weniger an medizinischer Macht?}

Die Entscheidungsfreiheit, die den Frauen mit dem Veil-Gesetz eingeräumt wird, bedeutet allerdings, mit Foucault verstanden, nicht ein Weniger an medizinischer Macht, wie Dominique Memmi herausgearbeitet hat. Sie verortet die Verwaltung der Geburt in einem Transformationsprozess der politischen Kontroll- und Steuerungsmodi seit den 1960er Jahren und der sich verändernden Formen des Umgangs des Individuums mit seinem Körper, von Verbot und Strafe hin zu einer Entkriminalisierung und Bioindividuation, was sie die delegierte Biopolitik nennt (Memmi 2003a: 292f; 2005). Diese Bioindividuation bedeutet, körperliche Bedingungen auszubalancieren, Risiken zu kalkulieren und die Kosten der eigenen Gesundheit und ihrer Verbesserung zu evaluieren (ebd.). Die Führung dieser Art der Selbstregierung findet weniger durch Verbote oder Strafe statt, sondern durch eine von Experten und Expertinnen angeleitete Art und Weise des Sprechens, das governing through speech (Memmi 2003c). Dessen wesentliches Merkmal ist die Stimulation einer individuellen und »rationalen« Selbstkontrolle (Memmi 2003c: 656). Im Kontext der Abtreibung haben in diesem Transformationsprozess die sozialen Bewegungen eine wesentliche Bedeutung, so Memmi. Bereits mit der Legalisierung der Kontrazeptiva sind durch den Mouvement francais pour le planning familial - die französische Bewegung für Familienplanung private Verhütungspraktiken wie Kräuter, Kondome oder Abstinenz öffentlich geworden. Mit den 
Verhütungspraktiken gehen Entscheidungen einher, die gegen staatliche Regulierungen und gegen die Kirche stattgefunden haben. Der Prozess der Bioindividuation, der erst gegen jede Instanz und gegen den Staat gerichtet war, wendet sich ab Ende der 1960er Jahre an ihn. »Das, was enorm schockiert hat, war, dass wir es gesagt haben, dass wir das Recht auf Abtreibung einforderten«, wie Gisele Halimi bestätigt, eine Hauptakteurin im Abtreibungskonflikt (Halimi (2001) »Je déclare avoir avorté«, in: Le Monde 8./9.04.: 14, zit. nach Memmi 2005: 11/Übers. S.K.). Die vormals heimliche Praxis wird zur Forderung an den Staat und gelangt in den öffentlichen Diskurs. Im Kontext der Abtreibung und des Veil-Gesetzes wird die Art und Weise des Sprechens durch den Mediziner oder die Medizinerin angeleitet bzw überwacht - die medizinische Beratung ist gesetzlich vorgeschrieben (ebd.). Die Frau, die durch die Abtreibungsfrist zeitlich unter Druck steht, wird ermuntert zu sprechen, selbst wenn sie durcheinander ist, leidet oder Angst hat, sie muss ihre Entscheidung begründen. Auch der Mediziner oder die Medizinerin muss sprechen, erklären und informieren. Er oder sie kann nicht ohne Zustimmung der schwangeren Frau über deren Körper verfügen. Die Mediziner und Medizinerinnen müssen zeigen, dass sie ihre Regungen beherrschen, zivilisiert sind. Die Entscheidung über einen Schwangerschaftsabbruch wird vom Staat durch das Gesetz an die individuelle Frau delegiert. Es handelt sich um eine Regierung, die sich wenig autoritär gibt und auf eine begleitende Selbstkontrolle durch eine gelehrte medizinische Expertise setzt (ebd.: 2f; vgl. Memmi 2003b: 975f).

Auch mit dem Ethikkomitee vom INSERM scheint sich tendenziell eine von Experten und Expertinnen angeleitete Form der Regierung, des governing through speech durchzusetzen. Und zwar insofern, als der Mediziner oder die Medizinerin sich gegenüber dieser Instanz, sofern er oder sie eine Finanzierung möchte, »richtig sprechen« muss. Begleitet wird das »richtige Sprechen« durch die gelehrte Expertise in Form des Komitees. Im Kontrast zum Abtreibungsdiskurs und insbesondere seiner konservativ-moralischen Rahmung erscheint die Ethik-Rahmung in Form des Komitees als modern, antipaternalistisch oder antiautoritär und demokratisch. Sowohl für Forscher und Forscherinnen als auch für Frauen, die eine Schwangerschaft abbrechen möchten, scheint sich eine begleitende Selbstkontrolle durch eine gelehrte Expertise durchzusetzen - in Form des Ethikkomitees und in Form der medizinischen Beratung. Mit der Legalisierung der Abtreibung verliert der Ordre des médecins weiter seine Definitionsmacht. Dies wirkt sich auch auf seine Definitionsmacht im Hinblick auf die Humanexperimente aus, so Maio: »Ab dem Moment, wo die Standesvertreter ihre Definitionsmacht in der Bewertung des Humanexperimentes verloren, war der Weg für eine >neue< Bewertung geebnet « (Maio 2000: 78/Herv. i.O.). Der Weg für diese neue, nicht-moralische sondern ethische Bewertung führt in die Einrichtung des Ethikkomitees vom INSERM. Es 
wird aber nicht nur eine bestimmte Moral verurteilt, sondern parallel eine bestimmte moralische Haltung befürwortet. Wie die Gesundheitsministerin Veil 1974 in ihrer Rede zum 10. Jahrestag vom INSERM sagt, sei die Kritik an der Medizin übereilt und aufgebauscht, an einer medizinischen Forschung, der sie nicht allein eine Schlüsselfunktion in der Lenkung, eine Führungsposition der zukünftigen wissenschaftlichen Entwicklungen zuschreibt, sondern zudem eine humanisierende Wirkung (Veil 1974). Veil zeigt implizit auf, dass sich die Kritikerinnen und Kritiker dieser humanisierenden Wirkung, einer menschenwürdiger machende Entwicklung verschließen. Nur eine Haltung, die eine Führungsposition medizinischer Forschung akzeptiert, scheint moralisch.

\section{ZUSAMMENFASSUNG}

Ethische und moralische Rahmungen tauchen in der französischen Nachkriegszeit insbesondere im Kontext der Diskussion um Humanexperimente, der Medizinkritik, die aus den 1968ern hervorgeht und der Kontroversen um die Legalisierung des Schwangerschaftsabbruchs auf. Medikamentenskandale Mitte der 1950er und Anfang der 1960er Jahre werden nicht mit Humanexperimenten oder Experimentieranstalten verknüpft. Sie sind nicht ethisch gerahmt, sondern als Fehler oder Unfall. Diesen kann innerwissenschaftlich oder gesundheitspolitisch mit besseren Evaluationen im Labor begegnet werden oder mit staatlichen Regelungswerken zur Medikamentenfreigabe. Vor dem Hintergrund der Rahmungen als Fehler oder Unfall erscheint die Ethik-Rahmung als vertrauensbildend und verantwortungsvoll die Forschungspläne werden geprüft; ihre Bewertung bleibt allerdings innerwissenschaftlich. Im Frankreich der 1960er und 70er Jahre setzen mehrere Diskurse ein, deren Schnittmenge vor allem in der Problematisierung und Verteidigung medizinischer Autonomie und Autorität liegt. Es handelt sich nicht allein um emanzipatorische soziale Bewegungen, die die Frage der medizinischen Autorität stellen, sondern auch um innermedizinische. In den 1960er Jahren werden der ärztliche Paternalismus und insbesondere das Verhalten des Ordre des médecins, der französischen Vertretung der Ärztinnen und Ärzte, im Kontext der Legalisierung der Kontrazeptiva politisch und öffentlich kritisiert. Im Kontext der Legalisierung der Abtreibung steht den konservativen Medizinern und Medizinerinnen ab Anfang der 1970er Jahre die Forderung einer politischen Selbstbestimmung von Frauen gegenüber. Durch die Legalisierung sowohl der Kontrazeptiva als auch der Abtreibung verlieren insbesondere der Ordre an Definitionsmacht und mit ihm konservativ-medizinische Vorstellungen. Weitere Kritik an der medizinischen Autonomie entsteht ab den 1970er Jahren durch den GIS. Der GIS kritisiert zudem den Fort- 
schrittsmythos und versteht Medizin im Rahmen der kapitalistischen Gesellschaft. Meine These ist, dass Simone Veil in ihrer Rede zum 10. Jahrestag vom INSERM im Jahr 1974 aufgrund der medizin- und kapitalismuskritischen Thematisierungen, biomedizinische Forschung nicht vordergründig mit Wohlstand verknüpft, sondern mit nationalem Prestige. So wird sie weniger angreifbar. In den innermedizinischen Diskursen um Humanexperimente der Nachkriegszeit und im Abtreibungskonflikt zeigt sich vor allem eine moralische Rahmung, die unterschiedlich zum Einsatz kommt. Sie zeigt sich im Kontext einer eher konservativen ärztlichen Standespolitik, sowohl als Kritik an US-amerikanischen Humanexperimenten und solcher der NS-Medizin, als auch zur Sicherung der medizinischen Autorität. Und sie zeigt sich als Abwehr eben dieser ärztlichen Autorität im Kontext des Abtreibungsdiskurses, indem die moralische Autorität kritisiert wird. Mit dem Ethikkomitee scheint eine neue Definitionsmöglichkeit des Humanexperiments eröffnet zu werden. Durch die Verknüpfung von nicht-therapeutischen Versuchen und NS sowie mit ihrer moralischen Verurteilung durch den Ordre war es schwierig, nichttherapeutische Versuche zu thematisieren. Indem der Terminus Ethik mit Wissenschaftlichkeit und Fortschritt verknüpft wird, eröffnet sich ein neuer Raum, um über Humanexperimente zu sprechen. Die eher konservative moralische Rahmung wird zudem durch die Kritik an der konservativ-bürgerlichen Moral im Zuge des medizinkritischen Diskurses des GIS sowie des Abtreibungskonfliktes angegriffen und durch die Legalisierung der Abtreibung geschwächt. Die neue Ethik-Rahmung scheint sich mithilfe staatlicher Einflussnahme in Person der Gesundheitsministerin durchzusetzen, sei es durch die Legalisierung der Abtreibung mit dem VeilGesetz oder durch die Einrichtung des Ethikkomitees vom INSERM. Wie eingangs zitiert, besteht nach Noëlle Lenoir der Grund für die Einrichtung von Ethikinstitutionen im »Bewusstwerden über ethische Probleme« in Folge der NS-Medizin und ihrer Experimente (Lenoir 1991). Ethikinstitutionen erscheinen in dieser Reihung als notwendige Folge von Problemen medizinischer Forschung. Die Verknüpfung von Ethik mit medizinischer Forschung ist in dieser Logik unhinterfragbar, ein ethischer Umgang selbstverständlich. Das Thematisieren der »skandalösen Experimente von Hitlers Medizinern«, wie Jean Bernard sagt oder dieses »Bewusstwerden über ethische Probleme« (Lenoir) bedeutet allerdings nicht, dass es eine Reflexion, eine kollektive Aufarbeitung medizinpolitischer Entwicklungen gegeben hätte, aus der das Ethikkomitee des INSERM als Lösung hervorgeht. Weder findet solch eine Aufarbeitung statt noch ist die Einrichtung des Ethikkomitees die Lösung für nur ein Problem, sondern für mehrere: Es verspricht Schutz für medizinische Autonomie und Forschungsfreiheit, für eine Forschung die in einer linearen Entwicklung mit wissenschaftlichem Fortschritt, Humanität und nationalem Prestige gesehen wird. Darüber hinaus erscheint es als Schutz des Ansehens der 
Wissenschaft. Medizinische Forschung und insbesondere die Versuche am Menschen, die mit der NS-Medizin verknüpft werden, bekommen ein ideologiefreies, antipaternalistisches und antiautoritäres Antlitz, indem sich das Verhältnis zwischen Forscher oder Forscherin und Patient oder Patientin zu einem ausgedehnten Verfahren medizinischer Selbstkontrolle durch eine begleitende Expertise entwickelt. 


\section{Die Sprache der Ethik als Antwort auf Problematisierungen (gen)wissenschaftlicher Autonomie}

Im Jahr 1974 erreicht die Ethik-Rahmung ein weiteres Mal die Ebene staatlicher Politik und zwar in Form einer Ethikkommission, die bei der Délégation Générale à la Recherche Scientifique et Technique (DGRST) eingerichtet wird. Die DGRST entsteht im Jahr 1961 im Kontext der planification scientifique, zu der die Förderung der Molekularbiologie, Medizin und Genetik gehört. Die DGRST existiert bis 1981 und geht dann in der Struktur des unter François Mitterrand eingerichteten Forschungsministeriums auf. Die Entstehung der Ethikkommission bei der DGRST steht in Zusammenhang mit den Diskursen um Gentechnologie. Um die Frage zu beantworten, auf welche Problematisierungen diese Ethikkommission antwortet, wird, ausgehend von ihrer Initiierung, die Sprache der Ethik in den Diskurs um Gentechnologie zurïckverfolgt.

\section{Die EThikKommission der DÉLÉGation GÉNÉRALE À LA RECHERCHE SCIENTIFIQUE ET TECHNIQUE}

$\mathrm{Zu}$ der Ethikkommission bei der DGRST liegen keinerlei offizielle Dokumente vor, wie François Alias in seiner Dissertation über den CCNE festhält, in welcher er diese Kommission berücksichtigt (Alias 1991: 123). Auch jüngere Untersuchungen verweisen auf keine Originaldokumente. Jedoch wurde diese Ethikkommission auf Basis von Interviews mit forschungspolitischen Akteurinnen und Akteuren in der Studie »Governing Molecules« untersucht (Gottweis 1998). Bei der von dem österreichischen Politikwissenschaftler Herbert Gottweis durchgeführten Studie handelt es sich um eine vergleichende Länderstudie zu den USA und Europa. Gottweis arbeitet unter Berücksichtigung der sozio- und forschungspolitischen 
Situation heraus, wie Gentechnologie zu einem Bereich staatlicher Intervention wurde. In seinem vom Poststrukturalismus und den STS geprägten Ansatz liegt der Fokus auf Sprache, die er als konstitutiv im Politikprozess versteht. Er untersucht, wie Diskurse und Narrative das Politikfeld der Gentechnologie konstituieren. Er berücksichtigt vor allem die Frage, wie Risiko als Hauptthema in der Gentechnologiepolitik auftauchte (ebd.: 3ff, 11). Auf diese Studie greife ich im Folgenden zur Untersuchung der Ethikkommission der DGRST vor allem zurück.

Die Ethikkommission der DGRST entsteht auf Anfrage französischer Wissenschaftler und Wissenschaftlerinnen aus dem Bereich der Genforschung. Im November 1974 richten sie diese Anfrage an das nationale Forschungsinstitut, den Centre National de la Recherche Scientifique (CNRS). Sie fragen danach, ein Kontrollsystem für die gentechnologische Forschung und Entwicklung einzurichten. Ihre Anfrage wird an die DGRST geleitet (Alias 1991: 123). Die DGRST richtet in Folge dieser Anfrage eine Ethikkommission ein. Auf Empfehlung der Ethikkommission wird eine technische Kommission zur Evaluierung von Forschungsprojekten aus dem Bereich der Gentechnologie eingerichtet. Die technische Kommission wiederum wird zur Commission Nationale de Classement - zur Nationalen Klassifizierungskommission umbenannt (Duteil 1990: 18/Übers. S.K.). Sie tagt zum ersten Mal im März 1975. Ihre Aufgabe ist es, Forschungsprojekte auf Grundlage von Kriterien zu evaluieren, die aus einer internationalen, wissenschaftlichen Tagung, der Asilomar-Konferenz, im Februar 1975 resultieren (Gottweis 1998: 141). »The task of this commission was to elaborate French guidelines for recombinant DNA ${ }^{1}$ research and to evaluate and classify research projects involving such work « (ebd.; vgl. Duteil 1990: 18). Wissenschaftlerinnen und Wissenschaftler öffentlicher und privater Forschungsinstitutionen, die ein Forschungsvorhaben planten, das die Arbeit mit rekombinanter DNA (rDNA) beinhaltete, mussten ihr Projekt der Kommission vorlegen und zwar in Form eines ausgefüllten standardisierten Fragebogens (Gottweis 1998: 142). In der Commission Nationale de Classement sind ausschließlich Wissenschaftlerinnen und Wissenschaftler u.a. aus den Disziplinen Mikrobiologie, mikrobiologische Genetik, Molekularbiologie und Epidemiologie sowie Repräsentanten und Repräsentantinnen u.a. des CNRS, INSERM und des Institut Pasteur vertreten (ebd.: 143). Was die Zusammenset-

1 Unter rekombinanter DNA (rDNA) wird ein künstlich erzeugtes DNA-Molekül verstanden. DNA bedeutet deoxyribonucleic acid (dt. Desoxyribonukleinsäure). Das Molekül wird durch gentechnologische Methoden hergestellt, indem fremde DNA in eine Bakterienzelle integriert wird. Zum ersten Mal wird rDNA im Jahr 1973 von den US-amerikanischen Genetikern und Biochemikern Stanley Norman Cohen und Herbert Wayne Boyer hergestellt. 
zung der Ethikkommission betrifft, schreibt Gottweis, dass im Kontrast zur Commission Nationale de Classement auch Nicht-Wissenschaftlerinnen und -Wissenschaftler beteiligt sind (ebd.). Alias führt aus, dass die Beteiligung von Jean Bernard, Präsident des Ethikkomitees vom INSERM und späterer erster Präsident des CCNE, und von den Biochemikern bzw. Genetikern Jacques Monod und François Jacob bekannt ist (Alias 1991: 123). Die Ethikkommission »was to be involved, as a sort of court of appeals, in the case of an ethical problem arising during the deliberations « (Gottweis 1998: 142) - sie sollte einbezogen werden im Fall eines »ethischen Problems«, das bei der Evaluierung der Forschungsprojekte in der Commission Nationale de Classement auftreten könnte. De facto wird die Ethikkommission allerdings nicht einbezogen: Bis 1978 werden rund 70 Projekte evaluiert, ohne je die Ethikkommission in das Verfahren einzubinden (ebd.: 143). Wenn die Ethikkommission bei der Evaluation von Forschungsprojekten nicht einbezogen wird, inwiefern scheint die Einrichtung solch einer Kommission im Kontext gentechnologischer Entwicklungen dann überhaupt notwendig? Einer der Hauptakteure im französischen Diskurs um Gentechnologie, der französische Biologe und Genetiker Philippe Kourilsky, äußert sich wie folgt: Es war wichtig, dass die Mitglieder der Ethikkommission der Meinung sind, »to control recombinant DNA experiments, that the conclusions of the Asilomar conference were okay, and that these should be followed up« (Interview mit Ph. Kourilsky von H. Gottweis zit. nach ebd.: 140f). ${ }^{2}$ Die Ethikkommission scheint der Commission Nationale de Classement übergeordnet, ein »court of appeals«, der im Fall eines »ethischen Problems« mit dem Ziel einberufen wird, die Einhaltung der Ergebnisse der Asilomar-Konferenz ${ }^{3}$ zu sichern. Diese internationale Konferenz, benannt nach ihrem Veranstaltungsort, dem kalifornischen Asilomar, geht auf einen internationalen, vorrangig US-amerikanisch geprägten Diskurs um Gentechnologie zurück.

Um zu verstehen, inwiefern es notwendig schien, Forschungsvorhaben in Frankreich ethisch prüfen zu lassen, wird nicht allein der Gentechnologiediskurs in Frankreich, sondern zuerst der internationale Diskurs um Gentechnologie dargestellt. Da dieser Diskurs vorrangig US-amerikanisch geprägt ist und die AsilomarKonferenz in den USA stattfindet, wird auch der länderspezifische Kontext des

2 Die Interviews wurden 1988-1992 durchgeführt (vgl. Gottweis 1998: 369f).

3 In Asilomar finden in diesem Kontext zwei Konferenzen statt. Die Konferenz, von der hier gesprochen wird, ist die zweite. Die erste Konferenz wird 1973 abgehalten. Es handelt sich hierbei um ein Treffen von US-amerikanischen Wissenschaftlern und Wissenschaftlerinnen zur Vorbereitung der Konferenz im Jahr 1975 (Krimsky 1982: 62). Da insbesondere die zweite Tagung in Asilomar internationale Auswirkungen hatte, auch in Frankreich, wird die erste Tagung in der vorliegenden Studie nicht weiter berücksichtigt. 
US-amerikanischen Diskurses um Gentechnologie berücksichtigt. Wie ist die Asilomar-Konferenz entstanden und was beinhalten die Ergebnisse dieser Konferenz? Inwiefern tritt sowohl in den Diskursen um Gentechnologie in Frankreich als auch in dem internationalen, US-amerikanisch geprägten Diskurs die Sprache der Ethik in Erscheinung? Ich gehe nicht davon aus, dass die (ethischen) Rahmungen, die im US-amerikanischen oder internationalen Diskurs um Gentechnologie entstanden sind, mit den Rahmungen im französischen Diskurs um Gentechnologie identisch sein müssen. Es werden also die länderspezifischen Charakteristika der jeweiligen Diskurse um Gentechnologie herausgearbeitet. Dies ermöglicht zudem die (ethischen) Rahmungen im französischen gentechnologischen Diskurs mit den Rahmungen im US-amerikanischen Diskurs zu kontrastieren.

Von Gottweis werden nicht nur die Ethikkommission der DGRST, sondern auch die Asilomar-Konferenz und ihre Auswirkungen in Europa und den USA in seiner Studie »Governing Molecules« untersucht. Wie bereits erwähnt, berïcksichtigt er vor allem die Frage, wie Risiko zum Hauptthema in der Gentechnologiepolitik wird (Gottweis 1998: 3ff, 11). Der Gentechnologiediskurs in den USA und die Asilomar-Konferenz wurden zudem durch zahlreiche Studien, hauptsächlich aus dem Bereich der STS, analysiert. Auch hier wird u.a. herausgearbeitet, dass die Risiko-Thematik bereits während der Asilomar-Konferenz dominiert (vgl. Evans 2002; Gisler/Kurath 2010; Krimsky 1982, 2005; Nelkin 1979, 1992; Stevens 2000; Wright 1994). Ob und inwiefern im Kontext und während der Konferenz die Sprache der Ethik in Erscheinung tritt und, wenn ja, inwiefern sie von der Sprache des Risikos marginalisiert wird, ist allerdings nicht Gegenstand der Untersuchungen. Zwar wird von Braun und ihren Kolleginnen und Kollegen aufgezeigt, dass Ethik als diskursives Rahmenwerk aus der Risiko-Rahmung von Asilomar hervorging in Frankreich wird die Ethikkommission bei der DGRST eingerichtet, in Großbritannien wird von der Regierung der Ashby-Report zur Prüfung ethischer und sozialer Fragen der rDNA in Auftrag gegeben (Braun et al. 2010a: 516f; Braun et al. 2008: 225). Jedoch liegt der Schwerpunkt der Untersuchung nicht darauf, inwiefern die Problematisierungen sozialer Proteste Auswirkungen auf die RisikoRahmung bzw. auf die Ethik-Rahmung in Form der Institutionalisierung der Ethikkommission in Frankreich haben. Im Kontext der USA wird insbesondere anhand der responsible science movement (Stevens 2000) bzw. der critical science movement (Nelkin 1979, 1992), die aus der Kritik an Atomenergie entstanden ist, aufgezeigt, inwiefern sie den Gentechnologiediskurs mit ihrem Konzept von gesellschaftlicher Verantwortung beeinflusst. Inwiefern es in Frankreich solche wissenschaftskritischen Diskurse gab und sie die Institutionalisierung der Ethikkommission bei der DGRST beeinflussten, wurde bisher nicht berücksichtigt. 


\section{DER INTERNATIONALE GENWISSENSCHAFTLICHE DISKURS DER 1970ER JAHRE - AUSTAUSCH IN EINEM 》EXKLUSIVEN CLUB « 4}

Zu Beginn der 1970er Jahre handelt es sich bei den am Gentechnologiediskurs beteiligten Akteurinnen und Akteuren um ein kleines, internationales wissenschaftliches Netzwerk: Sie stehen in engem Austausch und treffen sich auf Tagungen, Konferenzen oder zu persönlichen Gesprächen (vgl. Wright 1994: 135). Der französische Genetiker François Jacob schreibt in seiner Autobiografie, dass es sich bei diesem Netzwerk um eine Struktur handelt, die bereits seit den 1950er Jahren gewachsen ist. Er bezeichnet sie als »exklusiven Club«, dem zu Beginn weltweit ein Dutzend Laboratorien angehörten (Jacob 1988: 286). ${ }^{5}$ Ausgehend von US-amerikanischen Wissenschaftlerinnen und Wissenschaftlern erscheinen Anfang der 1970er Jahre die ersten Publikationen zu gentechnologischer Forschung, ihren möglichen Risiken und den Schwierigkeiten ihrer Bewertung. Im April 1974 rufen namhafte US-amerikanische Wissenschaftlerinnen und Wissenschaftler zu einem freiwilligen internationalen Moratorium für gentechnologische Forschung, die rDNA-Technologie, auf (Gisler/Kurath 2010: 214). Es werden internationale Tagungen organisiert, darunter insbesondere die Asilomar-Konferenz, auf der der Fortgang der Genforschung besprochen, Richtlinien verabschiedet und das Moratorium beendet wird. Von den USA wird der Diskurs in verschiedene Länder getragen, so auch nach Frankreich (Wright 1994: 137ff; Gottweis 1998: 85ff, 138ff; Weiner 2001: 215).

\subsection{Der Hintergrund der US-amerikanischen Kontroverse um Gentechnologie: Öffentliche Kontextualisierung wissenschaftlich-technologischer Entwicklungen}

Der US-amerikanische Diskurs um Gentechnologie in den 1970er Jahren ist von mehreren Ereignissen und Themen geprägt: Zum einen von der Watergate-Affäre, die 1974 zum ersten Rücktritt eines US-amerikanischen Präsidenten, Richard M. Nixon, führt. Der politische Skandal, der zu Beginn der 1970er Jahre publik wird, geht mit einer neuen »Sensibilität« (Krimsky 2005: 310/Übers. S.K.) und einem Vertrauensverlust der Öffentlichkeit gegenüber der US-amerikanischen Regierung

4 Jacob 1988: 286.

$5 \mathrm{Zu}$ dem Austausch zwischen US-amerikanischer und französischer Forschung in der Zeit nach dem Zweiten Weltkrieg vgl. die Untersuchung von Jean-Paul Gaudillière (2002b). 
einher (Dunham/Mauss 1976; Bernstein 1976). Zudem hat durch die WatergateAffäre eine regierungskritische Berichterstattung in der Presse Aufschwung bekommen, insbesondere durch einen investigativen Journalismus - zwei Journalisten hatten den Skandal aufgedeckt (Toinet 1973: 1044). »The Watergate scandal became the symbol of the power of the press in American society (Schudson 2004: 1234). Bis dahin gelten die nationalen Medien als »a bulwark of legitimacy and stability« des US-amerikanischen Systems (Bernstein 1976: 60). Zum anderen rückt nicht zuletzt durch die Anti-Kriegsbewegung die Frage der Verwendung wissenschaftlicher Ergebnisse für Kriegszwecke in den öffentlichen Raum: Sei es durch den Einsatz von Bio- und Nuklearwaffen wie durch die Atombombenabwürfe auf Hiroshima und Nagasaki (1945), oder sei es durch den Einsatz biochemischer Waffen wie Agent Orange während des Vietnamkrieges ${ }^{6}$ oder auch durch die US-amerikanischen Pläne für den Einsatz von Biobomben in Form von AnthraxBazillen während des Zweiten Weltkrieges (Wright 1994: 37ff; Krimsky 1982: 13ff; vgl. Radkau 1988: 330). Thematisiert werden mögliche negative soziale Auswirkungen wissenschaftlich-technologischer Entwicklungen für ganze soziale Gruppen oder auch für die Gesellschaft oder die Menschheit insgesamt. Der Diskurs um die Auswirkungen konzentriert sich allerdings nicht allein darauf, sondern auch auf deren Auswirkungen für die Umwelt (Wright 1994: 116, 121). Die Umwelt- oder ökologische Perspektive wird durch die Umweltbewegung in den USA bestärkt, welche Mitte der 1970er Jahre einen Aufschwung erfährt (Krimsky 1982: 340). Popularisiert wird die ökologische Perspektive durch den Club of Ro$\mathrm{me}^{7}$-Bericht »Die Grenzen des Wachstums«, der im Jahr 1972 erscheint (Meadows et al. 1972). Kerngedanke des Berichts, nach seinen Autorinnen und Autoren auch Meadows-Studie genannt, ist die Vorstellung von der Welt als einem einheitlichen Modell - das des Planeten Erde als geschlossenes Ökosystem. Mit diesem Bericht bildet sich eine »global-ökologische Sichtweise« heraus (Eblinghaus/Stickler 1998: 27). Damit einher geht eine wachstumskritische Diskussion und eine Kritik am Ressourcenverbrauch der Industrieländer. Thematisiert werden die Erschöpfung der natürlichen Ressourcen und vor allem die Umweltzerstörung als Problem für

6 Da mit Vietnamkrieg meist die US-amerikanische Intervention in Vietnam (1965-1975) verbunden wird, gehe ich davon aus, dass in der von mir verwendeten Literatur eben diese Intervention gemeint ist. Denn biochemische Waffen, wie Agent Orange, wurden ab 1965 eingesetzt. Der Vietnamkrieg wird darüber hinaus auch in zwei Phasen eingeteilt, die so genannte französische Phase (Indochinakrieg 1945-1965) und die darauf folgende US-amerikanische Phase.

7 Der internationale Club wurde im Jahr 1968 in Rom gegründet. Seine Mitglieder setzen sich u.a. aus Industrie, Diplomatie und Wissenschaft zusammen. 
die Menschheit (Schlebusch 1994: 54; Könninger 2001: 11ff). Darüber hinaus gerät durch publik gewordene Medizinskandale in den USA, wie die so genannte Tuskegee-Studie, insbesondere die medizinische Forschung in den Blick der Öffentlichkeit. Bei der Studie handelt sich um eine von 1932-1972 am TuskegeeInstitut durchgeführte medizinische Studie an rund 400 afroamerikanischen Männern. In dieser vom U.S. Public Health Service initiierten Langzeitstudie sollte der »natürliche Verlauf« der Syphillis-Erkrankung untersucht werden (Brandt 1978: 21/Übers. S.K.). Weder hatten die Teilnehmer der Studie die Möglichkeit der informierten Einwilligung, noch wurden sie dann behandelt, als entsprechende Medikamente zur Verfügung standen (ebd.). ${ }^{8}$ Nach Bekanntwerden der Studie entsteht in den USA die erste Ethikinstitution, die National Commission for the Protection of Human Subjects of Biomedical and Behavioral Research (1974-1978) (vgl. Kohlen 2009: 65).

Mehrere Ereignisse lösen zu Beginn der 1970er Jahre Fragen aus, die die USamerikanische Öffentlichkeit gegenüber der Politik sensibilisieren und die Glaubwürdigkeit der Politik berühren. Darüber hinaus wird auch die Vorstellung (möglicher) gesellschaftlicher Auswirkungen medizinischer und wissenschaftlichtechnologischer Forschungen und Entwicklungen berührt. Diese Auswirkungen sind bis dahin vor allem mit Wohlstand und ökonomischem Wachstum verknüpft. Diese Verknüpfung hat sich nach dem Zweiten Weltkrieg in den USA insbesondere durch den Bericht »Science - the Endless Frontier« aus dem Jahr 1945 manifestiert. In diesem Bericht des wissenschaftlichen Beraters Vannevar Bush an den damaligen Präsidenten Franklin D. Roosevelt heißt es:

»Without scientific progress the national health would deteriorate; without scientific progress we could not hope for improvement in our standard of living or for an increased number of jobs for our citizens; and without scientific progress we could not have maintained our liberties against tyranny«(Bush 1945: 11).

Und darüber hinaus heißt es in Bushs Bericht, wissenschaftlicher Fortschritt »will promote conservation of our limited national resources« (ebd.: 74). Die allumfassende Lösungskompetenz, die der Wissenschaft hier zugeschrieben wird (Lebensstandard und nationale Gesundheit erhöhen, Ressourcen schützen, militärische Verteidigung), geht mit einem Postulat der Forschungsfreiheit einher. Bush meint:

»We must $[\ldots]$ recover freedom of inquiry and that healthy competitive scientific spirit so necessary for expansion of the frontiers of scientific knowledge. Scientific progress on a

8 Explizit zur so genannten Tuskegee-Studie vgl. die Studie von Susan Reverby (2009). 
broad front results from the free play of free intellects, working on subjects of their own choice, in the manner dictated by their curiosity for exploration of the unknown. Freedom of inquiry must be preserved under any plan for Government support of science« (ebd.: 12).

Diese Vorstellung einer linearen Entwicklung wissenschaftlich-technologischer Forschung und (nationalem) Wohlstand auf Basis der Forschungsfreiheit dominiert in der Nachkriegszeit. Bereits nach dem Zweiten Weltkrieg wird zwar im Kontext der Atombombe die Verwendung von Forschung für Kriegszwecke kritisch hinterfragt, deutlich wird diese Kritik allerdings ab Ende der 1960er Jahre im Kontext des Vietnamkrieges. Es bildet sich eine new critical science movement heraus und mit ihr rückt die Frage der Selbststeuerung wissenschaftlicher und technologischer Entwicklungen in den Diskurs um die gesellschaftlichen Auswirkungen dieser Entwicklungen.

\subsection{Die new critical science movement: Wissenschaftliche Verantwortung als politische Perspektive?}

Viele Wissenschaftlerinnen und Wissenschaftler in den USA sind, so Krimsky und Radkau in ihren Arbeiten zur Geschichte und zum Diskurs der Gentechnologie, für die Problematik der Verwendung bio- und nuklearwissenschaftlicher Ergebnisse für Kriegszwecke sensibilisiert (Krimsky 2005: 310; 1982: 340; Radkau 1988: 334). Ein Ereignis, das sich bis in den Gentechnologiediskurs in den 1970er Jahren auswirkt, ist die öffentliche (Selbst-)Kritik von Wissenschaftlerinnen und Wissenschaftlern im Kontext der Nuklearforschung. Diese Kritik wird bereits in der unmittelbaren Zeit nach dem Zweiten Weltkrieg geäußert. Wissenschaftlerinnen und Wissenschaftler, die am Manhattan-Projekt beteiligt sind, stehen nach Beendigung des Projektes Mitte der 1940er Jahre ihrem Werk durchaus kritisch gegenüber. ${ }^{9}$ So auch der Physiker Robert Oppenheimer (*1904-†1967), der so genannte Vater der Atombombe. Das Projekt trägt zur Entwicklung der Atombombe ab 1942 in den USA bei. Wie der portugiesische Sozialwissenschaftler Fernando Cascais schreibt, wirkt sich die (Selbst-)Kritik der Wissenschaftlerinnen und Wissenschaftler aus: »Awareness of the hazards of technoscientific development had grown since Robert Oppenheimer, president of Manhattan Project, rebuffed goodness of nuclear physics applied to warfare« (Cascais o.J.: 17).

9 Vgl. weiterführend den Rückblick von R. Wilson, einem der beteiligten Wissenschaftler (Wilson 1996). 
Vor dem Hintergrund des Zweiten Weltkrieges und ab den 1960er Jahren vor dem Hintergrund des Vietnamkrieges entwickeln sich im US-amerikanischen Raum verschiedene Konzepte von Verantwortung der Wissenschaft gegenüber der Gesellschaft. Die US-amerikanische Wissenschaftssoziologin Dorothy Nelkin behandelt diese verschiedenen Vorstellungen von Verantwortung und die korrespondierenden wissenschaftliche Strömungen (Nelkin 1979): Die Nachkriegswissenschaftlerinnen und -wissenschaftler, die insbesondere die Verwendung ihrer Forschung für Kriegszwecke kritisieren, bezeichnet sie als »movement for social responsibility«. Die Wissenschaftlerinnen und Wissenschaftler, die ab den späten 1960er Jahren aktiv werden, bezeichnet sie als »new critical science movement $«$. In der Bewegung für soziale Verantwortung der Nachkriegszeit ist vor allem die Information der Öffentlichkeit über die Implikationen der Atomenergie zentral. Diese ist gleichzeitig mit der Hoffnung verbunden, die staatliche Politik zu beeinflussen. Autonomie und Selbststeuerung der Wissenschaft sollten aber geschützt bleiben. Die Bewegung ist unorganisiert, sie setzt sich aus Individuen zusammen, die sich persönlich gegenüber der Öffentlichkeit verantwortlich fühlen (ebd.: 177f). Ende der 1960er Jahre weiten sich der politische Radius und die politischen Aktivitäten der Wissenschaftlerinnen und Wissenschaftler über militärische Themen auf die Umwelt- und Anti-Atombewegung und auf Fragen biomedizinischer und genetischer Forschung aus. Es entsteht die new critical science movement, mit der sich Wissenschaftlerinnen und Wissenschaftler verschiedener politischer Orientierungen identifizieren (ebd.). Auch hier handelt es sich nicht um eine soziale Bewegung mit einem gewissen Organisationsgrad (vgl. Krimsky 1982: 340). Nelkin unterscheidet im Wesentlichen zwei politische Orientierungen innerhalb der new critical science movement: die Gruppe der »critical science « und der »public interest science« (Nelkin 1979: 178). Die Gruppe der critical science ist eng mit Wissenschaftlerinnen und Wissenschaftlern verknüpft, die sich während des Vietnamkrieges politisieren und einen gesellschaftlichen und politischen Wandel anstreben (ebd.). $\mathrm{Zu}$ diesem Teil der Bewegung gehören Organisationen politisch aktiver Wissenschaftlerinnen und Wissenschaftler, die zu unterschiedlichen Themenbereichen wie Umwelt, Nuklear- oder Gentechnologie arbeiten. Hierzu zählen die Organisationen Science for the People oder Scientists and Engineers for Social and Political Action (ebd.: 179; Krimsky 2005: 311). Das Konzept der Verantwortung der critical science entwickelt sich zu einer politischen Perspektive (Nelkin 1979: 177f). Es beinhaltet die Thematisierung der Frage, wer über Forschungsrichtungen und die Anwendung von Wissenschaft entscheidet. Die Gruppe der public interest scientists stammt direkt aus der Tradition der movement for social responsibility der Nachkriegszeit. Wie schon in der Bewegung der Nachkriegszeit basiert ihr Konzept der wissenschaftlichen Verantwortung auf der Überzeugung von der poli- 
tischen Wirkungskraft der Erziehung und Information der Öffentlichkeit und vor allem auf der Selbstregulierung der Wissenschaft (ebd.: 178; Krimsky 1982: 94f, 340; Radkau 1988: 329). Das neue Konzept von Verantwortung der critical science steht dem der movement for social responsibility der Nachkriegszeit bzw. den public interest scientists gegenüber. Während das Nachkriegskonzept m.E. ganz im Sinne des Berichtes »Science - the Endless Frontier« (Bush 1945: 12) darauf insistiert, Forschung von politischer Kontrolle fern zu halten, geht es den kritischen Wissenschaftlerinnen und Wissenschaftlern gerade darum, politische Einflussnahme vor dem Hintergrund ihres Konzeptes der gesellschaftlichen Verantwortung zu erhöhen: Entmystifizierung von Wissenschaft, Rechenschaftspflicht und Partizipation sind Schlüsselbegriffe dieses kritischen Konzeptes (Nelkin 1979: 179f). Wie insbesondere Susan Wright in ihrer Studie »Molecular Politics« herausarbeitet, ist Partizipation allerdings nicht allein Schlüsselbegriff des kritischen wissenschaftlichen Diskurses, sie wird ebenso von der staatlichen Forschungspolitik aufgegriffen (Wright 1994: 122). Das Konzept der Partizipation dient hier als möglicher Umgang mit anti-wissenschaftlichen und anti-fortschrittlichen Haltungen, die mit den critical scientists in Zusammenhang gebracht werden. Die kritischen Wissenschaftlerinnen und Wissenschaftler werden, wie Wright schreibt, mit der Anti-Kriegsund Umweltbewegung insgesamt assoziiert und ihre Haltung als »ideology of antiscience $«$ oder »Luddism ${ }^{10}$ bezeichnet, als Wissenschaftlerinnen und Wissenschaftler, die sich von Fakten und Vernunft entfernen (ebd.: 51, 122). Pejorativ, wie in dem Zitat, wird der Begriff Luddismus m.E. gleichbedeutend mit Technik- und Fortschrittsfeindlichkeit verwendet. Wie es die Nationalen Akademie der Wissenschaften im Jahr 1969 formuliert, soll öffentliche Partizipation im Technikfolgenabschätzungsprozess gefördert werden, allerdings in einem klar abgesteckten Rahmen, mit Mechanismen, die »irresponsible claims« herausfiltern (National Academy of Sciences (1969) zit. nach Wright 1994: 52). Bei Wright wird deutlich, dass forschungspolitische Akteurinnen und Akteuren eine kontrollierte öffentliche Teilnahme an der Abschätzung von Wissenschaft- und Technikentwicklung thematisieren. Kontrolliert bedeutet, dass sie nicht das Ziel verfolgen, dass eine Partizipation der Öffentlichkeit letztlich zu einem Forschungsstopp führt (Wright 1994: 50ff). Schlüsselbegriffe wie Partizipation werden sowohl von kritischen Wissenschaftlerinnen und Wissenschaftlern als auch von forschungspolitischen Akteuren

10 Luddismus bezeichnet eine bestimmte Form des Arbeiterinnen- und Arbeiterkampfes im Europa des 17. und 18. Jahrhunderts. Die Ludditinnen und Ludditen, auch Maschinenstürmerinnen und Maschinenstürmer genannt, protestierten durch das Zerstören von Maschinen gegen die zunehmende Technisierung und gegen die ihrer Meinung nach damit einhergehende Verelendung der Bevölkerung (vgl. Randall 1995). 
und Akteurinnen aufgegriffen und entsprechend inhaltlich gefüllt, um die Selbststeuerung von Wissenschafts- und Technikentwicklungen in Frage zu stellen und um diese Art der Steuerung zu sichern. So soll mit dem forschungspolitischen Konzept der Partizipation die Teilnahme an der Abschätzung von Wissenschaftsund Technikentwicklungen bedacht, aber nicht grundlegend die Beteiligung an Entscheidungen über diese Entwicklungen ermöglicht werden.

Mit den unterschiedlichen Konzepten von Verantwortung innerhalb der new critical science movement stehen sich auch unterschiedliche Vorstellungen über die Steuerung von Wissenschafts- und Technikentwicklungen gegenüber. Allein durch diese offenkundige Uneinigkeit der wissenschaftlichen Community ist das Bild der politischen und normativen Neutralität, das die Selbststeuerung der Wissenschaft in der Nachkriegszeit rechtfertigte, in Frage gestellt (vgl. Nelkin 1979: 181). In der Kontroverse zwischen kritischen Wissenschaftlerinnen und Wissenschaftlern sowie forschungspolitischen Akteurinnen und Akteuren rückt gerade diese Frage der Neutralität in den Fokus, nicht das Konzept der Verantwortung. Indem die kritischen Wissenschaftlerinnen und Wissenschaftler als ideologisch, anti-wissenschaftlich und anti-fortschrittlich bezeichnet werden, zeigt sich m.E., welches Bild von Wissenschaft sich im Sinne der forschungspolitischen Akteurinnen und Akteuren etablieren soll. Ein Bild von Wissenschaft als politisch neutral, objektiv und fortschrittlich, einer Wissenschaft, die auf Fakten und Vernunft basiert. Wissenschaft soll als ein Bereich, der eigentlich politisch nicht reguliert zu werden braucht, verstanden werden, da er frei von jeglichen Interessen oder Ideologien sein soll. Wissenschaft wird mit Fortschritt gleichgesetzt, der wiederum per se als gut betrachtet wird - er beinhaltet im Sinne der Nachkriegsvorstellung nationalen Wohlstand. Kurz gesagt: Durch diese Abgrenzung vom Luddismus rückt in dem Konflikt die Frage nach einer neutralen Wissenschaft in den Fokus - nicht die der Verantwortung. Wenn die Vorstellung einer neutralen Wissenschaft dominant bleibt, dann bleibt dieser Logik gemäß die Forschungsfreiheit unangetastet. Die Problematisierung der Selbststeuerung, die mit dem neuen Konzept der Verantwortung der kritischen Wissenschaftlerinnen und Wissenschaftler einhergeht, wird m.E. auf die Frage der Neutralität der Wissenschaft verschoben.

Wie im wissenschaftskritischen Diskurs um gesellschaftliche Verantwortung in den USA insgesamt, so ist auch im Gentechnologiediskurs zu Beginn der 1970er Jahre Verantwortung ein zentrales Konzept.

\subsubsection{Genwissenschaftliche Verantwortung als politische Perspektive?}

Das Konzept der Verantwortung kommt mit der new critical science movement in den Diskurs um Gentechnologie. Einer der prominentesten Akteure der critical science ist der Genetiker und Mikrobiologe Jonathan Beckwith von der Harvard 
Medical School in Boston (USA). Ein Vortrag, den Beckwith im Jahr 1970 anlässlich der Verleihung des Wissenschaftspreises Eli Lilly Award hält, sorgt medial für Aufmerksamkeit, vor allem, da er angibt, das Preisgeld politisch zu verwenden und der afroamerikanischen Bürgerinnen- und Bürgerrechtsbewegung Black Panther Party zu spenden (Beckwith 1970: 225; 2002: 63). Beckwith thematisiert die Rolle von Wissenschaftlerinnen und Wissenschaftlern in der Gesellschaft und insbesondere ihre gesellschaftliche Verantwortung, die sich aus der Verwendung ihrer Arbeit ergebe: »we have a special responsibility in this society because of the way we and our work are used« (Beckwith 1970: 224). Er thematisiert die Verwendung wissenschaftlichen Wissens für die Entwicklung von Atom- und Biowaffen sowie die Möglichkeit der Eugenik im Kontext der Gentechnologie (ebd.: 225). Eine Ursache des »Missbrauchs « der Wissenschaft sieht Beckwith in einem »system which is based on maximum profit« (ebd.: 226/Übers. S.K.). Die Profitorientierung ist nicht nur Ursache für den Missbrauch der Wissenschaft, so Beckwith, sondern auch ein wesentliches Problem für die Umweltverschmutzung: »The solution to the problem of pollution will not come until the leaders of industry decide that profits are not important. I'm afraid they're going to need a little help on that problem《 (ebd.). Darüber hinaus kritisiert er, welche Forschung finanziert wird, und hinterfragt ihre Verwendung: »Do we scientists really want to be responsible for the one-third of a billion dollars wasted on the recent moon trip when people are starving and receiving grossly inadequate health care in this country?« (ebd.). Die Lösung sieht Beckwith darin, das »capitalist system《 zu überwinden (ebd.: 227). Notwendig sei ein radikaler gesellschaftlicher Wandel: »If you agree that we have a responsibility because of the misuse of our work, then, I believe, it must be recognized that there must be a radical change in society before we will have an opportunity to be absolved of our responsibility« (ebd.: 226). Ein Missbrauch wissenschaftlicher Arbeit kann nach Beckwith also nur mit einem radikalen gesellschaftlichen Wandel verhindert werden. Erste Schritte in Richtung dieses gesellschaftlichen Wandels sieht der Genetiker in einem Bewusstwerden der Wissenschaftlerinnen und Wissenschaftler über diesen (möglichen) Missbrauch (ebd.: 225). Und er sieht die ersten Schritte darin, dass diese ihre elitäre Haltung hinterfragen (ebd.: 227), denn sie besäßen nicht unbedingt die Fähigkeit, über gesellschaftliche Probleme zu urteilen:

»I do not think scientists have a superior ability to judge what the problems are in our society and how they can be solved. Instead, scientists, recognizing their responsibility, must ally with other workers, the poor people, and other oppressed groups to work together for meaningful radical political change (ebd.: 226). 
Beckwiths Ausführungen zeigen ein politisches Konzept von Verantwortung, das auf einer kapitalismus- und elitekritischen Perspektive basiert. In dieser Perspektive sind wissenschaftliche Entwicklungen politisch nicht neutral oder führen per se zu Fortschritt oder Wohlstand. Da sie mit einer Profitorientierung verflochten sind, die zu gesellschaftlichen Missständen führen wie »inadequate health care« (ebd.), oder da die wissenschaftlichen Entwicklungen in Kriegszwecke, z.B. Atomwaffen (ebd.: 225) eingebunden sind, muss sich nach Beckwith gerade diese Profitorientierung ändern. Ein erster Schritt in diese Richtung ist für ihn nicht eine reformerische Art der Partizipation von Nicht-Wissenschaftlerinnen und -Wissenschaftlern an der Abschätzung von Wissenschaft- und Technikentwicklung. Das Ziel ist nicht eine andere Art der Evaluation zur Sicherung wissenschaftlicher und technologischer Entwicklungen, sondern der Zusammenschluss mit Armen, Unterdrückten, Arbeitern und Arbeiterinnen mit dem Ziel des gesellschaftlichen Wandels.

\subsection{Die genwissenschaftliche Community und ihre Zwickmühle}

Vor dem Hintergrund der Thematisierung wissenschaftlicher Verantwortung gegenüber der Gesellschaft, von Forschung für Kriegszwecke sowie der Thematisierung ökologischer Probleme steht die wissenschaftliche Community im Hinblick auf die gentechnologischen Entwicklungen unter Druck, einen Umgang mit ihrer Forschung zu finden. Nicht allein Forscher und Forscherinnen, auch die US-Armee hat ein besonderes Interesse an genetischer Forschung, da die bisherigen Biowaffen als militärisch ineffektiv gelten (Wright 1994: 118). Praktische Ergebnisse aus der Genforschung fordern zudem die US-amerikanische Regierung und forschungspolitische Instanzen im Hinblick auf den internationalen Wettbewerb und im Hinblick auf die weitere Legitimation von Fördergeldern. Wright führt aus: »For scentists, the techniques promised not only the novel direction in research and the fast results by serial-competitive funding arrangements, but also a powerful justification for continued support« (ebd.: 114f). Die genwissenschaftliche Community ist in einer Zwickmühle: Sie steht unter dem Druck, so schnell wie möglich anwendungsorientierte Ergebnisse zu liefern. Wie Wright schreibt, bedeutet dies finanzielle Förderung und die Möglichkeit, zu forschen. Gleichzeitig werden die möglichen politischen, sozialen und ökologischen Auswirkungen wissenschaftlichtechnischer Entwicklungen hinterfragt. Zwickmühlenartig wird die Situation dadurch, dass die Ansichten innerhalb der wissenschaftlichen Community zu Genforschung nicht homogen sind. Sie reichen von einer forschungsbefürwortenden über eine -skeptische bis zu einer forschungsverweigernden Haltung. Einige kritische Wissenschaftler und Wissenschaftlerinnen ziehen sich aus der Forschung zurück 
bzw. warnen, wie Beckwith, vor ihrer militärischen Anwendung (ebd.: 118f; Radkau 1988: 329; Krimsky 1982: 31; Barben 1997: 385). Ein erster Schritt aus dieser Zwickmühle ist, das Thema Gentechnologie und mögliche Auswirkungen dieser Technologie einem internationalen Publikum offenzulegen.

\subsection{Der Asilomar-Prozess (1973-1975)}

An eine breitere internationale Öffentlichkeit gelangt der Diskurs um Gentechnologie und ihrer Auswirkungen erstmals durch zwei im Wissenschaftsmagazin Science publizierte Briefe einzelner Wissenschaftler und Wissenschaftlerinnen. Science ist die Fachzeitschrift der Amerikanischen Gesellschaft zur Förderung der Naturwissenschaften. Die beiden Briefe werden in der Literatur meist nach ihren jeweiligen Hauptautoren und -autorinnen benannt: Der Singer-Brief und der BergBrief. ${ }^{11}$ Mit einer breiteren Öffentlichkeit ist an dieser Stelle ein eher akademisches Publikum gemeint. Die Briefe sind in Fachtermini gehalten und somit nicht allgemein verständlich (Krimsky 1982: 78; Weiner 2001). Grund dafür, dass die Briefe fast ausschließlich in akademischen Kreisen rezipiert werden, ist aber m.E. auch, dass sie in englischsprachigen Wissenschaftsmagazinen publiziert wurden, die keine breitere Leserinnen- und Leserschaft erreichen. Der Singer-Brief geht aus der Gordon-Konferenz zu Nukleinsäuren hervor, die im Jahr 1973 stattfand. GordonKonferenzen, die von einer gleichnamigen NGO bis heute organisiert werden, finden in den Disziplinen Chemie, Biologie und Physik statt und sind geprägt durch ihren informellen Charakter, der explizit von den Veranstaltern und Veranstalterinnen gewünscht ist (Krimsky 1982: 70f). Auf der Gordon-Konferenz im Jahr 1973 sind ungefähr 140 Teilnehmende aus elf Ländern anwesend, darunter auch Frankreich (ebd.: 71). Thematisch werden u.a. die neuen Entwicklungen in der gentechnologischen Forschung und insbesondere der rDNA-Technologie präsentiert. In Folge dieser Präsentationen formulieren einige Forscher und Forscherinnen »Bedenken «, wie die Molekularbiologin Maxine Singer, eine der Vorsitzenden der Gordon-Konferenz, im Jahr 1975 rückblickend auf die Konferenz in einem Interview meint (Interview mit M. Singer von R. Goodell, 31.07.1975 zit. nach ebd.: 75). Jüngere Wissenschaftler und Wissenschaftlerinnen wollen eine Diskussion, in der gesellschaftliche Fragen einbezogen werden, ältere befürchteten Einschrän-

11 Der Singer Brief wurde verfasst von Maxine Singer und Dieter Soll (Singer/Soll 1973). Der Berg-Brief von Paul Berg, David Baltimore, Herbert W. Boyer, Stanley N. Cohen, Ronald W. Davis, David S. Hogness, Daniel Nathans, Richard Roblin, James D. Watson, Sherman Weissman und Norton D. Zinder (Berg et al. 1974). 
kungen dieser Forschung, so Singer (ebd.). Noch während der Gordon-Konferenz initiiert Singer einen kollektiven Brief, der an die US-amerikanische Nationale Akademie der Wissenschaften und an die Nationale Akademie der Medizin geschickt und schließlich im September 1973 in der Fachzeitschrift Science publiziert wird (Singer/Soll 1973; Krimsky 1982: 74ff). Dieser Singer-Brief enthält die Forderung an diese Akademien, ein Komitee zur Untersuchung der Risiken der Gentechnologie und zur Ausarbeitung von Richtlinien für die weitere gentechnologische Forschung einzurichten (Singer/Soll 1973). Mit diesem Akt wenden sich Wissenschaftlerinnen und Wissenschaftler an staatliche Instanzen und legen damit Probleme der Selbstregulierung offen. Eine Notwendigkeit für diese Offenlegung kann vor dem Hintergrund des Watergate-Skandals gesehen werden: Wie der USamerikanische Politologe Ira Carmen vermutet, waren die Wissenschaftler und Wissenschaftlerinnen insofern von dem Skandal motiviert, da sie von der Öffentlichkeit nicht der Vertuschung beschuldigt werden wollten, wie es der US-amerikanischen Regierung im Kontext des Watergate-Skandals geschah (Carmen 1985: 63). Aus dem im Anschluss an den Singer-Brief eingesetzten US-amerikanischen Untersuchungskomitee zu den Risiken der rDNA-Technologie resultiert der im Jahr 1974 ebenfalls in Science publizierte Berg-Brief (Berg et al. 1974). Dieser beinhaltet, wie zu Beginn des Kapitels bereits erwähnt, die Forderung nach einem freiwilligen internationalen Moratorium zu rDNA-Experimenten, welches bis $\mathrm{zu}$ einer bereits geplanten internationalen Tagung in Asilomar eingehalten werden sollte und letztlich auch wird. ${ }^{12}$ Ziel der Tagung ist, den weiteren Fortgang gentechnologischer Forschung und Entwicklung zu diskutieren (ebd.). Sie wird schließlich im Februar 1975 mit 150 internationalen Teilnehmenden abgehalten die so genannte Asilomar-Konferenz. Frankreich ist durch den Biologen und Genetiker Philippe Kourilsky vertreten, der zu der Zeit am Institut Pasteur tätig ist (vgl. Kamel et al. 2002).

Im Zeitraum vom Singer-Brief (1973) bis zur Tagung in Asilomar (1975) - im Folgenden kurz Asilomar-Prozess genannt - dominiert zwar die Risiko-Rahmung. Jedoch zeigt sich bei näherer Untersuchung explizit die Sprache der Ethik. Darauf, was die dominierende Risiko-Rahmung beinhaltet und wie im Asilomar-Prozess die Sprache der Ethik auftaucht, wird im Folgenden eingegangen.

12 Dass im Zeitraum des Moratoriums trotzdem gentechnologische Experimente durchgeführt wurden, ist sicherlich nicht ausgeschlossen. In den für diese Studie analysierten Dokumenten wurde diesbezüglich nichts erwähnt. 


\subsubsection{Risiko im Asilomar-Prozess}

Die STS-Studien zum Asilomar-Prozess zeigen, dass es sich um einen Prozess handelt, in dem das Anliegen im Vordergrund steht, die Selbststeuerung von Wissenschafts- und Technikentwicklungen zu bewahren und staatliche Einflussnahme zu verhindern: Das Hauptaugenmerk wird auf Risiken und Sicherheitsthemen gelegt, wodurch andere Themen ausgeklammert werden (Gisler/Kurath 2010), seien es politische, ökonomische oder ökologische Themen (Radkau 1988: 335). »This kept the debate on a technical level - whether certain procedures were risky or not, and how risk could be minimized - and therefore justified keeping decision making entirely in the hands of the scientists (Evans 2002: 95; vgl. Gottweis 1998: 90; Barben 1997: 385). Indem Risiken technisch gerahmt werden, bleibt die Lösungskompetenz wissenschaftlich definiert. Zudem werden durch die RisikoRahmung, durch die technische Ebene, nicht allein politische oder ökologische Themen ausgeklammert, sondern darüber hinaus Diskussionen über die Verantwortung der Wissenschaft gegenüber der Gesellschaft; Themen die durch die new critical science movement in die Diskussion um Gentechnologie gebracht wurden. Nelkin interpretiert die Asilomar-Konferenz als »a striking example of an organized professional response to pressures for social responsibility« (Nelkin 1979: 180). Organisiert ist die Antwort insofern, als den Organisatoren und Organisatorinnen bereits im Vorfeld von der US-amerikanischen Nationalen Akademie der Wissenschaften gesagt wird, sie sollen darauf achten, dass die Debatte innerwissenschaftlich bleibe (Wright 1994: 137). Dies wird berücksichtigt: die Tagung findet hinter verschlossenen Türen statt, und sowohl die Teilnehmenden des Komitees, das die Konferenz organisierte, als auch die Konferenzteilnehmenden und die Pressevertreter und -vertreterinnen wurden ausgewählt. Kritische Wissenschaftler und Wissenschaftlerinnen oder Technikskeptiker und -skeptikerinnen sind nicht vertreten (ebd.: 137, 146). Vor dem Hintergrund der Watergate-Affäre betrachtet, kann mit Carmen die Asilomar-Konferenz als Öffnung verstanden werden, um nicht der Vertuschung beschuldigt zu werden (Carmen 1985: 63). Durch die Auswahl der Teilnehmenden, insbesondere der Presse, der im Kontext des WatergateSkandals und seiner Aufdeckung eine tragende Rolle zukam, zeigt sich m.E. deutlich, dass es sich um eine organisierte Öffnung handelt. Die Begrenzung der Teilnehmenden hat auch Einfluss auf die Agenda und die Rahmung der Themen. Die Rahmung, die sich bereits in der Vorbereitungsphase der Konferenz von Asilomar deutlich abzeichnet, ist die Risiko-Rahmung. Einer der Hauptakteure des Asilomar-Prozesses, u.a. Organisator der Konferenz und Autor des nach ihm benannten Berg-Briefes, der US-amerikanische Biochemiker und spätere Nobelpreisträger für Chemie (1980), Paul Berg, sagt rückblickend in einem Interview über die Konferenz: 
»What I realized is that there was a whole question of biohazards that many of us were not facing up to, and they needed discussion. And so I and a group of people decided that we ought to have two conferences to deal with the problem of biohazards; the first one would be a fact-gathering meeting at which we would discuss what is known about the potential biohazards of tumor viruses; then, following that a second conference to discuss the kinds of experiments that people are doing, whether they pose any hazard, how you would find out if they posed a hazard, what we would do while we were waiting? « (Interview mit P. Berg von Ch. Weiner, 17.05.1975 zit. nach Krimsky 1982: 61f).

In seinem Rückblick thematisiert Paul Berg »Fakten«, auf denen die Risikoabschätzung basiert. Die Risiko-Rahmung dominiert sowohl in der Vorbereitungsphase als auch während der Asilomar-Konferenz bis in das Abschlussdokument der Konferenz hinein.

Ziel der Konferenz ist es, einen Konsens zu finden wie auch Empfehlungen abzugeben. Paul Berg sagt hierzu während der Konferenz: »We can't say that 150 scientists spent four days at Asilomar and all of them agreed that there was a hazard - and that they still couldn't come up with a single suggestion. That's telling the government to do it for us« (P. Berg zit. nach Evans 2002: 96). Anhand des Statements zeigt sich, dass durch ein einheitliches Meinungsbild und durch die Ausarbeitung von Empfehlungen staatliche Einflussnahme reduziert und wissenschaftliche Autonomie gesichert werden soll. Mit einem einheitlichen Meinungsbild wird der Uneinigkeit, die innerhalb der wissenschaftlichen Community herrscht, begegnet. Es ist eine Uneinigkeit, die das Bild der politischen und normativen Neutralität der Wissenschaft ins Wanken brächte und damit ihre Selbststeuerung gefährde, ausgelöst durch Kontroversen um gesellschaftliche Verantwortung. Sie wird im Asilomar-Prozess und damit im Kontext der Gentechnologie auf zweierlei Ebenen ausgeklammert: Zum einen durch die Auswahl einer homogenen Teilnehmendenschaft und zum anderen durch den Konsens im Abschlussdokument der Konferenz. Dieser Konsens beinhaltet, dass Risiken existieren und ein Verfahren der Risikobewertung notwendig ist. Die Risikobewertung wiederum wird in einen Prozess überführt. Im Abschlussdokument wird festgehalten: »The means for assessing and balancing risks with appropriate levels of containment will need to be reexamined from time to time « (Berg et al. 1975: 1981). Nicht die Risikobewertung an sich, sondern die Mittel der Risikobewertung sollen wiederkehrend überprüft werden. So wird ermöglicht, dass auch zukünftig die Lösung in der Risikobewertung liegt, die auf »Fakten« basiert und damit in wissenschaftlichen Händen bleiben kann. Gentechnologische Forschung ist damit gesichert. Zudem wird mit der dauerhaften Risikobewertung auch in Zukunft die Möglichkeit, andere Themen 
außerhalb der Risiko-Rahmung in die Diskussion zu bringen, erschwert, etwa, ob diese Forschung überhaupt durchgeführt werden soll.

Durch die Dominanz der Risiko-Rahmung werden Kontroversen um die Selbststeuerung von Wissenschaft und Forschung marginalisiert. Das Bild wissenschaftlicher Einigkeit und politischer Neutralität wird dadurch wieder hergestellt. Eine Marginalisierung von Themen jenseits des Themas der Risiken zeigt sich gleich zu Beginn der Konferenz in einer Eröffnungsrede. So heißt es von dem USamerikanischen Biologen David Baltimore, die Diskussion über Gentechnologie führe $\mathrm{zu} \gg$ complicated questions of what's right and what's wrong - complicated questions of political motivation - and [of] which I do not think this is the right time [for discussion]« (D. Baltimore zit. nach Wright 1994: 149/Hinzufügungen i.O.). Durch die Ausklammerung politisch motivierter Themen und die Betonung von Risiken wird das Bild der Neutralität manifestiert, denn die Evaluation von Risiken basiert auf »Fakten«, die wiederum als neutral gelten. Es wird eine Trennung zwischen Fakten auf der einen und Werten und politischen Fragen auf der anderen Seite errichtet. Weiter zeigt sich im Abschlussdokument der Konferenz, dass auf den staatlichen Druck auf die Wissenschaft, zu praktischen Ergebnissen zu kommen, reagiert wird: »The use of recombinant DNA methodology promises to revolutionize the practice of molecular biology« und »there is every reason to believe that they will have significant practical utility in the future « (Berg et al. 1975: 1981). Ebenso wird erklärt, dass keinerlei Risiken für die Ökosysteme bestünden (ebd.). Wie Radkau feststellt, werden ökologische Themen auf der Konferenz nicht diskutiert (Radkau 1988: 335). Trotzdem werden »die Ökosysteme« in das Abschlussdokument aufgenommen. Die Notwendigkeit, diese anzusprechen, kann m.E. sowohl in einem ökologischen Bewusstsein der Teilnehmenden der Konferenz liegen, bzw. darin auf umweltmotivierte Kritiken reagieren zu müssen - die Umweltbewegung hatte einen Aufschwung - als auch darin, die Umweltpolitik der US-Regierung aufzugreifen. Die US-Regierung hatte Umweltfragen bereits in ihre Politik aufgenommen. Im Jahr 1970 wird die Umweltschutzbehörde US Environmental Protection Agency eingerichtet, der Umwelttag Earth Day eingeführt und Umweltverträglichkeitsuntersuchungen bei Technikbewertungen halten Einzug in bundesstaatliche Programme (Krimsky 2005: 310). Indem erklärt wird, dass für die Ökosysteme kein Risiko bestehe, wird m.E. ein Kritikpunkt der critical scientists entkräftet und der regierungspolitische ökologische Trend berücksichtigt, der wichtig ist, um Forschungsgelder zu erhalten. In abgewandelter Form zeigt sich im Abschlussdokument der Asilomar-Konferenz darüber hinaus die Vorstellung einer linearen Entwicklung von Wissenschaft und Fortschritt. Während in der Nachkriegszeit noch davon die Rede ist, wissenschaftlicher Fortschritt führe zu Wohlstand, wird nun nicht mehr explizit davon gesprochen. Der »scientific progress in 
research on recombinant DNA molecules «, liege darin, dass die Molekularbiologie mit der rDNA-Technologie revolutioniert wird und »significant practical utility« bringe (Berg et al. 1975: 1981). Wobei gentechnologische Entwicklungen nur als fortschrittlich gelten, sofern sie keine Risiken für die Ökosysteme darstellen. Die Vorstellung von Fortschritt wird damit m.E. um ein ökologisches Leitbild ergänzt. Mit der innerwissenschaftlichen Risikobewertung und der Betonung technischer Risiken im Abschlussdokument wird eine staatliche Einflussnahme auf die Selbststeuerung von Wissenschafts- und Technikentwicklungen zurückgewiesen. Indem im Abschlussdokument nicht nur von den Ökosystemen gesprochen wird, für die keine Risiken bestehen, sondern auch von dem praktischen Nutzen gentechnologischer Entwicklungen, wird m.E. dem staatlichen Interesse der Wettbewerbsfähigkeit entgegen gekommen. Die Aussicht auf Wettbewerbsfähigkeit impliziert wiederum Fördergelder für die Forschung. Indem Risiken technisch gerahmt werden, wird eine Diskussion um gesellschaftliche Verantwortung, wie sie durch die critical scientists befördert wird, und die damit einhergehende Forderung eines »radical change in society« (Beckwith 1970: 226) nicht thematisiert. Obwohl sich die Risiko-Rahmung im Asilomar-Prozess deutlich durchsetzt und die Diskussion um Verantwortung der Wissenschaft gegenüber der Gesellschaft, die zu einigen Kontroversen führt, außen vor bleibt, taucht ein Konzept von Verantwortung im Abschlussdokument der Tagung auf, und zwar ein in die Risiko-Rahmung integriertes Konzept von Verantwortung.

\subsubsection{Verantwortung = Risikoabschätzung}

Im Abschlussdokument der Tagung wird die Verantwortung des Forschers oder der Forscherin erwähnt: »each investigator bears a responsibility for determining whether, in his particular case, special circumstances warrant a higher level of containment than is suggested here « (Berg et al. 1975: 1982). Verantwortung ist hier kein Konzept der wissenschaftlichen Community, das wissenschaftliche und technologische Entwicklungen in Frage stellt oder gar etwas, das einen »radical change in society« (Beckwith 1970: 226) beinhaltet, sondern etwas, das der individuelle Forscher oder die Forscherin trägt und je nach Experiment variieren kann. Der Handlungsrahmen wird zwar durch die empfohlenen Sicherheitsmaßnahmen abgesteckt, die zugrundeliegende Risikoabschätzung wird aber wiederum in einen Prozess überführt und somit auch die Sicherheitsmaßnahmen und mit ihnen die Verantwortung. Das Konzept der individuellen Verantwortung wird in die Sicherheitsmaßnahmen, die Risiko-Rahmung integriert. Obwohl bzw. weil die RisikoRahmung dominiert, zeichnet sich innerhalb dieser Rahmung zum Ende des Asilomar-Prozesses eine Individualisierung von Verantwortung ab. 
Die Asilomar-Konferenz ist eine Antwort auf die Kontroverse um die Verantwortung der Wissenschaft gegenüber der Gesellschaft und der damit einhergehenden Problematisierung der Selbststeuerung genwissenschaftlicher und -technologischer Entwicklungen. Als Lösung für diese Problematisierung erscheint eine technische Risikoabschätzung. Sowohl der Singer-Brief als auch der Berg-Brief und die Konferenz von Asilomar sind hauptsächlich in der Sprache des Risikos gehalten. Jedoch zeigt sich die Sprache der Ethik explizit im Asilomar-Prozess.

\subsection{Ethik im Asilomar-Prozess}

Die Sprache der Ethik zeigt sich zweimal bereits im Vorfeld der AsilomarKonferenz. Und zwar in einem Entwurf des Singer-Briefes sowie in einem Beitrag Paul Bergs auf einer internationalen Konferenz in Davos. Ein weiteres Mal zeigt sie sich bei der Konferenz von Asilomar im Jahr 1975. Hier gibt es ein Forum, das $\mathrm{zu}$ »ethical and legal dimensions« tagt (Wright 1994: 146).

\subsubsection{Der Singer-Brief - »moral and ethical issues ${ }^{13}$ ?}

In dem Entwurf des Singer-Briefes vom 21. Juni 1973 schreibt Maxine Singer in Bezug auf die Genforschung Folgendes an die Teilnehmenden der Gordon Konferenz:

»The cause of the excitement and enthusiasm is two-fold. First there is our fascination with an evolving understanding of these amazing molecules and their biological action and second, there is the idea that such manipulations may lead to useful tools for the alleviation of human health problems. Nevertheless we are all aware that such experiments raise moral and ethical issues because of the potential hazards such molecules may engender « (Entwurf des Singer-Briefes (1973) zit. nach Krimsky 1982: 76).

In der Endversion des Briefes findet sich die ethische Rahmung nicht mehr, obwohl sich, wie Singer sagt, alle Wissenschaftler und Wissenschaftlerinnen über »moralische und ethische Themen « bewusst sind, die sich aus den Experimenten ergeben. An der entsprechenden Textstelle heißt es nun: »Certain such hybrid molecules may prove hazardous to laboratory workers and to the public« (Singer/Soll 1973). Thematisiert wird in der Endversion nicht mehr das Bewusstsein der Wissenschaftler und Wissenschaftlerinnen über »moralische und ethische Themen«, die aus ihren Experimenten resultieren können, sondern (Gesundheits-)Gefahren 
für Laborarbeiter und -arbeiterinnen und die Öffentlichkeit. Die Aussage, dass gentechnologische Forschung der »alleviation of human health problems« diene, findet sich in beiden Dokumenten, dem Entwurf sowie der Endversion des SingerBriefes (Entwurf des Singer-Briefes (1973) zit. nach Krimsky 1982: 76; Singer/Soll 1973). Diese Vorstellung, gentechnologische Forschung führe zu einer Verminderung von Gesundheitsproblemen des Menschen und damit zu einer Verbesserung des Gesundheitszustandes des Menschen, korrespondiert mit der Vorstellung des linearen Modells im wissenschaftspolitischen Bericht Vannevar Bushs. Mit Krimsky gehe ich davon aus, dass die im Entwurf des Singer-Briefes benannten »moral and ethical issues «, denen sich alle Wissenschaftler und Wissenschaftlerinnen bewusst sind, »moral and ethical issues « auf gesellschaftlicher Seite bezeichnen und nicht auf wissenschaftlicher (Krimsky 1982: 77). Krimskys Einschätzung wird bei näherer Betrachtung der Person Maxine Singer verständlich (vgl. Krimsky 1982: 73f). Singer war insofern für ethische Themen sensibilisiert, als ihr Mann Daniel Singer Vizepräsident des Institute of Society, Ethics and the Life Sciences des Hastings Center war. Das Hastings Center ist ein privates, unabhängiges Forschungsinstitut in den USA, das im Jahr 1969 gegründet wurde. Es ist eines der ersten US-amerikanischen Institute, die in der Entwicklung der akademischen Bioethik ausschlaggebend sind (Düwell/Steigleder 2003: 20). Es beschäftigt sich mit der Untersuchung der »social impact of biological revolution«, wie es in einer Veröffentlichung des Hastings Center aus dem Jahr 1973 heißt (The Hastings Center 1973/Herv. i.O.). Tina Stevens beschreibt das Center wie folgt: »It was, and remains, an effort to grapple with a disturbing array of ethical dilemmas generated by technologies that are seen as value-neutral in their creation even while they are problem-causing in their outcomes« (Stevens 2000: 47). In der Logik dieser Bioethik bestehen »ethische Dilemmata« dann, wenn technologische Entwicklungen in die Gesellschaft kommen. Diese Vorstellung entspricht m.E. einem räumlichen Denkmodell: Während im Labor Wissenschaft politisch und normativ neutral ist, kommt sie, sobald sie in den gesellschaftlichen Raum tritt, mit Werten in Kontakt und kann zu »Dilemmata« führen. In dieser Perspektive wird eine Grenze zwischen Wissenschaft und Gesellschaft gezogen. Ethische Themen scheinen für das Hastings Center nur außerhalb des Labors relevant zu werden, sobald Wissenschaft die wertneutrale Welt des Labors verlässt. Krimskys Einschätzung in Bezug auf die »moral and ethical issues « aus dem Entwurf des Singer-Briefes ist insofern schlüssig, als in der Endversion an der entsprechenden Textstelle von der Öffentlichkeit gesprochen wird. Insofern entspricht die ethische Rahmung im Entwurf des Singer-Briefes der Vorstellung der damaligen akademischen Bioethik des Hastings Center. In der Endversion des Singer-Briefes werden »ethical issues « überhaupt nicht mehr thematisiert, weder auf gesellschaftlicher Ebene noch auf der 
Ebene der Forschung. Dass »moralische und ethische Themen « von Wissenschaftlerinnen und Wissenschaftlern außerhalb einer auf Fakten basierenden Forschung gesehen werden und gleichzeitig kein Thema sein sollen, zeigt sich auch anhand Paul Bergs Vorstellung von Ethik.

\subsubsection{Paul Berg - »not a problem of science ethics « ${ }^{14}$}

Wie die Ethik-Rahmung im Vorfeld der Tagung von Asilomar marginalisiert wird, zeigt sich ein weiteres Mal anhand eines Statements von Paul Berg auf einer internationalen Tagung zu Gentechnologie im Oktober 1974 im schweizerischen Davos. Die Tagung mit dem Titel »Genetic Engineering. Its Applications and Limitations « wird von der schweizerischen Gesellschaft für Molekularbiologie und dem Gottlieb Duttweiler Institut organisiert. Das internationale Moratorium zu gentechnologischer Forschung, gefordert im April 1974, sowie die Planung der AsilomarKonferenz für das Jahr 1975 fällt in die Vorbereitungsphase dieser Tagung und beeinflusst sie: Die Organisatorinnen und Organisatoren kritisieren die Planung der Asilomar-Konferenz insofern, als keine Nicht-Wissenschaftler und -wissenschaftlerinnen vertreten sein sollen. Im Kontrast zu dem Vorhaben in Asilomar soll die Davoser Tagung die Möglichkeit zum interdisziplinären Austausch bieten und auch zur Kommunikation mit der Öffentlichkeit. Es werden sowohl Wissenschaftlerinnen und Wissenschaftler verschiedener Disziplinen als auch Medienvertreterinnen und -vertreter eingeladen (Wheeler 1974: 156; Lewin 1974). Wissenschaft wird explizit als Teil von Gesellschaft verstanden: »Science is a social issue « und somit Grund der Tagung, wie der US-amerikanische Politologe John Harvey Wheeler meint (Wheeler 1974: 158). Diskutiert werden nicht allein die Entwicklungen der Gentechnologie, sondern darüber hinaus Fragen der Steuerung (gen-)wissenschaftlicher Entwicklungen, die Möglichkeiten des Dialogs mit der Öffentlichkeit und der Bürgerinnen- und Bürgerbeteiligung (Weber 1974: 148; Wheeler 1974: 158; Jungk 1974: 145).

Berg trägt auf der Davoser Tagung zu seiner Arbeit mit rDNA-Technologie vor und äußert sich zu dem Moratorium. Im Tagungsband ${ }^{15}$ wird festgehalten:

»Berg made it quite clear that he is not at all interested in ethical scruples in connection with gene transplantation; the only purpose of his ${ }^{16}$ moratorium is to prevent the emergence of

14 Berg (1974) zit. nach Weber 1974: 149.

15 Sein Vortrag ist in dem Tagungsband nicht komplett publiziert, sondern nur partiell referiert oder kommentiert. 
new pathogenetic, possibly carcinogenic germs or germs that are particularly resistent to antibiotics, if the experiments are not carefully designed. This is not a problem of science ethics, but a problem of public hygiene« (Weber 1974: 149).

Ähnlich heißt es auch in einem Tagungsbericht aus der internationalen, populärwissenschaftlichen Fachzeitschrift New Scientist von 1974, in der Berg zitiert wird: die Forschung sei »simply a public health problem« und habe nichts mit Ethik zu tun (Lewin 1974).

Wie bereits im Singer-Brief, der ein Jahr zuvor publiziert wird, in welchem im Kontrast zu seinem Entwurf ebenfalls nicht mehr von »moralischen und ethischen Themen«, sondern von Gefahren für Laborarbeiter und -arbeiterinnen und für die Öffentlichkeit gesprochen wird, stellt Berg fest, dass es sich bei Gentransplantationen nicht um ein »ethisches Problem« handle, sondern um ein Problem der öffentlichen Gesundheit oder Hygiene. Als ein Beispiel dafür, wie dieses Problem gelöst werden kann, führt Berg die Quarantänemaßnahmen für die Apollo-Astronauten ${ }^{17}$ an (Weber 1974: 149). Noch deutlicher als im Singer-Brief zeigt sich hier m.E., dass es sich bei der rDNA-Technologie nicht um ein ethisches Problem handeln soll, sondern um ein Risiko-Problem, das mit Quarantänemaßnahmen, sprich Sicherheitsvorkehrungen gelöst werden kann.

Was Paul Berg unter Ethik versteht, zeigt sich anhand seiner Antwort auf eine Publikumsfrage, die in dem Tagungsartikel aus dem New Scientist erwähnt wird. Auf die Frage, was Berg tun würde, wenn er in sein Labor zurückkehre und die nun besser informierte Öffentlichkeit proklamiere, dass sie seine Forschung nicht will, sagt er: »I'd stop if there is a sound practical reason, but not if it were an ethical judgement« (Berg zit. nach Lewin 1974). Eine Äußerung der Öffentlichkeit $\mathrm{zu}$ seiner Forschung ist für Berg eine »ethische Beurteilung «. Diese werden wie die »moralischen und ethischen Themen« im Entwurf des Singer-Briefes auf gesellschaftlicher Seite, nicht auf wissenschaftlicher identifiziert. Dies bedeutet zudem, wie schon im Kontext des Singer-Briefes ersichtlich ist, dass Berg Wissenschaft als etwas nicht Gesellschaftliches betrachtet. Indem Berg die »ethische Beurteilung« in Kontrast zu einem »sound practical reason« - einem stichhaltigen, vernünftigen und technischen Grund setzt, wird deutlich, dass er eine ethische und damit gesellschaftliche Beurteilung als unvernünftig und unzureichend sieht. Berg wird auf der Tagung, wie weiter in dem Artikel ausgeführt wird, von Charles Weissmann, Insti-

16 »His« ist so zu erklären, dass das Moratorium eng mit der Person Paul Berg verknüpft wird. Er war einer der maßgeblichen Initiatoren des Moratoriums. Es wird in der Literatur auch als Berg-Moratorium bezeichnet.

17 Apollo war ein US-amerikanisches Raumfahrtprojekt von 1961-1972. 
tut für Molekularbiologie, Zürich, unterstützt. Weissmann meint, dass Grundlagenforschung damit zu tun hätte, Fakten zu ergründen. Er konkretisiert, dass »facts have no ethical connotation«, aber dass »ethics change with time« (ebd.). Die Auffassung von Ethik erscheint im Kontrast zu Fakten als etwas Fluides, sich temporär Änderndes. Das, was unter Fakten verstanden wird, scheint stabil und wertfrei. Fakten sind von Werten und Wissenschaft von Gesellschaft getrennt. Die Möglichkeit, gesellschaftliche Einflussnahme zu diskutieren, wird in dieser Argumentation begrenzt. Oder wie es in dem Artikel aus dem New Scientist heißt: »Asserting that ethics have nothing to do with facts is simply another way of claiming that scientists have an absolute right to continue with their research unhindered by public influence« (ebd.). Nicht nur gesellschaftliche Einflussnahme, auch die Diskussion über die Selbststeuerung wissenschaftlicher und technischer Entwicklungen ist in Bergs Argumentation ausgeschlossen.

Die Diskussion über Gentechnologie im Asilomar-Prozess ist somit bereits im Vorfeld der Konferenz geprägt von Risiken im Rahmen eines »public health problem«. Wie im Singer-Brief oder Bergs Tagungsbeitrag deutlich wird, wird von neuen möglichen Gefahren für Laborarbeiterinnen und -arbeiter durch die Arbeit mit genmanipulierten Viren gesprochen. Und auch mögliche Gefahren für die Öffentlichkeit durch Entweichung dieser Viren aus dem Labor werden benannt (vgl. Singer/Soll 1973; Weber 1974: 149). Die Wissenschaftshistorikerin und Politologin Susan Wright bringt es folgendermaßen auf den Punkt: »Thus the genetic engineering >problem< was reprojected almost exclusively in terms of producing suitable safety precautions to contain hazards. In other words, it was reduced and redefined in terms that make it suscepible to a technical >solution«" (Wright 1994: 158/ Herv. i.O.). Es geht um Sicherheitsprobleme, die durch Sicherheitsstandards gelöst werden können - besteht die Sicherheit am Arbeitsplatz, besteht kein Risiko für die Arbeiter und Arbeiterinnen und die Öffentlichkeit. Das explizite Benennen moralischer und ethischer Themen oder Probleme auf gesellschaftlicher Seite - denn nur hier existieren solche Probleme in der Logik des Entwurfs des Singer-Briefes und Bergs Tagungsbeitrag - hätte m.E. möglicherweise eine Debatte über die genwissenschaftliche Community hinaus ausgelöst, gerade vor dem Hintergrund der Debatte um Verantwortung der Wissenschaft gegenüber der Gesellschaft. Solch eine Debatte war nicht erwünscht. Das Vorbereitungskomitee der Asilomar-Konferenz wurde von der US-amerikanischen Nationalen Akademie der Wissenschaften dazu angehalten, die Debatte innerwissenschaftlich zu führen (ebd.: 137). Inwiefern dies notwendig schien, zeigt sich anhand eines Briefes des US-amerikanischen Biologen Bernard Davis an Paul Berg im Vorfeld der Asilomar-Konferenz. Der Biologe sah eine »Gefahr der Überreaktion in der Öffentlichkeit« (Evans 2002: 95/Übers. S.K.). Diese Überreaktion »could lead to renewal of fear of genetic engineering in 
man, and perhaps to fear of molecular genetics in general « (B. Davis zit. nach ebd.: 95). Eine mögliche öffentliche Debatte wird als etwas Emotionales (»fear«), als Überreaktion gesehen, etwas, das unkalkulierbar werden kann, das aber durch eine innerwissenschaftliche, auf Fakten basierenden, wertneutrale Debatte verhindert werden kann. Bereits im Vorfeld der Tagung sollte und wurde durch die Dominanz der technischen Risiko-Rahmung in der innerwissenschaftlichen Debatte eine öffentliche Debatte verhindert.

\subsubsection{Die Asilomar-Konferenz - »Ethical and legal dimensions “ ${ }^{18}$}

Nicht nur im Vorfeld, auch auf der Konferenz in Asilomar selbst wird eine ethische Rahmung marginalisiert. Jedoch gibt es einen organisierten Raum auf der Tagung, in dem normative Fragen explizit erwünscht zu sein scheinen, und zwar in dem Forum zu »ethical and legal dimensions« (Wright 1994: 146). Die Frage, warum überhaupt ein solches Forum organisiert wurde, lässt sich mit Wright damit beantworten, dass dies auf Anregung und Rat von Maxine und Daniel Singer geschah (ebd.). Daniel Singer, Vizepräsident des Institute of Society, Ethics and the Life Sciences des Hastings Center, setzt diese Anregung selbst um und organisiert das Forum (Krimsky 1982: 139). Dass das Forum organisatorisch getrennt von der als wissenschaftlich verstandenen Debatte stattfindet, entspricht bildlich gesehen der Vorstellung von Bioethik des Hastings Center. Ethische Themen und Diskussionen scheinen hier wie bereits im Entwurf des Singer-Briefes und in Bergs Tagungsbeitrag in Davos etwas zu sein, das räumlich getrennt zu behandeln ist. Obwohl das Forum nur am Rande der Konferenz zusammenkommt (Wright 1994: 146), wird in dem Wissenschaftsmagazin Science davon berichtet (Wade 1975: 935). Geschildert wird eine Stellungnahme des US-amerikanischen Juristen Roger Dworkin. Sein Vortrag erreicht höchste Aufmerksamkeit in dem Ethik-Forum (Krimsky 1982: 141f). Der Jurist der Universität von Indiana alarmiert die Wissenschaftler und Wissenschaftlerinnen, dass Richtlinien Forscher und Forscherinnen nicht davor schützen würden, in einem Schadensfall haftbar gemacht zu werden (ebd.: 141). Denn: »any appearance of self-serving will sacrifice the reservoir of respect that scientists have and will bring disaster on them « (R. Dworkin zit. nach Wade 1975: 935). Wie dieses Desaster aussehen könnte, wird von Dworkin mit den Worten konkretisiert, das Recht ${ }^{19}$ habe eine Tradition, dass sich Berufsgruppen selbst regulieren. Wenn die Gruppen allerdings diese Entscheidungsfreiheit missbrauchen, wie es die Berufsgruppe der Mediziner und Medizinerinnen getan habe, fänden sie

18 Wright 1994: 146.

19 Um welches Recht, bspw. das amerikanische oder internationale, es sich handelt, wird nicht gesagt. 
sich vor Gericht als »massakriert« wieder (Wade 1975: 935/Übers. S.K.). Dworkin meint m.E., das Ergebnis der Konferenz dürfe nicht eigennützig aussehen, damit die Entscheidungsfreiheit, die Freiheit der Forschung weiterhin legitimiert werden könne. Als negatives Beispiel nimmt er auf die Berufsgruppe der Mediziner und Medizinerinnen Bezug, die ihre Entscheidungsfreiheit missbraucht und darum vor Gericht gestanden hätten. Mit diesem Beispiel warnt Dworkin vor ähnlichen Konsequenzen für die Genwissenschaftler und -wissenschaftlerinnen: sofern diese ihre Entscheidungsfreiheit, die sie durch die Selbstregulierung haben, missbrauchen, können sie mit juristischen Sanktionen konfrontiert sein bzw. werden vor Gericht »massakriert«, wie Dworkin sagt. Durch den Gebrauch des Terminus »massakriert « werden sowohl mögliche Anklagen gegen Wissenschaftler und Wissenschaftlerinnen als auch die Prozesse gegen Mediziner und Medizinerinnen als übertrieben dargestellt. Um welchen Prozess es sich handelt, wird in dem Tagungsbericht der Science nicht konkretisiert. Ein Anhaltspunkt hierzu findet sich in der französischen Publikation »Les manipulations génétiques « - »Die genetischen Manipulationen « (Mendel 1980/Übers. S.K.). Berichtet wird hier nicht explizit von dem Beitrag Dworkins, sondern von einem Juristen. Dieser habe den Fall eines Augenarztes aus Oregon (USA) erwähnt, der einen Glaukom-Test an einem Patienten durchgeführt habe, obwohl dieser kein Symptom dieser Krankheit aufwies. Es gab einen Prozess wegen Fahrlässigkeit, den der Augenarzt verlor (ebd.: 44). In der anschließenden Diskussion auf der Tagung werden weitere Experimente, wie solche an Föten und Gefangenen, aufgeführt (ebd.: 45). Zu Beginn der 1970er Jahre sind insbesondere zwei Studien an Gefangenen publik geworden, die bereits erwähnten Studien in Stateville zu Malaria und zu Krebs am Ohio State Prison (Hornblum 1997: 1438f; Alving et al. 1440, 1948). Ob und inwiefern der Nürnberger Ärzteprozess in dem Ethik-Forum auf der Asilomar-Konferenz explizit benannt wurde oder nur implizit durch die Stateville-Studie, die beim Nürnberger Ärzteprozess thematisiert wurde, ist unklar. Wobei m.E. in der Assoziationskette von »Berufsgruppe der Mediziner « und »Gericht« unweigerlich der Nürnberger Ärzteprozess aufkommt. Durch die Warnung, »vor Gericht massakriert zu werden« wird zu vorsichtigem Handeln geraten. »Vor Gericht massakriert « erscheint als Folge eines Missbrauchs von Entscheidungsfreiheit übertrieben, die Mediziner und Medizinerinnen werden als Opfer der Justiz dargestellt. Deutlich wird, dass das Gericht als etwas gedeutet wird, das »massakriert«, also überreagiert und letztlich zur Gefahr für die wissenschaftliche Selbststeuerung werden kann. Die Betonung liegt bei Dworkin auf der Profession der Mediziner und Medizinerinnen und der missbrauchten Entscheidungsfreiheit, ob dies nun im Kontext der NS-Medizin steht oder nicht, nicht aber auf den Folgen. Er thematisiert nicht den Missbrauch von Menschen, von Betroffenen, nicht die Opfer von Wissenschaft und Medizin. Nicht 
die Taten werden von Dworkin im Ethik-Rahmen des Forums thematisiert, sondern die Entscheidungsfreiheit, die durch die Selbstregulierung ermöglicht wird. Die Entscheidungsfreiheit wird aber nicht grundlegend angezweifelt, nur ihr Missbrauch. So hat die Diskussion um die Selbstregulierung der Wissenschaft und um eine kollektive wissenschaftliche Verantwortung in dem Ethik-Forum keinen Raum. Das Problem wird auf rechtlicher Seite gesehen. Das Gericht kann zur Bedrohung werden, da ihm die Fähigkeit zugeschrieben wird, zu massakrieren. Was in dem Forum zu »ethical and legal dimensions« von Dworkin besprochen wird, mutet wie strategische Überlegungen zur Verhinderung der Einflussnahme auf wissenschaftliche Autonomie an oder, umgekehrt, zur Rettung der Forschungsfreiheit.

Ethische Rahmungen treten im Asilomar-Prozess nur am Rande in Erscheinung, sei es im Entwurf des Singer-Briefes, in Paul Bergs Tagungsbeitrag in Davos oder in dem Forum zu »ethical and legal dimensions « auf der Asilomar-Konferenz. Die Konflikte um die Verantwortung der Wissenschaft gegenüber der Gesellschaft bekommen keinen Raum auf der Konferenz. Dies wird schon dadurch verhindert, dass das Sprechen über »ethical dimensions« als etwas von der Wissenschaft Getrenntes organisiert wird. Ethische Themen werden im gesellschaftlichen Raum verortet, und Gesellschaft und Wissenschaft werden als zwei getrennte Bereiche konzipiert. Mit der Nicht-Thematisierung dieser Konflikte wird eine Diskussion um eine öffentliche Einflussnahme auf die Steuerung von Wissenschafts- und Technikentwicklungen verhindert. Die Bedeutung von Verantwortung war im Vorfeld der Konferenz umstritten. Auf der Asilomar-Konferenz setzt sich dann eine bestimmte Bedeutung durch, nämlich die der individuellen Verantwortung des Wissenschaftlers oder der Wissenschaftlerin. Diese wird als entscheidender Mechanismus innerhalb einer Strategie der Risikominimierung konzipiert. Der Möglichkeitsraum, wie über gentechnologische Forschung, die rDNA-Technologie gesprochen werden kann, hat sich verengt. Wurden insbesondere durch die new critical science movement Themen wie Ökologie, Politik oder Antimilitarismus in die Diskussion um Gentechnologie gebracht und damit einhergehend die Selbststeuerung wissenschaftlicher und technologischer Entwicklungen problematisiert, scheint es auf der Asilomar-Konferenz nur noch möglich, über Risiken und Sicherheitsvorkehrungen zu sprechen. Durch das Ausklammern bestimmter Themen und Diskussionen, etwa Ökologie oder Politik, durch die Auswahl der Teilnehmenden und das Abschlussdokument, das sich als Konsens darstellt, bleibt die Thematik der wissenschaftlichen Selbststeuerung unangetastet. Neutralität und Einigkeit der (gen)wissenschaftlichen Community wird durch das konsensuale Ergebnis der Konferenz symbolisiert und durch die Betonung von »Fakten« (Berg) hergestellt. Dominant zeigt sich im Asilomar-Prozess die Sprache des technischen Risikos. Sie zeigt sich sowohl als Abwehr einer möglichen ethischen, öffentlichen Diskussion 
als auch als Abwehr staatlicher Einflussnahme auf die wissenschaftliche Selbststeuerung im Bereich gentechnologischer Forschung und Entwicklung.

Während sich im US-amerikanisch dominierten, internationalen AsilomarProzess eine Risiko-Rahmung durchsetzt, zeigt sich in Frankreich als Folge von Asilomar eine explizit ethische Rahmung auf forschungspolitischer Ebene in Form der Ethikkommission bei der DGRST. Wie lässt sich die Einrichtung der Ethikkommission vor dem Hintergrund des Asilomar-Prozesses verstehen?

\section{ETHIKKOMMISSION UND RISIKOABSCHÄTZUNG: Die AusWirkungen des Asilomar-Prozesses in FRANKREICH}

Die Ethikkommission bei der DGRST, die im November 1974 eingerichtet wird, verfügt, wie zu Beginn des Kapitels erwähnt, über eine technische Kommission bzw. eine Commission Nationale de Classement zur Evaluierung von Forschungsprojekten aus dem Bereich der Gentechnologie. Die Ethikkommission soll die Ergebnisse der Asilomar-Konferenz sichern. Wie einer der französischen Hauptakteure im Gentechnologiediskurs, Philippe Kourilsky, meint, ist es wichtig, dass die Mitglieder der Kommission mit den Ergebnissen der Konferenz, den Empfehlungen zum Umgang mit gentechnologischen Experimenten übereinstimmen. Darüber hinaus hatte die Ethikkommission vor allem symbolischen Charakter, denn Kourilsky meint weiter:

»who can decide on ethics, what does it mean? In practical terms, it had importance in that the people on this committee are rather famous, at least locally [...]. The major point was to show that the heads of the scientific community here were in agreement with what the technical committee was going to do« (Interview mit Ph. Kourilsky von H. Gottweis zit. nach Gottweis 1998: 140f).

Die Ethikkommission scheint der Commission Nationale de Classement übergeordnet, ein »court of appeals« (ebd.: 142). Sie soll einberufen werden, sofern es bei der Evaluierung der Forschungsprojekte ein »ethisches Problem« gibt (ebd.). Ziel der Ethikkommission ist, die Einhaltung der Ergebnisse der internationalen Asilomar-Konferenz, die Risikoabschätzung zu sichern. Insofern kann die Ethikkommission als eine Art übergeordnete Sicherheitsvorkehrung für die Risikoabschätzung verstanden werden - Vorkehrung, da sie nie einberufen wird. Die Risikoabschätzung innerhalb der übergeordneten ethischen Sicherheitsvorkehrung beinhaltet die Zuweisung von Verantwortung an den individuellen Forscher oder die 
individuelle Forscherin. Ethik scheint aber etwas zu sein, das nicht festgelegt oder entschieden werden kann, dessen Bedeutung unklar ist. Sicher an dieser ethischen Sicherheitsvorkehrung ist also nur, dass sie die Risikoabschätzung inklusive individualisierter Verantwortung ermöglicht, aber keine grundsätzliche Diskussion über Inhalt und Bedeutung von Ethik voraussetzt. Wie es in dem Zitat heißt, war ein Ziel, dass die »heads of the scientific community«, die Mitglieder der Ethikkommission, mit den Tätigkeiten der technischen Kommission übereinstimmen (Interview mit Ph. Kourilsky von H. Gottweis zit. nach ebd.: 140f). Dies bedeutet m.E. die Vermittlung eines harmonischen Bildes zwischen Ethikkommission und technischer Kommission, also zwischen Ethik und Technik, Werten und Fakten. Durch dieses Bild zeigt sich die wissenschaftliche Community einig. Die Notwendigkeit der Vermittlung von Einigkeit resultierte im US-amerikanisch geprägten Diskurs um Gentechnologie aus den Kontroversen um die gesellschaftliche Verantwortung der Wissenschaft. Inwiefern ist diese Vermittlung im französischen Diskurs um Gentechnologie notwendig?

\subsection{Technokratiekritik und Wissenschaftsskepsis in der französischen Öffentlichkeit}

Wie in den USA entwickelt sich auch in Frankreich in den 1970er Jahren eine zunehmend skeptische Öffentlichkeit, nicht allein gegenüber medizinischer Forschung und Autonomie, sondern ebenso gegenüber wissenschaftlicher Forschung und Autonomie allgemein.

Frankreich ist zu Beginn der 1970er Jahre geprägt von einer zunehmend technologie- und wissenschaftsskeptischen Öffentlichkeit. Nach dem Zweiten Weltkrieg ist eine Kritik an der Vorstellung einer linearen Entwicklung von Wissenschaft, Technologie, Wachstum und Wohlstand im öffentlichen Raum nicht vorzufinden. Auch im Kontext des Atombombenabwurfs auf Hiroshima wird bspw. lediglich die falsche Anwendung von Wissenschaft thematisiert, nicht aber die Wissenschaft selbst (Bonneuil 2004: 20f; Petitjean 1998; Topçu 2007: 3). In den Trentes Glorieuses, ${ }^{20}$ insbesondere seit dem »Colloque de Caen « in den 1950er Jahren, wird wissenschaftlicher und technologischer Fortschritt als Kernelement der nationalen Entwicklungsfähigkeit gesehen, die sich in ökonomischem Wachstum oder in medizinischen Entwicklungen zeigt (Gottweis 1998: 52). Erst in den 1970er Jahren und konkret mit den Post-68er-Bewegungen wird Kritik am Ver-

20 Eine Bezeichnung für die 30 Jahre ökonomischen Wachstums in Frankreich (19451975). 
hältnis zwischen Wissenschaft, Politik und Öffentlichkeit laut. Mit diesen Bewegungen kommt nicht allein eine Technologieskepsis auf, sondern eine Kritik an staatlicher Technokratie (Petitjean 1998; vgl. Bonneuil 2004: 16f). Eine der wesentlichen technologiekritischen Bewegungen ist vor dem Hintergrund einer französischen Energiepolitik, die ihren Schwerpunkt auf Kernenergie setzt, zu Beginn der 1970er Jahre die Anti-Atombewegung. Ähnlich wie in den USA beeinflusst sie maßgeblich den Diskurs um Gentechnologie.

\subsection{Die Konflikte um Atomenergie}

Während Regierung und forschungspolitische Instanzen in Frankreich bereits seit den 1950er Jahren der Genforschung in Hinblick auf die internationale Wettbewerbsfähigkeit große Bedeutung zuschreiben (Gottweis 1998: 56ff), dominiert im öffentlichen Diskurs der 1970er Jahre die Atompolitik. Wissenschaft und Technik und insbesondere Atomtechnologie, von Charles de Gaulle im Nachkriegsfrankreich gefördert, standen in der Logik der Mobilisierung für den Wiederaufbau der französischen Nation und einer positiven nationalen Identität (Bonneuil 2004: 20; Hecht 1994: 659f; 2009). Im Jahr 1973 verkündet der damalige Premierminister Pierre Messmer (1972-1974) während der Präsidentschaft George Pompidous (1969-1974) und vor dem Hintergrund der Energiekrise ${ }^{21}$ seinen Plan zur Errichtung von rund 200 Atomkraftwerken $^{22}$ (AKW) bis zum Jahr 2000 (von Oppeln 1989: 28f; Topçu 2007: 5). Ein Jahr später reagiert Frankreich auf die Vervierfachung des Erdölpreises im Kontext der Energiekrise mit der Beschleunigung des Atomenergieprogrammes. Der so genannte Messmer-Plan entsteht. In diesem Kontext wirbt die Électricité de France, die staatliche französische Elektrizitätsgesellschaft, mit dem Motto: »tout électrique - tout nucléaire!« - »alles elektrisch - alles nuklear!« (Hegedus/Touraine 1982: 37ff/Übers. S.K.). Vor dem Hintergrund des Vietnamkrieges und beeinflusst von der 1968er- und der Umweltschutzbewegung hatte die Anti-Atombewegung in dieser Zeit ihren ersten Aufschwung.

21 Ausgelöst wird die Energie- bzw. Ölkrise durch den so genannten Jom-Kippur-Krieg, dem vierten arabisch-israelischen Krieg im Rahmen des Nahostkonfliktes.

22 Die Anzahl der Kernkraftwerke, die bis zum Jahr 2000 gebaut werden sollten, divergiert in der Literatur: Sabine von Oppeln spricht von 200, Sezin Topçu von 170 AKW (von Oppeln 1989: 28f; Topçu 2007: 5). 


\subsubsection{Die Anti-Atombewegung}

Die Anti-Atombewegung ist von Beginn an in die Umweltschutzbewegung ${ }^{23}$ integriert, deren organisatorische Strukturen sich in Frankreich Ende der 1960er Jahre festigen. Die Umweltschutzbewegung gewinnt im Zuge des Mai '68 mehr Anhängerinnen und Anhänger und politisiert sich: Vom Naturschutz zur Verteidigung der Natur. Die Verteidigung der Natur war mit einer Kritik an der kapitalistischen Konsumgesellschaft verknüpft (von Oppeln 1989: 27). Die Proteste gegen Atomenergie beginnen bereits im Jahr 1970 mit Aktionen gegen das Kernkraftwerk im elsässischen Fessenheim und das Kernkraftwerk Bugey in der Nähe von Lyon (ebd.). Im Jahr 1973 wird von Atomkritikern und -kritikerinnen die Forderung eines fünfjährigen Moratoriums für Atomanlagen gestellt und die Einstellung der militärischen Atomwaffentests im Pazifik gefordert (Topçu 2007: 4; Hegedus/Touraine 1982: 44). Zwischen 1966 und 1974 führt Frankreich über 40 Atomwaffentests im Pazifik (Französisch Polynesien) durch. Kritische Veröffentlichungen der Atomkraftgegner und -gegnerinnen über die Risiken des Kernenergieprogramms schaffen es allerdings zuerst nicht, die Öffentlichkeit zu mobilisieren, so heißt es in dem Buch »Die anti-nukleare Prophetie«, herausgegeben u.a. von dem französischen Sozialwissenschaftler Alain Touraine (Hegedus/Touraine 1982: 43f). Erst die Veröffentlichung des Standortplanes der AKW löst eine breite Bewegung aus: Erste BauplatzBesetzungen finden statt, die eine mobilisierende Wirkung in weiteren Regionen haben (ebd.: 44). In Folge dieser Proteste verschiebt die Électricité de France ihre Projekte von einem Standort zum anderen (ebd.: 46). Die wohl berühmtesten Aktionen finden 1976-1977 in Malville gegen den schnellen Brüter, den Superphénix, statt. Ihnen wird mit einem gewaltsamen Vorgehen der Polizei begegnet. Malville wird zum Symbol des Anti-AKW-Kampfes auch über die Grenzen Frankreichs hinaus. Zudem wird durch den Streik der Arbeiter und Arbeiterinnen im Jahr 1976 in der Wiederaufbereitungsanlage im nordfranzösischen La Hague die Öffentlichkeit über die Folgen der Arbeitsbedingungen in der Atomindustrie informiert. Trotz der Mobilisierungskraft von Malville fehlt es den unterschiedlichen Aktivistinnen

$23 \mathrm{Zu}$ den politisch einflussreichsten Organisationen gehört die Fédération Nationale des Sociétés de Protection de la Nature, ein Zusammenschluss mehrerer Umweltschutzverbände, der sich als konservativ und apolitisch versteht. Sie ist Hauptansprechpartnerin für die Regierung, da sie, wie von Oppeln meint, einen weniger politischen, sondern eher technokratischen Umgang mit Fragen des Umweltschutzes hat. Zu dem politisch radikalen Teil der Bewegung gehören die Amis de la Terre (von Oppeln 1989: 28). Radikal sind sie insofern, als sie »eine Verbindung zwischen antikapitalistischer, basisdemokratischer Gesellschaftskonzeption und Ökologie suchten« (Doll/Schmidt 2006: 340). 
und Aktivisten an einer gemeinsamen Zielsetzung und an Organisierung. In Folge dessen zersplittert die Bewegung (ebd.: 50; vgl. Doll/Schmidt 2006: 339f).

\subsubsection{Die (Anti-)Atompolitik}

Bereits zu Beginn der 1970er Jahre wird seitens der Regierung versucht, den aufkommenden Protesten entgegenzuwirken: Im Jahr 1970 werden die »100 Maßnahmen für den Umweltschutz« von Georges Pompidou - Nachfolger von Präsident Charles de Gaulle - propagiert und im Jahr 1971 wird ein Umweltministerium eingerichtet. Viele Aktivisten und Aktivistinnen, die Écologistes, sehen die Bewegung und damit den Kampf gegen Kernenergie als Fortsetzung der Mairevolten von 1968 (von Oppeln 1989: 27). Zentral ist in den Revolten eine grundsätzliche Kritik an der Gesellschaft und an deren wirtschaftlicher und politischer Organisation. Sie richten sich gegen den allmächtigen Staat, den der damalige konservative Staatspräsident Charles de Gaulle symbolisiert (1959-1969), sowie gegen hierarchische und autoritäre Strukturen in Schule, Betrieb und Universität (vgl. Doll 2006: 607). So ist auch die Ökologiebewegung stark auf die Politik und die Thematisierung von Strukturproblemen und Politikstrategien ausgerichtet (Rucht 1994: 247). Entsprechend geht es im Anti-Atomkampf nicht nur gegen Atomenergie, sondern gegen Atompolitik und somit um eine Fortsetzung der 1968erBewegung gegen die gaullistische Herrschaft (Hegedus/Touraine 1982: 35).

Die Kritik an der Technokratie entwickelt sich in einem intellektuellen Milieu. Der Anti-Atomdiskurs ist zu Beginn der 1970er Jahre von einer »intellektuellen Elite« geprägt, die sich aus Wissenschaftlern und Wissenschaftlerinnen verschiedener Disziplinen, Journalisten und Journalistinnen, Lehrern und Lehrerinnen sowie aus Rechtsanwälten und Rechtsanwältinnen zusammensetzt (Topçu 2007: 4/Übers. S.K.; Rucht 1994: 242). Aus dieser Elite geht eine Reihe an Publikationen hervor. Hierzu zählen u.a. die Zeitschriften Survivre, Impasciences und Politique Hebdo $^{24}$ (Topçu 2007: 3f, 8; Quet 2009). Zudem gibt es die Reihe Science Ouverte - Offene Wissenschaft, die vom Verlag Seuil unter der Federführung des Physikers Jean-Marc Lévy-Leblond publiziert wird. Hierzu zählt u.a. die Textsammlung »(Auto)critique de la science « - »(Selbst)Kritik der Wissenschaft«, die Mitte der 1970er Jahre veröffentlicht wird (Jaubert/Lévy-Leblond 1975/Übers. S.K.). Zudem entsteht der atompolitikkritische Zusammenschluss linker Wissenschaftlerinnen und Wissenschaftler, der Groupement des Scientifiques pour l'Information sur

24 Die Wochenzeitschrift Politique Hebdo stand den gauchistes, der linksradikalen Bewegung in Frankreich nahe. Sie wird zwischen 1970 und 1978 publiziert. Impascience existiert von 1975-1977. Es gibt insgesamt sieben Ausgaben der Zeitschrift. Survivre existiert von 1970-1975. 
l'Énergie Nucléaire - Zusammenschluss von Wissenschaftlern und Wissenschaftlerinnen für die Information zur Atomenergie (Topçu 2007: 4; Bonneuil 2004: 21; Hegedus/Touraine 1982: 42). Zwar bezeichnet sich der Zusammenschluss nicht als »groupe«, es handelt sich aber um eine der Informationsgruppen, wie sie in Anlehnung an die von Michel Foucault initiierte Gefängnisinformationsgruppe entstanden sind (Topçu 2007: 9). Diese neue Generation an Wissenschaftlerinnen und Wissenschaftlern

»macht es sich zur Aufgabe die Wissenschaft selbst zu hinterfragen und zu politisieren und verweigert ihr den Anspruch auf irgendeine überlegene und neutrale Sichtweise auf die Welt. Sie stellt die bestehenden Herrschaftsstrukturen [dominations existantes, S.K.] innerhalb der wissenschaftlichen Gemeinschaft in Frage - Hierarchien, Arbeitsteilung, Stellung der Frauen etc. - stellt die Funktion in Frage, die die Wissenschaft während des Krieges spielt - wir sind mitten im Vietnam-Krieg ${ }^{25}$ - und in der Produktion und Legitimation einer $>$ kapitalistischen Gesellschaftsordnung« (Bonneuil 2004: 21/Herv. i.O., Übers. S.K.; vgl. Topçu 2007: 3; Quet 2009: 147; Chavot/Masseran 2003c: 78).

Es wird eine neue Wissenschaft proklamiert, die eine »Wissenschaft des Volkes« und nicht eine »für das Volk sein« sollte: »La Nouvelle Science peut se définir comme la science du Peuple et non pas la Science pour le Peuple - »Die Neue Wissenschaft kann als Wissenschaft des Volkes definiert werden, nicht als Wissenschaft für das Volk« (Survivre (1971), n 10: 19 zit. nach Quet 2009: 177/Übers. S.K.). Diese Wissenschaft des Volkes stellt die Wissenschaftsgläubigkeit in Frage, greift die Macht wissenschaftlicher Experten und Expertinnen an, ihre positivistische Ideologie, kritisiert die Ausbeutung am Arbeitsplatz und schlägt vor, die Wissenschaft in die Hände des Volkes zu legen (Quet 2009: 148; 2014: 323f). Dieser Vorschlag stellt die Selbststeuerung von Wissenschafts- und Technikentwicklungen der Nachkriegszeit in Frage und damit auch die Vorstellung einer linearen Entwicklung von Wissenschaft, Fortschritt und Wohlstand (vgl. Bonneuil 2004: 20f). Ein Schritt in Richtung dieser neuen Wissenschaft ist die Forderung an die Wissenschaftler und Wissenschaftlerinnen, sich für ihre Gesellschaft $\mathrm{zu}$ interessieren: »gegen den Obskurantismus der wissenschaftlichen Milieus zu kämpfen, Wissenschaftlern zu helfen, sich für ihre eigene Gesellschaft zu interessieren, sie [die Gesellschaft, S.K.] zu verstehen und sie zu ändern« (Survivre (1970), n 6: 11 zit. nach Topçu 2007: 4/Übers. S.K.). Ein wesentlicher Punkt dabei ist, gegen den

25 Nicht allein der Vietnamkrieg initiierte in Frankreich eine antimilitaristische Kritik, sondern zuvor bereits der Algerienkrieg (1954-1962), der den Mai'68 beeinflusste (vgl. Quet 2014: 631). 
Obskurantismus vorzugehen. In Frankreich gewinnt der Begriff des Obskurantismus im Zeitalter der Aufklärung an Bedeutung. Er bezeichnet Aufklärungs- und Fortschrittsfeindlichkeit in dem Sinn, dass Menschen unwissend bleiben sollen (vgl. Petitjean 1998). Obskurantismus wird nun den Wissenschaftlern und Wissenschaftlerinnen selbst vorgeworfen, einer Wissenschaft, die die Popularisierung von Wissen verhindere. Wie diese Popularisierung umgesetzt werden soll, wird innerhalb des wissenschaftskritischen Diskurses heterogen beantwortet, so Mathieu Quet in seiner Dissertation über die Beziehungen zwischen Wissenschaft, Technologie und Partizipation von 1968-1983 in Frankreich (Quet 2009). Das Konzept der Science du Peuple bleibt vage. Es wird von Aneignung und Wiederaneignung von Wissen gesprochen und somit implizit von Partizipation. Der Begriff Partizipation wird explizit kaum benannt, da er durch seine "gaullistischen Verwendungen « negativ konnotiert ist (ebd.: 140f/Übers. S.K.; 2014: 634): Ein wesentliches Konzept Charles de Gaulles Politik des so genannten dritten Weges - zwischen Kommunismus und Liberalismus - war die Partizipation der Arbeiter und Arbeiterinnen, und zwar durch Gewinnbeteiligung und Mitbestimmung in den Betrieben. Hierdurch wollte er u.a. dem Arbeiterinnen- und Arbeiterkampf entgegentreten (Godfrain 2000). Bei Demonstrationen im Mai '68, die wesentlich die gaullistische Regierung angriffen, lautete bspw. eine Parole »Participation, the new opium of the people« (Quet 2014: 634/Übers. Quet). Partizipation, einer der Schlüsselbegriffe im US-amerikanischen Diskurs, ist somit negativ konnotiert, da er als Regierungskonzept dazu dient, politische Konflikte zu verhindern. Über diese intellektuelle wissenschafts- und technokratiekritische Elite hinaus prägt zudem eine der großen französischen Gewerkschaften den Anti-Atomdiskurs, die sozialistische Conféderation francaise démocratique du travail (CFDT). Das gewerkschaftliche Engagement im Anti-Atomkampf sei einzigartig im Vergleich zu anderen Ländern, so Zsuzsa Hegedus und ihr Kollege Alain Touraine (1982: 43). Die CFDT lehnt die Kernenergie nicht ab, wehrt sich aber gegen die sozialen, wirtschaftlichen und technischen Folgen dieser Entwicklung des »tout nucléaire«. Von der Gewerkschaft werden besonders die Gefahren für die Arbeiter und Arbeiterinnen in Kernkraftwerken hervorgehoben, so Touraine und Hegedus. Zudem lehnt sie die Entscheidungsverfahren in Energie-Fragen ab (ebd.: 43). Diese Ablehnung ist darauf zurückzuführen, dass die CFDT in Anlehnung an den Mai '68 ihrem Programm das Prinzip der Selbstverwaltung für Unternehmen zugrunde legt (CFDT 2013; vgl. Doll 2006: 609). Somit wird m.E. die hierarchische Form der Entscheidungsverfahren innerhalb der Atomkraftwerke selbst abgelehnt. Da die CFDT Stellung zum Atomstromprogramm der Regierung bezieht, wird auch Kritik an staatlichen Entscheidungen in Energie-Fragen geübt. 
Thematisiert werden in der Atompolitikkontroverse der 1970er Jahre Technokratie, staatliche Entscheidungen wie auch hierarchische Strukturen und Entscheidungsverfahren innerhalb der Atomindustrie und Sicherheitsfragen am Arbeitsplatz. Die Rahmung dieser Themen wird im Folgenden untersucht.

\subsubsection{Vom technisch-wissenschaftlichen und vom politisierten Risikobegriff im Atomkonflikt}

Die Argumentation der Atomkraftgegner und -gegnerinnen, insbesondere der Écologistes, richtet sich »gegen eine technizistische, zentralistische und autoritäre Konzeption von Gesellschaft«, so Hegedus und Touraine (1982: 43). Probleme und Gefahren der Atomindustrie und des damit einhergehenden Wachstumstypus werden als soziale und politische Probleme angesehen (ebd.: 44). Die atompolitikkritische Zeitschrift Impascience nimmt dementsprechend 1975 eine Umkehrung des Mottos der Électricité de France vor: »tout électrique - tout nucléaire « heißt: »tout nucléaire - tout politique« (N.N. 1975d). Die zentralen Themen der AntiAtombewegung sind nach Touraine und Hegedus: »Ablehnung der als unvertretbar betrachteten Gefahren und Risiken, Anfechtung der Zuverlässigkeit der Nukleartechnologie und gar der Rationalität einer jeden Entwicklung, die auf die Kernenergie setzt « (Hegedus/Touraine 1982: 40f). Innerhalb der Anti-Atombewegung wird bezweifelt, so Hegedus und Touraine, dass die Atomindustrie eine neutrale und fortschrittliche Lösung wäre. Es wird aufgezeigt, dass mit ihr ein spezifischer Gesellschaftstypus zusammenhängt, der »immer zentralisierter und überwachter wird « und dessen extremer Ausdruck die »)Atomgesellschaft«« sei (ebd.: 43f/Herv. i.O.). Nicht allein die Atomindustrie wird als nicht neutral angesehen, sondern die Wissenschaft selbst (Bonneuil 2004: 21). Beide werden als nicht fortschrittlich verstanden, wodurch das Modell der linearen Entwicklung von Wissenschaft, Fortschritt und Wohlstand angegriffen wird.

Im Februar 1975 richten sich 400 Wissenschaftlerinnen und Wissenschaftler mit einem Appell, der nach einem Jahr 4000 Unterschriften zählt, an die Öffentlichkeit (Hegedus/Touraine 1982: 42). Mit dem »Appel des scientifiques à propos du programme nucléaire français « - »Appell der Wissenschaftler in puncto des französischen Nuklearprogramms« (N.N. 1975a/Übers. S.K.) machen sie auf die »Unüberlegtheit« und die »schwerwiegenden Konsequenzen« der Entscheidungen der Messmer-Regierung aufmerksam und fordern eine »wahre Debatte« (N.N. 1975a/Übers. S.K.; Hegedus/Touraine 1982: 42). Es heißt: »il s'agit d'une décision irréfléchie dont les conséquences risquent d'être grave - »es handelt sich um eine unüberlegte Entscheidung, aus der schwerwiegende Konsequenzen folgen können « (N.N. 1975a/Übers. S.K.). Die aktuelle Politik berücksichtige nicht die Interessen der Bevölkerung und die der zukünftigen Generationen (ebd.). Die Risiken seien 
vielfältig, so heißt es weiter in dem Appell, sie umfassten die Sicherheitsrisiken der AKW, die Risiken der Umweltverschmutzung oder auch die Risiken beim Transport radioaktiven Materials (ebd.). Touraine und Hegedus interpretieren den Appell wie folgt: Er »übt im Namen eben der Wissenschaft und Rationalität, die dieses Kernenergieprogramm für sich reklamiert, Kritik an ihm « (Hegedus/Touraine 1982: 42). Diese Kritik äußert sich in dem Appell folgendermaßen: »Systematisch werden Risiken klein geredet, mögliche Konsequenzen verschwiegen, beruhigt. Die Abweichungen zwischen den Studien, die Unsicherheiten in den öffentlichen Berichten zeigen jedoch gut, dass Risiken existieren« (N.N. 1975a/Übers. S.K.). Die »unüberlegten Konsequenzen« in dem Appell der Wissenschaftlerinnen und Wissenschaftler beziehen sich auf die Risiken des Kernenergieprogramms und die Gefahren für die »générations futurs « - die »zukünftigen Generationen « (ebd./ Übers. S.K.). Mit der Aufklärung der Öffentlichkeit ist die Aufklärung über die Risiken gemeint. Touraine und Hegedus sagen zudem, die Wissenschaftler und Wissenschaftlerinnen bringen $»$ in den Anti-AKW-Protest ihr Wissen als notwendige Kompetenz zur Bekämpfung des Kernenergieprogramms ein « (Hegedus/ Touraine 1982: 42). Dies erinnert an das Konzept Wissen in den Dienst der Menschen zu stellen, das zeitgleich im medizinkritischen Diskurs und im Diskurs um den Schwangerschaftsabbruch aufkommt. Das Konzept wurde von der GIS formuliert, die in Folge des Mai '68 entsteht. Mit eben diesem Konzept bleibt die wissenschaftlich-medizinische Kompetenz im medizinkritischen Diskurs und im Diskurs um den Schwangerschaftsabbruch zu Beginn der 1970er unangetastet. Wie Gaudillière meint, werden die Aktivitäten der Gruppe durch ein Wissen gerechtfertigt, das sich die Mitglieder an der medizinischen Fakultät angeeignet haben. Der Inhalt dieses Wissens wird aber kaum kritisiert, sondern als Kampfmittel eingesetzt (Gaudillière 2006a: 245f). Ähnliches zeichnet sich im Anti-Atomdiskurs ab. Auch von den Atomkritikerinnen und -kritikern wird m.E. Wissen als politisches Instrument eingesetzt, das die politische Steuerung von Wissenschafts- und Technikentwicklungen angreift. Mit Wissen oder Wissenschaft im Anti-Atomdiskurs ist m.E. ein politisiertes Risiko-Wissen gemeint ist. Zu einem ähnlichen Schluss kommt der französische Soziologe Michel Wieviorka in seiner Analyse des Anti-Atomkampfes in Frankreich (Wieviorka 1982). Er sagt, dass bei dem Teil der AntiAtombewegung mit wissenschaftlichem Hintergrund eine »rationale Version der Angst « vorzufinden sei. Darunter versteht Wieviorka die Möglichkeit: »eine statistische Berechnung einer anderen gegenüberzustellen, der mit geringen Wahrscheinlichkeiten operierenden $>$ Sicherheitsphilosophie< der Betreiber die Unermeßlichkeit der Risikodimension hier und heute oder die Anzahl potentieller Opfer entgegenzuhalten « (ebd.: 57f/Herv. i.O.). Dies bedeutet m.E., dass von den Atomkritikerinnen und -kritikern, besonders von jenen mit einem wissenschaftlichen 
Hintergrund, die Risiko-Argumentation im Atomkonflikt verwendet wurde, aber mit einem politisierten technisch-wissenschaftlichen Risikobegriff. Technischwissenschaftlich meint, dass das Risiko auf einer wissenschaftlichen Rationalität basiert, auf Wahrscheinlichkeitskalkulationen, die als wahr und vernünftig gelten. Diese Kalkulationen haben bisher die Atompolitik legitimiert. Indem nun die wissenschaftlichen Atomkritikerinnen und -kritiker ebenfalls Risiken kalkulieren, stellen sie eine Gegen-Wahrheit auf. Sie greifen hierbei auf das vorgängige Wissen, die Risiko- oder Wahrscheinlichkeitskalkulation zurück, so wird ihre Kritik überhaupt als Kritik wahrnehmbar (Bröckling/Krasmann 2010: 30). Das Referenzwissen bleibt unkritisiert, die Interpretation und Bedeutung, die ihm von den Kritikerinnen und Kritikern zugeschrieben wird, verändern sich: das Wissen wird politisch gegen die Atompolitik eingesetzt - in diesem Sinn handelt es sich um einen politisierten technisch-wissenschaftlichen Risikobegriff.

Anhand der Kritik an Atomenergie wird deutlich, dass sich hier keine expliziten ethischen Rahmungen abzeichnen, sondern vor allem eine politisierte RisikoRahmung. Staatspolitisch wird nicht etwa mit der Einrichtung einer Ethikinstitution reagiert, sondern mit »100 Maßnahmen für den Umweltschutz« (1970) von Präsident Georges Pompidou und einem Umweltministerium (1971). Da letzteres über wenige finanzielle Mittel und Kompetenzen verfügt, wird es in der Öffentlichkeit als »Alibi-Ministerium« kritisiert (Schmidt 2006a: 334f). Im Anti-Atomdiskurs in Frankreich steht weniger ein Konzept der Verantwortung der Wissenschaft gegenüber der Gesellschaft im Vordergrund, wie im wissenschaftskritischen Diskurs in den USA, sondern eine gesellschaftspolitische Perspektive. Sie beinhaltet eine Kritik an Technokratie und an der Art und Weise, wie Entscheidungen über die Entwicklung der Forschung getroffen werden. Außerdem entsteht die Forderung nach Wissensverbreitung und -aneignung und implizit nach Partizipation. Sie wendet sich gegen die Neutralität der Wissenschaft und thematisiert, dass eben diese in eine bestimmte Vorstellung von Gesellschaft eingebettet ist. Wie beeinflusst der Anti-Atom- den Gentechnologiediskurs?

\subsection{Die Kontroversen um Gentechnologie im Kontext des Asilomar-Prozesses}

Sozialwissenschaftliche Untersuchungen, insbesondere auch in den französischen STS, berücksichtigen bisher die Konflikte um Gentechnologie Mitte der 1970er Jahre in Frankreich wenig. Ausnahmen sind ein Aufsatz des französischen Wissenschaftshistorikers Christophe Bonneuil über das öffentliche Engagement von Forscherinnen und Forschern in den Kontroversen um Genetisch Manipulierte Orga- 
nismen (GMO) (Bonneuil 2006), sowie die Publikationen »Governing Molecules« (1998) und »Gene, Hochtechnologie-Narrative und Politik in Frankreich« (1997) von Herbert Gottweis. Das Hauptaugenmerk sozialwissenschaftlicher Untersuchungen liegt meist auf den Atompolitikkonflikten und dem sich verändernden Verhältnis von Wissenschaft und Gesellschaft in Frankreich. Erst mit der Zunahme der Konflikte um Gentechnologie in den 1990er Jahren, hauptsächlich um die GMO im Agrarbereich, gibt es zu der Thematik mehr sozialwissenschaftliche Untersuchungen. Sie konzentrieren sich allerdings auf die Konflikte in den 1990er Jahren (vgl. bspw. Bonneuil et al. 2008; Bonneuil 2004; Joly et al. 2000). Im Folgenden greife ich vor allem auf die Publikation »Les manipulations génétiques «»Die genetischen Manipulationen« aus dem Jahr 1980 zurück (Mendel 1980/ Übers. S.K.). Es handelt sich hierbei um ein Buch, das von einem Autorinnen- und Autorenkollektiv von Gentechnologiekritikerinnen und -kritikern verfasst und mit dem Ziel der Information der Öffentlichkeit über Gentechnologie veröffentlicht wird. Publiziert wird es unter dem Pseudonym Agata Mendel in der Reihe Science Ouverte. Da es sich nach meiner Recherche um die einzige Publikation handelt, die gentechnologiekritische Positionen in Frankreich ausführlich darstellt und darin Flugblätter und Appelle abgedruckt sind, bietet die Publikation wichtige Dokumente der Primärliteratur. Gleichzeitig wird aber das Kollektiv in meiner Ausführung als eigenständiger Akteur berücksichtigt.

Mendel verwendet den Begriff genetische Manipulationen, um sich von dem Begriff »génie génétique« abzugrenzen. Dieser werde von Genforscherinnen und -forschern verwendet und mit falschen Versprechen verbunden, wie mit dem Slogan »l'âge d'or de la révolution bio-industrielle « - »das goldene Zeitalter der bioindustriellen Revolution « (Mendel 1980: 13/Übers. S.K.). Der Begriff génie génétique steht im Französischen für Gentechnologie. ${ }^{26}$ Dass der Begriff mit falschen Versprechen verbunden sei, wie Mendel meint, rührt m.E. daher, dass der Begriff génie mehrdeutig ist. Génie kann auch Genie, Genialität, im militärischen Zusammenhang auch Pioniertruppe bedeuten. So gesehen kann génie génétique bspw. auch als genetisches Genie verstanden werden, und ist damit eher positiv konnotiert. Anhand der Dokumente wird zwar deutlich, dass der Begriff genetische Manipulationen Mitte der 1970er Jahre sowohl von Wissenschaftlerinnen und Wissenschaftlern sowie der Presse - auch von der eher forschungsfreundlichen Le Monde - verwendet wird. Dennoch hat sich der Begriff génie génétique mittlerweile durchgesetzt. Daran, dass Mendel es im Jahr 1980 überhaupt für notwendig hält,

26 Génie steht für einen Wissensbereich, der mit einer bestimmten Technik verbunden ist. Wird er in diesem Sinn verwendet, folgt dem Begriff ein Adjektiv, bspw. génie atomique - Kerntechnik oder génie maritime - Schiffsbau (vgl. PONS 2003: 420). 
sich begrifflich abzugrenzen, zeigt sich, dass die Verwendung des Begriffs génie génétique in dieser Zeit noch ungewöhnlich war. Der Name Agata Mendel ist ein Pseudonym; die fiktive Schwester von Gregor Mendel, dem so genannten Vater der Genetik. Hinter dem Pseudonym stehen folgende Autorinnen und Autoren: Catherine Bousquet (Wissenschaftsjournalistin und Biologin), Jean Deutsch (Genetiker), Geneviève Gonzy (Biochemikerin), Françoise Laborie (Soziologin), Michel Steinmetz (Mikrobiologe) und John Stewart (Genetiker und Soziologe).

Die Konflikte, die es Mitte der 1970er Jahre um die Genforschung gibt, finden vor allem innerhalb von Forschungsinstituten statt. Über mehrere Jahre hinweg gibt es am Institut Pasteur und an anderen Forschungsinstituten teils heftige, hauptsächlich interne Einwände (CNRS 2004). Eine Debatte hat bis zu dieser Zeit im Parlament nicht stattgefunden, auch in der Öffentlichkeit kaum, und von einer Anti-GMO-Bewegung kann nicht gesprochen werden. Einen wahrnehmbaren Widerstand in der Öffentlichkeit gibt es, wie erwähnt, erst ab Mitte der 1990er Jahre, und zwar gegen die Anwendung genetisch manipulierter Pflanzen (Perret et al. 2004; Joly et al. 2000; Bonneuil et al. 2008). Auslöser ist, dass eine kleine Gruppe von Aktivistinnen und Aktivisten beginnt, Felder mit genetisch manipulierten Pflanzen zu mähen (Bonneuil et al. 2008: 203, 219). Hauptantriebskraft in diesem Widerstand ist die Gewerkschaft Conféderation Paysanne rund um den Bauernführer José Bové (Perret et al. 2004: 124ff; vgl. Bonneuil et al. 2008; Bonneuil 2006). Die Bewegung konzentriert sich auf die Anwendung von Gentechnologie im Umweltbereich. Die Konflikte um Gentechnologie Mitte der 1970er Jahre finden hauptsächlich wissenschaftsintern statt. Durch eine intensive mediale Berichterstattung erreichen sie zwar eine breitere Öffentlichkeit, aber dies betrifft nur einen sehr kurzen Zeitraum. Insgesamt haben sie dennoch ihre Auswirkungen.

Die Konflikte um Gentechnologie entwickeln sich mit gentechnologischen Versuchen, die am Institut Pasteur und dem Institut de Biologie Moléculaire ${ }^{27}$ der Universität Jussieu in Paris geplant werden. Dies geschieht vor dem Hintergrund des Moratoriums im Kontext des Asilomar-Prozesses. Es werden sowohl gentechnologische Experimente als auch die ersten Kontrollgremien für gentechnologische Forschung laut Mendel geplant (Mendel 1980: 215f): Im November 1974 richten sich Forscherinnen und Forscher an den CNRS mit der Anfrage, ein Kontrollsystem für gentechnologische Forschung einzurichten. Aus dieser Anfrage entsteht letztlich die Ethikkommission bei der DGRST, deren Commission Nationale de Classement zum ersten Mal im März 1975 tagt, also kurz nach Beendigung des internationalen Moratoriums. Die französischen Forscher und Forscherinnen stehen

27 Das Institut wurde im Jahr 1979 in Institut de Recherches en Biologie Moléculaire umbenannt. Seit 1982 heißt es Institut Jacques Monod. 
in den Startlöchern, um nach dem Moratorium ihre Forschung fortsetzen zu können. Am Institut Pasteur formiert sich Mitte der 1970er Jahre die technologiekritische Gruppe, der Groupe d'Information Biologie (GIB). Es handelt sich wieder um eine der Informationsgruppen, die in Anlehnung an die von Michel Foucault initiierte Gefängnisinformationsgruppe entstanden sind. Der GIB erhofft sich, der Öffentlichkeit wissenschaftliche Informationen über die Genforschung zu vermitteln, da die Informationen ihres Erachtens nicht in einem, wie sie es formulieren, »wissenschaftlichen Ghetto « bleiben sollten (Mendel 1980: 216/Übers. S.K.). Es soll sich in ihrem Sinn um eine Gegen-Information und nicht um eine Gegen-Expertise handeln (ebd.): »nous refusons de nous constituer en contre-experts ou de réclamer un pouvoir de décision pour nous-mêmes « - »wir lehnen es ab, uns als GegenExperten darzustellen oder eine Entscheidungsmacht für uns selbst zu beanstanden«, so heißt es in einem Artikel in der Tageszeitung Le Monde im Jahr 1975 (Le Monde 24.07.1975 zit. nach Bonneuil 2006: 259/Übers. S.K.). Diese GegenInformation hat sowohl zum Ziel, die Debatte zu öffnen als auch das Ziel, dass die Öffentlichkeit selbst in diesem Bereich Entscheidungen treffen kann. Diese Zielsetzung überschneidet sich mit der der Atompolitikkritiker und -kritikerinnen, da diese auch für eine Popularisierung und Aneignung von Wissenschaft wie auch für eine Wissenschaft des Volkes plädieren. Der GIB will Anlaufstelle für Arbeiter und Arbeiterinnen sowie Gewerkschafter und Gewerkschafterinnen sein (Mendel 1980: 216). Obwohl eines der Betätigungsfelder der Gruppe die Arbeitsbedingungen sein sollen, konzentriert sich der GIB schließlich auf die Genforschung allgemein. Dies führt zu einer Spaltung der Gruppe, denn mit dieser Schwerpunktsetzung gerät sie direkt in Konflikte mit dem Institut Pasteur und dessen neuen Orientierungen in der Forschung, so Mendel. Das Institut erhofft sich durch die gentechnologische Forschung und die entsprechende Förderung, seine finanziellen Probleme lösen zu können (ebd.: 218). Die Ausführung von Mendel ist m.E. so zu verstehen, dass das Thema Arbeitsbedingungen und die Kritik daran weniger grundlegend ist, als die Genforschung selbst in Frage zu stellen. Eine Kritik an Arbeitsbedingungen beinhaltet nicht unbedingt eine Kritik an der Forschung und ihrer Entwicklungen. Eine grundlegende Kritik an Genforschung wollen nicht alle Mitglieder des GIB teilen, da sie damit die Forschungsorientierung des Instituts angreifen. Vermutlich führt dies nicht zuletzt auch zu der Spaltung der Gruppe, da das Institut Arbeitgeber der Mitglieder des GIB war. Der Institut Pasteur ist, so Mendel, nicht allein mit dem GIB, sondern auch mit der Gewerkschaft des Instituts konfrontiert, die sich personell überschneiden. Diese Gewerkschaft ist die sozialistische CFDT, die auch zum Atomenergieprogramm Stellung bezogen hat. Wie auch im Kontext der Atomenergie kritisiert die Gewerkschaft die Entscheidungsverfahren und fordert entsprechend eine Diskussion darüber: »Qui décide qu'une recherche est plus importante 
qu'une autre? « - Wer entscheidet, dass eine Forschung wichtiger ist als eine andere?« (ebd./Übers. S.K.). Abgesehen von der Kritik am Entscheidungsverfahren, äußert sich die CFDT aufgrund der Möglichkeit katastrophaler Epidemien gegen genetische Forschung (ebd.: 217f). Um diesen Grundsatzfragen zu begegnen, organisiert der Institut Pasteur eine offizielle Diskussions- und Informationsveranstaltung im Jahr 1975. Allerdings hat er nicht mit einem so großen Zulauf gerechnet. Außer Forscherinnen und Forschern kommen Techniker und Technikerinnen, Sekretäre und Sekretärinnen, Arbeiter und Arbeiterinnen sowie Menschen von außerhalb des Instituts. Wie von Genforscherinnen und Genforschern die damalige Situation eingeschätzt wird, zeigt sich an einem Interview: »Es gab Konferenzen am Pasteur-Institut, bei denen Leute von der Straße eingefallen sind, die gestört haben [...], die das Wort an sich gerissen haben [...] ohne dass man sie zu Versammlungen eingeladen hat (Int F VII/Übers. S.K.). Auf die Frage, wie es dazu kam, heißt es »die ganze Genetik wurde als suspekt angesehen« (Int F VII). Ähnlich heißt es auch in einem Interview mit Philippe Kourilsky aus Gottweis Studie »Governing Molecules«: »people got extremely neurotic about the whole business « (Interview mit Ph. Kourilsky von H. Gottweis zit. nach Gottweis 1998: 139). Während der offiziellen Veranstaltung von 1975 bleiben die Vorträge theoretisch und in technischem Vokabular, wie ein Journalist der linken Wochenzeitschrift Politique Hebdo feststellt (Mendel 1980: 220). Bei folgenden Veranstaltungen werden externe Teilnehmende nicht mehr zugelassen, es gibt nur noch Debatten innerhalb des Instituts, die nach Mendel stark kontrolliert werden (ebd.: 219). Inwiefern die Veranstaltungen kontrolliert waren, führt Mendel nicht aus. Der GIB verfolgt weiterhin seine Öffentlichkeitsarbeit: Er veröffentlicht Dossiers in den Zeitschriften La Gueule ouverte ${ }^{28}$ und Politique Hebdo mit dem Ziel, die Debatte zu öffnen. In Anlehnung an den Appell der Atomphysiker und -physikerinnen vom Februar 1975, in dem auf die »schwerwiegenden Konsequenzen« der Entscheidungen der Messmer-Regierung aufmerksam gemacht wird, wird ein Appell verfasst, der im Juni 1975 in Le Monde und in der populärwissenschaftlichen Monatszeitschrift La Recherche mit über 500 Unterschriften erscheint (ebd.: 223). In dem Appell werden vor allem unkontrollierbare Epidemien hervorgehoben, die durch die Ausstreuung neuer Krankheitskeime entstehen könnten. Kritisiert wird außerdem die Orientierung der Forschungsinstitute an wirtschaftlichen Interessen. Es wird die sofortige Einstellung gentechnologischer Versuche gefordert und eine Kontrolle der Versuche durch alle Arbeiterinnen und Arbeiter und nicht durch selbsternannte Expertinnen und Experten. Darüber hinaus werden Informationen

28 La Gueule ouverte war eine Monatszeitschrift zum Thema Ökologie. Sie existiert von 1972-1980. Ihr Gründer, Pierre Fournier, engagierte sich im Anti-Atomkampf. 
gefordert, die allen zugänglich sind, sowie die öffentliche Diskussion von Forschungsprojekten und ihrer möglichen Risiken. Denn, so heißt es zum Schluss des Appells: »Ces manipulations nous concernent tous. Les décisions doivent être prises par tous « - $\gg$ Diese Manipulationen betreffen uns alle. Die Entscheidungen müssen von allen getroffen werden « (GIB (1975) appel zit. nach ebd.: 224/Übers. S.K.). Wie im atompolitikkritischen Diskurs steht auch hier die Entscheidungsfrage im Mittelpunkt. Die Entscheidungsfindung basiert ebenso auf einem politisierten technisch-wissenschaftlichen Risikobegriff. Mögliche Risiken der Forschungsprojekte sollen in öffentlichen Debatten diskutiert werden, solche Risiken nämlich, die »den Urhebern dieser Entdeckungen selbst nicht entgangen sind, [...] die entschieden haben, ihre Forschung auszusetzen« (ebd./Übers. S.K.). Gemeint ist hier m.E. der Asilomar-Prozess bzw. das internationale Moratorium, in welchem sich eine technische Risiko-Rahmung durchgesetzt hat. Die Sprache des Risikos wird nun politisch verwendet, indem sie als Gegen-Information zur Einstellung und nicht zur Förderung gentechnologischer Forschung eingesetzt wird. Anhand der Risiken gentechnologischer Forschungen wird die Entscheidungsfrage abgeleitet. Abgesehen von letztlich ungefähr 1000 Unterschriften erreicht der Appell nicht seine Ziele, eine öffentliche Debatte anzuregen oder gar die Experimente zu stoppen, so Mendel (ebd.). Allerdings ruft der Appell nur fünf Tage nach seiner Publikation eine Reaktion der Direktion des Institut Pasteur hervor. Sie veröffentlicht am 17. Juni 1975 einen Zeitungsartikel in Le Monde unter dem Titel »Des assertions dénuées de tout fondement « - »Behauptungen ohne jegliche Grundlage « (Le Monde 17.06.1975 zit. nach ebd./Übers. S.K.). Bei diesem Artikel handelt es sich, so Mendel, um eine Chancen-Risiken-Aufstellung, wobei gleichzeitig Risiken abgestritten und die bestehenden Kontrollmechanismen hervorgehoben werden. Die Direktion des Instituts betont die möglichen Anwendungsgebiete und die zu erwartenden Wohltaten für die Gesellschaft, wie Krankheiten durch Impfstoffe heilen oder Hunger durch Verbesserung der landwirtschaftlichen Erträge bekämpfen (ebd.: 224f). Die Vorstellung einer linearen Entwicklung von Forschung und Wohlstand wird in der Darstellung des Institut Pasteur aufrecht erhalten. Dem GIB wird unterstellt, ein Klima der Unruhe und Panik in der Öffentlichkeit schaffen zu wollen. Der Gruppe wird eine »profonde irresponsabilité « - »tiefgreifende Verantwortungslosigkeit« zugeschrieben (ebd./Übers. S.K.). Im Anschluss an die Veröffentlichung der Direktion des Institut Pasteur erreicht der GIB, dass die forschungsfreundliche Le Monde den »Controverses sur les manipulations génétiques « »Kontroversen über genetische Manipulationen« im Juli 1975 eine komplette Seite widmet. Hier werden Artikel der beiden Konfliktparteien veröffentlicht. Auch Le Monde selbst nimmt hier eine klare Position ein - die Zeitung unterstützt die Seite der Genforscher und -forscherinnen, in diesem Fall die Direktion des Institut Pas- 
teur. Ebenso wie beim Institut Pasteur entstehen auch am Institut de Biologie Moléculaire (IBM) der Universität Jussieu Konflikte. Das Institut wird im Jahr 1966 gegründet und dem Biologen Jacques Monod (Institut Pasteur) gewidmet. Monod und sein Kollege François Jacob, die zusammen im Jahr 1964 den Nobelpreis erhalten haben, bilden das Leitungskomitee des IBM (Institut Jacques Monod 2013). Vorrangig auf Initiative junger Forscherinnen und Forscher, geht es am IBM um die Entscheidungsfrage über die Forschung und um die Thematisierung möglicher Katastrophen durch Genforschung. In einer Broschüre fordern sie den Stopp aller Manipulationen, bis die Sicherheitsbedingungen erarbeitet, veröffentlicht, diskutiert und von allen akzeptiert sind. Für Aufruhr sorgt hier ein veröffentlichtes Foto einer weggeworfenen Petrischale als Beweis für die mangelnden Sicherheitsvorkehrungen in den Laboratorien. Am IBM gibt es in der Folgezeit immer wieder Konflikte zwischen Gewerkschaft und Direktion über die Sicherheitsvorkehrungen. Diese Konflikte eskalieren im Jahr 1977: Das Personal protestiert und infolgedessen reagiert das IBM mit Entlassungen (Mendel 1980: 228ff).

Diese Vorfälle erreichen kaum eine größere Öffentlichkeit. Wie Bonneuil meint, verschwinden die Konflikte aus den Medien bereits im Herbst 1975 (Bonneuil 2006: 259), also kurz nach den Kontroversen, über die in Le Monde berichtet wurde. Der Genforschung wird Mitte der 1970er Jahre seitens der Forschungsinstitute besondere Bedeutung zugeschrieben, da sie finanziellen Aufschwung verspricht. Den Störfaktoren dieser Forschung wird begegnet, indem sie als Panikmacher und -macherinnen dargestellt werden, wie bspw. in Le Monde. Die jungen, kritischen Forscherinnen und Forscher werden als verantwortungslos präsentiert, während sich die so genannten manipulateurs als verantwortungsvoll und kontrolliert zeigen. Auch durch den Ausschluss der Öffentlichkeit bei den Veranstaltungen des Institut Pasteur und durch die Entlassungen am IBM werden m.E. die Störfaktoren für die Genforschung beseitigt. Mendel stellt fest, »dass die wissenschaftliche Institution eine sehr große Kapazität hat, Einwände aus der eigenen Mitte zu verdauen und zu neutralisieren« (Mendel 1980: 231/Übers. S.K.). Mendel meint, es geht im Anschluss an diese Vorfälle an den Instituten nicht mehr um die Entscheidungsfrage, sondern um die Einhaltung von Sicherheitsvorkehrungen für die angeblich unbewiesenen Risiken (ebd.: 228ff).

Die Ergebnisse der Asilomar-Konferenz werden von den Gentechnologiekritikern und -kritikerinnen wahrgenommen. Zentral in der Kritik ist das Thema der Selbststeuerung von Wissenschaft und gentechnologischer Entwicklungen. Die atom- und auch gentechnikkritische Zeitschrift Impascience kommentiert das Ergebnis der Asilomar-Konferenz 1975 wie folgt: »was soll man davon halten [...]? Zweifellos zwei Dinge. Zuerst, dass es unendlich besser ist, dass sie [die Wissenschaftlerinnen und Wissenschaftler, S.K.] diese Verantwortung übernehmen anstatt 
dass sie sie nicht übernehmen. Sodann, dass dies radikal unzureichend ist « (N.N. 1975b: 48/Übers. S.K.). Unzureichend sei dies, so heißt es weiter, da niemand sich selbst kontrollieren kann, auch keine »wissenschaftlichen Elite«. Außerdem würden die Berufsgruppen, die sich darauf berufen, sich selbst zu kontrollieren, wie Mediziner und Medizinerinnen oder Pharmafirmen, nur ihren Machtbereich sichern wollen. Es sei notwendig, jenseits einer »wissenschaftlichen Elite« über kollektive Formen der Kontrolle nachzudenken, denn es handle sich nicht um technische Probleme, sondern um soziale und politische (ebd.: 48f). Anhand des Artikels aus Impascience zeigt sich m.E., dass die Zeitschrift der wissenschaftlichen Elite durchaus eine Lösungskompetenz zuschreibt - und zwar vor allem für technische Probleme. Denn die Begründung, dass die wissenschaftliche Elite für diese Probleme nicht zuständig sei, lautet, dass es es sich nicht um technische Probleme handele. Es zeigt sich, dass es Mitte der 1970er Jahre umstritten ist, um was für ein Problem es sich bei der rDNA-Technologie handelt, wie es definiert ist - technisch oder sozial und politisch. Damit verbunden ist die Frage, wer die Lösungskompetenz für das Problem hat.

»Die Forschung wurde unter guten Sicherheitsbedingungen wieder aufgenommen«, so schätzt Jean Bernard, Mitglied der Ethikkommission der DGRST, die Situation in Frankreich nach der Konferenz in Asilomar und der Beendigung des Moratoriums für rDNA-Technologie ein (Bernard 1986/1987: 32/Übers. S.K.). Die Ethikkommission ist als eine Art »court of appeals« für »ethische Probleme« zuständig, sofern diese bei der Evaluierung der Forschungsprojekte durch die Commission Nationale de Classement auftauchen (Gottweis 1998: 142). Diese Evaluation basiert auf Risiken. Die Probleme sind als technische Risiko-Probleme gerahmt, die mit der Einhaltung entsprechender Sicherheitsmaßnahmen gelöst werden können. Durch die technische Rahmung bleibt die Lösungskompetenz innerwissenschaftlich. Keinen Raum haben soziale und politische Probleme, wie sie von den Gentechnologiekritikerinnen und -kritikern definiert werden. Ihre Lösungsvorstellungen, die auf Formen kollektiver Kontrolle der rDNA-Experimente basieren, werden entsprechend nicht thematisiert. Politische Einwände oder Konflikte, als verantwortungslos dargestellte Panikmacher und -macherinnen, haben keinen Platz in der Commission Nationale de Classement und somit auch nicht in der Ethikkommission. Diese Ethikkommission soll dafür Sorge tragen, dass die Ergebnisse der Asilomar-Konferenz eingehalten werden. Sie erscheint im Kontrast zu den Panikmachern und Panikmacherinnen als verantwortungsvolle Lösung zur Sicherung technischer Risiken, und damit auch als verantwortungsvolle Lösung der gentechnologischen Forschung und wissenschaftlicher Selbststeuerung. Andere Problematisierungen und Lösungsmöglichkeiten werden ausgeschlossen oder, um Mendels Begrifflichkeit zu verwenden: »verdaut«. 


\section{Zusammenfassung}

In dem eher US-amerikanisch geprägten internationalen Asilomar-Prozess hat sich der Möglichkeitsraum, um über gentechnologische Forschung zu sprechen, verengt. Die Asilomar-Tagung kann als Ereignis verstanden werden, das den Schritt in Richtung US-staatlicher Instanzen, der durch den Singer-Brief eingeleitet wird und Probleme wissenschaftlicher Selbstregulierung offen legt, weitgehend rückgängig macht. Zudem kann der Asilomar-Prozess als Antwort auf die Kontroverse um die Verantwortung der Wissenschaft gegenüber der Gesellschaft gesehen werden, die in den USA geführt wurde. Innerhalb dieses Prozesses findet eine Verschiebung statt: Aus einem politischen Konzept der Verantwortung, das die wissenschaftliche Selbststeuerung angreift, wird ein Konzept der Verantwortung, das durch seine technische Risiko-Rahmung auf einer technischen und individuellen Ebene gehalten wird. Mit der Problematisierung von technischen Risiken werden extra-wissenschaftliche Formen der Steuerung wissenschaftlicher und technologischer Entwicklungen erfolgreich abgewehrt. Risikoabschätzung ist insofern im USamerikanischen wie auch im französischen Diskurs um Gentechnologie die Lösung für die Problematisierung wissenschaftlicher Selbststeuerung. Der politisierte Risikobegriff und die Frage wissenschaftlicher Selbststeuerung tauchen sowohl in den USA als auch in Frankreich im Kontext der Konflikte um Atomenergie auf und übertragen sich auf die gentechnologischen Diskurse. Die Ethikkommission der DGRST zeigt sich als eine Art ethische Sicherheitsvorkehrung für eine Risikoabschätzung im Sinne Asilomars. Während in den USA die Risiko-Rahmung die Antwort auf eine kontroverse Diskussion um Verantwortung und wissenschaftliche Selbststeuerung ist, ist in Frankreich die Ethik-Rahmung die Antwort auf einen politisierten Risikobegriff, auf die Konflikte um die Entscheidungsfrage und damit auf die Diskussion um wissenschaftliche Selbststeuerung. Wenn die Ethikkommission Risikokommission genannt worden wäre, was inhaltlich naheliegend scheint, hätte es die Kritikerinnen und Kritiker gegebenenfalls bestätigt und eine politisierte Risikokontroverse gefördert.

Der Asilomar-Prozess führte in Frankreich zur Einrichtung der Ethikkommission bei der DGRST. Was durch die Initiative der Wissenschaftlerinnen und Wissenschaftler Mitte der 1970er Jahre kenntlich wird, sind Bemühungen, in Frankreich einen verantwortungsvollen Umgang mit Forschung zu zeigen. In diesem Kontext taucht eine der ersten expliziten Ethikinstitutionen auf Politikebene auf. Diese Institution ist mit »heads of scientific community« besetzt. Gottweis sagt zwar, dass die Ethikkommission auch mit Nicht-Wissenschaftlern und -wissenschaftlerinnen besetzt gewesen ist, sprich zu den »heads « zählten nicht nur WissenschaftlerInnen und Wissenschaftler. Die Commission Nationale 
de Classement war aber rein wissenschaftlich besetzt. Da die Ethikkommission nicht einberufen wird, bleibt so die Steuerung gentechnologischer Forschung und Entwicklung in den eigenen Händen. Der Ethik-Rahmen beinhaltet hier eine Risikoabschätzung inklusive individualisierter Verantwortung. Die Risikoabschätzung, basierend auf den Ergebnissen von Asilomar, ist als Prozess konzipiert; die Kriterien der Risikominimierung werden immer wieder überdacht, entsprechend mit ihr die Verantwortung. Das Infragestellen der Forschung selbst ist in diesem Rahmen nicht vorgesehen. Die Kritik an Gentechnologiepolitik ist in Frankreich Anfang/Mitte der 1970er Jahre nicht ethisch gerahmt. Sie beinhaltet die Kritik an Technokratie, an hierarchischen Entscheidungsverfahren, dem linearen Modell von Wissenschaft und Fortschritt, den Risiken. Mit der Ethikkommission sowie mit der Commission Nationale de Classement hat sich das Verfahren der Selbststeuerung von Wissenschafts- und Technikentwicklungen ausgedehnt. Vor allem die Commission wird für die Kontrolle von Forschungsvorhaben in Anspruch genommen, die Ethikkommission nicht. Die Ethikkommission kann, als »court of appeals « gesehen werden, als Berufungsgericht für den Fall eines »ethischen Problems « (Gottweis 1998: 142), als eine Art ungewisses Damoklesschwert für den Forscher oder die Forscherin. Ungewiss ist dies deshalb, da unklar ist, was Ethik bedeutet: »what does it mean? « (Interview mit Ph. Kourilsky von $\mathrm{H}$. Gottweis zit. nach ebd.: 140). Der undefinierte Ethik-Rahmen in Form der Kommission kann so als Bedrohung verstanden werden, die hintergründig im Raum steht, sofern der standardisierte Fragebogen, der bei der technischen Kommission vorgelegt werden muss, nicht richtig ausgefüllt wurde, oder als präventive Selbstdisziplinierung. Mit der Einrichtung der technischen Kommission und der Ethikkommission findet eine offenere Diskussion über einzelne Forschungsprojekte statt. Eine öffentliche Diskussion über Forschungsziele und damit einhergehend über gesellschaftspolitische Ziele oder auch darüber, was Ethik bedeutet, hat dort allerdings keinen Raum. 


\section{Die Sprache der Ethik als Ursache für Problematisierungen biologischer und lebenswissenschaftlicher Autonomie}

Im Jahr 1974 erreicht, wie in den letzten Kapiteln ausgeführt, die Ethik-Rahmung zweimal die Ebene staatlicher Politik: in Form des Ethikkomitees beim INSERM und der Ethikkommission der DGRST. Im selben Jahr taucht ein Konzept der wissenschaftlichen Verantwortung auf, und zwar in Form der unabhängigen Vereinigung Mouvement Universel de la Responsabilité Scientifique (MURS) - Universelle Bewegung für wissenschaftliche Verantwortung. Der MURS entsteht 1974 auf einer internationalen Tagung, die an der Sorbonne-Universität in Paris stattfindet. Sie hat den Titel »Biologie et devenir de l'homme« - »Biologie und die zukünftige Entwicklung des Menschen«. Im Unterschied zu den Ethikinstitutionen, die als Antworten auf Problematisierungen der Selbststeuerung medizinischer oder gentechnologischer Entwicklungen entstehen, erscheint der MURS im Kontext einer disziplinenübergreifenden Problematisierung. Innerhalb dieser Problematisierung erscheint nicht nur das Konzept von Verantwortung, sondern auch die Sprache der Ethik. Ebenso im Kontext einer disziplinenübergreifenden Problematisierung erscheint die Sprache der Ethik ein weiteres Mal im Jahr 1979, nämlich in einem Bericht mit dem Titel »Sciences de la vie et société« - »Lebenswissenschaften und Gesellschaft«, den der damalige Staatspräsident Valéry Giscard d'Estaing bei Wissenschaftlern in Auftrag gibt.

Im Folgenden stelle ich den MURS vor und gehe anhand der Tagung den Fragen nach, inwiefern solch eine Bewegung für wissenschaftliche Verantwortung notwendig scheint, wie in diesem Kontext die Sprache der Ethik auftaucht und was dieses Konzept von Verantwortung beinhaltet. Im Anschluss daran gehe ich der Sprache der Ethik in dem Bericht »Lebenswissenschaften und Gesellschaft« nach.

Sozialwissenschaftliche Untersuchungen zu der Tagung »Biologie und die zukünftige Entwicklung des Menschen« und zu ihrem 600seitigen Tagungsband 
(Galpérine 1976a), der im Jahr 1976 erscheint, sowie zu dem Bericht »Lebenswissenschaften und Gesellschaft« (Gros et al. 1979) von 1979 liegen nach meiner Recherche nicht vor. Von dem kanadischen Theologen und Bioethiker Guy Durand wird sie als erste internationale Tagung benannt, die ethische Themen biomedizinischer Forschung behandelt (Durand 1999: 476). Die französische Philosophin Anne Fagot-Largeault, ehemaliges Mitglied des CCNE (1990-1998), sieht die Tagung und den MURS als wegbereitend für den CCNE (Fagot-Largeault 1985). Die französische Medizinerin und ehemalige Mitarbeiterin des Dokumentationszentrums des CCNE, Claire Ambroselli, bewertet zu Beginn der 1990er Jahre die Tagung rückblickend als »erste internationale Debatte in Frankreich über ethische Fragen, die durch die biomedizinische Forschung aufgeworfen wurden« (Ambroselli 1990: 106/Übers. S.K.). Auch in dem von der belgischen Sozialwissenschaftlerin Madeleine Moulin herausgegebenen Sammelband zu Ethikkomitees heißt es, die Tagung gelte als wegbereitend für den CCNE (Kenis/Heuskin 1990: 65). Inwiefern sie oder der MURS als wegbereitend gelten, wird allerdings nicht ausgeführt.

\title{
1 Der Mouvement Universel de la Responsabilité SCIENTIFIQUE
}

\begin{abstract}
»Wir unterstützen vernünftige Entwicklungen, wir bestärken sinnvolle Vorhersagen [...], um der Sache des Verstands zu dienen und, wenn es nötig ist, einer Moral, die der unverzichtbaren Objektivität der Wissenschaft nicht widerspricht. Die Gründung des Ethikkomitees in Frankreich ist dafür der Beweis« (Mallet 1986/1987: 21f/Übers. S.K.).
\end{abstract}

»Wir«, das sind die Mitglieder des MURS, für die Robert Mallet hier stellvertretend anlässlich des zehnjährigen Bestehens des Mouvement spricht. Mallet (*1915-†2002), französischer Schriftsteller und damaliger Rektor der Académie de Paris, ${ }^{1}$ war Mitbegründer und erster Präsident des MURS. Der MURS ist Resultat einer internationalen Tagung, die im September 1974 an der Sorbonne abgehalten wird. Mallet, damals Initiator der Tagung, sieht den MURS rückblickend als Weg-

1 Die Akademie ist der Schul- und Universitätsbezirk von Paris. Der Rektor oder die Rektorin ist u.a. Kanzler oder Kanzlerin der Universitäten von Paris, ausgenommen der École Normale Supérieure, der École Nationale des Chartes und der École des Hautes Études en Sciences Sociale. Letztere sind unabhängig von dieser Verwaltungsstruktur. 
bereiter für die Einrichtung des nationalen Ethikkomitees, des CCNE. Diese Ansicht teilt er nicht nur mit den o.g. Autorinnen und Autoren, sondern auch mit Jean Bernard. Bernard ist nicht nur Mitglied der Ethikkommission der DGRST und des Ethikkomitees vom INSERM und ab 1983 erster Präsident des CCNE, sondern ebenfalls Mitglied des MURS. Er sieht die NS-Medizin, wie in Kapitel III aufgezeigt, sowie die Revolution und Fortschritte in der Biologie als Ursprung für die Ethik in der Medizin und Biologie. Zu dieser Revolution zählen für ihn Gentechnologie oder auch Reproduktionstechnologien (Bernard 1986/1987: 27ff; vgl. Bernard 1994: 321). Explizit bezogen auf die Entstehung von Ethikinstitutionen bezeichnet er die Reflexion, die in Frankreich mit dem MURS begonnen habe, als Inspiration:

»Diese Reflexion hat in Frankreich 1974 an der Sorbonne mit der Gründung des Mouvement Universel de la Responsabilité Scientifique durch den Rektor Robert Mallet begonnen [...]. Sie hat sich in mehreren Ländern weiter entwickelt, insbesondere in den Vereinigten Staaten. In unserem Land hat sie die Entstehung von Ethikkomitees an den großen wissenschaftlichen Instituten inspiriert (Délégation Générale à la Recherche Scientifique et Technique, Institut National de la Santé et de la Recherche Médicale) [...]. Eine neue Etappe wurde in Frankreich 1983 mit der Gründung eines Comité National Consultatif d'Éthique des Sciences de la Vie et de la Santé erreicht« (Bernard 1986/1987: 34/Herv. hinzugefügt, Übers. S.K.).

Gegen die Ausführung von Jean Bernard, der MURS habe ab 1974 mit seiner Reflexion die Entstehung des Ethikkomitees vom INSERM inspiriert, steht, dass dieser Comité bereits einige Monate vor der Tagung an der Sorbonne eingerichtet wird. Das Komitee wird im Februar 1974 eingerichtet, die Tagung findet im September statt. Allerdings tagt das Ethikkomitee zum ersten Mal erst im Jahr 1976. Der Antrag für die Ethikkommission, die bei der DGRST eingerichtet wird, wird im November 1974 beim CNRS eingereicht, also kurz nach der Tagung an der Sorbonne. So könnte es sein, dass die Reflexion des MURS die Einrichtung dieser Ethikkommission im Jahr 1975 inspiriert, neben dem Asilomar-Prozess, wie in vorigem Kapitel ausgeführt. De facto wird diese Kommission im Bewertungsverfahren zu Forschungsvorhaben mit rDNA allerdings nie konsultiert (Gottweis 1998: 143). Ein Komitee existiert also bereits vor dem MURS, eine Kommission wird aber nie aktiv. Bis auf das nationale Ethikkomitee, das im Jahr 1983 ins Leben gerufen wird, können die Ethikinstitutionen nicht (allein) in Konsequenz der Reflexionen des MURS eingerichtet worden sein. Dennoch sprechen Bernard und Mallet ihnen eine inspirierende Bedeutung zu. Was beinhalten die Reflexionen, die 1974 mit der internationalen Tagung an der Sorbonne beginnen und das Konzept von Verantwortung des MURS? 
Die internationale Tagung »Biologie et devenir de l'homme «, aus der der MURS hervorgeht, wird vom 18. bis 24. September 1974 abgehalten (Galpérine 1976b: VIII). ${ }^{2}$ Die Organisation und Konzeption des MURS wird auf der Tagung präsentiert und per Resolution verabschiedet (Mallet 1976c). Der Mouvement ist als unabhängige Vereinigung mit dem Ziel konzipiert, eine internationale Plattform für Wissenschaftler und Wissenschaftlerinnen aller Disziplinen sowie Interessierte zu sein. Die Vereinigug hat das Ziel Probleme, die aus der wissenschaftlichen Entwicklung und ihrer Anwendung für die Menschheit entstehen können, zu diskutieren und offen zu legen (Statuts de la Fondation, MURS zit. nach Galpérine 1976a: 551/Übers. S.K.; MURS 2005). Ebenso soll sie auf Anfrage internationale Organisationen, Regierungen und NGOs beraten sowie die Öffentlichkeit informieren, sei es durch Publikationen oder Tagungen (Résolution, MURS zit. nach Galpérine 1976a: 545; vgl. N.N. 1974). Ziel ist es zudem, so heißt es im Gründungsstatut, »[à, S.K.] stimuler une réflexion prospective rigoureuse sur l'homme et la planète « - »eine zukunftsorientierte, genaue Reflexion über den Menschen und den Planeten zu stimulieren « (Statuts de la Fondation, MURS zit. nach Galpérine 1976a: 551/Übers. S.K.). Verbindendes Element der Teilnehmenden am Mouvement ist, so Mallet in seiner Präsentation des MURS auf der Tagung, das Bewusstsein individueller und kollektiver Verantwortung: »The consciousness of individual and collective responsibility will draw together the participants of the >Movement « « (Mallet 1976c: 542/Herv. i.O.). Es bestehe eine »Pflicht« der Wissenschaftler und Wissenschaftlerinnen, so heißt es in der Resolution: »to contribute by developing apropriate means to express and to satisfy the needs and interests of society, to promote informed public opinion, and to elaborate decisions which affect the world community« (Résolution, MURS zit. nach Galpérine 1976a: 545/Übers. S.K.). Diskutiert werden sollen vor allem die (möglichen) Auswirkungen wissenschaftlicher Entwicklungen: »There's no doubt that science comes from experiment and observation without reference to morals. But its implications, conditioning inevitably the spiritual and social life of Man, will be studied« (Mallet 1976c: 542). Untersucht werden sollen »des bienfaits et des risques « - »Wohltaten und Risiken « dieser Entwicklungen für die Menschheit (Statuts de la Fondation, MURS zit. nach Galpérine 1976a: 551/Übers. S.K.; MURS 2005). Die Verantwortung des Wissenschaftlers oder der Wissenschaftlerin ist bei der Untersuchung »entière « - »umfassend « und liegt »in der Auswertung der Versuche, in der Bewertung nicht messbarer Risiken neuer Techniken« (Galpérine 1976b: IX/Übers. S.K.). Mit dem MURS

2 Die Beiträge in dem Tagungsband sind zum Teil auf Französisch oder auf Englisch, zum Teil sind die Beiträge in beiden Sprachen abgedruckt. Liegt eine englische Übersetzung vor, wird diese im Folgenden verwendet. 
soll ein permanentes Instrumentarium geschaffen werden, um »rethinking free from all pressures and vested interests; it will act as an expression of the common weal and can be accepted and consulted by politicians« (Mallet 1976d: 565).

Geschaffen werden soll mit dem MURS also ein permanentes Instrumentarium, das eine unabhängige Reflexion über wissenschaftliche Entwicklungen und ihre (möglichen) Auswirkungen auf den Menschen und die Zukunft des Planeten ermöglicht. Diskutiert werden sollen auf Basis eines individuellen und kollektiven Verantwortungsbewusstseins die Risiken und Wohltaten dieser Entwicklung. Wissenschaft wird als etwas gesehen, das nicht mit Moral verknüpft ist, also wertfrei scheint. Auch hier zeigt sich ein gleichsam räumlich trennendes Denken wie im Asilomar-Prozess, denn die Pflicht der Wissenschaftlerinnen und Wissenschaftler, die (möglichen) Auswirkungen wissenschaftlicher Entwicklungen zu untersuchen, scheint erst außerhalb des Labors einzusetzen. Inwiefern scheint es notwendig, solch eine Bewegung für wissenschaftliche Verantwortung ins Leben zu rufen, die Wissenschaftler und Wissenschaftlerinnen »verpflichtet«, die Konsequenzen ihrer Arbeit zu untersuchen? Was wurde auf der internationalen Tagung »Biologie und die zukünftige Entwicklung des Menschen« besprochen und was nicht?

\subsection{Die internationale Tagung »Biologie und die zukünftige Entwicklung des Menschen ${ }^{3}$}

Während die Tagung »Biologie und die zukünftige Entwicklung des Menschen« geplant und durchgeführt wird, ist der Organisationsprozess für die Konferenz in Asilomar im Gange. Das freiwillige, internationale Moratorium zu rDNA-Technologie ist noch nicht beendet. Wissenschafts- und medizinkritische Diskurse zeigen sich zu dieser Zeit sowohl in den USA als auch in Frankreich, sei es durch die new critical science movement oder durch die Science du Peuple. Und die Konflikte an den französischen Forschungsinstituten $\mathrm{zu}$ gentechnologischer Forschung sind ebenso aktiv wie die Diskurse um die Legalisierung der Abtreibung, um $\mathrm{Hu}-$ manexperimente und Atompolitik.

Die Tagung findet mit rund 170 Teilnehmenden statt, vertreten sind 37 Nationen (Mallet 1976d: 563). Die Schirmherrschaft für diese Tagung setzt sich aus einem Komitee verschiedener sowohl staatlicher als auch nicht-staatlicher (forschungs-)politischer französischer Institutionen und internationaler Organisationen zusammen, u.a. dem Institut National d'Études Démographiques, dem CNRS und INSERM, der DGRST, dem Institut Pasteur und der UNESCO (Galpérine 1976b:

3 Galpérine 1976a/Übers. S.K.. 
X). Im Organisationskomitee, das sich aus französischen Mitgliedern zusammensetzt, sind u.a. Robert Mallet, Jean Bernard, der französische Philosoph und Soziologe Raymond Aron $(* 1905-\uparrow 1983)$ und sein Studienkollege, der damals bereits emeritierte Sorbonne-Professor, Philosoph und Mediziner Georges Canguilhem $\left({ }^{*} 1904-\dagger 1995\right){ }^{4}$ Ebenso im Organisationskomitee sind Jacques Monod und François Jacob. Organisiert wird die Tagung u.a. in Kooperation mit Paul Berg und dem Hastings Center (ebd.: IXf). Beide sind maßgebliche Akteure im zeitgleich stattfindenden Asilomar-Prozess. Anwesend sind sie allerdings auf der Tagung nicht, die Kooperation findet ausschließlich im Vorbereitungsprozess statt. Unter den Anwesenden sind nicht nur internationale Vertreter und Vertreterinnen verschiedener wissenschaftlicher Disziplinen, sondern auch aus dem Gesundheitsbereich wie der Weltgesundheitsorganisation und aus der französischen Politik, darunter befindet sich auch der damalige Staatspräsident Giscard d'Estaing (Galpérine 1976a: 589-600). Zu den vertretenen Disziplinen gehören laut Teilnehmendenliste des Tagungsbandes überwiegend Medizin, Biologie und Genetik, aber auch Geschichtswissenschaft, Philosophie, Anthropologie und Soziologie, die letzteren sind zahlenmäßig allerdings geringer vertreten (ebd.: 589-600). Journalisten und Journalistinnen sind in der Liste nicht aufgeführt. Zwar gibt es in den französischen Abendnachrichten des öffentlich-rechtlichen Fernsehens einen Beitrag über die Tagung (Laury 1974) ${ }^{5}$, ebenso in der internationalen, populärwissenschaftlichen Zeitschrift New Scientist (N.N. 1974). Ob allerdings Journalistinnen und Journalisten kontinuierlich an der Tagung teilnehmen konnten, wird aus dem Tagungsband nicht ersichtlich. Augenscheinlich sind wissenschaftskritische Teilnehmende, sei es aus dem Spektrum der US-amerikanischen new critical science movement oder des französischen Science du Peuple, nicht vertreten. Ausnahmen kritischer Teilnehmender, die sich allerdings nicht, wie die gerade genannten, strikt im außerparlamentarischen Spektrum verorten lassen, sind die Franzosen René Dumont und Brice Lolande. Dumont $(* 1904-\dagger 2001)$, Professor für Agrarwissenschaft und Soziologe, ist im Jahr 1974 auf Initiative der Amis de la Terre (Friends of the Earth) und mit persönlicher Unterstützung von Lalonde als Kandidat für das

4 Zu Leben, Werk und Rezeption von Canguilhem, Foucaults Doktorvater, vgl. weiterführend das Schwerpunktheft von »Economy and Society«; darin für einen Überblick insbesondere den Artikel von David Macey (1998).

5 Diese Abendnachrichten wurden im Archiv des Institut National de l'Audiovisuel auf den 29. Januar 1974 datiert. Vermutlich wurde das Dokument falsch archiviert, denn der Nachrichtenbeitrag kann erst im September gesendet worden sein, da die Tagung erst im September, nicht im Januar stattfindet. Zudem wird in dem Bericht selbst der Monat September genannt und es werden Tagungsbilder eingeblendet. 
Amt des Staatspräsidenten angetreten. Die Amis de la Terre versuchen eine antikapitalistische, basisdemokratische Konzeption von Gesellschaft mit Ökologie zu verknüpfen (Doll/Schmidt 2006: 340). Dumont wird später zudem Mitbegründer von Les Verts - der grünen Partei, die 1984 entsteht. Im Tagungsband taucht er allerdings nicht als Vertreter der Amis de la Terre auf; benannt wird seine Zugehörigkeit zum nationalen agrarwissenschaftlichen Institut (Galpérine 1976a: 592). Lolande, écologiste, Mitstreiter der 1968er-Bewegung, im Jahr 1981 ebenfalls Präsidentschaftskandidat und von 1988-1992 Umweltminister, ist Anfang der 1970er Jahre vor allem im Anti-Atomkampf aktiv. Atomenergie oder -politik steht jedoch nicht auf der Agenda der Tagung. Lalonde ist laut Tagungsband als USamerikanischer Vertreter ${ }^{6}$ der Amis de la Terre auf der Tagung (ebd.: 595).

\subsection{Ein »Ort des Zusammenflusses « ${ }^{7}$}

Gegenstand der Tagung ist, wie der Titel »Biologie und die zukünftige Entwicklung des Menschen« zeigt, die Biologie, und zwar verstanden als »lieu de confluence « - »Ort des Zusammenflusses« von Fragen, die das Verhältnis des Lebenden, des Menschen zu seiner Umgebung betreffen (Galpérine 1976b: VIII/ Übers. S.K.). Die Tagung wird rückblickend als ein Ort verstanden, an dem sich durch den Austausch Disziplinen und Sichtweisen mischen konnten (ebd.: IX). Die Themen, die auf der Agenda stehen, werden in zwölf Diskussionsrunden besprochen. Hierzu zählen bspw. die Überalterung der Bevölkerung, therapeutische $\mathrm{Hu}$ manexperimente, Organtransplantation, Reproduktion und Genetik, Ökotoxikologie oder Umweltschutz (Galpérine 1976a: Vf). Eingeteilt sind die Diskussionsrunden in drei Themenkomplexe: »Interventions and Balance«, »Variation and Number« sowie »Quality of life. Dignity of death« (ebd.). Wie waren die Themen gerahmt und inwiefern tauchen diese Rahmungen in der Konzeption des MURS auf?

Leitsatz der Tagung ist »Nouveaux pouvoirs de la science, nouveaux devoirs de l'homme« - »Neue Fähigkeiten der Wissenschaft, neue Pflichten für den Menschen« (Mallet 1976a: VIII/Übers. S.K.). Das Credo ist also, dass sich neue Pflichten für den Menschen aus den neuen wissenschaftlichen Fähigkeiten ergeben. Robert Mallet konkretisiert das Ziel der Tagung im Vorwort des Tagungsbandes: es

6 Wieso Lalonde als US-amerikanischer und nicht als französischer Vertreter der Amis de la Terre auf der Tagung war, ist unklar. Sie existierten zu diesem Zeitpunkt bereits in Frankreich (vgl. Topçu 2007: 4).

7 Galpérine 1976b: VIII/Übers. S.K.. 
soll nicht nur die Frage gestellt werden, was der Mensch sein will, sondern auch, was er sein kann (Mallet 1976b/Übers. S.K.). Die Logik dieser Perspektive liegt m.E. darin, wissenschaftliche Entwicklungen als unbestreitbares Kontinuum zu sehen, an die sich der Mensch entsprechend permanent anpassen soll und aus denen ebenso permanent neue Pflichten entstehen. Zu den Pflichten gehört es nicht, Fragen zu stellen, wie: »Welche Forschung wollen wir in unserer Gesellschaft« und: »Welche Gesellschaft wollen wir?«. Diese Fragen lassen sich nicht aus dem Leitsatz der Tagung ableiten. Es ergibt sich m.E. nicht die Pflicht, eine Position zur wissenschaftlichen Entwicklung einzunehmen, sondern die Pflicht, sich innerhalb der wissenschaftlichen Entwicklungen zu denken. Die neuen Fähigkeiten ergeben sich aus der wissenschaftlichen Entwicklung, aus der sich nicht allein Fragen über die Zukunft des Menschen ableiten lassen, sondern auch über seine Entwicklung im Titel der Tagung heißt es explizit nicht »Biologie et avenir de l'homme«, sondern »devenir de l'homme«. Während »avenir« Zukunft bedeutet, steht »devenir« zwar ebenso für Zukunft oder zukünftige Entwicklung, aber darüber hinaus für Werden, Entstehen und Entwicklung (vgl. PONS 2003: 273). Die zukünftige Entwicklung des Menschen, sein Werden und Entstehen, was er sein kann, soll m.E. in der biologischen Perspektive gefasst werden. Um diese wissenschaftlichen Entwicklungen geht es auf der Tagung. Inwiefern sich dieser Leitsatz niederschlägt, zeigt das folgende Beispiel.

\section{$1.3 »$ Moral and ethical aspects « - "partly emotional ${ }^{8}$}

Als Beispiel dient die Diskussionsrunde zum Thema »Responsibility and decision in the orientation and genetic control of human procreation «, das Subthema des Themenkomplexes »Quality of life. Dignity of death« ist (Galpérine 1976a: 373ff). In diesem Themenkomplex werden Fragen zur Kontrolle menschlicher Zeugung diskutiert, zur medizinischen Verantwortung gegenüber Behinderten ebenso wie Fragen dazu, wie Mediziner und Medizinerinnen sich gegenüber Sterbenden verhalten, die ihr Leben selbst beenden wollen (Galpérine 1976b: IX). Eingeleitet wird die Diskussionsrunde zur genetischen Kontrolle menschlicher Zeugung mit einer Kurzdarstellung des britischen Physiologen Robert G. Edwards (Edwards 1974). ${ }^{9}$ Edwards ist Pionier im Bereich der Reproduktionsmedizin und praktiziert

8 Edwards 1974: 382.

9 Publiziert ist in dem Tagungsband nicht der Vortrag, sondern ein Artikel von Edwards, der im selben Jahr in Quarterly Review of Biology, Vol. 49 erschien (vgl. Fußnote Edwards 1974: 377). Ich gehe davon aus, dass er auf der Tagung einen Vortrag gehalten hat, 
zusammen mit dem Gynäkologen Patrick Steptoe weltweit zum ersten Mal erfolgreich die Methode der In-vitro-Fertilisation (IVF). Als die Tagung stattfindet, ist das erste so genannte IVF-Baby, Louise Brown, noch nicht geboren. Dies ereignet sich erst im Jahr 1978. Unter dem Titel »Moral and ethical aspects « (Edwards 1974: 382) heißt es in Edwards Darstellung:

»The impact of human research can obviously be wider than merely affecting patients and doctors, and many of the themes running through debates on fertilization in vitro also arise in connection with abortion, contraception and artificial insemination from a donor (AID). The idea of initiating human life in vitro will probably be unacceptable in principle to some people, even for the cure of infertility. This response is partly emotional and might be modified as the notion becomes more familiar and the benefits clearer, just as previous debates have led to the acceptance of new attitudes towards various other aspects of human reproduction and sexuality [...]. Certain other well-known concepts stimulating a great deal of discussion concern the moment when human life begins and the >rights $<$ of embryos, fetuses, and neonates $[\ldots .$.$] . Fears of genetic engineering also enter prominently into these debates « (ebd./$ Herv. i.O.).

Festgestellt wird von Edwards, dass sich die Auswirkungen der IVF-Forschung nicht allein auf Mediziner und Medizinerinnen oder Patienten und Patientinnen beschränken. Auf wen diese Forschung darüber hinaus Auswirkungen hat, wird nicht konkretisiert. Es wird auch nicht konkretisiert, welche Themen in die Debatte wirken. Es sind nicht Konflikte oder Positionen, die in die Debatte einwirken, wie zu Abtreibung oder Verhütung, obwohl die Proteste und die Kritik gerade um den Schwangerschaftsabbruch zu dieser Zeit nicht nur in Frankreich aktiv und fassbar sind - sie werden nicht explizit thematisiert. Die Haltung derer, für die die IVF nicht akzeptabel ist, wird als »teilweise emotional « bezeichnet. Diese Emotionen könnten, so Edwards, durch Gewöhnung gemindert werden, wenn die »Vorteile« der IVF akzeptiert werden. Ebenso können »Ängste« gegenüber der Gentechnologie durch Gewöhnung gemindert werden - der »instinctive appeal of those who are fearful « oder »the pessimistic assumption that the worst will inevitably happen« (ebd.: 385). Edwards geht also entsprechend dem Leitsatz der Tagung davon aus, dass sich Menschen (früher oder später) den wissenschaftlichen Entwicklungen anpassen, wie es schon bei anderen Aspekten, wie der menschlichen Fortpflanzung und Sexualität der Fall gewesen sei. Das Problem, das unter dem Titel »moralischen und ethischen Aspekten « gefasst wird, scheint zu sein, mit einer Reihe an Ir-

der auf dem Artikel basiert. Eine Anmerkung dazu gibt es in dem Tagungsband nicht. Das folgende Zitat stammt entsprechend aus dem Artikel. 
rationalitäten, mit Emotionen oder instinktiven Handlungen umgehen zu müssen, um für die IVF Akzeptanz zu gewinnen und sie umzusetzen.

Wie Wissenschaft verstanden wird, zeigt sich in der im Anschluss an Edwards Darstellung geführten Diskussion. Unter den Diskutierenden ist der französische Mediziner und Genetiker Jerôme Lejeune, Lebensschützer und Gründer der konservativen Organisation Laissez-les vivre. Er sieht in der Methode der IVF die Möglichkeit genetischer Manipulation am Menschen, die er kritisiert, und stellt die Frage nach dem Beginn des Lebens. Der Molekularbiologe François Jacob, Mitglied des Organisationskomitees der Tagung und zukünftiges Mitglied der Ethikkommission der DGRST, reagiert im Kontext dieser Diskussion wie folgt:

»Nicht biologische Überlegungen sind in diesem Bereich wichtig, sondern Überlegungen, die man moralisch oder gesellschaftlich nennen kann. Was mir infolgedessen inakzeptabel erscheint, ist, persönliche Meinungen und das Gebot zu verwechseln, andere zwingen zu wollen, seinen eigenen persönlichen Meinungen zu folgen« (Galpérine 1976a: 409/Übers. S.K.).

Indem Jacob sagt, dass es sich um moralische und gesellschaftliche Überlegungen handelt, weist er Lejeune implizit darauf hin, dass sein Beitrag eher moralisch oder gesellschaftlich gehalten ist und nicht bio-logisch. Biologie scheint in der Ausführung von Jacob getrennt von Moral und Gesellschaft. Eine Moral, die eine klare Haltung zum Beginn des Lebens hat, gegenüber der Abtreibung und der Forschung am Ungeborenen, eine Position, die möglicherweise andere überzeugen will, erscheint inakzeptabel. Möglicher Verstärker dafür, die »moralischen Überlegungen« Lejeunes als »zwingend « zu kritisieren, ist der Diskurs um Abtreibung, der zeitlich parallel stattfindet. Lejeunes Laissez-les vivre fordert u.a. eine moralische und staatsbürgerliche Erziehung der Abtreibungsbefürworterinnen und -befürworter (Ferrand-Picard 1982: 387). Jacob ist insofern nicht alleine mit seiner Moral-Kritik an Lejeune, da sich die Frauen in ihrem »Manifest der 343« aus dem Jahr 1971 gegen einen »moralischen Druck« wenden und dabei namentlich Lejeune nennen (vgl. Abba-Sidick et al. 1971). Das Beispiel verdeutlicht, dass auf der Tagung eine persönliche Meinungsäußerung zwar akzeptiert wird, nicht aber eine Position, die überzeugen will. An der Art und Weise, wie gesprochen werden kann und soll, zeigt sich zum einen, dass das Infragestellen wissenschaftlicher Entwicklungen ebenso wenig erwünscht ist, wie der Versuch, andere zu überzeugen. Denn die »moralischen und gesellschaftlichen Überlegungen« scheinen zum Problem werden zu können. Zum anderen zeigt sich auch, dass eine Trennlinie zwischen Wissenschaft und Moral bzw. Gesellschaft gezogen wird. Zwar sollte die Tagung ein Ort sein, an dem sich durch den Austausch die Disziplinen und die Sichtweisen mi- 
schen konnten (Galpérine 1976b: IX), aber scheinbar unter dem Vorbehalt, dass Biologie, Moral und Gesellschaft getrennte Bereiche sind.

Jacob spricht zwar nur von »Moral«, Edwards von »moralischen und ethischen Aspekten«, beide Perspektiven erinnern m.E. aber an eine éthique à l'américaine in Form der Ethik des US-amerikanischen Hastings Center bzw. des Institute of Society, Ethics and the Life Sciences, die im zeitlich parallel stattfindenden Asilomar-Prozess zum Tragen kommt. Indem davon ausgegangen wird, dass wissenschaftliche Entwicklungen erst dann, wenn sie aus dem Labor in den gesellschaftlichen Raum eintreten, auch mit Moral oder Ethik in Kontakt kommen, erscheinen sie zuvor als neutral oder objektiv (vgl. Stevens 2000: 47). Die Perspektive erinnert auch an den Entwurf des Singer-Briefes, der während des AsilomarProzesses entsteht. In diesem Entwurf werden »moral and ethical issues« auf gesellschaftlicher Seite verortet, nicht auf wissenschaftlicher. Wie Jacob und Edwards Moral und/oder Ethik auffassen, erinnert darüber hinaus an die Perspektive Paul Bergs auf die »ethische Beurteilung «, wie er sie auf einer internationalen Tagung zu Gentechnologie im Oktober 1974 im schweizerischen Davos verdeutlicht. Sie stehen für ihn im Kontrast zu einem wissenschaftlichen »sound practical reason « einem stichhaltigen, vernünftigen und technischen Grund (vgl. Lewin 1974). Der Kontext der Aussage war, welchen Grund es für Berg geben könne, seine Forschung zu Gentechnologie zu stoppen - eine »ethische Beurteilung« von gesellschaftlicher Seite zählen für ihn nicht.

Was sich in dem Leitsatz der Tagung an der Sorbonne »Neue Fähigkeiten der Wissenschaft, neue Pflichten für den Menschen« (Mallet 1976a: VIII/Übers. S.K.) zeigt, und auch in der Art und Weise, wie sich akzeptierte Sprecher- und Sprecherinnenpositionen herausbilden, ist, dass biologische Entwicklungen bzw. die IVFForschung nicht in Frage gestellt werden sollen. Ebenso nicht in Frage gestellt werden soll, dass sich Menschen und ethische, moralische oder gesellschaftliche Beurteilungen früher oder später an wissenschaftliche und technologische Entwicklungen anpassen (können und sollen). Ethische und moralische Rahmungen tauchen nicht allein im medizinischen oder gentechnologischen Diskurs auf, sondern auch im biologischen/interdisziplinären. Im medizinischen und gentechnologischen Diskurs zeigen sie sich als eine Antwort auf Problematisierungen der Selbststeuerung wissenschaftlicher und technologischer Entwicklungen. Im biologischen/interdisziplinären Diskurs wird aber nun keine Ethikinstitution eingerichtet, sondern der MURS ins Leben gerufen und mit ihm ein Konzept der wissenschaftlichen Verantwortung. Inwiefern schien es notwendig, sich auf Verantwortung zu berufen? Antworten auf diese Frage finden sich in den Tagungsbeiträgen und der Presse, auf die ich im Folgenden eingehe. 


\subsection{Wissenschaftliche Verantwortung $=$ Wohltaten und Risiken}

In den Abendnachrichten des öffentlich-rechtlichen Fernsehens wird über die Tagung berichtet. Der Bericht wird damit eingeleitet, dass Wissenschaftler und Wissenschaftlerinnen sowie Nobelpreisträger und -trägerinnen aus der ganzen Welt an der Sorbonne zusammengekommen seien, um über die Konsequenzen des wissenschaftlichen Fortschritts zu sprechen (Laury 1974). In seinem Interviewbeitrag in diesen Abendnachrichten nennt Raymond Aron, Mitglied des Organisationskomitees, die Beunruhigungen der Wissenschaftler und Wissenschaftlerinnen in Bezug auf die Zukunft der Menschheit: Beunruhigungen aufgrund der schnell wachsenden Bevölkerung, des biologischen Ungleichgewichts ${ }^{10}$ und der Möglichkeiten der Gentechnologie, das menschliche Erbgut zu manipulieren (ebd.). Angesichts dieser Entwicklungen hebt Mallet, der ebenfalls für die Abendnachrichten interviewt wird, ausdrücklich die Kompetenz der Wissenschaft hervor sowie die Notwendigkeit politische Entscheidungsträgerinnen und -träger wissenschaftlich zu informieren (ebd.). Wie Wissenschaft verstanden werden soll, verdeutlicht Mallet in seinem Beitrag aus der Abschlussrunde der Tagung. Er sagt:

»Thus we are all convinced that political power divorced from moral duty [of science, S.K.] is bound to land in tyrannical anarchy. Yet this realization, backed as it is by so many disastrous experiences, has still not penetrated into the realm of science. In this special field [...], there are far too many who still delude themselves into believing that >revelatory< science can spread abroad its influence simply by virtue of its objective research and discoveries in a kind of neutrality and independance [sic! S.K.] that shelter it from the greed of power and protect it from its own power temptations, thus conferring on it a true built-in immunity. [...] this kind of science has nothing to do with our Conference« (Mallet 1976d: 563/Herv. i.O.).

Neu ist an Mallets Beitrag, dass außerhalb des Spektrums der new critical science movement oder der Science du Peuple eine sich neutral und objektiv verstehende Wissenschaft vehement kritisiert wird. Mallet sagt, dass die Trennung von politischer Macht und moralischer Pflicht der Wissenschaft zu tyrannischer Anarchie führe, wobei der Begriff Anarchie m.E. allein durch das Adjektiv tyrannisch negativ konnotiert wird. Damit spricht er sich gegen diese Trennung aus. Während der Tagung aber betont er, dass Moral nicht mit der wissenschaftlichen Praxis ver-

10 Was Aron unter dem Ausdruck »biologisches Ungleichgewicht « versteht, sagt er in den Abendnachrichten nicht. Im Tagungsband wird aber deutlich, dass damit bspw. das gestörte Gleichgewicht mikrobieller Populationen in Boden und Wasser aufgrund chemischer Belastungen durch Abfall gemeint ist. 
knüpft sei: »There's no doubt that science comes from experiment and observation without reference to morals. But its implications, conditioning inevitably the spiritual and social life of Man, will be studied« (Mallet 1976c: 542). Die »umfassende« Verantwortung der Wissenschaftlerinnen und Wissenschaftler sowie der Medizinerinnen und Mediziner liegt in der Bewertung nicht messbarer Risiken neuer Techniken (Galpérine 1976b: IX/Übers. S.K.). Dennoch gibt es für Mallet eine Moral. Er spricht rückblickend, wie eingangs bereits erwähnt, »von einer Moral, die der unverzichtbaren Objektivität der Wissenschaft nicht widerspricht « (Mallet 1986/1987: 21f/Übers. S.K.). Moral bedeutet in Mallets Perspektive nicht, wissenschaftliche Praxis moralisch zu sehen, sondern gerade ihre Objektivität zu schützen. Dementsprechend gehe ich davon aus, dass er, wenn er von Risiken spricht, eine technische Vorstellung von Risiken hat, nicht eine politische. Welchen Kontext Mallet vor Augen hat, wenn er davon spricht, dass die Trennung von politischer Macht und moralischer Pflicht der Wissenschaft zu tyrannischer Anarchie führe und was tyrannische Anarchie überhaupt ist, wird nicht ausgeführt. Auch die »desaströsen Erfahrungen« werden nicht konkretisiert. Es handelt sich m.E. bei dieser bedeutungsvoll klingenden Terminologie eher um stilistische Mittel, um eine Wissenschaft, die ihre gesellschaftlichen Auswirkungen als neutral und objektiv erachtet, zu kritisieren und gegen eine immune, von der Politik abgegrenzte Wissenschaft zu appellieren. Insofern nicht abgegrenzt, da es um die Notwendigkeit geht, politische Entscheidungen wissenschaftlich zu informieren, wie Mallet in den Abendnachrichten sagt (Laury 1974). Es scheint also Mallet zufolge, dass wissenschaftliche Praxis nichts mit Moral zu tun habe, dass es aber eine moralische Pflicht der Wissenschaft gegenüber der Politik gebe, die desaströse Erfahrungen verhindern kann. Die Verantwortung der Wissenschaft scheint darin zu bestehen die Wohltaten und Risiken (möglicher) wissenschaftlicher Auswirkungen zu evaluieren und die Politik zu informieren.

\subsection{Mehr »shifted responsibilities " $^{11}$ und weniger Moral}

Die Vorstellung, dass desaströse Erfahrungen durch Moral verhindert werden können, erinnert an die Vorstellung von Moral im medizinischen Diskurs in Bezug auf die Verbrechen der NS-Medizin. Im Jahr 1949 empfiehlt die nationale Medizinakademie in Frankreich, eine »Kommission zur Förderung der moralischen Bildung des Arztes« einzurichten. Im Nachrichtenblatt der Akademie heißt es, dass die moralische Bildung des Arztes in unserer Zeit wichtiger sei als je zuvor 
(Académie Nationale de Médecine 1949: 231). Auch in einer Diskussionsrunde auf der Tagung an der Sorbonne zum Thema »Problems posed by therapeutic experimentation on man « geht es um eine Moral zum Schutz vor etwas: In Anbetracht möglicher Exzesse wissenschaftlicher Entwicklungen werden moralische Verhaltensregeln gefordert, an die sich jeder zukünftige Forscher und jede Forscherin halten kann, so Carlos Chagas in seiner Einleitung zur Diskussionsrunde (Chagas 1976: 34). Chagas $(* 1910-\uparrow 2000)$, brasilianischer Mediziner und Biophysiker, Direktor des biophysikalischen Instituts der Universität von Rio de Janeiro ist zum Zeitpunkt der Tagung Direktor der Päpstlichen Akademie der Wissenschaften. Um welche Exzesse es sich handelt, präzisiert er an dieser Stelle nicht, zitiert aber in seinem Vortrag den Nürnberger Kodex und bezieht sich auf die Deklaration von Helsinki. Entsprechend vermute ich, er nimmt mit »Exzessen« Bezug auf die Experimente der NS-Medizin. Darüber hinaus bezieht sich Chagas auf Ethikkomitees, die bereits an Forschungsinstituten bestünden und spricht sich für die Einrichtung weiterer Ethikkomitees aus. Für ihn sind sie eine mögliche Antwort auf problematische Situationen im Verhältnis zwischen Arzt oder Ärztin und Patient oder Patientin im Kontext der Zustimmung des Patienten oder der Patientin zu einem medizinischen Versuch. $\mathrm{Zu}$ diesen problematischen Situationen wirft er folgende Fragen auf: »Soll man es also so verstehen, dass die Zustimmung, so wie sie im Dekalog von Nürnberg gefordert ist, die alleinige Verantwortung des Individuums ist, das das Experiment durchführt? Kann diese Verantwortung nicht delegiert werden?« (ebd.: 33/Übers. S.K.).

Die »shifted responsibilities«, die die deutsche Sozialwissenschaftlerin Helen Kohlen im Kontext von Ethikkomitees an Krankenhäusern in den USA und Deutschland beobachtet (Kohlen 2011: 127), werden im Kontext medizinischer Forschung auf der Tagung an der Sorbonne explizit gefordert. Wie in Kapitel III aufgezeigt, kann auch im Kontext des Ethikkomitees von INSERM von »shifted responsibilities « gesprochen werden: Nicht mehr allein die Verantwortung des Mediziners oder der Medizinerin ist für das Durchführen eines Versuchs relevant, sondern nun auch eine übergeordnete Instanz in Form eines Expertinnen- und Expertenkomitees. Dieses übergeordnete kollektive Gewissen prüft das individuelle Gewissen des Arztes oder der Ärztin; es findet also eine Art antipaternalistischer Entzerrung des Verhälnisses zwischen Arzt oder Ärztin und Patient oder Patientin statt. Die »shifted responsibilities« zeigen sich während der Tagung in Chagas Vortrag in Form von Ethikkomitees und auch bei der Präsentation der Diskussionsergebnisse $\mathrm{zu}$ therapeutischen Experimenten in der Abschlussrunde. In letztgenannter Runde wird sich sowohl für transnationale als auch nationale Komitees ausgesprochen »in order to prevent the carrying out of human experimentation which would be dangerous in Man, dangerous to the community and dangerous in 
the long run« (Milliez 1976: 488f). Teilnehmen sollten nicht nur Ärztinnen und Ärzte oder Wissenschaftlerinnen und Wissenschaftler, sondern darüber hinaus Psychologinnen und Psychologen, Ökonominnen und Ökonomen, Soziologinnen und Soziologen sowie Patientinnen und Patienten (ebd.: 489). Allerdings werden diese Komitees in der Abschlussrunde nicht Ethikkomitees genannt, sondern schlicht Komitees. Auch die Moral in Form der vorgeschlagenen moralischen Verhaltensregeln für Forscher und Forscherinnen wird nicht thematisiert. Es wird nicht mehr von »Exzessen«, sondern von »Versuchen«, die für den Menschen gefährlich sein könnten, gesprochen. Diese Gefahren könnten durch Komitees gebannt werden. Im Kontext der Diskussion um therapeutische Experimente scheinen am Ende der Tagung die Sprache der Moral und der Ethik zu verschwinden.

\section{$1.6 »[R]$ esponsibility to and for science « $?^{12}$}

Medizinische Exzesse werden zwar in der Diskussionsrunde zu therapeutischen Experimenten benannt, aber nicht mehr in der gemeinsamen Abschlussrunde der Tagung. Was vermutlich mit dem Begriff tyrannische Anarchie gemeint ist oder um welche desaströsen Erfahrungen es sich handelt, die Mallet anspricht, wird in einer der Abschlussreden deutlich. In dieser erinnert Staatspräsident Giscard d'Estaing daran, dass es eine Entscheidung eines amerikanischen Präsidenten und eine Gleichung von Einstein war, die Hiroshima zerstörte und wodurch die Beziehung von Wissenschaft und Politik problematisch wurde: »that the crucial issue of the relationship between science and politics was now definetely in the open « (Monsieur le Président de la République Giscard d'Estaing 1976: 583). Nicht allein Einsteins Gleichung und der Atombombenabwurf auf Hiroshima sind für Giscard d'Estaing die Ursache dafür, dass die Beziehung von Wissenschaft und Politik problematisch wurde, sondern die Entscheidung des amerikanischen Präsidenten. Ebenso bezieht sich der Franzose René Maheu (*1905-†1975), Generaldirektor der UNESCO, in seinem Tagungsbeitrag auf Hiroshima (Maheu 1976: 576) - der Atombombenabwurf auf Nagasaki wird weder von ihm noch von Giscard d'Estaing thematisiert. Maheu betont ein weiteres Problem. Der französische Philosoph meint:

»Down the centuries there has been none like ours to witness such a great, wide-ranging and crucial progress of science. [...] The triumphs of technology as derived from science are $[\ldots]$ spectacular. [...] And yet, in this very era of triumphs to which nothing in the past can com-

12 Monsieur le Président de la République Giscard d'Estaing 1976: 585. 
pare, we witness the spectacle of science being challenged, partly by a rather undefined disenchantment, partly by declared opposition - an onslaught mainly on the part of exactly those who have derived the greatest benefit from its intellectual and material achievements. There is every indication that science has lost [...] some of its one-time prestige, and even more of the trust once reposed in its ability to ensure the progress and the happiness of human race $\ll$ (ebd.: 574).

Maheu verweist auf einen Prestigeverlust und ein mangelndes Vertrauen in die Fähigkeit der Wissenschaft, Fortschritt zu sichern und der Menschheit Glück zu bringen. Giscard d'Estaing hingegen thematisiert die politische Entscheidungsgewalt eines Einzelnen. Er konzentriert sich zudem auf die negativen Auswirkungen der Wissenschaft selbst, hier die Atombombe, als Ursache für die problematisch gewordene Beziehung von Wissenschaft und Politik. Die Art und Weise der Thematisierung Hiroshimas ist insofern neu, als zu dem Zeitpunkt, an dem der Atombombenabwurf 1945 geschah, in Frankreich lediglich die falsche Anwendung von Wissenschaft thematisiert wurde, nicht aber die Wissenschaft selbst (vgl. Bonneuil 2004: 20f). Vermutlich zeigt sich hier ein Einfluss aus dem atomkritischen Diskurs, denn erst mit ihm kommt in den 1970er Jahren eine Wissenschaftskritik auf. Die Lösungsvorschläge von Maheu und D'Estaing sehen entsprechend unterschiedlich aus. Der Staatspräsident meint:

»Basically, on my own attitude, as I have said, inclines towards an approach of collective responsibility to and for science. Accordingly, I think that progress will result from a joining of forces between scientists and the rest of mankind, with special reference to politicians, rather than from an in camera brains trust of a close shop of scientists « (Monsieur le Président de la République Giscard d'Estaing 1976: 585/Herv. i.O.).

Der Staatspräsident hält gerade die kollektive Verantwortung für die Wissenschaft für wichtig, nicht allein eine Verantwortung der Wissenschaftlerinnen und Wissenschaftler. Das Konzept der Verantwortung für etwas beinhaltet m.E. die Idee, Wissenschaft nicht sich selbst zu überlassen, sie zu beobachten, zu lenken, ggf. einzuschreiten, damit kein Schaden entsteht. Giscard d'Estaing richtet sich damit implizit gegen das Prinzip der Selbststeuerung von Wissenschaft und Technik, aber auch gegen die Entscheidungsgewalt eines einzelnen Staatsoberhauptes über die Anwendung von Technologien. Indem Verantwortung für die Wissenschaft übernommen werden soll, zweifelt der Staatspräsident zudem die Autorität der Wissenschaft an, (allein) zur Problemlösung beizutragen. Fortschritt kann nach Giscard d'Estaing nur erreicht werden, wenn es einen Zusammenschluss gibt, insbesondere mit der Politik, er entsteht nicht mehr per se aus wissenschaftlichen und technolo- 
gischen Entwicklungen. Maheu, der von einem Vertrauensverlust in die Wissenschaft ausgeht, hebt wiederum, wie schon Mallet, die besondere wissenschaftliche Kompetenz in der Lösung gegenwärtiger Probleme hervor:

»Moreover, it stands to reason that, since the crisis arose out of the advances made by science, the scientists are in the most propitious position to define its nature and find the requisite answers and remedies « oder »this duty is above all incumbent by the scientists who are in a better position than anyone else to demonstrate the importance of reason to humanity « (Maheu 1976: 575f).

Die Pflicht, von der Maheu spricht, oder die moralische Pflicht der Wissenschaft, von der Mallet spricht, besteht also darin, zur Lösung der Probleme, die aus wissenschaftlichen und technischen Entwicklungen entstehen, beizutragen. Die Position und Fähigkeit, die den Wissenschaftlerinnen und Wissenschaftlern zugeschrieben wird, wird von einem Tagungsteilnehmer kritisch hinterfragt, Georges Canguilhem. Er bezieht sich auf den Leitsatz der Tagung »New powers of Science, new duties of men « und sagt in seiner Zusammenfassung zu dem Themenschwerpunkt »Quality of life. Dignity of death« in der Abschlussrunde:

»Finally the more general theme of this Conference: New powers of Science, new duties of Man, then becomes: New duties of scientists as to the power they exercise. Do biologists and physicians think that, in their own way they are men of power? If they recognize their power, are they prepared to exercise it? Are they ready to cooperate with other power bodies, and which ones? Has the moment not arrived, for the scientists, to recognize that the scientific discourse is not enough to solve the problems of which, thanks to their scientific knowledge, they have a lucid awareness, but which concern them as men, like all men « (Canguilhem 1976: 537/Herv. i.O.).

Neu an Canguilhems Beitrag ist, dass er im Kontrast zu Maheu und Mallet nicht von Pflichten der Wissenschaft in Anbetracht ihrer neuen Fähigkeiten spricht. Genauso wenig spricht er von den, wie es in dem Leitsatz der Tagung heißt, neuen Pflichten für den Menschen (Man). Vielmehr thematisiert er die Macht der Wissenschaftlerinnen und Wissenschaftler als Menschen (men of power) und ihre Pflichten. Für Canguilhem steht Wissenschaft nicht außerhalb von Machtbeziehungen. Diese Perspektive beinhaltet m.E. kein räumlich trennendes Denken in dem Sinn, dass Wissenschaft erst außerhalb des Labors nicht mehr neutral ist oder sich über ihre Auswirkungen Gedanken gemacht werden muss - Wissen ist in seinen Augen per se mit Macht verknüpft. Daher rührt auch die Frage Canguilhems, mit welchen 
anderen Machtbereichen Wissenschaftler und Wissenschaftlerinnen bereit sind zu kooperieren - Wissenschaft versteht er als einen Machtbereich von vielen.

Auf der Tagung zeigen sich verschiedene Problematisierungen: Problematisiert wird das Verhältnis von Wissenschaft und Politik (D'Estaing und Mallet) und ihre Selbststeuerung (D'Estaing), die Krise der Wissenschaft seit dem Bombenabwurf auf Hiroshima und der Vertrauensverlust der Öffentlichkeit in die Wissenschaft (Maheu) sowie die alleinige Lösungskompetenz der Wissenschaft (D'Estaing und Canguilhem). Auf welche Problematisierungen antwortet nun der MURS?

\section{Der Mouvement Universel de la Responsabilité SCIENTIFIQUE: LÖSUNG WOFÜR?}

Dem MURS wird eine umfassende Lösungskompetenz zugeschrieben. Wie Maheu meint: »A Movement like that $[\ldots]$ sets out its highly desirable aims in precise terms, seems to me to meet all the requirements of the situation as well as the expectation of the public « (Maheu 1976: 577). Der MURS erscheint hier als etwas, dass »to meet all the requirements « - »alle Anforderungen erfüllen kann«. Wenn Maheu von »all« spricht, bedeutet dies, dass er dem MURS die Kompetenz zuspricht, zur Lösung der Krise beizutragen, in der sich die Wissenschaft seit dem Atombomenabwurf auf Hiroshima befinde. Darüber hinaus spricht er der Wissenschaft die Fähigkeit zu, zur Lösung des von ihm benannten Vertrauensverlustes in die Fähigkeit der Wissenschaft, Fortschritt zu sichern und der Menschheit Glück zu bringen, beizutragen. Dieser Vertrauensverlust ist nach Maheu durch eine Opposition hervorgerufen worden, die selbst von den geistigen und materiellen Errungenschaften der Wissenschaft profitiert habe (ebd.: 574). Es handelt sich hierbei m.E. um eine Anspielung auf das wissenschaftskritische Spektrum der Science $d u$ Peuple, das sich vor allem aus Akademikern und Akademikerinnen zusammensetzt. Möglicherweise bezieht sich Maheu auch international auf die new critical science movement. Maheu und auch Mallet schreiben den Wissenschaftlern und Wissenschaftlerinnen eine außerordentliche Fähigkeit zu, der Menschheit die Wichtigkeit der Vernunft zu demonstrieren (ebd.: 576). Indem die Wissenschaftler und Wissenschaftlerinnen, die sich mit dieser Position und dem MURS identifizieren, sich somit im Rahmen wissenschaftlicher Verantwortung bewegen und der Vernunft verpflichtet sind, wird die Opposition, die die Wissenschaft angreift (»onslaught« ebd.: 574), als unvernünftig und verantwortungslos gegenüber der Menschheit und der Zukunft des Planeten dargestellt. Mit dem MURS wird eine Öffnung des wissenschaftsinternen Diskurses demonstriert. Dieser wissenschaftsinterne Diskurs wird sowohl von Giscard d'Estaing als auch von Georges Canguil- 
hem kritisiert. Teilnehmen sollen im MURS nicht ausschließlich Wissenschaftler und Wissenschaftlerinnen aller Disziplinen, sondern auch Interessierte. Auch die Pflicht der Wissenschaftlerinnen und Wissenschaftler wird aufgegriffen, die sowohl von Canguilhem, Mallet und Maheu erwähnt wird. Allerdings setzt sich mit dem MURS eine Form der Pflicht durch, die darin besteht, eine informierte öffentliche Meinung zu fördern und keine Pflicht, sich mit der eigenen Machtposition auseinanderzusetzen, wie Canguilhem es für notwendig hält. Informiert werden soll über die Wohltaten und Risiken wissenschaftlicher Entwicklungen. Deren Definition soll in wissenschaftlichen Händen sein, da den Wissenschaftlern und Wissenschaftlerinnen diese Kompetenz zugesprochen wird. In dem Konzept der wissenschaftlichen Verantwortung erscheint vor allem die Pflicht des Wissenschaftlers oder der Wissenschaftlerin, mögliche Auswirkungen wissenschaftlicher Entwicklungen zu evaluieren. Evaluiert werden sollen die Wohltaten und Risiken, die selbst definiert werden. Während auf der Tagung noch von moralischer Pflicht der Wissenschaft gesprochen wurde oder auch von moralischen Verhaltensregeln für Forscherinnen und Forscher im Kontext therapeutischer Versuche, taucht die Sprache der Moral sowohl in der Resolution als auch in dem Statut des MURS nicht mehr auf. Nicht einmal mehr in Form von »moralischen und ethischen Aspekten«, die auf Seite der Gesellschaft verortet werden, wie in der vorgestellten Diskussionsrunde zur IVF, wird die Sprache der Moral und Ethik noch sichtbar.

Dies erinnert an den Entstehungsprozess des Singer-Briefes, an seinen Entwurf und dessen Überarbeitung im Asilomar-Prozess. Die Endversion des Briefes geht an die US-amerikanische Nationale Akademie der Medizin sowie die Nationale Akademie der Wissenschaften und wird im September 1973 in dem Wissenschaftsmagazin Science publiziert. Gefordert wird in der Endversion des Briefes, ein Komitee zur Untersuchung der Risiken der Gentechnologie und zur Ausarbeitung von Richtlinien für die weitere gentechnologische Forschung einzurichten (Singer/Soll 1973). Während in dem Entwurf des Singer-Briefes noch von »moral and ethical issues « die Rede ist, wird in der Endversion des Briefes von Gefahren für Laborarbeiter und -arbeiterinnen gesprochen. Letztlich dominiert im Abschlussdokument der Asilomar-Konferenz die Risiko-Rahmung. Darüber hinaus erinnert der MURS an die Ethikkommission der DGRST, die ebenso im Kontext des Asilomar-Prozesses eingerichtet wurde. Ethik beinhaltet hier, die Richtlinien von Asilomar zur Risikobewertung einzuhalten. Diese Ethikkommission und die Konzeption des MURS stimmen in ihrer Aufgabe, der Risikobewertung, überein. Nur ist die übergeordnete Rahmung der Risikobewertung einmal Ethik, einmal Verantwortung, einmal ist ihr Feld Gentechnologie, einmal Biologie. Ethikkommission und Konzept des MURS stimmen auch darin überein, dass Fragen darüber, ob wissenschaftliche Entwicklungen überhaupt durchgeführt werden sollen, nicht 
thematisiert werden sollen. Denn zentral soll die Diskussion von Wohltaten und Risiken wissenschaftlicher Entwicklungen sein, nicht die Entwicklung selbst. Sowohl das Infragestellen wissenschaftlicher und technischer Entwicklungen als auch ihre Steuerung sind Themen der wissenschaftskritischen Science du Peuple. Aus diesem Spektrum gibt es - wie im Folgenden ausgeführt wird - Reaktionen auf die Tagung an der Sorbonne und das Konzept von Verantwortung.

\subsection{Technokratiekritik: »[D]ie Wissenschaft de-moralisieren ${ }^{13}$}

Der MURS und die internationale Tagung »Biologie und die zukünftige Entwicklung des Menschen « bleiben nicht unkritisiert. Ihnen widmet sich ein Artikel in der ersten Ausgabe der technokratiekritischen Zeitschrift Impascience im Jahr 1975 (N.N. 1975c). Impascience ist wie Survivre oder Politique Hebdo eine der Zeitschriften, die im wissenschaftskritischen, insbesondere atomkritischen Milieu entstanden sind. Das Milieu ist von einer »intellektuellen Elite « geprägt, von Wissenschaftlern und Wissenschaftlerinnen, Juristen und Juristinnen, Journalisten und Journalistinnen sowie Lehrern und Lehrerinnen (Topçu 2007: 4/Übers. S.K.). Es handelt sich m.E. hierbei um die Opposition, von der Maheu auf der Tagung an der Sorbonne spricht: Diese Opposition habe, so Maheu, selbst von den geistigen und materiellen Errungenschaften der Wissenschaft profitiert und sei die Ursache für den Vertrauensverlust in die Wissenschaft (Maheu 1976: 574). Der Artikel in Impascience titelt und diskutiert anhand der Sorbonne-Tagung »le $>$ changement $<$ de l'idéologie de la science « - »die >Wende < in der Ideologie der Wissenschaft « (N.N. 1975c/Herv. i.O., Übers. S.K.). Es entwickle sich, so die Vermutung in Impascience, ein neuer technokratischer Diskurs. Neben rechte, gaullistische, zentralistische Positionen träten nun Positionen, die selbstkritisch scheinen und die versuchten ihre politische Position zu sichern. Diesen Positionen habe die Universitätsreform den Weg geebnet.

Die Universitätsreform, von der hier gesprochen wird, wird nach den Ausschreitungen im Mai '68 initiiert. Sie wird parteiübergreifend unterstützt und »was seen as a means of achieving social peace« (Patterson 1972: 286). Die Reform wird mit dem nach dem damaligen Bildungsminister im Kabinett De Gaulles, Edgar Faure (*1908-†1988), genannten Loi Faure - Faure-Gesetz - umgesetzt. Es beinhaltet »Universitätsautonomie, Mitbestimmung aller Universitätsmitglieder, Pluridisziplinarität durch Auflösung der bisherigen Fakultäten sowie Einführung relativ autonomer Fachbereiche« (Doll/Taubert 2006: 579). Die Mitbestimmung ist 
ursprünglich paritätisch gedacht, erfährt allerdings durch bestimmte Durchführungsbestimmungen Einschränkungen (ebd.). Die Reform umfasst nicht die Grandes Écoles, die in Frankreich für eine Eliteausbildung stehen (Patterson 1972: 281). Erschwert wird die Umsetzung der Universitätsreform durch die staatliche Sparpolitik ab 1974. Darüber hinaus misstraut die konservative Regierung zunehmend den Neuerungen an den Universitäten und versucht, die Entwicklungen stärker zu beeinflussen (Doll/Taubert 2006: 580). Die Verknüpfung in dem Artikel aus Impascience zur Universitätsreform liegt insofern nahe, da Mallet, Initiator der Tagung an der Sorbonne, als Rektor der Académie de Paris, dem Schul- und Universitätsbezirk von Paris, an der Umsetzung der Universitätsreform beteiligt ist (Forest 1990). Ebenso beteiligt ist Michel Alliot, Präsident der Universität VII von Paris, der im Organisationskomitee der Tagung ist (Galpérine 1976a: o.S.). Auch Raymond Aron, ebenfalls im Organisationskomitee, ist zu den Reformerinnen und Reformern zu zählen. Im Kontext des Mai '68, den er als »Psychodrama« bezeichnet, äußert er sich für ein »comité de défense et de rénovation de l'université française « - »Komitee zur Verteidigung und Erneuerung der französischen Universität« (Baverez 1993: 394/Übers. S.K.).

Inwiefern nun die Universitätsreform eine neue Technokratie hervorbringt, wird in dem Artikel in der Zeitschrift Impascience nicht konkretisiert. Dies ist vermutlich darauf zurückzuführen, dass es sich um eine Reform handelt, die im Sinn des Impascience-Artikels nicht elitekritisch war, da bspw. die Grandes Écoles von der Reform ausgenommen und die Mitbestimmungsmöglichkeiten an den Universitäten begrenzt sind. Das Konzept der Mitbestimmung oder Partizipation ist zudem seit De Gaulles Partizipationspolitik mit dem Ziel der Entpolitisierung von Konflikten negativ konnotiert (vgl. Godfrain 2000; Quet 2009: 140f). Die Haltung der gauchistes, der Linksradikalen, interpretiert Michelle Patterson in einer sozialwissenschaftlichen Untersuchung zur Universitätsreform wie folgt: »To participate in its managment, they felt, would force them to be co-opted into the bourgeois state« (Patterson 1972: 293). Sie bevorzugen es, zu protestieren und nicht zu partizipieren (ebd.). Zwar spricht Patterson nicht von der Zeitschrift Impascience oder dem technokratiekritischen Spektrum, sondern von den gauchistes, ich vermute aber, dass die Kritik eine ähnliche ist und dahin geht, die Art der Partizipationspolitik zu kritisieren.

In dem Artikel aus der Zeitschrift Impascience wird diese Entwicklung hin zu einem neuen technokratischen Diskurs exemplarisch anhand der Positionen von Michel Debré, René Maheu, Robert Mallet und Jean Bernard belegt. Debré, Premierminister unter Charles de Gaulle, steht für die konservative, gaullistische Position. Zitiert wird ein Beitrag von ihm aus der Tageszeitung Le Monde von 1974, in dem er sagt: »Savants et chercheurs sont indispensable pour développer notre 
capacité industrielle « - »Gelehrte und Forscher sind unabdingbar, um unsere wirtschaftliche Leistungsfähigkeit zu entwickeln« (Le Monde 28.08.1974 zit. nach N.N. 1975c). Im Kontrast dazu werden die Positionen Mallets, Bernards und Maheus als neu bezeichnet, da sie selbstkritisch scheinen, indem sie einen Vertrauensverlust in die Wissenschaft feststellen oder negative Auswirkungen der Wissenschaft thematisieren, wie Umweltverschmutzung. Es handle sich aber nicht um eine Selbstkritik, so in dem Impascience-Artikel, sondern um die Sicherung politischer Positionen, und zwar insofern, als unter Giscard d'Estaing Forschungsgelder gekürzt werden und es wichtig scheint, für die Forschung Anerkennung zu gewinnen. In dem Artikel werden die Tagung an der Sorbonne und die Entstehung des MURS wie folgt interpretiert:

»Meines Wissens haben niemals Intellektuelle so zynisch ihre Rolle als Wissenspolizisten [frz. scientiflics, S.K.] reklamiert. Die Argumentation ist einfach: Da ja die Wissenschaft gefährlich ist, sind die Wissenschaftler verantwortlich, darum muss man ihnen also erlauben diese Verantwortung zu übernehmen, indem man sie direkt an der politischen Macht teilhaben lässt« (N.N. 1975c: 17/Übers. S.K.).

Kritisiert wird, dass Ursache und Lösung in ein und derselben Personengruppe, den Wissenschaftlern und Wissenschaftlerinnen anhaftet. Und weiter heißt es: »Wir könnten hinter dem Lauf der Geschichte beträchtlich zurück liegen, einem Diskurs gefällig zu sein, der viel zu oft moralistisch und ich-bezogen ist: Wir müssen die Wissenschaft de-moralisieren auf die Gefahr hin, einige Wissenschaftler zu demoralisieren « (ebd.: 18/Übers. S.K.). Während im Tagungsverlauf an der Sorbonne die Sprache der Moral und der Ethik marginalisiert werden, bezeichnet Impascience den neuen technokratischen Diskurs gerade als moralistisch - gewissermaßen als Re-moralisierung. Dieser neue Diskurs soll mit seinem Konzept von Verantwortung durch eine De-Moralisierung angegriffen werden. Eine Re-moralisierung im Sinne des Impascience-Artikels kann sich allerdings nicht durchsetzen, wie noch im folgenden Kapitel (VI) gezeigt wird.

\subsection{Vertrauensverlust der Öffentlichkeit - eine »natural evolution « $?^{14}$}

Anhand der Untersuchung der Tagung an der Sorbonne wird deutlich, dass zum ersten Mal ein Vertrauensverlust der Öffentlichkeit in die Wissenschaft festgestellt 
wird (Maheu 1976: 574). Dieser Vertrauensverlust führte zur Krise der Selbststeuerung der Wissenschaft, so die Problematisierung. Die Lösung liegt in der Öffnung der Reflexion über wissenschaftliche und technologische Entwicklungen.

Von einem Vertrauensverlust der Öffentlichkeit in wissenschaftliche Expertise sprechen auch einige Vertreterinnen und Vertreter der STS. Aus diesem Vertrauensrückgang seien neue Formen der »public justification« der Wissenschaft hervorgegangen, insbesondere partizipative Verfahren (Jasanoff 2003: 226). Zeitlich eingeordnet wird der Vertrauensrückgang der Öffentlichkeit meist in die 1980er und 1990er Jahre, einhergehend mit der Tschernobyl-Katastrophe und der BSE-Krise (Lengwiler 2008: 194; vgl. Callon 1999: 38f; Jasanoff 2003: 223). Wie Irwin in seiner Untersuchung zu GMO-Kontroversen Ende der 1990er Jahre in Großbritannien zeigt, wird die Vertrauenskrise der Öffentlichkeit in die Wissenschaft von Politikern und Politikerinnen festgestellt. Diese sehen den Weg aus der Vertrauenskrise u.a. in Offenheit, Transparenz, Kommunikation und Dialog zwischen Wissenschaft und Öffentlichkeit (Irwin 2006: 307f). Irwin beobachtet eine Abkehr vom alten, technokratischen Modell der Politikberatung und die Entstehung einer neuen »politics of talk «, die sich aber gleichzeitig mit alten Annahmen des Defizitmodells verbindet (ebd.). Defizitmodell meint, dass die Öffentlichkeit »as deficient and misguided in its present >lack of uptake of science«« (Irwin/Wynne 1996: 154/Herv. i.O.) interpretiert wird. In Frankreich zeigt sich, dass im biologischen Diskurs ein Vertrauensverlust bereits Mitte der 1970er Jahre von forschungspolitischen Akteuren und Akteurinnen konstatiert wird. Auch hier formiert sich eine Politik des Sprechens in Form des MURS und seiner Reflexionen, eine Offenheit und die Möglichkeit der Partizipation, hier von Interessierten. Diese Interessierten werden allerdings (noch) nicht als defizitär wahrgenommen.

Auch die internationalen Bildungs- und Wissenschaftsforscherinnen und -forscher Michael Gibbons, Helga Nowotny und ihre Kolleginnen und Kollegen konstatieren in ihren Werken zur »new production of knowledge « einen Vertrauensrückgang der Öffentlichkeit in wissenschaftliche Expertise (Nowotny et al. 2003: 184; vgl. Nowotny 2003; 2008; Gibbons et al. 1994). U.a. mit dem Vertrauensrückgang der Öffentlichkeit sehen die Autorinnen und Autoren einen neuen Modus der Wissensproduktion aufkommen, den sie Modus 2 nennen. Er steht im Kontrast zum Modus 1, der im Wesentlichen als hierarchisch, disziplinär, homogen und akademisch charakterisiert wird. Merkmal von Modus 1 ist zudem eine strikte Trennung von gesellschaftlichen und wissenschaftlichen Akteurinnen und Akteuren. »Mode 1 problems are set and solved in a context governed by the, largely academic, interests of a specific community« (Gibbons et al. 1994: 3). Ziel von Modus 1 ist es, wissenschaftlich abgesichertes Wissen zu erzeugen. Der Übergang von Modus 1 zu 2 wird als fließend beschrieben. Modus 2, die neue Art der Wis- 
sensproduktion, ist als transdisziplinär, heterogen, weniger hierarchisch und kontextabhängig gekennzeichnet. Gesellschaft, Markt und weitere Akteurinnen und Akteure sind in die Produktion des Wissens integriert. Die neue Wissensproduktion stellt sich der gesellschaftlichen Verantwortung und einer breit gefächerten Qualitätskontrolle, die somit nicht mehr innerwissenschaftlich sei. Ziel ist nicht nur sicheres, sondern gesellschaftlich robustes Wissen. Nowotny et al. gehen davon aus, dass Modus 2 durch die enge Beziehung zwischen Wissenschaft und Gesellschaft, Möglichkeiten der Demokratisierung von Expertise biete. Gesellschaft wird zur aktiven Partnerin im Sinne von »we all are experts now« (Nowotny 2003: 155f).

Zurück zur Tagung an der Sorbonne: Was sich hier - mit Nowotny et al. gedacht - zeigt, ist eine Öffnung der wissenschaftlichen Community im Sinne einer weniger strikten Trennung von gesellschaftlichen und wissenschaftlichen Akteurinnen und Akteuren - es sind verschiedene Disziplinen vertreten, NGOs, Politiker und Politikerinnen. Die Diskussion ist transdisziplinär ausgerichtet, auch die Qualitätskontrolle von Wissen, versteht man den MURS als solche, steht Interessierten offen. Insofern wird die Gesellschaft als aktive Partnerin konzipiert. In dieser Hinsicht kann die Tagung als ein Schritt in den Übergang von Modus 1 zu 2 verstanden werden. Unberücksichtigt bleibt in den Werken zur neuen Wissensproduktion jedoch, und hier schließe ich mich der Kritik von Dominique Pestre an, ${ }^{15}$ dass dieser Übergangsprozess von Modus 1 zu Modus 2 schlicht festgestellt wird. Unberücksichtigt bleiben so die Konflikte, Kontroversen, die Frage, welche Alternativen in diesem Prozess zum Umgang mit dem Verhältnis von Wissenschaft und Gesellschaft formuliert werden, welche Lösungsvorschläge es gibt und welche Lösungen letztlich dominieren (Pestre 2003: 247):

»the authors may have underestimated the extent to which these transformations have been the results of political and social choices. This would mean recognizing that the developments they describe are not cases of natural evolution, which have simply to be identified and acknowledged, but are, rather, articulated with alternative and conflicting social, economic, and political projects. In cruder terms, by not sufficiently stressing the oppositions and the social forces at work, these books might tend to naturalize the process of change, and thus deprive social actors of the tools needed for criticism and the construction of alternative ways of managing society and science« (ebd.: 246/Herv. i.O.).

15 Die Werke zur neuen Wissensproduktion führten zu einigen Diskussionen und wurden vielfältig kritisiert (vgl. bspw. Godin 1994; Fuller 2000). Für einen Überblick der wissenschaftlichen Rezeption vgl. insbesondere Hessels und van Lente (2008). 
Vor dem Hintergrund von Pestres Kritik und von Irwins Ergebnissen zeigt sich, dass der konstatierte Vertrauensverlust oder Vertrauenskrise eine bestimmte Problematisierung von bestimmten Akteuren und Akteurinnen ist, die mit bestimmten Lösungen einhergeht. Mit der Problematisierung Vertrauensverlust scheint es nicht mehr notwendig, über die Kritiken und Alternativen, wie die der Science du Peuple sprechen zu müssen. Auch kann die gesellschaftliche Verantwortung, der sich die Wissenschaft stellt, wie Nowotny et al. den Modus 2 charakterisieren, selbst als umstrittenes Konzept verstanden werden: So hält Giscard d'Estaing es bspw. für notwendig, Verantwortung für die Wissenschaft zu übernehmen; wissenschaftskritische Akteure und Akteurinnen wiederum kritisieren die Verantwortung des MURS als Teil eines neuen technokratischen Diskurses.

Was sich in meinem Untersuchungskontext abzeichnet, ist, dass sich in Form des MURS ein kollektives Konzept von Verantwortung durchsetzt. Diese kollektive Verantwortung besteht in der Pflicht über Wohltaten und Risiken wissenschaftlicher Entwicklungen für die Menschheit zu reflektieren. Es geht also nicht, wie bspw. von der US-amerikanischen new critical science movement gefordert, um ein Konzept von Verantwortung, das mit einer Elitekritik einhergeht, sowie mit einem Zusammenschluss mit Nicht-Wissenschaftlerinnen und -Wissenschaftlern wie Armen, Unterdrückten, Arbeiterinnen und Arbeitern. Am MURS teilnehmen sollen Wissenschaftler und Wissenschaftlerinnen sowie Interessierte, d.h. Menschen mit einem Interesse an wissenschaftlichen Entwicklungen und mit einem Interesse an der Diskussion über die Wohltaten und Risiken dieser Entwicklungen. Es geht nicht um eine Wissenschaft, die einen grundlegenden gesellschaftlichen Wandel will: Von der US-amerikanischen new critical science movement wird nicht eine neue Form der Evaluation zur Sicherung wissenschaftlicher und technologischer Entwicklungen gefordert, sondern ein »radical change in society« (Beckwith 1970: 226). Mit dem MURS erscheint ein Konzept von Verantwortung, das im Kontrast zum Konzept von Verantwortung im US-amerikanischen wissenschaftskritischen Diskurs nicht die Thematisierung der Frage beinhaltet, wer über Forschungsrichtungen und die Anwendung von Wissenschaft entscheidet. Im französischen Diskurs ist es neu, auch im wissenschaftskritischen. Eine vordergründige Rolle spielt hier nicht die Frage der Verantwortung, sondern Technokratiekritik und die Wiederaneignung von Wissen.

In dieser Zeit sind gerade wissenschafts- und medizinkritische Diskurse aktuell, insbesondere die Kritik an Technokratie. Wissenschaftlicher und technologischer Fortschritt in Frankreich wird nun staatspolitisch nicht mehr als Kernelement nationaler Entwicklungsfähigkeit betrachtet. Vor diesem Hintergrund gesehen, kann die Tagung als Versuch verstanden werden, wissenschaftliche Expertise in ein positives Licht $\mathrm{zu}$ stellen. Ihr wird eine umfassende Lösungskompetenz zuge- 
schrieben, nicht allein für nationale Probleme, sondern auch für Probleme, die die Menschheit betreffen. Neu ist, dass sowohl mit der Tagung als auch mit dem MURS eine Öffnung im Hinblick auf die Diskussion von Implikationen wissenschaftlicher Entwicklungen für die Gesellschaft stattfindet - die Diskussion ist nicht mehr allein innerwissenschaftlich, wie dies auf der Asilomar-Konferenz der Fall ist. Vertreterinnen und Vertreter aus Wissenschaft, Politik und NGOs zeigen öffentlich Verantwortung. Allerdings ist dies eine Form von Verantwortung, die darauf basiert, in einer Reflexionsgruppe technische Risiken zu evaluieren.

Während im linearen Modell wissenschaftliche Entwicklungen per se Fortschritt, ökonomisches Wachstum und Allgemeinwohl bedeuten, mit einer Betonung auf der unabhängigen, von der Politik getrennten Wissenschaft, gibt es verschiedene Stimmen auf der Tagung, die sich gegen eine von der Politik getrennte Wissenschaft aussprechen. Es geht zudem nicht mehr um das Allgemeinwohl einer Gesellschaft oder Nation, sondern um Wohltaten und übergreifend um das Überleben der Menschheit und des Planeten Erde. Allgemeinwohl bzw. Wohltaten entstehen allerdings nicht mehr per se aus der Wissenschaft, sondern aus der unabhängigen wissenschaftlichen Reflexion. Inhalt der Reflexion ist, wie letztlich auch im Abschlussdokument der Asilomar-Konferenz, die Bewertung von technischen Risiken. Sowohl im Kontext von Asilomar in Form der Risikobewertung von Versuchen mit rDNA-Technologie als auch im Kontext der Tagung an der Sorbonne in Form des MURS wird ein permanentes Instrumentarium zur Reflexion und zur Stimulierung einer Reflexion in der Öffentlichkeit eingerichtet. Im Kontrast zum Abschlussdokument der Asilomar-Konferenz, das eine Individualisierung der Verantwortung beinhaltet, eine Verantwortung, die der Forscher oder die Forscherin trägt, gibt es in der Konzeption des MURS zudem eine kollektive Verantwortung: »The consciousness of individual and collective responsibility will draw together the participants of the >Movement « (Mallet 1976c: 542/Herv. i.O.).

Die Idee der Reflexion oder einer Reflexionsgruppe taucht nicht allein im Kontext des MURS auf, sondern ein weiteres Mal wenige Jahre später im Jahr 1979 in einem Bericht, den der damalige Staatspräsident Giscard d'Estaing in Auftrag gibt. Es ist nicht allein die Idee einer Reflexionsgruppe, die aufkommt, sondern darüber hinaus die Idee des Dialogs mit der Gesellschaft. Allerdings sind diese Reflexion und der Dialog nicht mit Verantwortung verknüpft, sondern mit Ethik. 


\section{Reflexion und Dialog als antwort auf ethische Problematisierungen UND ZUM SCHUTZ DER FORSCHUNGSFREIHEIT}

Im Jahr 1978 gibt Giscard d'Estaing einen Bericht in Auftrag, der 1979 mit dem Titel »Sciences de la vie et société« - »Lebenswissenschaften und Gesellschaft« (Gros et al. 1979/Übers. S.K.) publiziert wird. Beauftragt werden drei Wissenschaftler: der Molekularbiologe François Gros, zu dieser Zeit Direktor des Institut Pasteur, Professor am Collège de France und späteres Mitglied des CCNE, sein Kollege am Institut Pasteur und am Collège de France, der Nobelpreisträger François Jacob sowie der Mediziner und Universitätsprofessor Pierre Royer, Kollege Robert Debrés, späteres Mitglied des CCNE und zu dieser Zeit Präsident des Verwaltungsrates des Institut Pasteur sowie Berater der DGRST. Was führte zu dem Bericht?

\subsection{Der Bericht »Lebenswissenschaften und Gesellschaft « ${ }^{16}$}

Staatspräsident Giscard d'Estaing vermutet für die Lebenswissenschaften eine »bioindustrielle Revolution« (Le Président de la République M. Valéry Giscard d'Estaing 1978: 3/Übers. S.K.) und eine ähnliche Entwicklung wie in den Physikwissenschaften. Er formuliert in dem Anschreiben an die Wissenschaftler:

»So, wie die Physikwissenschaften dazu beitragen, die gesellschaftliche und industrielle Organisation zu gestalten, besonders durch ihre Anwendungen im Bereich der Energie, des Verkehrs, der Kommunikation, der Elektrophonik ${ }^{17}$ und der Informatik, so sind die Lebenswissenschaften aufgerufen, einen maßgeblichen Einfluss auszuüben, insbesondere durch ihre Auswirkungen auf die Medizin, die Pharmazie, die Chemie, die Landwirtschaft, die Ernährung, die Energieproduktion und auf den Umweltschutz« (ebd.: 3/Übers. S.K.).

Der Staatspräsident vertraut Gros, Jacob und Royer die Aufgabe an,

»gesellschaftliche Konsequenzen der Entdeckungen der modernen Biologie [zu untersuchen, S.K.], die imstande sind, Auswirkungen auf die Organisation und die Funktionsweise der Gesellschaft zu haben, die nützlichen biotechnologischen Anwendungen für den Fortschritt

16 Gros et al. 1979/Übers. S.K..

17 Frz. électrophonique - ein veralteter Begriff, hierzu zählen bspw. Schallplattenspieler. 
und das menschliche Wohl zu erfassen und eigene Mittel für die Umsetzung dieser Anwendungen vorzuschlagen« (ebd.: 4/Übers. S.K.).

Im Kontrast zur Tagung »Biologie und die zukünftige Entwicklung des Menschen« im Jahr 1974 scheint sich Giscard d'Estaing im Jahr 1978 auf das lineare Modell wissenschaftlicher und technischer Entwicklungen zurück zu besinnen. Nicht nur der Atombombenabwurf auf Nagasaki, der bereits auf der Tagung an der Sorbonne nicht erwähnt wird, nun scheint auch der Atombombenabwurf auf Hiroshima vergessen: Während Giscard d'Estaing auf der Tagung an der Sorbonne mit der Bezugnahme auf Einstein und Hiroshima das Verhältnis von Wissenschaft und Politik anzweifelt, scheint dies für ihn kein Problem mehr zu sein. Er nimmt sogar explizit Bezug auf die Physikwissenschaften und ihren industriellen und gesellschaftlichen Nutzen im Bereich der Energie. Entsprechend geht es ihm nicht mehr um die Auswirkungen der Atombombe auf die Menschheit, sondern um den industriellen und gesellschaftlichen Nutzen für Frankreich: »Je souhaite que notre pays occupe une place de premier rang « - »Ich wünsche, dass unser Land einen Platz in der ersten Reihe einnimmt « (ebd.: 3/Übers. S.K.). In seiner Amtszeit verfolgt Giscard d'Estaing den Ansatz, auf dem Weltmarkt Nischen für Frankreich zu finden, um international Position zu gewinnen (Gottweis 1997, 327). Allerdings vertraut er der Wissenschaft nicht insofern, als aus ihr per se Fortschritt und menschliches Wohl entsteht - genau dieses muss erst wissenschaftlich untersucht werden. Was wird vor diesem Hintergrund in dem Bericht thematisiert und inwiefern taucht die Sprache der Ethik auf?

Der Bericht beinhaltet einen über 200seitigen Überblick über lebenswissenschaftliche Entwicklungen, Vorschläge zu deren Förderung und ein abschließendes Kapitel zur Wechselwirkung von Biologie und Gesellschaft. Die demografischen Auswirkungen einer zukünftig möglichen Geschlechtswahl durch Präimplantationsdiagnostik werden ebenso thematisiert wie die demografischen Auswirkungen durch das Überaltern der Industriegesellschaften. Gegen den Hunger der Welt biete die Genetische Revolution eine Antwort. Die Qualität des Alters werde durch die medizinische Entwicklung garantiert. Wie schon auf der Tagung an der Sorbonne in Edwards Beitrag ist die Reproduktionstechnologie, wie bspw. die IVF, ein Thema, das explizit ethisch gerahmt ist. Sie werden in dem Kapitel »Mœurs et Pratiques Sociales« $-»$ Sitten und soziale Praktiken« (Gros et al. 1979: 266/Übers. S.K.) behandelt. 


\subsection{Die Lebenswissenschaften und die Befreiung der Frau}

In dem Kapitel »Sitten und soziale Praktiken« werden im Kontext neuer Reproduktionstechnologien perspektivisch folgende Veränderungen gesehen: »alle diese Techniken sind dabei, vollständig die traditionellen Verhaltensweisen im Bereich der Fortpflanzung und der Sexualität zu verändern« (Gros et al. 1979: 266/Übers. S.K.). In ihrer Prognose für die neuen Reproduktionstechnologien greifen die Autoren auf die gesellschaftlichen Auswirkungen zurück, die die Anti-Baby-Pille gehabt habe. Hier heißt es: »Die Errungenschaften der Biologie haben folglich stark dazu beigetragen, die Frau von ihren Verpflichtungen im Haushalt zu befreien« (ebd.: 266/Übers. S.K.). Und weiter heißt es, sie hätten zu einem massiven Eintritt der Frauen in die Arbeitswelt geführt, was soziobiologische Konsequenzen für die Kindererziehung sowie für die intellektuelle und affektive Entwicklung des Kindes habe. Dies könne durch Halbtagsarbeit eines Elternteils gelöst werden (ebd.: 266). Indem auf die Anti-Baby-Pille positiv Bezug genommen wird, als Beitrag zur Befreiung der Frau, erscheint der Beitrag, den die Reproduktionstechnologien leisten könnten, ebenso positiv. Soziobiologische Probleme, die aus der Befreiung entstehen, wie die Konsequenzen für die Kindererziehung, scheinen lösbar. Eine Verknüpfung wird hier zwischen Reproduktionstechnologien und Kontrazeptiva hergestellt. Deutlich wird, dass vor allem vom sozialen Nutzen der neuen Reproduktionstechnologie die Rede ist. Abtreibung, wie noch 1974 in dem Beitrag von Edwards auf der Tagung an der Sorbonne, wird nicht thematisiert. Dies ist vermutlich darauf zurückzuführen, dass der Abtreibungskonflikt in Frankreich durch das VeilGesetz im Jahr 1975 gelöst scheint. Auch eine mögliche Inakzeptanz, Ängste oder Emotionen gegenüber den neuen Reproduktionstechnologien wie noch in Edwards Beitrag werden nicht thematisiert. Ein (möglicher) Konflikt wird hier nicht wahrgenommen. Indem im Zusammenhang mit neuen Reproduktionstechnologien explizit von sich ändernden traditionellen Verhaltensweisen in der Sexualität und Reproduktion, von der Befreiung der Frauen gesprochen wird, verengt sich der Raum, wie über dieses Thema gesprochen werden kann, es erfolgt eine Einordnung. Positionen, wie die von Jerôme Lejeune, Lebensschützer und Gründer der konservativen Organisation Laissez-les vivre, werden ausgeschlossen. Dieser kritisiert noch auf der Tagung 1974 an der Sorbonne die Methode der IVF aufgrund der Möglichkeit genetischer Manipulation am Menschen. Jacob, nun Mitautor des Berichts, hat damals auf der Tagung Lejeunes Beitrag als moralische Position kritisiert, die andere zwingen will, ihr zu folgen. Diese Art der Moral wird in dem Bericht nicht erwähnt, lediglich einige Widerstände im Kontext der Anti-Baby-Pille: »Trotz einiger Widerstände wurde die chemische Verhütung als einflussreicher Faktor der Befreiung aufgenommen« (ebd.: 282/Übers. S.K.). »Widerstände« ge- 
gen die Anti-Baby-Pille gibt es insbesondere durch den Ordre des médecins im Kontext der Legalisierung von Verhütungsmitteln durch das Neuwirth-Gesetz im Jahr 1967. Dessen Definitionsmacht von Moral wird im Kontext der Legalisierung der Abtreibung 1975 zunehmend geschwächt. Diese Moralvorstellung wie auch die Lejeunes werden nicht explizit thematisiert, zentral ist die Befreiung der Frau.

\subsection{Die Lebenswissenschaften und ihre "humanitäre Rolle « ${ }^{18}$}

Im Unterschied zu den Reproduktionstechnologien werden für den Bereich der Gentechnologie, jetzt »génie génétique « genannt, Kontroversen festgestellt: »In der Biologie haben die Entdeckung und die Anwendung genetischer Techniken zum ersten Mal öffentliche Kontroversen über ihre möglichen Gefahren ausgelöst« (Gros et al. 1979: 282/Übers. S.K.). Ob mit der Formulierung »öffentliche Kontroversen« die Kontroversen im internationalen Kontext von Asilomar gemeint sind oder/und speziell die Kontroversen, die in Frankreich Mitte der 1970er Jahre hauptsächlich an den Forschungsinstituten stattfinden, wird in dem Bericht nicht deutlich. Jedoch zeigt sich anhand der Befürchtungen, die die Autoren mit den Kontroversen verbinden, um welche Art Kontroversen es sich handelt. Die Kontroversen, so heißt es in dem Bericht, könnten den Platz der Lebenswissenschaften in der Gesellschaft gefährden: »Was bisher als humanitäre Rolle betrachtet wurde, könnte jetzt häufig als ein Alibi für einen Profit- und Machtwillen erscheinen « (ebd.: 279f/Übers. S.K.). Der Profit- und Machtwille, den die Autoren benennen, ist m.E. eine Anspielung auf die Gentechnologiekritikerinnen und -kritiker, seien es solche aus der amerikanischen new critical science movement oder der französischen Science du Peuple - beide kritisieren die Gefahren, die Profitorientierung und die Entscheidungsverfahren in der Forschung und Entwicklung. Gleichzeitig ist der Profit- und Machtwille einer der Hauptkritikpunkte der französischen Atomkraftgegnerinnen und -gegner, die sich gegen den Messmer-Plan und das Motto »tout électrique - tout nucléaire!« richten, der Frankreich zur Atommacht bringen soll. Als der Bericht »Lebenswissenschaften und Gesellschaft« im Jahr 1979 erscheint, erreicht die Anti-Atombewegung in Frankreich kurz zuvor durch die Demonstrationen gegen den Superphénix in Malville im Jahr 1977 einen Höhepunkt.

18 Gros et al. 1979: 279f/Übers. S.K.. 


\subsection{Die Lebenswissenschaften und die "Gunst der Öffentlichkeit “ ${ }^{19}$}

Atomenergie bzw. Physiktechnologien werden in dem Bericht aber nicht im Kontext der Gentechnologie und ihrer Kontroversen thematisiert, sondern im Kontext der Lebenswissenschaften: »Die Lebenswissenschaften erhalten die Gunst der Öffentlichkeit. Das Wissen über das Leben und der Kampf gegen die Krankheit werden oft als Gegengewicht zu den Exzessen in der Physiktechnologie wahrgenommen« (Gros et al. 1979: 282/Übers. S.K.). Was mit »Exzessen in der Physiktechnologie « gemeint ist, wird von den Autoren nicht konkretisiert. Vor dem Hintergrund betrachtet, dass der Bericht im Auftrag des Staatspräsidenten verfasst wurde, vermute ich, dass damit nicht die französische Atompolitik gemeint ist, auch nicht die Atomwaffentests Frankreichs im Pazifik, sondern wiederum eher, wie auf der Tagung an der Sorbonne, der Atombombenabwurf auf Hiroshima. Die negativen Auswirkungen, die »Exzessen in der Physiktechnologie«, werden in Kontrast zum Kampf gegen Krankheiten gestellt. Sie werden in Kontrast zu den positiven Auswirkungen der Forschung gesetzt, die in der Öffentlichkeit anerkannt sind. Positive Auswirkungen der Lebenswissenschaften insgesamt, nicht nur der Gentechnologie, werden im Kontext der Atomtechnologie thematisiert. So erscheinen Lebenswissenschaften als Gegengewicht zu den »Exzessen in der Physiktechnologie« und nicht etwa die Gentechnologie und ihre Kontroversen als Pendant zur nicht akzeptierten Atomtechnologie. Die Gentechnologiekontroversen werden unter dem Begriff Lebenswissenschaften subsumiert und scheinen nicht mehr nennenswert, sie verschwinden hinter dem neuen Begriff. Im Kontext der Reproduktionstechnologien wird von einigen Widerständen gesprochen, im Kontext der Gentechnologie von Kontroversen; die Lebenswissenschaften und insbesondere der Kampf gegen Krankheiten stehe in der Gunst der Öffentlichkeit. Diese Gunst scheint den Autoren jedoch nicht dauerhaft sicher:

»Bis jetzt war die Entwicklung der Lebenswissenschaften und ihrer Anwendungen sich selbst überlassen: sie schien ganz natürlich an den Wachstum der Humanität und der Förderung des Wohlergehens angepasst. Mediziner und Biologen nahmen eine Art der Selbstregulierung wahr, sie legten Regeln für Experimente am Menschen oder für die Gentechnologie fest. Früher oder später jedoch wird sich die Entwicklung dieser Wissenschaften noch direkter mit der Entwicklung der Gesellschaft, ihren Hoffnungen, ihrer Ethik verbinden müssen « (ebd.: 280/Übers. S.K.).

19 Gros et al. 1979: 282/Übers. S.K.. 
Die Autoren stellen eine mögliche zukünftige Krise der bisher praktizierten professionellen Selbstregulierung fest. Allerdings ist mit der Verbindung von wissenschaftlichen und gesellschaftlichen Entwicklungen nicht eine Ausrichtung wissenschaftlicher Entwicklungen an eine »Ethik « oder an »Hoffnungen « in der Gesellschaft gemeint. Denn es heißt weiter in dem Bericht: »Mit der Zeit nehmen die Zwänge, die auf der wissenschaftlichen Forschung lasten, zu: Ethische und soziale Zwänge auf der einen Seite; technologische und industrielle auf der anderen Seite « (ebd.: 283/Übers. S.K.). Ethik erscheint nun wieder als etwas, das räumlich getrennt auf gesellschaftlicher Seite verortet wird, im Sinne einer éthique à l'américaine. Die Lösung sehen die Autoren im Dialog: »Es ist wichtig, dass der wissenschaftliche Dialog mit der Gesellschaft einerseits und mit der Verwaltung und der Politik andererseits zusammenkommt« (ebd.: 283/Übers. S.K.). Voraussetzung für einen Dialog sei allerdings die Bildung und Information der Öffentlichkeit: »Es wird zuerst eine gesteigerte Anstrengung der Wissenschaftler für die Bildung und Information der Öffentlichkeit nötig sein« (ebd.: 282). Das Wissen der Öffentlichkeit wird als defizitär wahrgenommen. Zwar wird auch aus dem technokratiekritischen Spektrum, wie der GIB, eine Information der Öffentlichkeit gefordert, in Form einer Gegen-Information. Der Unterschied liegt allerdings darin, dass die Bürger und Bürgerinnen mit dem Ziel informiert werden sollen, eine politische Diskussion über die Entscheidungen zu wissenschaftlichen und technischen Entwicklungen führen zu können. Die Partizipation an Entscheidungsverfahren über die wissenschaftlichen Entwicklungen wird in dem Bericht allerdings nicht thematisiert. Die Autoren meinen also, dass mit der Entwicklung der wissenschaftlichen Forschung »ethische und soziale Zwänge « zunehmen. Aufgrund dessen ist es in ihrer Perspektive wichtig, in Dialog zu treten. Zum Zweck des Dialogs heißt es weiter in dem Bericht: Er bewahre »der Forschung das Stück der Freiheit und des Unvorhergesehenen, ohne die sie zugleich ihre Legitimität und Effizienz verlieren würde« (ebd./Übers. S.K.). Die »ethischen Zwänge«, die von gesellschaftlicher Seite ausgeübt werden, werden in diesem Zitat als Störfaktor für die Freiheit der Forschung gesehen, der durch eine Bildung der Öffentlichkeit und durch die Teilnahme im Dialog aufgelöst werden könne. Der Dialog wird in der Perspektive der Autoren zum Garant für die Freiheit und zur Legitimitätssicherung der Forschung. Ethik wird in diesem Bericht als »Zwang « dargestellt, der von der Gesellschaft auf die Forschung ausgeübt wird und die wissenschaftliche Selbstregulierung in Frage stellen könnte. Abgesehen von der Förderung des Dialogs wird zudem die Einrichtung einer Reflexionsgruppe vorgeschlagen:

»Es ist vor allem eine sorgfältige und kontinuierliche Reflexion durch eine Gruppe notwendig, die nicht nur mit Wissenschaftlern und Politikern zusammengesetzt ist, sondern mit Per- 
sönlichkeiten verschiedener Kompetenzen. [...] So ist es ein wenig die Angelegenheit aller. Aber diese Angelegenheit impliziert [...] eine ausreichende Anzahl gut informierter Bürger, die fähig sind mit den Wissenschaftlern zu kommunizieren« (ebd.: 280/Übers. S.K.).

Die Idee einer Reflexionsgruppe taucht hier im Jahr 1979 zum zweiten Mal auf. Während im Jahr 1974 in der Konzeption des MURS noch von Interessierten gesprochen wird, die abgesehen von Wissenschaftlern und Wissenschaftlerinnen teilnehmen sollen, wird nun auch die Partizipation von Bürgern und Bürgerinnen vorgeschlagen. Mit der »neuen Wissensproduktion« gedacht, werden die Bürgerinnen und Bürger zu aktiven Partnerinnen und Partnern, denn die Reflexion sei »ein wenig die Angelegenheit aller«. Allerdings sind sie nicht als Experten oder Expertinnen im Sinne eines »we are all experts« konzipiert, sondern werden im Kontrast zu den Wissenschaftlern und Wissenschaftlerinnen als defizitär gesehen. Denn Bürger und Bürgerinnen müssen erst informiert werden, um befähigt zu sein, mit den Wissenschaftlern und Wissenschaftlerinnen zu sprechen. Zudem sollen explizit Politiker und Politikerinnen teilnehmen. Während in der Konzeption des MURS Politikerinnen und Politiker bzw. Regierungen beraten werden sollen, ist nun eine gemeinsame Reflexion vorgesehen. Dies entspricht dem Vorschlag Giscard d'Estaings von 1974 auf der Sorbonne-Tagung, in dem er formuliert, dass ein Zusammenschluss mit der Politik notwendig sei (vgl. Monsieur le Président de la République Giscard d'Estaing 1976: 585). Offen bleibt noch, ob und inwiefern nun die Art der Reflexion des MURS und/oder die in dem Bericht vorgeschlagene Reflexion letztlich das Konzept des CCNE inspirieren. Diese Frage wird im Kontext des CCNE wieder aufgegriffen (Kapitel VI).

\section{ZuSAMmENFASSUNG}

Meine These ist, dass für die Autoren des Berichts durch die Aufmerksamkeit der Politik bzw. durch das wirtschaftliche Interesse des Staatspräsidenten eine mögliche Förderung ihrer Forschung absehbar ist oder sie sich diese erhoffen. Indem Giscard d'Estaing auf den gesellschaftlichen und industriellen Nutzen der Physikwissenschaft verweist, liegt dies nahe. Wenn die Lebenswissenschaften in einem ähnlichen Ausmaß zu einem Regierungsprogramm würden wie der Messmer-Plan für die Atomenergie, wären öffentliche Konflikte absehbar, die bereits im Kontext der Gentechnologie an den Forschungsinstituten begonnen haben. Zwei der Autoren des Berichts arbeiten zu dieser Zeit am Institut Pasteur, wo es Mitte der 1970er Jahre Konflikte um Gentechnologie gibt. Die Kritik an einem solchen Regierungsprogramm wäre ebenso möglich gewesen, wie ein Zusammenschluss von Kritikerinnen und Kritikern 
der Gen- und Atompolitik. Beide verwenden einen politisierten Risikobegriff. Indem aber nun in dem Bericht nicht von gesellschaftlichen Risiken, sondern »ethischen Zwängen« gesprochen wird, erscheint eine Entpolitisierung oder Ethisierung politischer Risiko-Konflikte. Konflikte sollen vermieden werden. Die Autoren des Berichts schlagen der Politik einen Mechanismus vor, um die wissenschaftliche Entwicklung weiter zu ermöglichen. Dies stellt eine Art Konfliktprävention dar: Die Sprache der Ethik erscheint hier im Kontext der Antizipation gesellschaftlicher Kritik und offeriert m.E. präventiv politische Handlungsoptionen für die Steuerung der Forschung mit dem Ziel, deren Freiheit zu garantieren. Während sich im internationalen, US-amerikanisch geprägten Asilomar-Prozess eine deutliche Risiko-Rahmung abzeichnet und Ethik-Rahmungen nur marginal erscheinen, zeigt sich in Frankreich eine etwas andere Entwicklung. Hier entsteht in diesem Kontext explizit eine Ethikund nicht eine Risikokommission. Im medizinischen und im gentechnologischen Diskurs erscheinen Ethik-Rahmungen als Antwort auf Problematisierungen wissenschaftlicher Autonomie. In den interdisziplinären Diskursen um Biologie oder Lebenswissenschaften scheint Ethik zunehmend zum Problem zu werden. Auf der Tagung »Biologie und die zukünftige Entwicklung des Menschen« im Jahr 1974 sind es noch Irrationalitäten und Emotionen, die als »ethische und moralische Aspekte« diskutiert werden, die sich aber mit der Zeit mindern können. Im Jahr 1979, in dem Bericht »Lebenswissenschaften und Gesellschaft« sind es »ethische Zwänge«, die auf gesellschaftlicher Seite identifiziert werden. Mit einer zunehmenden Öffnung der Selbststeuerung wissenschaftlicher und technologischer Entwicklungen scheint es eine qualitative Zunahme an Problemen zu geben, die als ethische verstanden und in der Gesellschaft verortet werden. Explizit werden wissenschafts- und medizinkritische Positionen - etwa von Seiten der Science du Peuple, der Abtreibungsbefürworter und -befürworterinnen oder auch der Abtreibungsgegner und -gegnerinnen - nicht thematisiert. (Mögliche) politische Konflikte werden als ethische aufgefasst bzw. ethisiert - im wissenschafts- und medizinkritischen Spektrum, das aus den 1968erBewegungen hervorgeht, findet sich die Sprache der Ethik nicht. Auch eine bestimmte Moral hat zunehmend weniger Raum, sei es die der Lebensschützer und -schützerinnen oder des Ordre des médecins - auch Moral wird ethisiert. 


\section{Die Sprache der Ethik als Antwort auf Problematisierungen lebenswissenschaftlicher Autonomie}

Im Jahr 1983 erscheint nun zum ersten Mal auf nationaler Ebene und staatlich initiiert ein Ethikkomitee in Frankreich. Der CCNE, das nationale konsultative Ethikkomitee für Lebenswissenschaften und Gesundheit, wird eingerichtet.

In den in Kapitel III und IV untersuchten Diskursen, dem medizinischen und dem gentechnologischen Diskurs, erscheint die Ethik-Rahmung als Antwort auf Problematisierungen wissenschaftlicher und medizinischer Autonomie. Ein Ethikkomitee beim INSERM und eine Ethikkommission bei der DGRST werden eingerichtet. Zunehmend zum Problem wird Ethik in den interdisziplinären Diskursen um Biologie und Lebenswissenschaften, wie in Kapitel V aufgezeigt. Hierbei zeigen sich in den wissenschafts- und medizinkritischen Diskursen keine ethischen Rahmungen, sondern die Sprache der Moral und der Politik. Moralische und politische Positionen werden als etwas wahrgenommen, das die Steuerung von medizinischen und technologischen Entwicklungen in Frage stellen kann. Eine Tendenz zur Ethisierung wird sichtbar: politische und moralische Probleme von forschungspolitischen Akteurinnen und Akteuren werden als ethische gerahmt. Wofür ist nun das nationale Ethikkomitee die Lösung?

Bevor diese Frage beantwortet wird, wird der forschungspolitische Hintergrund dargestellt. Bereits hier zeigen sich die Sprache der Ethik und Formen des governing at a distance im Sinne von Rose. Im Anschluss an den forschungspolitischen Hintergrund wird der CCNE vorgestellt, seine Aufgaben, Arbeitsweise und Zusammensetzung. Bei der daran anknüpfenden Beantwortung der Frage, worauf der CCNE die Antwort ist, wird berücksichtigt, inwiefern die medizin-, wissenschafts- und technokratiekritischen Problematisierungen und der Konflikt um den Schwangerschaftsabbruch zum Tragen kommen und auch, inwiefern es neue Problematisierungen gibt. Die Frage, inwiefern die Problematisierungen zum Tragen 
kommen, ist mit der Frage verbunden, welche Themen als ethische verstanden werden und welche nicht. Ein Thema, das als ethisches Thema verstanden wird, ist die Gentechnologie. Sie wird allerdings nicht nur als ethisches Thema verstanden, sondern ebenso als Risiko-Problem. Dies wird anhand des Aufgabenbereichs der parlamentarischen Technikfolgenabschätzungsbehörde deutlich, die im selben Jahr wie der CCNE eingerichtet wird. Kontrastiert wird anhand des Aufgabenbereichs dieser Behörde, welche Themen als ethische und somit als Aufgabenbereich des CCNE verstanden werden und inwiefern diese Rahmung Auswirkungen auf die Kritik an Gentechnologie hat. Nicht zuletzt wird in diesem Kapitel berücksichtigt, was im institutionellen Ethik-Rahmen unter Ethik verstanden wird, ob sich dieses Verständnis über die Zeit ändert und welche Sprecherinnen- und Sprecherpositionen sich im Ethik-Rahmen herausbilden.

\section{Die Forschungspolitik ZU BEgINN DER 1980ER JAHRE - »EIN ABSOLUT ANDERER ESPRIT « ${ }^{1}$}

Die Einrichtung des CCNE findet vor dem Hintergrund eines Wandels in der staatlichen Forschungspolitik statt, die sich mit dem Regierungswechsel von 1981 vollzieht. Nach über 20 Jahren konservativer Präsidentschaften von Charles de Gaulle, Georges Pompidou und zuletzt von Valéry Giscard d'Estaing übernimmt der Sozialist François Mitterrand das Amt des Staatspräsidenten. Der Wandel in der Forschungspolitik findet im Rahmen einer Reformpolitik statt, die von der sozialistischen Regierung eingeschlagen wird. Während der Präsidentschaft von Giscard d'Estaing sind die Ausgaben für Forschung und Entwicklung stark zurückgegangen (Gottweis 1997: 327). Der Premier- und Wirtschaftsminister unter Giscard d'Estaing, der liberale Ökonom Raymond Barre, verfolgte insgesamt eine Austeritätspolitik. Ziel war es, mit den Folgen der Ölkrisen von 1973 und 1979, der Inflation und einer anwachsenden Erwerbslosigkeit umzugehen. $\mathrm{Zu}$ seiner energischen Sparpolitik zählte, die Staatsausgaben zu verringern, die Wettbewerbsfähigkeit zu stärken, d.h. Großkonzerne zu begünstigen oder Lohnerhöhungen zu verhindern (vgl. Denord 2005; Montbrial 1979). Nach dem Regierungswechsel von 1981 setzen umfangreiche wirtschafts- und sozialpolitische Reformen ein. Die sozialistische Reformpolitik hält allerdings nur einige Jahre an (Gottweis 1997: 330f). Der gesetzliche Mindestlohn wird u.a. erhöht, die Arbeitszeit ohne Lohnabzug verkürzt, Verstaatlichungen in der Großindustrie und im Finanzsektor werden in Angriff genommen (Schmidt/Fekl 2006: 186f). Die Entwicklung der Forschung

1 Int. F IX/Übers. S.K.. 
und Technologie wird nach dem Regierungswechsel zur nationalen Priorität erklärt (Chèvenement 1982c: 5; Mitterrand 1982: 73; vgl. Krauss 1996: 54; Gottweis 1997). Die Forschungsförderung und explizit die Förderung biologischer und medizinischer Forschung sind bereits 1972 in dem so genannten gemeinsamen Regierungsprogramm des Parti Socialiste (PS) und des Parti Communiste (PC) aufgenommen (Parti Socialiste/Parti Communiste 1972: 5). Regierungsprogramm meint nicht das Programm einer tatsächlichen Regierung, sondern das Programm für eine zukünftige Regierung - auf diesem Regierungsprogramm basieren die gemeinsamen Wahlkämpfe der beiden Parteien. Die PS und die PC bilden zusammen die Union de la Gauche - Linksunion. Auf Basis dieses Programms kämpft Mitterrand als Kandidat der Linksunion bereits 1974 vergeblich um das Amt der Präsidentschaft. Im Wahlprogramm festgehalten sind nicht allein die Forschungsförderung, sondern auch die Demokratisierung u.a. der Universitäten, Forschungszentren und forschungspolitischer Institutionen, wie der DGRST und dem CNRS, außerdem die Frauenförderung und der Kampf gegen Umweltverschmutzung (ebd.: 3, 5f). Die Forschungspolitik, die sich mit dem Regierungswechsel durchsetzt, ist im Vergleich zu der Forschungspolitik der vorangegangenen Jahre ein »absolut anderer Esprit« (Int. F IX/Übers. S.K.). Was beinhaltete dieser Esprit?

\section{1 »Der Weg aus der Krise $«^{2}$}

Ziel von Mitterrands Forschungs- und Technologiepolitik zu Beginn der 1980er Jahre ist es, die ökonomische Krise zu überwinden (Chavot/Masseran 2003c: 79). Schwerpunkt der nationalen Priorität ist, wie erwähnt, die biologische Forschung. Vorsatz der Forschungspolitik Mitterrands ist, dass Frankreichs Anteil am Biotechnologieweltmarkt auf zehn Prozent anwächst. Eine umfassende Förderung vom Labor bis zum fertigen Produkt soll vollzogen werden (Gottweis 1997: 328). Wie Bonneuil in seiner Untersuchung zum Verhältnis von Wissenschaft und Gesellschaft in Frankreich meint, zeichnet sich der Wandel in der Forschungspolitik sowohl durch eine Art Erneuerung des wissenschaftlichen Colbertismus als auch durch eine neue Offenheit gegenüber der Gesellschaft aus (Bonneuil 2004: 22). Erneuerung des Colbertismus insofern, da die Forschungspolitik der Nachkriegszeit als colbertistisch verstanden wird. Gemeint ist eine eng mit der Wirtschaft zusammenarbeitende, staatlich gelenkte Forschung und Wissenschaft (ebd.). Ein neues Forschungsgesetz wird im Jahr 1982 verabschiedet (Loi n 82-610 1982) und

2 Membres du comité d'organisation du Colloque national 1982: 17/Herv. i.O., Übers. S.K.. 
diverse Institutionen entstehen. Ein Forschungsministerium wird 1981 eingerichtet, in dessen Struktur die DGRST aufgeht, die 1961 während der Präsidentschaft De Gaulles eingerichtet wird. Forschungsminister ist Jean-Pierre Chèvenement. Eine parlamentarische Technikfolgenabschätzungsbehörde entsteht 1983, der Office Parlementaire d'Évaluation des Choix Scientifiques et Technologiques. Außerdem wird ebenfalls 1983 der CCNE eingerichtet. Allerdings ist dieser im Rahmen der neuen Forschungspolitik nicht vorgesehen, er entsteht per Dekret. ${ }^{3}$ Die neue Offenheit bezieht sich sowohl auf bestimmte Formen der Einbeziehung der Öffentlichkeit, die mit der neuen Forschungspolitik umgesetzt werden, als auch auf die Einbeziehung verschiedener Akteure und Akteurinnen in die Ausgestaltung dieser neuen Politik und des Forschungsgesetzes: Noch bevor das neue Forschungsgesetz 1982 umgesetzt wird, findet ein breit angelegtes nationales Kolloquium zu »Recherche et Technologie « - »Forschung und Technologie « statt, geplant von Chèvenement. Zurückgegriffen wird auf dem Forschungskolloquium auf den Bericht »Lebenswissenschaften und Gesellschaft« von 1979 (vgl. Ministère de la Recherche et de la Technologie 1982: 35). Er wurde zwar von Giscard d'Estaing, der die Potentiale der Biotechnologie als Zukunftstechnologie entdeckte, am Ende seiner Amtszeit in Auftrag gegeben, aufgegriffen wird er aber schließlich von Mitterrand (Gottweis 1998: 213). Einer der Autoren des Berichts, François Gros, wird sein wissenschaftlicher Berater (Rouban 1988b: 162).

\section{2 "Bewegung von unten « $^{4}$ : das nationale Kolloquium zu Forschung und Technologie}

Das nationale Kolloquium besteht aus 31 Tagungen, die sowohl auf regionaler Ebene in Frankreich als auch in Paris und in den französischen Überseegebieten von 1981-1982 stattfinden. Zu den Organisatorinnen und Organisatoren zählen u.a. François Gros, der Soziologe Michel Callon, der Physiker Pierre Papon und Philippe Lazar, der 1982 Generaldirektor vom INSERM wird (Gros 1982a: 8; Théry 2001: 12). Die rund 10.000 Teilnehmenden kommen aus Forschungseinrichtungen, Universitäten, Unternehmen, Gewerkschaften, Berufsorganisationen und Politik (Chèvenement 1982c: 5). Verstanden wird das nationale Kolloquium als »Mouvement d'en bas « - »Bewegung von unten «, da eine Masse an Forscherinnen und Forschern zusammenkommt (Bois 1982: 100; Chèvenement 1982b: 56/Übers.

3 Dekrete können in Frankreich vom Staatspräsidenten oder dem Premierminister erlassen werden (vgl. Loewe 2006a).

4 Chèvenement 1982b: 56/Übers. S.K.. 
S.K.). »Bewegung von unten« bezieht sich vermutlich auf den Umstand, dass die Forschungspolitik der 1970er Jahre die Forscherinnen und Forscher marginalisiert hat, sie nicht gefördert hat, wie es auf dem Kolloquium heißt, und diese nun »von unten« zusammenkommen (Chèvenement 1982b: 56/Übers. S.K.; vgl. Membres du comité d'organisation du Colloque national 1982: 14). Ziel des Kolloquiums ist es, wie der Forschungsminister meint, sowohl in der wissenschaftlichen Community als auch im ganzen Land Bedingungen zu schaffen, die das Aufkommen neuer Ideen und Innovationen begünstigen (Chèvenement 1982c: 5). Was unter dem Begriff Innovation verstanden wird, zeigt sich an einem Beitrag von François Gros:

»Für den Unternehmenssektor ist die Herausforderung beachtlich. Die industrielle Innovation $[\ldots]$ ist zu einer > wichtigen Waffe im Wirtschaftskrieg, den die Unternehmen und die Nationen führen geworden. Viele Unternehmen, kleine und große, wissen nunmehr, dass die Lösung der Wirtschaftskrise, der industrielle Aufschwung, stark abhängig von der Forschung ist (Gros 1982b: 64/Herv. i.O., Übers. S.K.).

In Anlehnung an das »Colloque de Caen« von 1956 soll das nationale Kolloquium der 1980er Jahre zu »programmes mobilisateurs « - »Mobilisierungsprogrammen« führen (Chèvenement 1982b: 57f/Übers. S.K.). Ähnlich wie bei dem »Colloque de Caen«, wo der liberale Kapitalismus der Zwischenkriegszeit als Ursache für die ökonomischen Probleme gesehen wurde, wird auf dem nationalen Kolloquium die staatliche Politik der 1970er Jahre für die Krise verantwortlich gemacht (Bonneuil 2004: 22). Sie habe die wissenschaftliche und technologische Forschung nicht in die Politik integriert (Membres du comité d'organisation du Colloque national 1982: 14). Forschung, Innovation und wirtschaftlicher Aufschwung werden als Schlüssel gesehen, um die Wirtschaftskrise, die zunehmende Erwerbslosigkeit und die Inflation zu überwinden. Grundsätzlich abhängig sind Innovation und Aufschwung von den Entwicklungen, vom Fortschritt in Forschung und Wissenschaft. Dieser Fortschritt geschieht aber nicht von alleine, wie Mitterrand festhält: »>[D]ie Wissenschaft< kann und soll einen außerordentlichen Fortschritt für den Menschen bewirken [...]. Aber ich bin vollkommen davon überzeugt, dass das nicht von alleine geschieht. [...] Die Wissenschaft im Dienste des Menschen, ja. Nicht das Gegenteil« (Mitterrand 1982: 67/Herv. i.O., Übers. S.K.). Es soll sich um eine Wissenschaft handeln, die sich ihrer Rationalität ebenso bewusst ist, wie ihrer Grenzen (Fossaert 1982: 92). Der »nicht von alleine« geschehende Fortschritt der Wissenschaft geht mit einer Einbeziehung der Öffentlichkeit einher: Ein »dialogue permanent « - »dauerhafter Dialog« (Mermaz 1982: 75), ein »vrai dialogue« - »wahrer Dialog«, ein »dialogue engagé« - »engagierter Dialog« (Gros 1982a: 7; 1982b: 63) soll eingerichtet werden, wie auf dem nationalen Kolloquium mehrfach in ver- 
schiedenen Varianten betont wird. Jede Bürgerin und jeder Bürger soll zukünftig als potentielle Forscherin oder potentieller Forscher angesehen werden, so das Organisationskomitee (Membres du comité d'organisation du Colloque national 1982: 31). Dieser Dialog gehört zu dem Konzept der Verbreitung der »culture scientifique, technique et industrielle « - »wissenschaftlichen, technischen und industriellen Kultur «. Eine Rolle in der Vermittlung dieser Kultur wird den Geistesund Sozialwissenschaften zugeschrieben. Diese besteht darin, den Dialog zwischen Wissenschaft und Gesellschaft wiederherzustellen, wie es in dem Forschungsgesetz heißt (Loi n ${ }^{\circ}$ 82-610 1982: 2271, 2276). Konkretisiert wird diese Rolle in dem Gesetz nicht. Allerdings heißt es während des Kolloquiums, dass die Sozial- und Geisteswissenschaften zu einem besseren Verständnis des Verhaltens des Menschen und der Gesellschaft beitragen, wie auch zur Analyse der wirtschaftlichen und gesellschaftlichen Bedürfnisse (Demazure 1982: 110, 112). Sie können auch dazu beitragen, den technischen Fortschritt tatsächlich in den Dienst des Menschen zu stellen, so der Bericht der Kommission zu Sozial- und Geisteswissenschaften (Bois 1982: 96). Vermittelt werden soll diese Kultur zudem durch Einrichtungen, die mit der neuen Forschungspolitik umgesetzt werden, wie durch die Cité des Sciences et de l'Industrie in Paris oder auf regionaler Ebene durch die Centre de Culture Scientifique, Technique et Industrielle (CCSTI) - Zentren der wissenschaftlichen, technischen und industriellen Kultur (Chavot/Masseran 2003c: 79/Übers. S.K.; vgl. Loi n 82-610 1982: 2276). Zur Förderung dieser Einrichtungen wird ein Conseil National de la Culture Scientifique, Technique et Industrielle eingerichtet, der nationale Rat für wissenschaftliche, technische und industrielle Kultur (Chavot/Masseran 2003c: 80). Die Cité des Sciences ist eine Art populärwissenschaftliches Museum. Sie verkörpert eine der »majestic spaces, >cathedrals built to celebrate products of science and technology « (Chavot/Masseran 2003b: 536/Herv. i.O.). Aufgabe der CCSTI ist sowohl die Information der Öffentlichkeit über wissenschaftliche Entwicklungen, die Organisation von Dialogen zwischen Wissenschaft, Industrie und Öffentlichkeit als auch eine aktive Bürgerinnen- und Bürgerschaft zu fördern (Association nationale des CCSTI 2014b, a; vgl. Chavot/Masseran 2003a: 244f). Die Information der Öffentlichkeit wird, wie es auf dem nationalen Kolloquium heißt, als eine Art Demokratisierung verstanden: Wissenschaftliche Informationen zu teilen bedeutet, Macht zu teilen, und bereichert die Demokratie (Dessieux 1982: 162). Information oder Bildung und das Konzept des Dialogs mit der Gesellschaft sind nicht neu, auch wenn sie bisher nicht im Kontext einer Demokratisierung standen. Der Dialog wird bereits Ende der 1970er Jahre in dem Bericht »Lebenswissenschaften und Gesellschaft« von den Autoren François Gros, François Jacob sowie Pierre Royer vorgeschlagen. François Gros, nun wissenschaftlicher Berater Mitterrands, vertritt dieses Konzept auch auf dem nationa- 
len Kolloquium. Wie in Kapitel V ausgeführt, erscheint in dem Bericht der Dialog als Lösung für »ethische Zwänge«. Sie werden als Störfaktoren für die Freiheit der Forschung gesehen und auf gesellschaftlicher Seite verortet. Aufgelöst werden können sie durch die Bildung der Öffentlichkeit und durch Teilnahme am Dialog. Dialog wird zum Garant der Forschungsfreiheit, er sichert ihre Legitimität. Es stellt sich die Frage, wofür der Dialog in der culture scientifique die Lösung ist.

\subsection{Ethik - vom gesellschaftlichen Problem zur Aufgabe der Bürgerin und des Bürgers}

Auch im Kontext der neuen Forschungspolitik zu Beginn der 1980er Jahre zeigt sich die Notwendigkeit der Bildung oder Information der Öffentlichkeit in Form der Cité des Sciences oder der CCSTI. Es handelt sich, so Bonneuil, um einen linearen und pädagogischen Ansatz vom Staat abwärts hin zur Öffentlichkeit. Die Öffentlichkeit wird hier als eine begriffen, die sich in Distanz zum Fortschritt befindet. Die Distanz wird mit einem Defizit an wissenschaftlichem Wissen begründet (Bonneuil 2004: 23).

»Nur mit einem umfangreichen Einsatz der Wissensverbreitung [...] können wir bestimmte Vorurteile gegenüber der Wissenschaft zurückdrängen, die anti-wissenschaftlichen Bewegungen eindämmen und die Bürger in die Lage versetzen, die wissenschaftlichen und technologischen Herausforderungen besser zu erfassen«, so der Forschungsminister (Chèvenement 1982a: 205/Übers. S.K.).

Nicht Ethik in Form »ethischer Zwänge« sind das Problem, sondern Vorurteile und die anti-wissenschaftlichen Bewegungen, die vor allem durch Wissensverbreitung und einen informierten Dialog gelöst werden können. Unter anti-wissenschaftliche Bewegungen werden, so Bonneuil, sowohl Wahrsager und Wahrsagerinnen ${ }^{5}$ als auch Ökologen und Ökologinnen verstanden (Bonneuil 2004: 22; vgl. Testart 2006). Da die Ökologinnen und Ökologen oder die Umweltbewegung mit der Anti-Atombewegung verknüpft ist und letztere wiederum mit der Kritik an Gentechnologie, lässt sich m.E. auch vermuten, dass die Science $d u$ Peuple insgesamt dazu gezählt werden kann. Indem diese als anti-wissenschaftlich verstanden werden, als Bewegungen, die sich einer Wissenschaft gegenüber stellen, die sich als rational

$5 \mathrm{Zu}$ dieser Zeit ist in Frankreich die Wahrsagerin Madame Soleil bekannt und erfolgreich. Sie hat eine Fernsehsendung in einem Pariser Privatsender. Konsultiert wird sie von Klientinnen und Klienten aus Kunst, Politik und Wirtschaft (vgl. Cicco 1993). 
versteht (Fossaert 1982: 93), können sie nur eines sein, nämlich »irrationel « - »irrational«, wie es auf dem Kolloquium heißt (Bois 1982: 102/Übers. S.K.). Auch wenn die Sprache der Ethik insgesamt auf dem nationalen Kolloquium kaum auftaucht, erscheint Ethik jedoch schon - allerdings nicht mehr als Problem, sondern als Teil der Lösung. Letztere beinhaltet ein Konzept von Verantwortung. Es heißt, man müsse sich insgesamt über die gesellschaftliche Verantwortung des Wissenschaftlers oder der Wissenschaftlerin einig sein, und zwar als Verantwortung

»eines Bürgers, der von seinen Aufgaben gelenkt ist, eine wesentliche anti-technokratische Rolle zu spielen, das heißt, dabei zu helfen, Fragen jeglicher Natur, die alle Beteiligten betreffen, technische oder ethische, ausgelöst von der Entwicklung des Wissens und den technologischen Möglichkeiten, zu formulieren und zu verbreiten. Eine der größten Herausforderungen unserer Unternehmung stellt sich darin dar, der >Anti-Wissenschafts $<-B e w e g u n g$ Einhalt zu gebieten, deren Entwicklung mit der Krise fortgeschrittener kapitalistischer Gesellschaften zusammenfällt« (Ministère de la Recherche et de la Technologie 1982: 38/ Herv. i.O., Übers. S.K.).

Sowohl Wissenschaftlerinnen und Wissenschaftler werden als aktive Bürgerinnen und Bürger konzipiert als auch die Öffentlichkeit. Bürgerinnen und Bürger werden als Akteurinnen und Akteure verstanden, die aktiv zur Innovation und zur Überwindung der ökonomischen Krise beitragen können, die sie selbst betrifft, bspw. in Form von Erwerbslosigkeit. Grundlegend ist, dass die wissenschaftlichen Entwicklungen verstanden werden. Einhalt geboten werden soll der anti-wissenschaftlichen Bewegung. Sie wird mit der Krise des Kapitalismus verknüpft. Diese Krise basiere auf einer mangelhaften Forschungspolitik. Es scheint fast so, als könne, wenn die Forschungspolitik falsch ist, auch die Kritik daran nur falsch sein kann. Sowohl die Krise als auch die so genannte anti-wissenschaftliche Bewegung sind negativ konnotiert, als Zustand, der überwunden werden muss. Schlüssel hierfür sind Bildung und Information. Die Information geht von den Wissenschaftlerinnen und Wissenschaftlern verstanden als Bürgerinnen und Bürger aus, die definieren, welche Fragen durch die wissenschaftlichen und technologischen Entwicklungen aufgeworfen werden. Das Verbreiten und Teilen dieser Information wird als Machtteilung verstanden und letztlich als Bereicherung der Demokratie. Wissen wird m.E. als Machtbereich anerkannt und es wird davon ausgegangen, dass das Teilen von Wissen ein Weniger an Macht oder eine bessere Macht bedeutet - ein Mehr an Demokratie. Die »Wissenschaft im Dienst des Menschen«, wie Mitterrand sagt, erinnert an das Konzept der Informationsgruppen Wissen in den Dienst der Menschen zu stellen. Es geht mit dem Konzept des »spezifischen Intellektuellen« einher, das Foucault 1976 ausarbeitet (Gaudillière 2006a: 245). Es beinhaltet die Idee, sich po- 
litisch und im jeweiligen Spezialgebiet zu engagieren und gleichzeitig das Fachwissen zu verwenden, um dessen Praktiken anzuprangern (Artières 2002: 38). In der neuen Forschungspolitik geht es allerdings nicht darum, die Praktiken der Wissenschaft anzuprangern oder - wie bei der Science du Peuple - um die Beteiligung an Entscheidungen über wissenschaftliche und technologische Entwicklungen. Während die Informationsgruppen ihr Wissen als Kampfmittel einsetzen, um es denen zur Verfügung zu stellen, die es brauchen, erscheint die Wissenschaft Mitterrands als Kampfmittel gegen die ökonomische Krise, deren Überwindung ein gesellschaftliches Projekt wird. In der Wissensverbreitung geht es darum, Informationen oder Fragen der wissenschaftlichen und technologischen Entwicklungen, die von Wissenschaftlerinnen und Wissenschaftlern definiert und verbreitet werden, $\mathrm{zu}$ diskutieren. Die Definition dessen, worüber gesprochen wird, was ethische oder technische Fragen sind, liegt in wissenschaftlichen Händen. Während in dem Bericht »Lebenswissenschaften und Gesellschaft« politische Konflikte ethisiert und zum Problem werden, gibt es jetzt »ethische Fragen«, die der Wissenschaftler oder die Wissenschaftlerin formuliert. Zuvor werden »ethische Fragen oder Zwänge« räumlich getrennt auf gesellschaftlicher Seite verortet. Indem m.E. Wissenschaftlerinnen und Wissenschaftler als Bürgerinnen und Bürger konzipiert werden, gewinnen sie eine Kompetenz für »ethische Fragen«. Ihnen wird also nicht nur die Kompetenz für technische Fragen, also für facts zugeschrieben, sondern auch für $v a$ lues. Der oder die Intellektuelle entspricht weder dem Konzept des oder der spezifischen noch des oder der universellen Intellektuellen, der oder die die Wahrheit für alle spricht. Der oder die neue Intellektuelle als Bürger oder Bürgerin vermittelt und diskutiert Fragen, die er oder sie definiert und formuliert. Politische oder ökonomische Fragen werden in diesem Kontext allerdings nicht thematisiert. Ökonomische Themen werden nur thematisiert, wenn es darum geht, dass Sozial- und Geisteswissenschaftlerinnen und -wissenschaftlern die Aufgabe zukommt bzw. sie diese selbst formulieren, wirtschaftliche und gesellschaftliche Bedürfnisse zu analysieren, um den technischen Fortschritt in den Dienst des Menschen zu stellen (vgl. Demazure 1982: 110, 112; Bois 1982: 96). Bei der Rolle, die den Sozial- und Geisteswissenschaften zukommt, scheint es sich um eine Art Begleitforschung zur Innovation zu handeln. Das Wissen der Bürgerinnen und Bürger wird einerseits als defizitär konstruiert, gleichzeitig werden sie als potentielle Forscherinnen und Forscher konzeptioniert. Zwar kann dieser Ansatz als linear vom Staate abwärts verstanden werden, wie Bonneuil meint, parallel erscheint aber der Bürger oder die Bürgerin als gleichgestellter Teilnehmer oder gleichgestellte Teilnehmerin. Im Sinne Nowotnys und ihrer Kolleginnen und Kollegen zeigt sich hier ein Übergang zu Modus 2, und zwar sowohl durch die transdisziplinäre und heterogene Ausrichtung der Teilnehmerinnen- und Teilnehmerschaft des Kolloquiums als auch durch das 
Konzept des Bürgers oder der Bürgerin. Als potentieller Forscher oder potentielle Forscherin werden sie als eine Form von Experten oder Expertinnen konzeptioniert (vgl. Nowotny 2003: 155f). Dabei ist eine Dialog-Beteiligung unter der Voraussetzung eines bestimmten wissenschaftlichen Wissens erwünscht - eben als potentielle Forscherin oder potentieller Forscher und nicht als potentielle Arbeiterin, Hausfrau oder Vater und vor allem nicht als Wahrsager oder Ökologin.

Mit dem nationalen Kolloquium zu Forschung und Technologie in den Jahren 1981-1982 endet, so der Wissenschaftshistoriker Patrick Petitjean, die wissenschafts- und technokratiekritische Debatte der Post-68er (Petitjean 1998; vgl. Chavot/Masseran 2003a: 244; Bonneuil 2004: 23). Jedoch ist ein Ende meistens nicht abrupt.

\subsection{Das Verschwinden der wissenschaftskritischen Debatte}

Es zeichnet sich m.E. ab, dass Konzepte wie Dialog und Verantwortung auf staatspolitischer Ebene eingeführt werden. Gleichzeitig aber werden auch Protestformen, Begriffe und Konzepte des Mai '68, der wissenschafts- und technokratiekritischen Bewegungen, auch ihre Akteure und Akteurinnen in die culture scientifique übernommen - die Politik der sozialistischen Regierung versteht sich selbst als antitechnokratisch. Wie dies geschieht, wird im Folgenden nur beispielhaft aufgeführt und später in diesem Kapitel, im Kontext der Einrichtung des CCNE, konkretisiert.

Bereits das nationale Forschungskolloquium wird als Bewegung von unten verstanden oder auch als »véritables sétats généraux de la recherche et de la technologie « - »echte >Generalstände zu Forschung und Technologie««, wie der Forschungsminister meint (Chèvenement 1982b: 56/Herv. i.O., Übers. S.K.). États généraux werden in Frankreich in unterschiedlicher Form praktiziert. Historisch geht das Konzept der États généraux in das 14. Jahrhundert zurück. Sie setzen sich aus Adel, Klerus und Drittem Stand zusammen und werden vom König einberufen. Aufgabe der Generalstände in der Monarchie ist die Beratung des Königs in Krisensituationen; sie werden aber auch genutzt, um Einfluss auf dessen Gesetzgebung zu nehmen (Loewe 2006b: 840ff). ${ }^{6}$ Einige hundert Jahre später, Ende der 1960er Jahre wird das Konzept der Generalstände allerdings als Protestform verwendet - sie sind nicht vom König bzw. Staat initiiert, sondern selbstorganisiert, um gegen staatliche Politik zu protestieren. Mit dem Begriff États généraux wer-

6 Am 17.06.1789 erklärt sich der Generalstand zur Nationalversammlung, aus der die Erklärung der Menschen- und Bürgerrechte hervorgeht. Ausführlich vgl. Loewe (2006b: $840 \mathrm{ff})$. 
den Versammlungen oder Zusammenkünfte bezeichnet, die durch eine große Anzahl von Akteurinnen und Akteuren gekennzeichnet sind. Im Mai '68 finden bspw. États généraux du cinéma français - Generalstände des französischen Kinos statt. Ihr Ziel ist u.a. die Abschaffung von Zensur und Monopolen sowie die Einrichtung selbstverwalteter Strukturen (Doll 2006: 606). Ob nun die »echten Generalstände«, wie das nationale Kolloquium zu Forschung und Technologie verstanden wird, im Kontrast zu den Generalständen während der Monarchie oder zum Mai '68 stehen, wird von Chèvenement nicht gesagt. $\mathrm{Zu}$ den Generalständen während der Monarchie zeigen sie Parallelen aufgrund der Krisensituation und der Möglichkeit, die Gesetzgebung zu beeinflussen - das neue Forschungsgesetz entsteht aus dem nationalen Kolloquium. »Echt« hieße dann, nicht mehr zur Monarchie, sondern zur Demokratie beizutragen. Zum Mai '68 zeigt sich die Parallele insofern, als sich das Kolloquium als Bewegung von unten versteht. »Echt « hieße in diesem Fall, tatsächlich Einfluss auf die Gesetzgebung zu haben oder breiter mobilisiert und mehr Menschen einbezogen zu haben. Auch die Boutiques des Sciences - Wissenschaftsläden gehen in der culture scientifique auf. Noch zu Beginn der 1980er Jahre bilden sie sich aus der wissenschaftskritischen Bewegung. Sie haben das Ziel, Bürger und Bürgerinnen bei Institutionengängen, rechtlichen und medizinischen Fragen zu helfen: »to listen to citizens' demands and provide counter-expertise that would challenge expertise offered by industries and institutions: this way, they would help people to defend themselves against risks related to scientific, technological and industrial developments« (Chavot/Masseran 2003c: 79). Die Boutiques verschwinden bis auf einige wenige Mitte der 1980er Jahre mit der neuen culture scientifique und insbesondere der CCSTI: »The critical movements became progressively institutionalised and lost some of their radicalism « (Chavot/Masseran 2003b: 536). Akteure und Akteurinnen aus dem wissenschafts- und technokratiekritischen Spektrum der Post-68er gehen den Weg in die Institutionen, so bspw. der Physiker Jean-Marc Levy-Leblond, der Ende der 1960er Jahre beim Verlag Seuil die wissenschaftskritische Reihe Science Ouverte herausgibt. Er wird Präsident des Conseil National de la Culture Scientifique, Technique et Industrielle (Chavot/Masseran 2003c: 80). In einem Interview wird mir erklärt, dass die neue Forschungspolitik gerade für linke Forscherinnen und Forscher attraktiv war,

»weil der politische Geist sich geändert hat. Man hat nicht mehr von Steuerung gesprochen, sondern von Partnerschaft. Man hat in Frankreich [...] die Industrie verstaatlicht. Die Forscher wurden sich darüber klar, Industrie ist nicht Synonym mit Kapital und Profit. Man kann mit der Industrie zusammenarbeiten, ohne einen Pakt mit dem Teufel einzugehen« (Int. F IX/Übers. S.K.). 
Die Themen und Ziele der kritischen Bewegungen werden zunehmend institutionalisiert. Kritik wie die am Profit- und Machtwillen, die insbesondere atom-, genund medizinkritische Bewegungen aufwerfen, wird entkräftet. Dass mit dem Regierungswechsel von 1981 für viele Forscherinnen, Forscher, Wissenschaftlerinnen und Wissenschaftler Hoffnungen verknüpft sind, ist verständlich. Die Forschung erhält nationale Anerkennung, Stellen werden geschaffen, Bildung kommt eine besondere Bedeutung zu, es werden Strukturen eingerichtet und Möglichkeiten des Dialogs, die als demokratisch verstanden werden - es herrscht ein »anderer Esprit« (Int. F IX/Übers. S.K.). Bereits mit dem Wahlprogram greifen die PS und die PC eine Reihe an Themen auf, die Teil der Politik wissenschaftskritischer außerparlamentarischer Bewegungen sind, seien es Umweltverschmutzung oder Frauenrechte. Die Themen werden nicht unbedingt vereinnahmt, denn sie sind bereits im Wahlprogramm von 1972 enthalten. Sie werden aber nun staatspolitisch ver- und gewendet - zur Sicherung der Innovation und der Entwicklung von Forschung und Technologie.

Mit der neuen Forschungspolitik kommt ein Konzept des Bürgers oder der Bürgerin als potentielle Forscherin oder potentieller Forscher auf, ebenso des Wissenschaftlers oder der Wissenschaftlerin als Bürger oder Bürgerin. Bürgerinnen und Bürger sollen sich aktiv am wirtschaftlichen Innovationsprozess beteiligen, indem sie an Dialogveranstaltungen zu Entwicklungen in Forschung und Technologie teilnehmen und letztlich zur Krisenlösung beitragen. Zeichnen sich mit der neuen Forschungspolitik Formen des governing at a distance des advanced liberalism im Sinne von Rose ab?

\subsection{Dialog und Innovation als Krisenlösung - eine Form des advanced liberal government? ${ }^{7}$}

Neu ist zu Beginn der 1980er Jahre das Konzept der Innovation als Krisenlösung. In zwei Publikationen aus dem Jahr 1988 sieht der französische Politikwissenschaftler Luc Rouban den Innovationsdiskurs sowohl als Symptom für die ökonomische Krise als auch für die Krise des modernen Staates im Kontext der Beziehung von Wissenschaft, Politik und Gesellschaft (Rouban 1988a: 327; 1988b: 196ff). Diese Beziehung werde neu geordnet, zum Vorteil eines neuen Modells, das Marktmechanismen bevorzugt, so Rouban (1988a: 332). Die Innovationspolitik entsteht aus der Kritik am Wohlfahrtsstaat und seiner Wissenschaftspolitik und kann als Versuch verstanden werden, das Legitimitätsdefizit zu füllen, das durch

7 Rose/Miller 1992; Rose 1996. 
die Unsicherheit technologischer Entwicklungen hervorgerufen wird (ebd.: 327). In dieser Innovationspolitik zeige sich die Frage der Entwicklung von Wissenschaft und Technologie nicht mehr als übergreifende Aufgabe des Wohlfahrtsstaates (Rouban 1988b: 196). Sie ist weniger Gegenstand staatlicher Politik, sondern vielmehr gesellschaftliche Aufgabe (Rouban 1988a: 330). Denn sie geht mit einer Einbeziehung weiterer Akteure und Akteurinnen in die wissenschaftliche und technologische Entwicklung einher, mit einer neuen Kultur der Verbreitung wissenschaftlicher Information und mit der Einrichtung von intermediären Strukturen (Rouban 1988b: 196, 200).

Was Rouban verdeutlicht, kann in der Perspektive der Gouvernementalität m.E. als eine bestimmte Form der Problematisierung verstanden werden und als Aufkommen einer neoliberalen Rationalität. Der Wohlfahrtsstaat wird in Frage gestellt und mit dem Innovationsdiskurs zeichnet sich eine Ausrichtung auf marktund wettbewerbsorientierte Formen des Regierens ab. In den Worten Lemkes formuliert, findet eine Restrukturierung staatlicher Regierungstechniken statt, und zwar in dem Sinn, dass die Regulierungskompetenz des Staates auf verantwortliche Individuen verschoben wird (Lemke 2001: 202f). Dies geschieht indem bspw. die Frage der Erwerbslosigkeit von der Innovation abhängig gemacht wird, die wiederum vom Fortschritt der Forschung abhängt. Zugespitzt formuliert bedeutet dies: stelle ich mich gegen wissenschaftliche und technologische Entwicklungen, bin ich (mit-)verantwortlich für die ökonomische Krise und meine Erwerbslosigkeit. Die Verantwortung für soziale Risiken, wie Erwerbslosigkeit, wird indirekt in den Bereich des verantwortlichen Individuums verschoben. Dialogveranstaltungen, in denen wissenschaftliche und technologische Entwicklungen diskutiert werden, stellen die Möglichkeit her, sich aktiv an der Lösung zu beteiligen und indirekt zur ökonomischen Akteurin oder zum ökonomischen Akteur zu werden. Es handelt sich um Regierungstechniken, die im Sinne von Rose eine Distanz zwischen sozialen Akteurinnen und Akteuren und den Entscheidungen formaler politischer Institutionen schaffen. Oder, wie Rouban 1988 in Bezug auf (wohlfahrts-)staatliches Handeln meint: »es geht nicht mehr darum, auf das Wesen des Allgemeingutes einzuwirken, sondern auf die Struktur des Handlungssystems, die dem Verhalten, den Erwartungen [...] der Individuen zugrunde liegt« (Rouban 1988a: 341/Übers. S.K.). Die Techniken des governing at a distance versuchen, auf das Individuum einzuwirken, indem sie bestimmte Verhaltensformen wahrscheinlicher machen (Rose 1996: 53f; Rose/Miller 1992; Bröckling/Krasmann 2010: 29). Das Verhalten, das sie hier insbesondere wahrscheinlicher machen, ist, Akzeptanz für wissenschaftliche und technologische Entwicklungen zu erlangen. Die Frage, inwiefern es sich beim CCNE um eine Technik des Regierens auf Distanz handelt, bleibt noch zu beantworten. Hierfür wird er zuerst einmal vorgestellt. 


\section{Der Comité Consultatif National d'Éthique}

Der CCNE ist eine vom damaligen Staatspräsidenten Mitterrand und dem Minister für Forschung und Technologie, Chèvenement, am 23. Februar 1983 per Dekret permanent eingerichtete konsultative Institution (Décret $n^{\circ} 83-132$ 1983). Es gilt als weltweit erstes nationales Ethikkomitee, dem eine Vorbildfunktion für die Einrichtung solcher Komitees zugeschrieben wird (Fuchs 2001a: 3; 2005: 13). Sein Sitz ist in Paris.

\subsection{Aufgaben}

Der CCNE versteht sich als unabhängige Stelle, die über keine Entscheidungsgewalt verfügt. Sie dient der Politikberatung und kann Denkanstöße für eine ethische Debatte geben (Michaud 1990: 180; 2002; vgl. Braun et al. 2002: 21; Fuchs 2001b: 86ff; Fuchs 2005: 13). Sowohl der Status des Komitees als auch seine Aufgabe ändern sich über die Jahre. ${ }^{8}$ Bei seiner Einrichtung im Jahr 1983 hat es, wie es im Dekret heißt, die Aufgabe »seine Meinung zu moralischen Problemen abzugeben, die durch die Forschung in den Bereichen der Biologie, der Medizin und der Gesundheit aufgeworfen werden, ob diese Probleme den Menschen, gesellschaftliche Gruppen oder die gesamte Gesellschaft betreffen« (Décret n 83-132 1983: Art. prem./Übers. S.K.). Im Jahr 1994 wird der Status des CCNE im Gesetz zu Organspende, medizinisch assistierter Fortpflanzung und Pränataldiagnostik, dem meist so genannten ersten Bioethikgesetz, verankert. In dem Gesetz wird nicht mehr von moralischen Problemen gesprochen, sondern von »problèmes éthiques« - »ethischen Problemen «, die nicht durch die Forschung, wie im Dekret von 1983, sondern durch die »progrès de la connaissance - »wissenschaftlichen Fortschritte « im Bereich der Medizin, Biologie und Gesundheit aufgeworfen werden. Zudem soll er nicht mehr allein avis ${ }^{9}$ - Meinungen, sondern auch Empfehlungen veröffentlichen (Loi n 94-654 1994: Art. 23/Übers. S.K.; vgl. Michaud 2002). Und schließlich hat der CCNE seit dem Bioethikgesetz von 2004 die Aufgabe, seine

8 Die Dekrete und Gesetze, welche Aufgaben, Tätigkeitsbereich und Art und Weise der Zusammensetzung des CCNE behandeln, sind die Dekrete von 1983, 1997 und 2005 (Décret n 83-132 1983; Décret n 97-555 1997; Décret n 2005-390 2005) sowie die Gesetze von 1994, 2004 und 2011 (Loi n ${ }^{\circ}$ 94-654 1994; Loi n 2004-800 2004; Loi n ${ }^{\circ}$ 2011-814 2011).

9 Im Folgenden wird für Meinung der französische Begriff avis verwendet, da der Begriff Meinung im Deutschen je nach Kontext missverständlich sein kann. 
Meinung abzugeben $\mathrm{zu}$ »problèmes éthiques et les questions de société soulevés par les progrès de la connaissance « - »ethischen Problemen und gesellschaftlichen Fragen, die durch die wissenschaftlichen Fortschritte aufgeworfen werden « (Loi n ${ }^{\circ}$ 2004-800 2004: Art. L. 1412-1). Es zeichnet sich m.E. eine Ausdehnung im Aufgabenbereich des CCNE ab: von Problemen, die als moralisch bzw. ethisch bezeichnet werden, hin zu »ethischen und gesellschaftlichen Problemen«, wie es im Bioethikgesetz von 2004 heißt. Die Frage, ob und inwiefern sich diese Ausdehnung in der Tätigkeit des CCNE, in den über 120 avis und Empfehlungen über die Zeit niederschlägt, wäre Anlass für eine eigene Untersuchung. Zu dieser Frage stelle ich in meiner Untersuchung verschiedene Thesen auf.

\subsection{Zusammensetzung}

Bei dem Komitee handelt es sich vorrangig um ein Expertinnen- und Expertenkomitee, das sich aus rund 40 Mitgliedern zusammensetzt. Formal gesehen vertreten diese kein Interesse, sondern sollen unabhängig sein (Int. F IV, IX). Bei der Zusammensetzung des Komitees wird auf Pluridisziplinarität und Pluralismus Wert gelegt:

»Die Mitglieder der Kommission verkörpern ein breites Spektrum unterschiedlicher Erfahrungen und Disziplinen. Dieser Pluralismus ist von entscheidender Bedeutung. Da die Kommission weder ein Organ wissenschaftlicher Bewertung noch der Rechtsprechung ist, liegt gerade in ihrem Pluralismus ihre Besonderheit, ihre Existenzberechtigung « (Michaud 1990: 179; vgl. Michaud 2002).

Es besteht der Versuch, ein breites Spektrum sowohl wissenschaftlicher Disziplinen, Weltanschauungen als auch aus der Politik zu vereinen. Jedoch ist der CCNE nicht repräsentativ; gesellschaftliche Mehrheitsverhältnisse werden nicht widergespiegelt (Braun et al. 2002: 22). Das Komitee ist darüber hinaus insofern nicht demokratisch legitimiert, als seine Mitglieder nicht gewählt werden - sie werden benannt und sind alle unentgeltlich tätig (Michaud 2002; Décret $n^{\circ}$ 2005-390 2005; Int. F IV, X): Der Präsident oder die Präsidentin des CCNE wird von dem Staatspräsidenten oder der Staatspräsidentin für die Dauer von zwei Jahren benannt. Es besteht die Möglichkeit der Verlängerung um weitere zwei Jahre. Ein Vizepräsident oder eine Vizepräsidentin wird vom CCNE selbst benannt (Décret $n^{\circ} 2005$ 390 2005: Art. R. 1412-5). Außerdem benennt die Staatspräsidentin oder der Staatspräsident fünf Mitglieder aus den wichtigsten »familles philosophiques et spirituelles « - »philosophischen und geistlichen Familien« (Loi n ${ }^{\circ}$ 2004-800 
2004: Art. L. 1412-2.1). Um welche es sich handelt, wird weder im Dekret von 1983 noch im entsprechenden aktuellen Gesetz konkretisiert (Décret n ${ }^{\circ}$ 83-132 1983; Loi n 2004-800 2004; Loi n 2011-814 2011). Praktisch nehmen aber Zugehörige verschiedener Religionen teil: des Katholizismus, des Protestantismus, des Judentums und des Islam. Zudem nimmt eine Atheistin oder ein Atheist teil bzw. jemand, der oder die aufgrund seiner oder ihrer Nicht-Religiosität benannt wird, wie mir in einem Interview erklärt wurde (Int. F X; vgl. Michaud 2002). ${ }^{10}$ Gemeint sind also mit »philosophischen und geistlichen Familien« m.E. die religiösen und weltanschaulichen Spektren. 15 der Mitglieder kommen aus dem Forschungssektor. Dazu zählen u.a. Forscherinnen und Forscher vom INSERM, dem CNRS und den CHU wie auch Mitglieder der Académie des Sciences und dem Institut Pasteur, die von ihren jeweiligen Präsidentinnen oder Präsidenten, Direktorinnen oder Direktoren benannt werden (Loi n 2004-800 2004: Art. L. 1412-2f). 19 Mitglieder werden aufgrund ihrer Kompetenz oder ihres Interesses an »ethischen Problemen « für das Gremium benannt. Hierzu gehören Repräsentanten und Repräsentantinnen des Senats und der Nationalversammlung; weitere Mitglieder werden von den diversen Ministerien benannt wie auch vom Premierminister oder der Premierministerin (ebd./Übers.S.K.).

\subsection{Arbeitsweise}

Der CCNE kann sich selbst beauftragen oder von Politik und Wissenschaft beauftragt werden wie durch den Staatspräsidenten oder die Staatspräsidentin, durch den Präsidenten oder der Präsidentin des Senats oder der Nationalversammlung, außerdem durch ein Regierungsmitglied oder eine Forschungsanstalt (Décret $n^{\circ} 2005$ 390 2005: Art. R. 1412-4). Die Sitzungen sind nicht öffentlich. Sie unterteilen sich in Sitzungen des »comité plénier«, den Vollversammlungen und Sitzungen der technischen Sektion (CCNE 2014). In der technischen Sektion werden Anfragen, sofern möglich, direkt beantwortet oder, wenn sie den Mitgliedern komplexer erscheinen, als Thema für die Vollversammlung vorgeschlagen. Die Vollversammlung trifft sich einmal pro Monat und veröffentlicht letztlich die avis oder Empfeh-

10 Manche Autoren und Autorinnen nennen an dieser Stelle nicht den Atheismus, sondern den Marxismus (Michaud 1990: 178; Fuchs 2001b: 86; 2005: 14). Dies rührt vermutlich daher, dass das erste Mitglied des CCNE, das als Angehöriger des Atheismus im Komitee war, ein marxistischer Philosoph war. Konzeptioniert ist es laut Interviewauskunft in der Form, dass ein Mitglied als Zugehöriger oder Zugehörige des Atheismus aufgrund der laizistischen Tradition Frankreichs teilnimmt (Int. F VIII). 
lungen (ebd.; Michaud 2002). Die technische Sektion mit ihren zwölf Mitgliedern trifft sich ebenfalls monatlich (Michaud 2002; vgl. Int. F IV, X). Zudem bestehen Arbeitsgruppen, die ein Thema für die Vollversammlung vorbereiten. Für diese Gruppen können externe Expertinnen und Experten eingeladen werden, um ihre Perspektive auf das jeweilige Thema vorzustellen (CCNE 2014; vgl. Fuchs 2001b: 87; Michaud 2002; Braun et al. 2002: 23). Die Arbeitsgruppen treffen sich je nach Bedarf und nach den Kapazitäten der Mitglieder (Michaud 2002; Int. F IV). Die Mitglieder haben die Möglichkeit, auf das Dokumentationszentrum des CCNE zurückzugreifen, das beim INSERM angesiedelt ist (Décret $n^{\circ}$ 97-555 1997: Art. 10). Es verfügt über einen Bestand von über 8000 Publikationen zu bioethischen Themen. Nach Terminabsprache ist das Dokumentationszentrum nicht nur den Mitgliedern des CCNE, sondern auch öffentlich zugänglich; Entleihungen sind aber nicht möglich.

\section{4 Öffentlichkeitsarbeit}

Vierteljährlich erscheinen die »Cahiers du CCNE« - Hefte, die einen Überblick über die laufenden Arbeiten geben sollen (CCNE 2004). In den Cahiers werden offizielle Dokumente, Informationen über Reglementierungen im Ausland, Aktivitäten der Mitglieder des CCNE sowie Berichte von internationalen Zusammenkünften nationaler Ethikkomitees oder -kommissionen publiziert (Braun et al. 2002: 23). Informationen über den CCNE finden sich auf dessen Homepage. ${ }^{11}$ Die meisten Informationen ebenso wie die avis und Empfehlungen sind mittlerweile auch auf Englisch verfügbar. ${ }^{12}$ Der CCNE veröffentlicht, abgesehen von den Cahiers, seinen avis und Empfehlungen, jährlich einen Tätigkeitsbericht (Loi n ${ }^{\circ}$ 2004-800 2004: Art. L. 1412-2f). Mehrmals jährlich, nach der Veröffentlichung einer avis oder Empfehlung, hält das Komitee eine Pressekonferenz ab (Fuchs 2001b: 88). Mittlerweile wurden über 120 avis oder Empfehlungen abgegeben. Sie sind in Frankreich meist, aber nicht zwangsläufig, Konsenspapiere, es können ebenso divergierende Standpunkte abgegeben werden, d.h. es handelt sich nicht um einen eindeutigen Rat mit einer Handlungsoption, sondern vielmehr darum, durch die avis und Empfehlungen eine Debatte und zur Reflexion anzuregen bzw. Meinungsoptionen für eine öffentliche Debatte zu liefern (Sicard 2001: 15; vgl. Fuchs

11 Vgl. http://www.ccne-ethique.fr/, 01.02.2014.

12 Sofern eine englische Übersetzung vorliegt, werden diese im Folgenden zitiert. In einigen Ausnahmen wird auf die französischen Dokumente zurückgegriffen, wenn Begriffe oder Formulierungen dort präziser eingeschätzt wurden. 
2001b: 88). Zudem organisiert der CCNE einmal jährlich eine öffentliche Tagung, die Journées annuelles d'éthique. Sie findet meist in Paris statt, wurde aber auch schon in anderen Städten Frankreichs abgehalten (Michaud 1990: 181f). Bei dieser zweitägigen Veranstaltung werden avis und Empfehlungen des CCNE vorgestellt, seit einigen Jahren auch noch nicht abschließend diskutierte Themen. Raum für Präsentationen zu einem als ethisch verstandenen Thema bekommen zudem Schülerinnen und Schüler verschiedener Schulen, meist Gymnasien, aus ganz Frankreich. Sieben bis acht Schulklassen bereiten Präsentationen mit ihren Lehrerinnen und Lehrern vor. Vorgestellt werden die Themen dem Publikum der Journées von jeweils rund fünf Schülerinnen und Schülern pro Klasse und Thema (Int. F IV). ${ }^{13}$

Die Ausgestaltung des CCNE beinhaltet m.E. mehrere Konzepte und Ideen, die bereits ab Mitte der 1970er Jahre mit der Einrichtung des Ethikkomitees vom INSERM, der Ethikkommission bei der DGRST, dem MURS und dem Bericht »Lebenswissenschaften und Gesellschaft« aufkommen. Die beiden Ethikgremien sowie das Konzept des Dialogs und der Reflexion erscheinen als Antworten auf (antizipierte) Problematisierungen medizinischer, gentechnologischer, biologischer und lebenswissenschaftlicher Autonomie. Sie erscheinen als Sicherung der Selbststeuerung wissenschaftlicher und technologischer Entwicklungen. Ob und inwiefern der CCNE als Sicherung dieser Selbststeuerung erscheint, ist davon abhängig, auf welche Problematisierungen er antwortet und was unter Ethik verstanden wird. Auf die Frage, was das auslösende Problem für die Einrichtung des CCNE ist, wird zuerst eingegangen.

\section{Wofür war der Comité Consultatif National D'ÉTHIQUE DIE LÖSUNG?}

Als Vorläufer des CCNE gilt das Ethikkomitee vom INSERM sowohl aufgrund personeller als auch aufgrund konzeptioneller Überschneidungen. Personelle Überschneidungen existieren insofern, als Jean Bernard als Präsident für den CCNE gleichsam übernommen wird. Konzeptionelle Überschneidungen ergeben sich daraus, dass Bernard 1980 Reformvorschläge für das Komitee vom INSERM ausarbeitet, die dann mit dem CCNE umgesetzt werden (Alias 1991: 134ff; Memmi

13 Formal festgehalten ist die Anzahl der Schulklassen nicht. Die Angaben basieren auf einem Interview, Stichproben der Programme der Journées annuelles (CCNE 2005, 2009, 2013) - wobei in älteren Programmen die Schulen nicht explizit aufgeführt werden sowie auf der Teilnehmenden Beobachtung der Journées annuelles d'éthique (16./17.11.2004), Université Paris V René Descartes, Grand Amphithéâtre, Paris. 
1996: 53ff). Zu den Reformvorschlägen gehört das Einrichten einer Reflexionsgruppe und damit einhergehend die Öffnung des Komitees für andere Professionen, um bei »ethischen Fragen« aus dem Bereich der Biomedizin weitere Ansichten mit einbeziehen zu können. Vorgeschlagen wird zudem eine Gruppe von Delegierten aus dem Komitee. Mit dem Ziel der Arbeitserleichterung soll sie sich öfter treffen als die Reflexionsgruppe: Das Delegierten-Komitee soll Anfragen von Forscherinnen und Forschern direkt beantworten; nur wenn diese Anfragen negativ bewertet werden, werden sie an die Reflexionsgruppe weiter geleitet. $\mathrm{Zu}$ den Vorschlägen gehört darüber hinaus die Einrichtung eines Bioethik-Zentrums zu Dokumentationszwecken, zur Organisation von Tagungen und Seminaren. Damit soll eine öffentliche Debatte zur biomedizinischen Ethik stimuliert werden. Zudem ist eine Dezentralisierung in lokale Komitees vorgesehen (Alias 1991: 134ff). Zu der Frage, welchen Anlass es für die Reformvorschläge gibt, erwähnt Alias, dass dies aufgrund der Zunahme externer Anfragen an das Komitee geschieht (ebd.). Dass sich Jean Bernards Reformvorschläge für das Ethikkomitee vom INSERM von 1980 in der Ausgestaltung des CCNE niederschlagen, liegt darin begründet, dass er an dieser Ausgestaltung beteiligt ist: Im Jahr 1982 richtet Forschungsminister Chevènement, veranlasst durch den Staatspräsidenten, eine direkte Anfrage an den Generalsekretär vom INSERM, Philippe Lazar. Sie beinhaltet, konkrete Vorschläge für ein nationales Komitee auszuarbeiten (Didrich-Tabaste 1994: 95; Lazar 2003: 957f). Lazar konzeptioniert schließlich zusammen mit Bernard das nationale Ethikkomitee. Aber nicht nur die Zunahme an externen Anfragen an das Ethikkomitee vom INSERM sind 1982 Auslöser dafür, ein nationales Komitee zu initiieren, sondern auch die Geburt des ersten Reagenzglasbabys in Frankreich.

\subsection{Das erste Reagenzglasbaby - Eine Bedrohung der sozialen Ordnung?}

Die Einrichtung des nationalen Ethikkomitees wird gegenwärtig als Folge der Debatte um das erste Reagenzglasbaby dargestellt. In den Tageszeitungen Le Monde und Le Figaro heißt es im Jahr 2013 in Artikeln, die anlässlich des 30jährigen Bestehens des CCNE verfasst werden: »Die Gründung des CCNE wurde am 23. Februar 1983 von François Mitterrand beschlossen, nachdem eine heftige Debatte über die Geburt des ersten >Reagenzglasbabys< stattgefunden hat « (Piel 2013/Herv. i.O., Übers. S.K). Und in Le Figaro ist zu lesen: »Der Comité national consultatif d'éthique (CCNE) wurde 1983 von François Mitterrand ins Leben gerufen, um auf eine heftige Auseinandersetzung zu antworten, die auf die Geburt von Amandine folgte, dem ersten Reagenzglasbaby« (Duportail 2013/Übers. S.K.). 
»Le premier bébé eprouvette« - »Das erste Reagenzglasbaby« Frankreichs, Amandine, kommt am 24. Februar 1982 zur Welt. Verwirklicht wird die IVF von dem Gynäkologen René Frydman und dem Biologen und Agrarwissenschaftler Jacques Testart. ${ }^{14}$ Zwei Tage nach der Geburt von Amandine, am 26. Februar 1982, richtet Forschungsminister Chèvenement ein Schreiben an Lazar, den Generalsekretär vom INSERM. Er schreibt: »Ich weiß, dass sich der Comité consultatif d'éthique médicale vom INSERM schon mit diesen Problemen befasst hat (die mit der Entwicklung der In-vitro-Fertilisation zusammenhängen). Es erscheint mir dennoch, dass eine Erweiterung dieser Instanz notwendig geworden ist « (Brief von J.-P. Chèvenement an Ph. Lazar, 26.02.1982 zit. nach Memmi 1996: 52/Herv. i.O. u. hinzugefügt, Übers. S.K.). Gegen die Ausführung in Le Monde und Le Figaro, der CCNE sei in Folge einer »heftigen Auseinandersetzung « nach der Geburt Amandines entstanden, steht, dass der Forschungsminister sich bereits zwei Tage nach der Geburt an Lazar wendet. Chèvenement agiert somit noch vor einer öffentlichen Debatte, die m.E. in zwei Tagen nicht entstanden sein kann, auch wenn die Geburt medial einige Tage zuvor angekündigt wird, wie bspw. in der Pariser Tageszeitung Le Parisien (Dauzats 1982). Die Entscheidung, ein nationales Ethikkomitee einzurichten, ist auch nicht die Folge einer Diskussion auf parlamentarischer Ebene (Senat und Nationalversammlung). Wie es in einem Interview von einem ehemaligen Senatsmitglied und ehemaligen Mitglied des CCNE auf diese Frage heißt: »Nein. Mitterrand hat ihn per Dekret eingerichtet. Es gab keine Konsultation des Parlaments. Er [der CCNE, S.K.] ging von der Autorität des Präsidenten aus« (Int. F I/Übers. S.K.). Die Entscheidung, das nationale Ethikkomitee einzurichten, wird, so lautet es in einem weiteren Interview, im »Elysée« (Int. F VI) getroffen - der Elysée-Palast in Paris ist Amtssitz des französischen Staatspräsidenten. In einem weiteren Interview mit einem der ersten Mitglieder des CCNE wird gesagt: »Die Entscheidung, ein Ethikkomitee einzurichten? Weiß ich nicht, ich gehörte nicht zu den Eingeweihten. Ich weiß nicht genau, wie das genau ausgearbeitet wurde. [...] Es ist ein Werk des Präsidenten und des Forschungsministers

14 Frydman war in den 1970er Jahren in der medizinkritischen Gruppe GIS tätig, die für die Legalisierung der Abtreibung aktiv war. 1986-1990 ist er Mitglied des CCNE. Er zählt zu dem Spektrum, dessen Mitglieder aufgrund ihrer Kompetenz oder ihres Interesses an ethischen Fragen benannt werden. Testart entwickelt sich ab Mitte der 1980er zum Wissenschaftskritiker in den Bereichen Biomedizin, insbesondere Reproduktionstechnologien, Gentechnologie sowie Nanotechnologie (vgl. Testart/Serres 1988; Edelman et al. 1989). Vgl. weiterführend auch die Homepage von Testart, auf der sich eine Reihe seiner Texte befinden: http://jacques.testart.free.fr/, 12.09.2014. 
dieser Zeit!« (Int. F II/Übers. S.K.). Was die »Eingeweihten« als Problem im Kontext der IVF sehen, zeigt das folgende Zitat:

»Wenn man die menschliche Reproduktion berührt, die Sexualität auch, indirekt, löst man viele Fragen aus. [...] Und dann ging das [gemeint ist die IVF, S.K.] einen Schritt weiter in der Trennung von Sexualität und Zeugung. Die Verhütung war schon massiv, das war eine wichtige Entwicklung für die gesellschaftliche Situation der Frau und ein wichtiges Element der Trennung von Sexualität und Befruchtung. In dem Moment, wo man sich darüber bewusst geworden ist, dass man in den Prozess der Zeugung eingreifen kann, wurde man sich klar, dass Dinge passieren, die die Frage der Sexualität und der Zeugung völlig durcheinander bringen« (Int. F IX/Übers. S.K.).

Die Äußerung in dem Interview erinnert an die Problematisierung in dem Bericht »Lebenswissenschaften und Gesellschaft« von 1979. In dem Kapitel »Sitten und soziale Praktiken« wird im Kontext neuer Reproduktionstechnologien prognostiziert, dass diese Techniken »vollständig die traditionellen Verhaltensweisen im Bereich der Fortpflanzung und der Sexualität« umformen (Gros et al. 1979: 266/Übers. S.K.). In der Eröffnungsrede Mitterrands zur Einrichtung des CCNE heißt es im Kontext der neuen Reproduktionstechnologien und speziell zur Trennung von Sexualität und Zeugung: »im Hinblick auf unsere Gewissheiten und unsere Werte erschüttert diese Trennung eine der grundlegenden Beziehungen unserer Identität [die biologische Beziehung zwischen Eltern und Kind, S.K.], Gründerin der Familie und der Gesellschaft« (Mitterrand 1987: 86/Übers. S.K.). Wie Braun et al. schreiben, »wird eine gewisse Bedrohung der sozialen Ordnung und ihrer sexual-moralischen Grundlagen gesehen« (Braun et al. 2008: 228; vgl. Braun et al. 2010a: 7; Braun et al. 2010b: 518). Auslösendes Problem ist also nicht eine »heftige Debatte « um das erste Reagenzglasbaby, sondern dass eine Bedrohung der sexual-moralischen Grundlagen der französischen Gesellschaft gesehen wird. Ethik in Form des CCNE ist also die Lösung für mögliche, als moralisch wahrgenommene Konflikte durch die IVF - »man löst viele Fragen aus«, wie es in dem Interviewauszug heißt.

Die Thematisierung moralischer Probleme im Kontext der IVF zeigt sich zuletzt 1974 auf der Tagung »Biologie und die zukünftige Entwicklung des Menschen «. Auf dieser Tagung wird die Diskussion um IVF mit der Abtreibungsthematik verknüpft. $\mathrm{Zu}$ dieser Zeit ist die Diskussion um den Schwangerschaftsabbruch aktuell. Im Jahr 1979, in dem Bericht »Lebenswissenschaften und Gesellschaft«, geschieht dies nicht mehr. Die neuen Reproduktionstechnologien werden mit den Kontrazeptiva verknüpft. Erwähnt werden zwar einige Widerstände gegenüber der Anti-Baby-Pille, betont wird aber die Konsequenz, die veränderte gesellschaftliche 
Position der Frauen und die Befreiung vom Haushalt (Gros et al. 1979: 266). Als der Bericht 1978 in Auftrag gegeben wird, scheint der Abtreibungskonflikt gesetzlich gelöst. Die Vermutung liegt nahe, dass die Thematisierung moralischer Probleme im Kontext der IVF dann auftaucht, wenn der Konflikt um die Legalisierung des Schwangerschaftsabbruchs aktuell ist. Dieser These wird nun nachgegangen.

\subsubsection{Der Schwangerschaftsabbruch: vom Wiederaufleben und Verschwinden eines Konflikts}

Das Wiederaufleben und Verschwinden des Abtreibungskonfliktes kann an mehreren Eckpunkten festgemacht werden: An den Auseinandersetzungen um das VeilGesetz nach 1975, das als Probegesetz nach fünf Jahren erneut im Parlament verhandelt wird, an der Entwicklung der Frauenbewegung und am Regierungswechsel von 1981.

Nach den Konflikten um den Schwangerschaftsabbruch und der Verabschiedung des Veil-Gesetzes im Jahr 1975 gibt es gegen Ende der 1970er Jahre erneut Auseinandersetzungen. Nach anfänglichen Versuchen von Konservativen sowie Katholikinnen und Katholiken, gegen das Probegesetz vorzugehen, kommt es zunächst zu einer Ruhephase. Gegen Ende der Probezeit des Gesetzes gibt es jedoch erneut Konflikte. Der deutsche Sozialwissenschaftler Dieter Rucht identifiziert in seiner Untersuchung zu neuen sozialen Bewegungen, Gegenbewegungen und dem Staat im Kontext des Abtreibungskonfliktes zwei Fraktionen in Frankreich: Auf der einen Seite sieht er linke, feministische oder liberale Gruppen, die eine bedingungslose Freigabe der Abtreibung und die Kostenübernahme durch den Staat fordern. Auf der anderen Seite verortet er hauptsächlich katholisch orientierte Lebensrechtgruppen. Im Oktober 1979 kommt es schließlich zu einer der bis dahin größten Frauendemonstrationen in der Geschichte Frankreichs (Rucht 1991: 36). Die Frauen demonstrieren für die völlige Freigabe der Abtreibung und für die Übernahme der Kosten durch den Staat. Im Jahr 1980 geht das befristete Probegesetz mit einschränkenden Zusatzbedingungen, auch mit den Mehrheiten der konservativen Parteien parlamentarisch beschlossen, in ein Dauergesetz über (ebd.). Allerdings werden die Kosten vom Staat nicht komplett übernommen, auch gibt es keine absolute Freigabe, die Beratungspflicht bleibt. Mit dem Regierungswechsel von 1981 erhoffen sich die Aktivistinnen und Aktivisten die Aufnahme der Kostenübernahme in das Gesetz. Die PS und PC machen diesbezüglich Wahlversprechen, die sie jedoch nach dem Wahlerfolg nicht einhalten (ebd.; Ottomeyer-Hervieu 2006b: 586). So kommt es im Oktober 1982 zu einer weiteren großen Demonstration in Paris, an der sich 10.000 Menschen beteiligen. Nach einigen Kontroversen im Parlament über den Gesetzentwurf zur Kostenübernahme des Schwangerschaftsabbruchs setzt sich diese Ende Dezember 1982 zu 80 Prozent durch. Der 
Begriff der Notsituation und die Beratungspflicht werden von dem Gesetz nicht berührt. Bei dem Gesetz handelt es sich um das meist nach der Frauenministerin Yvette Roudy benannte Roudy-Gesetz (Loi Roudy) (Loi n 82-1172 1982). In den folgenden Jahren kommt es zwar weiterhin zu Kritiken am Veil-Gesetz, sowohl von den Abtreibungsbefürworterinnen und -befürwortern als auch von den -gegnerinnen und -gegnern. Es mangelt jedoch an »politischem Gewicht«, so Rucht: »Es scheint so, als habe sich bei anhaltender Kritik von beiden Polen des Meinungsspektrums eine relativ stabile Pattsituation eingependelt « (Rucht 1991: 36). Ein Aspekt dieser Pattsituation ist m.E., dass die Rolle einer der wesentlichen Akteurinnen und Akteure der Abtreibungsgegner und -gegnerinnen, der Ordre des médecins, nicht nur durch den Abtreibungskonflikt geschwächt ist. Bereits im Jahr 1975 gibt es einen Gesetzesvorschlag zur Auflösung des Ordre. Weitere Bemühungen gibt es nach dem Regierungswechsel von 1981. Die neue sozialistische Regierung begründet ihr Vorgehen mit dem Misstrauen, das in der Öffentlichkeit gegenüber dem Ordre des médecins vorhanden sei und kritisiert ihn aufgrund seiner veralteten und undemokratischen Strukturen (Maio 2000: 55, 58f). Ein weiterer Aspekt ist m.E., dass die Frauenbewegung insgesamt weniger sichtbar ist. Zum einen, so die französische Soziologin Françoise Laborie, konzentrieren sich die bewegten Frauen vermehrt an den Universitäten und in der Forschung - deren Forschungsergebnisse erreichen nur eine kleine Öffentlichkeit. ${ }^{15}$ Zum anderen gibt es nach dem Regierungswechsel eine offizielle Anerkennung frauenpolitischer Themen durch die sozialistische Regierung: Es wird ein Ministère des Droits des femmes - Ministerium für Frauenrechte eingerichtet. Frauenministerin ist die o.g. Yvette Roudy. Auf Initiative dieses Ministeriums wird bspw. die Öffentlichkeit über Frauenrechte informiert. Außerdem wird mit dem Ziel, insbesondere junge Menschen zu erreichen, eine breit angelegte Kampagne über Verhütungsmittel verwirklicht (Laborie 1995: 2; Duchen 1986: 128; vgl. Ottomeyer-Hervieu 2006a: 385). Zudem bringt der Wechsel zu einer sozialistischen Regierung die Aktivistinnen in eine neue Position gegenüber der Regierung, wie in der Untersuchung der Frauenforscherin Claire Duchen über den Feminismus in Frankreich deutlich wird (Duchen 1986). Der Wechsel führt in den Resten der Frauenbewegung zu Diskussionen über das Verhältnis zur Regierung und zu politischen Institutionen, »strategy and power, alliances and co-option, autonomy and dependence « insbesondere in Bezug auf das neu eingerichtete Frauenministerium (ebd.: viii). Wie in den von Duchen im Jahr 1984 geführten Interviews mit Aktivistinnen der französischen Frauenbewegung betont wird, war die Frauenbewegung vor 1981 eine Bewegung, die in Opposition

15 Für einen Überblick zum Beginn der Frauenforschung in Frankreich vgl. OttomeyerHervieu (2006a: 385). 
zur Regierung stand (ebd.: ixf, 136ff). Zwar bestehe ab 1981 weiterhin Kritik auch an der sozialistischen Regierung, so eine Interviewpartnerin Duchens, aber die Herausforderung, an einer Regierung Kritik zu üben, die eine Plattform für Frauen bietet, bringe viele zum Schweigen oder in Loyalitätskonflikte (ebd.: 138f). Thematisiert werden in den Interviews zudem der fehlende Dialog zwischen Frauenbewegung und dem Ministerium und die dadurch zunehmende Kluft. Die italienisch-australische feministische Theoretikerin und Philosophin Rosi Braidotti, die zu Beginn der 1980er Jahre an der Sorbonne in Paris promoviert, fasst dies in einem Interview mit Duchen folgendermaßen zusammen: »What we have done is establish a femocracy without thinking about what we're doing « (Interview mit R. Braidotti von C. Duchen 1984 zit. nach ebd.: 140). Es fehlt zudem, wie in der Untersuchung Duchens deutlich wird, ein gemeinsames und mobilisierendes Thema, wie es noch im Kampf für die Legalisierung der Abtreibung gegeben war. So beginnt einerseits die Suche nach einem übergreifenden Thema und andererseits auch die Diskussion über die eigenen Strukturen und die Organisierung (ebd.: 133). Dies hatte bereits ab Ende der 1970er Jahre zu ideologischen und organisatorischen Zerstückelungen geführt (Rucht 1991: 36; vgl. Ottomeyer-Hervieu 2006a: 384f). Ein Thema, das von Feministinnen aufgegriffen wird, ist das Thema der Reproduktionstechnologien. Dies geschieht allerdings erst in den 1980er Jahren nach der Einrichtung des CCNE. Anders als in der BRD hatte die Thematik bis dahin in Frankreich keine mobilisierende Wirkung. ${ }^{16}$ International gibt es Mitte der 1980er Jahre mit Beteiligung von Frauen aus Frankreich eine Serie von Zusammentreffen im Kontext von FINRRAGE, dem Feminist International Network of Resistance against Reproductive and Genetic Engineering, um Informationen und Analysen zu verbreiten und um eine öffentliche Debatte anzuregen. Zur Frage des feministischen Widerstands gegen neue Reproduktionstechnologien in Frankreich heißt es 1985 von Anne-Marie de Vilaine auf einer Tagung des Feminist Network on the New Reproductive Technologies, später FINRRAGE:

»We cannot speak of any active resistance of women against NRT [New Reproductive Technologies, S.K.], or of any elaborate or unanimous feminist position about NRT. Among partial considerations and reflexions on the subject, a split is appearing. Some women have a critical approach on NRT. They think they are increasing medical and social control over women - and are shading maternity and reproduction into a male, mechanistic and profit making process. Others see NRT as a possible liberation for women who could be freed from

16 Zur feministischen Kritik an den neuen Reproduktionstechnologien in der BRD vgl. Susanne Schultz (1996). 
their biological destiny or able to plan better their pregnancies and conciliate career and motherhood« (de Vilaine 1985).

Mit Ausnahme einiger Artikel in feministischen Zeitschriften sei die Sensibilisierung in Frankreich allerdings gescheitert, so Françoise Laborie rückblickend im Jahr 1995. Die große Mehrheit scheint gleichgültig und wenig interessiert. Von feministischer Seite setzen sich nur fünf bis sechs Frauen mit der Entwicklung der neuen Reproduktionstechnologien im französischsprachigen Raum auseinander, obwohl es in Frankreich eine enorme Entwicklung der Reproduktionstechnologien gibt (Laborie 1995: 2): Im Mai 1985 wird das 100. Reagenzglasbaby allein in einem IVF-Zentrum in Frankreich geboren, während es in den gesamten USA 100 Reagenzglasbabys im selben Jahr sind. 1986 gibt es 100 IVF-Zentren in Frankreich, in den USA 125 (Laborie 1988: o.S.).

Als das erste IVF Baby in Frankreich zur Welt kommt und der CCNE entsteht, lebt der Konflikt um den Schwangerschaftsabbruch noch einmal auf. Insofern bestätigt sich die Vermutung, dass moralische Probleme im Kontext der IVF dann thematisiert werden, wenn dieser Konflikt aktuell ist. Bei der Entstehung des CCNE werden m.E. im Kontext der IVF moralische Konflikte in der Qualität des Konfliktes um den Schwangerschaftsabbruch befürchtet. Zwar ist das erste Reagenzglasbaby Auslöser für die Initiierung des nationalen Ethikkomitees, jedoch antwortet das Komitee auf weitere Probleme.

\subsection{Ethik als Lösung für die "schwindelerregenden Fortschritte ${ }^{17}$}

Weitere Probleme sind solche, die der Fortschritt in der Forschung mit sich bringt, wie in der Eröffnungsrede von Mitterrand aus dem Jahr 1983 deutlich wird:

»Diese Beunruhigungen, diese Unsicherheiten sind zuerst die der Forscher und Mediziner, deren Verantwortung unmittelbar und tagtäglich gefragt ist. Welchen Risiken wird die Menschheit durch meine Arbeiten ausgesetzt? Dieser Frage kann kein Gelehrter entgehen, nicht mehr nur der Atomphysiker, bevor er Nuklearengerie freisetzt, auch der Biologe, bevor er mit genetischen Manipulationen beginnt. Auch die gesamte Gesellschaft stellt sich Fragen [...]« über die Pränataldiagnostik, die künstliche Befruchtung, über Medikamentenversuche, Humanexperimente und die neuen Möglichkeiten der Genetik (Mitterrand 1987: 85/Übers. S.K.).

17 Mitterrand 1987: 87/Übers. S.K.. 
Die Lösung für die Unsicherheiten, Beunruhigungen und Fragen sieht nach Mitterrand wie folgt aus:

»Ich wünsche mir, dass Ihr Komitee auf eine dreifache Erwartung antwortet: die der Bürger, die Anhaltspunkte in den manchmal schwindelerregenden Fortschritten der Wissenschaften suchen [...]; die der Forscher und Praktiker, die sich gegenüber den gigantischen Konsequenzen ihrer Reflexionen und ihrer Arbeiten zu alleine fühlen; die der Staatsorgane, die eine Meinung brauchen, Ratschläge, Empfehlungen« (ebd.: 87).

Während noch Mitte bis Ende der 1970er Jahre zunehmend ethische Probleme auf gesellschaftlicher Seite identifiziert werden, werden jetzt übergreifend sowohl für Wissenschaftler und Wissenschaftlerinnen sowie Bürger und Bürgerinnen Unsicherheiten, Beunruhigungen und Fragen identifiziert. In dieser Perspektive scheint es einen Bedarf an Anhaltspunkten und Empfehlungen zu geben, auch für die Politik. Nicht die »schwindelerregenden Fortschritte « scheinen das Problem, sondern, wie Anhaltspunkte geboten werden können, die die Unsicherheiten und die Beunruhigungen mindern. Der CCNE antwortet auf zwei Problematisierungen: Zum einen auf die Problematisierung, dass neue medizinische und technologische Entwicklungen Beunruhigungen und Unsicherheiten hervorrufen und dass die Selbststeuerung medizinischer und technologischer Entwicklungen angezweifelt wird. Zum anderen antwortet der CCNE auf das Problem der Bedrohung der sexualmoralischen Grundlagen der französischen Gesellschaft und der Befürchtung von moralischen Konflikten. Dem Problem der Bedrohung sexual-moralischer Grundlagen kann m.E. nach den Emanzipationsbewegungen der 1968er nicht mehr restriktiv begegnet werden. Denn das hieße, sich gegen die Befreiung der Frau vom Haushalt zu stellen oder gegen die Freiheit der Frauen, ihre Mutterschaft zu planen, wie es sowohl forschungspolitische Akteurinnen und Akteure als auch Feministinnen formulieren. Eine allgemein-verbindliche Moral als Bezugsrahmen politischer Regulierung ist problematisch geworden (Braun et al. 2008: 238). Es handelt sich um Probleme, die nicht allein auf individueller Ebene entschieden werden sollen (IVF) oder mit der medizinischen und wissenschaftlichen Selbstregulierung zu lösen sind.

Die Themen, die Mitterrand in seiner Eröffnungsrede anspricht, umfassen Pränataldiagnostik, künstliche Befruchtung, Medikamentenversuche, Humanexperimente und Genetik. Jedoch ist nicht jedes Thema ein Thema für den CCNE, wie im Folgenden gezeigt wird. 


\section{Der Comité Consultatif national d'Éthique Und SEIN VERSTÄNDNIS VON ETHISCHEN UND NICHT ETHISCHEN THEMEN}

Laut Dekret und Gesetzen soll sich der CCNE mit den Lebenswissenschaften und der Gesundheit befassen, konkret mit den Forschungen in Biologie und Medizin (vgl. Décret n 83-132 1983; Loi n 94-654 1994; Loi n 2004-800 2004). Noch Mitte bis Ende der 1970er Jahre, sowohl auf der Tagung »Biologie und die zukünftige Entwicklung des Menschen« von 1974 als auch in dem Bericht »Lebenswissenschaften und Gesellschaft« von 1979, werden unter den Begriffen Biologie und Lebenswissenschaften Themen aus dem Bereich der Reproduktionstechnologien und der Genetik verstanden, aber auch Themen aus dem Bereich der Ökotoxikologie oder des Umweltschutzes (vgl. Galpérine 1976a: Vf; Gros et al. 1979). Dies ändert sich mit der Einrichtung des CCNE.

\subsection{Zur Aufteilung von facts und values}

Nicht alle Themen sind ethische Themen, wie mir in einem Interview anhand des Unterschieds zwischen dem Office Parlementaire d'Évaluation des Choix Scientifiques et Technologiques (OPECST), der parlamentarischen Technikfolgenabschätzungsbehörde, und dem CCNE erklärt wird. Der OPECST wird ebenfalls 1983 eingerichtet. Er hat die Aufgabe, Parlamentarierinnen und Parlamentarier zu informieren sowie Vorschläge und Empfehlungen auszusprechen (Sénat 2006). Der Office erarbeitet Berichte, die direkt dem Gesetzgebungsprozess dienen. In dem Interview mit einem Mitglied sowohl vom CCNE als auch vom OPECST, heißt es:

»Der OPECST ist ein wissenschaftliches Komitee, es geht um Wissen und Entscheidung. Hier kann die ethische Dimension existieren, aber sie ist nicht ausschlaggebend. Viele Themen sind keine aus dem ethischen Bereich. Wenn man von Atomen und Atomenergie, auch GMO und der Art und Weise der Verbesserung von Lauch spricht [...]. Das sind typische Themen für den OPECST « (Int. F V/Übers. S.K.).

Ausgeschlossen aus der Ethik-Rahmung ist also genmanipulierter Lauch, sprich Biotechnologie im Agrarbereich oder Atomtechnologie, die in das Aufgabenfeld vom OPECST fallen. Was sich 1983 abzeichnet, ist eine Ein- und Aufteilung bestimmter Technologien in den Zuständigkeitsbereich vom OPECST und den Zuständigkeitsbereich des CCNE. Die Aufgabenfelder vom OPECST liegen zu Be- 
ginn im Bereich Umwelt, Atomkraft und Informationstechnologien. Begründet wird die Einrichtung der Behörde wie folgt:

»[Die Wissenschaft, S.K.] hat neue Probleme und neue Unruhen hervorgerufen. Gestern noch ließ man sie sich ungezügelt und ohne Sicherheitsmaßnahmen in der Funktion des Wohlstands entwickeln, den sie eigentlich gewährleisten sollte, heute verlangt man von ihr den Beweis ihrer Unschuld im Voraus zu bringen« (Sénat 2006).

Mit dem OPECST sollen Mechanismen umgesetzt werden, die den technischen Fortschritt steuern, indem die Technikfolgen antizipiert werden (ebd.). Der Punkt ist, dass hier eine institutionelle Trennung zwischen den Zuständigkeiten für facts und für values vorgenommen wird und nur bestimmte Technologien im EthikRahmen verhandelt werden. Während Atomtechnologie zu den facts zählt und damit zum OPECST, ebenso wie Biotechnologie im Agrarbereich, zählen biotechnologische Entwicklungen in der Medizin zu den values und damit zum CCNE. Das Themenfeld Biotechnologie taucht ab den 1990er Jahren in den Berichten vom OPECST auf. ${ }^{18}$ Zwar beschäftigt sich der OPECST erst Mitte der 1990er Jahre mit dem Thema Biotechnologie im Agrarbereich, da aber die Biotechnologie in der Medizin zu den values, dem Aufgabengebiet des CCNE gezählt wird, findet m.E. implizit bereits eine Aufteilung statt.

Mit der Einrichtung vom OPECST und dem CCNE gibt es nicht nur eine Einteilung der Diskussion verschiedener Technologien in facts und values. Auch die Gen- bzw. Biotechnologie, die zuvor als eine Technologie gedacht wird, wird m.E. aufgeteilt. Diese Aufteilung entspricht, auch wenn dies nicht explizit so genannt wird, der aktuellen Bezeichnung von grüner und roter Biotechnologie. Unter dem Begriff grüne Gen- bzw. Biotechnologie wird aktuell die Anwendung gentechnischer Methoden im Agrar- und Lebensmittelbereich gefasst, unter roter Gentechnologie wird der Einsatz gentechnischer Methoden in Medizin und biomedizinischer Forschung verstanden. Mitte der 1970er Jahre wird diese Unterteilung noch nicht vorgenommen. In der Kontroverse, die in Le Monde zwischen dem Institut Pasteur und dem GIB ausgetragen wird, wird sowohl von der Herstellung von Impfstoffen und der Möglichkeit Krankheiten zu heilen gesprochen als auch von der Verbesserung landwirtschaftlicher Erträge durch Gentechnologie. Auch die Genkritikerinnen und -kritiker sprechen von den genetischen Manipulationen, und zwar auch noch zu Beginn der 1980er Jahre. Wie die Gen- bzw. Biotechnologie in wissen-

18 Im Juni 1998 organisiert der OPECST eine Bürgerinnen- und Bürgerkonferenz zu GMO im Agrar- und Ernährungsbereich (OPECST 1998). Vgl. weiterführend Callon et al. (2005), Joly et al. (2000), Marris und Joly (1999) sowie Bonneuil et al. (2008). 
schaftskritischen Veröffentlichungen zu Beginn der 1980er Jahre gedacht und gerahmt wird, zeigt die Publikation »Les manipulations génétiques« - »Die genetischen Manipulationen«, die 1980 erscheint.

\subsubsection{Politische oder technische Probleme? - Vom Verschwinden der Gentechnologiekritik}

Das Buch »Die genetischen Manipulationen« wird von einem Autorinnen- und Autorenkollektiv unter dem Pseudonym Agata Mendel geschrieben (Mendel 1980/Übers. S.K.). Darin werden zum einen die gentechnologiekritischen Positionen in Frankreich ab Mitte der 1970er Jahre ausführlich dargestellt, außerdem wird die Entwicklung der Proteste anhand von Flugblättern und Appellen dokumentiert. Zum anderen ist das Ziel des Kollektivs die Information der Öffentlichkeit über Gentechnologie, die Öffnung der vor allem wissenschaftlich geführten Debatte und die Beteiligung der Öffentlichkeit an Entscheidungsfragen über technologische und wissenschaftliche Entwicklungen:

»Wenn wir dieses Buch veröffentlichen, so machen wir dies - und wir hoffen, es ist nicht zu spät - , damit die Debatte wieder aufgenommen wird und sich verbreitet und um daran zu erinnern, dass das Problem nicht nur technisch, sondern auch politisch ist. Wir denken, dass die Öffentlichkeit sowohl von den unmittelbaren Risiken genetischer Manipulationen betroffen ist als auch von den Langzeitfolgen und dass sie sich äußern können muss « (ebd.: 22/Übers. S.K.).

Unter politisch wird von Agata Mendel nicht nur die Meinungsbildung und Entscheidungsfrage über technologische und wissenschaftliche Entwicklungen verstanden. Sie hinterfragt die ihres Erachtens in der Debatte dominierende technische Problemdefinition und Lösungskompetenz der Wissenschaftlerinnen und Wissenschaftler politisch: »Man verspricht uns Getreide, das ohne Stickstoffdünger wächst und das deswegen das Problem des Hungers in der Welt lösen könnte; hat das Beispiel der grünen Revolution nicht weitreichend vor Augen geführt, dass dieses Problem weniger technisch als politisch ist?« (ebd.: 19/Übers. S.K.).

Als Grüne Revolution wird die Industrialisierung der Landwirtschaft bezeichnet, die in den 1960er Jahren in Indien und anderen so genannten Entwicklungsländern beginnt. Vordergründig hat sie sich mit dem Ziel durchgesetzt, der Nahrungsmittelknappheit und dem Hunger in Gebieten der so genannten Entwicklungsländer entgegen zu wirken. Die Weltbank, die US-amerikanische Ford- und 
die Rockefeller Stiftung und auch die US Agency for International Development ${ }^{19}$ erhoffen sich mit der Grünen Revolution jedoch auch, die Forderungen nach Umverteilung von Land und Ressourcen zu entschärfen und den ländlichen Raum politisch zu stabilisieren (Shiva 1991: 58). Mit der Grünen Revolution gehen verschiedene Prozesse einher: die Verwendung von gezüchtetem Hochleistungssaatgut, der Anbau von Monokulturen, die Anwendung mineralischer Dünge- und Pflanzenschutzmittel. Die landwirtschaftlichen Erträge können zwar erheblich gesteigert werden, im Gegenzug allerdings geht die Sortenvielfalt zurück, die Anfälligkeit für Schädlingsbefall steigt und die ursprüngliche Fruchtbarkeit der Böden sinkt. Darüber hinaus finden sich die lokalen Bäuerinnen und Bauern durch die Grüne Revolution in Abhängigkeitsverhältnissen zu internationalen Agrar- und Chemiekonzernen wieder, da sie auf deren Saatgut, Dünge- und Pflanzenschutzmittel angewiesen sind, so die indische Wissenschaftlerin, Umweltaktivistin und Frauenrechtlerin Vandana Shiva (ebd.: 60). Mit den Möglichkeiten der Biotechnologie und insbesondere der Gentechnologie beginnt schließlich die häufig so genannte zweite Grüne Revolution.

Indem Agata Mendel auf das Beispiel der Grünen Revolution Bezug nimmt und sie als politisches Problem bezeichnet, versucht sie Probleme und deren Auswirkungen jenseits technischer Möglichkeiten zu denken und eine historische Perspektive sowie den gesellschaftspolitischen Kontext in die Diskussion um Gentechnologie einzubeziehen. Dies bezieht sich allerdings nicht allein auf die Diskussion der Grünen Revolution, also auf die Gentechnologie im Agrarbereich, sondern auf weitere Bereiche der Gentechnologie. So heißt es z.B.:

»Mit Hilfe von Manipulationen verspricht man uns haufenweise billige Medikamente, Impfstoffe, Hormone. Ist es wirklich das, was wir brauchen? [...] Man verspricht uns schließlich die Korrektur erblicher Belastungen, sprich die Kontrolle der Evolution, die Verbesserung der menschlichen Gattung. Erinnert Sie das nicht an etwas? (Mendel 1980: 19/Übers. S.K.).

Agata Mendel orientiert sich mit der Frage »was brauchen wir? « an der Frage der gesellschaftlichen Bedürfnisse. Zudem eröffnet sie eine historische Perspektive: Mit »Verbesserung der menschlichen Gattung « und der Frage, woran dies erinnert, stellt sie eine Verknüpfung zwischen Gentechnologie und Eugenik her. Mendel thematisiert die NS-Eugenik, stellt eine länderübergreifende Renaissance der Eugenikbewegung fest und thematisiert Eugenikpolitiken in weiteren Ländern, insbesondere im Kontext des Schwangerschaftsabbruchs. Sie äußert die Befürchtung,

19 Die Agency ist eine der weltweit größten Entwicklungsorganisationen und dem USamerikanischen Außenministerium unterstellt. 
dass mit zunehmenden genetischen Untersuchungen, wie der Untersuchung von Aggressivität bei Ratten, die Möglichkeiten eugenischer Maßnahmen beim Menschen erstarken können (ebd.: 319ff). Nach meiner Recherche hatte Agata Mendels Publikation nicht die gewünschte Wirkung, nämlich die Debatte zu öffnen oder eine politische Diskussion anzuregen. Vermutlich hatte sie diese Wirkung auch nicht, weil die politische Risiko-Rahmung der Gentechnologiekritikerinnen und -kritiker mit der Risiko-Rahmung der Atomtechnologiekritikerinnen und -kritiker verknüpft ist und auch diese Kritik verschwindet.

\subsection{2 ... und vom Verschwinden der Kritik an Atomtechnologie}

Auch Atomenergie wird mit der Einrichtung vom OPECST als technisch gerahmt. Maßgebliche Akteurin im Konflikt um Atomtechnologie ist die Anti-Atombewegung, die eng mit der Ökologiebewegung verknüpft ist. Sie verwendet vor allem einen politisierten Risikobegriff. Eine Erklärung für die Marginalisierung des politischen Risikobegriffs kann in der Entwicklung der Ökologiebewegung gesehen werden. Anders als in den meisten übrigen Ländern, so Rucht in seiner Untersuchung zu sozialen Bewegungen in Deutschland, Frankreich und den USA, festigt sich die französische Ökologiebewegung in den 1980er Jahren nicht (Rucht 1994; vgl. von Oppeln 1989): Die Écologistes, die an der Anti-Atombewegung beteiligt sind, sind im Jahr 1981 mit dem Kandidaten Brice Lalonde von Aujourd'hui l'Écologie bei den Präsidentschaftswahlen vertreten. Bereits im Jahr 1978 ziehen sie unter der Bezeichnung Écologie 78 in die Wahlen zur Nationalversammlung, obwohl der politische Zweck einer Wahlbeteiligung vorher nicht diskutiert wird und nicht auf breite Unterstützung stößt (Hegedus/Touraine 1982: 51). Die Grünen selbst gehen erst 1984 aus einer Fusion von Parti Écologiste und der Confédération Écologiste, die 1982 gegründet wird, hervor (Schmidt 2006b: 995f). Der Regierungswechsel von 1981 wird von vielen Écologistes unterstützt, zeigt sich aber nicht, wie erhofft, als Wende in der Umweltpolitik. Viele Aktivistinnen und Aktivisten fühlen sich von der sozialistischen Regierung verraten. Kleinere Gruppierungen zerfallen oder verlieren an Bedeutung, während die etablierten Umweltverbände und Les Verts an Boden gewinnen. Zu dieser Zeit wird erkennbar, so Rucht, dass der Kampf gegen die Atomenergie verloren ist (Rucht 1994: 243f).

Während sich Mitte bis Ende der 1970er Jahre ein politischer und technischer Risikobegriff gegenüberstehen, wird nun auf institutioneller Ebene mit dem OPECST ein technischer Risikobegriff sichtbar. Eine Behörde für Technikfolgen wird eingerichtet. Damit wird anerkannt, dass aus technologischen Entwicklungen nicht allein Wohltaten für die Menschheit entstehen. Das Thema der Technikfolgen als »Langzeitfolgen« ist zuvor Gegenstand der Atom- und Gentechnologiekritikerinnen und -kritiker, sie thematisieren die politischen Risiken der Folgen (Mendel 
1980: 22/Übers. S.K.). Dadurch, dass die Thematisierung von Folgen von einer Behörde übernommen wird, findet m.E. eine Thematik, die zuvor in der technologiekritischen Bewegung verhaftet war und in Opposition zur Regierung stand, Anerkennung von eben dieser. Dies führt m.E. zu einer Entpolitisierung: ein politisierter Risikobegriff wird technisiert und damit marginalisiert.

Mit der Forschungspolitik der 1980er Jahre, der Einrichtung des CCNE, vom OPECST oder der CCSTI verschwindet die wissenschaftskritische Debatte. Die Einrichtung des CCNE hat allerdings nicht dazu geführt, dass es keine Kritik mehr gibt, nur zeigt sie sich in anderer Form.

\subsubsection{Exkurs: eine neue Form der Kritik?}

Als der CCNE im Jahr 1983 eingerichtet wird, bleibt er in der Presse nicht unkritisiert. Die Kritik richtet sich insbesondere auf die Art und Weise der Zusammensetzung seiner Mitglieder: Von der linken Tageszeitung Libération, ${ }^{20}$ der Tageszeitung Le Quotidien de Paris und der Wochenzeitschrift Le Point wird bspw. festgehalten, dass unter den Mitgliedern des CCNE kein Repräsentant oder keine Repräsentantin des Ordre des médecins ist (Le Point 24.10.1983 zit. nach Alias 1991: 150; Conan 1983). In der Libération und in Le Monde ist ein weiterer Kritikpunkt, dass ein Marxist der PC Mitglied des CCNE wurde (Conan 1983; Valadier 1983). Es handelt sich um Lucien Sève, der 1983 als Angehöriger einer der so genannten wichtigsten philosophischen und geistlichen Familien Frankreichs benannt wird. Die Autorinnen und Autoren von Le Monde und der Libération mokieren, dass es sich beim Marxismus wohl um eine Religion handle (Conan 1983). Kritisiert wird zudem die Mehrheit an »Technokraten«, gemeint sind Forscherinnen und Forscher, Medizinerinnen und Mediziner (EscoffierLambiotte 1983/Übers. S.K.). Auch das Fehlen weiterer Berufsgruppen wird thematisiert, wie das Fehlen von Psychiatern oder Psychiaterinnen, Moralphilosophen oder -philosophinnen. Ebenso wird kritisiert, dass Juristen und Juristinnen sowie Soziologen und Soziologinnen nur in geringer Anzahl vertreten sind (ebd.). Le Monde kritisiert darüber hinaus das Benennungsverfahren insofern, als der Staatspräsident oder die Staatspräsidentin die Mitglieder aus den »philosophischen und geistlichen Familien « benennt und diese nicht von den »Familien« selbst benannt werden können (ebd./Übers. S.K.; Valadier 1983). Ein Kritikpunkt ist zudem, dass in der technischen Sektion keine Mitglieder vertreten sind, die aufgrund ihrer $\mathrm{Zu}$ gehörigkeit $\mathrm{zu}$ den wichtigsten weltanschaulichen und religiösen Spektren im

20 Die Tageszeitung Libération entsteht aus dem Kontext der 1968er-Bewegung und erscheint zum ersten Mal 1973. Ursprünglich als linke Tageszeitung konzipiert, vertritt sie mittlerweile eher linksliberale Positionen, vergleichbar mit der taz in Deutschland. 
CCNE sind. Nur Mitglieder aus dem Forschungsbereich und Mitglieder, die aufgrund ihrer ethischen Kompetenz im CCNE sind, sind vertreten. In Le Monde wird vermutet, dass sich so ein Übergewicht von Forschung und Wissenschaft ergibt, das sich auf die Ergebnisse des CCNE auswirken könnte (Valadier 1983).

Das Augenmerk meiner Studie zur Entstehung des CCNE liegt auf den Problematisierungen, aus welchen das Komitee als Lösung hervorgeht. Es geht mir weniger darum, welche Konflikte der CCNE bei seiner Einrichtung auslöst. Allerdings ist in diesem kurzen Ausschnitt der Presseartikel eines auffällig, das an dieser Stelle doch genannt werden soll: Es scheint sich eine Kritik an Regierungspraktiken zu entwickeln, die sich auf das Verfahren, die Zusammensetzung und die Benennungsmodi richtet. Der Punkt ist, dass die sozialistische Regierung sich selbst als anti-technokratisch versteht. Diese neue Kritik überprüft das Regierungshandeln nach diesen Maßstäben. Es wird sich bspw. nicht mehr für eine bestimmte technologische Entwicklung ausgesprochen, wie noch Mitte der 1970er in Le Monde im Kontext der Kontroversen um Gentechnologie, sondern implizit für das richtige oder bessere Verfahren im Umgang mit technologischen und wissenschaftlichen Entwicklungen. Es geht in diesen Zeitungsartikeln weniger darum, eine Position zu einer bestimmten Technologie einzunehmen, als vielmehr um die Verfahrensfrage.

Zurück zu den Themen, die als ethische Themen des CCNE verstanden werden. Es sind nicht nur Themen wie Atomtechnologie oder genmanipulierter Lauch, die nicht in den institutionalisierten Ethik-Rahmen gehören, auch Themen aus der medizinischen Praxis gehören nicht dazu.

\subsection{Von medizinischer Praxis und medizinischer Forschung - eine thematische Aufteilung}

Der CCNE beschäftigt sich explizit mit Themen aus der medizinischen und biologischen Forschung und nicht mit deren Praxis. Diese Aufteilung zwischen Forschung und Praxis erklärt sich wiederum anhand einer weiteren Institution, und zwar anhand des Ordre des médecins. Jean Bernard erklärt 1984, dass die Aufgabe des CCNE im Bereich der Moral der medizinischen Forschung liege und die Aufgabe des Ordre im Bereich der Moral im Kontext der Ausübung des medizinischen Berufs (Bernard (1984), in CFDT Magazine (nov.): o.S., zit. nach Alias 1991: 151f). Auch in einem Interview wird mir erklärt: »der Ordre beschäftigt sich mit der medizinischen Deontologie, d.h. mit akzeptablem oder inakzeptablem Verhalten der Mediziner [...]. Hier gibt es keinen Wertkonflikt, nicht in dem Sinn, wie ich Ethik verstehe. Wenn jemand falsch handelt, wird er sanktioniert. Das ist die Funktionsweise des Ordre«(Int. F IX/Übers. S.K.). Dass der Ordre des médecins 
sich mit der medizinischen Deontologie beschäftigt ist nicht neu. Neu ist allerdings, dass sich Themen, die zuvor in seinem Kompetenzbereich liegen, zu welchen er in der Öffentlichkeit Position bezogen hat, wie Humanexperimente, nun im institutionellen Ethik-Rahmen des CCNE wiederfinden. Die Themen werden nun in einem institutionellen Rahmen diskutiert, aus dem der Ordre ausgeschlossen ist - in der Zusammensetzung der Mitglieder des CCNE ist kein Mitglied des Ordre vorgesehen. Die Kompetenzbereiche und Themen verschieben sich vom Ordre zum CCNE. Dass dies ein Novum darstellt, wird in einem sozialwissenschaftlichen Artikel von François-André Isambert aus dem Jahr 1983 deutlich, der noch vor der Umsetzung des nationalen Ethikkomitees entstand. ${ }^{21}$

\subsection{1 "[E]in Bruch in der medizinischen Souveränität « ${ }^{22}$}

François-André Isambert stellt in seinem Artikel einige Überlegungen zum nationalen Ethikkomitee und dem ärztlichen Berufsstand an:

»Nun aber ist die ethische Kompetenz traditionell Bestandteil der medizinischen Kompetenz in Bezug auf alles, was therapeutische Entscheidungen betrifft. Das Teilen dieser Kompetenz mit Nicht-Medizinern wäre ein Bruch in der medizinischen Souveränität, was bisher in unserem Land kaum akzeptiert wird « (Isambert 1983: 680/Übers. S.K.).

Dass dieser »Bruch « akzeptiert werden muss, zeigt m.E. folgendes Zitat aus einem Interview: »Der CCNE wurde 12 Jahre von Jean Bernard präsidiert, der ein Papst der französischen Medizin war, das hat den CCNE in den ersten Jahren geschützt. Kein Präsident des Ordre hätte es gewagt, Jean Bernard anzugreifen « (Int. F IX/Übers. S.K.). Der Bruch in der medizinischen Souveränität, von dem Isambert spricht, zeichnet sich m.E. nicht erst 1983 mit der Einrichtung des CCNE ab, sondern bereits ab Mitte der 1960er Jahre. Im Kontext der Legalisierung der AntiBaby-Pille, im Kontext der Diskussion um Humanexperimente und der Kontroverse um den Schwangerschaftsabbruch verlor der Ordre des médecins bereits an moralischer Definitionsmacht. Dies bahnte den Weg für eine neue, nicht moralische, sondern ethische Bewertung von Humanexperimenten in Form des Ethikkomitees vom INSERM (Maio 2000: 78). Mit dem CCNE zeichnet sich der Bruch noch deutlicher ab. Nicht allein Humanexperimente, auch weitere Themen wie der Schwangerschaftsabbruch verschieben sich in den Kompetenzbereich des CCNE -

21 Dass der Artikel entstand, bevor der CCNE 1983 umgesetzt wird, wird in den Fußnoten 21 und 25 des Artikels deutlich (Isambert 1983: 679, 681).

22 Isambert 1983: 680/Übers. S.K.. 
von einem moralischen zu einem ethischen Verständnis. ${ }^{23}$ Wie in Anknüpfung an Kohlen herausgearbeitet, zeigen sich beim Ethikkomitee vom INSERM insofern »shifted responsibilities«, da nicht mehr allein die Verantwortung des Mediziners oder der Medizinerin ausschlaggebend für das Durchführen eines Versuchs ist, sondern nun auch eine übergeordnete Instanz in Form eines Expertinnen- und Expertenkomitees. In Frankreich ist diese Verschiebung m.E. möglich, da der Ordre an Einfluss verliert und so überhaupt erst eine neue Bewertung möglich wird. Der Verantwortungsbereich verschiebt sich also vom Ordre zum Ethikkomitee des INSERM. Mit dem CCNE verschiebt sich der Verantwortungsbereich noch einmal, und zwar nicht allein für Humanexperimente, sondern für weitere Themen, die zuvor im Kompetenzbereich des Ordre liegen.

Der Grund für die Entstehung von Ethikinstitutionen wird, wie in Kapitel III herausgearbeitet, im »Bewusstwerden über ethische Probleme« in Folge der NSMedizin und ihrer Experimente gesehen (Lenoir 1991: 16/Übers. S.K.). Ethikinstitutionen erscheinen als notwendige Folge von Problemen medizinischer Forschung, ein ethischer Umgang scheint selbstverständlich. Diese Ethik beinhaltet jedoch nicht eine historische Aufarbeitung der Humanexperimente im NS, wie anhand des Vergangenheitsdiskurses, in dem das Ethikkomitee des INSERM entsteht, aufgezeigt. Auch im Kontext des CCNE zeichnet sich diese Aufarbeitung nicht ab.

\subsubsection{NS-Medizin - »Dinge, für die man kein Recht hat, sie zu tun « ${ }^{24}$}

Auf meine Frage, ob die NS-Medizin in den Diskussionen des CCNE thematisiert wird, heißt es von einem Mitglied, das von Beginn an und über 15 Jahre an den Diskussionen teilnimmt:

»am Anfang hat das [die NS-Medizin, S.K.] eine wichtige Rolle gespielt. Ich erinnere mich, zu Beginn meiner Teilnahme am CCNE sind wir oft darauf zurückgekommen, sehr oft. Die großen internationalen Bestimmungen, die damit verbunden waren, waren der Sockel für den Respekt vor der menschlichen Person - für die Dinge, für die man kein Recht hat sie zu tun die freie und aufgeklärte Zustimmung als Voraussetzung zu Experimenten« (Int. F VIII, Übers. S.K.).

Mit den »großen internationalen Bestimmungen« sind m.E. die Deklaration von Helsinki und ihre überarbeiteten Versionen gemeint, da darin die »ethischen

23 Im Jahr 1986 publiziert der CCNE bspw. eine avis zur so genannten Abtreibungspille (CCNE 1986), im Jahr 2000 behandelt er das Gesetz zum Schwangerschaftsabbruch (CCNE 2000).

24 Int, F VIII/Übers. S.K.. 
Grundsätze« (WMA 2013/Übers. S.K.) für die Forschung am Menschen formuliert werden, auf die in dem Interviewausschnitt Bezug genommen wird. Zentral sind in dem Beitrag die internationalen Bestimmungen, nicht die NS-Medizin selbst oder ihre Entstehung, die Beteiligung, die internationalen Verflechtungen von Medizinerinnen und Medizinern oder die Förderung einer eugenisch und rassistisch motivierten Medizin. Auch eine Kritik, wie sie der Ordre an Humanexperimenten formuliert, die er in den Kontext der NS-Medizin stellt, ist insofern ausgeschlossen, da kein Mitglied des Ordre für den CCNE benannt ist. Es wird sich mit den Bestimmungen auseinandergesetzt. Das »Bewusstwerden über ethische Probleme« und die Einrichtung von Ethikinstitutionen als Folge der NS-Medizin bedeutet also auch im Kontext des CCNE nicht, dass es eine grundlegende historische Aufarbeitung medizinpolitischer Entwicklungen gegeben hätte.

Die Ethik-Rahmung schließt bestimmte Themen aus, wie Atomtechnologie oder grüne Biotechnologie. Zuvor als moralisch oder politisch verstandene Themen werden technisch gerahmt oder ethisiert. Die Frage, die nun beantwortet wird ist, was unter dem Begriff Ethik verstanden wird und, wie über die Themen gesprochen werden kann.

\section{Der Comité Consultatif national d'Éthique Und DIE BEDEUTUNG VON ETHIK}

Genau genommen wird zu Beginn des CCNE gar nicht von ethischen Problemen gesprochen, sondern von moralischen. Auf die Fragen, was unter Moral und Ethik verstanden wird und ob im Kontext des CCNE gar nicht von einer Ethisierung, sondern von einer Moralisierung gesprochen werden sollte, wird im Folgenden eingegangen.

\subsection{Die Moral der Ethik}

In einem Interview wird auf meine Frage, wieso zunächst von moralischen und später ethischen Problemen gesprochen wird, geantwortet, dass darüber nachgedacht wurde, ob man den Terminus Moral oder Ethik verwendet. Im Planungsprozess des CCNE fällt die Entscheidung von einem Ethikkomitee zu sprechen, das moralische Probleme behandelt. Und weiter heißt es:

»Das hat sich dann geändert. Das Wort Ethik [...] hat mehrfache Bedeutungen. Die Ethik des Spinoza ist nicht dasselbe wie das, wovon ich hier rede. Was heißt das genau, das Wort 
Ethik? Die Bezeichnung moralische Probleme ist verständlicher für die Bevölkerung und es war unsere Sichtweise, dass die Leute es verstehen. [...] Für eine Art intermediäre Instanz zwischen der Wissenschaft in ihrer Entwicklung und der Bevölkerung muss man Worte nehmen, die die Leute verstehen. [...] Es gibt Fragen moralischer Ordnung, die sich stellen, und die Leute sollen ein bisschen besser verstehen, wo wirklich die Fragen sind, um die Reflexion auf die Punkte zu konzentrieren, die wirklich ein Problem machen und um nicht auf zweitrangige Fragen auszuweichen« (Int. F IX/Übers. S.K.).

Die Verwendung des Terminus Moral erscheint als Mittel, um sich der Bevölkerung verständlich zu machen. Wichtig ist, dass die Leute »ein bisschen besser verstehen, wo wirklich die Fragen sind«. Ziel ist, »die Reflexion auf die Punkte zu konzentrieren, die wirklich ein Problem machen«. Ob es sich um moralische oder ethische Fragen handelt, scheint weniger zentral. Auch Mitterrand spricht 1983 in seiner Eröffnungsrede von Moral im Sinne einer Reflexions-Zeit: »Je schneller sich die Welt dreht, desto stärker ist die Versuchung des Unbekannten und desto mehr Zeit müssen wir uns nehmen: [...] die Zeit [...], die ich die Zeit des Austauschs und der Reflexion nennen möchte, das heißt die Zeit der Moral (Mitterrand 1987: 88/Übers. S.K.). Die Begriffe Ethik und Moral erscheinen hier nach meiner Ansicht austauschbar, sie werden nach ihrem Effekt auf die Öffentlichkeit eingesetzt. Gemeinsam ist den beiden Begriffen allerdings, die Reflexion anzuregen bzw. sie zu kanalisieren oder, wie es in dem Interviewauszug heißt, sie auf die problematischen Punkte zu konzentrieren. Der Terminus Moral erscheint hier als anfängliche Übergangslösung, die rhetorisch eingesetzt wird, um sich der Öffentlichkeit verständlich zu machen. Auch in den offiziellen Satzungen wird ersichtlich, dass die Bezeichnung Moral nur vorübergehend auftaucht. Entsprechend findet sich im Gründungsdekret des CCNE von 1983 noch die Formulierung, dass das Komitee seine avis zu moralischen Problemen, die sich aus der Entwicklung der Forschung im Bereich der Biologie, Medizin und Gesundheit stellen, abgeben soll (Décret $n^{\circ}$ 83-132 1983). Später gibt es diese Formulierung nicht mehr. Die Bezeichnung Moral verschwindet und zwar bereits in den ersten avis des CCNE. Dort wird nicht mehr von moralischen, sondern ethischen Problemen gesprochen: 1984 werden »ethical problems raised by >surrogate motherhood« « genannt (CCNE 1984a: o.S./Herv. i.O.), im gleichen Jahr »ethical issues raised by drugs trials on humans« (CCNE 1984b: o.S.). Sechs Jahre später, in der avis über die NichtKommerzialisierung des menschlichen Körpers, wird auch von einem »fundamental ethical criterion represented by the dignity of man « gesprochen (CCNE 1990b: o.S.). Wenn die Sprache der Moral hier eher ein Mittel zum Zweck ist, eine Übergangslösung, und der Terminus Moral durch den Terminus Ethik ersetzt werden kann, was wird dann von den Mitgliedern unter dem Begriff Ethik verstanden? 


\subsection{Definitionslose Ethik}

$\mathrm{Zu}$ der Frage, was unter Ethik zu verstehen ist, geben die Dokumente wenig Aufschluss und die (ehemaligen) Mitglieder des CCNE in den Interviews uneinheitliche und oft vage Antworten. Es zeigt sich, dass die Mitglieder keine gemeinsame Definition des Begriffs haben: »Was den Ethikrat und den Begriff Ethik betrifft: Ich denke, wenige Leute machen sich über das Wort Gedanken« (Int. F VIII/Übers. S.K.). Strategische Überlegungen, wie noch im Planungsprozess des CCNE, den verständlicheren Begriff Moral zu verwenden, gibt es nicht. Allerdings gibt es, wie in einem Interview ausgeführt wird, zu Beginn des CCNE eine Grundsatzentscheidung seiner Mitglieder über die Art und Weise des Diskussionsverfahrens, ausgelöst vom damaligen Präsidenten des CCNE, Jean Bernard. Die Frage lautet, ob schlicht mit der Diskussion der Themen begonnen oder zuerst eine allgemeine Prinzipiendiskussion geführt werden soll. Die Entscheidung fällt für den Beginn der Diskussion, da vermutet wird, dass man bei einer Prinzipiendiskussion nicht zu einem Ergebnis kommen würde, wie mir in zwei Interviews erzählt wird (vgl. Int F IX, VIII). In dieser Diskussion spielt aber wiederum der Terminus Moral eine Rolle, wie in folgendem Interviewausschnitt deutlich wird:

»Die meist praktizierte Form wird auch im Ethikkomitee verwendet, es sind keine Philosophen, sie suchen nicht die Präzision eines Konzeptes. Ethik bedeutet einfach, Moral in Aktion, Moral des Alltags, Moral gegenüber einem konkreten Fall. Moral ist die allgemeine Grundlage, Ethik ist der konkrete Fall, der diskutiert werden muss (Int. F VIII/Übers. S.K.).

Der Ethik-Rahmen kann also einerseits auch Moralvorstellungen beinhalten, etwa eine Moral, die ein Mitglied mit sich bringt, die aber nicht diskutiert wird, sondern als Grundlage angenommen wird. Sie scheint eher im Hintergrund zu stehen. Andererseits zeigt sich das Verständnis von Ethik aber auch gerade in Abgrenzung zu Moral: »Moral, das sind graue Haare, die alte Generation, Reaktionäre, die uns die Sachen, die sie für gut heißen, vorschreiben wollen. Ethik heißt, ich mache meine eigenen Lebensregeln, selbständig « (Int. F VIII/Übers. S.K.). In diesem Zitat zeigt sich Ethik in Abgrenzung zu Moral vor allem als individuelle Angelegenheit.

Wenn Moral im Hintergrund von Ethik steht oder Ethik im Kontrast zu einer veralteten Moral verstanden wird, was ist dann vordergründig?

»Die Leute sind aus persönlichen Gründen da, um zu versuchen, ein Modell für die Lösung eines ethischen Konfliktes zu sein, [ein Modell, S.K.] das repräsentativ für den Reichtum, [...] die Diversität der Nation ist. [...] Es scheint mir sehr vernünftig. Es gibt Repräsentanten der wichtigsten Religionen, der medizinischen und biologischen Professionen. [...] Zu ver- 
suchen, Leute zu nehmen, die aus eigenem Antrieb kommen und Leute, die zu dieser Diversität beitragen können« (Int. F V/Übers. S.K.).

Der CCNE ist in diesem Sinn ein Modell zur Konfliktlösung, eine Art nationales Vorreflexionskomitee mit engagierten Mitgliedern: diese kommen aus »eigenem Antrieb «. Das Vorreflexionskomitee liefert ein Modell oder einen Vorschlag für die »ethische« Konfliktlösung und zeigt die Möglichkeit auf, sich verständigen zu können. Der Anspruch, die Möglichkeit Verständigung vorzuleben, wird m.E. durch die Praxis, überwiegend konsensuale avis zu erstellen, unterstrichen. Für dieses Übereinkommen ist es wichtig, eine moderate Haltung zu haben, »weil die ethische Reflexion [...] meines Erachtens immer gemäßigt sein muss «, so in einem Interview (Int. F IV/Übers. S.K.). Auf meine Frage, ob es denn während der Diskussion zu Konflikten kommt, heißt die Antwort in einem anderen Interview: »Daran erinnere ich mich nicht, nein. [...] Nie, und wenn, dann absolut ausnahmsweise« (Int. F X/Übers. S.K.). Und ein weiteres Mitglied betont die Fähigkeit der Mitglieder des »Sich-Verstehen-und-Verständigen«-Könnens (Int. F. VIII/Übers. S.K.). Es scheint sich nicht so sehr um tatsächliche sondern eher um antizipierte Konflikte zwischen den Mitgliedern zu handeln. Ethik im Sinne des CCNE beinhaltet aber nicht nur gemäßigt und modellhaft vorzudenken, sondern auch, dass von allen mitgedacht werden soll, da »alle« verantwortlich sind.

\subsection{Verantwortung für alle}

Dieses Konzept von Verantwortung wird in der Rede Mitterrands zur Eröffnung des CCNE aus dem Jahr 1983 deutlich, die er an die Mitglieder des Komitees adressiert. Gegenstand der Arbeiten des CCNE sind, so Mitterrand

»Fragen, die manchmal schlimm sind, immer schwierig. Und ist es am Staat, sie alleine zu beantworten und Regeln zu erlassen, denen alle Wissenschaftler sich anpassen müssen? Das denke ich gewiss nicht. Soll man es den Wissenschaftlern überlassen, zu entscheiden? Soll man eine Verantwortung auf sie abschieben, die die aller ist? Delikate Haltung zwischen dem Dogmatismus und der Freigabe [...]. Ihr Ethikkomitee soll der Ort des Dialogs sein, der Konfrontation und der Reflexion, auch des Rats. Das ist seine Hauptrolle [...]« (Mitterrand 1987: 86/Übers. S.K.).

Weder die Wissenschaft noch die Politik kann Fragen, gemeint sind hier Fragen, die die biomedizinische Forschung und Anwendung betreffen, allein entscheiden. Mitterrand stellt damit die Selbststeuerung der Wissenschaft in Frage. Durch die 
Betonung der Verantwortung »aller« wird in der Rede Mitterrands die Verantwortung verschoben. Diese Konzept der Verantwortung beinhaltet allerdings nicht das Infragestellen biomedizinischer Entwicklungen, der »schwindelerregenden Fortschritte« (ebd.: 87/Übers. S.K.). Ähnlich dem Konzept der Verantwortung, das mit dem MURS Mitte der 1970er Jahre aufkommt, geht es vor allem darum, die möglichen Auswirkungen medizinischer und wissenschaftlicher Entwicklungen zu evaluieren. Allerdings umfasst das Konzept der Verantwortung nicht allein die Wissenschaftlerinnen und Wissenschaftler, wie im Kontext von MURS, sondern $»$ alle«.

Aus den Interviews mit (ehemaligen) Mitgliedern und in der Eröffnungsrede Mitterrands zum CCNE wird ersichtlich, dass die institutionalisierte Ethik das Ziel der (Vor-)Reflexion, aber auch der Vorauswahl der »wirklichen Probleme« hat (Int. F IX/Übers. S.K.). Es sind ausgewählte Probleme und Fragen, für die »alle« verantwortlich sind. Diese Vorauswahl basiert auf einem bestimmten Referenzwissen.

\subsection{Das Referenzwissen für die »wirklichen Probleme ${ }^{25}$}

Innerhalb des CCNE sitzen vorrangig Mediziner und Medizinerinnen sowie Forscher und Forscherinnen aus dem Bereich der Biologie, so ein Ergebnis von Memmi in ihrer Untersuchung zum CCNE (Memmi 1996: 48f). Von den Instanzen, die Mitglieder benennen, wird zwar nicht erwartet, Mediziner und Medizinerinnen sowie Forscher und Forscherinnen auszusuchen, in der Praxis wird dies aber meist getan, wie in einem Interview gesagt wird (Int. F IX). Memmi bestätigt dies in ihrer Untersuchung zum CCNE. Auch die staatlichen Instanzen, die Mitglieder benennen, suchen vorrangig Medizinerinnen und Mediziner oder Forscherinnen und Forscher aus. Die Mitglieder der weltanschaulichen und religiösen Spektren haben genauso überwiegend diesen professionellen Hintergrund wie die Präsidenten des CCNE (ebd.: 50). Ein medizinisch-naturwissenschaftliches Referenzwissen wird also als notwendig erachtet. Auch für Mitglieder, die nicht von vornherein über dieses Referenzwissen verfügen, hat es Bedeutung: »Das hat mich fasziniert. Ich habe eine Welt entdeckt, die ich nicht kannte. Die Philosophie, ein wenig die Biologie - ja, aber nicht die Welt der medizinischen Forschung. [...] Ich habe enorm gelernt, enorm gelernt. Das war eine der großen Erfahrungen in meinem Leben« (Int. F VIII/Übers. S.K.). Die Frage, ob ein biologisches oder medizinisches Wissen notwendig ist, um sich an der ethischen Diskussion zu beteiligen, wird nicht gestellt. 
Noch Mitte der 1970er Jahre auf der Tagung an der Sorbonne »Biologie und die zukünftige Entwicklung des Menschen« wird insbesondere die Kompetenz der Wissenschaftler und Wissenschaftlerinnen herausgestellt, die darin besteht, Probleme, die aus wissenschaftlichen und technologischen Entwicklungen entstehen, zu lösen. Wissenschaftliches Wissen wird für die Problemlösung als absolut notwendig erachtet. Aufgebrochen wird diese Perspektive punktuell von Georges Canguilhem, damals Tagungsteilnehmer. Er geht davon aus, dass Wissen ein Machtbereich von vielen ist (vgl. Canguilhem 1976: 537). Dass Wissen mit Macht verknüpft ist, wird mit der neuen Forschungspolitik zu Beginn der 1980er Jahre in gewisser Hinsicht anerkannt: indem Informationen geteilt werden, wird Macht geteilt. Die Annahme aber, dass Wissen oder Informationen notwendig sind, um sich an Debatten zu beteiligen, bleibt auch im Kontext des CCNE bestehen, wie sich an der Auswahl der Mitglieder zeigt.

\subsection{Reversible Ethik}

Das Referenzwissen, das medizinisch-biologische Wissen, ist allerdings kein stabiler Bezugsrahmen, sondern entwickelt sich permanent weiter, wie im nächsten Interviewauszug beschrieben:

»Umgekehrt, wenn sich nach unserer avis eine wahrhaftige Debatte einfindet, eine konstruktive - man muss sagen, dass wir unsere avis nicht als Wahrheit ansehen, wenn Sie so wollen, sind unsere avis prekär, weil wir zu einem bestimmten Zeitpunkt den Stand des Wissens diskutieren, das evolutionär ist, und zu einem Moment der sozialen Akzeptabilität, die sich auch permanent weiterentwickelt. Also unsere avis sind prekär und reversibel. Vielleicht sind sie im Jahr 2007 nicht mehr gerechtfertigt« (Int. F IV/Übers. S.K.).

Eine ethische Meinung in Form einer avis hängt genauso wie die soziale Akzeptabilität in dieser Vorstellung von den wissenschaftlichen Entwicklungen ab, die sich permanent weiterentwickeln. »Wir gehen davon aus, daß Ethik die Moral in Bewegung ist« (Michaud 1990: 180). Dieses permanente Fortschreiten medizinischer und wissenschaftlicher Entwicklungen wird nicht in Frage gestellt. Da sich die Wissenschaft permanent entwickelt, entwickelt sich auch die Vorstellung von Ethik, sie ist reversibel, eine Art Perpetuum mobile. Biomedizinisches Wissen wird, wie es in einem Interview heißt, als evolutionär, als permanent fortschreitend wahrgenommen. Dass es nicht das Ziel ist, Wissen bzw. wissenschaftliche Entwicklungen zu begrenzen, zeigt sich auch in dem Bericht des CCNE »Éthique et Connaissance « - »Ethik und Wissen« aus dem Jahr 1990: »Forschungseinrichtun- 
gen, Staatsorgane, die Gesellschaft insgesamt sollen sich bemühen, die falsche Vorstellung einer Ethik, die die Wissenschaft bremst, durch eine zu ersetzen, die es erlaubt, sie in das wissenschaftliche Fachwissen zu integrieren « (CCNE 1990a: 76/Übers. S.K.). Ein Infragestellen der Entwicklung der Wissenschaft und Forschung sollte von Beginn an nicht das Ziel des nationalen Ethikkomitees sein, denn, so formuliert es Mitterrand 1983 in einer Ansprache an die Mitglieder des CCNE: »Es ist um jeden Preis nötig, die Klippe der Unnachgiebigkeit zu umschiffen, die die Gefahr in sich bergen könnte, die Entwicklung der Forschung zu behindern oder der Gesellschaft Normen aufzudrücken und besonders der Gesellschaft der Gelehrten und Praktiker, die sie sind « (Mitterrand 1987: 87/Übers. S.K.). Wissenschaftliche und technologische Entwicklungen sollen also nicht behindert werden. Als Schlüssel hierfür wird gesehen, weder die Wahrheit zu verkünden, noch Normen aufzudrücken.

\subsection{Die Unabhängigkeit der Ethik}

Das Gegenargument zur norm- oder wahrheitsbringenden Instanz liegt seitens vieler interviewter (ehemaliger) Mitgliedern des CCNE in der Betonung eines bestimmten Kriteriums der Zusammensetzung des Ethikkomitees. Fünf Mitglieder werden aufgrund ihrer Zugehörigkeit zu den wichtigsten religiösen und weltanschaulichen Spektren Frankreichs für den CCNE benannt. Betont wird in den Interviews zudem, dass es sich um An- oder Zugehörige und nicht um Repräsentantinnen oder Repräsentanten der wichtigsten »philosophischen und geistlichen Familien« in Frankreich handelt. Eine Repräsentantin oder ein Repräsentant der katholischen Kirche bspw. würde die Meinung der Kirche vertreten, ihre Wert- und Glaubensvorstellung, was eine Angehörige oder ein Angehöriger einer Glaubensrichtung nicht tue (vgl. Int F III, VIII, IX). Es wäre m.E. in Frankreich schon deshalb nicht möglich, dass sich das Komitee aus expliziten Kirchenvertretern und -vertreterinnen zusammensetzt, da dies mit dem Laizismus, der strikten Trennung von Staat und Kirche, kollidieren würde. Aber nicht nur die Angehörigen der wichtigsten religiösen und weltanschaulichen Spektren sollen nicht als Repräsentantinnen und Repräsentanten verstanden werden, sondern die Mitglieder des CCNE insgesamt: Sie sollen nicht als solche verstanden werden und sich selbst nicht als solche verstehen, so Mitterrand 1983 in seiner Eröffnungsrede (Mitterrand 1987: 87). Sie sollen »nur sich selbst repräsentieren «, wie es in einem Interview heißt (Int. F X/Übers. S.K.). Ein weiteres Kriterium dafür keine norm- oder wahrheitsbringende Instanz zu sein, ist die intellektuelle und politische Unabhängigkeit: »Politische Unabhängigkeit und die Tatsache, dass die Mitglieder total frei sind, 
d.h. dass es keinen Imperativ gibt, jemand, der von einem Minister benannt ist, soll nicht einen Rat von ihm bekommen, bevor er in eine Versammlung geht. Politische und intellektuelle Unabhängigkeit«, wie in einem Interview gesagt wird (Int. F IV/Übers. S.K.). In einem weiteren Interview heißt es auf meine Frage, ob die Mitglieder mit einem bestimmten Mandat beauftragt werden: »Nein, das ist absolut nicht der Fall, wenn das so wäre, würde das gegen ihre Unabhängigkeit gehen. Ich hatte niemals Anfragen des Präsidenten des Cour de Cassation. ${ }^{26}$ Ich fühlte eine große Freiheit der Reflexion und der Entscheidung und des Handelns« (Int. F II/Übers. S.K.). Es zeigt sich, dass Ethik als individuelle Angelegenheit verstanden wird, selbständig seine »eigenen Lebensregeln« zu machen (Int. F IX/Übers. S.K.). Im institutionalisierten Ethik-Rahmen geht es weder darum, eine bestimmte Wertund Glaubensvorstellung zu vertreten, noch darum, Interessen einer politischen Instanz oder Partei durchzusetzen. Auch weitere Interessen wie z.B. die von Patientinnen- und Patientenvereinigungen sollen nicht vertreten werden - kein Mitglied ist aufgrund seiner Teilnahme an einer solchen Vereinigung benannt. Dies wird auch nicht für notwendig gehalten, denn, so heißt es in einem Interview mit einem CCNE-Mitglied »wir sind alle potentiell Patienten« (Int. F XI/Übers. S.K.).

Nicht das Konzept des potentiellen Forschers oder der potentiellen Forscherin, das mit der Forschungspolitik von 1981 aufkommt, scheint relevant, sondern vielmehr das des potentiellen Patienten oder der potentiellen Patientin. Obwohl »potentielle Patienten « nur in einem Interview benannt wird, scheinen mir hierzu doch einige Überlegungen interessant: Die Perspektive des potentiellen Patienten oder der potentiellen Patientin beinhaltet m.E., sich als potentiell betroffen oder krank zu fühlen, und zwar persönlich, auf einer individuellen Ebene. Es geht also nicht darum, gemeinsam oder als Vertreterin oder Vertreter einer Vereinigung für etwas zu kämpfen. Zudem beinhaltet diese Perspektive, gesund werden zu wollen. Gesundheit wird damit zum Ziel. Sich gegen medizinische und technologische Entwicklungen zu stellen, die Gesundheit bringen könnten, ist schwierig, vor allem aus der Perspektive des potentiellen Patienten oder der potentiellen Patientin. In dieser Perspektive geht es m.E. nicht um die Frage, in welcher Gesellschaft wir leben wollen, sondern darum, wie man Gesundheit erreichen kann. Darüber hinaus beinhaltet diese Perspektive den Gedanken, sich aktiv für Gesundheit einzusetzen - fühle ich mich gesund, muss ich nicht gesund werden. Eine Entwicklung hin zu aktiven Patientinnen und Patienten zeigt sich bereits in den 1960er und 70er Jahren wie bspw. im Kontext des Medikamentenführers von Pradal. Die Publikation kann als Zeugnis für das sich verändernde Verhältnis zwischen Ärztin oder Arzt und Pa-

26 Cour de Cassation bedeutet Kassationsgerichtshof. Er entspricht in etwa dem Bundesgerichtshof in Deutschland. 
tientin oder Patient, einem weniger paternalistischen Verhältnis verstanden werden. Folgt man dem Interviewbeitrag, kann auch der CCNE in diesem Sinn verstanden werden. Die Mitglieder des CCNE sprechen nicht aus der Perspektive des potentiellen Mediziners oder der potentiellen Medizinerin oder des Forschers oder der Forscherin, sondern aus der Perspektive von potentiellen Patientinnen und Patienten. Wesentliches Merkmal dieses sich verändernden Verhältnisses in den 1960er und 70er Jahren ist die Information des Patienten oder der Patientin über Medikamente oder medizinische Behandlungen. Dieses medizinische Wissen ist ebenso Referenzwissen der Mitglieder des CCNE. Die Teilnahme im institutionellen Ethik-Rahmen erfordert also Ethik als individuelle Angelegenheit, eine freie Reflexion zu verstehen, in der man sich selbst repräsentiert. Dies kann darüber hinaus in dem Sinn verstanden werden, dass man sich als informierte potentielle Patientin oder informierter potentieller Patient begreift.

Ziel des CCNE ist nicht, eine Wahrheit zu verkünden. Gegen eine wahrheitssprechende Instanz wird in den Interviews insofern argumentiert, als es das Ziel ist, durch die avis oder Empfehlung eine Debatte zu »provozieren« (Int. F III/Übers. S.K.) oder eine »wahrhaftige Debatte« (Int. F IV/Übers. S.K.) anzuregen:

»Die Idee war wirklich, die Reflexion und die Debatte auf die Ebene der Bevölkerung zu bringen, innerhalb des Versuchs der Erhöhung der Demokratie in unserer Gesellschaft, der demokratischen Funktionsweise unserer Gesellschaft« (Int. F IX/Übers. S.K.). »Man darf sich nicht täuschen, es ist kein normatives Komitee. Es soll ein Grundelement der demokratischen Debatte sein« (Int. F V/Übers. S.K.).

Anstatt einen Wahrheitsanspruch zu haben, was implizieren würde, andere Positionen seien falsch, zeichnet sich Ethik in Form der avis als eine Meinung aus, die ein moderates Übereinkommen symbolisiert, eine Koexistenz gemäßigter Positionen (vgl. Memmi 1996). Der Konsens der meisten avis und Empfehlungen des CCNE wird in dieser Vorstellung nicht als problematisch angesehen, denn selbst wenn eine avis oder Empfehlung des CCNE von der Öffentlichkeit als falsch, autoritär oder normgebend angesehen wird, diene dies trotzdem der Debatte, da diese durch die Kritik hervorgerufen wird (Int. F IV). Didier Sicard, ehemaliger Präsident des CCNE, schlägt vor, das Komitee solle »nationaler Beirat für ethische Verunsicherung « heißen (Sicard 2001).

Die meist konsensualen avis des französischen nationalen Ethikkomitees stehen in Kontrast zu den Dissensen, die den Empfehlungen der nationalen Ethikinstitutionen in Deutschland und Österreich zu Grunde liegen. Bogner und Menz haben herausgearbeitet, dass der Dissens ein wesentliches Merkmal der Ethisierung im 
Kontrast zur Moralisierung ist. Wie lassen sich vor diesem Hintergrund die meist konsensualen avis und Empfehlungen des CCNE verstehen?

\subsection{Moralisierung oder Ethisierung?}

In ihrer Untersuchung zu nationalen Ethikgremien in Österreich und Deutschland zeigen Bogner und Menz auf, wie in Kapitel I aufgeführt, dass die Empfehlungen dieser Kommissionen meist dissensual sind (Bogner/Menz 2010: 907; Bogner et al. 2008). Die divergierenden Empfehlungen von Ethik-Expertinnen und -Experten in Deutschland und Österreich scheinen aber kein Problem im Politikprozess darzustellen, so Bogner und Menz. Dass Expertise Wahrheit produziert, wird nicht (mehr) erwartet. Ist ein Wertkonflikt auf eine Konsensfindung aus, d.h. auf eine einzige Wahrheit, bestehe die Gefahr der Moralisierung. Ist der Dissens gewiss, können Wertkonflikte ethisiert sein, so in einer weiteren Arbeit von Bogner (Bogner 2013: 54). Die avis und Empfehlungen des CCNE sind nun aber meist konsensual. ${ }^{27}$ Bedeutet dies, es findet eine Moralisierung statt? Ich denke nein, denn der Punkt ist, dass zumindest auf programmatischer Ebene, im Sinne der Mitglieder des CCNE trotz Konsens keine Wahrheit produziert werden soll und der Pluralismus für notwendig erachtet wird. Auch wenn die Politik sich in der Praxis, im Gesetzgebungsprozess in Frankreich auf die avis und Empfehlungen des CCNE häufig beruft, sie einbezieht, sie als »Referenz dient« (Int. F III/Übers. S.K., vgl. Int. F II, IV), sie muss nicht. Im Unterschied allerdings zu Deutschland und Österreich gewinnt die Politik in Frankreich nicht mehrere Handlungs- und Legitimationsmöglichkeiten durch den Konsens des Ethikkomitees, sondern nur eine (vgl. Bogner et al. 2008: 263). Im Sinne von Bogners Ethisierungsbegriff gedacht, ist im Kontext des CCNE Ethisierung nicht abhängig von Dissens.

Auch im Sinn des in der vorliegenden Studie verwendeten Konzeptes der Ethisierung handelt es sich im Kontext des CCNE um eine solche und nicht um eine Moralisierung. Zwar wird in der Planungsphase und zu Beginn des CCNE von moralischen Problemen gesprochen, jedoch erscheint die Sprache der Moral eher als Mittel zum Zweck und als Übergangslösung. Der Zweck ist, sich der Öffentlichkeit verständlich zu machen. Moral und Ethik teilen das Ziel, eine Debatte anzuregen. Berücksichtigt man die Sprecherinnen- und Sprecherpositionen, die sich im institutionellen Ethik-Rahmen herausbilden, die Art und Weise, wie gesprochen

27 Der Konsens war und ist laut Interviewauskunft kein formell festgelegtes Ziel der Diskussion. Zurückgeführt wird er auf die pragmatische Herangehensweise des ersten Präsidenten des CCNE, Jean Bernard (Int F IX). 
werden kann, zeigt sich m.E. eine Ethisierung im Sinne einer Entpolitisierung oder Entmoralisierung. Eine moderate, gemäßigte Haltung ist ebenso Voraussetzung für die Teilnahme, wie die Fähigkeit zu reflektieren. Eine normative Position einzunehmen, scheint ebenso ausgeschlossen, wie die medizinischen und technologischen Entwicklungen in Frage zu stellen. Insofern scheinen auch moralische Positionen ausgeschlossen, wie sie vom Ordre des médecins oder von Lebensschützern und -schützerinnen eingenommen werden, ob sie persönlich im CCNE vertreten sind oder nicht. Zudem werden Themen, die zuvor im moralisch gerahmten Kompetenzbereich des Ordre liegen, vom CCNE aufgenommen und ethisch gerahmt. Auch politische Positionen wie die der Gentechnologiekritiker und -kritikerinnen scheinen keinen Raum zu haben - gentechnologische Entwicklungen könnten in Frage gestellt werden. In diesem Kontext zeigt sich eine Moralisierung. Wie mir eine Wissenschaftskritikerin oder ein Wissenschaftskritiker in einem Interview erklärt, ist die Ethik des CCNE nie von Dauer, weil davon ausgegangen wird, dass die Technologie immer voranschreitet: »Wenn du etwas anderes sagst, bist du Moralist« (Int. F XII/Übers. S.K.). Als Nebeneffekt der Ethisierung scheint eine Moralisierung wissenschaftskritischer politischer Positionen zu entstehen.

Es ergibt sich die Frage, welche Art der Sprecherinnen- und Sprecherpositionen im Kontext der Ausrichtung des CCNE auf die Öffentlichkeit entworfen werden. Auf diese Frage wird im Folgenden eingegangen.

\section{6 》FRAGEN ETHISCHER ORDNUNG AUF GESELLSCHAFTLICHEM NIVEAU « ${ }^{28}$}

Der CCNE zeichnet sich durch seine Ausrichtung auf die Öffentlichkeit aus. Die »Einleitung einer Debatte über Fragen ethischer Ordnung auf gesellschaftlichem Niveau« (Int. F IX/Übers. S.K.) ist das Ziel. Dies wird in einem Interview wie folgt beschrieben:

»wenn man will, dass die Debatte geführt wird, muss man sie nähren. Man muss ihr, man sagt auf Französisch >du grain à moudre «, damit die öffentliche Debatte mahlen kann, muss man ihr Korn zum Mahlen geben. Die Hauptrolle des CCNE ist es, der öffentlichen und demokratischen Debatte dieses zu mahlende Korn zu geben« (Int. F V/Übers. S.K.).

Es geht aber nicht um das gesamte »Korn«, sondern um das selektierte - um die »wirklichen Probleme«, wie es an anderer Stelle heißt (Int. F IX/Übers. S.K.). Dies 
erinnert m.E. an die Aufgabe, die dem Wissenschaftler oder der Wissenschaftlerin als Bürger oder Bürgerin in der Forschungspolitik Mitterrands Anfang der 1980er Jahre zukommt. Er oder sie soll technische und ethische Fragen formulieren und diese verbreiten. Die Definition der ethischen Fragen geht in wissenschaftliche Hände über. Im Kontrast zu diesem Konzept sind im CCNE nun aber nicht explizit Wissenschaftlerinnen und Wissenschaftler, sondern Repräsentantinnen und Repräsentanten seiner oder ihrer selbst vertreten, die (teils) die Perspektive von potentiellen Patientinnen oder Patienten einnehmen. Die Konstante ist allerdings das wissenschaftliche Referenzwissen. Zu der Vorstellung von Demokratie der 1980er Jahre, wie sie mit der sozialistischen Regierung aufkommt, gehört es, Wissen zu teilen, Debatten zu initiieren sowie Bürgerinnen und Bürger einzubeziehen. Bürgerinnen und Bürger, deren Wissen als defizitär konstruiert wird. Eine ähnliche Vorstellung zeichnet sich im Kontext des CCNE ab. Zum einen soll eine Debatte über »wirkliche Probleme« stimuliert werden, zum anderen soll die Öffentlichkeit überhaupt etwas verstehen: »die Leute sollen ein bisschen besser verstehen, wo wirklich die Fragen sind «(Int. F IX/Übers. S.K.). Wie schon Mitterrand 1983 sagt, geht es um Fragen, die sich die gesamte Gesellschaft stellt (Mitterrand 1987: 85). Es werden Sprecherinnen- und Sprecherpositionen entworfen, aus denen heraus Fragen gestellt werden, nicht aber Meinungen zu wissenschaftlichen und technologischen Entwicklungen geäußert werden sollen. Wenn gesagt wird, dass die Öffentlichkeit nicht alles versteht, Fragen hat, bedeutet dies, sie wird in irgendeiner Form als defizitär wahrgenommen. Wie es in einem Interview heißt:

»Anfänglich war es [das nationale Ethikkomitee, S.K.] konzipiert [...] [um] sich an die Öffentlichkeit zu wenden, die biologische und medizinische Information zu fördern [...] mit der Vorstellung, dass nach einer bestimmten Zeit die öffentliche Meinung informiert ist [...]. Die öffentliche Diskussion, um den Bürgern zu helfen, selbst zu werten. [...] Ein einfaches Beispiel: am Anfang, war eine große Menge der Franzosen für den Leih-Uterus, weil sie dachten, das sei ein großzügiger Akt [von Frauen, S.K.] für Frauen, die keine Kinder haben können, das Kind auszutragen. Das Komitee hat eine avis herausgebracht, die dagegen war, wegen der Möglichkeit der Kommerzialisierung [...]. Und die Meinung der französischen Öffentlichkeit hat sich umgekehrt. Diese Erfahrung zeigt, dass sie reif werden, selbst zu reflektieren« (Int. F VIII/Übers. S.K.).

Die Art und Weise, wie die Öffentlichkeit oder die Bürgerinnen und Bürger konstruiert sind, weist zwei Formen von Defiziten auf. Es handelt sich zum einen um ein Informationsdefizit, sprich um einen Mangel an einem bestimmten Wissen, nämlich dem biologischen und medizinischen. Dieses gilt als Referenzwissen, um sich an einer ethischen Debatte beteiligen zu können. Die Notwendigkeit dieses 
Referenzwissens wird also nicht allein für die Mitglieder des CCNE gesehen, sondern auch für die Öffentlichkeit bzw. die Bürgerinnen und Bürger. Darüber hinaus zeigt sich ein Defizit in der Fähigkeit der Reflexion: sie muss erst »reif werden « sie muss anwachsen, sich entwickeln und festigen. Nicht allein die Publikationen des CCNE, die avis und die Empfehlungen sollen der Öffentlichkeit, den Bürgerinnen und Bürgern in diesem Reifungsprozess helfen, sondern auch die Journées annuelles d'éthique.

\subsection{Die Journées annuelles d'éthique}

Praktiken, durch die eine breitere Öffentlichkeit erreicht werden soll, gibt es beim CCNE durch die einmal jährlich stattfindende mehrtägige öffentliche Tagung, die Journées annuelles d'éthique. Hier werden die über das Jahr verfassten avis und Empfehlungen des CCNE vorgestellt. Seit 2005 besteht für die Teilnehmenden auch die Möglichkeit an noch nicht verabschiedeten avis und Empfehlungen des CCNE mitzudiskutieren, d.h. die Teilnehmenden an den Journées annuelles werden in den Diskussionsprozess einbezogen:

»Die Journées annuelles bestehen im Rahmen des Möglichen darin, die Öffentlichkeit, die Gesellschaft Zeugen der Arbeit des Komitees werden zu lassen. Es gibt dazu [...] eine Debatte zwischen den Leuten, die im Raum sind und den Mitgliedern. Es gibt auch Beiträge von jungen Leuten aus Schulklassen von Gymnasien« (Int. F II/Übers. S.K.).

Früher sind die Journées annuelles laut Interview-Aussagen ausgesprochen gut besucht gewesen, was über die Zeit abgenommen habe. Darüber hinaus sind Mitglieder des CCNE früher selbst an Schulen gegangen, um über bioethische Themen zu sprechen und zu diskutieren. Seitens des CCNE wird dieser Rückgang an Öffentlichkeitsarbeit allerdings selbst als Mangel empfunden und auf organisatorische und finanzielle Schwächen zurückgeführt (Int. F VIII, IV).

\subsubsection{Ethische Multiplikation in Zeit und Raum}

Ein Schwerpunkt auf den Journées anuelles bilden die im Zitat genannten Vorträge von eingeladenen Schülerinnen und Schülern über bioethische Themen, die sie zuvor mit ihren Lehrerinnen und Lehrern ausgearbeitet haben. Dass die Lehrer und Lehrerinnen die Fächer Biologie und/oder Philosophie unterrichten, wird als ideal gesehen (Int. F IV). Auch hier zeigt sich m.E., dass die Notwendigkeit eines bestimmten Referenzwissens als Grundlage für ethische Reflexionen gesehen wird. Die Schüler und Schülerinnen werden als Multiplikatoren und Multiplikatorinnen 
betrachtet, in dem Sinn, dass sie ihre Reflexionen in ihre Familien tragen und sich die »Debatte über Fragen ethischer Ordnung« (Int. F IX/Übers. S.K.) damit ausweitet (Int. F IV). Sie weitet sich nicht nur auf weitere Akteure und Akteurinnen aus, auch die Zukunft der ethischen Reflexion spielt eine Rolle: »Ich habe mir immer gesagt, das, was wichtig ist, ist die zukünftige Generation, das sind nicht die alten Esel wie ich« (Int. F X/Übers. S.K.). Wie ein Mitglied des CCNE in einem Interview erklärt, sind die Schülerinnen und Schüler besonders wichtig, denn »[a]uf diese Art werden naive Fragen, Laienfragen gestellt, die die Reflexion des Ethikkomitees hervorrufen können. Es ist für den CCNE ein Gewinn, diese Journées zu haben, daran teilzunehmen« (Int. F II/Übers. S.K.). Die Naivität der Schüler und Schülerinnen wird als besondere Ressource für die Mitglieder des CCNE betrachtet, als »richesse pour le future « - »Reichtum für die Zukunft $«,{ }^{29}$ da sie Ideen und Perspektiven bieten, die spätere Debatten im Komitee stimulieren können. In einem weiteren Interviewbeitrag werden die Schülerinnen und Schüler als zukünftige Bürgerinnen und Bürger gesehen, die die ethische Reflexion üben müssen, indem sie auf der Veranstaltung eigene Themen präsentieren (Int. F IV). Die Schüler und Schülerinnen sichern in zweierlei Hinsicht die Zukunftsfähigkeit ethischer Debatten oder Reflexionen. Sie sind für den CCNE Ressourcen und geben neuen Input. Und sie sollen die ethische Reflexion üben - so ist die Art und Weise der Reflexion in der zukünftigen Generation gesichert.

\subsubsection{Das »richtige Sprechen ${ }^{30}$ und das governing through speech ${ }^{31}$}

Wie dieses Üben aussieht, zeigt das folgende Beispiel meiner Teilnehmenden Beobachtung der Journées annuelles von $2004 .^{32}$ Eine Schülerinnen- und Schülergruppe stellt das Thema »Euthanasie« - Sterbehilfe vor sowie ihre Diskussion, die sie vorbereitend geführt haben. Sie präsentieren Überlegungen zur Sterbehilfe beim Neugeborenen und bei einem alten Menschen. Beim Neugeborenen müsse man die Qualität seines zukünftigen Lebens kennen, so die Schülerinnen und Schüler. Auch sei es wichtig, die finanziellen Aspekte abzuwägen: Was kostet der Mensch zukünftig die Gesellschaft? Die Entscheidung für oder gegen Sterbehilfe läge bei den Eltern und dem Mediziner oder der Medizinerin, denn das Neugeborene selbst habe keine Alternative. Die letzte Entscheidung bleibe immer bei dem

29 Ein Mitglied des CCNE am 16.11.2004, Journées annuelles d'éthique, Teilnehmende Beobachtung, Übers. S.K..

30 Braun et al. 2008.

31 Memmi 2003c.

32 Die Journées fanden von 16. bis17.11.2004 in Paris an der Université Paris V René Descartes im Grand Amphithéâtre statt. 
Mediziner oder der Medizinerin, so die Schüler und Schülerinnen, auch bei der Sterbehilfe bei alten Menschen. Dass die Entscheidung bei dem Mediziner oder der Medizinerin liegt, sei insofern wichtig, als sich die Angehörigen des Patienten oder der Patientin aufgrund des anstehenden Erbes für Sterbehilfe entscheiden könnten. Auch könne es sein, dass sie sich für Sterbehilfe entscheiden, weil sie selbst das Leiden ihres Angehörigen nicht mehr ertragen. Nicht zuletzt, so ein weiterer Aspekt ihrer Überlegungen, sei aber auch die Palliativpflege für die Gesellschaft sehr teuer. Nach Abschluss der Präsentation wird die Diskussion mit dem Publikum eröffnet. Aus dem Publikum kommt die Frage, wie die Schülerinnen und Schüler persönlich zur Sterbehilfe stehen? Eine Schülerin beantwortet die Frage dahingehend, dass sie vom Sterben eines Großelternteils erzählt, das sie miterlebt habe. Ihre Antwort ist sehr persönlich, sie wirkt verunsichert und erklärt sich ausführlich. Sie wird nicht unterbrochen. Ein Mitglied des CCNE bestätigt abschließend den Schülerinnen und Schülern, das Problemfeld begriffen zu haben.

Im Sinne Memmis handelt es sich hier um eine angeleitete Form des Sprechens durch eine begleitende Expertise. Angeleitet insofern, als das Mitglied des CCNE nicht sagt, eine Überlegung der Schüler und Schülerinnen sei richtig oder falsch oder dass bemängelt wird, dass sie keine klare Position formulieren. Wichtig ist, dass die Probleme verstanden werden, die Art und Weise des Denkens und Sprechens, dass das persönliche In-Sich-Gehen stattfindet, die Bioindividuation. Körperliche Bedingungen und Gesundheitskosten werden evaluiert (Memmi 2003a: 292f; 2005). Mit Foucault und der Pastoralmacht verstanden, fühlt sich die Schülerin, die die Publikumsfrage beantwortet, in der Pflicht zu ergründen, was in ihr vorgeht. Sie ist gehalten ihr Inneres zu enthüllen, »gegen sich selbst auszusagen « (Foucault 1993b: 52). Allerdings tut die Schülerin dies nicht gegenüber dem Pastor, sondern vor dem Publikum der Journées annuelles - rund 100 Menschen an der Zahl, überwiegend älter und männlich. Es geht aber nicht wie bei der Beichte um das Heil im Jenseits, sondern um das irdische Heil. Dessen Bedeutung formulieren die Schülerinnen und Schüler selbst: die Qualität des (zukünftigen) Lebens und die Kosten für die Gesellschaft.

Die Perspektive, der Möglichkeitsraum, der sich für die Diskussion im CCNE eröffnet, denn die Beiträge der Schüler und Schülerinnen sollen die Diskussion des CCNE stimulieren, beinhaltet nicht die Frage, für welches Problem Sterbehilfe eine Lösung ist, welche gesellschaftlichen Bedingungen den Wunsch nach Sterbehilfe aufkommen lassen, oder wie es kommt, dass gerade die Medizin auf diesen 
Wunsch eine Antwort hat. ${ }^{33}$ Es geht darum, wie und von wem entschieden wird. In diesem Entscheidungsprozess dürfen Kosten eine Rolle spielen. Indem die Schülerinnen und Schüler darin bestätigt werden, das Problemfeld verstanden zu haben, wird das Sprechen über die Kosten, die ein Mensch der Gesellschaft verursacht, legitim.

Die Präsentation der Themen, die von den Schülerinnen und Schülern vorbereitet werden, wird als erfolgreich angesehen, wenn sie verstehen, dass Ethik bedeutet, das Problemfeld verstanden zu haben und auch, dass niemals endgültige Antworten gefunden werden können, wie ein weiteres Mitglied des CCNE bei den Journées meint. ${ }^{34}$ Auch bei den Journées zeigt sich somit, dass die Teilnahme am Ethikdiskurs des CCNE die Akzeptanz dafür verlangt, dass die Ergebnisse »evolutionär, reversibel und prekär« sind. Das Einüben des »richtigen Sprechens« bezieht sich nicht auf die Qualität substantieller Meinungen. Es bezieht auch nicht das Infragestellen medizinischer, wissenschaftlicher oder technologischer Entwicklungen mit ein, wie auch das folgende Beispiel zeigt: Bei den Journées annuelles 2004 wird der Beitrag eines Schülers von den Mitgliedern des CCNE übergangen, der nicht über die weitere Entwicklung gentechnologisch manipulierter Pflanzen ${ }^{35}$ sprechen wollte. Er thematisiert die Überproduktion in der Landwirtschaft sowie die internationale Verteilung von Nahrungsmitteln. Damit stellt er m.E. implizit die technologische Entwicklung in Frage. Im Gegensatz dazu steht der Kommentar eines Mitglieds des CCNE zu einem Schülerinnen- und Schülerbeitrag, der nicht die wissenschaftliche Entwicklung kritisiert: »Sie haben die Schwierigkeiten und die Problematik verstanden $\ll .{ }^{36}$ Diese erzieherische Dimension beinhaltet nicht, zu lernen, eine deutliche Position einzunehmen und zu verteidigen, sondern die richtige Art der Reflexion zu proben (vgl. Braun et al. 2008: 235f). Es geht um das angeleitete Einüben des »richtigen Sprechens«. Der übergangene Beitrag des Schülers zeigt, dass Diskussionen über die wissenschaftliche Entwicklung selbst nicht als zugehörig zur ethischen Debatten biotechnologischer Themen betrachtet werden. Auch die Wahrheit soll nicht vermittelt oder produziert werden, wie ein Beispiel von den Journées annuelles aus dem Jahr 1993 zeigt, das aus Memmis Untersu-

33 Auf einige dieser Fragen bietet die sozialwissenschaftliche Untersuchung zur Euthanasiedebatte und zur Selbstbestimmung am Lebensende von Isabella Jordan eine Antwort (Jordan 2007, 2011).

34 Journées annuelles d'éthique (16./17.11.2004), Teilnehmende Beobachtung, S.K..

35 Das Thema Gen-Pflanzen war bei den Journées m.E. ein Ausnahmethema. Der CCNE beschäftigt sich nicht mit grüner Gentechnologie. Die Ausnahme ist so zu erklären, dass die Schülerinnen und Schüler von einem agrarwissenschaftlichen Gymnasium kommen.

36 Journées annuelles d'éthique (16./17.11.2004), Teilnehmende Beobachtung, S.K.. 
chung stammt. Aus dem Publikum wird gefragt: »Ich weiß nicht, ob der Embryo eine Seele hat. [...] Ich weiß, dass es gefährlich ist, die Wahrheit zu kennen. Ich möchte die Wissenschaftler bitten: >Suchen Sie die Wahrheit<. Sagen Sie uns, ob dieser Embryo ein Mensch ist « (Publikumsfrage, Journées annuelles 8.02.1993 zit. nach Memmi 1996: 64/Herv. i.O., Übers. S.K.). Noëlle Lenoir, damals Leiterin des Bioethikkomitees der UNESCO, antwortet: »Ich würde Ihnen wie Pirandello ${ }^{37}$ antworten: >Jedem seine Wahrheit««. Und der Philosoph Jean-François Mattéi, Mitglied des CCNE (1993-1997), antwortet: »Was mich angeht, ich glaube, dass der Embryo ab der Empfängnis ein Mensch ist « (N. Lenoir/J.-F. Mattéi, Journées annuelles 8.02.1993 zit. ebd./Herv. i.O., Übers. S.K.).

Bei den Journées annuelles handelt es sich somit nicht einfach um eine Art, die Wahrheit oder wissenschaftliches Wissen zu kommunizieren, auch wenn die meisten Mitglieder beruflich einen medizinischen oder wissenschaftlichen Werdegang haben. Es geht auch nicht darum, bestimmte Positionen zu vermitteln, sondern eher darum, Teilnehmende zur Deliberation, zur Diskussion zu stimulieren, dazu ihre Argumente auszubalancieren und sich ihre eigenen individuellen, temporär gültigen Meinungen zu bilden, je nach Stand der wissenschaftlichen Entwicklungen. Es handelt sich mehr um eine Art, Reflexion in als richtig erachteter Weise zu managen: individuell, perpetuell und gemäßigt. Die Charakteristika des institutionalisierten Ethik-Rahmens sind das No-Go des Infragestellens wissenschaftlicher Entwicklung und der deutlichen Argumentation gegen diese. Die ethischen Sprecherinnen- und Sprecherpositionen, die sich im biomedizinischen und -technologischen Diskurs herausbilden, beinhalten, nicht eine substantielle Position einzunehmen (Braun et al. 2008: 235f; 2009: 44f; Braun et al. 2010a: 852f).

Der Möglichkeitsraum, worüber gesprochen werden kann, scheint sich stetig zu erweitern. Dies bezieht sich nicht nur darauf, dass kontinuierlich neue medizinische und technologische Entwicklungen besprochen werden, sondern auch, welche Aspekte in die Diskussion einbezogen werden dürfen, wie die Kostenabschätzung, die die Schülerinnen und Schüler eingebracht haben. Dieser ökonomische Aspekt wird auch in einem Interview im Hinblick darauf thematisiert, wie sich die Diskussionen innerhalb des Komitees verändert haben.

37 Luigi Pirandello (*1867-†1936) war italienischer Schriftsteller und Nobelpreisträger für Literatur. 


\section{ETHIK IM WANDEL?}

Die Frage, ob sich die Diskussionen im CCNE über die Zeit verändert haben, wird in einem Interview mit einem Mitglied, das über 15 Jahre im CCNE war, wie folgt beantwortet:

»Heute gibt es häufig die Haltung eines bestimmten wissenschaftlichen Optimismus, eine Haltung gegenüber der Rolle des Geldes, wie sie heute in einer neoliberalen Gesellschaft dominant ist. Vor allem hat sich in diesem Sinn die Richtung des CCNE bewegt. [...] Ich kannte noch ein Komitee mit der Sichtweise: große humanistische Mediziner, großes Anliegen an der menschlichen Person, Leute, die für den medizinischen und wissenschaftlichen Fortschritt waren, aber mit einem Anliegen [...] gegen das Geld, absolut gegen das Geld. [...] Jean Bernard war mit einer unglaublichen Heftigkeit gegen die Rolle des Geldes in solchen Angelegenheiten« (Int. F VIII/Übers. S.K.).

Wenn sich solch eine Verschiebung abzeichnet, zeigt sie sich auch in den Publikationen des CCNE? Wichtiges Element der Diskussionen des CCNE scheint lange Zeit die Nicht-Kommerzialisierung des menschlichen Körpers: Dieses Prinzip wird nicht nur in dem Bericht »Éthique et Connaissance - »Ethik und Wissen« aus dem Jahr 1990 erwähnt (CCNE 1990a), die Nicht-Kommerzialisierung ist zudem Thema mehrerer avis und Empfehlungen, wie bspw. im Jahr 1991 die avis zur Bluttransfusion für die eigens die Arbeitsgruppe »Ethics and money« eingerichtet wird (CCNE 1991a: o.S.) oder, ebenfalls 1991, die Nicht-Kommerzialisierung des menschlichen Genoms (CCNE 1991b). Auch die Leihmutterschaft darf nicht gewinnbringend sein und Organspende ebenso wenig (CCNE 1984a, 1990b). Der Ethik-Rahmen beinhaltet die Diskussion finanzieller Interessen nur im Hinblick auf die Abwehr der Kommerzialisierung des menschlichen Körpers. Diese Befürwortung der Nicht-Kommerzialisierung des menschlichen Körpers, seiner Elemente oder Substanzen, die im Bioethikgesetz von 1994 gesetzlich verankert wird (vgl. Loi $\mathrm{n}^{\circ}$ 94-654 1994), scheint sich aber zu ändern, wie exemplarisch die 93. avis aus dem Jahr 2006 zeigt (CCNE 2006b). In dieser avis werden die Schwierigkeiten einer eventuellen Kommerzialisierung von menschlichen, embryonalen und nichtembryonalen Stammzellen und anderer Zelllinien (bspw. aus Blut gewonnenen) behandelt. Ausdifferenziert wird hier der Begriff der Kommerzialisierung in dem Sinn, dass sich zwar gegen eine Forschung mit dem Ziel des Profits durch die Kommerzialisierung von Zellspenden gewendet wird, allerdings wird sich für eine akzeptable Kommerzialisierung ausgesprochen. Die Kommerzialisierung wird insofern akzeptabel, als sie eingegrenzt und reguliert wird. Als Regulierungsinstanz wird hierfür die durch das Bioethikgesetz 2004 eingerichtete Agence de la 
biomédecine - Biomedizin-Agentur ${ }^{38}$ vorgeschlagen (CCNE 2006a: 23). Für eine akzeptable Kommerzialisierung werden die Elemente des menschlichen Körpers ausdifferenziert. So wird in der avis darüber nachgedacht, bis zu welchem Stadium man bei einer Stammzelle überhaupt von einem Element des menschlichen Körpers sprechen kann. Zurückgegriffen wird innerhalb der Argumentation für die Kommerzialisierung auf das Beispiel der Blutspende. Diese ist zwar gratis, aus der Spende können allerdings nach einer Transformation Medikamente gewonnen werden, die wiederum kommerzialisiert werden (CCNE 2006b: 3). In diesem Sinn könnten Stammzellen, die zu »bio médicaments « - »Biomedikamenten« transformiert werden, kommerzialisiert werden. Allerdings kann dies nur geschehen, sofern sie nicht Teil eines elterlichen Projektes sind, was das Bioethikgesetz von 2004 verbietet (CCNE 2006a: 23/Übers. S.K.). Zur praktischen Umsetzung dieser Kommerzialisierung gibt es in der avis Überlegungen, eine Zellbank als »businessmodel « einzurichten, die den Zugriff auf die menschlichen Zellen unter bestimmten Kriterien ermöglicht (CCNE 2006b: 9/Herv. i.O.). Diese avis wird 2006 nicht einstimmig verabschiedet. Kritisiert wird sie von Mitgliedern wie bspw. der konservativen Senatorin Marie Thérèse Hermange, weil die empfohlenen Zellbanken als Aufruf für die Zellentnahme bei In-vitro-Embryonen interpretierbar wären. Eine andere Kritik kommt von dem Biologen und Mediziner Jean-Claude Ameisen, damals Mitglied des CCNE, 2003-2011 Präsident des Ethikkomitees vom INSERM und ab 2012 Präsident des CCNE. Er argumentiert, dass die Koexistenz verschiedener Arten von Wettbewerbssystemen, wie »gratuité « - Unentgeltlichkeit«, nicht gewinnbringender und gewinnbringender Verkauf, das Verantwortungsbewusstsein der Konsumentinnen und Konsumenten sowie der Herstellerinnen und Hersteller fördern würde. Dies verdeutlicht er am Beispiel der Software Linux, deren Hersteller sozusagen aus ethischem Bewusstsein heraus diese GratisSoftware anbieten würde, im Gegenzug zu der kommerziellen Software von Microsoft (CCNE 2006a: 27). Übertragen auf die Zellspende plädiert Ameisen dafür, dass die Zell-Spenderinnen und -Spender frei wählen können sollen, ob ihre Spende zu kommerziellen oder nicht-kommerziellen Zwecken verwendet werden soll. Im Sinne seines Beispiels bedeutet dies, die Spenderinnen und Spender werden mit der Entscheidung, ob sie für Linux oder für Microsoft sein wollen, zu den jeweils entsprechenden Akteuren und Akteurinnen einer »ethischen« Marktregulierung: »Donors of cells should be able to choose, on the basis of the information provided to them, the type of development and accessibility of innovations in which they

38 Die Biomedizin-Agentur fördert, evaluiert und kontrolliert Aktivitäten im Bereich des Transplantationswesens, der Fortpflanzungsmedizin, der Humangenetik und der Embryologie. Sie untersteht dem Gesundheitsministerium (Loi n ${ }^{\circ}$ 2004-800 2004). 
would like to participate, thereby becoming fully-fledged actors in the regulation of the ethical dimensions of the market « (CCNE 2006b: 26).

Der Markt wird nach dieser Aussage durch die Entscheidung des Spenders oder der Spenderin gesteuert und bspw. nicht durch staatliche Regulierungsinstanzen. Nicht nur der Markt kann durch die Zell-Spende reguliert werden, auch die Entwicklung der Innovation. Die Beteiligung an Innovation findet in dieser Vorstellung nicht mehr allein durch die Teilnahme an Diskussionsveranstaltungen zu wissenschaftlichen und technologischen Entwicklungen statt, wie zu Beginn der 1980er Jahre, sondern durch Zell-Spende. Es geht also nicht mehr allein um das Sprechen über wissenschaftliche und technologische Entwicklungen vor dem Hintergrund der Innovation, sondern sozusagen um den körperlichen Einsatz für eine Innovation. An dieser Stelle muss allerdings betont werden, dass es um eine Empfehlung geht, eine Möglichkeit, wie über Zell-Spende nachgedacht werden kann. Meine Vermutung ist an dieser Stelle, dass sich hier die Ausdehnung des Aufgabengebietes des CCNE zeigt - sie dehnt sich von »ethischen Problemen« hin zu »ethischen und gesellschaftlichen Problemen« aus. »Gesellschaftliche Probleme« beinhalten dann die »ethischen Dimensionen des Marktes« und mit ihnen die Entwicklung der Innovation. Die Tendenz, die sich abzeichnet, ist, dass das bisherige Postulat der Nicht-Kommerzialisierung des menschlichen Körpers durch die NeuDefinition, ob eine Stammzelle ein Element des menschlichen Körpers ist, aufgeweicht wird. Die Auffassung von Ethik ist reversibel, es entstehen neue Grenzen und Möglichkeiten. Der Möglichkeitsraum hat sich erweitert und beinhaltet auch, über die Möglichkeit nachzudenken, den Markt »ethisch« zu regulieren.

\section{ZuSAMmENFASSUNG}

Im Kontext des CCNE zeigt sich nicht nur, dass die Öffentlichkeit als informations-defizitär konstruiert wird. Ihr wird außerdem die Unfähigkeit zur richtigen ethischen Reflexion, dem »richtigen Sprechen« zugesprochen. Ein Merkmal dieser Reflexion ist, dass wissenschaftliche und technologische Entwicklungen nicht in Frage gestellt werden sollen. Dies ist auch eine wesentliche Überschneidung mit dem Konzept der Reflexion, wie es 1974 im Kontext des MURS entsteht. Die Frage, inwiefern die Reflexionen des MURS den CCNE beeinflussen, kann entsprechend in zweierlei Hinsicht beantwortet werden. Zum einen wird überhaupt über die gesellschaftlichen Konsequenzen, die aus wissenschaftlichen und technologischen Entwicklungen entstehen können, nachgedacht. Zum anderen zeigt sich der Einfluss in der Art und Weise der Reflexion, nämlich einer unabhängigen und indi- 
viduellen, innerhalb derer wissenschaftliche und technologische Entwicklungen als unumstrittenes Kontinuum verstanden werden.

Der CCNE antwortet nicht auf »ethische Probleme «. Ethik wandelt sich kurze Zeit vor seiner Einrichtung im Jahr 1983 mit der sozialistischen Forschungspolitik vom Problem, wie noch 1979, zum Teil der Lösung. Wissenschaftler und Wissenschaftlerinnen, nun als Bürger und Bürgerinnen konzipiert, bekommen die Kompetenz, nicht nur technische Fragen zu definieren, sondern auch als ethisch verstandene Themen. Diese Zuschreibung ethischer Kompetenz erscheint vor allem als Lösung dafür, die »anti-wissenschaftlichen Bewegungen« einzudämmen und Innovation zu sichern. Dieses Ziel, die Innovation, erscheint im Entstehungskontext des CCNE nicht, sie zeigt sich erst in der avis von 2006 zur Zell-Spende und steht vermutlich in Zusammenhang mit der Ausdehnung des Aufgabenbereichs des Komitees auf als gesellschaftlich betrachtete Probleme. Auch stellt Ethik in Form des CCNE nicht explizit die Lösung dafür dar, die anti-wissenschaftlichen bzw. wissenschaftskritischen Bewegungen einzudämmen. Eine Eindämmung findet eher insofern statt, als politisch verstandene Themen ethisiert bzw. im Kontext vom OPECST technisch gerahmt werden. Die Probleme, auf die der CCNE antwortet, sind mögliche moralische Konflikte, die im Kontext der IVF gesehen werden. Und er antwortet auf Beunruhigungen und Unsicherheiten, die neue medizinische und technologische Entwicklungen hervorrufen, und darauf, dass die wissenschaftliche Selbststeuerung dieser Entwicklungen angezweifelt wird. Im Sinne Deans werden Regierungsmechanismen, die Steuerung medizinischer und technologischer Entwicklungen selbst zum Gegenstand der Problematisierung.

Ethik in Form des nationalen Ethikkomitees erscheint im Kontext gegenläufiger Tendenzen - dem Infragestellen und der Stabilisierung wissenschaftlicher Selbststeuerung. Die Institutionalisierung von Ethik in Form des CCNE im Jahr 1983 erscheint als Resultat dieser gegenläufigen Tendenzen: Sie stellt eine Modifikation wissenschaftlicher Steuerung dar, ohne die wissenschaftliche Entwicklung zu verhindern. Die Lösung liegt in der institutionalisierten ethischen Reflexion als dauerhaftem Prozess (vgl. Braun et al. 2008: 228). Sie besteht nicht darin, dass direkt auf medizinische und technologische Entwicklungen eingewirkt wird, sondern darin, die Art und Weise des Sprechens über Probleme oder Fragen, die aus diesen Entwicklungen entstehen, anzuleiten. Insofern kann der CCNE als Form des governing at a distance (Rose) verstanden werden, das durch ein governing through speech (Memmi) charakterisiert ist. Dieses angeleitete Sprechen geht im Sinne Memmis mit einer Bioindividuation einher. Die Frage ist nun, ob sich dieses magistère bioéthique (Memmi) auch im Kontext der Nanoethik des CCNE zeigt und auch, ob es sich in diesem Kontext noch um ein reflexive government (Dean) handelt. 


\section{Die Sprache der Ethik als Antwort auf Problematisierungen nanotechnologischer Autonomie}

Im Jahr 2007 publiziert der CCNE seine avis zu Nanotechnologien (CCNE 2007). Bereits im Jahr 2004 hatte das Komitee eine Arbeitsgruppe zu dem Thema eingerichtet (vgl. Dupuy/Roure 2004: 25).

Wie im vorangegangenen Kapitel ausgeführt, weist der Ethik-Rahmen in Form des CCNE einige Charakteristika auf: Sprecherinnen- und Sprecherpositionen zeichnen sich nicht darin aus, eine deutliche Position einzunehmen, sondern in der Fähigkeit, eine bestimmte Art der Reflexion zu praktizieren oder zu lernen, das »richtige Sprechen«. Ein No-Go des »richtigen Sprechens« ist das Infragestellen wissenschaftlicher Entwicklungen bzw. deutlich dagegen zu argumentieren. Es handelt sich vielmehr um ein individuelles, perpetuelles und gemäßigtes Sprechen und Denken über diese Entwicklungen. Der CCNE, so zeigt sich, bietet eher einen Rahmen für die Anleitung, Produktion und Organisation von Diskursen als eine normative Handlungsorientierung. Das angeleitete Sprechen, das governing through speech, geht im Sinne Memmis mit einer Bioindividuation einher. Historisch gesehen, erschienen ethische Rahmungen im Kontext zweier gegenläufiger Tendenzen, dem Infragestellen einerseits und der Stabilisierung wissenschaftlicher Selbststeuerung andererseits. Im Sinne des reflexive government nach Dean wird Regierung problematisiert. Welche Problematisierungen zeigen sich im Diskurs um Nanotechnologie und lassen sich die Charakteristika der ethischen Rahmung auch hier finden? Und vor allem, inwiefern wird Nanotechnologie als gesundheitsrelevantes Thema verstanden? Handelt es sich um ein magistère bioéthique oder um ein magistère nanoéthique?

Berücksichtigt wird im Folgenden zuerst der forschungspolitische Hintergrund. Im Anschluss wird auf den US-amerikanischen Diskurs um Nanotechnologie eingegangen, denn hier zeigen sich chronologisch gesehen zuerst ethische Rahmun- 
gen. Sie gehen mit einer Kontroverse um die Seriosität nanotechnologischer Forschung und Entwicklung zu Beginn der 2000er Jahre einher. Zum einen tangieren diese Auseinandersetzungen den französischen Nanotechnologiediskurs, zum anderen stellt sich die Frage, ob und inwiefern sich die ersten ethischen Rahmungen im Diskurs um Nanotechnologie in Frankreich wiederfinden. Anschließend stelle ich den Diskurs und die Konflikte um Nanotechnologie in Frankreich dar. Anhand der avis des CCNE zu Nanotechnologie gehe ich darauf ein, auf welche Problematisierungen sie antwortet und inwiefern sich die ethische Rahmung ausdehnt. Zunächst aber skizziere ich das Problem der Definition der Nanotechnologie.

\section{Das Problem mit der Definition}

»Das interessante an der Nanotechnologie ist«, schreibt der deutsche Politikwissenschaftler und Biologe Joscha Wullweber, »dass niemand weiß, was die Nanotechnologie so genau ist « (Wullweber 2008a: o.S.). Zum Beispiel wird, so Wullweber, zwischen den Bereichen der Nanomaterialien, der Nanoelektronik, der Nanobiotechnologie, Nanomedizin oder Nanokosmetik und Anwendungen in den Informations- und Kommunikationstechnologien unterschieden. Die einen verstehen unter Nanotechnologie die kontrollierte Manipulation von Atomen und Molekülen. Die anderen verstehen darunter Möglichkeiten molekularer Fertigung: indem Atome gezielt und kontrolliert zu größeren Strukturen zusammengesetzt werden, könnte alles Atom für Atom aufgebaut werden, Proteine ebenso wie Computer oder Autos (ebd.). Aufgrund der verschiedenen Anwendungsbereiche der Nanotechnologie, der diversen Techniken und Instrumente wurde von der britischen Akademie der Wissenschaften, The Royal Society, vorgeschlagen, nicht von der Nanotechnologie, sondern von den Nanotechnologien zu sprechen (The Royal Society \& The Royal Academy of Engineering 2004: vii). Kurzum, es handelt sich bei der Nanotechnologie weder um ein spezifisches, klar abgegrenztes Forschungsfeld noch um eine einzelne Technologie (Davies et al. 2010). Das Kriterium der Nanotechnologie, auf das Wissenschaft und Politik sich meist beziehen, ist die Dimension von einem bis 100 Nanometer (Wullweber 2008b: 29; 2008a: o.S.; 2006: 100; Fogelberg/Glimell 2003: 10). Die Vorsilbe Nano stammt von dem griechischen Wort für Zwerg. Ein Nanometer entspricht dem Billionstel eines Meters (Wullweber 2006: 100; Kenneth 2008: 5). Die Fragen, warum es keine Definition der Nanotechnologie gibt, warum es schwierig scheint, eine zu finden oder was die fehlende Definition bewirkt, beschäftigen auch Untersuchungen in den Nano-STS (vgl. Baird et al. 2004; Fiedeler et al. 2010; Decker et al. 2004). Einige gehen davon aus, dass das Label, der »umbrella term« Nanotechnologie Wissenschaftlerin- 
nen und Wissenschaftlern dazu diene, Forschungsgelder zu erhalten (Schummer 2014: 23f; Glimell 2003: 71). Andere sehen die fehlende Definition als Charaktereigenschaft, die aus der Konvergenz von wissenschaftlichen Disziplinen und Technikfeldern entstammt, die traditionell getrennt waren (Paschen et al. 2003: 39). Wullweber vertritt die These, dass der Begriff Nanotechnologie ein umfassendes politisches Projekt kennzeichnet, eine »techno-socio-economical innovation strategy«, die gerade durch den »empty signifier« zusammengehalten wird (Wullweber 2008b: 41).

In der vorliegenden Untersuchung geht es nicht darum, wie sich die NichtDefinition der Nanotechnologie durchgesetzt hat. Ebenso wenig ist Gegenstand der Untersuchung, wie überhaupt der Diskurs um Nanotechnologie entstanden ist. Vielmehr geht es darum, wann explizit die Sprache der Ethik in diesem Diskurs auftaucht und was problematisiert wird. Da ethische Problematisierungen damit einhergehen (können), was unter der jeweiligen Wissenschaft oder Technologie verstanden wird, wird dies, ebenso wie in den vorangegangenen Kapiteln berücksichtigt. Anders gesagt, tangiert die Frage, was von bestimmten Akteurinnen und Akteuren in einem bestimmten Kontext unter Nanotechnologie verstanden wird, die Frage, was als jeweiliges ethisches Problem definiert wird.

\section{Forschungspolitischer HiNTERGRUND: DiE SCHLÜSSELTECHNOLOGIE DES 21. JAHRHUNDERTS}

Nanotechnologie gilt als die Technologie des 21. Jahrhunderts, als Schlüssel- oder auch als Querschnittstechnologie, die ähnlich der Informationstechnologie allgegenwärtig sein wird (vgl. Schaper-Rinkel 2006). In Europa beginnen die ersten konkreten nanotechnologiepolitischen Initiativen mit der Mitteilung der Kommission der Europäischen Union (EU) zu einer »europäischen Strategie der Nanotechnologie« im Jahr 2004 (Kommission der europäischen Gemeinschaften 2004). Bereits zwei Jahre zuvor stellt der damalige Kommissar für Forschung und Innovation der EU, der Belgier Philippe Busquin, auf einer Konferenz im französischen Grenoble fest: »La révolution des nanotechnologies a commencé « - »Die nanotechnologische Revolution hat begonnen« (Busquin 2002: 2/Übers. S.K.). Als problematisch wird in der Mitteilung der EU-Kommission eine mögliche zunehmende »Kluft« im internationalen Wettbewerb zwischen der EU und ihren »Hauptkonkurrenten«, insbesondere den USA und Japan gesehen (Kommission der europäischen Gemeinschaften 2004: 9). 
»Einer der wesentlichen Unterschiede zwischen der EU und ihren Hauptkonkurrenten besteht darin, dass sich die europäische FuE-Landschaft [FuE - Forschung und Entwicklung, S.K.] im Bereich der Nanotechnologie mit unterschiedlichen, rasch voranschreitenden Programmen und Finanzquellen relativ aufzusplittern droht. 2003 wurden 350 Millionen $€$ aus dem 6. Rahmenprogramm bereitgestellt, d.h. ein Drittel der europäischen Gesamtinvestitionen in Nanotechnologie « (ebd.).

Im Kontrast zur EU haben die USA bereits im Jahr 2001 die National Nanotechnology Initiative (NNI) ins Leben gerufen, eine Initiative zur Koordination von Forschungs- und Entwicklungsaktivitäten im Bereich der Nanotechnologie. ${ }^{1} \gg$ Auf dem heutigen Weltmarkt ist«, so heißt es in dem Strategiepapier der EU-Kommission, »Wirtschaftswachstum nicht ohne Innovation denkbar, die wiederum Forschung voraussetzt« (ebd.: 10). Die Nanowissenschaft verspreche »Innovationen, die zur Lösung zahlreicher Probleme der heutigen Gesellschaft beitragen können«, und zwar im medizinischen Bereich (z.B. synthetische Organtransplantation), in der Informationstechnologie (z.B. Datenträger mit größerer Kapazität), der Energieerzeugung (z.B. kostengünstige Solarzellen), in der Materialwissenschaft (z.B. kratzfeste Oberflächen), der Fertigung (z.B. Nachbildung von Strukturen auf Atom- und Molekularebene) oder im Bereich der Sicherheit (z.B. Schutz von Eigentum, wie Banknoten durch eine Nanoetikettierung) (ebd.: 5f). Zur »Förderung des Fortschritts « in Europa wird in dem Strategiepapier u.a. vorgeschlagen: die Koordinierung nationaler politischer Maßnahmen im Bereich FuE, die wettbewerbsorientierte Generierung neuer Kenntnisse zur Schaffung von Wohlstand und Arbeitsplätzen, die Einrichtung von Technologieplattformen sowie die öffentlichen Investitionen in die Nanotechnologie um ein Dreifaches zu erhöhen (ebd.: 10f, 13). Auch der französische Staatspräsident Jacques Chirac (1995-2007) spricht sich 2004 auf einer Botschafterinnen- und Botschafterkonferenz für eine massive Investition in die EU-Forschung aus: »Wir sollten große Wissenschaftsprojekte entwickeln. Gemeinsam können die Europäer alle Bereiche abdecken: Raumfahrt, Nanotechnologie, Umweltschutz, Biotechnologien, medizinische Forschung und viele andere (Chirac 2004).

In Frankreich gibt es bereits Ende der Jahrtausendwende nanotechnologiepolitische Initiativen. Im Jahr 1999 wird das Nationale Netzwerk für Mikro- und Nanotechnologien entwickelt, der Réseau National de Micro-NanoTechnologies, um die Zusammenarbeit zwischen öffentlicher und industrieller Forschung zu unter-

1 Zur NNI vgl. http://www.nano.gov/you/ethical-legal-issues, 06.11.2012. Ausführlich zu ihrer Entstehung vgl. Brice Laurent (2010: 21ff). 
stützen. Umfangreiche forschungspolitische Initiativen, so der französische Sozialwissenschaftler Brice Laurent in seinem Buch zur Politik der Nanotechnologie, beginnen im Jahr 2003 (Laurent 2010: 25). Hier beschäftigt sich die parlamentarische Technikfolgenabschätzungsbehörde OPECST zum ersten Mal mit dem Thema, insbesondere mit der Mikro- und Nanoelektronik (OPECST 2003). Der OPECST empfiehlt u.a. eine stärkere Koordination der Zusammenarbeit zwischen Forschung und Industrie (ebd.: 40). Im selben Jahr wird durch den Forschungsminister der Réseau National de Grandes Centrales Technologiques - das Nationale Netzwerk der großen Technologiezentren im Bereich nanotechnologischer Grundlagenforschung initiiert, die sich in Lille, Grenoble, Toulouse, Besançon und Saclay befinden. Seit der Einrichtung der Agence Nationale de la Recherche - der Nationalen Forschungsagentur im Jahr 2005 erscheint Nanotechnologie als forschungspolitische Priorität, so Laurent. Die Agentur finanziert Projekte öffentlicher und privater Forschungsgruppen und wird von dem Premierminister Jean-Pierre Raffarin (2002-2005) ins Leben gerufen. Zu ihren größten Forschungsprogrammen zählte das Nanotechnologie-Programm PNANO (Programme en Nanosciences et Nanotechnologies) (Laurent 2010: 25).

\section{DER NANOTECHNOLOGIEDISKURS: ETHIK UND »The Coming ERA OF NANOTECHNOLOGY $\ll^{2}$}

Erste ethische Rahmungen im Diskurs um Nanotechnologie erscheinen im Kontext des populärwissenschaftlichen Beststellers »Engines of creation. The Coming Era of Nanotechnology« des US-amerikanischen Physikers Eric Drexler aus dem Jahr 1986 (Drexler 2007). Drexler, der als Ideengeber der Nanotechnologie gilt, trägt mit seinen Publikationen, insbesondere seinem Bestseller, der in mehrere Sprachen übersetzt wird, zur Diskussion um Nanotechnologie bei, auch über die wissenschaftliche Community hinaus (Schaper-Rinkel 2006). Darüber hinaus werden Drexlers Vorstellungen über die Nanotechnologie dazu verwendet, den USamerikanischen Kongress $\mathrm{zu}$ überzeugen, die NNI zu finanzieren (Berube/ Shipman 2004: 23). Während seine Ideen 2001 noch zur forschungspolitischen Förderung der Nanotechnologie verwendet werden, führen sie kurze Zeit später zu einer Auseinandersetzung innerhalb der US-amerikanischen wissenschaftlichen Community darüber, was unter einer seriösen Nanotechnologie zu verstehen ist (vgl. Selin 2007; Berube/Shipman 2004).

2 Drexler 2007. 
Die Ideen und Visionen, die Drexler in »Engines of creation « publik macht, beziehen sich insbesondere auf das »molecular manufactoring «, das es jedem Mensch ermöglichen soll, Atome nach Wunsch anzuordnen und somit Dinge selbst herzustellen, wie Häuser, Computer oder Zahnbürsten (Drexler 2007: o.S.). Drexler thematisiert in diesem Kontext das Problem der knappen Ressourcen, welches durch die Nanotechnologie gelöst werden könne und wodurch der »ultimate moral imperative«, so wenig Ressourcen wie möglich zu verschwenden, nichtig werde. Drexler stellt sich zudem vor, dass so genannte Assembler kranke menschliche Zellen reparieren könnten (ebd.). In der molekularen Nanotechnologie wird unter einem Assembler ein Roboter verstanden, welcher hypothetisch einzelne Atome und Moleküle manipuliert. Damit können, so die Annahme, molekulare Strukturen erstellt werden, die nicht in der Natur vorkommen. Ethische Fragen sieht Drexler insbesondere im Hinblick auf die Medizinethik. Durch die mögliche Anwendung von »cell repair machines « kämen Fragen auf, so Drexler, die über die Fragestellungen in der aktuellen Medizinethik hinausgehen, $»$ which commonly involve dilemmas posed by scarce, costly, and half-effective treatments. They are instead questions involving the value of long, healthy lives achieved by inexpensive means « (ebd.). Unter »cell repair machines « werden im Sinne Drexlers Maschinen verstanden, die bspw. in menschliche Zellen eindringen können, um ein Enzymdefizit zu erkennen oder auch eine Beschädigung der DNA (ebd.).

Auf Drexlers Publikation folgt im Jahr 1989 die erste so genannten ForesightKonferenz, die namentlich auf das Institut, dem von Drexler in Palo Alto, Kalifornien, gegründeten Foresight Institute zurückgeht. Ziel des Institutes ist die Entwicklung von Richtlinien zum sicheren und verantwortungsvollen Umgang vor allem mit molekularer Nanotechnologie. Die Konferenz von 1989 verfolgt das Ziel, auf interdisziplinärer Ebene über die Herausforderungen und die sozialen Konsequenzen der Nanotechnologie zu sprechen (Crandall/Lewis 1992: vii). Die Ergebnisse dieser Konferenz, die in einem Tagungsband zusammengetragen wurden, präsentieren, so die Herausgeber, »technical as well as cultural and ethical considerations « (ebd.: viii). Auf der Konferenz wird ausgeführt, dass moderne Technologien und somit auch die Nanotechnologie zu dramatischen Veränderungen der Welt führen, insbesondere durch unkontrollierte, mutierte Selbstreplikationen von Assemblern. Es lägen potentielle Probleme in ihrem »mismanagement« oder Missbrauch. Als Beispiel hierfür dient die Chemiekatastrophe in Bhopal, Indien, eine Katastrophe bzw. ein »problem «, das in ähnlicher Qualität durch mutierte Assembler entstehen könnte (ebd.: 315). In Bhopal entweicht im Jahr 1984 aus dem Chemiewerk der Firma Union Carbide eine Giftgaswolke. 20.000 Menschen sterben bei der Katastrophe und an den Spätfolgen (Matern 2008). Auch von »mistakes « wie den Wasserstoffbomben, die am Grund des Ozeans lägen, wird ge- 
sprochen (Crandall/Lewis 1992: 321). Wichtig sei eine Kontrolle der Nanotechnologie. In der Abschlussdiskussion der Konferenz heißt es: »Nanotechnology has got to be controlled, and if it is not controlled, it will be abused in some way and injure all of us « (Drexler 2007: 316). Um einen Missbrauch zu verhindern, wird in der Abschlussdiskussion die Entwicklung einer planetarischen Politik vorgeschlagen, der ein planetarischer Konsens über die Notwendigkeit der Sicherung planetarischer Systeme vorangeht (Crandall/Lewis 1992: 316).

Die Nanotechnologie à la Drexler ist innerhalb der US-amerikanischen wissenschaftlichen Community umstritten. Nicht Drexlers Vorstellung im Hinblick auf den moralischen Imperativ der Ressourcenschonung oder die Auswirkungen auf die Medizinethik werden zu Beginn der 2000er Jahre diskutiert. Ebenso wenig wird, wie auf der Foresight-Konferenz aufgeworfen, die Kontrolle nanotechnologischer Entwicklungen thematisiert, basierend auf einem planetarischen Konsens und vor dem Hintergrund möglicher Katastrophen. Vielmehr geht es um die Frage der Seriosität von Drexlers Vorstellung, die vor allem innerhalb der US-amerikanischen wissenschaftlichen Community diskutiert wird.

\subsection{Nano-boundary work: von unrealistischen Fiktionen und realistischen Entwicklungen}

Bekannt wird vor allem die Kontroverse zwischen Drexler und dem US-amerikanischen Chemiker und Physiker Richard Smalley. Smalley, später Nobelpreisträger im Bereich Nanotechnologie, ist hinsichtlich seiner Forschung ursprünglich durch die Lektüre von Drexlers »Engines of Creation « inspiriert (Berube/Shipman 2004: 24). In der Kontroverse mit Drexler geht es darum, ob und inwiefern selbstreplizierende Assembler realisierbar sind. Der Disput beginnt im Jahr 2001 mit einem Artikel Smalleys in der populärwissenschaftlichen Zeitschrift Scientific American und wird zum Schluss, im Jahr 2003, in dem Wissenschaftsmagazin Chemical \& Engineering News, dem Magazin der American Chemical Society ausgetragen (Shew 2008: 395f; vgl. Baum 2003). Zuvor wird der Schriftwechsel zwischen den beiden Akteuren auf der Homepage des Foresight-Instituts veröffentlicht. Während Drexler selbstreplizierende Assembler für realisierbar hält, vor den Risiken in Anbetracht der globalen Sicherheit warnt und deshalb eine Diskussion vorschlägt, die über ein rein akademisches Interesse hinausgeht, stellt Smalley die Realisierbarkeit der Assembler in Frage. Zudem behauptet er, die Assembler wären Monster aus Drexlers Träumen, die nicht der Realität entsprächen und darum gefährlich seien. In den Chemical \& Engineering News heißt es von Smalley: 
»You and people around you have scared our children. I don't expect you to stop, but I hope others in the chemical community will join with me in turning on the light, and showing our children that, while our future in the real world will be challenging and there are real risks, there will be no such monster as the self-replicating mechanical nanobot of your dreams « (Smalley (2003) zit. nach Baum 2003).

Drexler meint:

»You apparently fear that my warnings of long-term dangers will hinder funding of current research [...] your attempt to calm the public through false claims of impossibility will inevitably fail, placing your colleagues at risk of a destructive backlash. [...] Endorsing calls for an independent scientific review of molecular manufacturing concepts would be constructive« (Drexler (2003) zit. nach ebd.).

Auch in der Folgezeit findet eine weitere Abgrenzung von den als utopisch und unrealistisch betrachteten Vorstellungen Drexlers statt. Vormals Hauptredner auf wissenschaftlichen Tagungen, wird seine Anwesenheit nun hinterfragt (Berube/Shipman 2004: 22). Von ihm geprägte Begriffe werden zum Teil in USForschungsprogrammen durch andere ausgewechselt (ebd.: 23); sein Ansatz wird marginalisiert (vgl. Selin 2007: 203). Von wissenschaftlicher Seite wird versucht, ihn als Schlüsselakteur für Nanotechnologie zu ersetzen, denn »serious scientists are simply burdened by his visions « (Berube/Shipman 2004: 23/Herv. i.O.). Um Investitionen wird gefürchtet, ebenso wie um Bedenken in der Öffentlichkeit.

Mit Thomas Gieryn und seinem Ansatz des boundary work gedacht, kann diese Auseinandersetzung als Abgrenzungstätigkeit verstanden werden (vgl. Gieryn 1983). Diese Abgrenzung findet gegenüber dem statt, was aus Perspektive der Wissenschaftler und Wissenschaftlerinnen als nicht seriöse Wissenschaft betrachtet wird bzw. sich als solche durchsetzt, um ihre professionelle Autonomie zu schützen. Die Politikwissenschaftlerin Petra Schaper-Rinkel bezeichnet diesen Vorgang in ihrer Untersuchung zur governance von Zukunftsversprechen im Kontext der Nanotechnologie als governance der Wahrheit: Mit den Grenzziehungen sind erste Konturen einer »politischen Ökonomie des Versprechens« entstanden, und zwar indem zwischen unrealistischen Fiktionen einerseits und wahrscheinlichen und realistischen Entwicklungen andererseits unterschieden wird (Schaper-Rinkel 2006: 481). Die Durchsetzung der Nanotechnologie als seriöse Wissenschaft, als realistische Entwicklung zeigt sich auch in der Herausbildung ihrer Standardgeschichte. 


\subsection{Die »standard story « ${ }^{3}$ über die Nanotechnologie}

Mittlerweile hat sich eine Standarderzählung der Nanotechnologie entwickelt, in der die Person Drexler zum Außenseiter wird und die Kontroverse um Drexlers Ansatz nicht thematisiert wird. Als Ursprungsfigur und Vater der Nanotechnologie gilt in der Standardgeschichte der US-amerikanische Physiker und Nobelpreisträger (1965) Richard Feynman (*1918-†1988) (Schaper-Rinkel 2006: 475f; vgl. Baird/Shew 2004; vgl. Fogelberg 2003: 30f; vgl. Woyke 2007: 333f; Shew 2008). Im Jahr 1959 hält Feynman auf dem Treffen der Amerikanischen Physikalischen Gesellschaft am California Institute of Technology den Vortrag »There's Plenty of Room at the Bottom « (Feynman 1959). Obwohl Feynman den Begriff Nanotechnologie nicht gebraucht - verwendet wird der Begriff erst 1974 von dem japanischen Wissenschaftler Norio Taniguchi - gilt er heute insofern als Vater der Nanotechnologie, als er in seinem Vortrag 1959 einen konzeptionellen Paradigmenwechsel der Miniaturisierung formuliert. Sein Gedanke ist, Dinge nicht nur zu miniaturisieren, sondern sie aus kleinsten Teilchen zu konstruieren (ebd.). Taniguchi kennzeichnet 1974 mit dem Begriff Nanotechnologie Produktionstechnologien im Nanometer-Bereich (Schaper-Rinkel 2006: 475). Zwar steht Drexlers Ansatz der Nanotechnologie insofern in einer Traditionslinie zu Feynmans Idee, da er sich auf sie bezieht und Feynmans Vortrag bekannt macht (Feynman verfolgte seine Ideen damals nicht weiter); jedoch findet ab den 1990er Jahren im technologiepolitischen Radius der führenden Industriestaaten durch Tagungen und Studien ein »diskursiver Selektionsprozess « statt, in welchem der Rekurs auf Feynman anwächst (ebd.: 476). Wie die Politikwissenschaftlerin Schaper-Rinkel meint,

»bietet sich der Rekurs auf einen Nobelpreisträger als Geburtsdokument eines Technologiefeldes an. Zugleich wird damit der Beginn in den USA, dem technologisch führenden Nationalstaat in der zweiten Hälfte des zwanzigsten Jahrhunderts, lokalisiert. Und schließlich kommt der Autor aus der Physik, der seinerzeitigen Leitwissenschaft« (ebd./Herv. i.O.).

Einerseits ist die Physik seinerzeit unumstrittene Leitwissenschaft, andererseits manifestiert sie sich m.E. rückwirkend als eben solche. Denn nicht thematisiert wird im Rekurs auf Feynman in der Standardgeschichte der Nanotechnologie seine Beteiligung am Manhattan-Projekt im Zweiten Weltkrieg, das zur Entwicklung der Atombombe beiträgt (vgl. Feynman 1976). Im Kontext des Manhattan-Projektes, an dem Feynman als junger Wissenschaftler beteiligt war, kam eine Selbstkritik der Wissenschaftlerinnen und Wissenschaftler im Hinblick auf die Verwendung wis-

3 Baird/Shew 2004: 151. 
senschaftlich technologischer Entwicklungen für Kriegszwecke auf. Die Standardgeschichte der Nanotechnologie zeichnet sich also nicht durch die Darstellung von Kontroversen oder durch das Infragestellen einer Technologie aus.

Drexlers Ansatz bleibt im Diskurs um Nanotechnologie dennoch weiter präsent. Im wissenschaftlichen Kontext bspw. beschäftigte sich im Jahr 2006 die NNI in ihrem Bericht "A matter of size « mit der Frage der Realisierbarkeit der »molecular self-assembly for manufacturing « (National Research Council 2006: 1). Im Genre des Science-Fiction zeigt sich Drexlers Ansatz bspw. in dem Roman »Prey« von Michael Crichton aus dem Jahr 2002. Bekannt wird der USamerikanische Bestseller-Autor u.a. durch den Roman »Jurassic Park« aus dem Jahr 1990, der später verfilmt wird. Ähnlich wie in seinem Roman »Jurassic Park«, in welchem Crichton anhand geklonter Dinosaurier auf die Gefahren der Gentechnologie hinweist, thematisiert er in dem Roman »Prey« die Unberechenbarkeit der Nanotechnologie. In dem Roman sind künstliche Maschinen-Organismen aus einem Unternehmen entwichen, das im Auftrag des US-Militärs Überwachungsanlagen auf Miniaturebene bauen soll. Diese sich selbstreplizierenden Organismen werden durch ihre unvorhersehbaren Anpassungsstrategien zur Gefahr für die Menschen - letztere werden selbst zur Beute (Prey) der Maschinen-Organismen. Der Science-Fiction popularisiert die Nanotechnologie über die wissenschaftliche Community hinaus.

Der Science-Fiction wird auch im Diskurs um Nanotechnologie in Frankreich thematisiert. Wie dies geschieht, wird im Folgenden aufgezeigt. Darüber hinaus liegt der Fokus darauf, wie sich der Diskurs entwickelt, und auf den Fragen, wann, wo und inwiefern die Sprache der Ethik in diesem Diskurs auftaucht.

\section{Der NanotechnologiediskuRs in FrankReich: VON RISIKO-PROBLEMEN UND ETHISCHEN IMPLIKATIONEN}

Der Diskurs um Nanotechnologie in Frankreich beginnt kurz nach der Jahrtausendwende und ist geprägt von einer Melange aus der Sprache des Risikos und der Sprache der Ethik. Schlüsselereignisse, die den öffentlichen Diskurs um Nanotechnologie initiierten, so der Wissenschaftssoziologe Dominique Vinck und seine Kolleginnen und Kollegen, sind eine öffentliche Rede des Thronfolgers des Vereinigten Königreichs von Großbritannien, Prinz Charles, sowie Publikationen internationaler NGOs, wie von der Action Group on Erosion, Technology and Concentration (ETC Group) und auch der Umweltorganisation Greenpeace (Vinck et al. 2009; Arnall 2003; ETC Group 2003). Prinz Charles warnt im Juli 2003 vor 
den möglichen Risiken der Nanotechnologie und ruft dazu auf, sie wissenschaftlich zu untersuchen. Seine Rede zieht eine heftige mediale Debatte nach sich, in der seine Warnung vor allem als Warnung vor dem so genannten Gray-Goo-Effekt (auch Grey-Goo-Effekt) verstanden wird (N.N. 2003). Gray goo beinhaltet die Vorstellung der Zerstörung allen Lebens durch die unkontrollierbare Ausbreitung so genannter selbstreplizierender nanobots (eine Art Roboter bzw. Assembler, SK). Der Begriff stammt aus Eric Drexlers Buch »Engines of Creation« (Drexler 2007). Der Grey Goo-Effekt wird bereits zwei Jahre vor Prinz Charles Rede medial thematisiert, und zwar im Kontext einer Forderung zum Verzicht auf Nanotechnologie: Im Jahr 2000 wird der Artikel »Why the Future doesn't need Us« im USamerikanischen Technologie-Magazin Wired (Joy 2000b) und auch in der französischen Tageszeitung Le Monde (Joy 2000a) veröffentlicht. Autor ist der USAmerikaner Bill Joy, Chief Scientist der Computer- und Softwarefirma Sun Microsystems, der zudem Teilnehmer der o.g. Foresight-Konferenz an Drexlers Institut war. Einleitend spricht er in seinem Artikel von »ethical dimensions«, die ihn beschäftigen, seit er in die Entwicklung neuer Technologien involviert ist (Joy 2000b). Joy konzentriert sich in seinem Artikel auf die Auswirkungen neuer Technologien wie Gentechnologie, Nanotechnologie und Robotik und ruft dazu auf, auf sie zu verzichten: In Anbetracht des unsicheren und begrenzten Wissens über den technologischen Fortschritt und die Potentiale der Nanotechnologie gäbe es Risiken, die nur durch Verzicht auf diese Technologien verhindert werden könnten (ebd.). Prinz Charles’ Rede geht allerdings nicht nur Joys Aufruf zum Verzicht voraus, sondern auch die Forderung eines Moratoriums für die kommerzielle Herstellung von Nanomaterialien. Die kritische Wissenschaftsassoziation ETC Group spricht sich auf dem »World Summit on Sustainable Development« in Johannesburg im Jahr 2002 aufgrund der unkalkulierbaren Risiken für Umwelt und Gesellschaft für ein Moratorium und gegen die kommerzielle Produktion neuer Nanomaterialien aus (Mnyusiwalla et al. 2003). Zu dem Moratorium heißt es in ihrer Publikation »The big down«:

»Given the concerns raised over nanoparticle contamination in living organisms, etc Group proposes that governments declare an immediate moratorium on commercial production of new nanomaterials and launch a transparent global process for evaluating the socioeconomic, health and environmental implications of the technology« (ETC Group 2003: 72).

Nanopartikel bestehen in der Natur, werden aber auch künstlich hergestellt und bspw. vielfach in der Kosmetik, etwa in Sonnencremes, verwendet. Sie haben dann neue physikalische Eigenschaften, deren Auswirkungen unklar sind (vgl. BUND 2013). Die Kritkerinnen und Kritiker empfehlen eine »International Convention for 
the Evaluation of New Technologies« sowie die Beteiligung von Bürgerinnen und Bürgern in offenen Debatten im Kontext der Evaluation der Technologien (ETC Group 2003: 72). Abgesehen von ihrer Forderung eines Moratoriums, geht die ETC Group davon aus, dass das »molecular manifacturing « zwar nicht unbedingt im Drexlerschen Sinn, aber in naher Zukunft umgesetzt werde (ebd.: 29).

Als direkte Reaktion auf die Forderung eines Moratoriums der ETC Group erscheint der Artikel »Mind the gap« von Anisa Mnyusiwalla, Abdallah Daar und Peter A. Singer der Universität von Toronto, USA (Mnyusiwalla et al. 2003). In dem Artikel werden einige Befürchtungen geäußert: »These [...] trends seem to be on a collision course towards a showdown of the type that we saw with GM crops « (ebd.: R9). Zudem wird diagnostiziert »the science leaps ahead, the ethics lags behind «. Sie appellieren, diese Kluft zu schließen. Denn, so die Autorinnen und Autoren, um ein Moratorium zu verhindern und um zu verhindern, dass sich wissenschaftliche Entwicklungen verlangsamen, sind Untersuchungen zu ethischen und sozialen Implikationen nanotechnologischer Entwicklungen notwendig (ebd.). Der Appell hat, wie im Kapitel zum Forschungsstand aufgeführt, eine Fülle an Untersuchungen initiiert.

Im Unterschied zu Bill Joys Artikel, in dem für das Problem der Risiken die Lösung des Verzichtes auf Nanotechnologie als ethische Dimension gerahmt wird, spricht die ETC Group zwar ebenfalls von Risiken, aber von einem Moratorium als Lösung. Nicht die von Joy und der ETC Group thematisierten Risiken werden als Problem gesehen, sondern die Lösungsmöglichkeiten, also der Verzicht auf Nanotechnologie oder ein Moratorium, die die wissenschaftlichen Entwicklungen behindern könnten. Um wissenschaftliche Entwicklungen nicht zu behindern, erscheint die Untersuchung »ethischer und sozialer Implikationen« als Lösung (vgl. ebd.). Da diese überhaupt untersucht werden müssen, scheint es genau hier Probleme zu geben, die die wissenschaftlichen Entwicklungen behindern - im gesellschaftlichen Raum. Risiko-Implikationen für die Gesellschaft werden nicht benannt. In diesem Sinn werden die von Joy und der ETC Group definierten Probleme, die als Risiken gerahmt sind, verdrängt oder ethisiert. Diese Ethik-Rahmung beinhaltet nicht die Diskussion über einen Verzicht auf Nanotechnologie, sondern garantiert nanotechnologische Entwicklungen. Einerseits werden Risiken ethisiert. Andererseits werden m.E. bestimmte ethische Themen marginalisiert, nämlich solche, die mit einer Vorstellung von Nanotechnologie als nicht seriös einhergehen. So werden etwa die ethischen Fragen, die Drexler im Kontext der Medizinethik aufwirft, verdrängt, genauso wie die Forderung nach einem Verzicht auf Nanotechnologie (Joy). Im Kontext des geforderten Moratoriums wird im Kontrast zu dem Moratorium im Kontext des Gentechnologiediskurses nicht explizit gefordert, die Forschung an sich zu stoppen, sondern lediglich die kommerzielle Produktion. 
Das Moratorium richtet sich m.E. zwar auf die Nanotechnologieindustrie, spricht aber implizit die Frage der Selbststeuerung wissenschaftlicher und technologischer Entwicklungen an. Da Nanotechnologie forschungspolitisch so ausgerichtet ist, dass sie die Zusammenarbeit von Forschung und Industrie unterstützt, würde mit einem Moratorium für die kommerzielle Produktion auch die Forschung gestoppt. Abgesehen von der ETC Group, Bill Joy und Prinz Charles, deren Kritiken nicht allein in Frankreich den Diskurs um Nanotechnologie förderten, kommt eine spezifische französische Kritik hinzu.

\section{1 »Nekrotechnologie ${ }^{4}$}

Im Jahr 2002 beginnen auf regionaler Ebene in Grenoble Proteste gegen nanotechnologische Forschung und Entwicklung (vgl. Vinck et al. 2009). Aktivistinnen und Aktivisten rund um die Gruppe mit dem Namen Pièces et main d'oeuvre (PMO) richten ihre Kritik symbolisch auf das Forschungs- und Innovationszentrum Minatec. ${ }^{6}$ Das Zentrum verbindet Forschung und Industrie und hat das Ziel, zu Europas führendem Zentrum für Innovation und Expertise im Bereich der Nanotechnologie zu werden (Laurent 2007: 345; 2008: 5). Die Nanokritikerinnen und -kritiker rund um PMO sprechen nicht von Nanotechnologie, sondern von Nekrotechnologie im Sinne einer Todestechnologie (Simples Citoyens 2003). »For the activists, nanotechnology research is a manifestation of [...] political, scientific, military and economic interests, which leads to decisions based on military or market interests, and, therefore, opposed to the general interest « (Laurent 2007: 345). Die Mitglieder der Gruppe sind zuvor in der Anti-Atom- und Anti-GMOBewegung in Frankreich aktiv, so Laurent. Er meint, dass die Kritik an der Nanotechnologie sich aus der Kritik, die in den beiden Bewegungen formuliert wurde, zusammensetzt. Im Unterschied zu diesen Bewegungen wird allerdings nicht nur der technokratische Staat kritisiert, wie im Kontext der Anti-Atombewegung, sondern auch multinationale Konzerne bzw. Marktinteressen. Denn die Anti-GMO-

4 Simples Citoyens 2003/Übers. S.K..

5 Es gibt weitere Gruppen und Aktivistinnen und Aktivisten in Grenoble, die sich meist auf PMO beziehen, die die einflussreichste Gruppe ist (Laurent 2008: 5). Da die einzelnen Akteurinnen und Akteure schwer zu differenzieren sind, wird im Folgenden nur PMO genannt.

6 Minatec wird im Jahr 2006 eröffnet; Pläne existierten bereits seit 1998. Zur Entstehung des Zentrums vgl. Callon et al. (2005) sowie Laurent (2007: 345) und Vinck et al. (2009). 
Proteste, die sich in Frankreich Mitte der 1990er Jahre entwickeln, sind mit der Anti-Globalisierungsbewegung verknüpft, deren zentrales Thema multinationale Konzerne sind (ebd.). In der Kritik von PMO wird nicht nur eine technologieskeptische Haltung deutlich, wie sie im Kontext der Kritik an GMO und Atomtechnologie besteht, sondern ebenso Kritik an der Entscheidungsfindung über wissenschaftliche und technologische Entwicklungen (ebd.). In einem Interview wird mir die Position von PMO wie folgt erklärt (teils wird hierbei die Perspektive von PMO eingenommen):

»das ist eine Kritik an den Entscheidungsmodi, d.h. wer entscheidet worüber, wie wird entschieden, und wie kommt es, dass wir nie auf dem Laufenden sind, dass wir immer erst im Nachhinein von etwas hören und nie vorher. Sie prangern die Art der governance an und den Zusammenschluss zwischen finanziellen Interessen und wissenschaftlichen, all das ist [im Sinn von PMO, S.K.] eine Mafia« (Int. F XIII/Übers. S.K.).

Nicht nur das Dominieren der Debatten durch Experten und Expertinnen und kapitalistische Interessen in der Entscheidungsfindung werden thematisiert, sondern auch die Gefahren für die Gesellschaft, die aus nanotechnologischen Entwicklungen entstehen (N.N. 2004, 2010). PMO führt einige Aktionen durch: sie besetzt während der Bauphase von Minatec einen Baukran, organisiert Demonstrationen mit ungefähr 1000 Teilnehmenden, stört öffentliche Debatten und hält alternative Diskussionsveranstaltungen ab (Laurent 2007: 345; Vinck et al. 2009; Opposition Grenobloise aux Nécrotechnologies 2006). Öffentliche Debatten werden 2005 vom Gemeindeverband, der Communauté d'agglomération Grenoble Alpes Métropole (La Metro), organisiert. Auf der zweitägigen Veranstaltung mit dem Titel »Forum Wissenschaft und Demokratie« diskutieren Vertreterinnen und Vertreter aus Wissenschaft, Lokalpolitik und von Umweltorganisationen zu Themen wie »Wissenschaft und Ethik « und beantworteten Publikumsfragen (Laurent 2008: 8/Übers. S.K.). Vom Präsident von La Metro wird dieses Forum als »offenes und partizipatorisches Ereignis« bezeichnet (ebd./Übers. S.K.). Diesem Forum folgt einige Kritik, auch von PMO. Sie kritisieren die Foren als »demokratische Parodie« (ebd.: 9/Übers. S.K.), da die Öffentlichkeit nicht an Entscheidungen über wissenschaftliche und technologische Entwicklungen beteiligt wird. In Folge gibt La Metro einen Bericht bei einer Gruppe von Wissenschaftlerinnen und Wissenschaftlern aus dem Bereich der STS in Auftrag. Ziel ist es, Empfehlungen zu partizipatorischen Mechanismen zu erarbeiten. In dem Bericht werden Bürgerinnen- und Bürgerkonferenzen empfohlen, in welchen über zukünftige Nanotechnologie-Projekte in Grenoble entschieden werden soll (Callon et al. 2005). Es folgen weitere Dialogveranstaltungen wie die Bürgerinnen- und Bürgerdialoge im Jahr 2006, die von dem 
Centre de Culture Scientifique, Technique et Industrielle in Grenoble organisiert werden (Laurent 2008: 9). Die Dialogveranstaltungen, wie auch der Bericht, werden von PMO kritisiert, da sie nur zur sozialen Akzeptanz der Technologie beitrügen und grundlegende Entscheidungen für die Entwicklung von Minatec bereits getroffen worden seien (ebd.: 10). Es ist also auch diese »Art der governance«, die von $\mathrm{PMO}$ »angeprangert« wird (Int. F. XIII/Übers. S.K.).

Ähnlich wie bei der Kritik, die sich in den 1970er Jahren im Kontext der wissenschafts- und technologiekritischen Science du Peuple formiert, wird das Konzept der Partizipation abgelehnt, da die Beteiligung an Entscheidungen über wissenschaftliche und technologische Entwicklungen in diesem Konzept ausgeschlossen ist. Im Kontrast zur Kritik der 1970er Jahre, in der die Sprache der Ethik nicht auftaucht, zeigt sie sich in der Kritik an Nanotechnologie.

\subsection{Von $»\left[\right.$ E]thischen ‘ Zierereien $\aleph^{7}$}

Neu im nanotechnologischen Diskurs ist im Kontrast zum medizinischen und gentechnologischen Diskurs, dass Kritikerinnen und Kritiker Ethik-Rahmungen angreifen: »Man riecht, dass die >ethischen $<$ Zierereien [...] nicht mehr als rein formale Verschleierungen der nächsten vollendeten Tatsachen sind « (Simples Citoyens 2003: 17/Herv. i.O., Übers. S.K.). Mit »Verschleierungen von vollendeten Tatsachen « sind die Entscheidungen über wissenschaftliche und technologische Entwicklungen gemeint (ebd.). Die Kritikerinnen und Kritiker betonen, dass die Themen politisch, sozial und ökologisch sind (ebd.; vgl. Pièces et Main d'Oeuvre 2006). Laurent meint, die Aktivisten und Aktivistinnen »refuse to accept the classification of ethics as a form of expert knowledge « (Laurent 2007: 345). Im Unterschied zu den Konflikten der 1970er Jahre wird nicht nur die Rolle der Experten und Expertinnen in der Steuerung wissenschaftlicher und technologischer Entwicklungen angegriffen - auch ihr »ethisches Wissen« wird abgelehnt. Indem die Kritikerinnen und Kritiker nanotechnologische Themen als politische, soziale oder ökologische Themen verstehen, wird darüber hinaus die Definition nanotechnologischer Themen als ethische angegriffen.

Im Kontrast zu den 1970er Jahren finden sich ethische Rahmungen in einer Fülle an Dokumenten aus Forschung und Politik, auch jenseits des CCNE.

7 Thomasson 2006/Herv. i.O., Übers. S.K.. 


\section{3 »Ethics lags behind « ?}

Insbesondere nach der Forderung eines Moratoriums und nach der Publikation »Mind the gap« (Mnyusiwalla et al. 2003) aus dem Jahr 2003 scheint Ethik nicht nur ein Thema für wissenschaftliche Untersuchungen zu werden, sondern auch zu einem forschungspolitischen Thema auf europäischer, internationaler und nationaler Ebene. In der bereits zitierten Mitteilung der EU-Kommission zu einer »europäischen Strategie der Nanotechnologie « aus dem Jahr 2004 wird festgehalten, dass ein wesentlicher Aspekt bei der Entwicklung der Nanotechnologie sei, sie »sicher « und »verantwortungsbewusst « zu entwickeln und »ethische Grundsätze« einzuhalten (Kommission der europäischen Gemeinschaften 2004: 3). Zu den »grundlegenden ethischen Werten« zählen, so heißt es unter der Überschrift »Verantwortungsbewusste Entwicklung der Nanotechnologie«, die »Achtung der Menschenwürde, die Unabhängigkeit des Einzelnen, Gerechtigkeit und Wohlbefinden, die Freiheit der Forschung und das Prinzip der Verhältnismäßigkeit« (ebd.: 22). Maßgebende Bedeutung habe, so früh wie möglich »die Einbeziehung der gesellschaftlichen Dimension«, der Dialog mit der Öffentlichkeit, »da die Aufmerksamkeit auf Fragen von echtem Belang und nicht auf >Science-Fiction-Szenarios< zu lenken ist« (ebd.: 21f, 3/Herv. i.O.). ${ }^{9}$

Die Notwendigkeit der Einbeziehung von Ethik wird ebenfalls im Jahr 2004 auf einem internationalen informellen Treffen gesehen, dem »International Dialogue on Responsible Research and Development of Nanotechnology«. Der Dialog findet in Alexandria, Virginia, auf Einladung der US-amerikanischen NNI statt (Tomellini 2004). Auf dem multidisziplinären Treffen, auf dem Expertinnen und Experten aus 25 Ländern vertreten sind, darunter Frankreich, werden insbesondere »socio-economic and ethical implications « der Nanotechnologien diskutiert. Die Teilnehmenden stimmen darin überein, »to continue the process and to explore ways of encouraging broader social dialogue « (ebd.). Frankreich wird auf dem informellen Treffen durch den Conseil Général des Technologies et de l'Information (CGTI) präsentiert. Im Jahr 2004 veröffentlicht dieser zusammen mit dem Conseil

8 Mnyusiwalla et al. 2003.

$9 \mathrm{Zu}$ dieser Zeit wird im Kontext der EU-Forschungspolitik insgesamt eher von responsible development und responsible research gesprochen. Obwohl sich einige Parallelen zeigen, entwickelt sich das Konzept der responsible research and innovation in der EU-Forschungspolitik erst ab 2010 und ist explizit seit 2014 in das Horizon 2020Förderprogramm für Forschung und Innovation aufgenommen (vgl. European Commission 2012). Zur Entwicklung des Konzeptes im EU-Policy-Diskurs vgl. Richard Owen et al. (2012) und weiterführend vgl. bspw. Ulrike Felt et al. (2013). 
Général des Mines den Bericht »Les nanotechnologies: éthique et prospective industrielle« - »Nanotechnologien: Ethik und industrielle Voraussicht« (Dupuy/ Roure 2004). Sowohl der Conseil Général des Mines als auch der CGTI existieren bis 2009 und fusionieren dann im Conseil Général de l'Économie, de l'Industrie, de l'Énergie et des Technologies, der wie seine Vorgänger, dem Wirtschafts-, Finanz-, und Industrieministerium untersteht. Verfasst wird der Bericht von dem französischen Philosophen Jean-Pierre Dupuy und der französischen Ökonomin Françoise Roure. Ziel des Berichtes ist es, Grundlagen zur Evaluierung der Nanotechnologie in der französischen Politik zu schaffen, insbesondere in Bezug auf die "gesellschaftlichen und ethischen Dimensionen« (ebd.: 1). In dem Bericht wird die Notwendigkeit einer Ethik festgehalten, die: »endlos anspruchsvoller ist als die Ethik, die sich heute langsam herausbildet, um den Rhythmus der Biotechnologien zu umfassen und die möglichen Abdriftungen einzudämmen« (ebd.: 22/Übers. S.K.). Darüber hinaus werden in dem Bericht staatliche Institutionen und Gremien ermutigt, Risiken und Ethik der Nanotechnologie auf ihre Agenda zu setzen. In Bezug auf den CCNE, so heißt es in den Empfehlungen des Berichtes, soll sichergestellt werden, dass er über ausreichend Mittel verfügt, um seine Aufgabe in Bezug auf die Nanotechnologien zu erfüllen (ebd.: 59). Das nationale Ethikkomitee richtet, ebenfalls im Jahr 2004, eine Arbeitsgruppe zu Nanotechnologie ein (ebd.: 25). Auch die Einbeziehung ethischer Themen als Prävention von Konflikten wie den GMO-Konflikten, wie sie in dem Artikel »Mind the gap« formuliert werden, ist in französischen forschungspolitischen Dokumenten zu finden. Ob dies als direkte Folge des Artikels oder auch als Reaktion auf die Proteste in Grenoble zu sehen ist, ist nicht immer nachzuvollziehen, da nicht in jedem Dokument deutlich wird, auf wen oder was Bezug genommen wird. Im Jahr 2006 wird ein Bericht des Centre d'Analyse Stratégique, der staatlichen Institution für strategische Analyse, ${ }^{10}$ mit dem Titel publiziert: »Nanotechnologies: anticiper pour gérér les risques «»Nanotechnologien: Vorausschauen, um Risiken zu erfassen « (Namir 2006: 3/Übers. S.K.). Darin werden mit Bezugnahme auf die Proteste in Grenoble »des craintes éthiques et sociales « - »ethische und gesellschaftliche Befürchtungen « antizipiert (ebd./Übers. S.K.). Die Befürchtungen, so der Bericht, könnten ähnlich wie bei den Kontroversen um die GMO »ein misstrauisches Verhalten gegenüber der Wissenschaft anheizen, die für ihre Entwicklung schädlich ist« (ebd./Herv. i.O., Übers. S.K.). Vom OPECST heißt es, es handle sich um »Des craintes diffuses - »diffuse Ängste in der Gesellschaft (OPECST 2006b: 3/

10 Der Centre d'Analyse Stratégique ist beim Premierminister Frankreichs angesiedelt und heißt seit 2013 Commissariat Général à la Stratégie et à la Prospective, France Stratégie. 
Herv. i.O., Übers. S.K.). In einer öffentlichen Anhörung vom OPECST zu Nanotechnologie im Jahr 2006 wird bezugnehmend auf die Proteste um Minatec erklärt, dass in der Öffentlichkeit kein Vertrauen gegenüber den Nanotechnologien bestehe und dass eine Art »Syndrome OGM $«$ - »GMO-Syndrom « im Vorfeld verhindert werden müsse (OPECST 2006a: 6/Herv. i.O., Übers. S.K.). Es wird betont: »In jedem Fall sind die Themen ethische, kommerzielle und Themen der öffentlichen Gesundheit«(OPECST 2006b: 46/Übers. S.K.).

Auf staatlicher, forschungspolitischer Ebene wird eine Entwicklung analog zu den Diskursen um GMO befürchtet, das heißt, Kontroversen, die für die wissenschaftlichen Entwicklungen »schädlich « sein könnten. Die Lösung wird im Dialog gesehen. Der Centre d'Analyse Stratégique schlägt bspw. einen Dialog zwischen Forscherinnen und Forschern sowie Akteurinnen und Akteuren der Zivilgesellschaft vor (Namir 2006: 3). Der OPECST schlägt einen Dialog zwischen Bürger und Bürgerinnen, Politik und Wissenschaft vor. Bei der Anhörung vom OPECST kommt zudem der Vorschlag, es müssten Wissenschaftlerinnen und Wissenschaftler gefunden werden, die fähig seien, mit den irrationalen und emotionalen Sichtweisen in öffentlichen Debatten umzugehen (OPECST 2006b: 126).

In diesem Prozess zeigt sich die Tendenz, dass die Kritik an nanotechnologischer und wissenschaftlicher Entwicklung und Entscheidungsfindung, wie sie insbesondere PMO äußern, ethisiert wird: Die Kritik wird zu »ethischen und gesellschaftlichen Befürchtungen «, »Ängsten « und Emotionen transformiert, die einer öffentlichen Debatte bedürfen. Die »ethischen Befürchtungen« und »Ängste« werden auf gesellschaftlicher Seite identifiziert und werden zu etwas, das durch die Einbeziehung der Öffentlichkeit in Debatten geheilt werden könne. Dass Emotionen auf gesellschaftlicher Seite identifiziert werden, ist nicht neu. Bereits im Asilomar-Prozess, im Kontext des Diskurses um rDNA-Technologie von 1973 bis 1975, wird eine öffentliche emotionale und damit unkontrollierbare Debatte befürchtet. Im Jahr 1974 auf der Tagung »Biologie und die zukünftige Entwicklung des Menschen« wird im Kontext der IVF und der Möglichkeit des Klonens ebenfalls von Emotionen und Ängsten geprochen, die durch Gewöhnung gemindert werden können. Im Unterschied zu den 1970er Jahren sprechen sich im Nanotechnologiediskurs Kritikerinnen und Kritiker gegen die Zuschreibung von Emotionalität aus: »Nous n'avons pas peur, nous sommes en colère « - »Wir haben keine Angst, wir sind wütend «, heißt es bspw. zynisch von einem Nanokritiker aus Grenoble (Thomasson 2006/Übers. S.K.). Nicht allein ethische Rahmungen der Nanotechnologie sind umstritten. Umstritten ist auch, wie in dem Zitat deutlich wird, die kritische Öffentlichkeit als emotional zu kategorisieren. Darüber hinaus problematisieren Kritikerinnen und Kritiker die »Art der governance " selbst (Int. F. XIII/Übers. S.K.). Hier zeigt sich das spezifische Konzept der Problematisierung 
von Dean, der davon ausgeht, dass Regierungsmechanismen selbst zum Gegenstand der Problematisierung werden (Dean 1999b: 38).

Welche Antwort hat der CCNE auf diese Problematisierung? Werden politische Probleme thematisiert? Oder geht es vielmehr um »ethische und gesellschaftliche Befürchtungen«, »Ängste«, Emotionen und das Problem, dass diese für die wissenschaftlichen und technologischen Entwicklungen »schädlich« sein könnten?

\title{
5 Die Nanoethik des nationalen Ethikkomitees
}

\begin{abstract}
»The difficulty is making uncertainty comprehensible without falling into disaster-scenario >grey goo< descriptions or at the other extreme, into scientism at its most careless or traditional. Perhaps nanomaterials and new manufactured nanosystems and their true purpose should be the subject of public debate before they are developed, and risk and biodegradability be included among the major ethical issues for discussion« (CCNE 2007: 12/Herv. i.O.).
\end{abstract}

Eine Schwierigkeit, die der CCNE in seiner avis zu Nanotechnologie aus dem Jahr 2007 sieht, ist es mit Unsicherheit umzugehen, ohne in ein »desaströses >Grey Goo-Szenario « zu verfallen. Das Grey Goo-Szenario bzw. der Grey Goo-Effekt geht auf Drexler zurück und kommt insbesondere durch Prinz Charles in den Nanotechnologiediskurs. Die Lösung sieht der CCNE in einer öffentlichen Debatte, in der das »echte Ziel« der Nanomaterialien und -systeme noch vor ihrer Entwicklung thematisiert wird und in der zu ethischen Themen insbesondere Risiken zählen.

\section{1 "Training in ethics ${ }^{11}$}

Die öffentliche Debatte, in der die »echten Ziele« besprochen werden sollen, präzisiert der CCNE an anderer Stelle wie folgt:

»Science opens up new vistas, broadens the concept of analysis, a process which presupposes that such innovation moves into a public forum accessible to everyone. This new culture of exchange with public opinion would require that pertinent training in ethics be on offer for researchers, engineers and economic decision-makers because the paradox is that democra- 
cies may be more sensitive to consumer arguments than to the ethics of responsible action and that the discussion on GMOs has locked both hard-line scientists and dogmatic opponents into a stalemate (CCNE 2007: 13).

»Ethisches Training« erscheint als Lösung, um eine Pattsituation wie in den Diskussionen um GMO zu verhindern. Eine Pattsituation oder Kontroversen wie sie auch in den Augen des Centre d'Analyse Stratégique verhindert werden müssen, um wissenschaftliche und technologische Entwicklungen nicht zu schädigen (Namir 2006: 3).

Neu ist das »ethische Training «. Im Kontext des lebenswissenschaftlichen Diskurses Ende der 1970er Jahre wird noch von der Notwendigkeit der Bildung und Information der Öffentlichkeit gesprochen, um mit der Wissenschaft in Dialog zu treten. Auch im Kontext des CCNE und seiner Journées annuelles d'éthique zeigte sich, dass die Öffentlichkeit und insbesondere die Schülerinnen und Schüler das »richtige Sprechen«, die richtige ethische Reflexion lernen und üben müssen. Nun wird aber nicht mehr das Wissen oder die Ethik der Öffentlichkeit in irgendeiner Form als defizitär wahrgenommen; es bedarf eines »ethischen Trainings « nicht nur der Forscher und Forscherinnen, sondern auch der Ingenieure und Ingenieurinnen sowie der ökonomischen Entscheidungsträger und -trägerinnen, um mit den »questions, expectations and fears« (CCNE 2007: 15) umzugehen. Ängste oder Emotionen scheinen auf Seite der Öffentlichkeit zu bestehen, denen mit dem entsprechenden »ethischen Training « begegnet werden könne. Politische Themen, wie sie von den Nanotechnologiekritikern und -kritikerinnen formuliert werden, scheinen keinen Raum zu haben. Bereits zu Beginn der 1980er Jahre wurde im Zuge der Forschungspolitik Mitterrands insbesondere den Wissenschaftlern und Wissenschaftlerinnen, konzipiert als Bürger und Bürgerinnen, eine ethische Kompetenz zugeschrieben. Mit der Zunahme (befürchteter) Konflikte scheint sich eine quantitative und qualitative Zunahme an Ethik-Rahmungen zu vollziehen. Ethik scheint sich nicht nur auf weitere Themen, sondern auch auf weitere Akteurinnen und Akteure auszudehnen, sowohl im Hinblick auf die Herausbildung weiterer EthikExpertinnen und Ethik-Experten - die Forscherinnen und Forscher, Ingenieurinnen und Ingenieure sowie Entscheidungsträgerinnen und -träger, als auch im Hinblick auf die Einbeziehung der Öffentlichkeit. Aber was beinhaltet diese EthikRahmung? 


\subsection{Mehr Forschung und mehr Ethik!}

Im Kontext bioethischer Themen wird das Infragestellen der technologischen Entwicklung ausgeschlossen - ähnlich auch im Kontext nanotechnologischer Themen. Ein Mitglied des CCNE sagt bei einer Anhörung vom OPECST: »The ethical reflection that we conduct especially within the CCNE isn't aimed at all at slowing down scientific progress, but to call for more science, more research « (OPECST 2006b: 52). In seiner avis zu Nanotechnologie sieht der CCNE eine »foremost ethical recommendation « in der Förderung der Grundlagenforschung. Seine Empfehlung leitet er aus dem Mangel an Publikationen und Informationen aus dem Bereich der Grundlagenforschung ab, insbesondere im Hinblick auf die mögliche Toxizität von Nanomaterialien. Es sei bspw. nicht geklärt, ob Nanomaterialien für Embryonen oder Föten toxisch sein können. »Should then women capable of producing offspring not be allowed to enter manufacturing centres or research laboratories working on nanomaterials? « (CCNE 2007: 10). Der CCNE empfiehlt konkret: »More support is urgently required for the development of fundamental research on nanosciences, without prejudice to the freedom of research. Ethical aspects must be evaluated in projects to be financed by national and European organisations and private foundations « (ebd.: 13). Es wird betont, dass Ethik nicht bedeutet, Forschung zu behindern, auch nicht durch die Evaluation »ethischer Aspekte«, denn es heißt: »In other words, the ethical attitude to nanosciences and nanotechnologies is not to stand in the way of science but on the contrary to ask for more science, more research, more reflection, more soulsearching covering research, transfer, innovation and industrial applications« (ebd.: 11). Die Nanoethik, die Ethik in der Nanowissenschaft, umfasst also nicht nur ein größeres Spektrum an Akteurinnen und Akteuren; die Reflexion umfasst auch einen größeren Themenpool, wie Innovation und industrielle Anwendungen. Sie bezieht sich nicht mehr allein auf Fragen, die aus dem wissenschaftlichen Fortschritt entstehen. In Kapitel VI habe ich die These aufgestellt, dass sich die Ausdehnung des Aufgabengebietes des CCNE von »ethischen Problemen« hin zu »ethischen und gesellschaftlichen Problemen « zeigt, etwa in der avis zu Zellspende und der Frage ihrer Kommerzialisierung. Dies bedeutet, dass die Rahmung »gesellschaftliche Probleme« die »ethischen Dimensionen des Marktes« und mit ihr die Entwicklung der Innovation beinhalten. Diese Vermutung scheint sich auch hier zu bestätigen, sie muss allerdings um die »industriellen Anwendungen « ergänzt werden. 


\section{3 »Echte « ethische Ziele ${ }^{12}$}

Die Fokussierung der Diskussion auf den »true purpose« der Nanotechnologie für die öffentliche Debatte in dem eingangs erwähnten Zitat (CCNE 2007: 12) erinnert an die Interviewaussage zur Entstehung des CCNE. Dort heißt es: »die Leute sollen ein bisschen besser verstehen, wo wirklich die Fragen sind, um die Reflexion auf die Punkte zu konzentrieren, die wirklich ein Problem machen und um nicht auf zweitrangige Fragen auszuweichen « (Int. F IX, Übers. S.K.). Die Themen und Probleme sollen in gewisser Hinsicht für die öffentliche Debatte ausgewählt werden, so das Ergebnis im Kontext der Untersuchung von Ethik-Rahmungen im biotechnologischen und -medizinischen Kontext. Außerdem soll die Diskussion in gemäßigter Art vorgelebt werden. Eine Mäßigung zeigt sich auch in der avis zu Nanotechnologie - es soll ein Desaster-Szenario verhindert werden, ebenso wie eine Pattsituation wie die im Kontext der GMO-Diskussion. Es wird an vielen Textstellen verdeutlicht, dass es sich bei Nanotechnologie und Nanowissenschaft nicht um einen »radically new approach " handle und die »novelty residing in its transdisciplinary nature (CCNE 2007: 3). Auch die Manipulation von Leben sei nicht revolutionär: »But manipulating living and inanimate matter on that scale is not in itself a revolution. For example, the DNA molecule, which only measures a few nanometres, has been manipulated with a great degree of precision for over 40 years by techniques which are in no way related to nanotechnology« (ebd.: 2). Es wird festgehalten $»$ we are not confronted apparently with a real scientific revolution « (ebd.: 6). Im Kontext der Nanotechnologie handle es sich um »a continuation, with new instruments, of the engineer's traditional dream « (ebd.: 4). Auch im Kontext der Konstruktion molekularer Objekte, die fähig sind, sich selbst zu replizieren, heißt es »synthetic biology raises the same issues « (ebd.). Und insgesamt wird verlautet: »The ethical issues in this respect are those generally raised by the development of technologies « (ebd.: 6).

Mit der Formulierung, nanotechnologische Entwicklungen und Innovationen seien nichts Neues, Revolutionäres, keine Entwicklungen, die neue »ethische Themen « mit sich bringen, wird m.E. suggeriert, dass alles in seinen gewohnten, traditionellen Bahnen läuft. Neue ethische Probleme, wie die, die auf der USamerikanischen Foresight-Konferenz angesprochen wurden, scheinen darum gar nicht thematisiert werden zu müssen oder können. Diese Probleme gingen mit der Überlegung einer planetarischen Kontrolle der Nanotechnologie einher, die auf einem planetarischen Konsens beruht. Dieser Lösungsvorschlag taucht nicht auf. Im 
Kontrast zu der Bedeutung von Ethik des CCNE hieße dieser Vorschlag, die Entwicklungen der Nanotechnologie ggf. in Frage zu stellen.

\subsection{Die Bedeutung von Nanoethik - Alles gesund?}

Ein weiteres Charakteristikum der institutionellen ethischen Rahmung durch den CCNE ist im Sinne Memmis das angeleitete Sprechen und die Konzentration auf die Bioindividuation (vgl. Memmi 2003a: 292f; 2005). Die Frage, die sich im Hinblick auf die Ausdehnung von Ethik-Rahmungen auf weitere Themen ergab, war, inwiefern Nanotechnologie als individuelles und gesundheitsrelevantes Thema verstanden wird.

Die avis des CCNE hat den Titel »Ethical Issues raised by Nanosciences, Nanotechnologies, and Health« (CCNE 2007). Nicht nur der Titel, auch das folgende Zitat zeigt, dass der CCNE »Ethik« und »Gesundheit« verknüpft, egal ob die Technologie mit Medizin zu tun hat oder nicht: »Ethics covers here a complex multidisciplinary array of issues ranging from the possible effects on health of nanoparticles used for non medical purposes through to the benefits and risks of nanomedicine and the human sciences « (ebd.: 7). Zudem wird problematisiert, dass Nanomaterialien für Embryonen oder Föten toxisch sein können, auch wird die Frage aufgeworfen, inwiefern gebärfähigen Frauen erlaubt werden soll, in Laboren zu arbeiten, die zu Nanomaterialien forschen (ebd.: 10). Zwar wird Nanotechnologie als gesundheitsrelevantes Thema verstanden, aber inwiefern handelt es sich um ein individuelles Thema? Gesundheit und Ethik werden insofern als individuelle Angelegenheit gerahmt, als empfohlen wird, dass Konsumenten und Konsumentinnen durch die Etikettierung von Nanoprodukten selbst entscheiden sollen, ob sie ein solches Produkt kaufen: »so that consumers can refuse to use them if they so wish « (ebd.: 15). Ob die Konsumentinnen und Konsumenten möglicherweise durch den Kauf von Nanoprodukten ihre Gesundheit schädigen, wird zur individuellen Abschätzung - die Frage wird an das Individuum delegiert. Im Sinne des CCNE ist Ethik nicht ein Infragestellen der ökonomischen Entwicklung, diese wird nicht diskutiert, sondern m.E. ein Stimulus für individuelle Entscheidungsfindung in Bezug auf die eigene Gesundheit. Meine These aus Kapitel VI lautet, dass sich der Möglichkeitsraum der Bioindividuation insofern auszudehnen scheint, als nicht allein die Verbesserung der eigenen Gesundheit evaluiert wird, sondern auch die des Marktes. Diese Tendenz zeigte sich in der avis zur kommerziellen Verwendung von Zellspenden aus dem Jahr 2006. Eine der Empfehlungen ist, dass ZellSpenderinnen und Zell-Spender frei wählen können sollen, ob ihre Spende zu kommerziellen oder nicht-kommerziellen Zwecken verwendet werden soll. Sie 
können zu »fully-fledged actors in the regulation of the ethical dimensions of the market « werden (CCNE 2006b: 26). Der Markt wird nach dieser Aussage durch die Entscheidung des Spenders oder der Spenderin reguliert, nicht durch staatliche Regulierungsinstanzen. Vor diesem Hintergrund kann die Frage, ob man Nanoprodukte konsumiert, nicht nur im Kontext des Managements der eigenen Gesundheit verstanden werden, sondern auch als Aspekt der »ethischen « Marktregulierung.

Darüber hinaus zeigt sich aber auch ein diffuses, offenes Verständnis von Ethik, das wie im Kontext biotechnologischer und -medizinischer Themen »evolutionär, reversible und prekär « ist. In einem Interview wird mir auf die Frage, was Ethik bedeutet, geantwortet, Ethik heiße »ein endloses Hinterfragen dessen, was wir als Werte ansehen « (Int. F X). Und es wird erklärt: »das Prinzip selbst der ethischen Reflexion bedeutet nicht zu sagen, was gut und was schlecht ist, nicht zu sagen, was erlaubt und was autorisiert ist « (Int. F X/Übers. S.K.). In anderen Worten, das Prinzip ethischer Reflexion sowohl im Kontext nanotechnologischer als auch biotechnologischer und -medizinischer Themen ist das Prinzip der endlosen Deliberation. Allerdings werden nun nicht nur die Entwicklung von Forschung sondern auch industrielle Anwendungen nicht infrage gestellt. Dass Ethik nicht substantiell mit bestimmten Themen verknüpft ist, zeigt ein weiterer Interviewauszug. Der Interviewpartner oder die Interviewpartnerin antwortet auf die Frage, wie es dazu kommt, dass der CCNE sich mit Nanotechnologie beschäftigt:

»Ich weiß nicht, ich erinnere mich nicht [...] sicher ist, dass der CCNE nicht bei den Debatten zu den GMO [im Agrarbereich, S.K.] präsent war. [...] Vielleicht hat das Gefühl, bei der Debatte über die GMO abwesend gewesen zu sein, den CCNE dazu ermutigt, sich selbst zu beauftragen « Und weiter heißt es: »Bioethik ist sehr weitgehend [und bedeutet, S.K.] sich für die technologischen Anwendungen zu interessieren in Bezug auf alles, was das Lebende angeht « (Int. F XI/Übers. S.K.).

Es scheint nicht die Frage wichtig zu sein, welches Thema im CCNE besprochen wird, sondern sich an der Debatte zu beteiligen. Es wird festgestellt, dass Bioethik sehr weitgehend ist. Sie beinhalte, sich für das zu interessieren, was das Lebende betrifft. Aber welche Technologie betrifft nicht das Lebende?

Dass Ethik flexibel ist betont auch Kathrin Braun in ihrer Untersuchung zur Ethik-Kommission Sichere Energieversorgung in Deutschland, die sich mit dem Ausstieg aus der Atomenergie beschäftigt. Gerade das diffuse Verständnis von Ethik ermögliche die Ausdehnung von Ethik auf neue Themenfelder wie das der Atomenergie, so Braun (2013: 102). Dieses diffuse Verständnis ist m.E. auch ein wesentliches Merkmal, das die Ausdehnung von Ethik-Rahmungen auf nanotechnologische Themen ermöglicht. Ein Unterschied zur Ethik-Kommission Sichere 
Energieversorgung ist m.E. darin zu sehen, dass sich dieselbe Institution mit nanotechnologischen Themen beschäftigt, die sich sonst mit medizinischen und biotechnologischen Themen beschäftigt. Bei beiden Themen besteht eine Verknüpfung mit individueller Gesundheit und damit einhergehend zur Bioindividuation. Anders formuliert: Damit Nanotechnologie zu einem ethischen Thema des CCNE wird, so meine Vermutung, muss sie mit Gesundheit verknüpft werden. Würde Atomtechnologie im CCNE besprochen, müsste sie als individuelles gesundheitliches Thema verstanden werden. Im Kontext der Ethikkommission zur Atomtechnologie in Deutschland liegt diese Verknüpfung nicht vor. Würde für die Nanotechnologie eine neue Ethikinstitution eingerichtet, wäre sie nicht unbedingt mit Gesundheit verknüpft. Die Ausdehnung auf weitere Themenfelder im Kontext des CCNE ist m.E. möglich, weil diese Ethik spezifisch mit individueller Gesundheit im Sinne der Bioindividuation von Memmi verknüpft ist.

\section{Ethische Ausblicke}

Obwohl es umstritten ist, ob es sich bei nanotechnologischen Themen um ethische oder politische handelt, scheint sich zumindest auf staatlicher Ebene deren Rahmung als ethisch und gesundheitlich durchzusetzen. Eine Entwicklung, die jenseits des Untersuchungszeitraumes dieser Studie liegt, ist an dieser Stelle noch erwähnenswert: Im Jahr 2009 werden in 17 Städten öffentliche Debatten abgehalten, organisiert von der Commission Nationale du Débat Public - der Nationalen Kommission für Öffentliche Debatten, in welchen »ethische Fragen« und »Problematiken « thematisiert werden sollten (Commission nationale du débat public 2009: 4f/Übers. S.K.). Insgesamt zeigt sich hier die Tendenz, ethische Debatten im öffentlichen Raum im Kontext nanotechnologischer Themen zu stimulieren. De facto wurden diese öffentlichen Debatten von Nanokritikerinnen und -kritikern zum Teil gestört oder fanden unter Ausschluss der Öffentlichkeit statt. Diese »Art der governance« bleibt also umstritten (Int. F XIII/Übers. S.K.).

Die Tendenz der weiteren Ausdehnung von Ethik-Rahmungen zeigt sich allerdings nicht nur im im Hinblick auf die Nanoethik, sondern auch in Bezug auf die Bioethik. Seit dem Bioethikgesetz aus dem Jahr 2004 ist die Einrichtung von »espaces de reflexion éthique - »ethischen Reflexionsräumen« an den CHU auf regionaler und interregionaler Ebene vorgesehen (Loi n 2004-800 2004: Art. L. 1412-6). Bisher bestehen einige dieser espaces wie der Espace Éthique Méditerranéen mit Sitz in Marseille, der Espace Éthique Azuréen in Nizza oder der Espace de réflexion éthique de la région Ile-de-France mit Sitz in Paris. Die espaces éthiques sollen an der Organisation öffentlicher Debatten mitwirken und 
die Information und Beratung der Bürgerinnen und Bürger in »ethischen Fragen « aus dem Gesundheitsbereich fördern (ebd./Übers. S.K.). Diese espaces stehen in Verbindung mit dem CCNE. Dieser hat laut dem Bioethikgesetz von 2004 die Aufgabe, seine Meinung zur Funktionsweise und Zusammensetzung der jeweiligen espaces dem Gesundheitsminister oder der Gesundheitsministerin zu übermitteln (ebd.). Seit dem Dekret von 2005 ist der CCNE angehalten, in Zusammenarbeit mit den espaces de reflexion éthique regionale Treffen zur ethischen Reflexion zu organisieren (Décret $\mathrm{n}^{\circ}$ 2005-390 2005). Wie mir in einem Interview im Jahr 2010 mitgeteilt wird, haben sich bisher kaum espace éthiques herausgebildet (Int. F X); eine Zusammenarbeit in Form regionaler Treffen zur ethischen Reflexion findet im Untersuchungszeitraum der vorliegenden Studie nicht statt. Laut Interviewauskunft bietet sich mit den espaces die Möglichkeit, eine breitere Öffentlichkeit zu ethischen Themen zu erreichen. Darüber hinaus bestehe die Möglichkeit, durch die Beziehung zwischen dem CCNE und den espaces die journée annuelle auf regionaler Ebene stattfinden zu lassen, was bisher kaum der Fall ist (Int. F IV).

Mit dem Bioethikgesetz von 2011 ist vorgesehen, dass der CCNE so genannte États généraux de la bioéthique organisiert. Noch nicht vom CCNE organisiert finden solche États généraux de la bioéthique bereits im Jahr 2009 statt. Sie werden damals per Dekret durch den Staatspräsidenten Nikolas Sarkozy (2007-2012) im Vorfeld der Reform des Bioethikgesetzes von 2004 veranlasst (Décret $\mathrm{n}^{\circ} 2008$ 1236 2008). Staatlich initiiert finden États généraux im Jahr 1998 zum ersten Mal zum Thema Gesundheit statt, ${ }^{13}$ und zwar die États généraux de la Santé (Chavot/Masseran 2003e: 379). Im Kontext der Bioethik wurden die États généraux im Jahr 2009 durch Sarkozy in Zusammenarbeit mit der Ministerin für Gesundheit und Sport Roselyne Bachelot-Narquin (2007-2010) per Dekret angeordnet, um die Entscheidungen der Regierung und der Volksvertreterinnen und -vertreter im Kontext der Revision des Bioethikgesetzes von 2004 durch eine Reflexion der Bürgerinnen und Bürger zu untermauern (Bachelot-Narquin 2009; Décret $n^{\circ}$ 2008-1236 2008). Die Revision des Bioethikgesetzes ist alle fünf bis sechs Jahre vorgesehen, »afin de prendre en compte les progrès scientifiques, les évolutions de la société et des mentalités « - »um den wissenschaftlichen Fortschritten, den Entwicklungen der Gesellschaft und den Denkweisen Rechnung zu tragen«, so Roselyne Bachelot-Narquin (Bachelot-Narquin 2009/Übers. S.K.; vgl. Loi $n^{\circ}$ 2011-814 2011: Art. 47 I.). Nach dem Motto »Informer, faire comprendre et

13 In Kapitel VI wird bereits einmal von États généraux gesprochen, im Kontext des nationalen Kolloquiums zu Forschung und Technologie nach dem Regierungswechsel von 1981. Allerdings wird das Kolloquium nur als États généraux verstanden, es war nicht explizit als solche konzipiert. 
faire réfléchir « - »Informieren, begreiflich machen und zu denken geben « (États géneraux de la bioéthique 2009/Übers. S.K.) werden u.a. in drei französischen Städten Bürgerinnen- und Bürgerforen und eine Abschlussveranstaltung in Paris abgehalten. Auch im Kontext dieser États généraux scheint sich eine reversible Auffassung von Ethik abzuzeichnen, die nicht darin besteht, wissenschaftliche und technologische Entwicklungen in Frage zu stellen. Zudem zeichnet sich auch hier ab, dass die Öffentlichkeit defizitär konstruiert ist. Die Ethik-Rahmung scheint sich nicht nur auf weitere Themen und Akteurinnen und Akteure, sondern auch auf weitere Institutionen und Praktiken auszudehnen - sie ist sehr flexibel.

\section{ZUSAMMENFASSUNG}

Die Nanoethik erscheint wie die Bioethik als Antwort auf die Problematisierung von Regierungsmechanismen. Die Frage, ob es sich im Kontext nanotechnologischer Entwicklungen um ethische oder politische Probleme handelt, ist umstritten. Dementsprechend ist auch die Lösung weiterhin umstritten. Gemeinsam ist dem Diskurs um Nanotechnologie mit den zuvor untersuchten Diskursen, dass ethische Rahmungen im Kontext zweier gegenläufiger Tendenzen erscheinen - einerseits im Infragestellen und andererseits in der Stabilisierung wissenschaftlicher Selbststeuerung. Sie erscheinen im Kontext der Antizipation bzw. Ethisierung technologie- und wissenschaftskritischer Meinungen und in der Kritik an wissenschaftlicher Entscheidungsfindung. Während im lebenswissenschaftlichen Diskurs »ethische und soziale Zwänge« auf gesellschaftlicher Seite gesehen werden, wird im Kontext der Nanotechnologie von »Syndromen, Ängsten und Emotionen« gesprochen. Die Art und Weise, wie Kritik oder Skepsis an wissenschaftlichen Entwicklungen wahrgenommen wird, zeigt eine Tendenz zur Pathologisierung (Syndrom) (vgl. Könninger 2013). Geheilt werden können die »Ängste« durch das »richtige Sprechen « in ethischen Debatten, und zwar durch den Dialog mit Ethikexpertinnen und -experten, die ausgebildet werden sollen. Der CCNE empfiehlt, dass weitere Akteurinnen und Akteure »ethisch« ausgebildet werden und eine gesteigerte Einbeziehung der Öffentlichkeit in Debatten stattfinden soll. Damit präsentiert er vielmehr eine Lösung zur Lösung von Konflikten dar. In Bezug auf den CCNE scheinen sowohl die Bioethik als auch die Nanoethik bestimmte Sprecherinnenund Sprecherpositionen auszuschließen, nämlich solche, die technologische und wissenschaftliche Entwicklungen anfechten und damit die Frage ansprechen, in welcher Gesellschaft wir leben wollen. Ansonsten ist keine Position per se falsch oder richtig. Das magistère bioéthique hat sich insofern auf nanotechnologische Themen ausgedehnt, als Nanoethik ebenso auf individuelle Entscheidungsfindung 
in Bezug auf die eigene Gesundheit fokussiert, indem Verantwortung auf das Individuum übertragen wird. Eine themen-flexible Reflexion scheint aufgrund des diffusen Verständnisses von Ethik möglich. Weder Normen noch Wahrheit, nicht das Gute oder Schlechte werden im Ethik-Rahmen produziert. Es gibt nur ein Prinzip: das »richtige Sprechen « unter der Prämisse der Unbestreitbarkeit von Forschung und Entwicklung. Ethik in Form des CCNE bietet eher einen Rahmen dafür, Diskurse anzuleiten, zu produzieren oder stimulieren und zu organisieren, als eine normative Handlungsorientierung. Sie scheint weniger substantiell, sondern vielmehr ein Modus der Bearbeitung politischer Konflikte. Bio- und nanotechnologische Diskurse teilen sich ein Charakteristikum: »Alles muss in diesem Rahmen möglich sein, nur eines nicht: Nein zu sagen« (Braun et al. 2009: 46). 


\section{Resümee}

»[D]enn, wie Sie wissen, bin ich wie ein Krebs, ich bewege mich seitwärts« (Foucault 2006 (1979): 116).

Als Krebsgang beschreibt Michel Foucault in einer seiner Vorlesungen am Collège de France im Jahr 1979 sein Vorgehen. In meiner Untersuchung habe ich auf die Analysebewegung Foucaults, die genealogische Herangehensweise, zurückgegriffen. Foucaults Bild des Krebses einmal übernommen, war meine Analysebewegung nicht seitwärts, sondern folgte einer absteigenden und einer aufsteigenden Spur. Absteigend war sie in dem Sinn, dass die Sprache der Ethik genealogisch zurückverfolgt wurde; aufsteigend in dem Sinn, dass nicht auf ein Großnarrativ fokussiert wurde, sondern in Abhängigkeit der Problematisierungen die Regierungstechniken herausgearbeitet wurden. Ziel war es, am Beispiel des CCNE, seiner Entstehungsgeschichte und seiner Institutionalisierung die Entwicklung zu untersuchen, wie Ethik in der Politik zu einem selbstverständlichen Rahmen wurde, welche Problematisierungen und Lösungsvorschläge sichtbar und welche unsichtbar wurden und inwiefern sich am Beispiel der Nanotechnologie diese Form der Steuerung von biomedizinischen und biotechnologischen auf weitere Themen ausdehnen kann.

Im medizinischen Diskurs erscheinen Ethik-Rahumgen insbesondere als Abkehr von einer als überholt und als paternalistisch empfundenen medizinischkonservativen Moralvorstellung. Diese Moralvorstellung wird von medizinkritischen Gruppen angegriffen und kommt zudem im Konflikt um den Schwangerschaftsabbruch zum Tragen. Darüber hinaus steht diese Moralvorstellung dem Konzept der medizinischen Forschung der Neo-Kliniker und -Klinikerinnen entgegen, das in der Nachkriegszeit aufkommt und sich in der Konzeption des INSERM wiederfindet, dem Institut, an dem in Frankreich eines der ersten Ethikkomitees eingerichtet wird. Hier zeigte sich, dass sich die Problematisierungen verschiedener Strömungen in der Kritik an einer Moralvorstellung zu einem bestimmten Zeitpunkt überschneiden. Mit dieser medizinisch-konservativen Moralvorstellung, die insbesondere mit der ärztlichen Standesvertretung, dem Ordre des médecins, ver- 
knüpft ist, scheinen bestimmte Forschungen wie Humanexperimente nicht möglich, da der Ordre sie in den Kontext der NS-Medizin stellt. Mit der Einrichtung des Ethikkomitees wird eine ethische Neubewertung medizinischer Forschung jenseits einer standespolitischen moralischen Bewertung, den Verflechtungen im Vichy-Regime und den Verknüpfungen zur NS-Medizin ermöglicht. Die Selbststeuerung medizinischer Forschung bekommt mit der Einrichtung des Ethikkomitees ein antipaternalistisches Antlitz insofern, als das Verhältnis zwischen forschender Medizinerin oder forschendem Mediziner und Patientin oder Patient entzerrt wird, hin zu einem ausgedehnten Verfahren medizinischer Selbstkontrolle durch eine begleitende Expertise. Es verschiebt sich nicht nur die medizinische Forschung vom Krankenbett ins Labor, sondern auch die Ethik: Nicht mehr allein die Verantwortung des Mediziners oder der Medizinerin ist ausschlaggebend für das Durchführen eines Versuchs, sondern nun auch eine übergeordnete Instanz in Form des Ethikkomitees des INSERM.

Im Kontext der Gentechnologie, so ein Ergebnis, sind die Diskurse länderübergreifend zwischen den USA und Frankreich verbunden. Es wurde deutlich, dass es Überschneidungen gibt, aber auch feine Unterschiede in den jeweiligen Rahmungen und Lösungen. Der Ethik-Rahmen erscheint in Form der Kommission der DGRST, der Vorgängerin des Forschungsministeriums, als Antwort auf einen politisierten Risikobegriff. Dieser Risikobegriff kommt durch Gentechnologiekritiker und -kritikerinnen und bestärkt durch die Kritik an Atomtechnologie auf. Während im US-amerikanischen Diskurs um Gentechnologie »ethische Probleme« auf gesellschaftlicher Seite identifiziert werden und die technische Risiko-Rahmung als Lösung erscheint, präsentiert sich in Frankreich gerade Ethik in Form eines übergeordneten Rahmens für eine technische Risikoabschätzung. Mit der Ethikkommission der DGRST dehnt sich das Verfahren der Selbststeuerung von Wissenschafts- und Technikentwicklungen auf konzeptioneller Ebene aus - sie wird nie einberufen. Sie kann somit als eine Art ausgedehnte präventive Selbstdisziplinierung für Forscherinnen und Forscher verstanden werden. Mit der Einrichtung der Ethikkommission bzw. der Commission de Classement findet eine offenere Diskussion über einzelne Forschungsprojekte statt; öffentliche Diskussionen über Forschungs- oder gesellschaftspolitische Ziele haben jedoch keinen Raum.

Während im US-amerikanischen Diskurs bereits im Asilomar-Prozess in den Jahren 1973 bis 1975 »ethische Probleme« auf gesellschaftlicher Seite identifiziert werden, geschieht dies in Frankreich erst deutlich 1979 in dem Bericht »Lebenswissenschaften und Gesellschaft« in Form von »ethischen Zwängen«. Bereits zuvor werden Probleme auf gesellschaftlicher Seite identifiziert, allerdings werden diese als moralisch oder ethisch gerahmt, wie auf der Tagung »Biologie und die zukünftige Entwicklung des Menschen« im Jahr 1974. Das räumlich trennende 
Denken vom Raum des Labors, in dem Wissenschaft politisch und normativ neutral ist, und dem gesellschaftlichen Raum, in dem sie mit Werten zusammentrifft, besteht also zeitgleich. Allerdings erscheint das Konzept Moral auf der Tagung 1974 nicht nur als Problem, sondern auch als Lösung: als eine bestimmte Moral, die die Objektivität der Wissenschaft nicht in Frage stellt. Diese Moral ist eingebettet in ein Konzept von Verantwortung und Reflexion in Form einer unabhängigen Vereinigung für wissenschaftliche Verantwortung, dem MURS. Dieser kann als Öffnung der Selbststeuerung wissenschaftlicher und technologischer Entwicklungen verstanden werden, da eine weniger strikte Trennung von gesellschaftlichen und wissenschaftlichen Akteurinnen und Akteuren besteht - auch Interessierte können sich an den Reflexionen beteiligen.

Nicht allein die Untersuchung von Strömungen, sondern explizit auch die Rahmungen von Akteuren und Akteurinnen, war insofern erhellend, als sichtbar gemacht werden konnte, dass zeitgleich wie bspw. in der Politik unterschiedliche Vorstellungen über die Steuerung wissenschaftlicher und technologischer Entwicklungen bestehen: Im Jahr 1974 werden Ethikinstitutionen, die als Sicherung der Selbststeuerung wissenschaftlicher und technologischer Entwicklungen erscheinen, bei staatlichen forschungspolitischen Institutionen eingerichtet, dem INSERM und der DGRST. Gleichzeitig wird diese Selbststeuerung von staatspolitischer Seite angezweifelt. Während die Gesundheitsministerin Simone Veil, die das Ethikkomitee des INSERM initiiert, medizinische Forschung in einer linearen Entwicklung zu Fortschritt, nationalem Prestige und Humanität sieht, äußert sich Staatspräsident Giscard d'Estaing im selben Jahr anders: Auf der Tagung »Biologie und die zukünftige Entwicklung des Menschen« zweifelt er gerade diese Linearität im Kontext der Atomtechnologie und des Atombombenabwurfs auf Hiroshima an. Zwar wird dieser Zweifel kurze Zeit später im Kontext des Berichtes »Lebenswissenschaften und Gesellschaft« revidiert. Dennoch wird sichtbar, dass in der Politik selbst zum gleichen Zeitpunkt unterschiedliche Vorstellungen über die Selbststeuerung wissenschaftlicher und technologischer Entwicklung bestanden: Während die Ethik-Rahmung in Form des Komitees beim INSERM und der Kommission der DGRST in Erscheinung tritt, um die Frage der Steuerung wissenschaftlicher und medizinischer Entwicklungen innerhalb der jeweiligen Professionen zu halten und um vor öffentlicher Kritik zu schützen, erscheint das Konzept der wissenschaftlichen Verantwortung des MURS als Antwort auf eine öffentliche und disziplinenübergreifende Problematisierung dieser Selbststeuerung.

Mit dem Konzept der Problematisierung konnte herausgearbeitet werden, dass zeitnah zwar Techniken des governing at a distance (Rose und Miller) erscheinen, diese jedoch auf unterschiedliche Probleme antworten. Während im Kontext der Forschungspolitik ab 1981 das Konzept des Dialogs erscheint, um Bürger und 
Bürgerinnen aktiv am Innovationsprozess zu beteiligen mit dem Ziel, sowohl die ökonomische Krise zu überwinden als auch wissenschaftskritische Bewegungen einzudämmen, zeigt sich diese Zielsetzung beim CCNE nicht. Die Probleme, auf die der CCNE antwortet, sind die möglichen moralischen Konflikte um die IVF sowie Unsicherheiten, die aus neuen wissenschaftlichen und technologischen Entwicklungen entstehen. Dass wirtschaftliche Innovation im Kontext des CCNE nicht zentral ist, spiegelt sich m.E. in dessen Abwehr der Kommerzialisierung körperlicher Elemente wider. Dieser Aspekt scheint sich allerdings zu ändern, insofern als der Möglichkeitsraum eröffnet wird, darüber nachzudenken, durch Zell-Spende oder durch den Konsum von Nano-Produkten Innovation und Markt »ethisch « zu beeinflussen.

Überschneidungen dieser Techniken des governing at a distance zeigen sich darin, wie die Öffentlichkeit konstruiert wird. Mit der zunehmenden Öffnung der Steuerung wissenschaftlicher und technologischer Entwicklungen, insbesondere im Kontext einer Demokratisierung der Forschungspolitik durch die sozialistische Regierung sowie in den Konzepten des Dialogs und der Reflexion, werden Öffentlichkeit oder Bürgerinnen und Bürger als informations-defizitär konstruiert. Im Kontext des CCNE zeigt sich nicht allein das Defizit der Information, sondern zudem die mangelnde Fähigkeit zur »richtigen« ethischen Reflexion. Ein Merkmal dieser Reflexion und des Dialogs ist, dass wissenschaftliche und technologische Entwicklungen nicht in Frage gestellt werden sollen. Diese Ausklammerung des Infragestellens wissenschaftlicher und technologischer Entwicklungen ist bereits ab Ende der 1970er Jahre zu erkennen, als das Konzept des Dialogs in dem Bericht »Lebenswissenschaften und Gesellschaft« vorgestellt wird.

Sowohl dem MURS als auch allen untersuchten Ethikinstitutionen ist gemeinsam, dass sie auf Probleme antworten, die von Wissenschaftlern und Wissenschaftlerinnen oder forschungspolitischen Akteuren und Akteurinnen als moralische oder ethische Probleme auf gesellschaftlicher Seite identifiziert werden. Hierzu zählen auch Emotionen oder Irrationalitäten. Sehr deutlich wird dies im Nanotechnologiediskurs. Hier sind es Ängste und Emotionen auf gesellschaftlicher Seite und das mögliche GMO-Syndrom, das durch die Einbeziehung in ethische Debatten verhindert werden soll. Interessant ist m.E., dass diese Probleme von forschungspolitischen Akteurinnen und Akteuren oder Ethikinstitutionen nie als politische Probleme identifiziert und verhandelt werden, wie sie von medizin- und wissenschaftskritischen Akteurinnen und Akteuren formuliert werden. Gerade die Untersuchungsperspektive auf die Problematisierungen sozialer Konflikte und Proteste zeigen, welche Lösungsvorschläge und Ziele über die Zeit unsichtbar werden: Es sind Lösungsvorschläge wie der Zusammenschluss von Forscherinnen und Forschern mit Armen, Unterdrückten sowie Arbeiterinnen und Arbeitern, wie im US-ame- 
rikanischen Diskurs um Gentechnologie in den 1970er Jahren, um die Profitorientierung der Forschung zu ändern und um einen gesellschaftlichen Wandel zu erreichen. Im französischen Gentechnologiediskurs wird kritisiert, wer über wissenschaftliche und technologische Entwicklungen zu entscheiden hat. Die Lösungsvorschläge der Science du Peuple bleiben zwar diffus, implizit beinhaltet die Kritik aber ein anderes Entscheidungsverfahren über wissenschaftliche und technologische Entwicklungen. Unsichtbar ist diese Kritik an Entscheidungsverfahren allerdings (noch) nicht im Kontext des Nanotechnologiediskurses.

Im Nanotechnologiediskurs zeigt sich, dass die ethische Steuerung selbst problematisiert wird. Im Sinne der Problematisierung nach Dean kann hier also von einem reflexive government gesprochen werden. Diese Problematisierung ist aber in dem Sinn offen, da es umstritten ist, um welche Art von Problem es sich bei der Nanotechnologie handelt: Um ein politisches Problem, das die Steuerung wissenschaftlicher und technologischer Entwicklungen angreift, oder um ein ethisches Problem, das durch eine Steigerung ethischer Techniken des governing at a distance gelöst werden kann. Bis zum Ende des Untersuchungszeitraums dieser Studie stellt sich die Lösung in einer Multiplikation von Ethik dar: Weitere Ethik-Akteure und -Akteurinnen sollen ausgebildet werden und Praktiken sollen entstehen, um die Öffentlichkeit vermehrt einzubeziehen.

Gerade das letzte Beispiel verdeutlicht, dass die neoliberale Rationalität sich nicht eins zu eins in einem linearen Prozess durchsetzt. Die Techniken des governing at a distance sind selbst umstritten. Die Frage, ob es sich bei der Nanotechnologie um ein ethisches, gesundheitliches oder politisches Problem handelt, ist Teil des »struggle over meaning «(Braun 2014: 79/Herv. i.O.). Dies konnte m.E. sichtbar gemacht werden, da nicht auf ein Großnarrativ - die neoliberale Gouvernementalität - fokussiert wurde, sondern verschiedene Rahmungen und Problematisierungen berücksichtigt wurden.

Mit der Verwendung zweier Konzepte der indirekten Regierung, der delegierten Biopolitik und dem governing at a distance, zeigt sich das Spezifische der Regierungsform des CCNE. Es ist nicht allein das diffuse Verständnis von Ethik, das die Einbindung weiterer Themen in den institutionellen Ethik-Rahmen ermöglicht oder allein die Offenheit für neue Themen, sondern die Offenheit, sie als individuelle gesundheitsrelevante Themen zu verstehen. Ein Merkmal dieser Regierungsform ist die damit einhergehende Bioindividuation im Sinne Memmis. Das ethische governing at a distance dehnt sich zwar auf weitere Bereiche aus, wie auf Nanotechnologie oder Atomtechnologie, doch ändern sich die Möglichkeiten der Ausdehnung je nach Thema und institutionellem Kontext. Sie können in dem diffusen Verständnis von Ethik liegen und/oder darin, sie als Thema der individuellen Gesundheit zu verstehen. 
Um dem nachgehen zu können, was im jeweiligen Kontext von den jeweiligen Akteurinnen und Akteuren unter Ethik verstanden wird, erwies sich das Konzept der Frame-Analyse als sinnvoll. Ebenso zielführend war, Ethik nominalistisch zu verstehen. Das bedeutete, sie als etwas zu begreifen oder auch, als etwas Seltsames. Gleichzeitig ermöglichte sie die notwendige Distanz, um die Selbstverständlichkeit von Ethik zu hinterfragen. Auch für die Interviews war die damit einhergehende offene Perspektive gegenüber dem Verständnis von Ethik der Gesprächspartnerinnen und -partner hilfreich. Sie reagierten auf Fragen wie z.B., ob in dem Ethikkomitee darüber gesprochen wird, was Ethik ist, oder was sie unter dem Begriff Ethik verstehen, zwar manchmal irritiert, antworteten aber mit großer Bereitschaft, auch auf die weiteren Fragen. Für diese große Bereitschaft, etwas erklären und verständlich machen zu wollen, können mehrere Gründe gesehen werden. Die Interviewpartnerinnen und -partner waren meist männlich und deutlich älter. Zum einen kam in der Interviewsituation sicherlich der so genannte Paternalismuseffekt zum Tragen, die Projektion auf die Interviewerin, die etwas aufgrund ihrer Alters oder ihres Geschlechts nicht wissen kann und der es deshalb erklärt werden muss (Abels/Behrens 2005: 182). Zum anderen war diese Bereitschaft m.E. auch mit der Annahme der Interviewpartner und -partnerinnen verknüpft, einer Ausländerin etwas erklären zu wollen, die etwas nicht wissen kann. Vereinzelt war auch mein Eindruck, dass aufgrund des hohen Alters der Gesprächspartnerinnen und -partner ein Bedürfnis da war, noch einmal alles erzählen zu können. Einige sahen in dem Interview die Möglichkeit, den CCNE im Ausland bekannt zu machen. Es zeigten sich also in einigen Interviewsituationen zwar Vorurteile, diese wirkten sich allerdings produktiv auf das Ergebnis aus.

Die Frame-Analyse wurde insofern praxisorientiert in meiner genealogischen Untersuchung verwendet, als sie dazu diente, aufzuzeigen, wie sich etwas als selbstverständlich etablieren konnte und sich daraus die Praxis einer anderen Denkweise ergeben kann. Ethikpolitiken sind nicht einfach da, sie entstehen aus einem kontingenten, konflikthaften Prozess, in dem sich bestimmte Problematisierungen bestimmter Akteurinnen und Akteure durchsetzen, wie gerade im Kontext der Nanoethik deutlich wurde. Die Studie kann, als Narrativ verstanden, in Anknüpfung an Dominique Pestres Aufforderung dazu beitragen, normative Tendenzen in den betreffenden Diskursen der Nano-STS, die in ethischer Steuerung eine »dialogical, open, legitimate and effective force (Rainey et al. 2012: 144) sehen, (selbst-)kritisch zu hinterfragen. Insofern wäre gerade die Untersuchung interessant, wie sich hier Ethik-Rahmungen als Lösung durchsetzen, für welches Problem sie als Lösung gesehen werden und wer dieses Problem definiert. Interessant ist zudem die Frage, inwiefern die STS-Diskurse um Ethik mit staatlich initiierten 
Ethikpolitiken korrespondieren und welche Regierungspraktiken sie befördern. In diesem Sinn schließe ich mit Dominique Pestre:

»it is time for us to become critical intellectuals once again and to consider the $[\ldots]$ regulations governing today's sciences and societies - because we are not doing it enough« (Pestre 2004a: 1). 



\section{ABKüRZUNGEN}

\begin{tabular}{|c|c|}
\hline AKW & Atomkraftwerk \\
\hline CCNE & $\begin{array}{l}\text { Comité Consultatif National d'Éthique pour les Sciences de la vie et } \\
\text { de la santé }\end{array}$ \\
\hline CCSTI & Centre de Culture Scientifique, Technique et Industrielle \\
\hline CGTI & Conseil Général des Technologies et de l'Information \\
\hline $\mathrm{CHU}$ & Centres Hospitaliers Universitaires \\
\hline CNRS & Centre National de la Recherche Scientifique \\
\hline DEEPEN & $\begin{array}{l}\text { Deepening Ethical Engagement and Participation in Emerging } \\
\text { Nanotechnologies }\end{array}$ \\
\hline DGRST & Délégation Générale à la Recherche Scientifique et Technique \\
\hline DNA & Deoxyribonucleic acid \\
\hline EABs & National Ethics Advisory Bodies \\
\hline EGAIS & Ethical GovernAnce of emergIng technologieS \\
\hline EGo & Ethical Governance \\
\hline ELSI & Ethical, Legal and Social Implications \\
\hline EU & Europäische Union \\
\hline FINRRAGE & $\begin{array}{l}\text { Feminist International Network of Resistance against Reproductive } \\
\text { and Genetic Engineering }\end{array}$ \\
\hline $\mathrm{FuE}$ & Forschung und Entwicklung \\
\hline GIB & Groupe d'Information Biologie \\
\hline GIS & Groupe d'Information Santé \\
\hline GMO & Genetisch Manipulierte Organismen \\
\hline GS & Gouvernementalitätsstudien \\
\hline IBM & Institut de Biologie Moléculaire \\
\hline INSERM & Institut National de la Santé et de la Recherche Médicale \\
\hline IPA & Interpretative Policy-Analyse \\
\hline IVF & In-vitro-Fertilisation \\
\hline MLAC & Mouvement pour la liberté de l'avortement et de la contraception \\
\hline MURS & Mouvement Universel de la Responsabilité Scientifique \\
\hline NGO & Non-governmental organization \\
\hline NNI & National Nanotechnology Initiative \\
\hline NS & Nationalsozialismus \\
\hline NSDAP & Nationalsozialistische Deutsche Arbeiterpartei \\
\hline OPECST & $\begin{array}{l}\text { Office Parlementaire d'Évaluation des Choix Scientifique et } \\
\text { Technologiques }\end{array}$ \\
\hline ORTF & Office de Radiodiffusion Télévision Française \\
\hline $\mathrm{PC}$ & Parti Communiste \\
\hline PS & Parti Socialiste \\
\hline STS & Science and Technology Studies \\
\hline UNESCO & United Nations Educational Scientific and Cultural Organization \\
\hline
\end{tabular}





\section{Literatur}

Abba-Sidick, J./Abdalleh, Janita/Anfredon, Monique et al. (1971): »Manifeste des 343 salopes«, in: Nouvel Observateur, 5 avril 1971, 334, http://tempsreel. nouvelobs.com/actualite/societe/20071127.OBS7018/le-manifeste-des-343salopes-paru-dans-le-nouvel-obs-en-1971.html, 16.03.2011.

Abels, Gabriele/Behrens, Maria (2005): »ExpertInnen-Interviews in der Politikwissenschaft. Geschlechtertheoretische und politikfeldanalytische Reflexion einer Methode«, in: Alexander Bogner et al. (Hg.): Das Experteninterview. Theorie, Methode, Anwendung. 2. Auflage. Wiesbaden: VS Verlag für Sozialwissenschaften, S. 173-190.

Académie Nationale de Médecine (1949): Bulletin de l'Académie Nationale de Médecine. 11 et $12.113 \mathrm{e}$ année, 3e série, tome 133 . Séances des 15 et 22 mars 1949. Paris: Masson et Cie.

Ach, Johann S./Runtenberg, Christa (2002): Bioethik: Disziplin und Diskurs. Zur Selbstaufklärung angewandter Ethik. Frankfurt a. M., New York: Campus.

Alias, François (1991): Le comité consultatif national d'éthique et l'institutionnalisation d'un débat public relatif à l'éthique biomédicale. Approche critique. Unveröffentlichte Dissertation. Toulouse, S. 364.

Alving, Alf S./Branch, Craige/Pullman, Theodore N. et al. (1948): »Procedures used at Stateville Penitentiary for the testing of potential antimalarial agents «, in: The Journal of Clinical Investigation, 27, 3, S. 2-5.

Ambroselli, Claire (1984): »Les institutions en question«, in: Après-demain: journal mensuel de documentation politique, 266, S. 5-10.

— (1987): »Présentation«, in: INSERM (Hg.): Comités d'éthique à travers le monde. Recherches en cours 1986. Paris: Éditions Tierce, S. 5-12.

— (1990): Le comité d'éthique. Paris: PUF. 
Arnall, Alexander Huw (2003): Future Technologies, Today's Choices. Nanotechnology, Artificial Intelligence and Robotics; A technical, political and institutional map of emerging technologies. Greenpeace Environmental Trust ( $\mathrm{Hg}$.), London, http://www.greenpeace.org.uk/files/pdfs/migrated/MultimediaFiles/ Live/FullReport/5886.pdf, 20.08.2008.

Arney, William Ray/Bergen, Bernard J. (1984): Medicine and the Management of Living. Taming the Last Great Beast. Chicago, London: The University of Chicago Press.

Artières, Philippe (2002): »1972: naissance de l'intellectuel spécifique«, in: Plein droit, 2, 53-54, S. 37-38.

Association nationale des CCSTI (2014a): La Charte. http://www.ccsti.fr/fr/acteurs /la_charte.html, 03.09.2014.

— (2014b): Les Objectifs. http://www.ccsti.fr/fr/acteurs/les_objectifs.html, 03.09.2014.

Bacchi, Carol (2012): »Why study Problematizations? Making Politics Visible«, in: Open Journal of Political Science, 2, 2, S. 1-8. http://www.scirp.org/ Journal/PaperInformation.aspx?paperID=18803, 12.08.2013.

Bachelot-Narquin, Roselyne (2009): Discours lors de la conférence de presse »Lancement des états généraux de la bioéthique«. Conférence de presse, 4.02.2009, Paris. http://www.sante-sports.gouv.fr/actualite-presse/presse-sante/ discours/lancement-etats-generaux-bioethique-discours-roselyne-bachelotnarquin.html, 01.07.2009.

Bachir-Benlahsen, Myriam (1995): Sagesse et Politique. L'impact des comités de sages sur les modes d'action et de légitimation politiques. Unveröffentlichte Dissertation. Amiens, S. 616.

Baier, Lothar (2001): »Fatal Histories«, in: der Freitag, 26.10.2001, http://www.freitag.de/autoren/der-freitag/fatal-histories, 25.01.2013.

Baird, Davis/Nordmann, Alfred/Schummer, Joachim (2004): Discovering the Nanoscale. Amsterdam: IOS Press.

Baird, Davis/Shew, Ashley (2004): »Probing the History of Scanning Tunneling Microscopy«, in: Davis Baird et al. (Hg.): Discovering the Nanoscale. Amsterdam: IOS Press, S. 145-156.

Barben, Daniel (1997): »Neoliberale Formierung der Biotechnologie? Zur Hegemoniegewinnung einer flexiblen Strategie«, in: Das Argument, 220, S. 383-395.

Barry, Andrew/Osborne, Thomas/Rose, Nikolas (1993): »Liberalism, neo-liberalism and governementality«, in: Economy and society [special edition], 22, 3, S. 265-407. 
- (2001): Foucault and political reason. Liberalism, neo-liberalism and rationalities of government. London, New York: Routledge.

Baruch, Marc Olivier (1999): Das Vichy-Regime. Stuttgart: Reclam.

Bashford, Alison/Levine, Philippa (2010): The Oxford Handbook of the History of Eugenics. Oxford, New York: Oxford University Press.

Bateman Novaes, Simone (1998): »La bioéthique comme objet sociologique «, in: Cahiers internationaux de Sociologie, 104, Figures de la connaissance, S. 5-32.

Baum, Rudy (2003): »Nanotechnology. Drexler and Smalley make the case for and against >molecular assemblers ««, in: Chemical \& Engineering News, 81, 48, S. 37-42. http://pubs.acs.org/cen/coverstory/8148/8148counterpoint.html, 07.05.2009.

Baumgartner, Christoph (2008): »Bionanotechnology: A New Challenge for Ethical Reflection?«, in: Fabrice Jotterand (Hg.): Emerging Conceptual, Ethical and Policy Issues in Bionanotechnology. Dordrecht: Springer, S. 67-84.

Baverez, Nikolas (1993): Raymond Aron: un moraliste au temps des idéologies. Paris: Flammarion.

Beckwith, Jonathan R. (1970): »Gene Expression in Bacteria and Some Concerns About the Misuse of Science «, in: Bacteriological Reviews, 34, 3, S. 222-227.

- (2002): Making Genes, Making Waves: A Social Activist in Science. Cambridge u.a.: Harvard University Press.

Beecher, Henry K. (1966): »Ethics and clinical research«, in: The New England Journal of Medicine, 274, 24, S. 367-372.

Berg, Paul/Baltimore, David/Boyer, Herbert W. et al. (1974): »Potential Biohazards of Recombinant DNA Molecules«, in: Science, 185, S. 303.

Berg, Paul/Baltimore, David/Brenner, Sydney et al. (1975): »Summary Statement of the Asilomar Conference on Recombinant DNA Molecules«, in: Proceedings of the National Academy of Sciences, 72, 6, S. 1981-1984.

Berlivet, Luc (2008): »Between Expertise and Biomedicine: Public Health Research in France after the Second World War«, in: Medical History, 52, 4, S. 471-492.

Bernard, Jean (1986/1987): »Biologie et morale. Naissance de la bio-éthique «, in:

Les Cahiers du MURS, 7, Hiver 1986/1987, S. 23-42. http://documents. irevues.inist.fr/bitstream/handle/2042/8159/MURS_1986_7_23.pdf? sequence $=1,29.20 .2013$.

- (1988): L'évolution de la bioéthique. Conférence prononcée le 14 novembre 1987 lors du Dies Academicus de 1'Université de Fribourg. Fribourg: Editions Universitaires.

— (1994): Médecin dans le siècle. Paris: Édition Robert Laffont. 
Bernstein, Barton J. (1976): »The Road to Watergate and Beyond: The Growth and Abuse of Executive Authority Since 1940«, in: Law and Contemporary Problems, 40, S. 58-86.

Berube, David/Shipman, J.D. (2004): »Denialism: Drexler vs. Roco«, in: Technology and Society Magazine, IEEE, 23, 4, S. 22-26. http://ieeexplore.ieee.org/ stamp/stamp.jsp?tp=\&arnumber=1371635\&isnumber=29989, 18.05.2009.

Bevir, Marc (2008): »What is Genealogy?«, in: Journal of the Philosophy of History, 2, S. 263-275.

Bidault, Ghislaine (1993): Les mémoires de la recherche. Etat des versements 1977-1989. Cahiers pour l'Histoire de la Recherche, Paris: CNRS Éditions.

Bioethikkommission beim Bundeskanzleramt (2007): Beschluss der Bioethikkommission beim Bundeskanzleramt vom 13. Juni 2007. Nanotechnologie, Katalog ethischer Probleme und Empfehlungen. S. 1-5. http://www.bundes kanzleramt.at/DocView.axd?CobId=23987, 27.09.2014.

Blondiaux, Loïc/Sintomer, Yves (2002): »L’impératif délibératif«, in: Politix, 15, 57, S. 17-35.

Bogner, Alexander (2009): »Ethisierung und die Marginalisierung der Ethik. Zur Mikropolitik der Ethik«, in: Soziale Welt. Zeitschrift für sozialwissenschaftliche Forschung und Praxis, 60, S. 3-21.

— (2013): »Ethisierung oder Moralisierung? Technikkontroversen als Wertkonflikte«, in: Alexander Bogner (Hg.): Ethisierung der Technik - Technisierung der Ethik: Der Ethik-Boom im Lichte der Wissenschafts- und Technikforschung. Baden-Baden: Nomos, S. 51-64.

Bogner, Alexander/Littig, Beate/Menz, Wolfgang (2005): Das Experteninterview. Theorie, Methode, Anwendung. 2. Auflage. Wiesbaden: VS Verlag für Sozialwissenschaften.

Bogner, Alexander/Menz, Wolfgang (2010): »How Politics Deals with Expert Dissent: The Case of Ethics Councils«, in: Science, Technology \& Human Values, 35, 6, S. 888-914.

Bogner, Alexander/Menz, Wolfgang/Schumm, Wilhelm (2008): »Ethikexpertise in Wertkonflikten. Zur Produktion und politischen Verwendung von Kommissionsethik in Deutschland und Österreich«, in: Renate Mayntz et al. (Hg.): Wissensproduktion und Wissenstransfer. Wissen im Spannungsfeld von Wissenschaft, Politik und Öffentlichkeit. Bielefeld: transcript, S. 243-268.

Bois, Guy (1982): »Sciences humaines, Commission no $1 \mathrm{~B} \ll$, in: Ministère de la Recherche et de la Technologie (Hg.): Recherche et Technologie, S. 95-108.

Boltanski, Luc/Chiapello, Ève (2003): Der neue Geist des Kapitalismus. Konstanz: UVK Verlagsgesellschaft. 
Bonah, Christian/Gaudillière, Jean-Paul (2007): »Faute, accident ou risque iatrogène? La régulation des événements indésirables du médicament à l'aune des affaires Stalinon et Distilbène«, in: Revue française des affaires sociales, 3, 3-4, S. 123-151.

Bonneuil, Christophe (2004): Les transformations des rapports entre sciences et société en France depuis la Seconde Guerre mondial: un essai de synthèse. Sciences, Médias et Société, École Normale Supérieure, Lettres et Sciences Humaines, Université de Lyon, 15.-17. Juin 2001. Actes. Publication électronique, S. 15-40. http://sciences-medias.ens-lyon.fr/IMG/pdf/actes.pdf, 28.08.2008.

- (2006): »Cultures épistémiques et engagement public des chercheurs dans la controverse OGM «, in: Natures Sciences Sociétés, 14, 3, S. 257-268.

Bonneuil, Christophe/Joly, Pierre-Benoît/Marris, Claire (2008): »Disentrenching Experiment: The Construction of GM Crop Field Trials As a Social Problem«, in: Science Technology \& Human Values, 33, 2, S. 201-229.

Braibant, Guy/Atlan, Henri/Langaney, André (1993): »Comités d'éthique et démocratie«, in: Raison présente. La maîtrise du vivant, 105, S. 161-184.

Brandt, Allan M. (1978): »Racism and Research: The Case of the Tuskegee Syphillis Study«, in: The Hastings Center Report, 8, 6, S. 21-29.

Braun, Kathrin (2009): Neotechnocratic governance and »The New spirit of Capitalism«. Unpublished paper presented at the 4th International Conference in Interpretive Policy Analysis: Discourse and Power in Critical Policy Studies, 25.27.06., Kassel. S. 1-13.

Braun, Kathrin (2011): Between Self-Determination and Social Technology. Medicine, Biopolitics and the New Techniques of Procedural Management. Bielefeld: transcript.

— (2013): »Ethics in Time - Ethikberatung und Zeitlichkeit in Kommissionen zu Biomedizin und Atomtechnologie«, in: Alexander Bogner (Hg.): Ethisierung der Technik - Technisierung der Ethik: Der Ethik-Boom im Lichte der Wissenschafts- und Technikforschung. Baden-Baden: Nomos, S. 93-114.

— (2014): »Im Kampf um Bedeutung. Diskurstheorie und Diskursanalyse in der interpretativen Policy Analyse«, in: Zeitschrift für Diskursforschung, 1, S. 77 101.

— (2015a): »Erbgesundheitsgesetz, Ächtung und Entschädigungsdebatten«, in: Torben Fischer/Matthias N. Lorenz (Hg.): Lexikon der »Vergangenheitsbewältigung « in Deutschland. Bielefeld: transcript, S. 345-348.

— (2015b): »Between Representation and Narration: Analyzing Policy Frames«, in: Frank Fischer et al. (Hg.): Handbook of Critical Policy Studies. Northampton, MA: Edward Elgar Publishing, S. 441-461. 
Braun, Kathrin/Herrmann, Svea/Könninger, Sabine (2002): Deliberative Modelle als Mittel der Demokratisierung von Bioethik- und Biomedizinpolitik. Gutachten für die Berlin Brandenburgische Akademie der Wissenschaften. Berlin.

Braun, Kathrin/Herrmann, Svea Luise/Brekke, Ole (2012): »Zwischen Gesetz und Gerechtigkeit. Staatliche Sterilisationspolitiken und der Kampf der Opfer um Wiedergutmachung«, in: Kritische Justiz, 3, S. 298-315.

Braun, Kathrin/Herrmann, Svea Luise/Könninger, Sabine et al. (2008): »Die Sprache der Ethik und die Politik des richtigen Sprechens? Ethikregime in Deutschland, Frankreich und Großbritannien«, in: Renate Mayntz et al. (Hg.): Wissensproduktion und Wissenstransfer. Wissen im Spannungsfeld von Wissenschaft, Politik und Öffentlichkeit. Bielefeld: transcript, S. 221-242.

— (2009): »Bioethik in der Politik«, in: Aus Politik und Zeitgeschichte, 8, 16. Februar 2009, S. 40-46.

Braun, Kathrin/Moore, Alfred/Herrmann, Svea Luise et al. (2010a): »'Ethical reflection must always be measured . Bioethics, Technologies of Humility and the Government of Government«, in: Science Technology \& Human Values, 35, 6, S. 839-864.

Braun, Kathrin/Moore, Alfred/Herrmann, Svea Luise et al. (2010b): »Science Governance and the Politics of Proper Talk: Governmental Bioethics as a New Technology of Reflexive Government«, in: Economy And Society, 39, 4, S. 510-533.

Braun, Kathrin/Schultz, Susanne (2010): »»... a certain kind of engineering involved $<$ : Constructing the public in participatory governance arrangements «, in: Public Understanding of Science, 19, 4, S. 403-419.

Bröckling, Ulrich/Krasmann, Susanne (2010): »Ni méthode, ni approche. Zur Forschungsperspektive der Gouvernementalitätsstudien - mit einem Seitenblick auf Konvergenzen und Divergenzen zur Diskursforschung«, in: Johannes Angermüller/Silke van Dyk (Hg.): Diskursanalyse meets Gouvernementalitätsforschung. Perspektiven auf das Verhältnis von Subjekt, Sprache, Macht und Wissen. Frankfurt a. M.: Campus, S. 23-42.

Bröckling, Ulrich/Krasmann, Susanne/Lemke, Thomas (2011): »From Foucault's Lectures at the Collège de France to Studies of Governmentality. An Introduction«, in: Ulrich Bröckling et al. (Hg.): Governmentality. Current Issues and Future Challenges. New York, Abingdon: Routledge, S. 1-33.

BUND (2013): Sonnencremes mit Nanopartikeln, ein ungeklärtes Risiko. http://www.bund.net/nc/service/oekotipps/detail/artikel/sonnencremes-mitnanopartikeln-ein-ungeklaertes-risiko/, 20.09.2014.

Burchell, Graham (1993): »Liberal government and techniques of the self «, in: Economy and Society, 22, 3, S. 267-282. 
Burchell, Graham/Gordon, Colin/Miller, Peter (1991): The Foucault Effect. Studies in Governmentality. Chicago: University of Chicago Press.

Bush, Vannevar (1945): Science. The endless frontier. A report to the President on a Program for Postwar Scientific Research. Reprinted 1960. Washington D. C.: National Science Foundation.

Busquin, Philippe (2002): L'intégration des efforts de recherche en nanotechnologie dans l'Espace Européen de la Recherche. Conférence Nanotechnology: A New Industrial Revolution? Challenges and Opportunities for Research and Innovation in Europe, 14.06.2002, Grenoble. Speech/02/286, http://europa.eu/ rapid/press-release_SPEECH-02-286_fr.htm, 10.05.2014.

Callon, Michel (1999): »Des différentes formes de démocratie techniques«, in: Les cahiers de la sécurité intérieure, 38, S. 37-54.

Callon, Michel/Dianoux, Laurent/Jean-Michel, Fourniau et al. (2005): Democratie locale et maîtrise sociale des nanotechnologies. Les publics grenoblois peuvent-ils participer aux choix scientifiques et techniques? 22 septembre 2005. In: La Métro, S. 1-58. http://sciencescitoyennes.org/IMG/pdf/Nano Grenoble_rapport_final_05_09_22.pdf, 12.10.2007.

Callon, Michel/Lascoumes, Pierre/Barthe, Yannick (2009): Acting in an Uncertain World. An Essay on Technical Democracy. Cambridge/London: The MITPress.

Canguilhem, Georges (1976): »Quality of life. Dignity of death. Summary«, in: Charles Galpérine (Hg.): Biologie et devenir de l'homme, S. 532-537.

Carmen, Ira H. (1985): Cloning and the Constitution. An Inquiry into Governmental Policymaking and Genetic Experimentation. Madison, London: The University of Wisconsin Press.

Carol, Anne (1995): Histoire de l'eugénisme en France. Les médecins et la procréation XIXe-XXe siècle. Paris: Édition du Seuil.

Carpentier, Jean (2001): »Aprenons à faire l'amour. Extrait de textes libres«, in: La lettre de l'enfance et de l'adolescence, 45, 3, S. 73-75.

Cascais, Fernando (o.J.): »Bioethics: From the Early Days to the Present «, in: Studia Bioetica, 3, http://utopia.duth.gr/ xirot/BIOETHICS/journal/vol03/ 01.doc, 4.01.2011.

Cassirer, Ernst (2004 (1930)): »Form und Technik«, in: Birgit Recki (Hg.): Ernst Cassirer, Gesammelte Werke. Hamburger Ausgabe. Band 17. Hamburg: Felix Meiner Verlag, S. 139-183.

CCNE (1984a): Opinion on ethical problems arising out of artificial reproductive techniques. No 3. Paris, http://www.ccne-ethique.fr/sites/default/files/publica tions/avis003en.pdf, 18.09.2014. 
- (1984b): Opinion on the testing of new treatments on humans. Considerations and proposals. No 2. Paris, http://www.ccne-ethique.fr/sites/default/files/ publications/avis002en.pdf, 18.09.2014.

- (1986): Opinion on the use of Mifepristone (RU 486). No 10. Paris, http://www.ccne-ethique.fr/sites/default/files/publications/avis010en.pdf, 18.09.2014.

— (1990a): Éthique et connaissance. Une réflexion sur l'éthique de la recherche biomédicale. Paris: La Documentation française.

- (1990b): Opinion that the human body should not be used for commercial purposes. No 21. Paris, http://www.ccne-ethique.fr/sites/default/files/publications/ avis021en.pdf, 18.09.2014.

- (1991a): Opinion on blood transfusion with reference to not making commercial use of the human body. No 28. Paris, http://www.ccne-ethique.fr/sites/ default/files/publications/avis028en.pdf, 19.09.2014.

- (1991b): Opinion that the human genome should not be used for commercial purposes. No 27. Paris, http://www.ccne-ethique.fr/sites/default/files/publica tions/avis027en.pdf, 19.09.2014.

- (2000): Réponse du CCNE aux saisines du Président du Sénat et du Président de l'Assemblée Nationale sur l'allongement du délai d'IVG. No 66. Paris, http://www.ccne-ethique.fr/fr/publications/reponse-du-ccne-aux-saisines-dupresident-du-senat-et-du-president-de-lassemblee, 01.08.2015.

- (2004): Cahiers du CCNE. http://www.ccne-ethique.fr/fr/pages/cahiers-du-ccne, 31.01.2014.

— (2005): Journées annuelles d'Éthique. Mardi 29 et Mercredi 30 novembre 2005. (Programme). Paris.

- (2006a): Commercialisation des cellules souches humaines et autres lignées cellulaires. No 93, In: CCNE, Paris, http://www.ccne-ethique.fr/sites/default/ files/publications/avis093.pdf, 19.09.2014.

- (2006b): Commercialisation of human stem cells and other cell lines. No 93. Paris, http://www.ccne-ethique.fr/sites/default/files/publications/avis093en.pdf, 12.05.2014.

- (2007): Ethical issues raised by nanosciences, nanotechnologies and health. No 96. Paris, S. 1-18. http://www.ccne-ethique.fr/docs/en/avis096.pdf, 3.06.2010.

- (2009): Programme. Journées annuelle d'éthique. Mardi 24 et mercredi 25 novembre 2009. »Pouvoir de, pouvoir sur, pouvoir pour: des limites de la liberté «. Paris.

- (2013): Journée publique de réflexion des lycéens. La place de la personne âgée dans la société. Samedi 23 mars 2013. (Programme). Paris, http://www.ccneethique.fr/sites/default/files/progr_journee_lyceens.pdf, 08.09.2014. 
— (2014): Le fonctionnement. http://www.ccne-ethique.fr/fr/pages/le-fonctionn ement, 31.01.2014.

CFDT (2013): Histoire de la CFDT. http://www.cfdt-sg.fr/Menu/Qui-sommesnous--/Notre-histoire/Histoire-de-la-CFDT/p-30.html, 24.07.2013.

Chagas, Carlos (1976): »Rapport introductif. Problèmes posés par les essais thérapeutiques chez l'homme«, in: Charles Galpérine (Hg.): Biologie et devenir de l'homme, S. 29-34.

Chambefort, Hélène (o.J.): Petite note historique avec repérage dans les archives de l'INSERM. http://www.vjf.cnrs.fr/histrecmed/documents-archives/rech erche-medicale/recherche-medicale-archives-inserm.html, 20.06.2011.

Chauveau, Sophie (2004): »Genèse de la >sécurité sanitaire <: les produits pharmaceutique en France aux XIXe et XXe siècles«, in: Revue d'histoire moderne et contemporaine, 2, 51-2, S. 88-117.

Chavot, Philippe/Masseran, Anne (2003a): »Exhibiting science and technology in France: Between education and leisure«, in: Ulrike Felt (Hg.): O.P.U.S., S. 237-261.

- (2003b): »An historical and political overview of PUS issues in France«, in: Ulrike Felt (Hg.): O.P.U.S., S. 530-542.

- (2003c): » La mise-en-culture of science: Public Understanding of Science in the French policy context«, in: Ulrike Felt (Hg.): O.P.U.S., S. 78-84.

— (2003d): »Non-governmental PUS initiatives in France: The social authority of technoscience under controversy «, in: Ulrike Felt (Hg.): O.P.U.S., S. 427-440.

— (2003e): »Public consultation and foresight exercise in France: In search for hybrid fora «, in: Ulrike Felt (Hg.): O.P.U.S., S. 347-382.

Chèvenement, Jean-Pierre (1982a): »Discours de Jean-Pierre Chèvenement, Ministre d'Etat, Ministre de la Recherche et de la Technologie«, in: Ministère de la Recherche et de la Technologie (Hg.): Recherche et Technologie, S. 193208.

— (1982b): »Discours introductif de M. Jean-Pierre Chevènement, Ministre d'Etat, Ministre de la Recherche et de la Technologie«, in: Ministère de la Recherche et de la Technologie (Hg.): Recherche et Technologie, S. 55-58.

— (1982c): »Préface«, in: Ministère de la Recherche et de la Technologie (Hg.): Recherche et Technologie, S. 5-6.

Chirac, Jacques (2004): Rede von Staatspräsident Jacques Chirac zum Abschluss der Botschafterkonferenz. 27. August 2004 - Auszüge. Présidence de la République: http://www.jacqueschirac-asso.fr/archives-elysee.fr/elysee/elysee.fr/ allemand/ansprachen_und_dokumente/2004/rede_von_staatspraesident_jacque s_chirac_zum_abschluss_der_botschafterkonferenz-auszuege-paris.295 69.html, 10.05.2014. 
Cicco, Anne (1993): »Madame Soleil prend la retraite«, in: L'Humanité, Jeudi, 2 Séptembre, http://www.humanite.fr/node/62499, 04.09.2014.

CNRS (2004): Biologie et société. Une nouvelle demande sociale. http://www. cnrs.fr/SDV/Dept/1980_2.html, 30.07.2004.

Commission nationale du débat public (2009): Débat public sur les options générales en matière de développement et de régulation des nanotechnologies. Dossier de presse. CNDP, Paris, S.1-13. http://www.debatpublic-nano.org/ presse/dossier_presse.html?id_document=38, 30.09.2009.

Conan, Eric (1983): »Une Haute Autorité pour la médecine«, in: Libération, 08./09.10.1983.

Conseil National de l'Ordre (2012): Code de déontologie médicale. Novembre 2012, S. 1-34. http://www.conseil-national.medecin.fr/article/le-code-de-deon tologie-medicale-915, 23.07.2013.

Crandall, BC/Lewis, James (1992): Nanotechnology. Research and Perspectives. Papers from the First Foresight Conference on Nanotechnology. Cambridge, London: MIT Press.

Dard, Olivier (2000): »Les technocrates: archéologie d'un concept, généalogie d'un groupe social«, in: Olivier Dard et al. (Hg.): L'occupation, l'État français et les entreprises. Actes du colloque organisé par l'Université de FrancheComté et le Musée de la Résistance et de la Déportation de Besançon. 24, 25 et 26 mars 1999 à Besançon. Paris: ADHE, S. 213-227.

Dauzats, Charles (1982): »Le premier bébé-éprouvette français naîtra ce mois-ci«, in: Le Parisien, 12.02.1982.

Davies, Sarah R./Fiedeler, Ulrich/Ferrari, Arianna et al. (2010): »Understanding Nanoscience and Emerging Technologies«, in: Ulrich Fiedeler et al. (Hg.): Understanding Nanotechnology: Philosophy, Policy, Publics. Heidelberg: Akademische Verlagsgesellschaft AKA GmbH, S. 1-8.

De Luca, Virginie (2003): »Pro-Natalism and Hygienism in France, 1900-1940. The Example of the Fight against Venereal Disease«, in: Population (English Edition), 64, 2009/3, S. 477-506.

de Vilaine, Anne-Marie (1985): France. Feminist Resistance. Feminist International Network on the New Reproductive Technologies. International Conference July 1985, Lund, Sweden. http://www.finrrage.org/pdf_files/Conference\% 20Reports/Finrrage_Conference_Lund_Sweden_1985.pdf, 14.01.2014.

Dean, Mitchell (1999a): Governmentality. Power and Rule in Modern Society. London: SAGE Publications.

— (1999b): »Risk, Calculable and Incalculable«, in: Soziale Welt. Zeitschrift für sozialwissenschaftliche Forschung und Praxis, Jahrgang 49, Heft 1, S. 25-42. 
Debru, Claude (2003): »Histoire des sciences. De la biologie à l'éthique«, in: Didier Sicard (Hg.): Travaux du Comité Consultatif National d'Éthique, S. 967973.

Decker, Michael/Fiedeler, Ulrich/Fleischer, Torsten (2004): »Ich sehe was, was du nicht siehst...zur Definition von Nanotechnologie«, in: Technikfolgenabschätzung - Theorie und Praxis, 2, 13 Jg. Juni, S. 10-16.

Décret n ${ }^{\circ}$ 83-132 (1983): Décret du 23 février 1983 portant création d'un Comité consultatif national d'éthique pour les sciences de la vie et de la santé. http://www.ccne-ethique.fr/fr/pages/1983-creation-du-ccne-par-decretpresidentiel-decret-ndeg-83-132\#.UTjlcNYzB8E, 31.01.2014.

Décret n ${ }^{\circ}$ 97-555 (1997): Décret relatif au Comité Consultatif National d'Éthique pour les sciences de la vie et de la santé. http://www.ccne-ethique.fr/fr/ pages/1997-decret-relatif-au-comite-consultatif-national-dethique-pour-lessciences-de-la-vie-et-de\#.UTjmCtYzCSo, 31.01.2014.

Décret $\mathrm{n}^{\circ}$ 2005-390 (2005): Décret sur les modalités d'organisation, de fonctionnement, de désignation des membres et de saisine CCNE. http:// www.ccne-ethique.fr/fr/pages/2005-decret-sur-les-modalites-dorganisation -de-fonctionnement-de-designation-des-membres-et-de\#UTjmbNYzB8E, 31.01.2014.

Décret n 2008-1236 (2008): Décret du 28 novembre 2008 instituant un comité de pilotage des états généraux de la bioéthique. http://www.legifrance.gouv.fr/ jopdf//jopdf/2008/1129/joe_20081129_0022.pdf, 06.02.2014.

DEEPEN (o.J.): Project Aims. http://www.geography.dur.ac.uk/projects/deepen/ Background/ProjectAims/tabid/1996/Default.aspx, 04.06.2014.

Del Re, Alisa (2000): »Avortement et contraception«, in: Groupe d'études sur la division sociale et sexuelle du travail du CNRS et de l'Université de Paris VIII (Hg.): Dictionnaire critique du féminisme. Paris: PUF, S. 1-6.

deLeon, Peter/Vogenbeck, Danielle M. (2007): »The Policy Sciences at the Crossroads«, in: Frank Fischer et al. (Hg.): Handbook of Public Policy Analysis. Theory, Politics and Methods. Boca Raton, London, New York: CRC Press, S. 3-14.

Demazure, Michel (1982): »Les grands équilibres et les domaines clés, Commission no 3 «, in: Ministère de la Recherche et de la Technologie (Hg.): Recherche et Technologie, S. 109-115.

Denord, François (2005): »La conversion au néo-liberalisme«, in: Mouvements, 5, 35, S. 17-23.

Dessieux, Gisèle (1982): »Recherche et communication, Commission no $10 \ll$, in: Ministère de la Recherche et de la Technologie (Hg.): Recherche et Technologie, S. 160-164. 
Devreux, Anne-Marie/Ferrand-Picard, Michèle (1982): »La loi sur l'avortement. Chronologies des événements et des prises de position«, in: Revue française de sociologie, XXIII, S. 503-518.

Didrich-Tabaste, Alix (1994): Éléments d'analyse pour la construction sociale d'une autorité administrative indépendante. Le comité consultatif national d'éthique saisi au sein de l'institutionnalisation du discours éthique. Unveröffentlichte Diplomarbeit. Paris, S. 177.

Doll, Jürgen (2006): »Mai 68«, in: Bernhard Schmidt et al. (Hg.): Frankreich Lexikon, S. 600-610.

Doll, Jürgen/Schmidt, Bernhard (2006): »Écologistes«, in: Bernhard Schmidt et al. (Hg.): Frankreich Lexikon, S. 338-341.

Doll, Jürgen/Taubert, Fritz (2006): »Loi d'orientation«, in: Bernhard Schmidt et al. (Hg.): Frankreich Lexikon, S. 578-581.

Donzelot, Jacques/Gordon, Colin (2008): »Governing Liberal Societies - the Foucault Effect in the English Speaking World «, in: Foucault Studies, 5, January, S. 48-62. http://ej.lib.cbs.dk/index.php/foucault-studies/article/view/1409/15 12, 06.08.2013.

Drexler, K. Eric (2007): Engines of Creation. The Coming Era of Nanotechnology (Free HTML version). http://e-drexler.com/p/06/00/EOC_Cover.html, 30.07.2008.

Drouard, Alain (1990): »La Fondation française pour l'étude des problèmes humains et l'organisation de la recherche en sciences sociales en France «, in: Reprint des Cahiers pour l'histoire du CNRS, 9, S. 1-18. http://www.vjf. cnrs.fr/histrecmed/pdf/drouard.pdf, 25.01.2013.

Duchen, Claire (1986): Feminism in France. From May'68 to Mitterrand. London u.a.: Routledge \& Kegan Paul.

Duclert, Vincent (2004): »La naissance de la délegation générale à la recherche scientifique et technique. La construction d'un modèle partagé de gouvernement dans les années soixante «, in: Revue française d'administration publique, 4, 112, S. 647-658.

— (2006): »Le colloque de Caen, second temps de l'engagement mendésiste «, in: Alain Chatriot/Vincent Duclert (Hg.): Le gouvernement de la recherche. Histoire d'un engagement politique, de Pierre Mendès France au général de Gaulle (1953-1969). Paris: La Découverte, S. 81-100.

Dunham, Roger G./Mauss, Armand L. (1976): »Waves from Watergate. Evidence concerning the Impact of the Watergate Scandal upon Political Legitimacy and Social Control«, in: The Pacific Sociological Review, 19, 4, S. 469-490.

Duportail, Judith (2013): »Quel est le rôle du Comité national d'éthique ?« in: Le Figaro, 04.02.2013. 
Dupuy, Jean-Pierre/Roure, Françoise (2004): Les Nanotechnologies: Éthique et prospectice industrielle. Tome 1, Section »Innovation et Entreprise«, 15.11.2004. In: Conseil Général des Mines, Conseil Général des Technologies de l'Information, S. 1-68. http://www.ladocumentationfrancaise.fr/var/sto rage/rapports-publics/054000313/0000.pdf, 21.07.08.

Durand, Guy (1999): Introduction générale à la bioéthique: histoire, concepts et outils. Montréal, Paris: Fides, Cerf.

Düsseldorf, G. P. (1961): »Mißgeburten durch Tabletten? Alarmierender Verdacht eines Arztes gegen ein weitverbreitetes Medikament«, in: Welt am Sonntag, 26.11.1961, S. 2.

Duteil, Myriam (1990): Comité consultatif national d'éthique pour les sciences de la vie et de la santé, la place de l'éthique dans le dispositif de la régulation social. Unveröffentlichte Diplomarbeit. Paris, S. 182.

Düwell, Marcus (2000): »Die Bedeutung ethischer Diskurse in einer wertepluralen Welt«, in: Matthias Kettner (Hg.): Angewandte Ethik als Politikum. Frankfurt a. M.: S. 76-114.

Düwell, Marcus/Steigleder, Klaus (2003): »Bioethik - Zu Geschichte, Bedeutung und Aufgaben«, in: Marcus Düwell/Klaus Steigleder (Hg.): Bioethik. Eine Einführung. Frankfurt a. M.: S. 12-37.

Ebbesen, Mette/Andersen, Svend/Besenbacher, Flemming (2006): »Ethics of Nanotechnology. Starting from Scratch?«, in: Bulletin of Science, Technology \& Society, 26, 6, S. 451-462.

Eblinghaus, Helga/Stickler, Armin (1998): Nachhaltigkeit und Macht. Zur Kritik von Sustainable Development. Frankfurt a. M.: IKO-Verlag für Interkulturelle Kommunikation.

Edelman, Bernard/Gavarani, Laurence/Hermitte, Marie-Angèle et al. (1989): »Pour des États généraux de la biomédecine«, in: Libération, 3.2.1989, S. 4.

Edge, David (1995): »Reinventing the Wheel«, in: Sheila Jasanoff et al. (Hg.): Handbook of Science and Technology Studies. Revised Edition. Thousand Oaks u.a.: Sage Publications, S. 3-23.

Edwards, Robert G. (1974): »Fertilization of Human Eggs in Vitro: Morals, Ethics and the Law «, in: Charles Galpérine (Hg.): Biologie et devenir de l'homme, S. 377-394.

Einsiedel, Edna F./Goldenberg, Linda (2004): »Dwarfing the social? Nanotechnology Lessons from the Biotechnology Front «, in: Bulletin of Science, Technology \& Society, 24, 1, S. 28-33.

Engelhardt, Viktor (1922): Weltanschauung und Technik. Leipzig: Felix Meiner Verlag. 
Équipe des »Cahiers de la République« (1956): »Colloque sur l'enseignement et la recherche scientifique«, in: Bulletin Quotidien d'Informations, 3, o.S. http://www.pasteur.fr/infosci/archives/mon/im_caen1.html, 27.02.2013.

Escoffier-Lambiotte (1983): »M. Mitterrand installe le Comité national d'éthique«, in: Le Monde, 03.12.1983, S. 12.

États géneraux de la bioéthique (2009): Qu'est-ce que les états généraux de la bioéthique? http://www.etatsgenerauxdelabioethique.fr/presentation-generale/ qu-est..., 16.02.2009.

ETC Group (2003): The Big Down. From Genomes to Atoms. Atomtech: Technologies Converging at the Nano-scale. Winnipeg. http://www.etcgroup.org/en/ materials/publications.html?pub_id=171, 28.03.2007.

Ethik-Kommission Sichere Energieversorgung (2011): Deutschlands Energiewende. Ein Gemeinschaftswerk für die Zukunft. 30.05.2011. Berlin, http://www.bundesregierung.de/ContentArchiv/DE/Archiv17/_Anlagen/2011/0 7/2011-07-28-abschlussbericht-ethikkommission.pdf?_blob=publicationFile\& $\mathrm{v}=4,02.10 .2014$.

European Commission (2012): Responsible Research and Innovation. Europe's ability to respond to societal challenges. http://ec.europa.eu/research/sciencesociety/document_library/pdf_06/responsible-research-and-innovationleaflet_en.pdf, 22.09.2014.

Evans, John H. (2002): Playing God: Human Genetic Engineering and the Rationalization of the Public Bioethical Debate. Chicago, London: University of Chicago Press.

Evleth, Donna (2006): »The Ordre des Médecins and the Jews in Vichy France, 1940-1944«, in: French History, 20, 2, S. 183-203.

Fagot-Largeault, Anne (1985): L'homme bioéthique, pour une déontologie de la recherche sur le vivant. Paris: Maloine.

Fassin, Didier (2011): »Coming Back to Life: An Anthropological Reassessment of Biopolitics and Governmentality«, in: Ulrich Bröckling et al. (Hg.): Governmentality. Current Issues and Future Challenges. New York, Abingdon: Routledge, S. 185-200.

Fassin, Didier/Memmi, Dominique (2004): Le gouvernement des corps. Paris: Éditions EHESS.

Fekl, Walther (2006a): »Occupation«, in: Bernhard Schmidt et al. (Hg.): Frankreich Lexikon, S. 681-686.

— (2006b): »Vichy«, in: Bernhard Schmidt et al. (Hg.): Frankreich Lexikon, S. 999-1007. 
Felt, Ulrike (2003): O.P.U.S. Optimising Public Understanding of Science and Technology. Final Report. Department for Philosophy of Science and Social Studies of Science, University of Vienna. http://www.univie.ac.at/virusss/opus/mpapers.html, 25.09.2008.

Felt, Ulrike/Barben, Daniel/Irwin, Alan et al. (2013): Science in Society: caring for our futures in turbulent times. European Science Foundation, Strasbourg, S. 1-36. http://www.esf.org/fileadmin/Public_documents/Publications/spb50ScienceInSociety.pdf, 22.09.2014.

Felt, Ulrike/Fochler, Maximilian (2010): »Machineries for Making Publics: Inscribing and De-scribing Public in Public Engegament«, in: Minerva, 48, S. 219-238.

Ferrand-Picard, Michèle (1982): »Médicalisation et contrôle social de l'avortement. Derrière la loi, les enjeux«, in: Revue française de sociologie, 23, 3, S. 383-396.

Fette, Julie (2012): Exclusions. Practicing Prejudice in French Law and Medicine, 1920-1945. Ithaka, London: Cornell University Press.

Feuerstein, Günter/Kollek, Regine (1999): »Flexibilisierung der Moral. Zum Verhältnis von biotechnischen Innovationen und ethischen Normen«, in: Claudia Honegger et al. (Hg.): Grenzenlose Gesellschaft? Verhandlungen des Deutschen Soziologentages. Kongressband Teil 2 des Freiburger Kongresses der DGS, ÖGS und SGS. Opladen: Leske+Budrich, S. 559-574.

Feynman, Richard P. (1959): Plenty of Room at the Bottom. http://www.its. caltech.edu/ feynman/plenty.html, 4.08.2008.

— (1976): »Los Alamos From Below. Reminiscences of 1943-1945«, in: Engineering and Science, January-February, S. 11-30. http://calteches.library. caltech.edu/3090/1/FeynmanLosAlamos.pdf, 21.09.2014.

Fiedeler, Ulrich/Coenen, Christopher/Davies, Sarah R. et al. (2010): Understanding Nanotechnology: Philosophy, Policy and Publics. Heidelberg: Akademische Verlagsgesellschaft AKA GmbH.

Fischer, Frank (1990): Technocracy and the Politics of Expertise. Newbury Park, London, New Dehli: Sage Publications.

— (2000): »Citizens and Experts in Biotechnology Policy: The Consensus Conference as Alternative Model«, in: Gabriele Abels/Daniel Barben (Hg.): Biotechnologie-Globalisierung-Demokratie. Politische Gestaltung transnationaler Technologieentwicklung. Berlin: edition sigma, S. 359-374.

- (2003): Reframing Public Policy. Discursive Politics and Deliberative Practices. Oxford: Oxford University Press.

Fischer, Frank/Forrester, John (1993): The Argumentative Turn in policy analysis and planning. Durham: Duke University Press. 
Fischer, Frank/Gottweis, Herbert (2012): The Argumentative Turn Revisited. Public Policy as Communicative Practice. Durham, London: Duke University Press.

Fischer, Frank/Miller, Gerald J./Sidney, Mara S. (2007): Handbook of Public Policy Analysis. Theory, Politics and Methods. Boca Raton u.a.: CRC Press.

Fischer, Frank/Torgerson, Douglas/Durnova, Anna (2015): Handbook of Critical Policy Studies. Northampton, MA: Edward Elgar Publishing.

Fisher, Erik (2005): »Lessons learned from the Ethical, Legal and Social Implications program (ELSI): Planning societal implications research for the National Nanotechnology Program«, in: Technology in Society, 27, S. 321-328.

Fogarty, Richard S./Osborne, Michael A. (2010): »Eugenics in France and the Colonies«, in: Alison Bashford/Philippa Levine (Hg.): The Oxford Handbook of The History of Eugenics. Oxford, New York: Oxford University Press, S. 332346.

Fogelberg, Hans (2003): »The Grand Politics of Technoscience: Contextualizing Nanotechnology«, in: Hans Glimell/Hans Fogelberg (Hg.): Bringing Visibility to the Invisible: Towards A Social Understanding of Nanotechnology. Göteborg: Göteborg University, S. 29-48. http://citeseerx.ist.psu.edu/viewdoc/ download?doi=10.1.1.198.7849\&rep=rep1\&type=pdf, 24.09.2009.

Fogelberg, Hans/Glimell, Hans (2003): »Molecular Matters: In Search for the Real Stuff «, in: Hans Glimell/Hans Fogelberg (Hg.): Bringing Visibility to the Invisible: Towards A Social Understanding of Nanotechnology. Göteborg: Göteborg University Press, S. 4-28. http://citeseerx.ist.psu.edu/viewdoc/download? doi=10.1.1.198.7849\&rep=rep1\&type=pdf, 24.09.2009.

Forest, Nadine (1990): Robert Mallet, Recteur de l'Académie de Paris de 19691980. Entretien, in: à dire. Journal de l'Université 7. Numéro spécial. Paris: http://www.univ-paris-diderot.fr/sc/site.php?bc=archivesP7\&np=Mallet, 04.12.2013.

Fossaert, Robert (1982): »L'apport culturel de la recherche scientifique et technologique, Commission no $1 \ll$, in: Ministère de la Recherche et de la Technologie (Hg.): Recherche et Technologie, S. 91-94.

Foucault, Michel (1976): Mikrophysik der Macht. Über Strafjustiz, Psychiatrie und Medizin. Berlin: Merve Verlag.

- (1977): Wahnsinn und Gesellschaft: Eine Geschichte des Wahns im Zeitalter der Vernunft. Frankfurt a. M.: Suhrkamp.

— (1988): Die Geburt der Klinik. Eine Archäologie des ärztlichen Blicks. Frankfurt a. M.: Fischer Taschenbuch Verlag. 
— (1990): »The Concern for Truth «, in: Lawrence D. Kritzman (Hg.): Politics, philosophy, culture: interviews and other writings of Michel Foucault, 19771984. New York: Routledge, S. 255-267.

— (1991): »Questions of method «, in: Graham Burchell et al. (Hg.): The Foucault effect. Studies in governmentality. London u.a.: Harvester Wheatsheaf, S. 7386.

— (1993a): »Leben machen und sterben lassen. Die Geburt des Rassismus«, in: DISS-Texte, 25, S. 27-50.

— (1993b): »Technologien des Selbst«, in: Luther H. Martin et al. (Hg.): Technologien des Selbst. Frankfurt a. M.: S. Fischer, S. 24-62.

— (1996): Diskurs und Wahrheit. Die Problematisierung der Parrhesia. 6 Vorlesungen, gehalten im Herbst 1983 an der Universität von Berkeley/Kalifornien. Berlin: Merve Verlag.

— (1997a): »The birth of biopolitics«, in: Paul Rabinow (Hg.): Essential works of Foucault 1954-1984. Ethics. Subjectivity and Truth. Vol. 1. New York: The New Press, S. 73-79.

— (1997b): »Polemics, Politics and Problematizations. An interview conducted by Paul Rabinow in May 1984«, in: Paul Rabinow (Hg.): Essential works of Foucault 1954-1984. Ethics. Subjectivity and Truth. Vol. 1. New York: The New Press, S. 111-119.

— (1998 (1970)): »Theatrum Philosophicum«, in: James D. Faubion (Hg.): Aesthetics, method and epistemology. Essential works of Foucault 1954-1984. Vol. 2. New York: New Press, S. 343-368.

- (1999): Der Wille zum Wissen: Sexualität und Wahrheit 1. Frankfurt a. M.: Suhrkamp Verlag.

— (2001 (1967)): »Nietzsche, Freud, Marx«, in: Daniel Defert/François Ewald (Hg.): Michel Foucault. Dits et écrits. Schriften. Erster Band. Frankfurt a. M.: Suhrkamp, S. 727-743.

— (2001 (1976)): La fonction politique de l'intellectuel. 1976. Foucault. Dits et Écrits. 1954-1988, II. Paris: Éditions Gallimard.

— (2003 (1976)-a): »Das Wissen als Verbrechen. Gespräch mit S. Terayama«, in: Daniel Defert/François Ewald (Hg.): Michel Foucault. Dits et écrits. Schriften. Dritter Band. Frankfurt a. M.: Suhrkamp, S. 105-115.

— (2003 (1976)-b): »Krise der Medizin oder Krise der Antimedizin?« in: Daniel Defert/François Ewald (Hg.): Michel Foucault. Dits et écrits. Schriften. Dritter Band. Frankfurt a. M.: Suhrkamp, S. 54-76.

— (2004 (1978)-a): »Vorlesung 4 (Sitzung vom 1. Februar 1978)«, in: Michel Sennelart (Hg.): Michel Foucault. Geschichte der Gouvernementalität I. Sicherheit, Territorium, Bevölkerung. Frankfurt a. M.: Suhrkamp, S. 134-172. 
— (2004 (1978)-b): »Vorlesung 6 (Sitzung vom 15. Februar 1978)«, in: Michel Sennelart (Hg.): Michel Foucault. Geschichte der Gouvernementalität I. Sicherheit, Territorium, Bevölkerung. Frankfurt a. M.: Suhrkamp, S. 201-238.

— (2004 (1978)-c): »Vorlesung 7 (Sitzung vom 22. Februar 1978)«, in: Michel Sennelart (Hg.): Michel Foucault. Geschichte der Gouvernementalität I. Sicherheit, Territorium, Bevölkerung. Frankfurt a. M.: Suhrkamp, S. 239-277.

—(2004 (1978)-d): »Vorlesung 9 (Sitzung vom 8. März 1978)«, in: Michel Sennelart (Hg.): Michel Foucault. Geschichte der Gouvernementalität I. Sicherheit, Territorium, Bevölkerung. Frankfurt a. M.: Suhrkamp, S. 331-368.

— (2005a): »Die Geburt der Biopolitik«, in: Daniel Defert/François Ewald (Hg.): Michel Foucault. Analytik der Macht. Frankfurt a. M.: Suhrkamp, S. 180-187.

— (2005b): »Die Machtverhältnisse gehen in das Innere der Körper über«, in: Daniel Defert/François Ewald (Hg.): Michel Foucault. Analytik der Macht. Frankfurt a. M.: Suhrkamp, S. 126-136.

— (2005c): »Subjekt und Macht«, in: Daniel Defert/François Ewald (Hg.): Michel Foucault. Analytik der Macht. Frankfurt a. M.: Suhrkamp, S. 240-263.

— (2005 (1984)): »Polemik, Politik und Problematisierung. Gespräch mit Paul Rabinow 1984«, in: Daniel Defert/François Ewald (Hg.): Michel Foucault. Dits et écrits. Schriften. Vierter Band. Frankfurt a. M.: Suhrkamp, S. 724-734.

— (2006 (1979)): »Vorlesung 4 (Sitzung vom 31. Januar 1979)«, in: Michel Sennelart (Hg.): Michel Foucault. Geschichte der Gouvernementalität II. Die Geburt der Biopolitik. Vorlesung am Collège de France 1978-1979. Frankfurt a. M.: Suhrkamp, S. 112-147.

Fuchs, Michael (2001a): Ethikräte im internationalen Vergleich. Modelle für Deutschland? 12/2001, Konrad-Adenauer-Stiftung. Sankt Augustin.

- (2001b): Internationaler Überblick zu Verfahren der Entscheidungsfindung bei ethischem Dissens. Gutachten für die Enquête-Kommission »Recht und Ethik der modernen Medizin« des Deutschen Bundestages. Arbeitsunterlage 14/55, Berlin. http://webarchiv.bundestag.de/archive/2007/0206/parlament/gremien/ kommissionen/archiv14/medi/medi_gut_fuchs.pdf, 31.07.2015.

- (2005): Nationale Ethikräte. Hintergünde, Funktionen und Arbeitsweisen im Vergleich. Berlin: http://www.ethikrat.org/dateien/pdf/nationale-ethikraete.pdf, 31.07.2015.

Fuller, Steve (2000): The Governance of Science. Buckinham: Open University Press. 
Galloux, Jean-Christophe/Mortensen, Arne Thing/De Cheveigné, Suzanne et al. (2002): »The institutions of bioethics«, in: Martin W. Bauer/George Gaskell (Hg.): Biotechnology. The Making of a Global Controversy. Cambridge: Cambridge University Press in Association with the Science Museum London, S. 129-148.

Galpérine, Charles (1976a): Actes du colloque mondial: Biologie et devenir de l'homme. Paris: McGraw Hill.

— (1976b): »Introduction«, in: Charles Galpérine (Hg.): Biologie et devenir de l'homme, S. VIII-X.

Garcia, Sandrine (2005): »Expertise scientifique et capital militant. Le rôle des médecins dans la lutte pour la législation de l'avortement «, in: Actes de la recherche en sciences sociales, 3, 158, S. 96-115.

Gaudillière, Jean-Paul (2000): »Rockefeller Strategies for Scientific Medicine: Molecular Machines, Viruses and Vaccines«, in: Studies in History and Philosophy of Science Part C: Studies in History and Philosophy of Biological and Biomedical Sciences, 31, 3, S. 491-509.

- (2002a): Inventer la biomédecine. La France, l'Amérique et la production des savoirs du vivant (1945-1965). Paris: La Découverte.

— (2002b): »Paris-New York roundtrip: transatlantic crossings and the reconstruction of the biological sciences in post-war France«, in: Studies in History and Philosophy of Biological and Biomedical Sciences, 33, S. 389-417.

- (2006a): »Intellectuels engagés et experts: biologistes et médecins dans la bataille de l'avortement «, in: Sciences Natures Sociétés, 14, 3, S. 239-248.

- (2006b): La médecine et les sciences. Paris: La Découverte.

Giami, Alain (2007): »Une histoire de l'éducation sexuelle en France: une médicalisation progressive de la sexualité (1945-1980)«, in: Sexologies, 16, 3, S. 219-229. http://www.hal.inserm.fr/inserm-00167498, 22.05.2015.

Gibbons, Michael/Limoges, Camille/Nowotny, Helga et al. (1994): The new production of knowledge. The dynamics of science and research in contemporary societies. London u.a.: Sage Publications.

Gieryn, Thomas F. (1983): »Boundary-Work and the Demarcation of Science from Non-Science: Strains and Interests in Professional Ideologies of Scientists«, in: American Sociological Review, 48, 6, S. 781-795.

Gill, Bernhard/Dreyer, Marion (2001): Internationaler Überblick zu Verfahren der Entscheidungsfindung bei ethischem Dissens. Gutachten im Auftrag der Enquête-Kommission »Recht und Ethik der modernen Medizin« des Deutschen Bundestages. Arbeitsunterlage 14/56, Berlin.

Gisler, Priska/Kurath, Monika (2010): »Paradise Lost? >Science< and >the Public< after Asilomar«, in: Science Technology \& Human Values, 36, 2, S. 213-243. 
Glimell, Hans (2003): »Dynamics of the Emerging Field of Nanoscience«, in: Hans Glimell/Hans Fogelberg (Hg.): Bringing Visibility to the Invisible: Towards A Social Understanding of Nanotechnology. Göteborg: Göteborg University Press, S. 70-75. http://citeseerx.ist.psu.edu/viewdoc/download?doi= 10.1.1.198.7849\&rep=rep1\&type=pdf, 24.09.2009.

Godfrain, Jacques (2000): »La participation, idée centrale de la pensée gaullienne«, in: Espoir, 125, http://www.charles-de-gaulle.org/pages/l-homme/dossiersthematiques/1958-1970-la-ve-republique/de-gaulle-et-la-participation/analyses/ la-participation-idee-centrale-de-la-pensee-gaullienne.php\#, 24.05.2011.

Godin, Benoit (1994): »Writing performative history: the new new Atlantis? «, in: Social Studies of Science, 28, S. 465-485.

Gottweis, Herbert (1997): »Gene, Hochtechnologie-Narrative und Politik in Frankreich «, in: Renate Martinsen (Hg.): Politik und Biotechnologie. Die Zumutung der Zukunft. Baden-Baden: Nomos, S. 321-340.

- (1998): Governing Molecules. The Discursive Politics of Genetic Engineering in Europe and the United States. Cambridge, London: The MIT Press.

— (2007): »Rhetoric in Policy Making: Between Logos, Ethos and Pathos«, in: Frank Fischer et al. (Hg.): Handbook of Public Policy Analysis. Theory, Policy and Methods. Boca Raton u.a.: CRC Press, S. 237-250.

Gros, François (1982a): »Avant-propos«, in: Ministère de la Recherche et de la Technologie (Hg.): Recherche et Technologie, S. 7-10.

— (1982b): »Discours du Professeur François Gros, Président du comité d'organisation du Colloque national«, in: Ministère de la Recherche et de la Technologie (Hg.): Recherche et Technologie, S. 59-65.

Gros, François/Jacob, François/Royer, Pierre (1979): Sciences de la vie et société. Rapport présenté à M. le Président de la République. Paris: La Documentation française.

Groupe d'information santé (1973): Oui nous avortons! Bulletin spécial. Paris: Edition Gît-le-coeur.

Grunwald, Armin (2004): »Ethische Aspekte der Nanotechnologie. Eine Felderkundung «, in: Technikfolgenabschätzung - Theorie und Praxis, 13, 2, S. 7178.

— (2005): »Nanotechnology - A New Field of Ethical Inquiry?«, in: Science and Engineering Ethics, 11, S. 187-201.

Guston, David H. (1999): »Stabilizing the Boundary between US Politics and Science: The Role of the Office of Technology Transfer as Boundary Organization «, in: Social Studies of Science, 29, 1, S. 87-111.

- (2000): Between Politics and Science. Assuring the Integrity and Productivity of Research. New York: Cambridge University Press. 
Hajer, Marten (1993): »Discourse Coalitions and the Institutionalization of Practice. The Case of Acid Rain in Britain«, in: Frank Fischer/John Forrester (Hg.): The Argumentative Turn in Policy Analysis and Planning. Durham: Duke University Press, S. 43-76.

Hecht, Gabrielle (1994): »Political Designs: Nuclear Reactors and National Policy in Postwar France«, in: Technology and Culture, 35, 4, S. 657-686.

- (2009): The Radiance of France. Nuclear Power and National Identity after World War II. Cambridge, London: MIT Press.

Hedgecoe, Adam M./Martin, Paul A. (2008): »Genomics, STS, and the Making of Sociotechnological Futures«, in: Edward J. Hackett et al. (Hg.): The Handbook of Science and Technology Studies. Cambridge, London: The MIT Press, S. 817-839.

Hegedus, Zsuzsa/Touraine, Alain (1982): »Der Anti-AKW-Kampf«, in: Alain Touraine (Hg.): Die antinukleare Prophetie. Zukunftsentwürfe einer sozialen Bewegung. Frankfurt a. M., New York: Campus, S. 33-52.

Herrmann, Svea (2011): »A speaking cure for conflicts: problematization, discourse stimulation and the ongoin of scientific progress «, in: Kathrin Braun (Hg.): Between Self-Determination and Social Technology, S. 159-188.

Herrmann, Svea/Könninger, Sabine (2008): »»... but you can not influence the direction of your thinking<: Guiding Self-Government in Bioethics Policy Discourse «, in: Elisabeth M. Armstrong et al. (Hg.): Advances in Medical Sociology. Bioethical Issues: Sociological Perspectives. 9. S. 205-223.

Herrmann, Svea Luise (2009): Policy Debates on Reprogenetics. The Problematisation of New Research in Great Britain and Germany. Frankfurt a. M., New York: Campus.

Herzlich, Claudine/Pierret, Janine (2010): »Au croisement de plusieurs mondes: la constitution de la sociologie de la santé en France (1950-1985)«, in: Revue française de sociologie, 51, 1, S. 121-148.

Hessels, Laurens K./van Lente, Harro (2008): Re-thinking new knowledge production: A literature review and a research agenda. In: Innovation Studies Utrecht. Working Paper 08.03. Universiteit Utrecht, Utrecht, S. http://www.geo.uu.nl/ isu/pdf/isu0803.pdf, 22.08.2014.

Hoeyer, Klaus (2005): »Studying Ethics as Policy. The Naming and Framing of Moral Problems in Genetic Research«, in: Current Anthropology, 46, Supplement, S. 71-90.

Hoffmann, Stanley (2000): »Vichy Studies in France: Before and After Paxton«, in: Sarah Fishman et al. (Hg.): France at War. Vichy and the Historians. Oxford, New York: Berg, S. 49-57. 
Holm, Søren (2014): »Does Nanotechnology Require a New >Nanoethics $<$ ? , in: Bert Gordijn/Anthony Mark Cutter (Hg.): In Pursuit of Nanoethics. Dordrecht u.a.: Springer, S. 31-38.

Horellou-Lafarge, Chantal (1982): »Une mutation dans les dispositifs de controle social: le cas de l'avortement«, in: Revue française de sociologie, 23, 3, S. 397-416.

Hornblum, Allen M (1997): »They Were Cheap and Available: Prisoners as Research Subjects in Twentieth Century America«, in: British Medical Journal, 315, S. 1437-1441.

Illich, Ivan (1976): Medical nemesis. The expropriation of health. New York: Pantheon Books.

Ilyes, Petra (2006): Zum Stand der Forschung des englischsprachigen »Science and Technology« (STS)-Diskurses. Teilstudie im Rahmen des Forschungsprojekts »Computersimulationen - Neue Instrumente der Wissensproduktion« im Rahmen der BMBF Initiative »Wissen für Entscheidungsprozesse« (BBAW). Institut für Kulturanthropologie und Europäische Ethnologie. Johann Wolfgang Goethe-Universität, Frankfurt a. M., S. 1-85. http://www.sciencepolicystudies. de/dok/STS-Forschungsstand-1.1.pdf, 28.09.2012.

INSERM (o.J.): De l'INH à l'INSERM. De la santé publique à la recherche médicale. http://histoire.inserm.fr/de-1-inh-a-l-inserm/1-inserm-de-1964-a-nosjours/de-1964-a-1970, 29.06.2015.

Institut Jacques Monod (2013): L'historique. http://www.ijm.fr/ijm/institut/ historique/, 24.07.2013.

Irwin, Alan (2001): »Constructing the scientific citizen: Science and democracy in the biosciences «, in: Public Understanding of Science, 10, 1, S. 1-18.

— (2006): »The Politics of Talk: Coming to Terms with >New< Scientific Governance $\ll$, in: Social Studies of Science, 36, 2, S. 299-320.

Irwin, Alan/Wynne, Brian (1996): Misunderstanding Science? Cambridge: Cambridge University Press.

Isambert, François-André (1983): »De la bio-éthique aux comités d'éthique«, in: Études, 358, 5, S. 671-683.

Jacob, François (1988): Die innere Statue. Autobiographie des Genbiologen und Nobelpreisträgers. Zürich: Ammann.

Jasanoff, Sheila (2003): »Technologies of humility: citizen participation in governing science «, in: Minerva, 41, S. 223-244.

— (2010): »A field of its own: the emergence of science and technology studies«, in: Robert Frodeman et al. (Hg.): The Oxford handbook of interdisciplinarity. Oxford u.a.: Oxford University Press, S. 191-205. 
— (2011): »Constitutional Moments in Governing Science and Technology«, in: Science and Engineering Ethics, 17, S. 621-638.

Jaubert, Alain/Lévy-Leblond, Jean-Marc (1975): (Auto)critique de la science. Paris: Éditions du Seuil.

Jelsøe, Erling/Mortensen, Arne Thing/Kamara, Mercy Wambui et al. (2006): »Moving the Goalposts in Bioethics«, in: George Gaskell/Martin W. Bauer (Hg.): Genomics and Society. Legal, Ethical and Social Dimensions. London: Earthscan, S. 44-59.

Joly, Laurent (2006): Vichy dans la »Solution finale«. Histoire du Commissariat général aux questions juives, 1941-1944. Paris: Bernard Grasset.

Joly, Pierre-Benoît/Gérald, Assouline/Kréziak, Dominique et al. (2000): L'innovation controversée: Le débat public sur les OGM en France. Projet de recherche financé par la DGAL (Contrat CM 97.15), Collectif sur les risques la décision et l'expertise: INRA, Grenoble, S. 1-188. http://www.grenoble.inra.fr/ Docs/pub/A2000/JOLY-OGM-Innov-Controverse.pdf, 17.03.2013.

Jordan, Isabella (2007): Hospizbewegung in Deutschland und den Niederlanden.

Palliativversorgung und Selbstbestimmung am Lebensende. Kultur der Medizin, Band 22. Frankfurt a. M., New York: Campus.

- (2011): »Planning death: Debates on euthanasia, end of life care and living wills in Germany since the 1970s«, in: Kathrin Braun (Hg.): Between SelfDetermination and Social Technology, S. 65-93.

Joy, Bill (2000a): »Restructurer le genre humain«, in: Le Monde, 5.07.2000.

— (2000b): »Why the future doesn't need us«, in: Wired, 8, 04, http://www.wired. com/wired/archive/8.04/joy_pr.html, 30.07.08.

Junge, Torsten (2008): Gouvernementalität der Wissensgesellschaft. Politik und Subjektivität unter dem Regime des Wissens. Bielefeld: transcript.

Jungk, Robert (1974): »Science, Society and Public Conscience«, in: Gottlieb Duttweiler Institute for Economic and Social Studies (Hg.): Genetic Engineering. Its Applications and Limitations. Proceedings of the Symposium held in Davos October 10-12, 1974. Rüschlikon Zürich: S. 141-146.

Kamel, Donney D./Picard, Jean-François/Givernaud, N. (2002): Entretien avec Philippe Kourilsky. Compilation de deux entretiens, 18 mars 2002 à l'Institut Pasteur. http://www.histrecmed.fr/?option=com_content\&view=article\&id=62: kourilsky-philippe\&catid=8:entretiens\&Itemid=129, 07.08.2014.

Kay, Lily E. (1997): »Rethinking Institutions: Philanthropy as a Historiographic Problem of Knowledge and Power«, in: Minerva, 35, S. 283-293.

Kearnes, Matthew/Grove-White, Robin/Macnaghten, Phil et al. (2006): »From Bio to Nano: Learning Lessons from the UK Agricultural Biotechnology Controversy«, in: Science as Culture, 15, 4, S. 291-307. 
Kelly, Susan E. (2003): »Public Bioethics and Publics: Consensus, Boundaries, and Participation in Biomedical Science Policy«, in: Science, Technology \& Human Values, 28, No. 3, S. 339-364.

Kenis, Yvon/Heuskin, Lucie (1990): »Le comité national d'éthique en France «, in: Madeleine Moulin (Hg.): Contrôler la science? La question des comités d'éthique. Bruxelles: De Boeck-Wesmael, S. 65-80.

Kenneth, David (2008): »Socio-Technical Analysis of those Concerned with Emerging Technology, Engagement and Governance«, in: David Kenneth/Paul B. Thompson (Hg.): What Can Nanotechnology Learn from Biotechnology? Social and Ethical Lessons for Nanoscience from the Debate over Agrifood Biotechnology and GMOs. Burlington u.a.: Elsevier, S. 3-30.

Kenneth, David/Thompson, Paul B. (2008): What Can Nanotechnology Learn From Biotechnology? Social and Ethical Lessons for Nanoscience from the Debate over Agrifood Biotechnology and GMOs. Burlington u.a.: Elsevier.

Kettner, Matthias (2000): »Einleitung «, in: Matthias Kettner (Hg.): Angewandte Ethik als Politikum. Frankfurt a. M.: S. 7-18.

Kohlen, Helen (2009): Conflicts of Care. Hospital Ethics Committees in the USA and Germany. Frankfurt a. M., New York: Campus.

— (2011): »Shifting responsibilities in the medical field: US-American bioethics and its move into the hospital setting«, in: Kathrin Braun (Hg.): Between SelfDetermination and Social Technology, S. 127-158.

Kommission der europäischen Gemeinschaften (2004): Mitteilung der Kommission. Auf dem Weg zu einer europäischen Strategie für Nanotechnologie. 338, 12.05.2004. Brüssel, http://ec.europa.eu/nanotechnology/pdf/nano_com_de. pdf, 10.05.2014.

Könninger, Sabine (2001): Das Konzept des Women's Empowerment in der internationalen bevölkerungspolitischen Debatte. Unveröffentlichte Magisterarbeit. Hannover, S. 91.

— (2009): >From Bioethics to Nanoethics?< - Development, Institutionalisation and Framing of Ethicpolitics in France. Unpublished paper presented at the 4th International Conference in Interpretive Policy Analysis: Discourse and Power in Critical Policy Studies, 25.-27.06. Methodological Workshop, Kassel.

— (2010): »From Bio to Nano? Governing through Ethical Speech in France $\ll$, in: Ulrich Fiedeler et al. (Hg.): Understanding Nanotechnology: Philosophy, Policy and Publics. Heidelberg, Amsterdam: AKA, S. 121-132.

- (2011): »Is everything in good health? From bio to nano-the proliferation of governmental ethics in France «, in: Kathrin Braun (Hg.): Between SelfDetermination and Social Technology, S. 215-238. 
— (2013): »Diskursforschung zur Ethikfalle«, in: Gen-ethischer Informationsdienst, 218, Kritische Sozialwissenschaft: Gesellschaftliche Intervention oder Elfenbeinturm, S. 18-20.

Könninger, Sabine/Ott, Ingrid/Papilloud, Christian et al. (2008): »Nanotechnologie - Herausforderung einer Zukunftstechnologie«, in: Update. Wissens-Service des HWWI, April, S. 3. http://www.hwwi.org/uploads/tx_wilpubdb/HWWI_ Update_04_08.pdf, 1.05.2008.

Könninger, Sabine/Ott, Ingrid/Zülsdorf, Torben et al. (2010): »Public reactions to the promotion of nanotechnologies in society«, in: International Journal of Nanotechnology, 7, 2-3, S. 265-279.

Krauss, Gerhard (1996): Forschung im unitaristischen Staat. Abhängigkeit und Autonomie der staatlich finanzierten Forschung in Frankreich. Frankfurt a. M.: Campus.

Krimsky, Sheldon (1982): Genetic Alchemy: The Social History of the Recombinant DNA Controversy. Cambridge u.a.: MIT Press.

- (2005): »From Asilomar to Industrial Biotechnology: Risks, Reductionism and Regulation«, in: Science as Culture, 14, 4, S. 309-323.

Kühl, Stefan (1997): Die Internationale der Rassisten. Aufstieg und Niedergang der internationalen Bewegung für Eugenik und Rassenhygiene im 20. Jahrhundert. Frankfurt a. M., New York: Campus.

Kuzma, Jennifer/Besley, John C. (2008): »Ethics of Risk Analysis and Regulatory Review: From Bio- to Nanotechnology«, in: NanoEthics, 2, 2, S. 149-162.

La documentation Française (2001): Contraception et IVG: Chronologie. http://www.ladocumentationfrancaise.fr/dossiers/ivg/chronologie.shtml, 08.11.2006.

Laborie, Françoise (1988): »New reproductive technologies: News from France and elsewhere «, in: Reproductive and Genetic Engineering: Journal of International Feminist Analysis, 1, 1, (online o.S.). http://www.finrrage.org/pdf_files/ RepTech\%20General/Repro_Technologies_News_from_France_and_ Elsewhere_1988.pdf, 23.01.2014.

— (1995): »Divergences entre féministes françaises sur l'analyse des nouvelles technologies de reproduction humaine (NTR)«, in: Arno Bammé et al. (Hg.): Klagenfurter Beiträge zur Technikdiskussion. 73. S. 1-15.

Lackerstein, Debbie (2012): National Regeneration in Vichy France. Ideas and Policies, 1930-1944. Surrey, Burlington: Ashgate Publishing.

Ladrière, Paul (1982): »Religion, morale et politique: le débat sur l'avortement«, in: Revue française de sociologie, 23, 3, S. 417-454.

Larner, Wendy (2000): »Neo-liberalism: Policy, Ideology, Governmentality«, in: Studies in Political Economy, 63, Autumn, S. 5-25. 
Laurent, Brice (2007): »Diverging Convergences«, in: Innovation: The European Journal of Social Science Research, 20, 4, S. 343-357.

- (2008): »Engaging the public in nanotechnology? Three visions of public engagement«, in: Papiers de recherche du CSI, 011, S. 1-29. http://hal.archivesouvertes.fr/docs/00/34/00/63/PDF/WP_CSI_011.pdf, 22.09.2014.

— (2010): Les politiques des nanotechnologies. Pour une traitement démocratique d'une science émergente. Paris: Édition Charles Léopold Mayer.

Lazar, Philippe (2003): »Jusqu'où l'éthique peut-elle être institutionnalisée? «, in: Didier Sicard (Hg.): Travaux du Comité Consultatif National d'Éthique, S. 957-961.

Le Bras, Hervé (1999): »Geburtenförderung und Bevölkerungspolitik in Frankreich: Wandel und Fortleben der Doktrinen «, in: The International Scope Review, 1, 2, S. 107-149. http://www.pierretap.com/pdfs/183a.pdf, 25.01.2013.

Le Bris, Sonia (1993): Les instances nationales d'éthique. Strasbourg: Edition du Conseil de l'Europe.

Le Bureau du Conseil national de l'ordre des médecins (1974): »L'ordre des médecins contre l'expérimentation humaine«, in: La Nouvelle Presse Médicale, 3, 6, S. 328.

Le Président de la République M. Valéry Giscard d'Estaing (1978): »Lettre aux scientifiques«, in: François Gros et al. (Hg.): Sciences de la vie et société. Rapport présenté à M. le Président de la République. Paris: La Documentation française, S. 3-4.

Leinhos, Mary (2005): »The US National Bioethics Advisory Commission as a boundary organization «, in: Science and Public Policy, 32, 6, S. 423-433.

Lemke, Thomas (1997): Eine Kritik der politischen Vernunft. Foucaults Analyse der modernen Gouvernementalität. Berlin, Hamburg: Argument Verlag.

— (1998): »Relativismus revisited. Foucault, die Genealogie und die Historie «, in: Jungle World, Dossier: Diskurs essen Linke auf, 12, http://jungleworld.com/artikel/1998/11/36737.html, 03.03.2013.

— (2000): »Neoliberalismus, Staat und Selbsttechnologien. Ein kritischer Überblick über die governmentality studies«, in: Politische Vierteljahresschrift, 41. Jg., 1, S. 31-47.

- (2001): » The birth of bio-politics<: Michel Foucault's lecture at the College de France on neo-liberal governmentality«, in: Economy and Society, 30, 2, S. 190-207.

Lemke, Thomas/Krasmann, Susanne/Bröckling, Ulrich (2000): »Gouvernementalität, Neoliberalismus und Selbsttechnologien. Eine Einleitung «, in: Ulrich Bröckling et al. (Hg.): Gouvernementalität der Gegenwart. Studien zur Ökonomisierung des Sozialen. Frankfurt a. M.: Suhrkamp, S. 7-40. 
Lengwiler, Martin (2008): »Participatory Approaches in Science and Technology. Historical Origins and Current Practices in Critical Perspective«, in: Science, Technology \& Human Values, 33, 2, S. 186-200.

Lenoir, Noelle (1991): Aux frontières de la vie. Une éthique biomédicale à la française. Paris: La documentation française.

- (1997): Nationale Ethikkomissionen in Europa - Möglichkeiten und Grenzen. Braucht Deutschland eine Bundes-Ethik-Kommission? Dokumentation des Expertengesprächs Gentechnik am 11. März 1997 in Bonn, Bonn. S. 5-27.

Lessenich, Stephan (2003): »Soziale Subjektivität. Die neue Regierung der Gesellschaft«, in: Mittelweg, 36, 4, S. 80-93.

Levidow, Les/Carr, Susan (1997): »How biotechnology regulation sets a risk/ethics boundary«, in: Agriculture and Human Values, 14, S. 29-43.

Lewin, Roger (1974): »Comment. Ethics and genetic engineering «, in: New Scientist, S. 163.

Lindsey, Nicola/Kamara, M. W./Jelsøe, E. et al. (2001): »Changing frames. The emergence of ethics in european policy on biotechnology«, in: Politeia, XVII, 63, S. 80-93.

Loewe, Siegfried (2006a): »Arrêté«, in: Bernhard Schmidt et al. (Hg.): Frankreich Lexikon, S. 83.

— (2006b): »Révolution française«, in: Bernhard Schmidt et al. (Hg.): Frankreich Lexikon, S. 839-845.

Loi $\mathrm{n}^{\circ}$ 75-17 (1975): Loi du 17 janvier 1975 relative à l'interruption volontaire de grossesse. In: Journal officiel de la République française: 18 janvier 1975.

S. 671. http://www.assemblee-nationale.fr/histoire/interruption/sommaire.asp\# Loi\%20n\%C2\%B0\%2075-17, 19.07.2013.

Loi $\mathrm{n}^{\circ}$ 82-610 (1982): Loi d'orientation et de programmation pour la recherche et le développement technologique de la France. 15 juillet 1982. http://www.legi france.gouv.fr/affichTexte.do?cidTexte=LEGITEXT000006068756\&dateTexte $=20110620,16.01 .2014$.

Loi no 82-1172 (1982): Loi du 31 décembre 1982 relative à la couverture des frais afférents à l'interruption volontaire de grossesse non thérapeutique et aux modalités de financement de cette mesure. http://www.legifrance.gouv.fr/ affichTexte.do?cidTexte $=$ JORFTEXT000000704429\&dateTexte=20140213, 13.02.2014.

Loi n ${ }^{\circ} 88-1138$ (1988): Loi relative à la protection des personnes se prêtant à la recherche biomédicale. http://legifrance.gouv.fr/affichTexte.do?cidTexte=JORF TEXT000000508831, 22.05.2015. 
Loi n 94-654 (1994): Loi du 29 juillet 1994 relative au don et à l'utilisation des éléments et produits du corps humain, à l'assistance médicale à la procréation et au diagnostic prénatal. http://www.ccne-ethique.fr/fr/pages/1994-inscriptiondu-ccne-dans-la-loi-du-29-juillet-1994-loi-du-29-juillet-1994\#.UTjlstYzB8E, 31.01.2014.

Loi $\mathrm{n}^{\circ} 300$ (1942): Loi du 15 février 1942 relative à la repression de l'avortement. In: Journal officiel de l'Etat français: No 57, 7 Mars 1942. S. 938.

Loi n ${ }^{\circ}$ 2004-800 (2004): Loi du 6 août 2004 relative à la bioéthique et liens vers les décrets d'application. http://www.legifrance.gouv.fr/html/actualite/actualite _legislative/decrets_application/2004-800.htm, 18.08.2004.

Loi $n^{\circ}$ 2007-1787 (2007): Loi du 20 décembre 2007 relative à la simplification du droit (1), Art. 8, Modifie Code civil Art. 63. http://www.legifrance.gouv.fr/ affichTexte.do?cidTexte=JORFTEXT000017727195, 16.07.2014.

Loi $n^{\circ}$ 2011-814 (2011): Loi du 7 juillet 2011 relative à la bioéthique. In: Journal officiel de la République Française: 8 juillet 2011. S. 1-163.

Loi $\mathrm{n}^{\circ} 4968$ (1941): Loi du 30 novembre 1941 créant un institut national d'hygiène. In: Journal officiel de l'Etat français: 6 décembre 1941. S. 5272.

Löwy, Ilana (2003): »La science dans son contexte: L'Institut national d'hygiène et la recherche mèdicale sous Vichy (Commentaire)«, in: Sciences Sociales et Santé, 21, 1, S. 27-30.

Löwy, Ilana/Zylberman, Patrick (2000): »Medicine as social instrument: Rockefeller Foundation, 1913-1945«, in: Studies in History and Philosophy of Science Part C: Studies in History and Philosophy of Biological and Biomedical Sciences, 31, 3, S. 365-379.

Macey, David (1998): »The honour of Georges Canguilhem«, in: Economy and Society, 27, 2-3, S. 171-181.

Macnaghten, Phil/Kearnes, Matthew/Wynne, Brian (2005): »Nanotechnology, Governance, and Public Deliberation: What Role for Social Sciences?« in: Science Communication, 27, 2, S. 268-291.

Maheu, René (1976): »Summary. Closing Session«, in: Charles Galpérine (Hg.): Biologie et devenir de l'homme, S. 574-577.

Maibaum, Thomas (2007): Die Führerschule der deutschen Ärzteschaft in AltRehse. Online veröffentlichte Dissertation. Hamburg-Eppendorf, S. 316. http://www.sub.uni-hamburg.de/opus/volltexte/2007/3457/pdf/Dissertation_Ma ibaum.pdf, 8.08.2012.

Maio, Giovanni (1994): »Forschung am Menschen. Eine französische Debatte«, in: Ethik in der Medizin, 6, S. 143-156. 
- (1995): »Die französische nationale Ethikkommission. Entstehungsgeschichte, Arbeitsweise und Bedeutung am Beispiel ihrer Empfehlung zur Embryonenforschung «, in: Zeitschrift für medizinische Ethik, 41, S. 291-299.

— (2000): »Ärztliche Ethik als Politikum. Zur französischen Diskussion um das Humanexperiment nach 1945«, in: Medizin Historisches Journal, 35, S. 35-80.

— (2001): »Zur Geschichte der Contergan-Katastrophe im Lichte der Arzneimittelgesetzgebung «, in: Deutsche Medizinische Wochenschrift, 126, 42, S. 1183 1186.

Mali, Franc/Pustovrh, Toni/Groboljsek, Blanka et al. (2012): »National Ethics Advisory Bodies in the Emerging Landscape of Responsible Research and Innovation«, in: Nanoethics, 6, S. 167-184.

Mallet, Robert (1976a): »Introduction«, in: Charles Galpérine (Hg.): Biologie et devenir de l'homme, S. VIII-X.

— (1976b): »Préface«, in: Charles Galpérine (Hg.): Biologie et devenir de l'homme, S. VII.

- (1976c): »Presentation. Creation of the universal movement of scientific responsibility«, in: Charles Galpérine (Hg.): Biologie et devenir de l'homme, S. 542.

— (1976d): »Séance de clôture. Allocution du Recteur Robert Mallet«, in: Charles Galpérine (Hg.): Biologie et devenir de l'homme, S. 557-567.

— (1986/1987): »Le M.U.R.S., dix ans déjà«, in: Les Cahiers du MURS, 7, Hiver 1986/1987, S. 13-22. http://documents.irevues.inist.fr/handle/2042/8160, 29.10.2013.

Marris, Claire/Joly, Pierre-Benoît (1999): »La gouvernance technocratique par consultation? Interrogation sur la première conférence de citoyen en France«, in: Les cahiers de la sécurité intérieure, Risque et démocratie. Savoirs, Pouvoirs, Participation. Vers un nouvel arbitrage?, S. 97-124.

Martinez, Éric (2004): Manuel du Comité Consultatif National d'Éthique. Bordeaux: Études Hospitalières Éditions.

Matern, Tobias (2008): »Gasunfall in Bhopal. Die Katastrophe, die nicht vergeht«, in: Sueddeutsche, 09.06.2008, http://www.sueddeutsche.de/politik/895/444633/ text/, 14.05.2009.

Meadows, Dennis/Meadows, Donella/Zahn, Erich et al. (1972): Die Grenzen des Wachstums: Bericht des Club of Rome zur Lage der Menschheit. Stuttgart: Dt. Verl.-Anst.

Mehta, Michael D. (2004): »From Biotechnology to Nanotechnology: What Can We Learn from Earlier Technologies? «, in: Bulletin of Science, Technology \& Society, 24, 1, S. 34-39. 
Membres du comité d'organisation du Colloque national (1982): »Un débat démocratique pour un grand enjeu «, in: Ministère de la Recherche et de la Technologie (Hg.): Recherche et Technologie, S. 13-32.

Memmi, Dominique (1996): Les gardiens du corps? Dix ans de magistère bioéthique. Paris: Éditions de l'École des Hautes Études en Sciences Sociales.

— (1999): »Que faire du corps aujourd'hui? La `bioéthiqueく, une autorité sociale en retrait«, in: Dominique Lecourt (Hg.): La bioéthique est-elle de mauvaise foi? Paris: PUF, S. 15-29.

— (2000): »Vers une confession laïque? La nouvelle administration étatique des corps «, in: Revue française de science politique, 50, 1, S. 3-19.

- (2003a): Faire vivre et laisser mourir. Le gouvernement contemporain de la naissance et de la mort. Paris: La Découverte.

- (2003b): »Gouverner par la parole: Le CCNE et la fabrication contemporaire du consensus «, in: Didier Sicard (Hg.): Travaux du Comité Consultatif National d'Éthique, S. 973-979.

- (2003c): »Governing through Speech. The New State Administration of Bodies«, in: Social Research, 70, 2, S. 645-658.

— (2004): »Administrer une matière sensible. Conduites raisonnables et pédagogie par corps autour de la naissance et de la mort«, in: Didier Fassin/Dominique Memmi (Hg.): Le gouvernement des corps. Paris: Éditions de l'École des Hautes Études en Sciences Sociales, S. 135-154.

— (2005): La »bio-politique déléguée«: L'institution serait-elle soluble dans le retour $\mathrm{du} »$ sujet $\ll$ ? Colloque international. Le politique vu avec Foucault. 6-8 janvier, Paris, IEP et CIR. Konferenzpapier, S. 1-16.

Mendel, Agata (1980): Les manipulations génétiques. Sciences ouvertes, Paris: Édition du Seuil.

Mermaz, Louis (1982): »Allocution de M. Louis Mermaz, Président de l'Assemblée nationale, réception à l'hôtel de Lassay, 14 janvier 1982«, in: Ministère de la Recherche et de la Technologie (Hg.): Recherche et Technologie, S. 75-77.

Michaud, Jean (1990): »Die französische Nationale Ethik-Kommission«, in: Georges Fülgraff/Annegret Falter (Hg.): Wissenschaft in der Verantwortung. Möglichkeiten der institutionellen Steuerung. Frankfurt a. M., New York: Campus, S. 178-188.

- (2002): Le Comité Consulatif National d'Éthique pour les sciences de la vie et de la santé. http://www.inserm.fr/ethique.cours.nsf, 16.05.2002.

— (2003): »Administration et Bioéthique«, in: Didier Sicard (Hg.): Travaux du Comité consultatif national d'Éthique, S. 824-827. 
Milliez, Paul (1976): »Therapeutic experimentation and respect towards man «, in: Charles Galpérine (Hg.): Biologie et devenir de l'homme, S. 487-489.

Ministère de la Recherche et de la Technologie (1982): Recherche et Technologie. Actes du Colloque national. 13-16 janvier 1982. Paris: La Documentation française.

Mitterrand, François (1982): »Discours de M. François Mitterrand, Président de la République française «, in: Ministère de la Recherche et de la Technologie (Hg.): Recherche et Technologie, S. 67-74.

— (1987): »Allocution prononcée par M. François Mitterrand, Président de la République française, à l'occasion de la mise en place du Comité consultatif national d'éthique pour les sciences de la vie et de la santé. Vendredi 2 décembre 1983«, in: Comités d'éthique à travers le monde. Recherches en cours 1986, S. 85-88.

Mnyusiwalla, Anisa/Abdallah, S. Daar/Singer, Peter A. (2003): »>Mind the gap science and ethics in nanotechnology«, in: Nanotechnology, 14, S. R9-R13.

Monsieur le Président de la République Giscard d'Estaing (1976): »Allocution«, in: Charles Galpérine (Hg.): Biologie et devenir de l'homme, S. 579-585.

Montbrial, Thierry de (1979): »Fondements de la politique économoique de Raymond Barre«, in: Commentaire, 6, S. 223-230. http://www.commentaire.fr/ pdf/articles/1979-2-006/1979-2-006_5p_0057_art1.pdf, 30.08.2014.

Moore, Alfred (2010): »Public Bioethics and Deliberative Democracy«, in: Political Studies, online in advance of print, S. 1-16.

Moulin, Madeleine (1990): Contrôler la science? La question des comités d'éthique. Bruxelles: De Boeck-Wesmael.

Muhle, Maria (2008): Eine Genealogie der Biopolitik. Zum Begriff des Lebens bei Foucault und Canguilhem. Bielefeld: transcript.

Murard, Lion/Zylberman, Patrick (2000): »Seeds for French Health Care: Did the Rockefeller Foundation Plant the Seeds between the Two World Wars«, in: Studies in History and Philosophy of Science Part C: Studies in History and Philosophy of Biological and Biomedical Sciences, 31, 3, S. 463-475.

MURS (2005): Qu'est ce que le M.U.R.S.? http://www.murs-france.asso.fr/Presen tation/C_present.html, 27.02.2005.

N.N. (1974): »World SSRS«, in: New Scientist, 3 October 1974, S. 45.

— (1975a): »Appel des scientifiques à propos du programme nucléaire français«, in: Impascience, 2, printemps-été, S. 22.

— (1975b): »Les enrages de Pasteur«, in: Impascience, 2, printemps-été, S. 46-49.

— (1975c): »Par delà le bien et le mal ou le >changement< dans l'idéologie de la science $\ll$, in: Impascience, 1, hiver, S. 14-18. 
— (1975d): »Tout nucléaire - tout politique«, in: Impascience, 2, printemps-été, S. 19.

- (2003): »Stay out of politics Charles, says Lord Sainsbury (the unelected billionaire Minister and major Labour donor)«, in: DailyMail, 17.06.2003, http://www.agbioworld.org/biotech-info/articles/biotech-art/princecharles.html, 21.05.2006.

- (2004): »Grenoble, occupation d'une grue du chantier Minatec «, in: cette semaine, o.S. http://cettesemaine.free.fr/spip/imprimersans.php3?id_article=31, 10.12.2007.

- (2010): Nano-débats pour maxi-arnaque. http://rebellyon.info/Nano-debatspour-maxi-arnaque.html, 16.02.2010.

Namir, Dominique (2006): »Nanotechnologies: Anticiper pour gérer les risques«, in: Centre d'analyse stratégique. La note de veille, $\mathrm{N}^{\circ} 27,25$ septembre 2006, S. 1-8. http://www.strategie.gouv.fr/IMG/pdf/noteveille27.pdf, 11.05.2008.

National Research Council (2006): A Matter of Size: Triennial Review of the National Nanotechnology Initiative. Washington: The National Academies Press.

Nelkin, Dorothy (1979): »The Social Responsibility of Scientists«, in: Annals of the New York Academy of Sciences, 334, S. 176-182.

— (1992): »Science, Technology, and Political Conflict: Analyzing the Issues«, in: Dorothy Nelkin (Hg.): Controversy. Politics of Technical Decision. Third Edition. Newbury Park u.a.: Sage Publications, S. ix-xxv.

Nordmann, Alfred/Rip, Arie (2009): »Mind the gap revisited «, in: Nature Nanotechnology, 4, 5, S. 271-274.

Nordmann, Alfred/Schwarz, Astrid (2010): »Lure of the >Yes<: The Seductive Power of Technoscience«, in: Mario Kaiser et al. (Hg.): Governing Future Technologies. Nanotechnology and the Rise of an Assessment Regime. Dordrecht u.a.: Springer, S. 255-277.

Nowotny, Helga (2003): »Dilemma of expertise. Democratising expertise and socially robust knowledge «, in: Science and Public Policy, 30, 3, S. 151-156.

Nowotny, Helga/Scott, Peter/Gibbons, Michael (2003): »Introduction. >Mode 2< Revisited: The New Production of Knowledge«, in: Minerva, 41, S. 179-194.

— (2008): Wissenschaft neu denken. Wissen und Öffentlichkeit in einem Zeitalter der Ungewißheit. Weilerswist: Velbrück Wissenschaft.

Nye, Robert A. (2006): »Médecins, éthique médicale et État en France 17891947«, in: Le Mouvement Social, 214, janvier-mars, S. 19-36.

OPECST (1998): Dossier de l'OPECST présentant la Conférence de Citoyens sur l'utilisation des organismes génétiquement modifiés (OGM) en agriculture et dans l'alimentation. Paris, http://www.agirpourlenvironnement.org/campagnes/ dossieropecst.htm, 1.11.2006. 
- (2003): Report on the evolution of the micro and nanoelectronics sector. Paris, http://www.assemblee-nationale.fr/13/rap-oecst/Rapport_nanotechnologie_UK. pdf, 10.05.2014.

- (2006a): Compte rendue de l'audition publique du 7 novembre. Les Nanotechnologies: Risques potentiels, enjeux éthiques. Paris, http://www.assembleenationale.fr/12/pdf/rap-off/i3658.pdf, 7.07.08.

- (2006b): Les nanotechnologies: risques potentiels, enjeux éthiques. Audition publique du mardi 7 novembre 2006. Synthèse. Paris, http://www.senat.fr/rap/ r06-208/r06-208-syn.pdf, 21.09.2014.

Opposition Grenobloise aux Nécrotechnologies (2006): 1000 personnes contre Minatec. La science sous escorte policière. http://ogn.ouvaton.org/docs/presse/ cp-OGN-20060601-manifestation.pdf, 07.07.2008.

Ordonnance $n^{\circ}$ 58-1373 (1958): Ordonnance du 30 décembre 1958 relative à la création de centres hospitaliers et universitaires, à la réforme de l'enseignement médical et au développement de la recherche médicale. http://www.snhu.com/ SNHU/Textes_officiels_files/ordonnance58.pdf, 10.07.2013.

Osborne, Thomas (2004): »Techniken und Subjekte: Von den >Governmentality Studies $<$ zu den >Studies of Governmentality««, in: Wolfgang Pircher/Ramón Reichert (Hg.): Governmentality Studies. Analysen liberal-demokratischer Gesellschaften im Anschluss an Michel Foucault. Münster: Lit Verlag, S. 33-42.

Ott, Ingrid/Könninger, Sabine/Zülsdorf, Torben et al. (2008): Public Image and Social Acceptance of Nanotechnologies (Part I). Challenge: Liberty\&Security, http://www.libertysecurity.org/IMG/pdf_NanoPublic08-1.pdf, 2.04.2008.

Ottomeyer-Hervieu, Christine (2006a): »Féminisme«, in: Bernhard Schmidt et al. (Hg.): Frankreich Lexikon, S. 382-386.

— (2006b): »Loi Veil«, in: Bernhard Schmidt et al. (Hg.): Frankreich Lexikon, S. 582-587.

Owen, Richard/Macnaghten, Phil/Stilgoe, Jack (2012): »Responsible Research and Innovation. From science in society to science for society, with society«, in: Science and Public Policy, 39, S. 751-760.

Papilloud, Christian (2010): Gouverner l'infiniment petit. Les nanotechnologies à Grenoble et Hambourg. Paris: L'Harmattan.

Parti Socialiste/Parti Communiste (1972): »Le programme commun de gouvernement. Parti socialiste - Parti communiste«, in: Bulletin Socialiste. Organe d'information du Parti Socialiste. Supplément de juin 1972, S. 1-16. 
Paschen, Herbert/Coenen, Christopher/Fleischer, Torsten et al. (2003): TA-Projekt Nanotechnologie. Endbericht. Büro für Technikfolgen-Abschätzung beim Deutschen Bundestag: Arbeitsbericht Nr. 92. Berlin, http://www.tab-beimbundestag.de/de/pdf/publikationen/berichte/TAB-Arbeitsbericht-ab092.pdf, 08.05.2014.

Patterson, Michel (1972): »French University Reform: Renaissance or Restauration?«, in: Comparative Education Review, 16, 2, S. 281-302.

Pavard, Bibia (2009): »Genre et militantisme dans le Mouvement pour la liberté de l'avortement et de la contraception. Pratiques des avortements (1973-1979)«, in: CLIO. Femme, Genre, Histoire, 29, S. 79-96.

Paxton, Robert O. (1982): Vichy France: old guard and new order. New York: Columbia University Press.

Perret, Horace/Bordogna Petriccione, Barbara/Kaufmann, Alain et al. (2004): »Disséminations expérimentales d'OGM en France et en Suisse: évolution du débat public«, in: Revue européenne des sciences sociales, XLII, 130, S. 121160.

Pestre, Dominique (2003): »Regimes of Knowledge Production in Society: Towards a More Political and Social Reading «, in: Minerva, 41, 3, S. 245-262.

- (2004a): Radically broadening the frame of our analyses, scientific intellectuals, the field of STS, and contemporary techno-science. Paper presented at the Conference: Shifting boundarys, 25.-26.06.2004, WZB, Berlin.

— (2004b): »Thirty years of science studies: knowledge, society and the political«, in: History and Technology, 20, 4, S. 351-369.

- (2008): »Challenges for the Democratic Management of Technoscience: Governance, Participation and the Political Today«, in: Science as Culture, 17, 2, S. 101-119.

- (2009a): A New Regime of Sciences in Society and Society in Science Today? A Reflection on the Last Three Decades. Discussion Paper SP III 2009-602. Tagung: Exzellenz durch Steuerung? Neue Herausforderungen für das deutsche und französische Wissenschaftssystem, 29.-30.11.07, WZB, Berlin, S. 47-69.

— (2009b): »Understanding the Forms of Government in Today's Liberal and Democratic Societies: An Introduction «, in: Minerva, 47, S. 243-260.

Petitjean, Patrick (1998): »La critique des sciences en France «, in: Alliage, 35-36, http://www.tribunes.com/tribune/alliage/35-36/06petit.htm\#1, 24.02.2011.

Picard, Jean-François (1999): La fondation Rockefeller et la recherche médicale. Science, histoire et société, Paris: PUF. 
- (2001): The Institut national d'hygiène and Public Health in France, 19401946. Paper presented at the Workshop European Health and the Second World War: Exile, Occupation and Post-War Reconstruction, Oxford (19-21 july). Histoire de la recherche médicale et en santé publique dans la France du XXe siècle, o.S. http://www.vjf.cnrs.fr/histrecmed/publications-electroniques/inhpicard.html, 10.02.2013.

— (2003): »Aux origines de l'Inserm: André Chevallier et l'Institut national d'hygiène«, in: Sciences Sociales et Santé, 21, 1, S. 5-25.

Pièces et Main d'Oeuvre (2006): Pièces et Main d'Oeuvre écrit aux députés. http://www.piecesetmaindoeuvre.com/spip.php?page=resume\&id_article=91, 27.04.2009.

Piel, Simon (2013): »Quel est le rôle du Comité consultatif national d'éthique?«, in: Le Monde, 08.02.2013.

Pollard, Miranda (1985): »Woman and the National Revolution«, in: Roderick Kedward/Roger Austin (Hg.): Vichy France and the Resistance. London, Sydney: Croom Helm, S. 36-47.

- (1993): Reign of Virtue. Mobilizing Gender in Vichy France. Chicago, London: University of Chicago Press.

— (2000): »Vichy and Abortion: Policing the Body and the New Moral Order in Everyday Life«, in: Sarah Fishman et al. (Hg.): France at War. Vichy and the Historians. Oxford, New York: Berg, S. 191-204.

PONS (2003): Wörterbuch für Schule und Studium Französisch-Deutsch. Studienausgabe. Stuttgart: Ernst Klett Verlag.

Pouillard, Jean (2005): »Historique du Conseil National de l'Ordre des Médecins (1845-1945)«, in: Histoires des Sciences Médicales, Tome XXXIX, 2, S. 213 223.

Pülzl, Helga/Wydra, Doris (2011): »Editorial - Public Policy Analysis und die interpretative Wende. Zur Erklärung von Politikwandel«, in: Österreichische Zeitschrift für Politikwissenschaft, 40. Jg., 4, S. 395-399.

Quéré, France (2003): »Un Comité National d'Éthique: Pourquoi? Comment?«, in: Didier Sicard (Hg.): Travaux du Comité Consultatif National d'Éthique, S. 4449.

Questiaux, Nicole (2003): »L'Éthique des Sciences de la vie, s'agit-il d'un champ normatif nouvaux?«, in: Didier Sicard (Hg.): Travaux du Comité Consultatif Naional d'Éthique, S. 23-32.

Quet, Mathieu (2009): Politique du Savoir. Une approche communicationnelle des rapports entre science, technologie et participation en France (1968-1983). Online publizierte Dissertation. Paris, S. 416. http://science.societe.free.fr/ documents/pdf/Quet_Politiques_du_savoir.pdf, 11.03.2011. 
— (2014): »Science to the people! (and experimental politics): Searching for the roots of participatory discourse in science and technology in the 1970s in France «, in: Public Understanding of Science, 23, 6, S. 628-645.

Quirke, Viviane/Gaudillière, Jean-Paul (2008): »The Era of Biomedicine: Science, Medicine, and Public Health in Britain and France after the Second World War «, in: Medical History, 52, 4, S. 441-452.

Radkau, Joachim (1988): »Hiroshima und Asilomar - Die Inszenierung des Diskurses über die Gentechnik vor dem Hintergrund der KernenergieKontroverse«, in: Geschichte und Gesellschaft. Zeitschrift für historische Sozialwissenschaft, 14, Zeitschriftenteil, S. 329-363.

Rainey, Steven/Goujon, Philippe (2011): Existing Solutions to the Ethical Governance Problem and Characterisation of their Limitations. S. 1-60. http://www.egais-project.eu/?q=node/8, 06.10.2012.

Rainey, Steven/Goujon, Philippe/Kurt, Aygen et al. (2012): New Guidelines Addressing the Problem of Integrating Ethics into Technical Development Projects. Deliverable 4.3, The Ethical GovernAnce of emergIng technologieSProject: funded by the European Commission under the Seventh Framework Programme (Science in Society). S. 1-161. http://www.egais-project.eu/ sites/default/files/deliverables/EGAIS_D4.3_29022012.pdf, 06.11.2012.

Randall, Adrian (1995): »Reinterpreting >Luddism $<$ resistance to new technology in the British Industrial Revolution «, in: Martin Bauer (Hg.): Resistance to new technology: nuclear power, information technology and biotechnology. Cambridge u.a.: Cambridge University Press, S. 57-79.

Reggiani, Andrés Horacio (2002): »Alexis Carrel, the Unknown: Eugenics and Population Research under Vichy«, in: French Historical Studies, 25, 2, S. 331-356.

Reichelt, Kathrin (2011): »Statut des Juifs«, in: Wolfgang Benz (Hg.): Handbuch des Antisemitismus. Judenfeindschaft in Geschichte und Gegenwart. Band 4. Ereignisse, Dekrete, Kontroversen. Berlin, Boston: De Gruyter, S. 396-399.

Rein, Martin/Schön, Donald (1993): »Reframing Policy Discourse«, in: Frank Fischer/John Forrester (Hg.): The Argumentative Turn in policy analysis and planning. Durham: Duke University Press, S. 145-166.

Reverby, Susan M. (2009): Examing Tuskegee: The Infamous Syphilis Study and Its Legacy. Chapel Hill, NC: The University of North Carolina Press.

Reynaud, Emmanuèle (1978): D'autres problèmes sociaux, d'autres formes d'organisations politique. Le cas de la santé. Rapport scientifique financée par le CORDES. Paris: Centre National de la Recherche Scientifique, Centre de Sociologie des Organsiations. 
Rickmann, Anahid S. (2002): »Rassenpflege im völkischen Staat«: Vom Verhältnis der Rassenhygiene zur nationalsozialistischen Politik. Online veröffentlichte Dissertation. Bonn, S. 373. http://hss.ulb.uni-bonn.de:90/2002/0091/0091.pdf, 08.08.2012.

Ridley, F. F. (1966): »French technocracy and comparative government«, in: Political Studies, XIV, 1, S. 34-52.

Rigal, Christelle S. (2008): »Neo-clinicians, Clinical Trials, and the Reorganization of Medical Research in Paris Hospitals after the Second World War: The Trajectory of Jean Bernard «, in: Medical History, 52, 4, S. 511-534.

Rose, Nikolas (1993): »Government, authority and expertise in advanced liberalism«, in: Economy and Society, 22, 2, S. 283-299.

— (1996): »Governing »advanced« liberal democracies«, in: Andrew Barry et al. (Hg.): Foucault and political reason. Liberalism, Neoliberalism and rationalities of government. Chicago, London: The University of Chicago Press, UCL Press, S. 37-64.

— (1999): Powers of freedom. Reframing political thought. Cambridge: Cambridge University Press.

Rose, Nikolas/Miller, Peter (1990): »Governing economic life «, in: Economy and Society, 19, 1, S. 1-31.

- (1992): »Political power beyond the state: Problematics of Government «, in: The British Journal of Sociology, 43, 2, S. 173-205.

Rosenthal, Paul-André (2003): L'intelligence démographique: sciences et politiques des populations en France (1930-1960). Paris: Odile Jacob.

Rouban, Luc (1988a): »Innovation, complexité et crise de l'État moderne«, in: Revue française de science politique, 38e année, 3, S. 325-347.

- (1988b): L'État et la science - la politique publique de la science et de la technologie. Paris: Éditions du CNRS.

Rucht, Dieter (1991): »Soziale Bewegungen, Gegenbewegungen und Staat: Der Abtreibungskonflikt in den USA, Frankreich und der Bundesrepublik Deutschland «, in: Forschungsjournal NSB, 2, S. 31-42.

— (1994): Modernisierung und neue soziale Bewegungen: Deutschland, Frankreich und USA im Vergleich. Frankfurt a. M.: Campus.

Saar, Martin (2008): »Understanding Genealogy. History, Power and the Self«, in: Journal of the Philosophy of History, 2, S. 295-314.

Salter, Brian/Jones, Mavis (2005): »Biobanks and bioethics: the politics of legitimation«, in: Journal of European Public Policy, 12, 4, S. 710-732.

Salter, Brian/Salter, Charlotte (2007): »Bioethics and the Global Moral Economy: The Cultural Politics of Human Embryonic Stem Cell Science«, in: Science, Technology \& Human Values, 32, 5, S. 554-581. 
Samerski, Silja (2011): »Genetic counseling and the fiction of choice: Taught selfdetermination as a new technique of social engineering «, in: Kathrin Braun (Hg.): Between Self-Determination and Social Technology, S. 95-126.

Sandler, Ronald (2014): »GM Food and Nanotechnology«, in: Bert Gordijn/ Anthony Mark Cutter (Hg.): In Pursuit of Nanoethics. Dordrecht u.a.: Springer, S. 39-57.

Saretzki, Thomas (2007): »The Policy Turn in German Political Science«, in: Frank Fischer et al. (Hg.): Handbook of Public Policy Ananlysis. Theory, Politics and Methods. Boca Raton u.a.: CRC Press, S. 587-602.

— (2009): »Aufklärung, Beteiligung und Kritik: Die >argumentative Wende < in der Policy-Analyse«, in: Klaus Schubert/Nils C. Bandelow (Hg.): Lehrbuch der Politikfeldanalyse 2.0. 2., vollständig überarbeitete und erweiterte Auflage. München: Oldenbourg Verlag, S. 431-456.

Schaper-Rinkel, Petra (2006): »Governance von Zukunftsversprechen: Zur politischen Ökonomie der Nanotechnologie«, in: PROKLA. Zeitschrift für kritische Sozialwissenschaft, 145, 36/4, S. 473-496.

Schlebusch, Cornelia (1994): Bevölkerungspolitik als Entwicklungsstrategie. Historisches und Aktuelles zu einem fragwürdigen Argument. Frankfurt a. M.: IKO-Verlag für Interkulturelle Kommunikation.

Schmidt, Bernhard (2006a): »Écologie«, in: Bernhard Schmidt et al. (Hg.): Frankreich Lexikon, S. 334-338.

— (2006b): »Les Verts«, in: Bernhard Schmidt et al. (Hg.): Frankreich Lexikon, S. 995-998.

Schmidt, Bernhard/Doll, Jürgen/Fekl, Walther et al. (2006): Frankreich Lexikon. Schlüsselbegriffe zu Wirtschaft, Gesellschaft, Politik, Geschichte, Kultur, Presse- und Bildungswesen. 2. überarbeitete und erweiterte Auflage. Berlin: Erich Schmidt Verlag.

Schmidt, Bernhard/Fekl, Walther (2006): »Cinquième République«, in: Bernhard Schmidt et al. (Hg.): Frankreich Lexikon, S. 183-193.

Schneider, William H. (1982): »Towards the Improvement of the Human Race. The History of Eugenics in France«, in: The Journal of Modern History, 54, 2, S. 268-291.

— (1990a): »The Eugenics Movement in France, 1890-1940«, in: Mark B. Adams (Hg.): The Wellborn Science. Eugenics in Germany, France, Brazil, and Russia. New York, Oxford: Oxford University Press, S. 69-109.

- (1990b): Quality and quantity: the quest for biological regeneration in twentieth-century France. Cambridge: Cambridge University Press.

- (2003): »War, Philantropy, and the National Institute of Hygiene in France «, in: Minerva, 41, S. 1-23. 
Schön, Donald/Rein, Martin (1994): Frame Reflection. Toward the Resolution of Intractable Policy Controversies. New York: Basic Books.

Schubert, Klaus/Bandelow, Nils C. (2009): »Politikfeldanalyse: Dimensionen und Fragestellungen«, in: Klaus Schubert/Nils C. Bandelow (Hg.): Lehrbuch der Politikfeldanalyse 2.0. 2., vollständig überarbeitete und erweiterte Auflage. München: Oldenbourg Verlag, S. 1-22.

Schudson, Michael (2004): »Notes on Scandal and the Watergate Legacy«, in: American Behavioral Scientist, 47, 9, S. 1231-1238.

Schultz, Susanne (1996): »Selbstbestimmtes Technopatriarchat? Sackgassen einer immanenten feministischen Kritik an den neuen Reproduktionstechnologien«, in: Susanne Schultz et al. (Hg.): Geld.Beat.Synthetik. Abwerten bio/technologischer Annahmen. Berlin: Edition ID-Archiv, S. 76-95.

Schulz, Kristina (2002): Der lange Atem der Provokation: Die Frauenbewegung in der Bundesrepublik und in Frankreich 1968-1976. Frankfurt a. M., New York: Campus.

Schummer, Joachim (2014): »On the Novelty of Nanotechnology: A Philosophical Essay«, in: Bert Gordijn/Anthony Mark Cutter (Hg.): In Pursuit of Nanoethics. Dordrecht u.a.: Springer, S. 15-29.

Selin, Cynthia (2007): »Expectations and the Emergence of Nanotechnology«, in: Science, Technology \& Human Values, 32, 2, S. 196-220.

Sénat (2006): Présentation de l'Office parlementaire d'évaluation des choix scientifique et technologiques. http://www.senat.fr/opecst/presentation.html, 30.06.2004.

Sennelart, Michel (2004a): Michel Foucault. Geschichte der Gouvernementalität I. Sicherheit, Territorium, Bevölkerung. Vorlesung am Collège de France 19771978. Frankfurt a. M.: Suhrkamp.

— (2004b): Michel Foucault. Geschichte der Gouvernementalität II. Die Geburt der Biopolitik. Vorlesung am Collège de France 1978-1979. Frankfurt a. M.: Suhrkamp.

Shew, Ashley (2008): »Nanotech's History: An Interesting, Interdisciplinary, Ideological Split«, in: Bulletin of Science, Technology \& Society, 28, 5, S. 390399.

Shiva, Vandana (1991): »The Green Revolution in the Punjab«, in: The Ecologist, 21, 2, S. 57-60.

Sicard, Didier (2001): Erfahrungsberichte. Ethikräte in Frankreich und der Europäischen Union. Berliner Dialog zu Biomedizin. März 2001, Berlin. S. 10-15. http://www.fes-landesbuero-berlin.de/doku/01_02.pdf, 12.03.2004.

Sicard, Didier (2003): Travaux du Comité Consultatif National d'Éthique. 20e anniversaire. Paris: PUF. 
Simonin, Anne (1997): »Le Comité Médical de la Résistance: un succès différé«, in: Le Mouvement Social, 180, Jul.-Sep., S. 159-178.

Simples Citoyens (2003): Nanotechnologies/Maxiservitude. Des contributions grenobloises à l'automatisation du cheptel humain. http://www.piecesetmain doeuvre.com/IMG/pdf/Nanotechnologies-maxiservitude.pdf, 22.07.2006.

Singer, Maxine/Soll, Dieter (1973): »Guidelines for DNA Hybrid Molecules«, in: Science, 4105, 21 September 1973, S. 1114.

Sismondo, Sergio (2008): »Science and Technology Studies and an Engaged Program«, in: Edward J. Hackett et al. (Hg.): The Handbook of Science and Technology Studies. Cambridge, London: The MIT Press, S. 13-31.

Stevens, Tina M. L. (2000): Bioethics in America. Origins and cultural politics. Baltimore, London: The John Hopkins University Press.

Stilgoe, Jack/Lock, Simon J./Wilsdon, James (2014): »Why should we promote public engagement with science $\ll$, in: Public Understanding of Science, 23, S. 4-15.

Stirling, Andy (2008): »>Opening Up< and >Closing Down $<$ Power, Participation, and Pluralism in the Social Appraisal of Technology«, in: Science, Technology \& Human Values, 33, 2, S. 262-294.

Strasser, Bruno J. (2002): »Institutionalizing molecular biology in post-war Europe: a comparative study«, in: Studies in History and Philosophy of Science Part C: Studies in History and Philosophy of Biological and Biomedical Sciences, 33, S. 515-546.

Taguieff, Pierre-André (1991): »L'introduction de l'eugénisme en France: du mot à l'idée«, in: Mots, Mars, 26, S. 23-45.

- (1994): »Eugénisme ou décadence? L'exception française«, in: Ethnologie française, 24, 1 (Penser l'hérédité), S. 81-103.

Testart, Jacques (2006): »Der Glaube im Labor. Der Befreiung der Menschheit kann die Wissenschaft nur dienen, wenn sie sich auf ihre emanzipative Kraft besinnt«, in: Le Monde diplomatique, http://www.monde-diplomatique.de/ pm/2006/02/10.mondeText.artikel,a0043.idx,11, 10.05.2014.

Testart, Jacques/Serres, Michel (1988): Das transparente Ei. Frankfurt a. M.: Schweitzer Verlag.

Thalmann, Rita/Dhoquois, Régine (1995): »La lutte pour le droit à l'IVG«, in: Les cahiers du CEDREF, 4-5, S. 97-102. http://cedref.revues.org/300\#text, 5.03.2013.

The Hastings Center (1973): The Hasting Center Studies. Institute of Society, Ethics and the Life Sciences. 1. 
The Rockefeller Foundation (1915): The Rockefeller Foundation. Annual Report. 1913-14. New York, S. 1-450. https://www.rockefellerfoundation.org/app/ uploads/RF-Annual-Report-1915.pdf, 22.05.2015.

— (1933): The Rockefeller Foundation. Annual Report 1933. New York, S. 1-477. https://www.rockefellerfoundation.org/app/uploads/Annual-Report-1933.pdf, 22.05.2015.

— (1940): The Rockefeller Foundation. Annual Report 1940. New York, S. 1-473. https://www.rockefellerfoundation.org/app/uploads/Annual-Report-1940.pdf, 22.05.2015.

— (1941): The Rockefeller Foundation. Annual Report 1941. New York, S. 1-425. https://www.rockefellerfoundation.org/app/uploads/Annual-Report-1941.pdf, 22.05.2015.

— (1942): The Rockefeller Foundation. Annual Report 1942. New York, S. 1-336. https://www.rockefellerfoundation.org/app/uploads/Annual-Report-1942.pdf, 22.05.2015.

— (1944): The Rockefeller Foundation. Annual Report 1944. New York, S. 1-334. http://www.rockefellerfoundation.org/app/uploads/Annual-Report-1944.pdf, 22.05.2015.

The Royal Society \& The Royal Academy of Engineering (2004): Nanoscience and nanotechnologies: opportunities and uncertainties. Science Policy Section, London, http://www.nanotec.org.uk/report/Nano\%20report\%202004\%20fin. pdf, 13.08.2008.

Théry, Jean-François (2001): »Aux origines de la Loi de 1982«, in: Jean-François Théry/Rémi Barré (Hg.): La loi sur la recherche de 1982. Origines, bilan et perspectives du »modèle français«. Paris: Edition Quæ, S. 9-36.

Thomasson, Sebastien (2006): Nous n'avons pas peur, nous sommes en colère. http://www.piecesetmaindoeuvre.com/IMG/pdf/Nous_n_avons_pas_peur.pdf, 14.05.2014.

Toinet, Marie-France (1973): »La liberté de la presse aux États-Unis: des documents du Pentagone au scandale du Watergate «, in: Revue française de science politique, 5, S. 1020-1045.

Tolmein, Oliver (2013): Contergan, Schadensersatz und Selbstbestimmungsrecht. http://blog.menschenundrechte.de/tag/contergan/, 6.03.2013.

Tomellini, Renzo (2004): International dialogue on responsible research and development of nanotechnology. 17/18 June. Alexandria (VA), USA, ftp://ftp.cordis.europa.eu/pub/nanotechnology/docs/alexandria062004.pdf, 09.06.2010. 
Topçu, Sezin (2007): »Les physiciens dans le mouvement antinucléaire: entre science, expertise et politique«, in: Cahiers d'histoire. Revue d'histoire critique, 102, S. 1-16.

Tournay, Virginie (2002): »Le contrôle institutionnel des facteurs d'incertitude dans la gestion du corps biologique: Le Comité Consultatif National d'Éthique, un dispositif résolutoire de l'incertitude écartelé entre les faits et les valeurs", in: Quaderni, 48, Automne, S. 101-110.

— (2003): »Le gouvernement contemporain des corps (1965-?): un nouveau type d'administration du vivant et des populations«, in: Quaderni, Hiver 2002/2003, 49, S. 31-39.

Tournès, Ludovic (2008): »La fondation Rockefeller et la construction d'une politique des sciences sociales en France (1918-1940)«, in: Annales: Histoire, Sciences Sociales., 6, 63, S. 1371-1402.

Turda, Marius (2010): Modernism and Eugenics. Modernism and... Houndmille u.a.: Palgrave Macmillan.

Valadier, Paul (1983): »Comité d'éthique< et technocratie«, in: Le Monde, 14.10.1983.

Vatter, Christoph (2009): Gedächtnismedium Film. Holocaust und Kollaboration in deutschen und französischen Spielfilmen nach 1945. Würzburg: Verlag Königshausen \& Neumann.

Veil, Simone (1974): Inauguration des nouveaux locaux de l'Inserm. Xème anniversaire de l'Inserm. Le 13 janvier 1974, Paris. http://infodoc.inserm.fr/ histoire/Histoire.nsf/8c74cc5c9c8f799bc1256c8b004c814e/2a6bb8be9fbe 7dfe80256ce200410baa?OpenDocument, 17.06.2011.

— (2007): Une vie. Paris: Édition Stock.

- (2009): Und dennoch leben: die Autobiographie der großen Europäerin. Berlin: Aufbau-Verlag.

Vinck, Dominique/Gallice, Perrine/Jouvenet, Morgan et al. (2009): Dynamique technologique controversée et débat démocratique: le cas des micro- et nanotechnologies. http://halshs.archives-ouvertes.fr/docs/00/36/00/74/PDF/060718_ ChapVinck_GOUJON_V1.pdf, 21.02.2010.

von Oppeln, Sabine; (1989): Die Linke im Kernenergiekonflikt. Deutschland und Frankreich im Vergleich. Frankfurt a. M., New York: Campus.

Voncken (1949): »La formation morale du médecin au point de vue international«, in: Académie Nationale de Médecine (Hg.): Bulletin de l'Académie Nationale de Médecine. 113e année, 3e série, tome 133. Séances des 15 et 22 mars 1949. Paris: Masson et Cie, S. 235-239. 
Wächter, Matthias (2006): Der Mythos des Gaullismus: Heldenkult, Geschichtspolitik und Ideologie 1940-1958. Moderne Zeit, Band 14. Göttingen: Wallstein Verlag.

Wade, Nicholas (1975): »Genetics: Conference Sets Strict Controls to Replace Moratorium «, in: Science, 187, 4180, S. 931-935.

Walter, Klaus Peter (2000): »Schwierige Vergangenheitsbewältigung. Die Okkupation Frankreichs (1940-1944) im Spiegel von Kinofilm und Roman«, in: Deutsch-Französisches Intitut et al. (Hg.): Frankreich-Jahrbuch 2000. Politik, Wirtschaft, Gesellschaft, Geschichte, Kultur. Opladen: Leske \& Budrich, S. 129-144.

Weber, Hans A. (1974): »Manipulation of the Genetic Substance: Imagination and Reality. Research Called in Question. Conference report on behalf of the Swiss Society for Cell and Molecular Biology«, in: Gottlieb Duttweiler Institute for Economic and Social Studies (Hg.): Genetic Engineering. Its Applications and Limitations. Proceedings of the Symposium held in Davos October 10-12, 1974. Rüschlikon Zürich: S. 147-152.

Wehling, Peter (2012): »From invited to uninvited paticipation (and back?): rethinking civil society engagement in technology assessment and development «, in: Poiesis Prax, 9, S. 43-60.

Weigel, Björn (2011): »Vélodrome d'Hiver Razzia (1942)«, in: Wolfgang Benz (Hg.): Handbuch des Antisemitismus. Judenfeindschaft in Geschichte und Gegenwart. Band 4. Ereignisse, Dekrete, Kontroversen. Berlin, Boston: De Gruyter, S. 413-415.

Weindling, Paul (1993): »Public health and political stabilisation: The Rockefeller Foundation in Central and Eastern Europe between the two world wars «, in: Minerva, 31, 3, S. 253-267.

- (1997): »Philantropy and World Health: The Rockefeller Foundation and the League of Nations Health Organisation«, in: Minerva, 35, S. 269-281.

Weiner, Charles (2001): »Drawing the Line in Genetic Engineering: Selfregulation and public participation«, in: Perspectives in Biology and Medicine, 44, 2, S. 208-220.

Weingart, Peter/Kroll, Jürgen/Bayertz, Kurt (1996): Rasse, Blut und Gene. Geschichte der Eugenik und Rassenhygiene in Deutschland. Frankfurt a. M.: Suhrkamp.

Weisz, George (1990): »The origins of medical ethics in France: The international congress of >Morale Medicale< of 1955«, in: George Weisz (Hg.): Social science perspectives on medical ethics. Dordrecht: Kluwer, S. 145-161. 
Wheeler, Harvey (1974): »The Challenge of Davos«, in: Gottlieb Duttweiler Institute for Economic and Social Studies (Hg.): Genetic Engineering. Its Applications and Limitations. Proceedings of the Symposium held in Davos October 10-12, 1974. Rüschlikon Zürich: S. 153-163.

Wieviorka, Michel (1982): »Der Bruch«, in: Alain Touraine (Hg.): Die antinukleare Prophetie. Zukunftsentwürfe einer sozialen Bewegung. Frankfurt a. M.: Campus, S. 55-102.

Wieviorka, Olivier (1989): »La génération de la résistance«, in: Vingième Siècle. Revue d'histoire, 22, avril-juin, S. 111-116.

Wilson, R. R. (1996): »Hiroshima: The Scientists' Social and Political Reaction«, in: Proceedings of the American Philosophical Society, 140, 3, S. 350-357.

WMA (2013): WMA Declaration of Helsinki - Ethical Principles for Medical Research Involving Human Subjects. http://www.wma.net/en/30publications/ 10policies/b3/index.html.pdf?print-media-type \&footer-right=[page]/[toPage], 23.07.2014.

Woyke, Andreas (2007): »>Nanotechnologie< als neue >Schlüsseltechnologie <? Versuch eines historischen und systematischen Vergleichs mit anderen Technologien«, in: Journal for General Philosophy of Science, 38, S. 329-345.

Wright, Susan (1994): Molecular Politics. Developing American and British Regulatory Policy for Genetic Engineering, 1972-1982. Chicago, London: University of Chicago Press.

Wullweber, Joscha (2006): »Der Mythos Nanotechnologie. Die Entstehung und Durchsetzung einer neuen Inwertsetzungstechnologie«, in: Peripherie, 101/102, 26, S. 99-118.

— (2008a): »Nanotechnologie: Innovationsprojekt ohne Widerspruch?«, in: Genethischer Informationsdienst, 191, Dezember, (online o.S.). http://www.genethisches-netzwerk.de/gid/191/wullweber/nanotechnologie-innovationsprojektohne-widerspruch, 10.03.2009.

— (2008b): »Nanotechnology - An Empty Signifier à venir? A Delineation of a Techno-socio-economical Innovation Strategy«, in: Science, Technology \& Innovation Studies, 4, 1, S. 27-45.

Wynne, Brian (2001): »Creating Public Alienation: Expert Cultures of Risk and Ethics on GMOs«, in: Sciences as Culture, 10, 4, S. 445-481. 


\section{Science Studies}

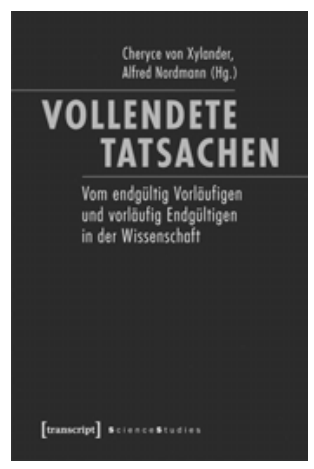

Cheryce von XYlander, Alfred Nordmann (Hg.)

Vollendete Tatsachen

Vom endgültig Vorläufigen

und vorläufig Endgültigen in der Wissenschaft

Dezember 20I6, ca. 320 Seiten, kart., ca. 34,99 €, ISBN 978-3-8376-2542-4

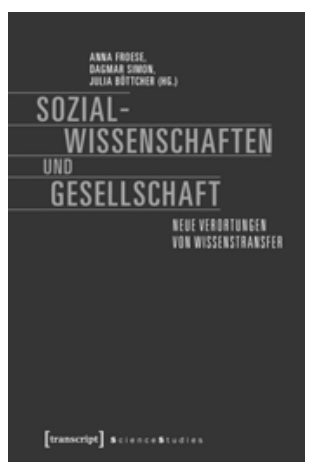

Anna Froese, Dagmar Simon, Julia Böttcher (Hg.) Sozialwissenschaften und Gesellschaft

Neue Verortungen von Wissenstransfer

Februar 2016, 342 Seiten, kart., 39,99€, ISBN 978-3-8376-3402-0

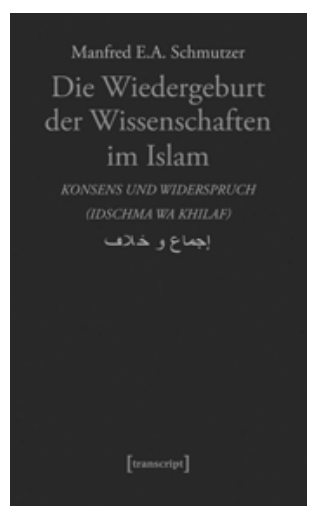

Manfred E.A. Schmutzer

Die Wiedergeburt der Wissenschaften im Islam Konsens und Widerspruch (idschma wa khilaf)

20I5, 544 Seiten, Hardcover, 49,99 €, ISBN 978-3-8376-3I96-8

Leseproben, weitere Informationen und Bestellmöglichkeiten finden Sie unter www.transcript-verlag.de 


\section{Science Studies}

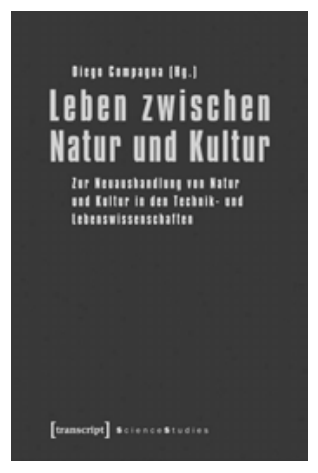

Diego Compagna (Hg.)

Leben zwischen Natur und Kultur

Zur Neuaushandlung von Natur und Kultur

in den Technik- und Lebenswissenschaften

2015, 272 Seiten, kart., 33,99€,

ISBN 978-3-8376-2009-2

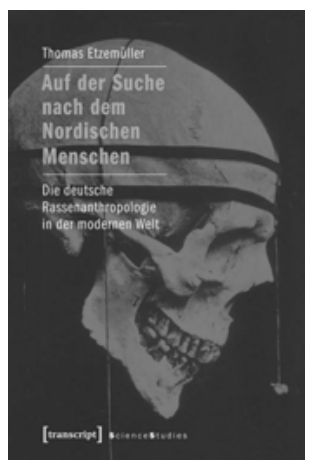

Thomas Etzemüller

Auf der Suche nach dem Nordischen Menschen

Die deutsche Rassenanthropologie

in der modernen Welt

20I5, 294 Seiten,

kart., zahlr. z.T. farb. Abb., 29,99 €,

ISBN 978-3-8376-3I83-8

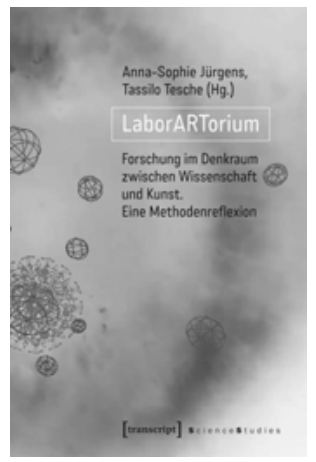

Anna-Sophie Jürgens, Tassilo Tesche (Hg.)

LaborARTorium

Forschung im Denkraum

zwischen Wissenschaft und Kunst.

Eine Methodenreflexion

2015, 336 Seiten, kart., zahlr. Abb., 34,99€,

ISBN 978-3-8376-2969-9

Leseproben, weitere Informationen und Bestellmöglichkeiten finden Sie unter www.transcript-verlag.de 


\section{Science Studies}

Christian Dieckhoff,

Anna Leuschner,

Frederike Neuber (Hg.)

Die Energiewende und ihre Modelle

Was uns Energieszenarien

sagen können - und was nicht

November 2016, ca. I60 Seiten,

kart., ca. 29,99€,

ISBN 978-3-8376-3I7I-5

Joanna Pfaff-Czarnecka (Hg.)

Das soziale Leben der Universität

Studentischer Alltag

zwischen Selbstfindung

und Fremdbestimmung

Oktober 20I6, ca. 270 Seiten,

kart., ca. $34,99 €$,

ISBN 978-3-8376-3348-I

Dania Achermann

Institutionelle Identität im Wandel

Zur Geschichte des Instituts

für Physik der Atmosphäre

in Oberpfaffenhofen

Februar 20I6, 3I2 Seiten,

kart., zahlr. Abb., 39,99 €,

ISBN 978-3-8376-3I42-5

\section{Christian Kehrt}

Mit Molekülen spielen

Wissenschaftskulturen

der Nanotechnologie

zwischen Politik und Medien

2015, 276 Seiten,

kart., zahlr. z.T. farb. Abb., 39,99€,

ISBN 978-3-8376-3202-6

Hildegard Matthies,

Dagmar Simon, Marc Torka (Hg.)

Die Responsivität der Wissenschaft

Wissenschaftliches Handeln

in Zeiten neuer Wissenschaftspolitik

2015, 268 Seiten, kart., 29,99€,

ISBN 978-3-8376-3298-9
Andreas Beaugrand (Hg.)

Bildung anführen

Über Hochschulmanagement

nach der Bologna-Reform

2015, 362 Seiten, kart., 39,99€,

ISBN 978-3-8376-3195-I

Christian Dieckhoff

Modellierte Zukunft

Energieszenarien

in der wissenschaftlichen Politikberatung

20I5, 284 Seiten, kart., 34,99€,

ISBN 978-3-8376-3097-8

Fabian Karsch

Medizin zwischen Markt und Moral

Zur Kommerzialisierung

ärztlicher Handlungsfelder

2015, 256 Seiten,

kart., zahlr. Abb., $32,99 €$,

ISBN 978-3-8376-2890-6

Matthias Groß

Experimentelles Nichtwissen

Umweltinnovationen und die Grenzen

sozial-ökologischer Resilienz

20I4, 202 Seiten, kart., 29,99€,

ISBN 978-3-8376-2855-5

Gert Dressel, Wilhelm Berger,

Katharina Heimerl, Verena Winiwarter (Hg.)

Interdisziplinär und

transdisziplinär forschen

Praktiken und Methoden

20I4, 366 Seiten, kart., 34,99 €,

ISBN 978-3-8376-2484-7

Oliver Ibert,

Felix C. Müller, Axel Stein

Produktive Differenzen

Eine dynamische Netzwerkanalyse

von Innovationsprozessen

20I4, 234 Seiten, kart., 32,99 €,

ISBN 978-3-8376-2699-5 
